ORP-56287

Revision 0

\title{
Final Report- Testing of Optimized Bubbler Configuration for HLW Melter VSL-13R2950-1, Rev. 0, dated 6/12/2013
}

Prepared for the U.S. Department of Energy

Assistant Secretary for Environmental Management

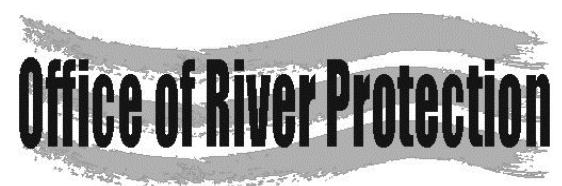

P.O. Box 450

Richland, Washington 99352 
ORP-56287

Revision 0

\section{Final Report- Testing of Optimized Bubbler Configuration for HLW Melter VSL-13R2950-1, Rev. 0, dated 6/12/2013}

A. A. Kruger

Department of Energy - Office of River Protection

I. L. Pegg

The Catholic University of America

R. A. Callow

The Catholic University of America
I. Joseph

The Catholic University of America

K. S. Matlack

The Catholic University of America

W. K. Kot

The Catholic University of America

Date Published

June 2013

Prepared for the U.S. Department of Energy

Assistant Secretary for Environmental Management

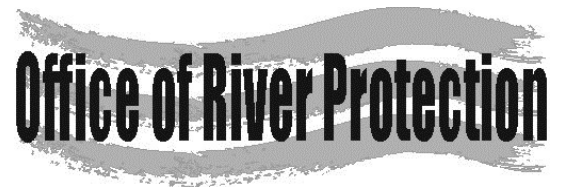

P.O. Box 450

Richland, Washington 99352

\section{APPROVED}

By Julia Raymer at 1:37 pm, Nov 13, 2013

Release Approval

Date 
ORP-56287

Revision 0

TRADEMARK DISCLAIMER

Reference herein to any specific commercial product, process, or service by tradename, trademark, manufacturer, or otherwise, does not necessarily constitute or imply its endorsement, recommendation, or favoring by the United States Government or any agency thereof or its contractors or subcontractors.

This report has been reproduced from the best available copy.

Printed in the United States of America 
VSL-13R2950-1

\title{
Final Report
}

Testing of Optimized Bubbler Configuration for HLW Melter

\author{
prepared by
}

\begin{abstract}
Keith S. Matlack, Wing K. Kot, Richard A. Callow, Innocent Joseph and Ian L. Pegg
\end{abstract}

\author{
Vitreous State Laboratory \\ The Catholic University of America \\ Washington, DC 20064 \\ and \\ EnergySolutions, Federal EPC Inc. \\ Columbia, MD 21046 \\ for \\ Department of Energy \\ Office of River Protection \\ Richland, WA
}

May 31, 2013

Rev. 0, 6/12/13 
Document Title:

\section{Document Number}

and Revision:

Issue Date:

Performing Organization: Vitreous State Laboratory, The Catholic University of America

Test Plan:
Testing of Optimized Bubbler Configuration for HLW Melter

VSL-13R2950-1, Rev. 0

$6 / 12 / 13$
Testing of Optimized Bubbler Configuration for HLW Melter,

This report describes the results of testing specified by the above Test Plan. The work was performed in compliance with the quality assurance requirements specified in the Test Plan. Results required by the Test Plan are reported. The test results and this report have been reviewed for correctness, technical adequacy, completeness, and accuracy. VSL-12T2950-1, Rev. 0

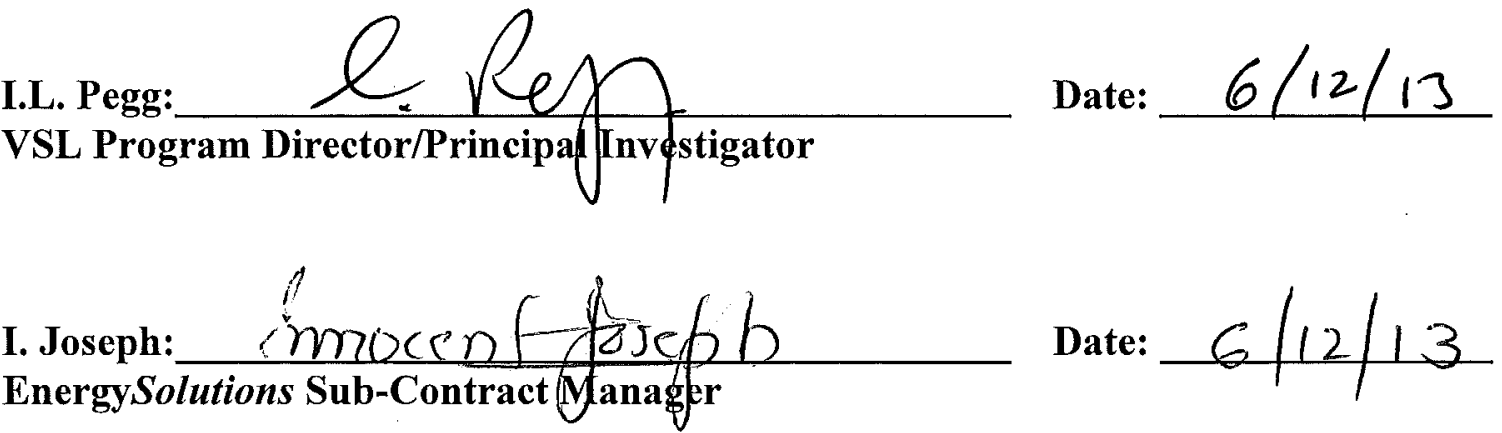


The Catholic University of America Vitreous State Laboratory
Testing of Optimized Bubbler Configuration for HLW Melter Final Report, VSL-13R2950-1, Rev. 0

\section{TABLE OF CONTENTS}

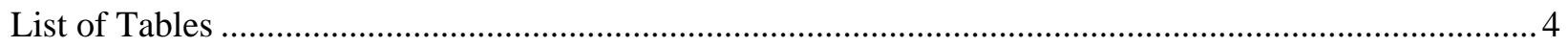

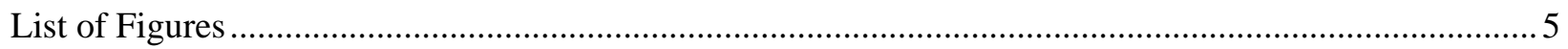

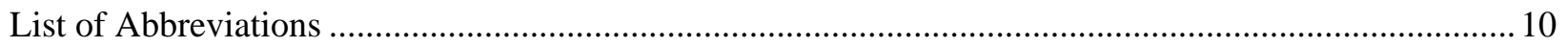

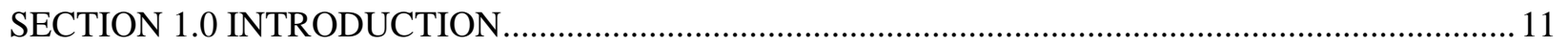

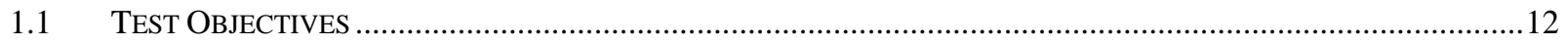

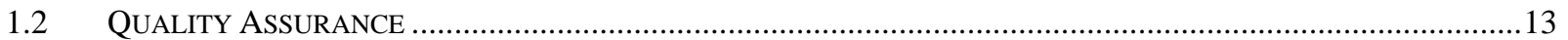

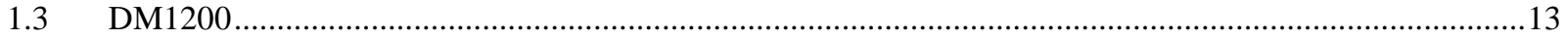

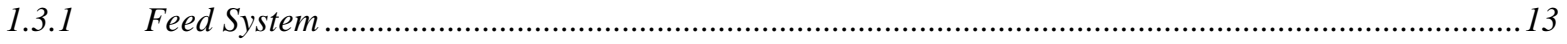

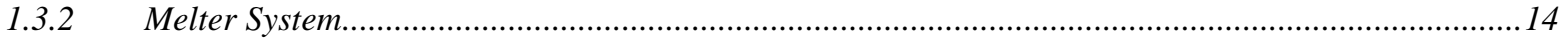

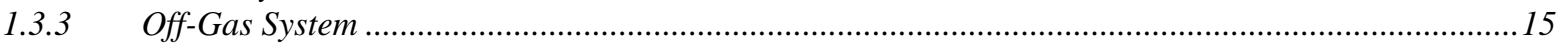

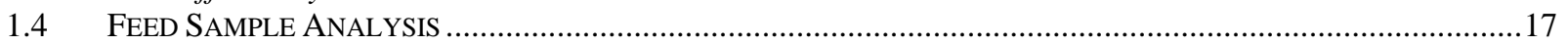

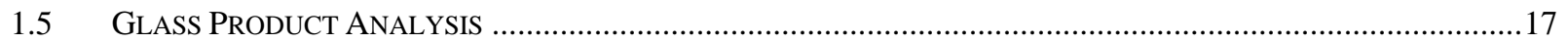

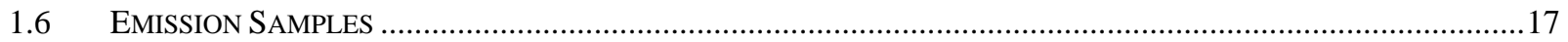

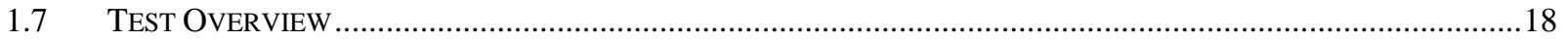

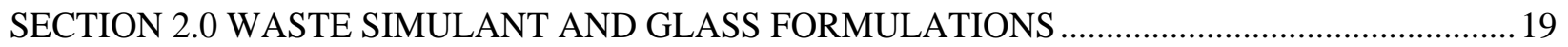

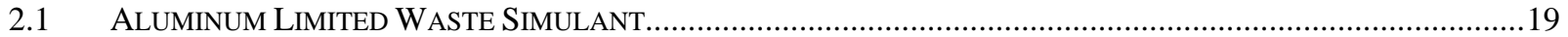

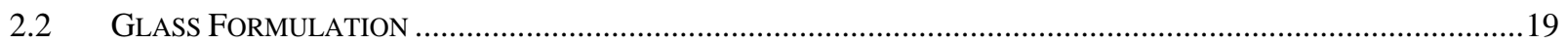

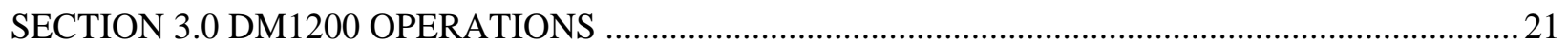

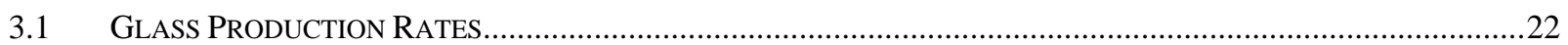

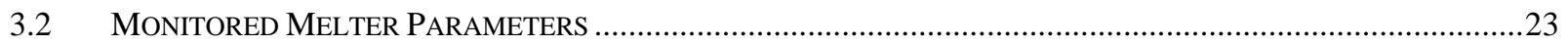

SECTION 4.0 DM1200 OFF-GAS SYSTEM PERFORMANCE .....................................................26

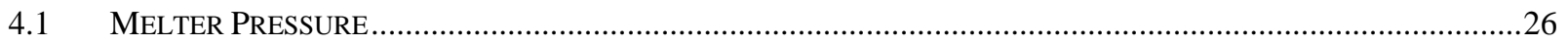

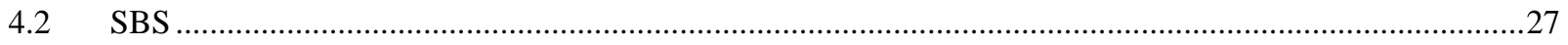

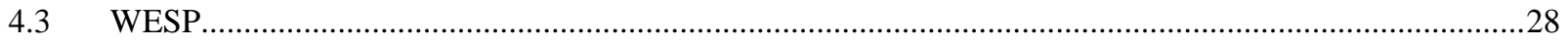

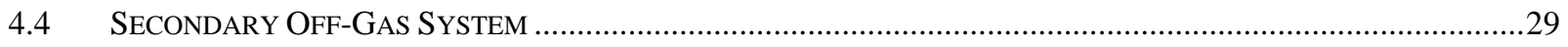

SECTION 5.0 FEED SAMPLE AND GLASS PRODUCT ANALYSIS ............................................31

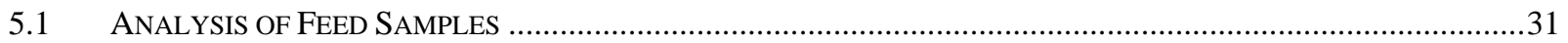

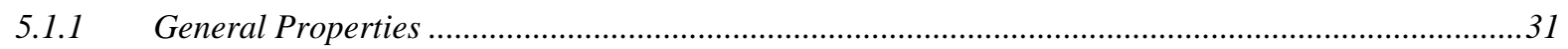

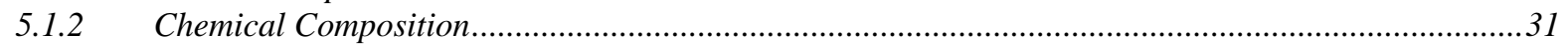

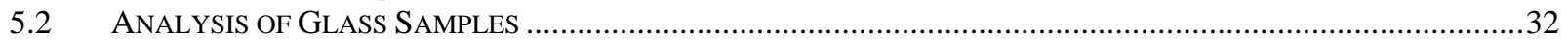

SECTION 6.0 MONITORED OFF-GAS EMISSIONS …..................................................................... 33

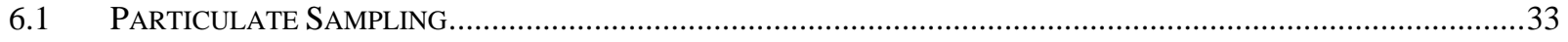

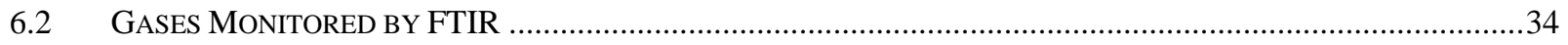

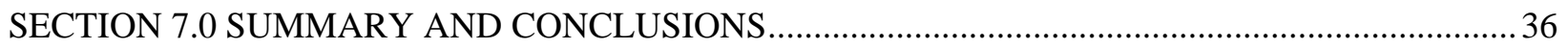

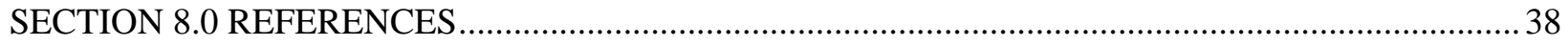


The Catholic University of America Vitreous State Laboratory
Testing of Optimized Bubbler Configuration for HLW Melter Final Report, VSL-13R2950-1, Rev. 0

\section{List of Tables}

Page No.

Table 2.1 Oxide Compositions of Limiting Waste Streams.

Table 2.2 Compositions of the Al-Limited Waste (Oxide Basis) and the HLW Waste

$\mathrm{T}-1$

$\mathrm{T}-2$

Simulant to Produce $100 \mathrm{~kg}$ of Waste Oxides (20 wt\% suspended solids).

Table 2.3 Composition and Properties of Aluminum Limited Waste and Glass Formulation $\quad$ T-3 HWI-Al-19 with 45\% Waste Loading (wt\%).

Table 2.4 Composition of Melter Feed to Produce $100 \mathrm{~kg}$ of Target Glass HWI-Al-19 T-4 (Target Glass Yield $=500 \mathrm{~g} / \mathrm{L}$ Feed) from the Al-Limited Waste Simulant

Table 3.1 Summary of Test Conditions and Results.

$\mathrm{T}-5$

Table 3.2 Summary of Operational Events. $\quad$ T-6

$\begin{array}{lll}\text { Table } 3.3 & \text { Cold Cap Observations. } & \text { T-47 }\end{array}$

Table 3.4 DM1200 Melter System Measured Parameters. $\quad$ T-72

Table 4.1 Measured DM1200 Off-Gas System Parameters. $\quad$ T-76

$\begin{array}{lll}\text { Table } 4.2 & \text { Off-Gas Fluid Volumes. } & \text { T-78 }\end{array}$

Table 5.1 Measured Feed Sample Properties. $\quad$ T-79

Table 5.2 XRF Analyzed Compositions for As-Received Vitrified Melter Feed Samples; $\quad$ T-80 HWI-Al-19 Composition (wt\%).

Table 5.3 XRF Analyzed Compositions of Vitrified Melter Feed Sampled During DM1200 T T-82 Tests (wt\%).

Table 5.4 Listing of Glass and Discharged Masses during DM1200 Tests. $\quad$ T-84

Table 5.5 XRF Analyzed Compositions for Glass Discharged During DM1200 Tests (wt\%). $\quad$ T-89

Table 6.1 Results from Melter Off-Gas Emission Samples. $\quad$ T-93

Table 6.2 Concentrations [ppmv] of Selected Species in Off-Gas Measured by FTIR $\quad$ T-95 Spectroscopy, Test 1.

Table 6.3 Concentrations [ppmv] of Selected Species in Off-Gas Measured by FTIR T-96 Spectroscopy, Test 2.

Table 6.4 Concentrations [ppmv] of Selected Species in Off-Gas Measured by FTIR $\quad$ T-97 Spectroscopy, Test 3.

Table 6.5 Concentrations [ppmv] of Selected Species in Off-Gas Measured by FTIR $\quad$ T-98 Spectroscopy, Test 4. 
The Catholic University of America Vitreous State Laboratory
Testing of Optimized Bubbler Configuration for HLW Melter Final Report, VSL-13R2950-1, Rev. 0

\section{List of Figures}

Figure 1.1 Lid diagram for WTP HLW Melter

Page No.

Figure 1.2

Figure 1.3

Figure 1.4

Figure 1.5

Figure 1.6

Figure 1.7

Figure 1.8

Figure 1.9

Figure 1.10

Figure 1.11

Figure 3.1.a

Figure 3.1.b

Figure 3.1.c

Figure 3.1.d

Figure 3.2

Figure 3.3

Figure 3.4.a

Figure 3.4.b

bubblers, Test 2.

Figure 3.4.c Plenum temperatures while processing with two double-outlet and two single-outlet bubblers, Test 3.

Figure 3.4.d Plenum temperatures while processing with two double-outlet and two single-outlet bubblers, Test 4.

Figure 3.5.a Average steady state plenum temperatures at monitoring locations; two double-outlet bubblers, Test 1.

Figure 3.5.b Average steady state plenum temperatures at monitoring locations; two double-outlet and one single-outlet bubblers, Test 2.

Figure 3.5.c Average steady state plenum temperatures at monitoring locations; two double-outlet and two single-outlet bubblers, Test 3.

Figure 3.5.d Average steady state plenum temperatures at monitoring locations; two double-outlet and two single-outlet bubblers, Test 4.

Figure 3.6.a Glass temperatures while processing with two double-outlet bubblers, Test 1.

Figure 3.6.b Glass temperatures while processing with two double-outlet and one single-outlet bubblers, Test 2.

Figure 3.6.c Glass temperatures while processing with two double-outlet and two single-outlet bubblers, Test 3.

Figure 3.6.d Glass temperatures while processing with two double-outlet and two single-outlet bubblers, Test 4.
F-1

F-2

F-3

F-4

F-5

F-6

F-7

F-8

F-9

F-10

F-11

F-12

F-13

F-14

F-15

F-16

F-17

F-18

F-19

F-20

F-21

F-22

F-23

F-24

F-25

F-26

F-27

F-28

F-29 
The Catholic University of America Vitreous State Laboratory
Testing of Optimized Bubbler Configuration for HLW Melter Final Report, VSL-13R2950-1, Rev. 0

Figure 3.7.a Electrode voltage and current while processing with two double-outlet

F-30 bubblers, Test 1.

Figure 3.7.b Electrode voltage and current while processing with two double-outlet and one

F-31 single-outlet bubblers, Test 2.

Figure 3.7.c Electrode voltage and current while processing with two double-outlet and two

F-32 single-outlet bubblers, Test 3.

Figure 3.7.d Electrode voltage and current while processing with two double-outlet and two $\quad$ F-33 single-outlet bubblers, Test 4.

Figure 3.8.a Electrode power and glass resistance while processing with two double-outlet $\quad$ F-34 bubblers, Test 1.

Figure 3.8.b Electrode power and glass resistance while processing with two double-outlet $\quad$ F-35 and one single-outlet bubblers, Test 2.

Figure 3.8.c Electrode power and glass resistance while processing with two double-outlet $\quad$ F-36 and two single-outlet bubblers, Test 3.

Figure 3.8.d Electrode power and glass resistance while processing with two double-outlet $\quad$ F-37 and two single-outlet bubblers, Test 4.

Figure 3.9.a Glass density and level while processing with two double-outlet bubblers, Test 1. F-38

Figure 3.9.b Glass density and level while processing with two double-outlet and one $\quad$ F-39 single-outlet bubblers, Test 2.

Figure 3.9.c Glass density and level while processing with two double-outlet and two $\quad$ F-40 single-outlet bubblers, Test 3.

Figure 3.9.d Glass density and level while processing with two double-outlet and two $\quad$ F-41 single-outlet bubblers, Test 4.

Figure 3.10.a Glass pool bubbling while processing with two double-outlet bubblers, Test $1 . \quad$ F-42

Figure 3.10.b Glass pool bubbling while processing with two double-outlet and one $\quad$ F-43 single-outlet bubblers, Test 2.

Figure 3.10.c Glass pool bubbling while processing with two double-outlet and two $\quad$ F-44 single-outlet bubblers, Test 3.

Figure 3.10.d Glass pool bubbling while processing with two double-outlet and two $\quad$ F-45 single-outlet bubblers, Test 4.

Figure 4.1 Average gas temperatures along the DM1200 off-gas train while processing $\quad$ F-46 with two double-outlet bubblers, Test 1.

Figure 4.2 Average gas temperatures along the DM1200 off-gas train while processing $\quad$ F-47 with two double-outlet and one single-outlet bubblers, Test 2.

Figure 4.3 Average gas temperatures along the DM1200 off-gas train while processing $\quad$ F-48 with two double-outlet and two single-outlet bubblers, Test 3.

Figure 4.4 Average gas temperatures along the DM1200 off-gas train rates while processing F-49 with two double-outlet and two single-outlet bubblers, Test 4.

Figure 4.5 Melter pressure while processing with two double-outlet bubblers, Test $1 . \quad$ F-50

Figure 4.6 Melter pressure while processing with two double-outlet and one single-outlet $\quad$ F-51 bubblers, Test 2.

Figure 4.7 Melter pressure while processing with two double-outlet and two single-outlet $\quad$ F-52 bubblers, Test 3.

Figure 4.8 Melter pressure while processing with two double-outlet and two single-outlet $\quad$ F-53 bubblers, Test 4.

Figure 4.9 Melter pressure at instrument port and control air flow rate while processing $\quad$ F-54 with two double-outlet bubblers, Test 1 .

Figure 4.10 Melter pressure at instrument port and control air flow rate while processing $\quad$ F-55 with two double-outlet and one single-outlet bubblers, Test 2. 
The Catholic University of America Vitreous State Laboratory
Testing of Optimized Bubbler Configuration for HLW Melter Final Report, VSL-13R2950-1, Rev. 0

Figure 4.11 Melter pressure at instrument port and control air flow rate while processing $\quad$ F-56 with two double-outlet and two single-outlet bubblers, Test 3.

Figure 4.12 Melter pressure at instrument port and control air flow rate while processing $\quad$ F-57 with two double-outlet and two single-outlet bubblers, Test 4.

Figure 4.13 Differential pressure across the film cooler while processing with two double-outlet F-58 bubblers, Test 1.

Figure 4.14 Differential pressure across the film cooler while processing with two double-outlet F-59 and one single-outlet bubblers, Test 2.

Figure 4.15 Differential pressure across the film cooler while processing with two double-outlet F-60 and two single-outlet bubblers, Test 3.

Figure 4.16 Differential pressure across the film cooler while processing with two double-outlet F-61 and two single-outlet bubblers, Test 4.

Figure 4.17 SBS inlet and outlet gas temperatures while processing with two double-outlet bubblers, Test 1 .

Figure 4.18 SBS inlet and outlet gas temperatures while processing with two double-outlet and one single-outlet bubblers, Test 2.

Figure 4.19 SBS inlet and outlet gas temperatures while processing with two double-outlet and two single-outlet bubblers, Test 3.

Figure 4.20 SBS inlet and outlet gas temperatures while processing with two double-outle and two single-outlet bubblers, Test 4 .

Figure 4.21 SBS inlet, outlet and differential pressures while processing with two double-outlet bubblers, Test 1 .

Figure 4.22 SBS inlet and differential pressures while processing with two double-outlet and one single-outlet bubblers, Test 2.

Figure 4.23 SBS inlet and differential pressures while processing with two double-outlet and two single-outlet bubblers, Test 3.

Figure 4.24 SBS inlet, outlet and differential pressures while processing with two double-outlet and two single-outlet bubblers, Test 4.

Figure 4.25 Off-gas temperatures in the SBS down-comer and sump water temperatures while processing with two double-outlet bubblers, Test 1 .

Figure 4.26 Off-gas temperatures in the SBS down-comer and sump water temperatures while processing with two double-outlet and one single-outlet bubblers, Test 2.

Figure 4.27 Off-gas temperatures in the SBS down-comer and sump water temperatures while processing with two double-outlet and two single-outlet bubblers, Test 3.

Figure 4.28 Off-gas temperatures in the SBS down-comer and sump water temperatures while processing with two double-outlet and two single-outlet bubblers, Test 4.

Figure 4.29 SBS cooling coil inlet, cooling coil outlet/jacket inlet and jacket outlet water temperatures while processing with two double-outlet bubblers, Test 1.

Figure 4.30 SBS cooling coil inlet, cooling coil outlet/jacket inlet and jacket outlet water temperatures while processing with two double-outlet and one single-outlet bubblers, Test 2 .

Figure 4.31 SBS cooling coil inlet, cooling coil outlet/jacket inlet and jacket outlet water temperatures while processing with two double-outlet and two single-outlet bubblers, Test 3.

Figure 4.32 SBS cooling coil inlet, cooling coil outlet/jacket inlet and jacket outlet water temperatures while processing with two double-outlet and two single-outlet bubblers, Test 4.

Figure 4.33 SBS cooling coil/jacket water flow rate while processing with two double-outlet bubblers, Test 1. 
The Catholic University of America Vitreous State Laboratory
Testing of Optimized Bubbler Configuration for HLW Melter Final Report, VSL-13R2950-1, Rev. 0

Figure 4.34 SBS cooling coil/jacket water flow rate during while processing with two double-outlet and one single-outlet bubblers, Test 2.

Figure 4.35 SBS cooling coil/jacket water flow rate while processing with two double-outlet

F-80 and two single-outlet bubblers, Test 3.

Figure 4.36 SBS cooling coil/jacket water flow rate while processing with two double-outlet and two single-outlet bubblers, Test 4.

Figure 4.37 Calculated heat loads on the inner coil and jacket while processing with two double-outlet bubblers, Test 1 .

Figure 4.38 Calculated heat loads on the inner coil and jacket while processing with two double-outlet and one single-outlet bubblers, Test 2.

Figure 4.39 Calculated heat loads on the inner coil and while processing with two double-outlet and two single-outlet bubblers, Test 3.

Figure 4.40 Calculated heat loads on the inner coil and jacket while processing with two double-outlet and two single-outlet bubblers, Test 4.

Figure 4.41 Accumulated SBS blow-down volume and accumulated feed water while processing with two double-outlet bubblers, Test 1.

Figure 4.42 Accumulated SBS blow-down volume and accumulated feed water while processing with two double-outlet and one single-outlet bubblers, Test 2.

Figure 4.43 Accumulated SBS blow-down volume and accumulated feed water while processing with two double-outlet and two single-outlet bubblers, Test 3.

Figure 4.44 Accumulated SBS blow-down volume and accumulated feed water while processing with two double-outlet and two single-outlet bubblers, Test 4 .

Figure 4.45 WESP inlet and outlet gas temperatures while processing with two double-outlet bubblers, Test 1 .

Figure 4.46 WESP inlet and outlet gas temperatures during while processing with two double-outlet and one single-outlet bubblers, Test 2.

Figure 4.47 WESP inlet and outlet gas temperatures while processing with two

F-81

F-82

F-83

F-84

F-85

F-86

F-87

F-88

F-89 double-outlet and two single-outlet bubblers, Test 3.

Figure 4.48 WESP inlet and outlet gas temperatures while processing with two F-93 double-outlet and two single-outlet bubblers, Test 4.

Figure 4.49 WESP outlet gas flow rate while processing with two double-outlet $\quad$ F-94 bubblers, Test 1.

Figure 4.50 WESP outlet gas flow rate while processing with two double-outlet and one $\quad$ F-95 single-outlet bubblers, Test 2.

Figure 4.51 WESP outlet gas flow rate while processing with two double-outlet and $\quad$ F-96 two single-outlet bubblers, Test 3.

Figure 4.52 WESP outlet gas flow rate while processing with two double-outlet and two $\quad$ F-97 single-outlet bubblers, Test 4.

Figure 4.53 Voltage and current across the WESP while processing with two double-outlet bubblers, Test 1.

Figure 4.54 Voltage and current across the WESP while processing with two double-outlet and one single-outlet bubblers, Test 2.

Figure 4.55 Voltage and current across the WESP while processing with two double-outlet $\quad$ F-100 and two single-outlet bubblers, Test 3.

Figure 4.56 Voltage and current across the WESP while processing with two double-outlet $\quad$ F-101 and two single-outlet bubblers, Test 4.

Figure 4.57 Outlet temperature and differential pressure for HEME \#1 while processing $\quad$ F-102 with two double-outlet bubblers, Test 1 . 
The Catholic University of America Vitreous State Laboratory
Testing of Optimized Bubbler Configuration for HLW Melter Final Report, VSL-13R2950-1, Rev. 0

Figure 4.58 Outlet temperature and differential pressure for HEME \#1 while processing

F-103 with two double-outlet and one single-outlet bubblers, Test 2.

Figure 4.59 Outlet temperature and differential pressure for HEME \#1 while processing

F-104 with two double-outlet and two single-outlet bubblers, Test 3 .

Figure 4.60 Outlet temperature and differential pressure for HEME \#1 while processing

F-105 with two double-outlet and two single-outlet bubblers, Test 4 .

Figure 4.61 Outlet temperature and differential pressure for HEPA \#1 while processing

F-106 with two double-outlet bubblers, Test 1.

Figure 4.62 Outlet temperature and differential pressure for HEPA \#1 while processing

F-107 with two double-outlet and one single-outlet bubblers, Test 2 .

Figure 4.63 Outlet temperature and differential pressure for HEPA \#1 while processing

F-108 with two double-outlet and two single-outlet bubblers, Test 3 .

Figure 4.64 Outlet temperature and differential pressure for HEPA \#1 while processing with two double-outlet and two single-outlet bubblers, Test 4.

Figure 4.65 Inlet gas temperature and differential pressure for PBS while processing with two double-outlet bubblers, Test 1.

Figure 4.66 Inlet gas temperature and differential pressure for PBS for while processing with two double-outlet and one single-outlet bubblers, Test 2 .

Figure 4.67 Inlet gas temperature and differential pressure for PBS while processing with two double-outlet and two single-outlet bubblers, Test 3.

Figure 4.68 Inlet gas temperature and differential pressure for PBS while processing with two double-outlet and two single-outlet bubblers, Test 4 .

Figure 4.69 $\mathrm{pH}$ for PBS while processing with two double-outlet bubblers, Test 1.

F-109

F-110

F-111 $\mathrm{pH}$ for PBS while processing with two double-outlet and one single-outlet bubblers, Test 2.

Figure 4.71 pH for PBS while processing with two double-outlet and two single-outlet bubblers, Test 3.

Figure 4.72 pH for PBS while processing with two double-outlet and two single-outlet bubblers, Test 4.

Figure 5.1.a

Figure 5.1.b DM1200 product and target glass compositions determined by XRF. DM1200 product and target glass compositions determined by XRF.

Figure 5.1.c DM1200 product and target glass compositions determined by XRF.

Figure 5.1.d DM1200 product and target glass compositions determined by XRF.

Figure 5.1.e DM1200 product and target glass compositions determined by XRF.

Figure 5.1.f

Figure 6.1.a DM1200 product and target glass compositions determined by XRF.

Figure 6.1.b FTIR Monitored water emissions during Test 1. FTIR Monitored water emissions during Test 2. FTIR Monitored water emissions during Test 3.

F-118

F-119

F-120

F-121

F-122

F-123

F-124

F-125

F-126

Figure 6.1.d FTIR Monitored water emissions during Test 4.

F-127 FTIR Monitored NO emissions during Test 1.

F-128

F-129 FTIR Monitored NO emissions during Test 2.

F-130 FTIR Monitored NO emissions during Test 3.

F-131 


\section{List of Abbreviations}

$\begin{array}{ll}\text { ADS } & \text { Air Displacement Slurry } \\ \text { AOD } & \text { Air Operated Diaphragm } \\ \text { ASME } & \text { American Society of Mechanical Engineers } \\ \text { BNI } & \text { Bechtel National, Inc. } \\ \text { DCP-AES } & \text { Direct Current Plasma - Atomic Emission Spectroscopy } \\ \text { DF } & \text { Decontamination Factors } \\ \text { DM } & \text { DuraMelter } \\ \text { DOE } & \text { Department of Energy } \\ \text { DWPF } & \text { Defense Waste Processing Facility } \\ \text { FTIR } & \text { Fourier Transform Infra-Red Spectroscopy } \\ \text { HEME } & \text { High-Efficiency Mist Eliminator } \\ \text { HEPA } & \text { High-Efficiency Particulate Air } \\ \text { HLW } & \text { High Level Waste } \\ \text { LAW } & \text { Low Activity Waste } \\ \text { NIST } & \text { National Institute of Standards and Technology } \\ \text { NQA } & \text { Nuclear Quality Assurance } \\ \text { ORP } & \text { Office of River Protection } \\ \text { PBS } & \text { Packed-Bed Scrubber } \\ \text { QARD } & \text { Quality Assurance Requirements and Description } \\ \text { SBS } & \text { Submerged Bed Scrubber } \\ \text { SCR } & \text { Selective Catalytic Reduction } \\ \text { SOP } & \text { Standard Operating Procedure } \\ \text { TCO } & \text { Thermal Catalytic Oxidation } \\ \text { VOC } & \text { Volatile Organic Compound } \\ \text { VSL } & \text { Vitreous State Laboratory } \\ \text { W.C. } & \text { Water Column } \\ \text { WESP } & \text { Wet Electrostatic Precipitator } \\ \text { WTP } & \text { Waste Treatment Immobilization Plant } \\ \text { XRF } & \text { X-ray Fluorescence Spectroscopy }\end{array}$


The Catholic University of America Vitreous State Laboratory
Testing of Optimized Bubbler Configuration for HLW Melter Final Report, VSL-13R2950-1, Rev. 0

\section{SECTION 1.0 INTRODUCTION}

Plans for the treatment of high level waste (HLW) at the Hanford Tank Waste Treatment and Immobilization Plant (WTP) are based upon the inventory of the tank wastes, the anticipated performance of the pretreatment processes, and current understanding of the capability of the borosilicate glass waste form [1]. The WTP HLW melter design, unlike earlier DOE melter designs, incorporates an active glass bubbler system. The bubblers create active glass pool mixing and thereby improve heat and mass transfer and increase glass melting rates. The WTP HLW melter has a melt surface area of $3.75 \mathrm{~m}^{2}$ and depth of $\sim 1.1 \mathrm{~m}$. The two melters in the HLW facility together are designed to produce up to 7.5 MT of glass per day at $100 \%$ availability. Further increases in HLW waste processing rates can potentially be achieved by increasing the melter operating temperature above $1150^{\circ} \mathrm{C}$ and by increasing the waste loading in the glass product. Increasing the waste loading also has the added benefit of decreasing the number of canisters for storage.

Development work for the WTP employed a "tiered" approach to vitrification testing involving computer-based glass formulation, glass property-composition models, crucible melts, and continuous melter tests of increasing, more realistic scales. Melter systems ranging from 0.02 to $1.2 \mathrm{~m}^{2}$ installed at the Vitreous State Laboratory (VSL) have been used for this purpose, which, in combination with the $3.3 \mathrm{~m}^{2}$ low activity waste (LAW) Pilot Melter operated by EnergySolutions, span more than two orders of magnitude in melt surface area. In this way, less-costly small-scale tests can be used to define the most appropriate tests to be conducted at the larger scales in order to extract maximum benefit from the large-scale tests. For HLW vitrification development, a key component in this approach is the one-third scale DuraMelter 1200 (DM1200), which is the HLW Pilot Melter that has been installed at VSL with an integrated prototypical off-gas treatment system. That system replaced the DM1000 system that was used for HLW throughput testing during Part B1 of the privatization contract [2]. Both melters have similar melt surface areas $\left(1.2 \mathrm{~m}^{2}\right)$, but the DM1200 is prototypical of the present WTP HLW melter design whereas the DM1000 was not. In particular, the DM1200 provides for testing on a vitrification system that includes the specific train of unit operations that has been selected for both HLW and LAW WTP off-gas treatment [3].

Over the course of testing on the DM1200 system, over one and a half million pounds of feed had been processed, producing almost 600,000 pounds of glass by the end of Bechtel national, Inc. (BNI) WTP testing in 2006 [4-19]. These tests were conducted to address several objectives, including determination of glass production rates and melt pool characteristics, as well as evaluation of the prototypical off-gas system. The compositions used for the extensive technology development and design work performed for the WTP baseline were iron limited with respect to waste loading (AZ-101, AZ-102, C-16/AY-102, and C-104/AY-101) [5, 6, 9, 11-15, 17, 18]. More recently however, the DM1200 has been used to process simulated high aluminum [20-22] and high bismuth [23] HLW streams identified by ORP [24]. In these tests, new high waste loading glass formulations 
The Catholic University of America Vitreous State Laboratory
Testing of Optimized Bubbler Configuration for HLW Melter Final Report, VSL-13R2950-1, Rev. 0

were developed and subjected to testing on the DM1200 system, which demonstrated processing rates above the WTP baseline requirement. In all DM1200 testing with HLW simulants to date, no more than four bubbling outlets were used in order to reflect the projected number of bubblers per unit surface area of the WTP HLW melter shown in Figure 1.1 [25]. In previous testing on the DM1200, increasing the number of bubbling outlets from two to four was demonstrated to dramatically increase production rates [15] such that the WTP contract glass production rate requirement could be met; these results were used to define the present WTP HLW melter bubbler configuration. While it would seem reasonable that a further increase in the number of bubbler outlets would lead to a further increase in production rates, this was not pursued under the BNI test program. Thus testing described in this report was designed to investigate the potential increase in production rates associated with increases in the number of bubbler outlets above the current WTP baseline. The work was described in a Test Plan [26], which is one of several prepared in response to a Scope of Work provided by the Department of Energy's (DOE) Office of River Protection (ORP) [27]. Results from the tests described herein are intended to support the option of a potential future re-design of the WTP HLW melter lid to allow for the additional bubbling outlets. If this option is pursued, it is anticipated that computer modeling of the WTP HLW melter with various possible bubbler configurations will be used as a tool to support the design effort in combination with the results from the present tests.

\section{$1.1 \quad$ Test Objectives}

The principal objective of this work was to determine the glass production rate increase and ancillary effects of adding more bubbler outlets to the current WTP HLW melter baseline. This was accomplished through testing on the HLW Pilot Melter (DM1200) at VSL. The DM1200 unit was selected for these tests since it was used previously with several HLW waste streams [5, 6, 9, 11-15, 17-23] including the four tank wastes proposed for initial processing at Hanford [5, 6, 9, 11-15, 17, 18]. This melter system was also used for the development and optimization of the present baseline WTP HLW bubbler configuration for the WTP HLW melter [15], as well as for MACT testing for both HLW and LAW [19]. Specific objectives of these tests were to:

- $\quad$ Conduct DM1200 melter testing with the baseline WTP bubbling configuration and as augmented with additional bubblers.

- $\quad$ Conduct DM1200 melter testing to differentiate the effects of total bubbler air flow and bubbler distribution on glass production rate and cold cap formation.

- $\quad$ Collect melter operating data including processing rate, temperatures at a variety of locations within the melter plenum space, melt pool temperature, glass melt density, and melter pressure with the baseline WTP bubbling configuration and as augmented with additional bubblers.

- $\quad$ Collect melter exhaust samples to compare particulate carryover for different bubbler configurations.

- Analyze all collected data to determine the effects of adding more bubblers to the WTP HLW melter to inform decisions regarding future lid re-designs. 
The Catholic University of America Vitreous State Laboratory
Testing of Optimized Bubbler Configuration for HLW Melter Final Report, VSL-13R2950-1, Rev. 0

The work used a high aluminum HLW stream composition defined by ORP [24], for which an appropriate simulant and high waste loading glass formulation were developed and have been previously processed on the DM1200 [20-22].

\subsection{Quality Assurance}

This work was conducted under a quality assurance program compliant with applicable criteria of 10 CFR 830.120; Office of Civilian Waste Management DOE/RW-0333P, Quality Assurance Requirements and Description (QARD) Revision 20; the American Society of Mechanical Engineers (ASME) Nuclear Quality Assurance (NQA)-1, 2004; and DOE Order 414.1 C, Quality Assurance. This program is supplemented by a Quality Assurance Project Plan for ORP work that is conducted at VSL [28]. Test and procedure requirements by which the testing activities are planned and controlled also are defined in this plan. The program is be supported by VSL standard operating procedures that were used for this work [29]. Since this work is not waste form quality affecting, the requirements of DOE/RW-0333P are not applicable to this work.

\section{$1.3 \quad$ DM1200}

\subsubsection{Feed System}

The feed material for these tests was prepared and controlled according to VSL specifications by a chemical supplier, as detailed in Section 2. Each batch of feed slurry was shipped to VSL in lined 55-gallon drums, which were staged for unloading into the mix tank. Both the mix tank and the feed tank are 750-gallon polyethylene tanks with conical bottoms that are fitted with mechanical agitators; the feed tank is also fitted with baffles to improve mixing. Any required feed additives can be added to the mix tank. Five calibrated load cells directly mounted on the legs of the feed tank are used to measure additions to, and removal from, the feed tank and are electronically monitored to determine the feed rate to the melter. The requisite amount of feed is pumped to the feed tank from the mix tank; measured amounts of water are combined by weight with the feed at this point to adjust the concentration of the melter feed. The material in the feed tank is constantly recirculated from the feed tank discharge outlet, at the tank bottom, to the tank inlet at the top, which provides additional mixing.

The feed was introduced into the melter using an air operated diaphragm (AOD) pump system in place of an air displacement slurry (ADS) pump. The AOD pump, which simulates the WTP baseline ADS pump, was used to prevent the clogging of the inlet screens observed in previous tests with the high aluminum waste [21, 22]. Only one feed tube is used to represent the planned number of feed tubes per unit melt surface area in the full-scale WTP HLW melter. The recirculation loop extends to the top of the melter where feed is diverted from the recirculation loop into the melter through a Teflon-lined feed line and water-cooled feed tube. Two computer-operated pinch valves, one on the feed line and one on the recirculation loop, are activated in a timed sequence to introduce feed into the melter at the desired rate. The feed rate is regulated by adjusting the length of each pulse, the time between each pulse, and the pressure applied to the recirculation loop. 
The Catholic University of America Vitreous State Laboratory
Testing of Optimized Bubbler Configuration for HLW Melter Final Report, VSL-13R2950-1, Rev. 0

\subsubsection{Melter System}

The DuraMelter 1200 (DM1200), which is the HLW Pilot Melter, was used for these tests. Cross-sectional diagrams of the melter illustrating the discharge chamber and electrode configuration are provided in Figures 1.2 and 1.3. The DM1200 is a Joule-heated melter with Inconel 690 electrodes and thus has an upper operating temperature of about $1200^{\circ} \mathrm{C}$. The melter shell is water-cooled and incorporates a jack-bolt thermal expansion system. The footprint of the melter is approximately $8 \mathrm{ft}$. by $6.5 \mathrm{ft}$. with a $4 \mathrm{ft}$. by $2.3 \mathrm{ft}$. air-lift discharge chamber appended to one end; the melter shell is almost $8 \mathrm{ft}$. tall. The melt surface area and the melt pool height are approximately 32 percent and 57 percent, respectively, of the corresponding values for the full-scale HLW melter. The discharge riser and trough are full-scale to verify pouring performance. Other aspects of the discharge system are also prototypical such as the chamber ventilation scheme. The glass contact refractory is Monofrax ${ }^{\circledR} \mathrm{K}-3$ while the plenum area walls are constructed of Monofrax ${ }^{\circledR}$ $\mathrm{H}$ refractory. The surface of the glass pool is 34" by 54" with a nominal glass depth of 25". The resultant melt volume is approximately 45,000 cubic inches (735 liters), which represents a glass tank capacity of more than 1.7 metric tons of glass. However, since the typical operating glass level is closer to 29 inches, the effective glass volume during testing is actually about 849 liters, giving an inventory of about 2.0 metric tons. The DuraMelter ${ }^{\mathrm{TM}} 1200$ is fitted with one pair of electrodes placed high on opposite walls of the melter as well as one bottom electrode. The side electrodes are 11 " by 34" giving an electrode area for the pair of about 750 sq. in. Depending on the glass level, the plenum space extends about 33" to 36" above the melt surface resulting in a plenum volume ranging from about 43 to $46 \mathrm{ft}^{3}$.

The single-phase power supply to the melter electrodes ( $250 \mathrm{~kW}$ design power) is derived from the DuraMelter 1000 transformers by wiring them in parallel and using a single large silicon controlled rectifier. Current can be passed either from the side electrodes to the bottom electrode or between the two side electrodes only, by rearranging jumpers; only side-to-side operation was used for the present tests. Programmable process controllers are installed and can be used to control temperature or power. The melt temperature is controlled by configuring the process controller to maintain constant power and adjusting the power set-point as needed to maintain the desired operating temperature. Alarms can be set to detect out-of-range temperatures or power in the melter. Backup process controllers are installed to be used in case of failure of the main controllers. The entire system is supported by a back-up generator that is tripped on in the event of a power outage.

The DuraMelter 1200 has several other features. The lid refractory is prototypic and also includes a two-piece construction, which simulates the seam needed for the LAW lid that was planned to be fabricated in three pieces. Nozzles are provided for the off-gas film cooler, a standby off-gas port, discharge airlift, along with 11 ports available for top-entering bubblers, start-up heaters and other components as needed. In addition, a bubbler arrangement is installed in the bottom electrode with the objective of developing permanent bubblers for possible use on future melters. The optimum bubbler configuration established during previous tests with HLW simulants [15], consisting of two double-outlet, top-entering bubblers located in positions to mimic conditions in the WTP HLW melter was used for the first test. Figure 1.4 shows a schematic diagram of the 
The Catholic University of America Vitreous State Laboratory
Testing of Optimized Bubbler Configuration for HLW Melter Final Report, VSL-13R2950-1, Rev. 0

prototypical double-outlet bubbler design that was based on the combination of the results from these DM1200 tests [15] and room-temperature tests that were performed in a transparent fluid simulating the properties of the glass melt [30]. A photograph of the double-outlet bubbler is provided in Figure 1.5. These bubblers have outlets 8 inches apart and were placed on the melter floor. The orientation of the bubblers in the melter, as shown in Figure 1.6, results in one of the bubbling outlets being 11.3 inches from the feed tube. The number of bubbling outlets was increased over the course of the tests by adding single out " $\mathrm{L}$ " bubblers, as pictured in Figure 1.7. The orientation of the double and single-outlet bubblers in the melter is shown for five and six total bubbling outlets in Figures 1.8 and 1.9, respectively. Notice that to evenly distribute bubbling outlets on each side of the melter, the double-outlet bubblers are rotated such that bubbling outlets come in closer proximity to the feed tube.

The DM1200 film cooler was replaced immediately prior to the previous tests [22]. The design of the new film cooler is very similar to that used for all previous testing on the DM1200 but it incorporates several changes that were made in the WTP HLW film cooler design after the installation of the DM1200 melter system. As a result, the new DM1200 film cooler is more prototypical of the present WTP HLW design. The original and new DM1200 film coolers are compared with a scaled version of the WTP HLW film cooler in Figure 1.10; it should be noted, however, that a simple directly scaled version would not maintain key air flow characteristics of the design, hence the differences between the new DM1200 film cooler and the scaled WTP HLW design. As compared to the original DM1200 film cooler, the new unit includes the prototypical louver on the outside edge, a modified hole size and pattern on the leading edge, fewer louvers (7 vs. 9), and a shorter louvered section (10” vs. 13").

\subsubsection{Off-Gas System}

The melter and entire off-gas treatment system are maintained under negative pressure by two Paxton external induced draft blowers. This negative pressure is necessary to direct the gases from the melter to the prototypical off-gas system. The off-gas treatment system, shown schematically in Figure 1.11, consists of a submerged bed scrubber (SBS); a wet electrostatic precipitator (WESP); a high-efficiency mist eliminator (HEME), a high-efficiency particulate air (HEPA) filter; a thermal catalytic oxidation unit (TCO); a $\mathrm{NO}_{\mathrm{x}}$ removal system (SCR); a caustic packed-bed scrubber (PBS); and a second HEME. Note that the PBS and the second HEME are not part of the WTP HLW off-gas train, which effectively ends at the SCR. The HEME is used to limit entrained particle carryover into the balance of the VSL ventilation system. The system can be functionally divided into four subsystems:

Particulate Removal: $\quad$ Components from the SBS to the HEPA serve to remove essentially all of the particulate from the gas stream with an estimated removal efficiency of greater than $99.9999 \%$ for particles greater than $0.3 \mu \mathrm{m}$ in size. In the WTP facility, this provision serves to segregate the radioactive from the non-radioactive components in the system for maintenance and handling purposes. 
The Catholic University of America Vitreous State Laboratory

VOC Control/Acid Gas:

Stack System:

Liquid Processing:
Testing of Optimized Bubbler Configuration for HLW Melter Final Report, VSL-13R2950-1, Rev. 0
The TCO unit is designed to oxidize any hazardous organics that are present in the off-gas stream. This is followed by a SCR to remove $\mathrm{NO}_{\mathrm{x}}$ gases and a PBS to remove remaining acid gases.

The emergency/bypass exhaust system, which includes a second HEPA, and the primary off-gas system both feed into the building stack system for exhausting to the atmosphere.

Components including the water spray lines, liquid sampling and water storage tanks, as well as the effluent evaporator, function to sample and process the system liquids for recycle or discharge.

With minor exceptions, the DM1200 off-gas system processing sequence follows the design for the full-scale WTP HLW melter system, except for cooling of the off-gas stream discharged from the SCR unit (which is present in the WTP off-gas train, but absent in the DM1200 system). Per WTP direction, the SBS unit that was used for previous DM1200 testing was modified in early 2004. Installation of the new system was completed in March 2004 and that unit was used for the present tests. The changes were implemented to reflect modifications to the WTP SBS design that have taken place since the original DM1200 unit was installed. These modifications included changes to the diffuser plate design, down-comer jacket and connection to the diffuser plate, bed diameter, bed packing materials, cooling coils, and liquid overflow level.

Initial quenching of the melter exhaust gas stream is effected by the film cooler. Immediately upstream of the film cooler is the injection point for control air, which is used to regulate melter pressure. The gas entering the balance of the off-gas system is at a temperature of about 250 to $350^{\circ} \mathrm{C}$ and a flow rate of about $100-250 \mathrm{scfm}$, of which about $10-80 \mathrm{scfm}$ is water vapor. The off-gas is then rapidly quenched by direct liquid water contact in the SBS, which also effects removal of most of the larger particulates. The piping between the film cooler and SBS has a high superficial gas velocity to minimize particulate deposition. The gas stream leaving the SBS is at a low temperature (typically between $40-50^{\circ} \mathrm{C}$ ). Further mist and particulate removal is effected in the WESP, HEME and HEPA. The TCO and SCR follow the particle removal components and serve to destroy organic compounds and nitrogen oxides. These two units were off-line during the present tests due to the low concentrations of these components in the exhaust stream. Finally, the PBS provides acid gas removal. Water sprays are located in the WESP, PBS, and facility HEME to wash down deposits and dissolved species into their respective collection sumps from which they can be sampled. The system components are fabricated from corrosion resistant materials, including AL6XN and 316L stainless steel, and various plastics in less demanding locations. There are extensive provisions for sampling both the gas and liquid streams throughout the system in order to collect mass balance information and removal efficiency data for each treatment stage.

The off-gas system maintains the melter plenum under slight negative pressure, typically about -5 in. water column (W.C.). The plenum pressure is controlled by means of an air injection system that introduces a controlled air flow into the off-gas jumper just after the film cooler. The air 
The Catholic University of America Vitreous State Laboratory
Testing of Optimized Bubbler Configuration for HLW Melter Final Report, VSL-13R2950-1, Rev. 0

is supplied by a blower through a diverter valve. The setting of the diverter valve, and therefore the air flow rate, is controlled by a process controller that responds to the signal from a melter pressure transducer. When the plenum pressure becomes more positive, the air injection flow rate is decreased, which tends to restore the pressure to the set-point. Conversely, the flow rate is increased when the plenum pressure becomes more negative.

\section{$1.4 \quad$ Feed Sample Analysis}

Feed samples were taken directly from the feed recirculation line during each test. Feed samples were poured into a platinum/gold crucible that was placed into a programmed furnace for drying and fusion to form a glass. The glass produced from this fusion was ground to less than 200 mesh and sealed in 20-ml vials for subsequent analysis by X-ray fluorescence spectroscopy (XRF), or by acid digestion followed by direct current plasma - atomic emission spectroscopy (DCP-AES) on the resulting solution. The feed samples were also characterized for their density, $\mathrm{pH}$, water content, and glass yield.

\subsection{Glass Product Analysis}

The glass product from the DM1200 tests was discharged from the melter into 55 gallon drums periodically using an air-lift system. The discharged product glass was sampled by removing sufficient glass from the top of the cans for compositional analysis after the cooling period and visual inspection (see Section 5.0). All of these procedures are routinely conducted at VSL and, therefore, standard operating procedures (SOPs) are in place. Sample preparation for chemical analysis typically involves size reduction and sieving. All samples were subjected to XRF to determine the concentration of all elements except boron and lithium. A series of National Institute of Standards and Technology (NIST) reference materials were used for confirmation of the XRF data. Boron and lithium were determined by total acid dissolution of ground glass samples in $\mathrm{HF} / \mathrm{HNO}_{3}$ and subjecting the resulting solutions to DCP-AES analysis.

\subsection{Emission Samples}

Melter emission fluxes were measured to complete the mass balance for each melter test. Isokinetic melter exhaust samples (exhaust gas flow velocity equal to velocity through the gas sample probe tip) were combined with the Fourier Transform Infra-Red Spectroscopy (FTIR) spectroscopy continuous monitoring data for gaseous species to characterize fluxes from the melter. In the DM1200 system, independent sampling ports for particulate and FTIR sampling are available throughout the off-gas treatment train (see Figure 1.11). Standard EPA isokinetic off-gas sampling

trains and methods (EPA Methods 1A, 2, 4, 5, 26, 29), composed of particulate filters and liquid impingers, were used to collect materials that were subjected to chemical and physical analyses using the techniques described in Sections 1.4 and 1.5. 
The Catholic University of America

Vitreous State Laboratory
Testing of Optimized Bubbler Configuration for HLW Melter Final Report, VSL-13R2950-1, Rev. 0

\subsection{Test Overview}

Melter testing was conducted in four fifty-hour test segments distinguished by the number of bubbling outlets employed and the total air flow rate applied to the bubblers. The waste and glass composition, feed solids content, glass temperature and extent of cold cap coverage were held constant throughout the tests while the number of bubbling outlets, bubbling outlet distribution, and bubbling rate were varied. The form of aluminum in the waste was aluminum oxide, which is the aluminum source that previous testing has shown to be the slowest to be incorporated into glass [20] and therefore provides the most conservative case for testing. The composition of the glass inventory in the DM1200 melter prior to testing was the same as that employed for the present tests (HWI-Al-19 [22]) and therefore no melt pool turnover was required. In the first test, two double-outlet bubblers were used in the prototypical locations used in previous tests [15, 18-23] to provide a baseline and to determine the amount of bubbling required to produce glass at the target rate of $1050 \mathrm{~kg} / \mathrm{m}^{2} / \mathrm{day}^{1}$. The total bubbling rate from this test was used in two of the subsequent tests as additional bubbling outlets were added to determine the effect of bubbling distribution on glass production rate. In another test, the six-outlet bubbling configuration was used and the bubbling rate was adjusted to achieve the target production rate of $1050 \mathrm{~kg} / \mathrm{m}^{2} /$ day. The melter feed rate was increased to achieve a complete cold cap throughout the tests, as verified by visual observations and corroborated by measured plenum temperatures. Observations of cold-cap coverage and characteristics were made frequently and are included in Section 3. Particular attention was paid to changes in plenum temperature distribution across the plenum space with changes in bubbling outlet distribution.

Throughout the tests, extensive melter operating data were collected to characterize the effects of bubbling outlet distribution and bubbling rate on measured parameters and implications for future designs of the melter lid. Melter exhaust samples were also taken to provide a connection between bubbling outlet distribution, bubbling rate, and particulate carryover during testing.

\footnotetext{
${ }^{1}$ The production rate of $1050 \mathrm{~kg} / \mathrm{m}^{2} /$ day was selected based on the previous requirement of $3 \mathrm{MT} /$ day for the WTP HLW melter and a scaling factor to account for differences in the number of bubbling outlets per unit area in the DM1200 and the WTP HLW melter.
} 
The Catholic University of America

Vitreous State Laboratory
Testing of Optimized Bubbler Configuration for HLW Melter

Final Report, VSL-13R2950-1, Rev. 0

\section{SECTION 2.0 \\ WASTE SIMULANT AND GLASS FORMULATIONS}

\subsection{Aluminum Limited Waste Simulant}

The waste stream compositions previously provided by ORP [24] are given in Table 2.1 on an oxide basis [31]. Of the four waste compositions listed, the present work focused exclusively on the aluminum limited waste stream as a result of previous processing experience on the DM1200 [20-22].

Actual Hanford tank HLW streams are aqueous solutions with suspended solids and dissolved salts including hydroxides, nitrates, nitrites, halides, and carbonates. For the purpose of the previous [20-22] and present work, the concentrations of the volatile components (i.e., carbonate, nitrite, nitrate, and organic carbon) are assumed to be similar to those found for the AZ-102 HLW [11]. With the waste compositions defined, formulation of the HLW simulant proceeds in a straightforward fashion. In general, oxides and hydroxides are used as the starting materials, with a slurry of iron (III) hydroxide (13\% by weight) as one of the major constituents. Volatile inorganic components are added as the sodium salts, whereas organic carbon is added as oxalic acid. Although crucible melts have been prepared using the appropriate radioactive components (i.e., thorium and uranium), substitution of non-radioactive starting materials were required in preparing the simulated waste for melter testing. The exact substitution depended on the measured properties of the radioactive glass prepared in a crucible melt and was determined on a case-by-case basis. Finally, water content was adjusted to target a glass yield of $500 \mathrm{~g}$ of glass per liter of feed. The composition of the waste simulant with aluminum oxide as the aluminum source formulated to produce $100 \mathrm{~kg}$ of waste oxides is given in Table 2.2. Aluminum oxide was chosen as the aluminum source because previous tests have shown this to produce the slowest melting melter feed as compared to gibbsite and boehmite [20]. A slower melting feed, in addition to providing the most conservative test case, allowed better estimation of the effect of bubbler configuration on feed processing rate without running into potential issues such as melter power limitations that could occur with a faster melting feed.

\subsection{Glass Formulation}

The HWI-Al-19 high waste loading glass formulation was developed for the high aluminum waste composition and was tested on both the DM100 and DM1200 melters to determine processing rates [20-22]. These tests demonstrated that the formulation exceeded WTP requirements with respect to glass production rate and processed at a faster rate than the previously developed formulation (HLW-E-Al-27 [31]) for the same waste, while maintaining the same $45 \mathrm{wt} \%$ waste loading. 
The composition and properties of the HWI-Al-19 formulation are listed in Table 2.3 and the melter feed composition with aluminum oxide as the aluminum source is shown in Table 2.4. Based on the results from small-scale melt rate testing, the formulation emphasized increased boron concentrations to improve melt rates and compensating changes to maintain other glass properties within acceptable ranges. The additional constituents required to form the target test glass from the HLW high aluminum waste simulant are boron, calcium, lithium, sodium, and silicon. The corresponding chemical additives that are the sources for these elements were selected based on previous testing and the current baseline chemicals for the WTP Project. The measured viscosity and conductivity of $\mathrm{HWI}-\mathrm{Al}-19$ at $1150^{\circ} \mathrm{C}$ are $33 \mathrm{P}$ and $0.27 \mathrm{~S} / \mathrm{cm}$, respectively. No crystalline phases were observed in the as-melted sample, and heat treatment for 72 hours at $950^{\circ} \mathrm{C}$ resulted in 1.3 vol\% crystals. Chemical durability was verified on crucible glasses and product melter glasses with leachate concentrations well below regulatory limits [20]. Melter feeds were produced by NOAH Technologies Corporation, the supplier of simulant and feed samples used in previous testing on the DM100 and DM1200 melter systems. Additional water to achieve the target glass yield of 500 g glass per liter was added to the feed at VSL. 
The Catholic University of America Vitreous State Laboratory
Testing of Optimized Bubbler Configuration for HLW Melter Final Report, VSL-13R2950-1, Rev. 0

\section{SECTION 3.0 DM1200 OPERATIONS}

A series of tests with high aluminum simulants were conducted between 1/15/13 and 2/8/13, producing nearly twelve metric tons of glass. The total duration of waste and water feeding, was 203 hours, during which over 33 metric tons of feed was processed. Summaries of the test conditions and results are provided in Table 3.1. The tests were conducted to determine the relationship between the number and locations of bubbler outlets and production rate. For each test, target or maximum production rates were indicated by complete cold cap coverage visually observed and confirmed by plenum temperature indications. Testing began with bubblers in a previously defined and tested bubbler configuration; this consisted of two double-outlet lance bubblers on the melter floor, 8" apart on the East and West side, with one bubbler outlet a horizontal distance of 11.3" from the feed tube location [4, 15, 18, 20-23]. As testing progressed, bubbling outlets were added while maintaining either constant bubbling rate or feed rate. The high aluminum feed employed glass formulation HWI-Al-19 and has been processed previously on the DM1200 [20- 22]. However, unlike the previous tests, the present feed employed aluminum oxide, which is the slowest melting aluminum source and which therefore provided the most conservative result for feed rates. Feed solids content was targeted at 500 g glass per liter and was confirmed by analysis of feed samples from each test. The tests are listed below in the order in which they were conducted:

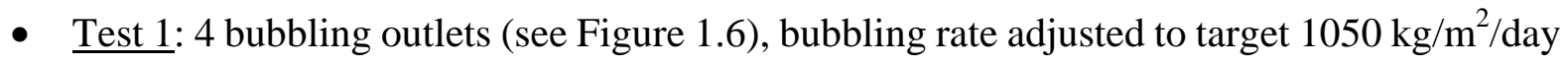
glass production rate.

- $\quad$ Test 2: 5 bubbling outlets (see Figure 1.8), bubbling rate of 78 lpm (steady state bubbling rate from Test 1 ) and feed rate adjusted to achieve maximum glass production rate.

- $\quad \underline{\text { Test 3: }} 6$ bubbling outlets (see Figure 1.9), bubbling rate adjusted to target $1289 \mathrm{~kg} / \mathrm{m}^{2} /$ day glass production rate (steady state rate from Test 2 ).

- $\quad$ Test 4: 6 bubbling outlets (see Figure 1.9), bubbling rate of 78 lpm (steady state bubbling rate from Test 1 ) and feed rate adjusted to achieve maximum glass production rate.

The tests employed an AOD feed system, a single feed tube in the center of the melter lid, a nominal glass temperature of $1150^{\circ} \mathrm{C}$ for all tests, and a side-to-side electrode firing pattern. The prototypical ADS feed system was not used due to screen clogging that was observed in previous tests while processing the high aluminum waste feed [20-22]; similar behavior was observed with high solids LAW Sub-Envelope B1 feed [10] and HLW feeds adjusted to higher feed viscosity [18]. Therefore the backup AOD feed system was used to process the high aluminum waste feed and performed without incident. Visual observations of the cold cap were made and recorded and were used as an indicator of complete cold cap coverage. A chronology of melter operations during the tests is provided in Table 3.2; a listing of all the cold cap observations is provided in Table 3.3. 
The Catholic University of America Vitreous State Laboratory
Testing of Optimized Bubbler Configuration for HLW Melter Final Report, VSL-13R2950-1, Rev. 0

All systems and major components of the melter and off gas systems performed well without need for repair during testing other than the trough in the discharge chamber. In the latter portion of Test 2 and throughout Test 3, spikes in current, voltage, and glass resistance were observed during each discharge. Lower than expected glass resistance resulted in higher than desirable current on the primary side of the electrodes. Also, one of the heating elements in the discharge chamber ceased to function. To address these conditions, the discharge chamber was cooled and opened up for inspection after Test 3. It was determined that the discharge trough had sagged down and was resting on one of the discharge heater sheathes. The affected heater sheath and heater were removed and replaced and the discharge trough was lifted onto additional refractory bricks inserted into the discharge chamber. Once returned to service, consistent supply of power was obtained throughout Test 4 with no difficulties maintaining the desired discharge chamber temperature. Although a portion of the available power was diverted during Tests 2 and 3, sufficient power was available to process feed to achieve steady state production rates in these tests.

\subsection{Glass Production Rates}

A primary objective of these tests was to measure glass production rates with added bubbling outlets. Glass production rates are illustrated in Figures 3.1.a - 3.1.d to address this question. Also of interest are the test average and steady state values, which are provided in Table 3.1. Steady state values were determined by eliminating portions of tests that were not indicative of the operating conditions, such as startup as the cold cap is developed and prior to achieving a consistent bubbling rate. The production rates were calculated from feed rate data and the analyzed solids content of the feed samples taken during each test (see Section 5.1). All of the measured feed solids contents approximated the target value except for the sample from the terminal portion of Test 1, which therefore is not considered as steady state. Evidently, the last feed transfer from the mix tank to the feed tank was lower in solids, presumably due to excess water with the transfer, which resulted in material with a lower solids content and lower glass yield being fed to the melter towards the end of Test 1. During this latter portion of Test 1 , the melter was fed at the target rate in terms of kilograms of feed per hour; however, since the amount of glass per unit mass of feed decreased, the glass production rate and the amount of bubbling required to maintain a complete cold cap decreased below the target and steady state values.

Feed and bubbling rates were increased gradually during the last test to achieve steady state conditions during the latter half of the test in order to achieve the highest production rate of the test series without exceeding the available power to the electrodes. Steady state operation with respect to processing rates and bubbling rates was achieved for at least 20 hours of each test.

The results from these tests clearly show increasing glass production rates with increasing number of bubbler outlets while processing at the same constant bubbling rate and with increasing bubbling rate when using the same number of bubbling outlets. Over Tests 1, 2, and 4, conducted at $78 \mathrm{lpm}$ bubbling, the glass production rate increased from about $1060 \mathrm{~kg} / \mathrm{m}^{2} /$ day using 4 bubbling outlets to about $1290 \mathrm{~kg} / \mathrm{m}^{2} /$ day using 5 outlets to nearly $1400 \mathrm{~kg} / \mathrm{m}^{2} /$ day using six outlets, as shown 
The Catholic University of America Vitreous State Laboratory
Testing of Optimized Bubbler Configuration for HLW Melter Final Report, VSL-13R2950-1, Rev. 0

in Figure 3.2. During Tests 3 and 4, conducted with 6 bubbling outlets, glass production rates increased from about $1250 \mathrm{~kg} / \mathrm{m}^{2} /$ day when flowing $60 \mathrm{lpm}$ air through the bubblers, to nearly $1400 \mathrm{~kg} / \mathrm{m}^{2} /$ day when flowing $78 \mathrm{lpm}$ through the bubblers. Specific increases in response to the varied test parameters were as follows:

- $32 \%$ increase in production rate (1062 vs. $1398 \mathrm{~kg} / \mathrm{m}^{2} /$ day) at constant bubbling rate in response to increasing the number of bubbling outlets from 4 to 6 .

- $21 \%$ increase in production rate (1062 vs. $1289 \mathrm{~kg} / \mathrm{m}^{2} /$ day) at constant bubbling rate in response to increasing the number of bubbling outlets from 4 to 5 .

- $8 \%$ increase in production rate (1289 vs. $1398 \mathrm{~kg} / \mathrm{m}^{2} /$ day) at constant bubbling rate in response to increasing the number of bubbling outlets from 5 to 6 .

- $12 \%$ increase in production rate (1251 vs. $1398 \mathrm{~kg} / \mathrm{m}^{2} /$ day) using 6 bubbling outlets in response to increasing the bubbling rate from 60 to $78 \mathrm{lpm}$.

It should be noted that bubbling rates were fixed at values obtained while processing with the fewest number of bubbling outlets and that bubbling rates were not optimized for any of the tests. If higher bubbling rates were used in these tests, higher production rates would likely have been obtained. In previous tests processing with the same glass composition and feeds containing faster melting forms of aluminum (hydroxide and boehmite) using four bubbling outlets, bubbling rates of 101 and $124 \mathrm{lpm}$ were required to achieve production rates between 1450 and $1500 \mathrm{~kg} / \mathrm{m}^{2} /$ day, respectively [20,21]. In comparison, the amount of bubbling distributed amongst 6 outlets required to achieve a production rate of nearly $1400 \mathrm{~kg} / \mathrm{m}^{2} /$ day with a slower melting form of aluminum was only $78 \mathrm{lpm}$. Increasing bubbling from $78 \mathrm{lpm}$ to 101 or $124 \mathrm{lpm}$ with the six bubbler outlet configuration can be expected to result in production rates well exceeding $1500 \mathrm{~kg} / \mathrm{m}^{2} /$ day given the improvement observed in response to the modest increase in bubbling from 60 to $78 \mathrm{lpm}$.

Comparison of the data from the current tests with data collected from previous tests processing high aluminum wastes [20,21] corroborates the relative melt rates of the various forms of aluminum. Depicted in Figure 3.3 is the amount of bubbling required to produce glass on the DM1200 melter at $1050 \mathrm{~kg} / \mathrm{m}^{2} /$ day using the nominal four bubbler outlet configuration using aluminum oxide, aluminum hydroxide, and boehmite as the aluminum source. All the tests targeted the same glass composition with a target concentration of $24 \mathrm{wt} \% \mathrm{Al}_{2} \mathrm{O}_{3}$. The amount of bubbling was the least with boehmite (62 lpm), most with aluminum oxide (78 lpm), and intermediate with aluminum hydroxide (71 lpm), indicating that boehmite is the easiest to process and aluminum oxide is the most difficult to process. This trend corroborates melt rate results obtained at constant bubbling rates on the DM100 melter [20, 21].

\subsection{Monitored Melter Parameters}

Measured plenum temperatures, given in Figure 3.4.a - 3.4.d, spanned a wide range during the testing, from near 300 to over $900^{\circ} \mathrm{C}$. Plenum temperatures measured at five of the six locations 
The Catholic University of America Vitreous State Laboratory
Testing of Optimized Bubbler Configuration for HLW Melter Final Report, VSL-13R2950-1, Rev. 0

were above $600^{\circ} \mathrm{C}$ only at the beginning of each test, as the cold cap was being formed, and at the conclusion of each test after feeding was discontinued as the cold was incorporated into the glass melt. Plenum temperatures were mostly below $550^{\circ} \mathrm{C}$ during steady state portions of the first two tests and mostly below $500^{\circ} \mathrm{C}$ during steady state portions of the last two tests, indicative of a complete cold cap in accordance with the visual observations recorded in Table 3.3. Measured plenum temperatures in Port B2 were about a hundred degrees higher than those measured in the other five locations, suggesting that Port B2 is in immediate proximity to an opening in the cold cap directly above a bubbler outlet. The test average steady state plenum temperatures are graphically illustrated in Figures 3.5.a - 3.5.d. The temperature gradient across the plenum space ranged from about 100 to $150^{\circ} \mathrm{C}$ and was relatively consistent over the four tests, suggesting that the addition of bubbling outlets did not result in openings in the cold cap around the added bubblers. It is important to note that with bubblers being added in Ports A1 and D3, there is no direct monitoring of plenum temperature in these corners of the melter. In the previous test sequence, which had monitoring in these ports [22], the highest plenum temperatures were measured in Port D3, near the exhaust outlet. The higher measured temperatures at Port D3 were not attributable to closer proximity to bubbling outlet locations but due to air from the airlift, which can flow between the melter wall and the cold cap to produce an opening in the cold cap and increased radiant heat at this location. It is suspected that measured temperatures around Port D3 in the current tests would also have been similarly elevated, thus obscuring any effect from the added bubblers.

A variety of other operational parameter measurements recorded during these tests, including temperatures throughout the melter system, are given in Table 3.4. The target glass temperature of $1150^{\circ} \mathrm{C}$ was successfully averaged for most of the glass pool during each test, as illustrated in Figures 3.6.a - 3.6.d. Exceptions were near the surface (27" from the floor), where temperatures were lower due to the thermocouples being in or near the cold cap. A $10-20^{\circ} \mathrm{C}$ gradient across the East and West side of the melter was observed throughout the tests, complicating power adjustments to maintain desired temperature. In the first three tests, the West side was hotter than the East; this trend was reversed in Test 4 after the discharge chamber was repaired. The East and West side electrode temperatures varied mostly over the narrow range of $1100-1160^{\circ} \mathrm{C}$ while feeding with an established cold cap; the two side electrode temperatures were the same in the first two tests while the East electrode was about $20^{\circ} \mathrm{C}$ higher than the West in tests employing six bubbling outlets. The bottom electrode, which was not powered in these tests, was about $90^{\circ} \mathrm{C}$ cooler than the West side electrode while feeding. The discharge chamber was largely maintained above $1000^{\circ} \mathrm{C}$ throughout most of the tests, with temperatures about $50^{\circ} \mathrm{C}$ higher after the discharge chamber was repaired after Test 3. Temperatures in one portion of the discharge chamber averaged only $941^{\circ} \mathrm{C}$ during Test 3 due to the loss of one of the heating elements.

Gas temperatures after the film cooler averaged between 235 and $385^{\circ} \mathrm{C}$ depending on the plenum temperature during each test segment. The film cooler was cleaned by a water spray every 12 hours during most of the testing, resulting in a short-duration reduction of about $75^{\circ} \mathrm{C}$ in the film cooler outlet temperature. 
The Catholic University of America Vitreous State Laboratory
Testing of Optimized Bubbler Configuration for HLW Melter Final Report, VSL-13R2950-1, Rev. 0

Conditions in the glass pool are illustrated for voltage and current in Figures 3.7.a - 3.7.d, electrode power and glass resistance in Figures 3.8.a - 3.8.d, and level and density in Figures 3.9.a 3.9.d. Voltage and current in the glass pool increased to steady state values over the course of each test; voltage to 120 -135 volts and current 1450 to 1700 amps, depending on feed rate and condition of the discharge trough. The spikes in voltage and drops in current in the latter portion of Test 2 and all of Test 3 are associated with the discharging of glass and the contact between the discharge trough and the heater sheath. Once the discharge chamber was repaired, the amount of current actually decreased from an average of about 1700 amps in Test 3 to about 1600 amps in Test 4, even though the production rate increased. Power supplied to the electrodes was relatively constant once the cold cap was established during steady state for each test. Power usage largely varied between 175 and $215 \mathrm{~kW}$, increasing with increasing production rate. This level of power utilization is consistent with the 200 and $225 \mathrm{~kW}$ used while processing the high aluminum waste at the higher feed rates [22]. Glass pool resistance decreased from 0.09 to 0.07 ohms over the course of the first three tests in part due to the shorting out of the glass during discharging as a result of the trough being in contact with the heater sheath in the discharge chamber. During Test 4, the glass resistance varied little from 0.08 ohms and better reflects the resistance based on the glass composition. The glass pool density varied little around the average value of $2.26 \mathrm{~g} / \mathrm{cc}$ due to the lack of compositional change during testing and the absence of foaming during the tests. The glass pool level varied between 28 and 31 inches, with frequent decreases in height of about two inches in response to glass discharging.

Bubbling rates used to provide melt pool agitation for the four tests are depicted in Figures 3.10.a - 3.10.d and listed in Table 3.4. In Tests 1 and 3, bubbling was adjusted to achieve a target production rate, while in Tests 2 and 4 bubbling was held constant at rates used to achieve a target specific production rate in a preceding test. The air flow through each outlet was the same within each test. A flow of $1.2 \mathrm{lpm}$ is assumed through the electrode bubblers throughout the tests. Bubbling rates from the two double ported bubblers were adjusted to a flow of $78 \mathrm{lpm}$ during the initial test to achieve a complete cold cap while processing feed at $1050 \mathrm{~kg} / \mathrm{m}^{2} /$ day. The target total flow rate was distributed between five and six bubbling outlets in Tests 2 and 4, respectively. Bubbling rates decreased in the latter portion of Test 1 as the solids content of the feed decreased. Target bubbling was achieved in Test 2 in a short amount of time, while during Test 4, bubbling and feed rates were increased gradually over the first day of testing to ensure that enough power was available to conduct the test. 
The Catholic University of America

Vitreous State Laboratory
Testing of Optimized Bubbler Configuration for HLW Melter

Final Report, VSL-13R2950-1, Rev. 0

\section{SECTION 4.0 \\ DM1200 OFF-GAS SYSTEM PERFORMANCE}

Tests on the DM1200 system at VSL have been used extensively to evaluate the performance of a pilot scale off-gas system that is prototypical of that designed for the WTP by BNI engineering [4-22]. In the present tests, the objective of the work was to determine the glass production rate increase and ancillary effects of adding more bubbler outlets to the current WTP HLW melter baseline. Thus, the performance of the off-gas system, although important to support the operation of the melter, was not a primary objective for investigation during the present tests. However, data for each of the off-gas system components were collected and evaluated and are provided in this report. Data are collected and electronically logged every two minutes and data and observations are also recorded manually throughout the tests. The average, minimum, and maximum values of the measured off-gas system parameters are given in Table 4.1. Target operational conditions for the system components such as sump temperatures, unit spray rates, and sump $\mathrm{pH}$ values that were not specified were adapted from previous tests conducted on the DM1200 [15]. For these tests the silver mordenite / activated carbon system was not used and the catalytic unit was bypassed.

Plots of the typical sequence of gas temperatures through the DM1200 off-gas system at various locations are given in Figures 4.1- 4.4 for the four tests. The SBS cooling system, as discussed below, acts to maintain SBS outlet temperatures at a selected operational value. In summary, plenum gas from the melter is cooled by dilution with film cooler air to about $364^{\circ} \mathrm{C}$, drops approximately another $69^{\circ} \mathrm{C}$ by control air dilution and heat loss along the transition line, is quenched to about $44^{\circ} \mathrm{C}$ in the SBS, and reheated to about $60^{\circ} \mathrm{C}$ to prevent condensation in the HEPA filtration unit. The exhaust is typically heated by another $25^{\circ} \mathrm{C}$ by the Paxton blowers. A slight piping heat loss occurs from that point to the PBS inlet. The gas temperature is about $81^{\circ} \mathrm{C}$ at the PBS inlet.

\subsection{Melter Pressure}

A vacuum on the melter of between two to three and a half inches of water was targeted and maintained throughout the majority of the tests. This is achieved by setting blower speeds and using a control air system that constantly monitors the vacuum on the melter and injects sufficient air into the transition line immediately downstream of the film cooler to maintain a relatively constant vacuum on the melter. The melter pressures measured at the instrument port and by the level detector are shown in Figures 4.5-4.8. The melter pressure fluctuated between 0.7 and -6.3 in W.C. throughout the tests in response to changes in feeding and cold cap conditions. During all four tests, positive melter pressure spikes were observed before and after off-gas sampling events when ports were opened. Melter pressure fluctuates constantly, mostly between -0.5 and -4 inches water, and does not directly correlate with feed rate or plenum temperature within the parameters used in these tests. Similarly, the calculated control air flow rates, as shown in Figures 4.9-4.12, do not appear to 
The Catholic University of America Vitreous State Laboratory
Testing of Optimized Bubbler Configuration for HLW Melter Final Report, VSL-13R2950-1, Rev. 0

directly correlate with melter feed rate or plenum temperature within the ranges investigated in these tests. The range of control air flow rates reflect the changes of melter exhaust volume in response to changes in the cold cap and feed rate, including pulsing of the feed throughout the tests.

Differential pressure measurements across the film cooler are provided in Figures 4.13- 4.16. During Test 2 at 10.1 hours of operations, the film cooler differential pressure exceeded 8.0 in W.C., and rodding of the film cooler was required. The single occurrence of clogging in the film cooler (for the entire four-test period) indicates relatively small amounts of solids carryover from the melter resulting from more complete cold cap coverage and/or a greater effectiveness of the new film cooler at preventing solids accumulations.

\subsection{SBS}

The SBS quenches the melter exhaust, condenses much of the water from the melter feed, and removes the majority of the particulate in the exhaust stream. Many parameters of the SBS were recorded during testing, including inlet and outlet gas temperatures, pressures, pressure drops, sump temperature, heat exchanger inlet and outlet water temperatures, and flow rates. The amounts of heat removed by the SBS jacket and the SBS inner cooling coil were calculated from the measured data using the hourly averaged cooling water temperature increases (outlet temperature minus supply temperature) across the SBS inner cooling coil and cooling jacket multiplied by the same timeaveraged water flow rate through each.

The SBS inlet and outlet gas temperatures are plotted in Figures 4.17-4.20. The average SBS inlet and outlet gas temperatures were $289^{\circ} \mathrm{C}$ and $45.3^{\circ} \mathrm{C}$ during Test $1,305^{\circ} \mathrm{C}$ and $43.6^{\circ} \mathrm{C}$ during Test $2,301^{\circ} \mathrm{C}$ and $43.7^{\circ} \mathrm{C}$ during Test 3 , and $286^{\circ} \mathrm{C}$ and $43.9^{\circ} \mathrm{C}$ during Test 4 . SBS inlet, outlet, and differential pressures are plotted in Figure 4.21 for Test 1 and in Figure 4.24 for Test 4 . The SBS outlet gas pressure indicator did not work properly during Tests 2 and 3. SBS inlet and differential pressures are plotted in Figure 4.22 for Test 2 and in Figure 4.23 for Test 3. Differential pressure averages were 33.3 and 41.1 in W.C., and inlet pressures averages were -9.6 and -7.9 in W.C. for Tests 2 and 3, respectively. Differential pressure averages were 30.1 and 30.5 in W.C., inlet pressures averages were -8.8 and -8.6 in W.C., and outlet pressures averages were -38.6 and -38.4 in W.C. for Tests 1 and 4, respectively.

The SBS off-gas temperatures in the down-comer measured at various depths (from 3 to 53 inches) and the SBS sump water temperature are given in Figures 4.25-4.28. During Test 3, after 31.9 hours of operations, the thermocouple at 43 inches failed. The average SBS sump temperatures were $40.1^{\circ} \mathrm{C}$ (Test 1 ), $37.7^{\circ} \mathrm{C}$ (Test 2), $37.4^{\circ} \mathrm{C}$ (Test 3 ) and $38.6^{\circ} \mathrm{C}$ (Tests 4 ), which are each about 5 to $6^{\circ} \mathrm{C}$ lower than the corresponding SBS outlet gas temperature. The measured off-gas temperatures decrease as the depth from the SBS lid increases due to cooling of the gas in the down-comer pipe by the surrounding SBS liquid. 
The Catholic University of America Vitreous State Laboratory
Testing of Optimized Bubbler Configuration for HLW Melter Final Report, VSL-13R2950-1, Rev. 0

Water temperatures at the SBS inner cooling coil inlet, inner cooling coil outlet/jacket inlet, and jacket outlet are given in Figures 4.29-4.32. The average water temperature differences were $20.0^{\circ} \mathrm{C}$ (Test 1$), 15.6^{\circ} \mathrm{C}$ (Test 2), $14.5^{\circ} \mathrm{C}$ (Test 3 ) and $17.2^{\circ} \mathrm{C}$ (Test 4 ) across the SBS inner cooling coil, and $2.3^{\circ} \mathrm{C}$ (Test 1 and Test 2), $2.8^{\circ} \mathrm{C}$ (Test 3) and $2.1^{\circ} \mathrm{C}$ (Test 4) across the jacket. The SBS cooling coil and jacket water flow rates are plotted in Figures 4.33- 4.36, and averaged $19.9 \mathrm{gal} / \mathrm{min}$ (Test 1), $33.0 \mathrm{gal} / \mathrm{min}$ (Test 2), $33.8 \mathrm{gal} / \mathrm{min}$ (Test 3), and $29.4 \mathrm{gal} / \mathrm{min}$ (Test 4).

Figures 4.37-4.40 show the calculated heat loads during all four tests. During Test 1, heat removal averaged $85.3 \mathrm{~kW}$ by the SBS inner cooling coil and $10.1 \mathrm{~kW}$ by the cooling jacket. This corresponds to about $89.4 \%$ of the heat load to the SBS being removed by the inner cooling coil and about $10.6 \%$ by the cooling jacket. During Test 2, heat removal averaged $109.0 \mathrm{~kW}$ by the SBS inner cooling coil and $16.6 \mathrm{~kW}$ by the cooling jacket. This corresponds to about $86.8 \%$ of the heat load to the SBS being removed by the inner cooling coil and about $13.2 \%$ by the cooling jacket. During Test 3, heat removal averaged $103.3 \mathrm{~kW}$ by the SBS inner cooling coil and $21.0 \mathrm{~kW}$ by the cooling jacket. This corresponds to about $83.1 \%$ of the heat load to the SBS being removed by the inner cooling coil and about $16.9 \%$ by the cooling jacket. During Test 4, heat removal averaged $105.8 \mathrm{~kW}$ by the SBS inner cooling coil and $14.2 \mathrm{~kW}$ by the cooling jacket. This corresponds to about $88.2 \%$ of the heat load to the SBS being removed by the inner cooling coil and about $11.8 \%$ by the cooling jacket.

One of the functions of the SBS is to condense water that originated in the melter feed. In Figures 4.41-4.44, the amount of water fed is compared to the total volumetric accumulations in the SBS during testing. The difference between the amounts of water coming from the feed and the amounts blown down from the SBS sump represent the amount of water carried out in the off-gas stream as a result of it being saturated at the SBS sump temperature, as well as a small amount of entrained droplets. This amount is largely determined by the SBS sump water temperature. In Test 1 , of the 1223 gal of water entering the SBS as part of the melter exhaust stream, 481 gal or $39 \%$ was condensed in the SBS. For Test 2, of the 1385 gal of water entering the SBS as part of the melter exhaust stream, 690 gal or $50 \%$ was condensed in the SBS. In Test 3, of the 1376 gal of water entering the SBS as part of the melter exhaust stream, 661 gal or $48 \%$ was condensed in the SBS. For Test 4, of the 1472 gal of water entering the SBS as part of the melter exhaust stream, 1000 gal or $68 \%$ was condensed in the SBS. Total blow-down volumes for the SBS (and other components) are summarized in Table 4.2. Since the sump temperature was relatively constant throughout the four tests, the percent of feed water condensed in the SBS increased with the feed rate.

\subsection{WESP}

The primary function of the WESP is to remove fine, often water soluble, particles from the exhaust stream that are not efficiently removed by the SBS. The inlet and outlet gas temperatures, differential pressure across the WESP, and the WESP current and voltage were measured and recorded by the computer data acquisition system. The WESP inlet and outlet gas temperatures for the test are plotted in Figures 4.45-4.48. Temperature increases of $0.2^{\circ} \mathrm{C}$ (Test 1 ), $1.5^{\circ} \mathrm{C}$ (Test 2), $3.0^{\circ} \mathrm{C}$ (Test 3 ) and $2.6^{\circ} \mathrm{C}$ (Test 4) were observed in the exhaust as gas passed through the WESP. 
The Catholic University of America Vitreous State Laboratory
Testing of Optimized Bubbler Configuration for HLW Melter Final Report, VSL-13R2950-1, Rev. 0

The periodic downward spikes in the WESP outlet temperature are a result of the daily deluge of the WESP to wash collected deposits off the electrodes and into the WESP sump. The WESP outlet gas flow rates are plotted in Figures 4.49-4.52. The measured differential pressure across the WESP averaged 3.6 in W.C. for Test 1, 4.0 in W.C. for Test 3, 3.2 in W.C. for Test 3, and 3.4 in W.C. for Test 4. The typical average wet gas flow rate exiting the WESP was between 233 and 259 scfm during these tests. The WESP voltage and current are plotted as functions of run time in Figures 4.53-4.56. During Test 1, the voltage averaged $29.6 \mathrm{kV}$ and the current averaged $8.9 \mathrm{~mA}$. During Test 2, the voltage averaged $30.4 \mathrm{kV}$ and the current averaged $10.8 \mathrm{~mA}$. During Test 3 , the voltage averaged $30.0 \mathrm{kV}$ and the current averaged $11.4 \mathrm{~mA}$. During Test 4, the voltage averaged $30.2 \mathrm{kV}$ and the current averaged $10.0 \mathrm{~mA}$. The current and voltage increased after deluges in the middle of each test. These increases were minimal after Test 1 suggesting a minimal amount of solids accumulation on the WESP elements at this stage of testing.

\subsection{Secondary Off-Gas System}

A HEME filtration unit (HEME 1) follows the WESP in the off-gas system to remove water droplets that may be present in the water-saturated gas exiting the WESP. The outlet gas temperature and differential pressure are plotted in Figures 4.57- 4.60. The typical pressure drop across HEME 1 during testing was between 1.6 and 1.9 in W.C.

The HEME is followed in the off-gas system by a heater, a HEPA filter (HEPA 1), and a Paxton blower (Blower 1). The purpose of the heater is to ensure that water-saturated gas exiting HEME 1 is heated above its dew point before passing through the HEPA filter in order to prevent moisture condensation in the HEPA filter. The outlet gas temperature and the pressure differential across the HEPA filter are the two parameters monitored by the off-gas data acquisition system; these are shown in Figures 4.61-4.64. During Test 4 the heater inadvertently was not turned on until 7.5 hours of run time. The typical pressure drop across the HEPA filter was 0.2 in W.C. throughout testing. A vacuum is maintained on the melter by a pair of redundant Paxton blowers (Blowers 701 and 702) immediately downstream of the HEPA filtration unit and a blower (Blower 801) downstream of the packed bed scrubber. The thermal catalytic oxidizer (TCO) and selective catalytic reduction unit (SCR) are located downstream of the HEPA filter and Paxton blowers in the off-gas train; however, these units were bypassed during the present tests.

A packed bed caustic scrubber (PBS) is used near the end of the off-gas train to remove acid gases from the off-gas stream. The PBS sump solution is derived from process water; caustic solution $(25 \% \mathrm{NaOH})$ is added to control the solids content and $\mathrm{pH}$ of the scrubber liquid. The PBS inlet gas temperatures and pressure drops across the PBS are shown in Figures 4.65-4.68. The average pressure drops across the PBS were 3.6 in W.C. during Test 1, 3.8 in W.C. during Test 2, 3.1 in W.C. during Test 3, and 3.2 in W.C. during Test 4. The average inlet gas temperature of about $77-84^{\circ} \mathrm{C}$ was quenched to about $26-28^{\circ} \mathrm{C}$ in the PBS during these tests. The $\mathrm{pH}$ for the PBS is plotted in Figures 4.69-4.72. In all four tests the $\mathrm{pH}$ was generally maintained above 8.2, as shown in Figures 4.69-4.72 with little addition of caustic needed. The PBS was periodically blown down as required to maintain constant volume. The PBS total blow-down volumes are included in Table 4.2. 
The Catholic University of America Testing of Optimized Bubbler Configuration for HLW Melter Vitreous State Laboratory

Final Report, VSL-13R2950-1, Rev. 0

A second HEME (HEME 2) is present near the end of the off-gas train, upstream of the stack blower, to prevent entrained water droplets from entering the stack. The HEME 2 total blow-down volumes are included in Table 4.2. 
The Catholic University of America

Vitreous State Laboratory
Testing of Optimized Bubbler Configuration for HLW Melter

Final Report, VSL-13R2950-1, Rev. 0

\section{SECTION 5.0 \\ FEED SAMPLE AND GLASS PRODUCT ANALYSIS}

\subsection{Analysis of Feed Samples}

\subsubsection{General Properties}

Samples from each of the six as-received feed batches were analyzed to adjust feed solids content and to verify chemical composition. Feed sampled while testing was also analyzed to confirm physical properties and chemical composition. Sample names, sampling dates, and measured properties are given in Table 5.1. Density, $\mathrm{pH}$, water content, glass conversion ratio, boron and lithium content by DCP, and oxide composition by XRF were measured for all samples. The average measured solids content of the as-received feed served as the basis for determining the amount of water required to achieve the target solids content of 500 g glass per liter of feed. The measured feed to glass ratios varied from 0.381 to 0.44 , illustrating the variability in solids content between the six as-received feed batches. This variability was addressed by transferring equal numbers of drums from each batch for each test to ensure uniformity in feed solids content over the four tests. The measured glass conversion ratios for feed samples taken during testing were relatively uniform, varying around 0.35 to 0.36 except for the sample taken at the end of Test 1 , which had a measured feed to glass conversion ratio of only 0.25 . Feed processing changed dramatically after the last feed transfer during Test 1, which is consistent with the addition of feed with a lower solids content (see Section 3.1) confirming the low solids analysis. Based on the feed sample analysis, feed to glass conversion ratios of 0.36 for Test 2 and most of Test $1,0.35$ for Tests 3 and 4 , and 0.25 for the latter portion of Test 1 were used to calculate feed rates during the melter tests. The measured water content and density are consistent with the solids content measured on a weight per weight basis; the as-received feed has a lower water content and higher density than the feed from tests, which was diluted with water to the target solids content. Measured $\mathrm{pH}$ values were about half a unit higher for diluted feed from tests than the as-received feed. The feed from the end of Test 1 had the highest $\mathrm{pH}$ as a result of the greater degree of dilution.

\subsubsection{Chemical Composition}

The methods used for analysis of feed sample chemical compositions are described in Section 1.4. The boron and lithium oxide concentrations measured using the DCP procedure and a fluorine concentration indicative of the typical retention in glass were used for normalizing the XRF data since their concentrations were not determined by XRF. These results, compared to the target composition in Tables 5.2 and 5.3, generally corroborate the consistency of the feed compositions and show good agreement with the target compositions for the major elements. Of the oxides with target concentrations greater than one percent, only average melter feed sample concentrations of bismuth exceed $10 \%$ from target. All other oxide concentrations deviated by less than five and a half 
The Catholic University of America Vitreous State Laboratory
Testing of Optimized Bubbler Configuration for HLW Melter Final Report, VSL-13R2950-1, Rev. 0

percent and aluminum deviated by less than three percent. The absolute deviations for bismuth are less than 0.14 percent. Other than the feed sample taken at the conclusion of Test 1 which contains an excess of aluminum and a greater than average deficiency of boron, there are no obvious differences between the analyses of as-received and test feed samples. The composition of this feed is further corroborated by comparison to the product glasses (see Section 5.2), which shows all oxides with concentrations greater than $1 \mathrm{wt} \%$ in the target composition to be within $9 \%$ of the target. Low concentrations of manganese were measured even though manganese was not included in the target composition. Also, magnesium and titanium were targeted at low concentrations and were well above these targets. These positive deviations are often observed in melter feeds due to the ubiquity of these elements in the raw materials used to make up the simulants and in the glass forming additives. None of these relatively small deviations would significantly affect the glass processing rates.

\subsection{Analysis of Glass Samples}

Over eleven and a half metric tons of glass was produced in the present tests. The glass was discharged from DM1200 into 55 gallon drums using an airlift system. The discharged product glass was sampled at the end of each test by removing sufficient glass from the top of the cans for total inorganic analysis. Product glass masses and discharge date are given in Table 5.4.

All discharge glass samples were crushed and analyzed directly by XRF. The measured boron and lithium concentrations for the glass pool prior to testing and the average measured concentrations for boron and lithium oxides in feed samples were used to calculate boron and lithium concentrations that were subsequently used for normalizing the XRF data to $100 \mathrm{wt} \%$. Fluorine analysis by XRF required a polished monolith as opposed to the standard ground glass preparation used for the other elements. Half the glass samples discharged were directly analyzed for fluorine; fluorine concentrations of other glasses were interpolated between the measured values. The XRF analyzed compositions of discharged glass samples are provided in Table 5.5. The melt pool composition at the beginning of testing was very similar to the target composition and therefore the composition of the discharged glasses approximated the target composition. The average XRF analysis of glass discharged over the course of the tests compared very favorably to the target values and feed sample analyses (see Section 5.1.2). Oxides with a target concentration greater than one weight percent all showed below 10\% deviation from the target values. Compositional trends for selected constituents shown in Figures 5.1.a - 5.1.f show the consistency in melt pool composition throughout the tests. At the onset of testing, small increases in chromium, nickel, and calcium at the expense of silicon and zirconium were observed as the glass pool even more closely approached the target composition. Sulfur and fluorine are below target for discharged glasses due to volatilization from the glass pool and cold cap. Over the course of the tests, fluorine and sulfur increase to steady state concentrations of about forty five and eighty percent of the target values, respectively. 
The Catholic University of America

Vitreous State Laboratory
Testing of Optimized Bubbler Configuration for HLW Melter

Final Report, VSL-13R2950-1, Rev. 0

\section{SECTION 6.0 \\ MONITORED OFF-GAS EMISSIONS}

\subsection{Particulate Sampling}

The melter exhaust was sampled for metals/particles according to 40-CFR-60 Methods 3, 5, and 29 at steady-state operating conditions during each test segment. The concentrations of off-gas species that are present as particulates and gaseous species that are collected in impinger solutions were derived from laboratory data on solutions extracted from air samples (filters and various solutions) together with measurements of the volume of air sampled. Particulate collection required isokinetic sampling, which entails removing gas from the exhaust at the same velocity that the air is flowing in the duct (40-CFR-60, Methods 1-5). Typically, a sample size of 30 dscf was taken at a rate of between 0.5 and $0.75 \mathrm{dscfm}$. Total particulate loading was determined by combining gravimetric analysis of the standard particle filter and chemical analysis of probe rinse solutions. An additional impinger containing $2 \mathrm{~N} \mathrm{NaOH}$ was added to the sampling train to ensure complete scrubbing of all acid gases. The collected materials were analyzed using direct current plasma atomic emission spectroscopy for the majority of the constituents and ion chromatography (IC) for anions. Melter emission fluxes are compared to feed fluxes in Table 6.1. Notice the distinction that is made between constituents sampled as particles and as "gas". The "gaseous" constituents are operationally defined as those species that are scrubbed in the impinger solutions after the air stream has passed through a $0.3 \mu \mathrm{m}$ heated filter. All samples are well within the $90-110 \%$ limits for isokinetic sampling.

Particulate emissions from the DM1200 constituted 0.13 to 0.18 percent of feed over the four tests. These results are within the range of 0.1 to 0.46 percent measured while processing the same waste and glass composition using the nominal four-outlet bubbler configuration over a variety of feed solids contents, glass temperatures, operational strategies, aluminum sources, and bubbling rates [20-22]. Particulate carryover while processing with the nominal four-outlet bubbler configuration at the production rate of $1050 \mathrm{~kg} / \mathrm{m}^{2} /$ day was 0.13 percent of feed, similar to the 0.1 percent of feed previously measured with feed containing aluminum hydroxide and boehmite as the aluminum source [20, 21]. At production rates between 1400 and $1500 \mathrm{~kg} / \mathrm{m}^{2} /$ day, particulate carryover while processing with six bubbling outlets in the current tests was only 0.17 percent of feed as compared to 0.16 and 0.46 percent of feed during previous tests processing with the nominal four-outlet bubbler configuration. At production rates around $1300 \mathrm{~kg} / \mathrm{m}^{2} /$ day, particulate carryover while processing with five and six bubbling outlets in the current tests was 0.14 and 0.18 percent of feed as compared to 0.12 percent of feed during previous tests processing with the nominal fouroutlet bubbler configuration [22]. These comparisons and the relatively narrow range of particulate carryover, $0.13-0.18$ percent of feed, suggest that the aluminum form and the number of bubbling outlets used have little effect on the amount of solids carryover from the melter. 
The Catholic University of America Vitreous State Laboratory
Testing of Optimized Bubbler Configuration for HLW Melter Final Report, VSL-13R2950-1, Rev. 0

As expected, the feed elements emitted at the lowest melter decontamination factors (DF) were clearly fluorine and sulfur. Other elements exhibiting some volatile behavior were boron, alkali metals, cadmium, chromium, and lead. The relative volatility of magnesium and titanium are difficult to evaluate due to their low target concentrations in the feed and the ubiquity of these constituents as trace level contaminants in additives and chemicals used to make the waste simulants. Boron, sulfur, and fluorine were the only elements detected in the impinger solutions collected downstream of the heated particle filter in the sampling train, which constitutes the "gas" fraction of the melter emissions.

\subsection{Gases Monitored by FTIR}

Melter emissions were monitored in each test for a variety of gaseous components, most notably CO and nitrogen species, by FTIR. The off-gas system temperature is maintained well above $100^{\circ} \mathrm{C}$ beyond the sampling port downstream of the DM100 HEPA filter to prevent analyte loss due to condensation prior to monitoring. The data, therefore, represent the relative concentrations of volatile gaseous species in the melter exhaust. The exhaust stream was sampled at the outlets of several prototypical components (melter, SBS, WESP, and HEPA filter) to discern the effect these components have on the volatiles in the exhaust stream. It should be noted, however, that the off-gas system component most responsible for the removal of nitrogen oxide and volatile organics, the TCO-SCR catalyst unit, was bypassed in these tests due to the relatively low concentrations of these components in the exhaust stream. Also, a single FTIR unit was used for all of the measurements and, therefore, locations were sampled sequentially and not simultaneously.

A summary of the range and average concentrations of gaseous species monitored is provided in Tables 6.2 - 6.5. The concentrations of two of the monitored species illustrating the two types of behavior observed during the tests are plotted in Figures 6.1.a - 6.2.d. The analytes listed in Tables 6.2 - 6.5 are those that were thought likely to be observed during the tests based on previous work; no other species were detected in the off-gas stream by FTIR. The concentration of water in the melter exhaust increased with increasing feed rate and was mostly consistent with the amounts determined using the Method 5-type sampling (see Section 6.1). Moisture is condensed in the SBS and therefore the water content measured downstream of the SBS depends on the SBS sump temperature. Small reductions in water concentrations are observed further downstream due to minor air dilution through the WESP and immediately upstream of the HEPA filter. Generally, emissions were relatively low as a result of the low concentrations of nitrogen, organic carbon, ammonia, and halogens in the feed. The most abundant nitrogen species monitored was $\mathrm{NO}$, with $\mathrm{NO}_{2}$ being about seven times lower in concentration than NO, which is in keeping with previous melter tests with both HLW and LAW feeds. Low concentrations of ammonia, $\mathrm{N}_{2} \mathrm{O}$, nitric acid, nitrous acid, and HCN were also observed in the tests. Consistent with the gaseous fluorine concentrations observed using the Method 5-type sampling (see Section 6.1), HF was observed throughout the testing by FTIR in response to fluorine being targeted at two thirds of a weight percent in the glass product.

Carbon monoxide was detected at around $2 \mathrm{ppm}$ as a byproduct of incomplete combustion of the feed carbon in the presence of nitrates. The variability in the $\mathrm{NO}$ and $\mathrm{NO}_{2}$ concentrations are attributable to the dynamic conditions in the cold cap and is in keeping with previous melter tests; 
the increase in concentration over the course of the tests reflects the increase in feed rate. Measured concentrations for most constituents at different locations in the DM1200 exhaust system were very similar. This confirms the expectation that the SBS, WESP, HEME, and HEPA do not remove significant proportions of nitrogen and carbon oxides. 
The Catholic University of America Vitreous State Laboratory
Testing of Optimized Bubbler Configuration for HLW Melter Final Report, VSL-13R2950-1, Rev. 0

\section{SECTION 7.0 SUMMARY AND CONCLUSIONS}

A series of tests was conducted on the DM1200 Pilot Melter system to determine the relationship between the number and locations of bubbler outlets and the glass production rate. The tests were performed with a high aluminum HLW stream with aluminum oxide as the aluminum source. Aluminum oxide was chosen as the aluminum source because previous tests have shown this to produce the slowest melting melter feed as compared to gibbsite and boehmite [20]. A slower melting feed, in addition to providing the most conservative test case, allowed better estimation of the effect of bubbler configuration on feed processing rate without running into potential issues such as melter power limitations that could occur with a faster melting feed. For each test, target or maximum production rates were indicated by complete cold cap coverage visually observed and confirmed by plenum temperature readings. Testing began with bubblers in a previously defined and tested bubbler configuration; this consisted of two double-outlet lance bubblers on the melter floor, 8" apart on the East and West side, with one bubbler outlet a horizontal distance of 11.3" from the feed tube location $[4,15,18,20-23]$. As testing progressed, bubbling outlets were added while maintaining either constant bubbling rate or constant feed rate. The bubbler air was equally divided between the bubbling outlets during each test. No significant processing problems were encountered during these tests. The DM1200 tests produced nearly twelve metric tons of glass from over 33 metric tons of feed.

The results from these tests clearly show increasing glass production rates with increasing number of bubbler outlets while processing at the same constant bubbling rate and with increasing bubbling rate when using the same number of bubbling outlets. During tests conducted at $78 \mathrm{lpm}$ total lance bubbling, the glass production rate increased from about $1060 \mathrm{~kg} / \mathrm{m}^{2} /$ day using four bubbling outlets to about $1290 \mathrm{~kg} / \mathrm{m}^{2} /$ day using five outlets and to nearly $1400 \mathrm{~kg} / \mathrm{m}^{2} /$ day using six outlets. During the test conducted with six bubbling outlets, the glass production rate increased from about $1250 \mathrm{~kg} / \mathrm{m}^{2} /$ day when flowing $60 \mathrm{lpm}$ air through the bubblers to nearly $1400 \mathrm{~kg} / \mathrm{m}^{2} /$ day when flowing $78 \mathrm{lpm}$ through the bubblers. Increasing the number of bubbling outlets from four to five and from four to six resulted in increases in production rate of 21 and 32\%, respectively. It should be noted that these increases are lower than can be realized with optimization of bubbler flow rates in conjunction with the addition of bubbling outlets. In the present tests, bubbling rates were fixed at values obtained while processing with the fewest number of bubbling outlets and not optimized for any of the tests. Considerably higher bubbling rates were used with four bubbling outlets in previous tests processing feeds containing easier forms of aluminum to melt than in present tests with six bubbling outlets to achieve comparable production rates. Comparison of the data from the current tests with data collected from previous tests processing high aluminum wastes [20, 21] corroborates the relative melt rates of the various forms of aluminum: aluminum oxide is the slowest to melt followed by aluminum hydroxide then boehmite.

Melter exhaust was sampled for particulate and gaseous species during each test to determine the effect of the number of bubbling outlets used and bubbler configuration on emissions. Total 
The Catholic University of America Vitreous State Laboratory
Testing of Optimized Bubbler Configuration for HLW Melter Final Report, VSL-13R2950-1, Rev. 0

particulate carryover into the off-gas stream ranged between 0.1 and 0.15 percent of feed and was within the range previously measured while processing the same glass composition. Collectively, the past and present emission monitoring data indicate no significant differences in percent carryover with the number of bubbling outlets and aluminum source in the waste. Melter DFs were determined for most elements in the feed. The most volatile species were sulfur and fluorine, which is typical. Gaseous emissions of nitrogen oxides and byproducts of incomplete combustion, such as carbon monoxide and ammonia, were very low due to low concentrations of nitrates and organic carbon in the feed.

The results of the testing presented herein clearly demonstrate the increases in glass production rates by adding more bubblers to WTP HLW melter. These data can be used to support a decision on potential future modifications to the WTP HLW melter system such as a re-design of the melter lid to allow the inclusion of additional bubblers. Such a change would increase the glass production rate that is achievable and add robustness to the WTP HLW vitrification system. Some additional testing would be useful to further underpin such a decision. Some of the elements recommended for development are summarized below.

- $\quad$ Other WTP HLW Waste Types: The present testing was based on one HLW high aluminum waste composition from the Hanford tanks. Waste and melter feed compositions have a significant effect on cold cap formation and processing rate. While these results are also relevant to waste from several tanks, the diversity of the Hanford tank wastes has the potential to result in a variety of different cold cap conditions and therefore glass production rates. Therefore the extent of production rate increase with the addition of more bubblers has yet to be demonstrated over the range of wastes and melter feed compositions projected for HLW WTP operations, particularly those that tend to be slower melting.

- $\quad$ Maximum Attainable Production Rates: The results from the present tests demonstrate that increases in production rate are possible at constant bubbling by adding bubbler outlets. However, it is known from previous testing that for a given number of bubbler outlets, optimization of the bubbler flow rate will lead to yet further increases in glass production rates. It would be useful to quantify the extent of that increase for each bubbler configuration in order to better define the potential glass production rate improvements that are possible.

- $\quad$ Lid Design for WTP HLW Melter: As noted above, inclusion of additional bubblers into the WTP HLW melter system would require a re-design of the melter lid. It is likely that such design work would be supported by computer modeling of the WTP HLW melter with various possible bubbler configurations, as was done for previous WTP melter designs as well as for the Defense Waste Processing Facility (DWPF) bubbler retro-fit. 
The Catholic University of America Vitreous State Laboratory
Testing of Optimized Bubbler Configuration for HLW Melter Final Report, VSL-13R2950-1, Rev. 0

\section{SECTION 8.0 REFERENCES}

[1] “River Protection Project System Plan 6,” P.J. Certa and P.A. Empey, ORP-11242, Rev. 6, Washington River Protection Solutions, LLC, Richland, WA, 99352, 10/5/11.

[2] "Determination of Processing Rate of RPP-WTP HLW Simulants using a DuraMelter 1000 Vitrification System,” K.S. Matlack, W.K. Kot, F. Perez-Cardenas, and I.L. Pegg, Final Report, VSL-00R2590-2, Rev. 0, Vitreous State Laboratory, The Catholic University of America, Washington, DC, 8/21/00.

[3] "Design and Installation of a Prototypical Off-Gas Treatment System for the DM1200 RPPWTP HLW Pilot Melter,” R.T. Anderson, M. Brandys, and R. Jung, Final Report, VSL01R2510-1, Rev. 0, Vitreous State Laboratory, The Catholic University of America, Washington, DC, 2/22/01.

[4] “Summary of DM1200 Operation at VSL,” K.S. Matlack, G. Diener, T. Bardakci, and I.L. Pegg, Final Report, VSL-06R6710-2, Rev. 0, Vitreous State Laboratory, The Catholic University of America, Washington, DC, 9/7/06.

[5] "Start-Up and Commissioning Tests on the DM1200 HLW Pilot Melter System Using AZ101 Waste Simulants," K.S. Matlack, M. Brandys, and I.L. Pegg, Final Report, VSL01R0100-2, Rev. 1, Vitreous State Laboratory, The Catholic University of America, Washington, DC, 10/31/01.

[6] "Tests on the DuraMelter 1200 HLW Pilot Melter System Using AZ-101 HLW Simulants," K.S. Matlack, W.K. Kot, T. Bardakci, T.R. Schatz, W. Gong, and I.L. Pegg, Final Report, VSL-02R0100-2, Rev. 0, Vitreous State Laboratory, The Catholic University of America, Washington, DC, 6/11/02.

[7] "Integrated Off-Gas System Tests on the DM1200 Melter with RPP-WTP LAW SubEnvelope C1 Simulants,” K.S. Matlack, W. Gong, T. Bardakci, N. D'Angelo, and I.L. Pegg, Final Report, VSL-02R8800-1, Rev. 1, Vitreous State Laboratory, The Catholic University of America, Washington, DC, 9/23/03.

[8] "Integrated Off-Gas System Tests on the DM1200 Melter with RPP-WTP LAW SubEnvelope A1 Simulants,” K.S. Matlack, W. Gong, T. Bardakci, N. D’Angelo, and I.L. Pegg, Final Report, VSL-02R8800-2, Rev. 0, Vitreous State Laboratory, The Catholic University of America, Washington, DC, 9/03/02.

[9] "DM1200 Tests with AZ-101 HLW Simulants," K.S. Matlack, W. Gong, T. Bardakci, N. D'Angelo, W.K. Kot, and I.L. Pegg, Final Report, VSL-03R3800-4, Rev. 0, Vitreous State Laboratory, The Catholic University of America, Washington, DC, 2/17/04. 
The Catholic University of America Vitreous State Laboratory
Testing of Optimized Bubbler Configuration for HLW Melter Final Report, VSL-13R2950-1, Rev. 0

[10] "Integrated Off-Gas System Tests on the DM1200 Melter with RPP-WTP LAW SubEnvelope B1 Simulants,” K.S. Matlack, W. Gong, T. Bardakci, N. D’Angelo, and I.L. Pegg, Final Report, VSL-03R3851-1, Rev. 0, Vitreous State Laboratory, The Catholic University of America, Washington, DC, 10/17/03.

[11] "Integrated DM1200 Melter Testing of HLW AZ-102 Compositions Using Bubblers," K.S. Matlack, W. Gong, T. Bardakci, N. D’Angelo, W. Kot and I.L. Pegg, Final Report, VSL03R3800-2, Rev. 0, Vitreous State Laboratory, The Catholic University of America, Washington, DC, 9/24/03.

[12] "Integrated DM1200 Melter Testing of HLW C-106/AY-102 Composition Using Bubblers," K.S. Matlack, W. Gong, T. Bardakci, N. D’Angelo, W. Kot and I.L. Pegg, Final Report, VSL-03R3800-1, Rev. 0, Vitreous State Laboratory, The Catholic University of America, Washington, DC, 9/15/03.

[13] "Integrated DM1200 Melter Testing of HLW C-104/AY-101 Compositions Using Bubblers,” K.S. Matlack, W. Gong, T. Bardakci, N. D’Angelo, W. Kot and I.L. Pegg, Final Report, VSL-03R3800-3, Rev. 0, Vitreous State Laboratory, The Catholic University of America, Washington, DC, 11/24/03.

[14] "Integrated DM1200 Melter Testing of Redox Effects Using HLW AZ-101 and C-106/AY102 Simulants," K.S. Matlack, W. Gong, T. Bardakci, N. D’Angelo, W. Lutze, P. M. Bizot, R. A. Callow, M. Brandys, W.K. Kot, and I.L. Pegg, Final Report, VSL-04R4800-1, Rev. 0, Vitreous State Laboratory, The Catholic University of America, Washington, DC, 5/6/04.

[15] "Integrated DM1200 Melter Testing of Bubbler Configurations Using HLW AZ-101 Simulants," K.S. Matlack, W. Gong, T. Bardakci, N. D’Angelo, W. Lutze, R. A. Callow, M. Brandys, W.K. Kot, and I.L. Pegg, Final Report, VSL-04R4800-4, Rev. 0, Vitreous State Laboratory, The Catholic University of America, Washington, DC, 10/5/04.

[16] "Bubbling Rate and Foaming Tests on the DuraMelter 1200 with LAWC22 and LAWA30 Glasses,” K.S. Matlack, W. Gong, T. Bardakci, N. D’Angelo, P.M. Bizot, R.A. Callow, M. Brandys, and I.L. Pegg, Final Report, VSL-04R4851-1, Rev. 0, Vitreous State Laboratory, The Catholic University of America, Washington, DC, 7/1/04.

[17] "Destruction of Alcohols in DM1200 Melter System During LAW and HLW Vitrification," K.S. Matlack and I.L. Pegg, Letter Report, VSL-03L4850-1, Rev. 0, Vitreous State Laboratory, The Catholic University of America, Washington, DC, 12/16/03.

[18] "Integrated DM1200 Melter Testing Using AZ-102 and C-106/AY-102 HLW Simulants: HLW Simulant Verification,” K.S. Matlack, W. Gong, T. Bardakci, N. D’Angelo, M. Brandys, W.K. Kot, and I.L. Pegg, Final Report, VSL-05R5800-1, Rev. 0, Vitreous State Laboratory, The Catholic University of America, Washington, DC, 6/27/05. 
The Catholic University of America Vitreous State Laboratory
Testing of Optimized Bubbler Configuration for HLW Melter Final Report, VSL-13R2950-1, Rev. 0

[19] "Regulatory Off-Gas Emissions Testing on the DM1200 Melter System Using HLW and LAW Simulants,” K.S. Matlack, W. Gong, T. Bardakci, N. D'Angelo, M. Brandys, W. Kot, and I.L. Pegg, Final Report VSL-05R5830-1, Rev. 0, Vitreous State Laboratory, The Catholic University of America, Washington, DC, 10/31/05.

[20] "Melt Rate Enhancement for High Aluminum HLW Glass Formulations,” K.S. Matlack, H. Gan, M. Chaudhuri, W.K Kot, W. Gong, T. Bardakci, I. Joseph, and I.L. Pegg, Final Report, VSL-08R1360-1, Rev. 0, Vitreous State Laboratory, The Catholic University of America, Washington, DC, 12/19/08.

[21] "DM100 and DM1200 Melter Testing with High Waste Loading Glass Formulations for Hanford High-Aluminum HLW Streams,” K.S. Matlack, H. Gan, M. Chaudhuri, W.K Kot, W. Gong, T. Bardakci, I. Joseph, and I.L. Pegg, Final Report, VSL-10R1690-1, Rev. A, Vitreous State Laboratory, The Catholic University of America, Washington, DC, 5/17/10.

[22] “HLW Melter Control Strategy Without Visual Feedback,” K.S. Matlack, W.K. Kot, H. Abramowitz, R. A. Callow, M. Brandys, I. Joseph, and I.L. Pegg, Final Report, VSL12R2500-1, Rev. 0, Vitreous State Laboratory, The Catholic University of America, Washington, DC, 11/9/11.

[23] “Tests with High-Bismuth HLW Glasses,” K.S. Matlack, H. Gan, W.K. Kot, M. Chaudhuri, R.K. Mohr, D. A. McKeown, T. Bardakci, W. Gong, A. C. Buechele, and I.L. Pegg, Final Report, VSL-10R1780-1, Rev. 0, Vitreous State Laboratory, The Catholic University of America, Washington, DC, 12/13/10.

[24] “Test and Evaluate High Level Waste (HLW) Vitrification System Improvements,” Contract Number DE-AC27-06RV14790, US Department of Energy, Office of River Protection, Richland, WA, April, 2006.

[25] “High Level Waste Vitrification Plant Enhancement Study,” C. Chapman., 24590-HLW RTP-PE-07-001, Rev 0, The River Protection Project, Waste Treatment Plant, Richland Washington, Feb. 282007.

[26] “Testing of Optimized Bubbler Configuration for HLW Melter,” K.S. Matlack, W.K. Kot, I.L. Pegg, and I. Joseph, Test Plan, VSL-12T2950-1, Rev. 0, Vitreous State Laboratory, The Catholic University of America, Washington, DC, 11/29/12.

[27] “Development and Testing of High-Level Waste Glass Compositions," Contract Number DE-EM0002103, US Department of Energy, Office of River Protection, Richland, WA.

[28] "Quality Assurance Project Plan for ORP/ RPP-WTP Support Activities Conducted by VSL,” Vitreous State Laboratory, QAPP-ORP, Rev. 3, Vitreous State Laboratory, The Catholic University of America, Washington, DC, 10/22/12. 
[29] “Master List of Controlled VSL Manuals and Standard Operating Procedures in Use,” QAMLCP, Rev. 90, Vitreous State Laboratory, The Catholic University of America, Washington, DC, 12/3/12.

[30] "High-Level Waste Melter Alternate Bubbler Configuration Testing," R.K Mohr, C.C. Chapman and I.L. Pegg, Final Report, VSL-04R4800-3, Rev. 0, Vitreous State Laboratory, The Catholic University of America, Washington, DC, 6/18/04.

[31] “High Level Waste Vitrification System Improvements,” K.S. Matlack, H. Gan, W. Gong, I.L. Pegg, C.C. Chapman and I. Joseph, VSL-07R1010-1, Rev. 0, Vitreous State Laboratory, The Catholic University of America, Washington, DC, 4/16/07. 
Table 2.1. Oxide Compositions of Limiting Waste Streams.

\begin{tabular}{|c|c|c|c|c|}
\hline $\begin{array}{c}\text { Waste } \\
\text { Component }\end{array}$ & $\begin{array}{c}\text { Bi Limited } \\
\text { Glass }\end{array}$ & $\begin{array}{c}\text { Cr Limited } \\
\text { Glass }\end{array}$ & $\begin{array}{l}\text { Al Limited } \\
\text { Glass }\end{array}$ & $\begin{array}{c}\text { Al and Na } \\
\text { Limited Glass }\end{array}$ \\
\hline $\mathrm{Al}_{2} \mathrm{O}_{3}$ & $22.45 \%$ & $25.53 \%$ & $49.21 \%$ & $43.30 \%$ \\
\hline $\mathrm{B}_{2} \mathrm{O}_{3}$ & $0.58 \%$ & $0.53 \%$ & $0.39 \%$ & $0.74 \%$ \\
\hline $\mathrm{CaO}$ & $1.61 \%$ & $2.47 \%$ & $2.21 \%$ & $1.47 \%$ \\
\hline $\mathrm{Fe}_{2} \mathrm{O}_{3}$ & $13.40 \%$ & $13.13 \%$ & $12.11 \%$ & $5.71 \%$ \\
\hline $\mathrm{Li}_{2} \mathrm{O}$ & $0.31 \%$ & $0.36 \%$ & $0.35 \%$ & $0.15 \%$ \\
\hline $\mathrm{MgO}$ & $0.82 \%$ & $0.16 \%$ & $0.24 \%$ & $0.44 \%$ \\
\hline $\mathrm{Na}_{2} \mathrm{O}$ & $12.97 \%$ & $20.09 \%$ & $7.35 \%$ & $25.79 \%$ \\
\hline $\mathrm{SiO}_{2}$ & $12.04 \%$ & $10.56 \%$ & $10.05 \%$ & $6.22 \%$ \\
\hline $\mathrm{TiO}_{2}$ & $0.30 \%$ & $0.01 \%$ & $0.02 \%$ & $0.35 \%$ \\
\hline $\mathrm{ZnO}$ & $0.31 \%$ & $0.25 \%$ & $0.17 \%$ & $0.36 \%$ \\
\hline $\mathrm{ZrO}_{2}$ & $0.40 \%$ & $0.11 \%$ & $0.81 \%$ & $0.25 \%$ \\
\hline $\mathrm{SO}_{3}$ & $0.91 \%$ & $1.52 \%$ & $0.41 \%$ & $0.44 \%$ \\
\hline $\mathrm{Bi}_{2} \mathrm{O}_{3}$ & $12.91 \%$ & $7.29 \%$ & $2.35 \%$ & $2.35 \%$ \\
\hline $\mathrm{ThO}_{2}$ & $0.25 \%$ & $0.04 \%$ & $0.37 \%$ & $0.04 \%$ \\
\hline $\mathrm{Cr}_{2} \mathrm{O}_{3}$ & $1.00 \%$ & $3.07 \%$ & $1.07 \%$ & $1.44 \%$ \\
\hline $\mathrm{K}_{2} \mathrm{O}$ & $0.89 \%$ & $0.37 \%$ & $0.29 \%$ & $1.34 \%$ \\
\hline $\mathrm{U}_{3} \mathrm{O}_{8}$ & $3.48 \%$ & $7.59 \%$ & $7.25 \%$ & $4.58 \%$ \\
\hline $\mathrm{BaO}$ & $0.02 \%$ & $0.03 \%$ & $0.11 \%$ & $0.06 \%$ \\
\hline $\mathrm{CdO}$ & $0.00 \%$ & $0.01 \%$ & $0.05 \%$ & $0.02 \%$ \\
\hline $\mathrm{NiO}$ & $3.71 \%$ & $1.06 \%$ & $0.82 \%$ & $0.20 \%$ \\
\hline $\mathrm{PbO}$ & $0.48 \%$ & $0.48 \%$ & $0.84 \%$ & $0.18 \%$ \\
\hline $\mathrm{P}_{2} \mathrm{O}_{5}$ & $9.60 \%$ & $3.34 \%$ & $2.16 \%$ & $4.10 \%$ \\
\hline F- & $1.58 \%$ & $2.00 \%$ & $1.37 \%$ & $0.46 \%$ \\
\hline Total & $100.00 \%$ & $100.00 \%$ & $100.00 \%$ & $100.00 \%$ \\
\hline
\end{tabular}


Table 2.2. Compositions of the Al-Limited Waste (Oxide Basis) and the HLW Waste Simulant to Produce $100 \mathrm{~kg}$ of Waste Oxides (20 wt\% suspended solids).

\begin{tabular}{|c|c|c|c|}
\hline \multicolumn{2}{|c|}{ Al-Limited Waste Composition } & \multicolumn{2}{|c|}{ Al-Limited HLW Waste Simulant } \\
\hline Waste Oxide & Wt\% & Starting Materials & Target Weight (kg) ${ }^{1}$ \\
\hline $\mathrm{Al}_{2} \mathrm{O}_{3}$ & $49.21 \%$ & $\mathrm{Al}_{2} \mathrm{O}_{3}$ & 49.707 \\
\hline $\mathrm{B}_{2} \mathrm{O}_{3}$ & $0.39 \%$ & $\mathrm{H}_{3} \mathrm{BO}_{3}$ & 0.700 \\
\hline $\mathrm{CaO}$ & $2.21 \%$ & $\mathrm{CaO}$ & 2.255 \\
\hline $\mathrm{Fe}_{2} \mathrm{O}_{3}$ & $12.11 \%$ & $\mathrm{Fe}(\mathrm{OH})_{3}(13 \%$ Slurry $)$ & 99.643 \\
\hline $\mathrm{Li}_{2} \mathrm{O}$ & $0.35 \%$ & $\mathrm{Li}_{2} \mathrm{CO}_{3}$ & 0.888 \\
\hline $\mathrm{MgO}$ & $0.24 \%$ & $\mathrm{MgO}$ & 0.253 \\
\hline $\mathrm{Na}_{2} \mathrm{O}$ & $7.35 \%$ & $\mathrm{NaOH}$ & 4.235 \\
\hline $\mathrm{SiO}_{2}$ & $10.05 \%$ & $\mathrm{SiO}_{2}$ & 10.152 \\
\hline $\mathrm{TiO}_{2}$ & $0.02 \%$ & $\mathrm{TiO}_{2}$ & 0.020 \\
\hline $\mathrm{ZnO}$ & $0.17 \%$ & $\mathrm{ZnO}$ & 0.172 \\
\hline $\mathrm{ZrO}_{2}$ & $0.81 \%$ & $\mathrm{Zr}(\mathrm{OH})_{4} \cdot x \mathrm{H}_{2} \mathrm{O}$ & 2.093 \\
\hline $\mathrm{SO}_{3}$ & $0.41 \%$ & $\mathrm{Na}_{2} \mathrm{SO}_{4}$ & 0.735 \\
\hline $\mathrm{Bi}_{2} \mathrm{O}_{3}$ & $2.35 \%$ & $\mathrm{Bi}_{2} \mathrm{O}_{3}$ & 2.374 \\
\hline $\mathrm{ThO}_{2}$ & $0.37 \%$ & \multicolumn{2}{|c|}{ Omitted } \\
\hline $\mathrm{Cr}_{2} \mathrm{O}_{3}$ & $1.07 \%$ & $\mathrm{Cr}_{2} \mathrm{O}_{3} \cdot 1.5 \mathrm{H}_{2} \mathrm{O}$ & 1.273 \\
\hline $\mathrm{K}_{2} \mathrm{O}$ & $0.29 \%$ & $\mathrm{KNO}_{3}$ & 0.632 \\
\hline $\mathrm{U}_{3} \mathrm{O}_{8}$ & $7.25 \%$ & \multicolumn{2}{|c|}{ Omitted } \\
\hline $\mathrm{BaO}$ & $0.11 \%$ & $\mathrm{BaCO}_{3}$ & 0.143 \\
\hline $\mathrm{CdO}$ & $0.05 \%$ & $\mathrm{CdO}$ & 0.051 \\
\hline $\mathrm{NiO}$ & $0.82 \%$ & $\mathrm{Ni}(\mathrm{OH})_{2}$ & 1.055 \\
\hline $\mathrm{PbO}$ & $0.84 \%$ & $\mathrm{PbO}$ & 0.848 \\
\hline $\mathrm{P}_{2} \mathrm{O}_{5}$ & $2.16 \%$ & $\mathrm{FePO}_{4} \cdot x \mathrm{H}_{2} \mathrm{O}$ & 5.738 \\
\hline $\mathrm{F}$ & $1.37 \%$ & $\mathrm{NaF}$ & 3.044 \\
\hline Carbonate & $1.20^{2}$ & $\mathrm{Na}_{2} \mathrm{CO}_{3}$ & 0.806 \\
\hline Nitrite & 0.50 & $\mathrm{NaNO}_{2}$ & 0.769 \\
\hline Nitrate & 2.00 & $\mathrm{NaNO}_{3}$ & 2.230 \\
\hline Organic Carbon & 0.05 & $\mathrm{H}_{2} \mathrm{C}_{2} \mathrm{O}_{4} \cdot 2 \mathrm{H}_{2} \mathrm{O}$ & 0.264 \\
\hline- & - & Water & 279.400 \\
\hline TOTAL & $100.0 \%$ & TOTAL & 469.478 \\
\hline
\end{tabular}

${ }^{1}$ Target weights adjusted for assay information of starting materials

${ }^{2}$ Unit for volatile components is $\mathrm{g} / 100 \mathrm{~g}$ of waste oxide

- Empty data field 
Table 2.3. Composition and Properties of Aluminum Limited Waste and Glass Formulation HWI-Al-19 with 45\% Waste Loading (wt\%).

\begin{tabular}{|c|c|c|c|c|}
\hline- & $\begin{array}{c}\text { Al-Limited } \\
\text { Waste }\end{array}$ & Waste in Glass & $\begin{array}{c}\text { Glass Forming } \\
\text { Additives }\end{array}$ & $\begin{array}{c}\text { Target Glass } \\
\text { HWI-Al-19 }\end{array}$ \\
\hline $\mathrm{Al}_{2} \mathrm{O}_{3}$ & 53.27 & 23.97 & - & 23.97 \\
\hline $\mathrm{B}_{2} \mathrm{O}_{3}$ & 0.42 & 0.19 & 19.00 & 19.19 \\
\hline $\mathrm{BaO}$ & 0.12 & 0.05 & - & 0.05 \\
\hline $\mathrm{Bi}_{2} \mathrm{O}_{3}$ & 2.54 & 1.14 & - & 1.14 \\
\hline $\mathrm{CaO}$ & 2.39 & 1.08 & 4.50 & 5.58 \\
\hline $\mathrm{CdO}$ & 0.05 & 0.02 & - & 0.02 \\
\hline $\mathrm{Cr}_{2} \mathrm{O}_{3}$ & 1.16 & 0.52 & & 0.52 \\
\hline $\mathrm{F}$ & 1.48 & 0.67 & - & 0.67 \\
\hline $\mathrm{Fe}_{2} \mathrm{O}_{3}$ & 13.11 & 5.90 & - & 5.90 \\
\hline $\mathrm{K}_{2} \mathrm{O}$ & 0.31 & 0.14 & - & 0.14 \\
\hline $\mathrm{Li}_{2} \mathrm{O}$ & 0.38 & 0.17 & 3.40 & 3.57 \\
\hline $\mathrm{MgO}$ & 0.26 & 0.12 & - & 0.12 \\
\hline $\mathrm{Na}_{2} \mathrm{O}$ & 7.96 & 3.58 & 6.00 & 9.58 \\
\hline $\mathrm{NiO}$ & 0.89 & 0.40 & - & 0.40 \\
\hline $\mathrm{P}_{2} \mathrm{O}_{5}$ & 2.34 & 1.05 & - & 1.05 \\
\hline $\mathrm{PbO}$ & 0.91 & 0.41 & - & 0.41 \\
\hline $\mathrm{SO}_{3}$ & 0.44 & 0.20 & - & 0.20 \\
\hline $\mathrm{SiO}_{2}$ & 10.88 & 4.90 & 22.10 & 27.00 \\
\hline $\mathrm{TiO}_{2}$ & 0.02 & 0.01 & - & 0.01 \\
\hline $\mathrm{ZnO}$ & 0.18 & 0.08 & - & 0.08 \\
\hline $\mathrm{ZrO}_{2}$ & 0.88 & 0.39 & - & 0.39 \\
\hline Sum & 100.0 & 45.0 & 55.0 & 100.0 \\
\hline
\end{tabular}

${ }^{*}$ Renormalized from Ref. [24] after removal of radioactive components.

\begin{tabular}{|c|c|c|c|}
\hline \multicolumn{3}{|c|}{ Viscosity@1150², $\mathrm{P}$} & 33 \\
\hline \multicolumn{3}{|c|}{ Conductivity@1150 $\mathrm{C}, \mathrm{S} / \mathrm{cm}$} & 0.27 \\
\hline \multicolumn{3}{|c|}{ Crystal Content, As Melted } & None \\
\hline \multicolumn{3}{|c|}{ Crystal Content, $72 \mathrm{hr}$ at $950^{\circ} \mathrm{C}$} & 1.3 \\
\hline \multicolumn{3}{|c|}{ Crystal Content, CCC } & 1.9 \\
\hline \multicolumn{3}{|c|}{ TCLP } & Pass \\
\hline \multirow{4}{*}{$\mathrm{PCT}, \mathrm{g} / \mathrm{L}$} & - & DWPF-EA & HWI-Al-19 \\
\hline & $\mathrm{B}$ & 16.7 & 0.654 \\
\hline & $\mathrm{Li}$ & 9.6 & 0.794 \\
\hline & $\mathrm{Na}$ & 13.3 & 0.624 \\
\hline
\end{tabular}

- Empty data field 
Table 2.4. Composition of Melter Feed to Produce $100 \mathrm{~kg}$ of Target Glass HWI-Al-19 (Target Glass Yield $=500 \mathrm{~g} / \mathrm{L}$ Feed) from the Al-Limited Waste Simulant.

\begin{tabular}{|c|c|c|c|}
\hline \multicolumn{2}{|c|}{ Al-Limited Waste Simulant } & \multicolumn{2}{|c|}{ Glass-Forming Additives } \\
\hline Starting Materials & Target Weight (kg) $^{*}$ & Starting Materials & Target Weight (kg) ${ }^{*}$ \\
\hline $\mathrm{Al}_{2} \mathrm{O}_{3}$ & 23.97 & - & - \\
\hline $\mathrm{H}_{3} \mathrm{BO}_{3}$ & 0.341 & $\mathrm{H}_{3} \mathrm{BO}_{3}$ & 34.089 \\
\hline $\mathrm{BaCO}_{3}$ & 0.070 & - & - \\
\hline $\mathrm{Bi}_{2} \mathrm{O}_{3}$ & 1.156 & - & - \\
\hline $\mathrm{CaO}$ & 1.099 & $\mathrm{CaSiO}_{3}$ (Wollastonite) & 9.798 \\
\hline $\mathrm{CdO}$ & 0.025 & - & - \\
\hline $\mathrm{Cr}_{2} \mathrm{O}_{3}$ & 0.532 & - & - \\
\hline $\mathrm{NaF}$ & 1.483 & - & - \\
\hline $\mathrm{Fe}(\mathrm{OH})_{3}(13 \%$ Slurry $)$ & 48.539 & - & - \\
\hline $\mathrm{KNO}_{3}$ & 0.308 & - & - \\
\hline $\mathrm{Li}_{2} \mathrm{CO}_{3}$ & 0.432 & $\mathrm{Li}_{2} \mathrm{CO}_{3}$ & 8.625 \\
\hline $\mathrm{MgO}$ & 0.121 & - & - \\
\hline $\mathrm{NaOH}$ & 2.190 & $\mathrm{Na}_{2} \mathrm{CO}_{3}$ & 10.364 \\
\hline $\mathrm{Ni}(\mathrm{OH})_{2}$ & 0.514 & - & - \\
\hline $\mathrm{FePO}_{4} \cdot x \mathrm{H}_{2} \mathrm{O}^{\$}$ & 2.795 & - & - \\
\hline $\mathrm{PbO}$ & 0.413 & - & - \\
\hline $\mathrm{Na}_{2} \mathrm{SO}_{4}$ & 0.358 & - & - \\
\hline $\mathrm{SiO}_{2}$ & 4.945 & $\mathrm{SiO}_{2}$ & 17.276 \\
\hline $\mathrm{TiO}_{2}$ & 0.010 & - & - \\
\hline $\mathrm{ZnO}$ & 0.084 & - & - \\
\hline $\mathrm{Zr}(\mathrm{OH})_{4} \cdot x \mathrm{H}_{2} \mathrm{O}^{\$}$ & 1.020 & - & - \\
\hline $\mathrm{H}_{2} \mathrm{O}$ & 97.687 & - & - \\
\hline $\mathrm{Na}_{2} \mathrm{CO}_{3}$ & 0.314 & - & - \\
\hline $\mathrm{NaNO}_{2}$ & 0.346 & - & - \\
\hline $\mathrm{NaNO}_{3}$ & 0.984 & - & - \\
\hline $\mathrm{H}_{2} \mathrm{C}_{2} \mathrm{O}_{4} \cdot 2 \mathrm{H}_{2} \mathrm{O}$ & 0.119 & - & - \\
\hline- & - & - & - \\
\hline Simulant Total & 189.855 & Additives Total & 80.152 \\
\hline- & - & FEED TOTAL & 277.300 \\
\hline
\end{tabular}

* Target weights adjusted for assay information of starting materials

\$ The water content of these compounds is variable; the recipe is based on the nominal assay for the particular source materials used, which is $20 \%$ water for the iron phosphate and $50 \%$ zirconium for the zirconium hydroxide

- Empty data field 
Table 3.1. Summary of Test Conditions and Results.

\begin{tabular}{|c|c|c|c|c|c|}
\hline \multicolumn{2}{|r|}{ Test } & 1 & 2 & 3 & 4 \\
\hline \multirow{3}{*}{ Time } & Feed Start & $\begin{array}{l}1 / 15 / 2013 \\
10: 05\end{array}$ & $\begin{array}{c}1 / 22 / 2013 \\
14: 00\end{array}$ & $\begin{array}{l}1 / 29 / 2013 \\
9: 30\end{array}$ & $\begin{array}{c}2 / 6 / 2013 \\
12: 00\end{array}$ \\
\hline & Feed End & $\begin{array}{c}1 / 17 / 2013 \\
12: 31\end{array}$ & $\begin{array}{c}1 / 24 / 2013 \\
17: 01\end{array}$ & $\begin{array}{c}1 / 31 / 2013 \\
10: 30\end{array}$ & $\begin{array}{c}2 / 8 / 2013 \\
17: 00\end{array}$ \\
\hline & Interval (hr) & 50.4 & 51.0 & 49.0 & 53.0 \\
\hline \multicolumn{2}{|c|}{ Water Feeding for Cold Cap (hr) } & 1.7 & 1.0 & 1.0 & 1.0 \\
\hline & Slurry Feeding (hr) & 48.7 & 50.0 & 48.0 & 52.0 \\
\hline \multicolumn{2}{|c|}{ Slurry Feeding at Steady State (hr) } & 20.9 & 44.3 & 28.9 & 29.6 \\
\hline \multicolumn{2}{|c|}{ Target Production Rate $\left(\mathrm{kg} / \mathrm{m}^{2} /\right.$ day $)$} & 1050 & None & 1289 & None \\
\hline \multirow{3}{*}{ Bubbling } & Outlets & 4 & 5 & 6 & 6 \\
\hline & Bubbler Set Point (lpm) & None & 78 & None & 78 \\
\hline & $\begin{array}{l}\text { Steady State Total Lance } \\
\text { Bubbling (lpm) }\end{array}$ & 78 & 78 & 60 & 78 \\
\hline \multicolumn{2}{|c|}{$\begin{array}{c}\text { Average Steady State Plenum Temperature } \\
\text { all } 6 \text { locations }\left({ }^{\circ} \mathrm{C}\right)\end{array}$} & 556 & 543 & 471 & 453 \\
\hline \multicolumn{2}{|c|}{$\begin{array}{l}\text { Average Steady State Glass Temperature } \\
\text { lower } 6 \text { locations }\left({ }^{\circ} \mathrm{C}\right)\end{array}$} & 1143 & 1143 & 1148 & 1155 \\
\hline \multirow{3}{*}{ Feed } & Used $(\mathrm{kg})$ & 7010 & 8823 & 8449 & 9054 \\
\hline & Measured Glass percentage & $0.36 / 0.25^{*}$ & 0.36 & 0.35 & 0.35 \\
\hline & Average Rate $(\mathrm{kg} / \mathrm{hr})$ & 95 & 94 & 109 & 79 \\
\hline \multirow{4}{*}{$\begin{array}{c}\text { Glass } \\
\text { Produced }\end{array}$} & Discharged & 2366 & 3255 & 3010.5 & 3188.5 \\
\hline & From Feed (kg) & 2276 & 3352 & 2957 & 3169 \\
\hline & Average Rate $\left(\mathrm{kg} / \mathrm{m}^{2} /\right.$ day $)$ & 935 & 1270 & 1232 & 1218 \\
\hline & $\begin{array}{c}\text { Average Steady state rate } \\
\left(\mathrm{kg} / \mathrm{m}^{2} / \text { day }\right)\end{array}$ & 1062 & 1289 & 1251 & 1398 \\
\hline
\end{tabular}

* At the end of Test 1 
Table 3.2. Summary of Operational Events.

\begin{tabular}{|c|c|c|c|c|}
\hline Test & Date & Time & $\begin{array}{l}\text { Run } \\
\text { Time } \\
\text { (hours) }\end{array}$ & Run time note \\
\hline \multirow{30}{*}{1} & \multirow{2}{*}{$1 / 14 / 13$} & 11:00 & - & $\begin{array}{c}\text { Transferred feed to mix tank. Tank mass is } 2949.5 \mathrm{~kg} \text {. This mass includes } 10 \mathrm{~kg} \\
\text { water. }\end{array}$ \\
\hline & & 19:20 & - & $\begin{array}{l}\text { Transferred feed from mix tank to feed tank. Net mass of feed transferred to feed } \\
\text { tank is } 3398.5 \mathrm{~kg} \text {. This mass includes } 498 \mathrm{~kg} \text { water. }\end{array}$ \\
\hline & \multirow{28}{*}{$1 / 15 / 13$} & $0: 40$ & - & $\begin{array}{l}\text { Completed feed transfer to mix tank. Tank mass at the start is } 55.0 \mathrm{~kg} \text {, tank mass } \\
\text { at the end is } 3005.5 \mathrm{~kg} \text {. Net mass of feed transferred to mix tank is } 2950.5 \mathrm{~kg} \text {. } \\
\text { This mass includes } 10 \mathrm{~kg} \text { flush water. }\end{array}$ \\
\hline & & 10:05 & 0.0 & Start water feeding at $0.5 \mathrm{liter} / \mathrm{min}$. \\
\hline & & 10:17 & 0.2 & Increased power from $90 \mathrm{~kW}$ to $100 \mathrm{~kW}$. Average glass temperature is $1155^{\circ} \mathrm{C}$. \\
\hline & & 10:19 & 0.2 & Increased bubbling on L1 and L2 from $1.5 \mathrm{lpm}$ to $2.5 \mathrm{lpm}$ each. \\
\hline & & 10:25 & 0.3 & Water flow rate was raised to 1.0 liter $/ \mathrm{min}$. \\
\hline & & 10.27 & 0.4 & Increased power from $100 \mathrm{~kW}$ to $110 \mathrm{~kW}$. Average glass temperature is $1144^{\circ} \mathrm{C}$. \\
\hline & & $10: 37$ & 0.5 & Increased power from $110 \mathrm{~kW}$ to $115 \mathrm{~kW}$. Average glass temperature is $1142^{\circ} \mathrm{C}$. \\
\hline & & $10: 45$ & 0.7 & Water flow rate was raised to 2.0 liter $/ \mathrm{min}$. \\
\hline & & 10:47 & 0.7 & Increased power from $115 \mathrm{~kW}$ to $125 \mathrm{~kW}$. \\
\hline & & 10:57 & 0.9 & Increased power from $125 \mathrm{~kW}$ to $130 \mathrm{~kW}$. \\
\hline & & 11:00 & 0.9 & Decreased bubbling on L1 and L2 from 2.5 to $2.0 \mathrm{lpm}$ each. \\
\hline & & $11: 12$ & 1.1 & Increased power from $130 \mathrm{~kW}$ to $135 \mathrm{~kW}$. \\
\hline & & $11: 25$ & 1.3 & Water flow rate was raised to 2.5 liter $/ \mathrm{min}$. \\
\hline & & $11: 43$ & 1.6 & $\begin{array}{l}\text { Water flow rate was raised to } 3.0 \text { liter/min. Average plenum temperature is } \\
\qquad 745^{\circ} \mathrm{C} .\end{array}$ \\
\hline & & $11: 50$ & 1.7 & $\begin{array}{l}\text { Secured feeding water. Started feeding HWI-Al-19 feed. Decreased power from } \\
135 \mathrm{~kW} \text { to } 130 \mathrm{~kW} .\end{array}$ \\
\hline & & $11: 55$ & 1.8 & Increased bubbling on L1 and L2 from 2.0 to $10.0 \mathrm{lpm}$ each. \\
\hline & & 12:02 & 1.9 & Increased power from $130 \mathrm{~kW}$ to $135 \mathrm{~kW}$. Average glass temperature is $1134^{\circ} \mathrm{C}$. \\
\hline & & $12: 12$ & 2.1 & Increased power from $130 \mathrm{~kW}$ to $135 \mathrm{~kW}$. Average glass temperature is $1142^{\circ} \mathrm{C}$. \\
\hline & & 12:14 & 2.1 & Decreased T4 to 50 seconds. \\
\hline & & $12: 25$ & 2.3 & Decreased T4 from 50 to 40 seconds. \\
\hline & & $12: 35$ & 2.5 & $\begin{array}{c}\text { Average glass temperature is } 1147^{\circ} \mathrm{C} \text {. Average power is } 139 \mathrm{~kW} \text { and current is } \\
1219 \text { Amps. Glass resistance is } 0.093 \Omega \text {. }\end{array}$ \\
\hline & & 12:41 & 2.6 & Increased $\mathrm{T} 1$ from 1.0 second to 1.2 seconds. \\
\hline & & 13:20 & 3.2 & Bubbling on L1 and L2 are @15.0 lpm each. \\
\hline & & 13:35 & 3.5 & Average glass temperature is $1149^{\circ} \mathrm{C}$. Plenum temperature is $666-707^{\circ} \mathrm{C}$. \\
\hline & & 13:50 & 3.7 & Average glass temperature is $1156^{\circ} \mathrm{C}$. Plenum temperature is $657-698^{\circ} \mathrm{C}$. \\
\hline & & 14:04 & 4.0 & Increased discharge power from 16 to $18 \mathrm{~kW}$. \\
\hline & & 14:12 & 4.1 & Reduced power from 140 to $135 \mathrm{~kW}$. \\
\hline & & $14: 20$ & 4.2 & Average glass temperature is $1159^{\circ} \mathrm{C}$. Plenum temperature is $644-665^{\circ} \mathrm{C}$. \\
\hline
\end{tabular}


Table 3.2. Summary of Operational Events (continued).

\begin{tabular}{|c|c|c|c|c|}
\hline Test & Date & Time & $\begin{array}{c}\text { Run } \\
\text { Time } \\
\text { (hours) }\end{array}$ & Run time note \\
\hline \multirow{31}{*}{1} & \multirow{31}{*}{$1 / 15 / 13$} & $14: 35$ & 4.5 & Average glass temperature is $1159^{\circ} \mathrm{C}$. \\
\hline & & $14: 46$ & 4.7 & Increased bubbling from 15 to $17 \mathrm{lpm}$. \\
\hline & & $14: 52$ & 4.8 & Plenum temperature is $617-640^{\circ} \mathrm{C}$. \\
\hline & & $15: 12$ & 5.1 & Reduced power to $133 \mathrm{~kW}$. Average glass temperature is $1158^{\circ} \mathrm{C}$. \\
\hline & & $15: 15$ & 5.2 & Plenum temperature is $576-615^{\circ} \mathrm{C}$. \\
\hline & & $15: 32$ & 5.4 & $\begin{array}{l}\text { Increased bubbling from } 17 \mathrm{lpm} \text { to } 20 \mathrm{lpm} \text {. Plenum temperature is } 573-599^{\circ} \mathrm{C} \text {. } \\
\text { Also adjusted Blower } \# 801 \text { from } 19 \text { to } 24 \mathrm{~Hz} \text {. }\end{array}$ \\
\hline & & $16: 20$ & 6.2 & Plenum temperature is $545-580^{\circ} \mathrm{C}$. \\
\hline & & $16: 42$ & 6.6 & Plenum temperature is $526-557^{\circ} \mathrm{C}$. \\
\hline & & $16: 53$ & 6.8 & $\begin{array}{l}\text { Reduced feeding by changing T4 from } 32 \text { to } 33 \text { seconds and increased bubbling } \\
\text { from } 20 \text { to } 22 \mathrm{lpm} \text {. Plenum temperature is } 519-549^{\circ} \mathrm{C} \text {. Feed rate is } 151 \mathrm{~kg} / \mathrm{hr} \text {. }\end{array}$ \\
\hline & & 17:01 & 6.9 & Increased power from $133 \mathrm{~kW}$ to $135 \mathrm{~kW}$. Average glass temperature is $1143^{\circ} \mathrm{C}$. \\
\hline & & 17:44 & 7.6 & $\begin{array}{l}\text { Dilution air on WESP was lowered from } 20 \mathrm{scfm} \text { to } 16 \mathrm{scfm} \text { for both south and } \\
\text { north air injectors. }\end{array}$ \\
\hline & & 17:51 & 7.8 & Increased bubbling on L1 and L2 from $22 \mathrm{lpm}$ to $25 \mathrm{lpm}$ respectively. \\
\hline & & 18:04 & 8.0 & Increased power from $135 \mathrm{~kW}$ to $140 \mathrm{~kW}$. \\
\hline & & 18:13 & 8.1 & Increased bubbling on L1 and L2 from $25 \mathrm{lpm}$ to $27 \mathrm{lpm}$ respectively. \\
\hline & & $18: 28$ & 8.4 & Increased power from $140 \mathrm{~kW}$ to $145 \mathrm{~kW}$. Average glass temperature is $1132^{\circ} \mathrm{C}$. \\
\hline & & $18: 55$ & 8.8 & Increased power from $145 \mathrm{~kW}$ to $150 \mathrm{~kW}$. \\
\hline & & 19:29 & 9.4 & Increased power from $150 \mathrm{~kW}$ to $155 \mathrm{~kW}$. Average glass temperature is $1127^{\circ} \mathrm{C}$. \\
\hline & & 19:46 & 9.7 & Performing film cooler rinse. \\
\hline & & 20:09 & 10.1 & Turned on Lances 1B and 2B mass flow controllers. \\
\hline & & $20: 18$ & 10.2 & Increased bubbling to $60 \mathrm{lpm}$ total. \\
\hline & & $20: 21$ & 10.3 & Increased power from $155 \mathrm{~kW}$ to $160 \mathrm{~kW}$. Average glass temperature is $1140^{\circ} \mathrm{C}$. \\
\hline & & $20: 23$ & 10.3 & Plenum temperature is $449-485^{\circ} \mathrm{C}$. \\
\hline & & 20:40 & 10.6 & $\begin{array}{l}\text { Increased bubbling on all four bubbler mass flow controllers from } 15 \text { to } 17 \mathrm{lpm} \text {, } \\
\text { total bubbling is at } 68 \mathrm{lpm} .\end{array}$ \\
\hline & & 21:05 & 11.0 & Plenum temperature is $457-479^{\circ} \mathrm{C}$. \\
\hline & & $21: 20$ & 11.2 & Increased power from $160 \mathrm{~kW}$ to $165 \mathrm{~kW}$. Average glass temperature is $1134^{\circ} \mathrm{C}$. \\
\hline & & $21: 27$ & 11.4 & Plenum temperature is $473-491^{\circ} \mathrm{C}$. \\
\hline & & $21: 37$ & 11.5 & Plenum temperature is $473-494^{\circ} \mathrm{C}$. \\
\hline & & $21: 52$ & 11.8 & Plenum temperature is $466-493^{\circ} \mathrm{C}$. \\
\hline & & $21: 59$ & 11.9 & Increased power from $165 \mathrm{~kW}$ to $167 \mathrm{~kW}$. Average glass temperature is $1140^{\circ} \mathrm{C}$ \\
\hline & & $22: 22$ & 12.3 & Plenum temperature is $475-495^{\circ} \mathrm{C}$. \\
\hline & & $22: 25$ & 12.3 & Feed tank recirculation pump is stopped. Increased pressure from 20 to 25 psi. \\
\hline
\end{tabular}


Table 3.2. Summary of Operational Events (continued).

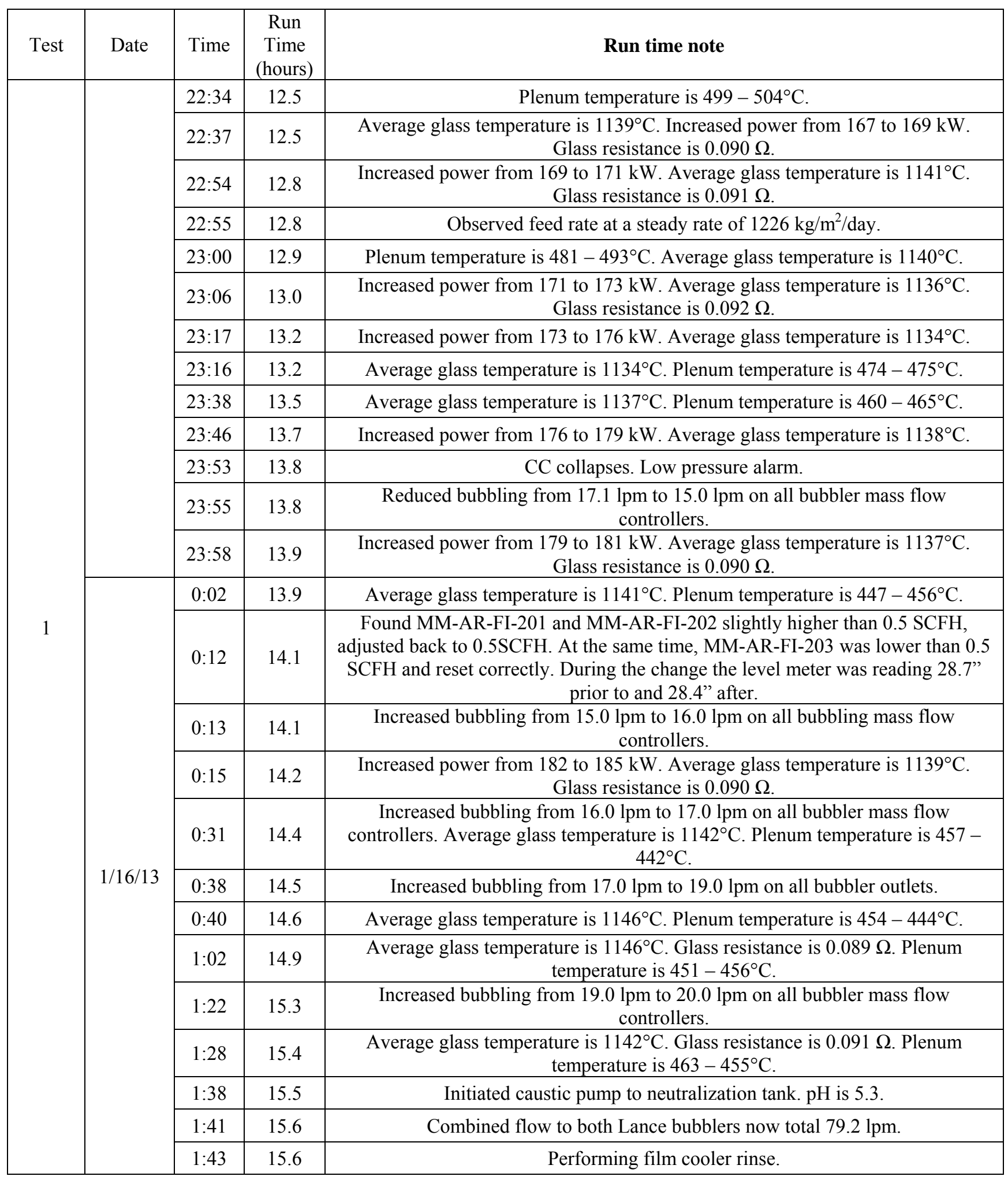


Table 3.2. Summary of Operational Events (continued).

\begin{tabular}{|c|c|c|c|c|}
\hline Test & Date & Time & $\begin{array}{l}\text { Run } \\
\text { Time } \\
\text { (hours) }\end{array}$ & Run time note \\
\hline \multirow{24}{*}{1} & \multirow{24}{*}{$1 / 16 / 13$} & $1: 45$ & 15.7 & Secured caustic pump, $\mathrm{pH}$ of neutralization tank is 7.0 . \\
\hline & & $1: 55$ & 15.8 & $\begin{array}{l}\text { Average glass temperature is } 1141^{\circ} \mathrm{C} \text {. Glass resistance is } 0.090 \Omega \text {. Plenum } \\
\text { temperature is } 470-453^{\circ} \mathrm{C} \text {. }\end{array}$ \\
\hline & & $1: 58$ & 15.9 & $\begin{array}{l}\text { Increased power from } 185 \text { to } 183 \times \mathrm{kW} \text {. T/W \#1 reads } 1109-1133^{\circ} \mathrm{C} . \mathrm{T} / \mathrm{W} \# 2 \\
\text { reads } 1125-1153^{\circ} \mathrm{C} \text {. Plenum temperature is } 450-469^{\circ} \mathrm{C} \text {. Glass resistance is } \\
0.091 \Omega \text {. }\end{array}$ \\
\hline & & $2: 15$ & 16.2 & $\begin{array}{l}\mathrm{T} / \mathrm{W} \# 1 \text { reads } 1105-1133^{\circ} \mathrm{C} . \mathrm{T} / \mathrm{W} \# 2 \text { reads } 1134-1157^{\circ} \mathrm{C} \text {. Plenum temperature is } \\
\qquad 452-460^{\circ} \mathrm{C} .\end{array}$ \\
\hline & & $2: 41$ & 16.6 & Mixer tank blade rotation is increased to $30 \mathrm{~Hz}$. \\
\hline & & $2: 43$ & 16.6 & $\begin{array}{c}\text { Average glass temperature is } 1143^{\circ} \mathrm{C} \text {. Glass resistance is } 0.090 \Omega \text {. Plenum } \\
\text { temperature is } 461-477^{\circ} \mathrm{C} \text {. }\end{array}$ \\
\hline & & $3: 01$ & 16.9 & $\begin{array}{c}\text { Average glass temperature is } 1144^{\circ} \mathrm{C} \text {. Glass resistance is } 0.089 \Omega \text {. Plenum } \\
\text { temperature is } 477-498^{\circ} \mathrm{C} \text {. }\end{array}$ \\
\hline & & $3: 19$ & 17.2 & Average glass temperature is $1137^{\circ} \mathrm{C}$. Plenum temperature is $485-536^{\circ} \mathrm{C}$. \\
\hline & & $3: 32$ & 17.4 & Plenum temperature is $493-534^{\circ} \mathrm{C}$. \\
\hline & & $3: 50$ & 17.7 & $\begin{array}{c}\text { Average glass temperature is } 1139^{\circ} \mathrm{C} \text {. Glass resistance is } 0.092 \Omega \text {. Plenum } \\
\text { temperature is } 501-550^{\circ} \mathrm{C} \text {. }\end{array}$ \\
\hline & & $4: 11$ & 18.1 & Average glass temperature is $1140^{\circ} \mathrm{C}$. Plenum temperature is $494-539^{\circ} \mathrm{C}$. \\
\hline & & $4: 24$ & 18.3 & Average glass temperature is $1140^{\circ} \mathrm{C}$. Plenum temperature is $491-527^{\circ} \mathrm{C}$. \\
\hline & & $4: 42$ & 18.6 & $\begin{array}{c}\text { Observed slight leak coming from SBS blow down valve, will continue to } \\
\text { monitor. }\end{array}$ \\
\hline & & $5: 08$ & 19.0 & Plenum temperature is $491-503^{\circ} \mathrm{C}$. \\
\hline & & $5: 10$ & 19.1 & $\begin{array}{l}\text { After investigation HEME \#2 differential pressure transmitter is non-functional. } \\
\text { Repair/replacement is pending. }\end{array}$ \\
\hline & & $5: 15$ & 19.2 & Repaired HEME\#2 differential transmitter. \\
\hline & & $5: 19$ & 19.2 & Average glass temperature is $1138^{\circ} \mathrm{C}$. Plenum temperature is $493-500^{\circ} \mathrm{C}$. \\
\hline & & $5: 34$ & 19.5 & Plenum temperature is $478-510^{\circ} \mathrm{C}$. \\
\hline & & $5: 48$ & 19.7 & Plenum temperature is $485-518^{\circ} \mathrm{C}$. \\
\hline & & 6:04 & 20.0 & Plenum temperature is $483-533^{\circ} \mathrm{C}$. \\
\hline & & $6: 19$ & 20.2 & Plenum temperature is $498-529^{\circ} \mathrm{C}$ \\
\hline & & $8: 09$ & 22.1 & Decreased from 34 to 33 seconds. Feed rate is $145.0 \mathrm{~kg} / \mathrm{hr}$. \\
\hline & & $8: 20$ & 22.2 & $\begin{array}{c}\text { Power is at } 186 \mathrm{~kW} \text {. Current is } 1449 \text { Amps. Total bubbling is } 80 \mathrm{lpm} \text {. Glass } \\
\text { resistance is } 0.089 \Omega .\end{array}$ \\
\hline & & $8: 40$ & 22.6 & $\begin{array}{c}\text { Power is at } 186 \mathrm{~kW}, 128 \text { volts. Current is } 1451 \text { Amps. Glass resistance is } 0.088 \\
\Omega .\end{array}$ \\
\hline
\end{tabular}


Table 3.2. Summary of Operational Events (continued).

\begin{tabular}{|c|c|c|c|c|}
\hline Test & Date & Time & $\begin{array}{l}\text { Run } \\
\text { Time } \\
\text { (hours) }\end{array}$ & Run time note \\
\hline \multirow{28}{*}{1} & \multirow{28}{*}{$1 / 16 / 13$} & $9: 10$ & 23.1 & $\begin{array}{l}\text { Transferred feed from mix tank to feed tank. Tank mass at the start is } 3011.0 \mathrm{~kg} \text {, } \\
\text { tank mass at the end is } 505.0 \mathrm{~kg} \text {. Net mass of feed transferred to feed tank is } \\
2506.0 \mathrm{~kg} \text {. Total of } 2936.0 \mathrm{~kg} \text { feed plus } 430 \mathrm{~kg} \text { water added to the feed rank. }\end{array}$ \\
\hline & & 10:05 & 24.0 & Average glass temperature is $1138^{\circ} \mathrm{C}$. \\
\hline & & $10: 20$ & 24.2 & Average glass temperature is $1142^{\circ} \mathrm{C}$. Plenum temperature is $509-526^{\circ} \mathrm{C}$ \\
\hline & & $10: 35$ & 24.5 & Average glass temperature is $1145^{\circ} \mathrm{C}$. \\
\hline & & $10: 50$ & 24.7 & Average glass temperature is $1143^{\circ} \mathrm{C}$. \\
\hline & & 11:05 & 25.0 & Average glass temperature is $1143^{\circ} \mathrm{C}$. \\
\hline & & $11: 39$ & 25.6 & Paused feeding for feed sample. \\
\hline & & $11: 49$ & 25.7 & Average glass temperature is $1143^{\circ} \mathrm{C}$. \\
\hline & & 12:05 & 26.0 & Performed WESP blow down, deluge and blow down. \\
\hline & & $12: 24$ & 26.3 & $\begin{array}{l}\text { Performed SBS blow down. Flow totalizer is not functioning. Broken lead } \\
\text { repaired. }\end{array}$ \\
\hline & & $13: 20$ & 27.2 & $\begin{array}{l}\text { Total bubbling is } 80 \mathrm{lpm} \text {, Power is at } 186 \mathrm{~kW} \text {. Current is } 1437 \text { Amps. Average } \\
\text { glass temperature is } 1142^{\circ} \mathrm{C} \text {. Glass resistance is } 0.090 \Omega \text {. }\end{array}$ \\
\hline & & $14: 23$ & 28.3 & Average glass temperature is $1146^{\circ} \mathrm{C}$. \\
\hline & & 14:41 & 28.6 & Plenum temperature is $539-548^{\circ} \mathrm{C}$ \\
\hline & & $14: 45$ & 28.7 & Equalized storage tanks, neutralization tank at 500gal. \\
\hline & & 15:05 & 29.0 & Plenum temperature is $520-543^{\circ} \mathrm{C}$ \\
\hline & & $15: 16$ & 29.2 & Increased power from 188 to $190 \mathrm{~kW}$. Average glass temperature is $1142^{\circ} \mathrm{C}$. \\
\hline & & $15: 31$ & 29.4 & $\begin{array}{c}\text { Due to melter pressure spikes higher than normal T1 has been changed from } 1.2 \\
\text { to } 1.1 \text { second and T4 from } 37 \text { to } 36 \text { seconds. }\end{array}$ \\
\hline & & $15: 49$ & 29.7 & Plenum temperature is $527-564^{\circ} \mathrm{C}$ \\
\hline & & $15: 58$ & 29.9 & $\begin{array}{l}\text { Reduced bubbling mass flow controllers from } 20 \mathrm{lpm} \text { to } 19 \mathrm{lpm} \text { each, total } \\
\text { bubbling from } 80 \mathrm{lpm} \text { to } 76 \mathrm{lpm} \text {. Plenum temperature is } 536-569^{\circ} \mathrm{C}\end{array}$ \\
\hline & & 16:09 & 30.1 & $\begin{array}{l}\text { Plenum temperature is } 542-576^{\circ} \mathrm{C} \text {. Increased feed rate by changing T4 from } 34 \\
\text { to } 33 \text { seconds. }\end{array}$ \\
\hline & & $16: 19$ & 30.2 & Plenum temperature is $550-578^{\circ} \mathrm{C}$. \\
\hline & & $16: 34$ & 30.5 & Plenum temperature is $537-573^{\circ} \mathrm{C}$. \\
\hline & & $16: 49$ & 30.7 & Plenum temperature is $534-582^{\circ} \mathrm{C}$. \\
\hline & & 17:04 & 31.0 & Plenum temperature is $545-589^{\circ} \mathrm{C}$ \\
\hline & & $17: 22$ & 31.3 & Plenum temperature is $539-592^{\circ} \mathrm{C}$. \\
\hline & & $17: 35$ & 31.5 & Plenum temperature is $539-584^{\circ} \mathrm{C}$. \\
\hline & & $17: 49$ & 31.7 & Plenum temperature is $541-575^{\circ} \mathrm{C}$. \\
\hline & & $17: 57$ & 31.9 & Opened pitot tube port in transition line. \\
\hline
\end{tabular}


Table 3.2. Summary of Operational Events (continued).

\begin{tabular}{|c|c|c|c|c|}
\hline Test & Date & Time & $\begin{array}{l}\text { Run } \\
\text { Time } \\
\text { (hours) }\end{array}$ & Run time note \\
\hline \multirow{28}{*}{1} & \multirow{28}{*}{$1 / 16 / 13$} & 18:11 & 32.1 & Plenum temperature is $535-581^{\circ} \mathrm{C}$. \\
\hline & & $18: 21$ & 32.3 & Off gas testing in transition line started. Emergency off gas, EOG is tripped. \\
\hline & & 18:28 & 32.4 & Plenum temperature is $545-573^{\circ} \mathrm{C}$. \\
\hline & & 18:37 & 32.5 & Plenum temperature is $536-560^{\circ} \mathrm{C}$. \\
\hline & & 18:52 & 32.8 & Plenum temperature is $546-576^{\circ} \mathrm{C}$. \\
\hline & & 19:09 & 33.1 & Plenum temperature is $528-584^{\circ} \mathrm{C}$. \\
\hline & & 19:21 & 33.3 & Off gas testing is done, EOG tripped again. \\
\hline & & 19:24 & 33.3 & $\begin{array}{l}\text { Transferred feed from mix tank to feed tank. Starting tank mass } 495.5 \mathrm{~kg} \text {, ending } \\
\text { tank mass } 53.0 \mathrm{~kg} \text {. Net mass of feed transferred to feed tank is } 442.5 \mathrm{~kg} \text {. Total of } \\
527.5 \mathrm{~kg} \text { feed including } 85 \mathrm{~kg} \text { water added to the feed rank. }\end{array}$ \\
\hline & & 20:04 & 34.0 & Performing film cooler rinse. \\
\hline & & 23:39 & 37.6 & $\begin{array}{l}\text { Electrode high temperature was alarmed. Power was reduced from } 192 \mathrm{~kW} \text { to } \\
\qquad 189 \mathrm{~kW} .\end{array}$ \\
\hline & & $23: 43$ & 37.6 & $\begin{array}{l}\text { Observed that lower level sight glass for HEME \#1 appears to have air bubbling } \\
\text { through it. Will continue to monitor. }\end{array}$ \\
\hline & & 19:45 & 33.7 & Plenum temperature is $537-583^{\circ} \mathrm{C}$. \\
\hline & & 19:56 & 33.8 & Increased feed tank mixer from 25 to $30 \mathrm{~Hz}$. \\
\hline & & 20:04 & 34.0 & Performed a film cooler rinse. \\
\hline & & $20: 12$ & 34.1 & Plenum temperature is $544-600^{\circ} \mathrm{C}$. \\
\hline & & $20: 27$ & 34.4 & Plenum temperature is $543-606^{\circ} \mathrm{C}$. \\
\hline & & $20: 53$ & 34.8 & Plenum temperature is $566-586^{\circ} \mathrm{C}$. \\
\hline & & 21:03 & 35.0 & Increased power from $190 \mathrm{~kW}$ to $192 \mathrm{~kW}$. Average glass temperature is $1145^{\circ} \mathrm{C}$. \\
\hline & & 21:09 & 35.1 & Plenum temperature is $584-588^{\circ} \mathrm{C}$. \\
\hline & & 21:13 & 35.1 & Feed system was paused. \\
\hline & & 21:21 & 35.3 & Resumed feeding. \\
\hline & & $21: 33$ & 35.5 & $\begin{array}{c}\text { Increased T1 from } 1.1 \text { to } 1.3 \text { second and T4 from } 32 \text { to } 28 \text { seconds for } 12 \text { minutes } \\
\text { to get the feed rate back up. }\end{array}$ \\
\hline & & $21: 38$ & 35.5 & $\begin{array}{l}\text { Average glass temperature is } 1150^{\circ} \mathrm{C} \text {. Glass resistance is } 0.085 \Omega \text {. Plenum } \\
\text { temperature is } 563-623^{\circ} \mathrm{C} \text {. }\end{array}$ \\
\hline & & 21:54 & 35.8 & $\begin{array}{c}\text { Average glass temperature is } 1148^{\circ} \mathrm{C} \text {. Glass resistance is } 0.086 \Omega \text {. Plenum } \\
\text { temperature is } 572-656^{\circ} \mathrm{C} \text {. }\end{array}$ \\
\hline & & 22:12 & 36.1 & Caustic pump to neutralization tank turned off. pH meter showed 7.7. \\
\hline & & 22:25 & 36.3 & $\begin{array}{c}\text { 1A }+1 \mathrm{~B} \text { bubbler mass flow controllers are reduced from } 19.0 \mathrm{lpm} \text { to } 17.0 \mathrm{lpm} \\
\text { each. } 2 \mathrm{~A}+2 \mathrm{~B} \text { bubbler mass flow controllers are reduced from } 19.0 \mathrm{lpm} \text { to } 17.0 \\
\text { lpm each. }\end{array}$ \\
\hline & & 22:26 & 36.3 & $\begin{array}{c}\text { Average glass temperature is } 1136^{\circ} \mathrm{C} \text {. Glass resistance is } 0.089 \Omega \text {. Plenum } \\
\text { temperature is } 550-639^{\circ} \mathrm{C} \text {. }\end{array}$ \\
\hline & & $22: 43$ & 36.6 & $\begin{array}{c}\text { Average glass temperature is } 1143^{\circ} \mathrm{C} \text {. Glass resistance is } 0.087 \Omega \text {. Plenum } \\
\text { temperature is } 567-621^{\circ} \mathrm{C} \text {. }\end{array}$ \\
\hline
\end{tabular}


Table 3.2. Summary of Operational Events (continued).

\begin{tabular}{|c|c|c|c|c|}
\hline Test & Date & Time & $\begin{array}{l}\text { Run } \\
\text { Time } \\
\text { (hours) }\end{array}$ & Run time note \\
\hline \multirow{24}{*}{1} & \multirow{11}{*}{$1 / 16 / 13$} & 23:03 & 37.0 & $\begin{array}{c}\text { Average glass temperature is } 1147^{\circ} \mathrm{C} \text {. Glass resistance is } 0.087 \Omega \text {. Plenum } \\
\text { temperature is } 568-598^{\circ} \mathrm{C}\end{array}$ \\
\hline & & 23:06 & 37.0 & $\begin{array}{l}1 \mathrm{~A}+1 \mathrm{~B} \text { bubbler mass flow controllers are reduced from } 17.0 \mathrm{lpm} \text { to } 16.0 \mathrm{lpm} \\
\text { each. } 2 \mathrm{~A}+2 \mathrm{~B} \text { bubbler mass flow controllers are reduced from } 17.0 \mathrm{lpm} \text { to } 16.0 \\
\text { lpm each. }\end{array}$ \\
\hline & & 23:10 & 37.1 & $\begin{array}{l}\text { 1A }+1 \mathrm{~B} \text { bubbler mass flow controllers are reduced from } 16.0 \mathrm{lpm} \text { to } 15.0 \mathrm{lpm} \\
\text { each. } 2 \mathrm{~A}+2 \mathrm{~B} \text { bubblers mass flow controller are reduced from } 16.0 \mathrm{lpm} \text { to } \\
15.0 \mathrm{lpm} \text { each. }\end{array}$ \\
\hline & & 23:15 & 37.2 & $\begin{array}{l}\text { Change/reduce SBS chill water temperature set point from } 45 \text { to } 42^{\circ} \mathrm{C} . \mathrm{CW} \\
\text { temperature presently } 48^{\circ} \mathrm{C} \text {. }\end{array}$ \\
\hline & & $23: 18$ & 37.2 & $\begin{array}{c}\text { Average glass temperature is } 1148^{\circ} \mathrm{C} \text {. Glass resistance is } 0.086 \Omega \text {. Plenum } \\
\text { temperature is } 577-588^{\circ} \mathrm{C}\end{array}$ \\
\hline & & $23: 39$ & 37.6 & $\begin{array}{c}\text { Electrode high alarm sounded. Reduced power from } 192 \mathrm{~kW} \text { to } 189 \mathrm{~kW} \text {. } \\
\text { Average glass temperature is } 1154^{\circ} \mathrm{C} \text {. }\end{array}$ \\
\hline & & 23:41 & 37.6 & Plenum temperature is $581-566^{\circ} \mathrm{C}$. \\
\hline & & $23: 43$ & 37.6 & $\begin{array}{c}\text { Observed lower level sight glass on HEME\#1 appears to have air bubbling } \\
\text { through it. Will continue to monitor. }\end{array}$ \\
\hline & & $23: 50$ & 37.7 & Reduced power from $189 \mathrm{~kW}$ to $186 \mathrm{~kW}$. Average glass temperature is $1152^{\circ} \mathrm{C}$ \\
\hline & & 23:59 & 37.9 & $\begin{array}{l}\text { 1A }+1 \mathrm{~B} \text { bubbler mass flow controllers are reduced from } 15.0 \mathrm{lpm} \text { to } 14.0 \mathrm{lpm} \\
\text { each. } 2 \mathrm{~A}+2 \mathrm{~B} \text { bubbler mass flow controllers are reduced from } 15.0 \mathrm{lpm} \text { to } \\
14.0 \mathrm{lpm} \text { each. }\end{array}$ \\
\hline & & 24:00 & 37.9 & $\begin{array}{l}\text { Reduced power from } 186 \mathrm{~kW} \text { to } 183 \mathrm{~kW} \text {. Average glass temperature is } \\
1155^{\circ} \mathrm{C} .\end{array}$ \\
\hline & \multirow{13}{*}{$1 / 17 / 13$} & 0:05 & 38.0 & Plenum temperature is $582-560^{\circ} \mathrm{C}$. \\
\hline & & 0:08 & 38.0 & $\begin{array}{l}\text { Electrode high temperature was alarm sounded. Power was reduced from } 183 \\
\mathrm{~kW} \text { to } 180 \mathrm{~kW} \text {. Average glass temperature is } 1157^{\circ} \mathrm{C} \text {. }\end{array}$ \\
\hline & & $0: 18$ & 38.2 & Reduced power from $180 \mathrm{~kW}$ to $177 \mathrm{~kW}$. Average glass temperature is $1158^{\circ} \mathrm{C}$ \\
\hline & & $0: 19$ & 38.2 & Plenum temperature is $568-576^{\circ} \mathrm{C}$. \\
\hline & & $0: 23$ & 38.3 & Changed T4 from 36 seconds to 38 seconds. \\
\hline & & $0: 28$ & 38.4 & $\begin{array}{l}\text { Reduced power from } 177 \mathrm{~kW} \text { to } 174 \mathrm{~kW} \text {. Average glass temperature is } \\
1159^{\circ} \mathrm{C} .\end{array}$ \\
\hline & & $0: 32$ & 38.4 & Changed T4 from 38 seconds to 40 seconds. \\
\hline & & $0: 39$ & 38.6 & $\begin{array}{l}\text { Reduced power from } 174 \mathrm{~kW} \text { to } 171 \mathrm{~kW} \text {. Average glass temperature is } \\
1157^{\circ} \mathrm{C} .\end{array}$ \\
\hline & & $0: 44$ & 38.6 & $\begin{array}{c}\text { Average glass temperature is } 1160^{\circ} \mathrm{C} \text {. Glass resistance is } 0.080 \Omega \text {. Plenum } \\
\text { temperature is } 559-562^{\circ} \mathrm{C}\end{array}$ \\
\hline & & $0: 55$ & 38.8 & Reduced power from $171 \mathrm{~kW}$ to $168 \mathrm{~kW}$. Average glass temperature is $1160^{\circ} \mathrm{C}$ \\
\hline & & $1: 02$ & 38.9 & $\begin{array}{l}1 \mathrm{~A}+1 \mathrm{~B} \text { bubbler mass flow controllers are reduced from } 14.0 \mathrm{lpm} \text { to } 12.0 \mathrm{lpm} \\
\text { each. } 2 \mathrm{~A}+2 \mathrm{~B} \text { bubbler mass flow controllers are reduced from } 14.0 \mathrm{lpm} \text { to } \\
12.01 \mathrm{pm} \text { each. }\end{array}$ \\
\hline & & $1: 04$ & 39.0 & $\begin{array}{c}\text { Average glass temperature is } 1159^{\circ} \mathrm{C} \text {. Glass resistance is } 0.080 \Omega \text {. Plenum } \\
\text { temperature is } 567-559^{\circ} \mathrm{C} \text {. }\end{array}$ \\
\hline & & $1: 07$ & 39.0 & $\begin{array}{l}\text { Reduced power from } 168 \mathrm{~kW} \text { to } 165 \mathrm{~kW} \text {. Average glass temperature is } \\
\qquad 1160^{\circ} \mathrm{C} .\end{array}$ \\
\hline
\end{tabular}


Table 3.2. Summary of Operational Events (continued).

\begin{tabular}{|c|c|c|c|c|}
\hline Test & Date & Time & $\begin{array}{l}\text { Run } \\
\text { Time } \\
\text { (hours) }\end{array}$ & Run time note \\
\hline \multirow{28}{*}{1} & \multirow{28}{*}{$1 / 17 / 13$} & $1: 09$ & 39.1 & Reduced T4 from 38 to 36 seconds. \\
\hline & & $1: 14$ & 39.1 & Reduced T4 from 36 to 33 seconds. \\
\hline & & $1: 16$ & 39.2 & $\begin{array}{l}\text { Increased 1A + 1B bubbler mass flow controllers from } 12.0 \mathrm{lpm} \text { to } 13.0 \mathrm{lpm} \\
\text { each. Increased } 2 \mathrm{~A}+2 \mathrm{~B} \text { bubbler mass flow controllers from } 12.0 \mathrm{lpm} \text { to } \\
13.0 \mathrm{lpm} \text { each. }\end{array}$ \\
\hline & & $1: 19$ & 39.2 & $\begin{array}{c}\text { Average glass temperature is } 1162^{\circ} \mathrm{C} \text {. Glass resistance is } 0.079 \Omega . \text { Plenum } \\
\text { temperature is } 562-546^{\circ} \mathrm{C}\end{array}$ \\
\hline & & $1: 23$ & 39.3 & $\begin{array}{l}\text { Reduced power from } 165 \mathrm{~kW} \text { to } 162 \mathrm{~kW} \text {. Average glass temperature is } \\
1161^{\circ} \mathrm{C} .\end{array}$ \\
\hline & & $1: 31$ & 39.4 & $\begin{array}{c}\text { Average glass temperature is } 1160^{\circ} \mathrm{C} \text {. Glass resistance is } 0.080 \Omega \text {. Plenum } \\
\text { temperature is } 562-541^{\circ} \mathrm{C}\end{array}$ \\
\hline & & $1: 34$ & 39.5 & Changed T4 from 33 seconds to 32 seconds. \\
\hline & & $1: 35$ & 39.5 & Reduced power from $162 \mathrm{~kW}$ to $159 \mathrm{~kW}$. Average glass temperature is $1159^{\circ} \mathrm{C}$ \\
\hline & & $1: 42$ & 39.6 & $\begin{array}{l}\text { Reduced 1A + 1B bubbler mass flow controllers from } 13.0 \mathrm{lpm} \text { to } 12.0 \mathrm{lpm} \text {. } \\
\text { Reduced } 2 \mathrm{~A}+2 \mathrm{~B} \text { bubbler mass flow controllers from } 13.0 \mathrm{lpm} \text { to } 12.0 \mathrm{lpm} \text {. } \\
\text { Increasing feed rate by changing T4 from } 32 \text { to } 31 \mathrm{~seconds} \text {. Targeting } 146 \\
\mathrm{~kg} / \mathrm{hr} \text {. Average glass temperature is } 1158^{\circ} \mathrm{C} \text {. Plenum temperature is } 565-534^{\circ} \mathrm{C}\end{array}$ \\
\hline & & $1: 47$ & 39.7 & Reduced power from $159 \mathrm{~kW}$ to $156 \mathrm{~kW}$. \\
\hline & & $1: 52$ & 39.8 & Average glass temperature is $1156^{\circ} \mathrm{C}$. Plenum temperature is $530-567^{\circ} \mathrm{C}$. \\
\hline & & $2: 10$ & 40.1 & Average glass temperature is $1150^{\circ} \mathrm{C}$. Plenum temperature is $520-537^{\circ} \mathrm{C}$. \\
\hline & & $2: 37$ & 40.5 & $\begin{array}{l}\text { Average glass temperature is } 1149^{\circ} \mathrm{C} \text {. Plenum temperature is } 502-507^{\circ} \mathrm{C} \text {. } \\
\text { Glass resistance is } 0.082 \Omega \text {. }\end{array}$ \\
\hline & & $2: 51$ & 40.8 & Plenum temperature is $495-499^{\circ} \mathrm{C}$. \\
\hline & & 3:02 & 40.9 & Average glass temperature is $1146^{\circ} \mathrm{C}$. \\
\hline & & 3:09 & 41.1 & Plenum temperature is $481-495^{\circ} \mathrm{C}$. \\
\hline & & $3: 25$ & 41.3 & Plenum temperature is $473-499^{\circ} \mathrm{C}$. \\
\hline & & $3: 31$ & 41.4 & $\begin{array}{l}\text { Increased power from } 156 \mathrm{~kW} \text { to } 159 \mathrm{~kW} \text {. Average glass temperature is } \\
1139^{\circ} \mathrm{C} .\end{array}$ \\
\hline & & $3: 41$ & 41.6 & Average glass temperature is $1139^{\circ} \mathrm{C}$. Plenum temperature is $461-483^{\circ} \mathrm{C}$. \\
\hline & & $3: 42$ & 41.6 & Plenum temperature is $460-480^{\circ} \mathrm{C}$. \\
\hline & & 4:05 & 42.0 & Glass level is at $30.2 "$. \\
\hline & & $4: 22$ & 42.3 & $\begin{array}{c}\text { Glass level is at } 27.8^{\prime \prime}, \text { Average glass temperature is } 1137^{\circ} \mathrm{C} \text {. Plenum } \\
\text { temperature is } 433-451^{\circ} \mathrm{C} \text {. }\end{array}$ \\
\hline & & $4: 32$ & 42.4 & Increased power from 159 to $162 \mathrm{~kW}$. All four Lances are at $11.0 \mathrm{lpm}$. \\
\hline & & $4: 47$ & 42.7 & Plenum temperature is $433-452^{\circ} \mathrm{C}$. \\
\hline & & $5: 10$ & 43.1 & Average glass temperature is $1150^{\circ} \mathrm{C}$. Plenum temperature is $427-445^{\circ} \mathrm{C}$. \\
\hline & & $5: 35$ & 43.5 & $\begin{array}{l}\text { Neutralizing neutralization tank, } \mathrm{pH} \text { is at 5.40. (All } 3 \text { tanks are equalized with } \\
\text { acid tank at } \mathrm{pH} \text { of } 7.63 \text { ) }\end{array}$ \\
\hline & & $5: 40$ & 43.6 & Stopped caustic pump to neutralization tank after $\mathrm{pH}$ increased from 5.4 to 6.8 . \\
\hline & & $5: 44$ & 43.6 & Average glass temperature is $1153^{\circ} \mathrm{C}$. Plenum temperature is $407-417^{\circ} \mathrm{C}$. \\
\hline
\end{tabular}


Table 3.2. Summary of Operational Events (continued).

\begin{tabular}{|c|c|c|c|c|}
\hline Test & Date & Time & $\begin{array}{l}\text { Run } \\
\text { Time } \\
\text { (hours) }\end{array}$ & Run time note \\
\hline \multirow{25}{*}{1} & \multirow{25}{*}{$1 / 17 / 13$} & $6: 42$ & 44.6 & Decreased power from 162 to $160 \mathrm{~kW}$. Average glass temperature is $1153^{\circ} \mathrm{C}$. \\
\hline & & $7: 06$ & 45.0 & Total bubbling is $44 \mathrm{lpm}$, feed rate is $144 \mathrm{~kg} / \mathrm{hr}$. \\
\hline & & $7: 08$ & 45.0 & $\begin{array}{l}\text { Decreased power from } 160 \mathrm{~kW} \text { to } 158 \mathrm{~kW} \text {. Average glass temperature is } \\
1158^{\circ} \mathrm{C}\end{array}$ \\
\hline & & $7: 51$ & 45.8 & $\begin{array}{c}\text { Average glass temperature is } 1154^{\circ} \mathrm{C} \text {. Feed rate is } 147.1 \mathrm{~kg} / \mathrm{hr} \text {. Power is } 157 \\
\mathrm{~kW} \text {, secondary current is } 1412 \mathrm{Amps} \text {. Total bubbling is } 44 \mathrm{lpm} \text {. Glass } \\
\text { resistance is } 0.079 \Omega \text {. Glass level is } 29.7^{\prime \prime}\end{array}$ \\
\hline & & 9:08 & 47.0 & Average glass temperature is $1150^{\circ} \mathrm{C}$. Glass level is $28.5^{\prime \prime}$. \\
\hline & & $9: 31$ & 47.4 & Average glass temperature is $1150^{\circ} \mathrm{C}$. Glass level is $29.3^{\prime \prime}$. \\
\hline & & 10:02 & 47.9 & Transferred feed $247.0 \mathrm{~kg}$ feed and $5 \mathrm{~kg}$ water to feed tank. \\
\hline & & $12: 30$ & 50.4 & Secured feeding, End of the test. No longer able to maintain feeding. \\
\hline & & $12: 35$ & 50.5 & $\begin{array}{c}\text { Feed tank mass in recirculation is } 34.5 \mathrm{~kg} \text {. Cleared recirculation line. Feed tank } \\
\text { mass is } 61.5 \mathrm{~kg} \text {. Flushed recirculation line. }\end{array}$ \\
\hline & & $12: 55$ & 50.8 & $\begin{array}{l}\text { Removed residual feed from feed tank. Starting mass is } 61.5 \mathrm{~kg} \text {. Ending mass is } \\
25.5 \mathrm{~kg} \text {. Total removed feed mass is } 36.0 \mathrm{~kg} \text {. }\end{array}$ \\
\hline & & $12: 47$ & 50.7 & $\begin{array}{l}\text { Decreased power from } 158 \mathrm{~kW} \text { to } 150 \mathrm{~kW} \text {. Average glass temperature is } \\
1155^{\circ} \mathrm{C} .\end{array}$ \\
\hline & & $12: 51$ & 50.8 & $\begin{array}{l}\text { Decreased power from } 150 \mathrm{~kW} \text { to } 140 \mathrm{~kW} \text {. Average glass temperature is } \\
1159^{\circ} \mathrm{C} .\end{array}$ \\
\hline & & $12: 55$ & 50.8 & $\begin{array}{l}\text { Removed residual feed from feed tank. Starting mass is } 61.5 \mathrm{~kg} \text {, end mass is } \\
25.5 \mathrm{~kg} \text {. Net mass removed is } 36.0 \mathrm{~kg} \text {. }\end{array}$ \\
\hline & & $12: 58$ & 50.9 & $\begin{array}{l}\text { Decreased power from } 140 \mathrm{~kW} \text { to } 130 \mathrm{~kW} \text {. Average glass temperature is } \\
1164^{\circ} \mathrm{C} .\end{array}$ \\
\hline & & 13:05 & 51.0 & $\begin{array}{c}\text { Decreased power from } 130 \mathrm{~kW} \text { to } 110 \mathrm{~kW} \text {. Average glass temperature is } \\
1161^{\circ} \mathrm{C} .\end{array}$ \\
\hline & & 13:15 & 51.2 & Decreased power from $110 \mathrm{~kW}$ to $90 \mathrm{~kW}$. Average glass temperature is $1162^{\circ} \mathrm{C}$ \\
\hline & & 14:00 & 51.9 & $\begin{array}{l}\text { Reduced bubbling from } 11 \mathrm{lpm} \text { on Lance Bubbler mass flow controllers L1 and } \\
\text { L2 to } 1.5 \mathrm{lpm} \text { on } 1 \mathrm{~A} \text {, and } 2 \mathrm{~A} \text {. De-energized 1B and 2B. }\end{array}$ \\
\hline & & $14: 20$ & 52.2 & Decreased power from $90 \mathrm{~kW}$ to $80 \mathrm{~kW}$. Average glass temperature is $1161^{\circ} \mathrm{C}$ \\
\hline & & 14:27 & 52.4 & $\begin{array}{l}\text { Increased bubbling mass flow controllers } 1 \mathrm{~A} \text { and } 2 \mathrm{~A} \text { to } 10 \mathrm{lpm} \text {. A cold cap has } \\
\text { reformed. }\end{array}$ \\
\hline & & 15:19 & 53.2 & Reduced bubbling on $1 \mathrm{~A}$ and $2 \mathrm{~A}$ from $10 \mathrm{lpm}$ to $5 \mathrm{lpm}$ each. \\
\hline & & 15:30 & 53.4 & $\mathrm{CC}$ is gone. \\
\hline & & $16: 30$ & 54.4 & Started melter and off gas shut down procedures. \\
\hline & & $17: 00$ & 54.9 & Performed WESP blow down, deluge and blow down. \\
\hline & & $17: 14$ & 55.1 & Reduced bubbling from 5 to $1.5 \mathrm{lpm}$ each. \\
\hline & & $18: 10$ & 56.1 & Melter and off gas shut downs are completed. \\
\hline 2 & $1 / 22 / 13$ & $10: 35$ & - & $\begin{array}{l}\text { Transferred feed from mix tank to feed tank. Tank mass at the start is } 40.5 \mathrm{~kg} \text {, } \\
\text { tank mass at the end is } 2951.5 \mathrm{~kg} \text {. Net mass of feed transferred to feed tank is } \\
2911.0 \mathrm{~kg} \text {. This mass includes } 500.7 \mathrm{~kg} \text { water. }\end{array}$ \\
\hline
\end{tabular}




\section{Table 3.2. Summary of Operational Events (continued).}

\begin{tabular}{|c|c|c|c|c|}
\hline Test & Date & Time & $\begin{array}{l}\text { Run } \\
\text { Time } \\
\text { (hours) }\end{array}$ & Run time note \\
\hline \multirow{27}{*}{2} & \multirow{27}{*}{$1 / 22 / 13$} & 13:15 & - & $\begin{array}{l}\text { Transferred feed to mix tank. Tank mass is } 3001.5 \mathrm{~kg} \text {. This mass includes } 10 \\
\mathrm{~kg} \text { water. Initial tank mass is } 40.5 \mathrm{~kg} \text {. Final tank mass is } 3001.0 \mathrm{~kg} \text {. Net mass } \\
\text { transferred is } 2960.5 \mathrm{~kg} \text {. }\end{array}$ \\
\hline & & 13:48 & - & Increased power to $140 \mathrm{~kW}$. Average glass temperature is $1142^{\circ} \mathrm{C}$. \\
\hline & & 14:00 & 0.0 & Started feeding water at $0.7 \mathrm{l} / \mathrm{min}$. \\
\hline & & $14: 15$ & 0.3 & Increased water flow rate to $1.4 \mathrm{l} / \mathrm{min}$. \\
\hline & & $14: 30$ & 0.5 & $\begin{array}{l}\text { Increased water flow rate to } 2.1 \mathrm{l} / \mathrm{min} \text {. Plenum temperature is } 941-946^{\circ} \mathrm{C} \text {. } \\
\text { Average glass temperature is } 1152^{\circ} \mathrm{C} \text {. Placed SBS cooling water booster pump } \\
\text { in service. }\end{array}$ \\
\hline & & $14: 37$ & 0.6 & Increased power from $140 \mathrm{~kW}$ to $145 \mathrm{~kW}$. Avg. glass temperature is $1147^{\circ} \mathrm{C}$. \\
\hline & & $14: 42$ & 0.7 & Increased water flow rate to $2.8 \mathrm{1} / \mathrm{min}$. \\
\hline & & $14: 52$ & 0.9 & Increased power from $145 \mathrm{~kW}$ to $155 \mathrm{~kW}$. Avg. glass temperature is $1143^{\circ} \mathrm{C}$. \\
\hline & & 15:00 & 1.0 & Secured water flow and starting feeding slurry. \\
\hline & & 15:05 & 1.1 & $\begin{array}{l}\text { Increased bubbling as follows: Lance } 1 \text { mass flow controllers A\&B changed } \\
\text { from } 1.5 \text { to } 5 \text {. lpm each, Lance } 2 \text { mass flow controllers A \& B changed from } \\
1.5 \text { to } 5 \mathrm{lpm} \text { each. Lance } 3 \text { mass flow controller A and B from } 1.5 \text { to } 5 \mathrm{lpm} \text {. } \\
\text { Total is } 30 \mathrm{lpm} \text {. }\end{array}$ \\
\hline & & 15:07 & 1.1 & Thermocouple TR- 08 not functional; it is out of service now. \\
\hline & & $15: 12$ & 1.2 & $\begin{array}{l}\text { Increased power from } 155 \mathrm{~kW} \text { to } 160 \mathrm{~kW} \text {. Average glass temperature is } \\
\qquad 1147^{\circ} \mathrm{C} .\end{array}$ \\
\hline & & $15: 27$ & 1.5 & $\begin{array}{c}\text { Increased T4 from } 28 \text { to } 32 \text { seconds and T1 from } 1.1 \text { to } 1.2 \text { second. Plenum } \\
\text { temperature is } 880-864^{\circ} \mathrm{C}\end{array}$ \\
\hline & & 15:44 & 1.7 & Plenum temperature is $768-806^{\circ} \mathrm{C}$. \\
\hline & & 16:03 & 2.1 & $\begin{array}{l}\text { Increased bubbling on Lance } 1 \text { mass flow controllers A\&B and Lance } 2 \text { mass } \\
\text { flow controllers A\&B from } 5 \text { to } 10 \mathrm{lpm} \text {, Lance } 3 \text { mass flow controllers A\&B } \\
\text { from } 5 \text { to } 7.5 \mathrm{lpm} \text {. Total bubbling is } 55 \mathrm{lpm} \text {. }\end{array}$ \\
\hline & & $16: 17$ & 2.3 & Increased power from 160 to $170 \mathrm{~kW}$. \\
\hline & & $16: 23$ & 2.4 & Plenum temperature is $718-748^{\circ} \mathrm{C}$. \\
\hline & & $16: 32$ & 2.5 & $\begin{array}{c}\text { Increased power from } 170 \text { to } 175 \mathrm{~kW} \text {. Average glass temperature is } 1143^{\circ} \mathrm{C} \text {. } \\
\text { Plenum temperature is } 691-714^{\circ} \mathrm{C} \text {. }\end{array}$ \\
\hline & & 17:07 & 3.1 & $\begin{array}{l}\text { Increased bubbling on Lances 1A, 2A (was } 10 \mathrm{lpm} \text { each), } 3 \mathrm{~A} \text { (was } 7.5 \mathrm{lpm} \text { ) to } \\
12.0 \mathrm{lpm} \text { and on Lances 1B, 2B from } 10.0 \text { to } 12.0 \mathrm{lpm} \text {. }\end{array}$ \\
\hline & & $17: 12$ & 3.2 & Plenum temperature is $657-695^{\circ} \mathrm{C}$. \\
\hline & & $17: 14$ & 3.2 & Increased power from 180 to $183 \mathrm{~kW}$. \\
\hline & & $17: 24$ & 3.4 & Plenum temperature is $666-710^{\circ} \mathrm{C}$. \\
\hline & & $17: 28$ & 3.5 & Increased power from 183 to $188 \mathrm{~kW}$. Average glass temperature is $1145^{\circ} \mathrm{C}$ \\
\hline & & $17: 38$ & 3.6 & Plenum temperature is $667-695^{\circ} \mathrm{C}$. \\
\hline & & $17: 42$ & 3.7 & Increased power from 188 to $193 \mathrm{~kW}$. Average glass temperature is $1138^{\circ} \mathrm{C}$. \\
\hline & & $17: 58$ & 4.0 & Plenum temperature is $660-682^{\circ} \mathrm{C}$. \\
\hline & & $17: 59$ & 4.0 & $\begin{array}{l}\text { Increased bubbling on Lance mass flow controllers } 1 \mathrm{~A}, 2 \mathrm{~A} \text { from } 12.0 \text { to } 16.0 \\
\mathrm{lpm} \text {. Total bubbling is } 78.5 \mathrm{lpm} \text {. Increased power from } 188 \text { to } 193 \mathrm{~kW} \text {. } \\
\text { Average glass temperature is } 1134^{\circ} \mathrm{C}\end{array}$ \\
\hline
\end{tabular}


Table 3.2. Summary of Operational Events (continued).

\begin{tabular}{|c|c|c|c|c|}
\hline Test & Date & Time & $\begin{array}{c}\text { Run } \\
\text { Time } \\
\text { (hours) }\end{array}$ & Run time note \\
\hline \multirow{29}{*}{2} & \multirow{23}{*}{$1 / 22 / 13$} & 18:30 & 4.5 & Increased power from 192 to $197 \mathrm{~kW}$. \\
\hline & & 19:04 & 5.1 & Plenum temperature is $632-675^{\circ} \mathrm{C}$. \\
\hline & & 19:20 & 5.3 & Paused feeding to collect feed sample. \\
\hline & & 19:26 & 5.4 & Plenum temperature is $650-669^{\circ} \mathrm{C}$. \\
\hline & & 19:34 & 5.6 & Plenum temperature is $647-686^{\circ} \mathrm{C}$. \\
\hline & & 19:48 & 5.8 & Plenum temperature is $645-683^{\circ} \mathrm{C}$. \\
\hline & & 20:00 & 6.0 & Adjusted T4 from 27 seconds to 25 seconds. \\
\hline & & $20: 20$ & 6.3 & Plenum temperature is $636-666^{\circ} \mathrm{C}$. \\
\hline & & $20: 35$ & 6.6 & Plenum temperature is $637-638^{\circ} \mathrm{C}$. \\
\hline & & 21:04 & 7.1 & Increased power from 197 to $202 \mathrm{~kW}$. Average glass temperature is $1135^{\circ} \mathrm{C}$ \\
\hline & & 21:09 & 7.2 & Plenum temperature is $604-645^{\circ} \mathrm{C}$. \\
\hline & & $21: 11$ & 7.2 & Increased power from 202 to $207 \mathrm{~kW}$. Average glass temperature is $1138^{\circ} \mathrm{C}$ \\
\hline & & $21: 19$ & 7.3 & Plugged pump for caustic tank, $\mathrm{pH}$ was 4.0 . \\
\hline & & 21:49 & 7.8 & $\begin{array}{c}\text { Average glass temperature is } 1143^{\circ} \mathrm{C} \text {. Glass resistance is } 0.085 \Omega \text {. Plenum } \\
\text { temperature is } 611-637^{\circ} \mathrm{C} \text {. }\end{array}$ \\
\hline & & 21:54 & 7.9 & Emergency off gas alarm is tripped. \\
\hline & & 22:04 & 8.1 & Average glass temperature is $1146^{\circ} \mathrm{C}$. Plenum temperature is $608-620^{\circ} \mathrm{C}$ \\
\hline & & 22:20 & 8.3 & $\begin{array}{l}\text { Average glass temperature is } 1147^{\circ} \mathrm{C} \text {. Glass resistance is } 0.085 \Omega \text {. Plenum } \\
\text { temperature is } 624-625^{\circ} \mathrm{C} \text {. }\end{array}$ \\
\hline & & $22: 36$ & 8.6 & $\begin{array}{c}\text { Average glass temperature is } 1149^{\circ} \mathrm{C} \text {. Glass resistance is } 0.083 \Omega \text {. Plenum } \\
\text { temperature is } 604-630^{\circ} \mathrm{C}\end{array}$ \\
\hline & & $22: 58$ & 9.0 & Plenum temperature is $598-624^{\circ} \mathrm{C}$ \\
\hline & & 23:03 & 9.1 & $\begin{array}{l}\text { Increased power from } 207 \text { to } 210 \mathrm{~kW} \text {. Average glass temperature is } 1140^{\circ} \mathrm{C} \text {. } \\
\text { Plenum temperature is } 593-613^{\circ} \mathrm{C} \text {. Feed rate is } \sim 180 \mathrm{~kg} / \mathrm{hr} \text {. }\end{array}$ \\
\hline & & 23:11 & 9.2 & Plenum temperature is $596-604^{\circ} \mathrm{C}$ \\
\hline & & 23:26 & 9.4 & Average glass temperature is $1148^{\circ} \mathrm{C}$. Plenum temperature is $594-610^{\circ} \mathrm{C}$. \\
\hline & & 23:41 & 9.7 & Plenum temperature is $581-602^{\circ} \mathrm{C}$ \\
\hline & \multirow{6}{*}{$1 / 23 / 13$} & 0:01 & 10.0 & $\begin{array}{l}\text { Reduced power from } 210 \text { to } 207 \mathrm{~kW} \text {. High temperature alarm. Average glass } \\
\text { temperature is } 1156^{\circ} \mathrm{C} \text {. Plenum temperature is } 597-613^{\circ} \mathrm{C} \text {. }\end{array}$ \\
\hline & & 0:05 & 10.1 & $\begin{array}{l}\text { Average glass temperature is } 1155^{\circ} \mathrm{C} \text {. Plenum temperature is } 596-611^{\circ} \mathrm{C} \text {. } \\
\text { Observed film cooler differential pressure is greater than } 8.0 \text { in W.C. Transition } \\
\text { line differential pressure is } \sim 25 \text { in W.C. Upon visual inspection found build up at } \\
\text { the bottom opening of the film cooler. Attempted to clear with two water flush } \\
\text { sequences. Attempt was not successful. }\end{array}$ \\
\hline & & $0: 11$ & 10.2 & $\begin{array}{l}\text { Film cooler rinse may have caused melter pressure spike. Emergency off gas } \\
\text { alarm is tripped. }\end{array}$ \\
\hline & & $0: 21$ & 10.4 & Average glass temperature is $1144^{\circ} \mathrm{C}$. Plenum temperature is $585-605^{\circ} \mathrm{C}$. \\
\hline & & $0: 29$ & 10.5 & Paused feeding, rodded film cooler. \\
\hline & & $0: 32$ & 10.5 & Resumed feeding. Film cooler differential pressure is 2.0 in W.C. \\
\hline
\end{tabular}


Table 3.2. Summary of Operational Events (continued).

\begin{tabular}{|c|c|c|c|c|}
\hline Test & Date & Time & $\begin{array}{c}\text { Run } \\
\text { Time } \\
\text { (hours) }\end{array}$ & Run time note \\
\hline \multirow{27}{*}{2} & \multirow{27}{*}{$1 / 23 / 13$} & $0: 39$ & 10.7 & Plenum temperature is $579-612^{\circ} \mathrm{C}$. \\
\hline & & $0: 52$ & 10.9 & Plenum temperature is $572-615^{\circ} \mathrm{C}$. \\
\hline & & $1: 06$ & 11.1 & Plenum temperature is $571-615^{\circ} \mathrm{C}$. \\
\hline & & $1: 21$ & 11.4 & Average glass temperature is $1152^{\circ} \mathrm{C}$. Plenum temperature is $565-606^{\circ} \mathrm{C}$. \\
\hline & & $1: 33$ & 11.6 & $\begin{array}{l}\text { Average glass temperature is } 1147^{\circ} \mathrm{C} \text {. Glass resistance is } 0.083 \Omega \text {. Plenum } \\
\text { temperature is } 564-588^{\circ} \mathrm{C} \text {. Glass density is } 2.27 \mathrm{~g} / \mathrm{cc} \text {. }\end{array}$ \\
\hline & & $1: 36$ & 11.6 & $\begin{array}{l}\text { Increased electric power from } 207 \text { to } 210 \mathrm{~kW} \text {. Average glass temperature is } \\
\qquad 1145^{\circ} \mathrm{C}\end{array}$ \\
\hline & & $1: 42$ & 11.7 & Changed T4 from 24 seconds to 25 seconds. \\
\hline & & $1: 43$ & 11.7 & $\begin{array}{l}\text { Average glass temperature is } 1141^{\circ} \mathrm{C} \text {. Glass resistance is } 0.085 \Omega \text {. Plenum } \\
\text { temperature is } 560-594^{\circ} \mathrm{C} \text {. }\end{array}$ \\
\hline & & $1: 47$ & 11.8 & Increased electrical power from $210 \mathrm{~kW}$ to $213 \mathrm{~kW}$. \\
\hline & & $1: 48$ & 11.8 & $\begin{array}{c}\text { Decreased electrical power from } 213 \text { to } 212 \mathrm{~kW} \text {. Electrical current is } 1577 \\
\text { Amps. }\end{array}$ \\
\hline & & $1: 50$ & 11.8 & Decreased T4 from 25 seconds to 24 seconds. \\
\hline & & 2:04 & 12.1 & $\begin{array}{c}\text { Average glass temperature is } 1141^{\circ} \mathrm{C} \text {. Glass resistance is } 0.085 \Omega \text {. Plenum } \\
\text { temperature is } 558-587^{\circ} \mathrm{C} \text {. }\end{array}$ \\
\hline & & $2: 20$ & 12.3 & $\begin{array}{l}\text { Average glass temperature is } 1146^{\circ} \mathrm{C} \text {. Glass resistance is } 0.082 \Omega \text {. Plenum } \\
\text { temperature is } 546-572^{\circ} \mathrm{C} \text {. }\end{array}$ \\
\hline & & $2: 37$ & 12.6 & $\begin{array}{l}\text { Average glass temperature is } 1149^{\circ} \mathrm{C} \text {. Glass resistance is } 0.082 \Omega \text {. Plenum } \\
\text { temperature is } 553-579^{\circ} \mathrm{C} \text {. }\end{array}$ \\
\hline & & $2: 45$ & 12.8 & Reduced power from 212 to $210 \mathrm{~kW}$. Electrical current is 1601 Amps. \\
\hline & & $2: 52$ & 12.9 & Plenum temperature is $544-574^{\circ} \mathrm{C}$. \\
\hline & & $3: 02$ & 13.0 & $\begin{array}{c}\text { Reduced power from } 210 \text { to } 207 \mathrm{~kW} \text {. Electrical current is } 1603 \text { Amps. Average } \\
\text { glass temperature is } 1153^{\circ} \mathrm{C}\end{array}$ \\
\hline & & $3: 17$ & 13.3 & $\begin{array}{l}\text { Average glass temperature is } 1144^{\circ} \mathrm{C} \text {. Plenum temperature is } 544-568^{\circ} \mathrm{C} \text {. Feed } \\
\text { rate is } \sim 180 \mathrm{~kg} / \mathrm{hr} \text {. }\end{array}$ \\
\hline & & $3: 27$ & 13.5 & $\begin{array}{l}\text { Reduced electrical power from } 207 \text { to } 205 \mathrm{~kW} \text {. Electrical current is above } 1571 \\
\text { Amps. Average glass temperature is } 1142^{\circ} \mathrm{C} \text {. Plenum temperature is } 548-567^{\circ} \mathrm{C}\end{array}$ \\
\hline & & $3: 38$ & 13.6 & $\begin{array}{c}\text { Reduced electrical power from } 205 \text { to } 203 \mathrm{~kW} \text {. Electrical current is } 1168 \mathrm{Amps} \text {. } \\
\text { Plenum temperature is } 557-573^{\circ} \mathrm{C} \text {. }\end{array}$ \\
\hline & & $3: 41$ & 13.7 & Average glass temperature is $1145^{\circ} \mathrm{C}$. Plenum temperature is $558-574^{\circ} \mathrm{C}$. \\
\hline & & 4:03 & 14.1 & Average glass temperature is $1150^{\circ} \mathrm{C}$. Plenum temperature is $559-574^{\circ} \mathrm{C}$. \\
\hline & & $4: 19$ & 14.3 & $\begin{array}{c}\text { Average glass temperature is } 1148^{\circ} \mathrm{C} \text {. Glass resistance is } 0.081 \Omega \text {. Plenum } \\
\text { temperature is } 567-571^{\circ} \mathrm{C} \text {. }\end{array}$ \\
\hline & & $4: 36$ & 14.6 & Average glass temperature is $1148^{\circ} \mathrm{C}$. Plenum temperature is $554-568^{\circ} \mathrm{C}$. \\
\hline & & $4: 51$ & 14.9 & $\begin{array}{c}\text { Average glass temperature is } 1145^{\circ} \mathrm{C} \text {. Glass resistance is } 0.083 \Omega \text {. Plenum } \\
\text { temperature is } 560-575^{\circ} \mathrm{C} \text {, glass density is } 2.28 \mathrm{~g} / \mathrm{cc} \text {. }\end{array}$ \\
\hline & & $5: 15$ & 15.3 & $\begin{array}{l}\text { Glass resistance is } 0.084 \Omega \text {. Plenum temperature is } 537-601^{\circ} \mathrm{C} \text {, glass density is } \\
\qquad 2.28 \mathrm{~g} / \mathrm{cc} \text {. }\end{array}$ \\
\hline & & $5: 32$ & 15.5 & Plenum temperature is $528-598^{\circ} \mathrm{C}$. \\
\hline
\end{tabular}


Table 3.2. Summary of Operational Events (continued).

\begin{tabular}{|c|c|c|c|c|}
\hline Test & Date & Time & $\begin{array}{l}\text { Run } \\
\text { Time } \\
\text { (hours) }\end{array}$ & Run time note \\
\hline \multirow{26}{*}{2} & \multirow{26}{*}{$1 / 23 / 13$} & $5: 39$ & 15.7 & Glass resistance is $0.085 \Omega$, glass density is $2.25 \mathrm{~g} / \mathrm{cc}$. \\
\hline & & $5: 44$ & 15.7 & $\begin{array}{c}\text { Increased power from } 203 \text { to } 206 \mathrm{~kW} \text {. Average glass temperature is } 1142^{\circ} \mathrm{C} \text {. } \\
\text { Electrical current is } 1540 \mathrm{Amps} \text {. Glass resistance is } 0.086 \Omega \text {. }\end{array}$ \\
\hline & & $5: 54$ & 15.9 & Plenum temperature is $501-566^{\circ} \mathrm{C}$. \\
\hline & & $6: 23$ & 16.4 & Increased power from 206 to $209 \mathrm{~kW}$. Average glass temperature is $1132^{\circ} \mathrm{C}$. \\
\hline & & $6: 40$ & 16.7 & $\begin{array}{c}\text { Average glass temperature is } 1137^{\circ} \mathrm{C} \text {. Glass resistance is } 0.083 \Omega \text {. Plenum } \\
\text { temperature is } 513-553^{\circ} \mathrm{C} \text {. }\end{array}$ \\
\hline & & $7: 02$ & 17.0 & Average glass temperature is $1141^{\circ} \mathrm{C}$. \\
\hline & & $7: 16$ & 17.3 & $\begin{array}{l}\text { Average glass temperature is } 1147^{\circ} \mathrm{C} \text {. Plenum temperature is } 515-545^{\circ} \mathrm{C} \text {. } \\
\text { Decreased power from } 209 \text { to } 205 \mathrm{~kW} \text {, the electrical current is } 1594 \mathrm{Amps} \text {. }\end{array}$ \\
\hline & & $7: 33$ & 17.6 & Plenum temperature is $497-539^{\circ} \mathrm{C}$. \\
\hline & & $7: 50$ & 17.8 & Plenum temperature is $529-553^{\circ} \mathrm{C}$. \\
\hline & & 8:06 & 18.1 & Plenum temperature is $533-584^{\circ} \mathrm{C}$. \\
\hline & & $8: 15$ & 18.3 & $\begin{array}{l}\text { Feed is stopped flowing into melter. Tank mass is steady at } 397.5 \mathrm{~kg} \text {, reduced } \\
\text { mixer speed to } 11 \mathrm{~Hz} \text {. AOD pump reset and pumping resumed at 8:21. }\end{array}$ \\
\hline & & $8: 21$ & 18.4 & Feeding is resumed. \\
\hline & & $8: 23$ & 18.4 & $\begin{array}{c}\text { Feed transfer is started. Beginning mass is } 3005.0 \mathrm{~kg} \text {. End mass is } 50.5 \mathrm{~kg} \text {. The } \\
\text { net mass transferred is } 2954.5 \mathrm{~kg} \text {. Adding } 493.0 \mathrm{~kg} \text { water to the feed tank. Total } \\
\text { mass transferred is } 3447.5 \mathrm{~kg} \text {. }\end{array}$ \\
\hline & & $9: 15$ & 19.3 & $\begin{array}{c}\text { Increased power from } 205 \text { to } 207 \mathrm{~kW} \text {. Electrical current is } 1510 \text { Amps. Average } \\
\text { glass temperature is } 1120^{\circ} \mathrm{C} \text {. Plenum temperature is } 457-513^{\circ} \mathrm{C} \text {. }\end{array}$ \\
\hline & & $9: 52$ & 19.9 & $\begin{array}{c}\text { Increased power from } 209 \text { to } 211 \mathrm{~kW} \text {. The electrical current is } 1547 \text { Amps. } \\
\text { Average glass temperature is } 1135^{\circ} \mathrm{C} \text {. }\end{array}$ \\
\hline & & $9: 55$ & 19.9 & $\begin{array}{l}\text { Average glass temperature is } 1146^{\circ} \mathrm{C} \text {. Glass resistance is } 0.083 \Omega \text {. Plenum } \\
\text { temperature is } 435-493^{\circ} \mathrm{C} \text {, electrical power is } 211 \mathrm{~kW} \text {, current is } 1568 \mathrm{Amps} \text {. }\end{array}$ \\
\hline & & $10: 05$ & 20.1 & $\begin{array}{c}\text { Average glass temperature is } 1145^{\circ} \mathrm{C} \text {. Plenum temperature is } 415-487^{\circ} \mathrm{C} \text {, } \\
\text { current is } 1573 \text { Amps. Feed rate is } \sim 180 \mathrm{~kg} / \mathrm{hr} \text {. }\end{array}$ \\
\hline & & $10: 20$ & 20.3 & $\begin{array}{l}\text { Average glass temperature is } 1145^{\circ} \mathrm{C} \text {. Plenum temperature is } 443-496^{\circ} \mathrm{C} \text {, } \\
\text { electrical power is } 210 \mathrm{~kW} \text {, current is } 1579 \text { Amps. Feed rate is } \sim 180 \mathrm{~kg} / \mathrm{hr} \text {. }\end{array}$ \\
\hline & & $10: 44$ & 20.7 & $\begin{array}{l}\text { Decreased power from } 211 \text { to } 209 \mathrm{~kW} \text {, current is } 1584 \text { Amps. Average glass } \\
\text { temperature is } 1149^{\circ} \mathrm{C} \text {. Feed rate is } \sim 180 \mathrm{~kg} / \mathrm{hr} \text {. LabVIEW rebooted. }\end{array}$ \\
\hline & & $11: 00$ & 21.0 & $\begin{array}{l}\text { After rebooting LabVIEW, average glass temperature is } 1156^{\circ} \mathrm{C} \text {. Power is } 211 \\
\mathrm{~kW} \text {, current is } 1630 \text { Amps. Decreased power from } 211 \text { to } 200 \mathrm{~kW} \text {. }\end{array}$ \\
\hline & & $11: 20$ & 21.3 & Average glass temperature is $1147^{\circ} \mathrm{C}$. \\
\hline & & $11: 35$ & 21.6 & Average glass temperature is $1147^{\circ} \mathrm{C}$. \\
\hline & & $11: 50$ & 21.8 & Average glass temperature is $1151^{\circ} \mathrm{C}$. \\
\hline & & 12:04 & 22.1 & Average glass temperature is $1155^{\circ} \mathrm{C}$. \\
\hline & & $12: 40$ & 22.7 & $\begin{array}{c}\text { Average glass temperature is } 1139^{\circ} \mathrm{C} \text {. Increased electrical power from } 200 \text { to } 203 \\
\mathrm{~kW} \text {, current is } 1530 \mathrm{Amps} \text {. Feed rate is } \sim 182 \mathrm{~kg} / \mathrm{hr} \text {. }\end{array}$ \\
\hline & & $13: 39$ & 23.7 & Plenum temperature is $418-513^{\circ} \mathrm{C}$. \\
\hline
\end{tabular}


Table 3.2. Summary of Operational Events (continued).

\begin{tabular}{|c|c|c|c|c|}
\hline Test & Date & Time & $\begin{array}{c}\text { Run } \\
\text { Time } \\
\text { (hours) }\end{array}$ & Run time note \\
\hline \multirow{29}{*}{2} & \multirow{29}{*}{$1 / 23 / 13$} & 13:54 & 23.9 & $\begin{array}{l}\text { Average glass temperature is } 1146^{\circ} \mathrm{C} \text {. Increased electrical power is } 201 \mathrm{~kW} \text {, } \\
\text { current is } 1564 \mathrm{Amps} \text {. Plenum temperature is } 448-493^{\circ} \mathrm{C} \text {. }\end{array}$ \\
\hline & & $14: 35$ & 24.6 & Average glass temperature is $1149^{\circ} \mathrm{C}$. \\
\hline & & $14: 44$ & 24.7 & $\begin{array}{l}\text { Transfer feed to mix tank. Starting mass is } 50.5 \mathrm{~kg} \text {, ending mass is } 2957 \mathrm{~kg} \text {. } \\
\text { Mixer is set @ } 30 \mathrm{~Hz} \text {. }\end{array}$ \\
\hline & & 15:04 & 25.1 & Plenum temperature is $496-531^{\circ} \mathrm{C}$. \\
\hline & & 15:19 & 25.3 & Plenum temperature is $506-520^{\circ} \mathrm{C}$. \\
\hline & & 15:39 & 25.7 & Plenum temperature is $514-539^{\circ} \mathrm{C}$. \\
\hline & & $15: 51$ & 25.9 & Increased power from 203 to $205 \mathrm{~kW}$. Average glass temperature is $1145^{\circ} \mathrm{C}$. \\
\hline & & 15:53 & 25.9 & Plenum temperature is $477-556^{\circ} \mathrm{C}$. \\
\hline & & 16:09 & 26.2 & Plenum temperature is $485-556^{\circ} \mathrm{C}$. \\
\hline & & $16: 18$ & 26.3 & Increased power from 205 to $208 \mathrm{~kW}$. Average glass temperature is $1141^{\circ} \mathrm{C}$. \\
\hline & & $16: 25$ & 26.4 & Performed WESP pre-deluge and blow downs. \\
\hline & & $16: 30$ & 26.5 & $\begin{array}{c}\text { Melter went positive and tripped emergency off gas, immediately reset, cause } \\
\text { unknown. }\end{array}$ \\
\hline & & $16: 39$ & 26.7 & $\begin{array}{c}\text { Increased power from } 208 \text { to } 209 \mathrm{~kW} \text {. Average glass temperature is } 1141^{\circ} \mathrm{C} \text {, the } \\
\text { current is } 1556 \text { Amps. }\end{array}$ \\
\hline & & $16: 49$ & 26.8 & Plenum temperature is $509-512^{\circ} \mathrm{C}$. \\
\hline & & 17:01 & 27.0 & $\begin{array}{c}\text { Reduced power from } 209 \text { to } 207 \mathrm{~kW} \text {. Average glass temperature is } 1148^{\circ} \mathrm{C} \text {. } \\
\text { Melter current is } 1584 \text { Amps. }\end{array}$ \\
\hline & & $17: 21$ & 27.4 & Average glass temperature is $1144^{\circ} \mathrm{C}$. Plenum temperature is $509-523^{\circ} \mathrm{C}$. \\
\hline & & 17:39 & 27.7 & Plenum temperature is $507-527^{\circ} \mathrm{C}$ \\
\hline & & $17: 46$ & 27.8 & $\begin{array}{l}\text { Melter pressure went positive and tripped emergency off gas, immediately reset, } \\
\text { caused by off gas sampling. }\end{array}$ \\
\hline & & $17: 50$ & 27.8 & Plenum temperature is $486-512^{\circ} \mathrm{C}$. \\
\hline & & 18:04 & 28.1 & Plenum temperature is $518-521^{\circ} \mathrm{C}$. Electrical current is $1572 \mathrm{Amps}$. \\
\hline & & 18:07 & 28.1 & $\begin{array}{c}\text { Stopped off gas sampling which caused melter pressure spike when probed } \\
\text { removed from transition line. }\end{array}$ \\
\hline & & 18:27 & 28.5 & Emergency off gas is tripped, $\mathrm{CC}$ is shifted. \\
\hline & & 18:39 & 28.7 & $\begin{array}{l}\text { Average glass temperature is } 1132^{\circ} \mathrm{C} \text {. Melter current is } 1163 \text { Amps. Plenum } \\
\text { temperature is } 517-524^{\circ} \mathrm{C} .\end{array}$ \\
\hline & & 18:49 & 28.8 & $\begin{array}{l}\text { Average glass temperature is } 1135^{\circ} \mathrm{C} \text {. Melter current is } 1571 \text { Amps. Plenum } \\
\text { temperature is } 523-525^{\circ} \mathrm{C} \text {. }\end{array}$ \\
\hline & & 19:04 & 29.1 & $\begin{array}{c}\text { Average glass temperature is } 1137^{\circ} \mathrm{C} \text {. Melter current is } 1571 \text { Amps. Plenum } \\
\text { temperature is } 524-526^{\circ} \mathrm{C} \text {. }\end{array}$ \\
\hline & & 19:20 & 29.3 & Plenum temperature is $514-525^{\circ} \mathrm{C}$. \\
\hline & & 19:29 & 29.5 & Paused feeding to collect feed sample. \\
\hline & & 19:35 & 29.6 & $\begin{array}{c}\text { Reduced power from } 207 \text { to } 204 \mathrm{~kW} \text {, current limited is } 1592 \text { Amps. Average } \\
\text { glass temperature is } 1144^{\circ} \mathrm{C} \text {. }\end{array}$ \\
\hline & & 19:39 & 29.7 & $\begin{array}{c}\text { Current is limited @ } 1575 \mathrm{Amps} \text {. Average glass temperature is } 1145^{\circ} \mathrm{C} \text {. Plenum } \\
\text { temperature is } 530-544^{\circ} \mathrm{C} \text {. }\end{array}$ \\
\hline
\end{tabular}




\section{Table 3.2. Summary of Operational Events (continued).}

\begin{tabular}{|c|c|c|c|c|}
\hline Test & Date & Time & $\begin{array}{c}\text { Run } \\
\text { Time } \\
\text { (hours) }\end{array}$ & Run time note \\
\hline \multirow{29}{*}{2} & \multirow{21}{*}{$1 / 23 / 13$} & 19:54 & 29.9 & $\begin{array}{c}\text { Average glass temperature is } 1147^{\circ} \mathrm{C} \text {. Melter current is } 1581 \text { Amps. Plenum } \\
\text { temperature is } 531-552^{\circ} \mathrm{C} \text {. }\end{array}$ \\
\hline & & 20:00 & 30.0 & $\begin{array}{c}\text { Reduced power from } 204 \text { to } 202 \mathrm{~kW} \text {. Average glass temperature is } 1147^{\circ} \mathrm{C} \text {. } \\
\text { Melter current is } 1574 \text { Amps }\end{array}$ \\
\hline & & $20: 12$ & 30.2 & Plenum temperature is $518-539^{\circ} \mathrm{C}$. \\
\hline & & $20: 26$ & 30.4 & Off gas sampling has started. \\
\hline & & $20: 49$ & 30.8 & $\begin{array}{c}\text { Average glass temperature is } 1152^{\circ} \mathrm{C} \text {. Melter current is } 1574 \text { Amps. Plenum } \\
\text { temperature is } 534-542^{\circ} \mathrm{C} \text {. }\end{array}$ \\
\hline & & 21:00 & 31.0 & End of off gas testing, melter pressure spiked. \\
\hline & & 21:04 & 31.1 & $\begin{array}{c}\text { Average glass temperature is } 1153^{\circ} \mathrm{C} \text {. Melter current is } 1574 \text { Amps. Plenum } \\
\text { temperature is } 527-546^{\circ} \mathrm{C} \text {. }\end{array}$ \\
\hline & & 21:07 & 31.1 & $\begin{array}{c}\text { Reduced power from } 202 \text { to } 201 \mathrm{~kW} \text {, current limited } 1574 \text { Amps. Average glass } \\
\text { temperature is } 1153^{\circ} \mathrm{C} \text {. }\end{array}$ \\
\hline & & $21: 25$ & 31.4 & Plenum temperature is $528-543^{\circ} \mathrm{C}$. \\
\hline & & $21: 39$ & 31.7 & Plenum temperature is $528-545^{\circ} \mathrm{C}$. \\
\hline & & $21: 54$ & 31.9 & Plenum temperature is $519-542^{\circ} \mathrm{C}$. \\
\hline & & $22: 08$ & 32.1 & Plenum temperature is $521-548^{\circ} \mathrm{C}$. \\
\hline & & $22: 24$ & 32.4 & Plenum temperature is $523-534^{\circ} \mathrm{C}$. \\
\hline & & $22: 44$ & 32.7 & Average glass temperature is $1150^{\circ} \mathrm{C}$. Glass density is $2.25 \mathrm{~g} / \mathrm{cc}$. \\
\hline & & $22: 48$ & 32.8 & Average glass temperature is $1144^{\circ} \mathrm{C}$. Plenum temperature is $513-533^{\circ} \mathrm{C}$. \\
\hline & & 23:08 & 33.1 & Plenum temperature is $512-537^{\circ} \mathrm{C}$. \\
\hline & & 23:23 & 33.4 & $\begin{array}{c}\text { Plenum temperature is } 512-548^{\circ} \mathrm{C} \text {. Average glass temperature is } 1145^{\circ} \mathrm{C} \text {. Glass } \\
\text { resistance is } 0.081 \Omega\end{array}$ \\
\hline & & $23: 38$ & 33.6 & Plenum temperature is $516-534^{\circ} \mathrm{C}$. Glass density is $2.24 \mathrm{~g} / \mathrm{cc}$. \\
\hline & & 23:42 & 33.7 & $\begin{array}{c}\text { SBS outlet gas pressure indicator is back in service after temporary problem. It is } \\
\text { fixed reading }-37.9 \text { in W.C. now. }\end{array}$ \\
\hline & & 23:50 & 33.8 & Plenum temperature is $524-536^{\circ} \mathrm{C}$. \\
\hline & & 23:54 & 33.9 & Glass density is $2.26 \mathrm{~g} / \mathrm{cc}$. \\
\hline & \multirow{8}{*}{$1 / 24 / 13$} & $0: 04$ & 34.1 & $\begin{array}{l}\text { Increased power from } 201 \text { to } 204 \mathrm{~kW} \text {, plenum temperature is } 525-545^{\circ} \mathrm{C} \text {. } \\
\text { Average glass temperature is } 1130^{\circ} \mathrm{C} \text {. Glass resistance is } 0.084 \Omega \text {. }\end{array}$ \\
\hline & & 0:08 & 34.1 & $\begin{array}{c}\text { Plenum temperature is } 524-544^{\circ} \mathrm{C} \text {. Average glass temperature is } 1132^{\circ} \mathrm{C} \text {. } \\
\text { Electrical current is } 1550 \text { Amps. }\end{array}$ \\
\hline & & $0: 14$ & 34.2 & $\begin{array}{l}\text { Increased power from } 204 \text { to } 206 \mathrm{~kW} \text {, current is } 1554 \text { Amps. Plenum temperature } \\
\text { is } 524-545^{\circ} \mathrm{C} \text {. Average glass temperature is } 1134^{\circ} \mathrm{C} \text {. Glass resistance is } 0.084 \Omega \text {. }\end{array}$ \\
\hline & & $0: 20$ & 34.3 & Plenum temperature is $524-540^{\circ} \mathrm{C}$. \\
\hline & & $0: 37$ & 34.6 & $\begin{array}{l}\text { Average glass temperature is } 1138^{\circ} \mathrm{C} \text {, current is } 1581 \text { Amps. Lowered T4 from } \\
\qquad 27.4 \text { to } 27.0 \text { seconds. }\end{array}$ \\
\hline & & $0: 39$ & 34.7 & $\begin{array}{c}\text { Reduced power from } 206 \text { to } 204 \mathrm{~kW} \text {, current is } 1580 \text { Amps, Average glass } \\
\text { temperature is } 1140^{\circ} \mathrm{C} \text {. }\end{array}$ \\
\hline & & $0: 43$ & 34.7 & Plenum temperature is $513-530^{\circ} \mathrm{C}$. \\
\hline & & $0: 54$ & 34.9 & Plenum temperature is $508-520^{\circ} \mathrm{C}$. \\
\hline
\end{tabular}


Table 3.2. Summary of Operational Events (continued).

\begin{tabular}{|c|c|c|c|c|}
\hline Test & Date & Time & $\begin{array}{l}\text { Run } \\
\text { Time } \\
\text { (hours) }\end{array}$ & Run time note \\
\hline \multirow{26}{*}{2} & \multirow{26}{*}{$1 / 24 / 13$} & $1: 04$ & 35.1 & $\begin{array}{l}\text { Average glass temperature is } 1140^{\circ} \mathrm{C} \text {. Plenum temperature is } 509-516^{\circ} \mathrm{C} \text {. Glass } \\
\text { resistance is } 0.081 \Omega \text {. Power is } 204 \mathrm{~kW} \text {, current is } 1568-1572 \mathrm{Amps} \text {. }\end{array}$ \\
\hline & & $1: 11$ & 35.2 & $\begin{array}{l}\text { Reducing power from } 204 \mathrm{~kW} \text { to } 202 \mathrm{~kW} \text {, current is } 1580 \text { Amps and average } \\
\text { glass temperature is } 1140^{\circ} \mathrm{C} \text {. Glass resistance is } 0.081 \Omega \text {. }\end{array}$ \\
\hline & & $1: 19$ & 35.3 & Plenum temperature is $514-520^{\circ} \mathrm{C}$, glass level is $29.8 \mathrm{in}$. \\
\hline & & $1: 27$ & 35.5 & LabVIEW re-started. \\
\hline & & $1: 43$ & 35.7 & Reduced electric power from 202 to $200 \mathrm{~kW}, 100$ Amps. \\
\hline & & $1: 45$ & 35.8 & $\begin{array}{c}\text { Average glass temperature is } 1142^{\circ} \mathrm{C} \text {. Plenum temperature is } 522-529^{\circ} \mathrm{C} \text {. Glass } \\
\text { resistance is } 0.078 \Omega \text {. Glass density is } 2.26 \mathrm{~g} / \mathrm{cc} \text {. }\end{array}$ \\
\hline & & $1: 46$ & 35.8 & Performed film cooler rinse. \\
\hline & & 2:06 & 36.1 & Glass density is $2.27 \mathrm{~g} / \mathrm{cc}$. Current is 1588 Amps. \\
\hline & & $2: 18$ & 36.3 & $\begin{array}{c}\text { Reduced power from } 200 \text { to } 198 \mathrm{~kW} \text {, current is } 1587 \text { Amps, Average glass } \\
\text { temperature is } 1147^{\circ} \mathrm{C} \text {. Glass resistance is } 0.078 \Omega \text {. }\end{array}$ \\
\hline & & $2: 19$ & 36.3 & Changed T4 from 27.0 to 26.5 seconds. \\
\hline & & $2: 34$ & 36.6 & $\begin{array}{l}\text { Plenum temperature is } 524-534^{\circ} \mathrm{C} \text {. Glass resistance is } 0.079 \Omega \text {. Glass density is } \\
2.27 \mathrm{~g} / \mathrm{cc} \text {, current is } 1579 \text { Amps. }\end{array}$ \\
\hline & & $2: 52$ & 36.9 & $\begin{array}{c}\text { Plenum temperature is } 528-533^{\circ} \mathrm{C} \text {. Current is } 1560 \text { Amps. Average glass } \\
\text { temperature is } 1139^{\circ} \mathrm{C} \text {. }\end{array}$ \\
\hline & & $3: 08$ & 37.1 & Changed T4 from 26.5 to 26.0 seconds. \\
\hline & & 3:09 & 37.2 & $\begin{array}{l}\text { Glass resistance is } 0.080 \Omega \text {. Current is } 1562 \text { Amps. Average glass temperature is } \\
1140^{\circ} \mathrm{C} \text {. }\end{array}$ \\
\hline & & $3: 30$ & 37.5 & $\begin{array}{l}\text { Mixer stopped, start transfer of feed to feed tank from mixing tank. Starting mass } \\
\text { of mixing tank is } 3003.5 \mathrm{~kg} \text {, end mass is } 73.0 \mathrm{~kg} \text {. Net mass transferred is } 2930.5 \\
\mathrm{~kg} \text {. Net amount of feed transferred is } 3428.5 \mathrm{~kg} \text { including } 498.0 \mathrm{~kg} \text { water. }\end{array}$ \\
\hline & & $3: 52$ & 37.9 & $\begin{array}{l}\text { Average glass temperature is } 1143^{\circ} \mathrm{C} \text {. Plenum temperature is } 510-533^{\circ} \mathrm{C} \text {. Glass } \\
\text { resistance is } 0.079 \Omega \text {. Current is } 1571 \text { Amps. Glass level is } 30.5 \mathrm{in} \text {. }\end{array}$ \\
\hline & & $4: 10$ & 38.2 & $\begin{array}{l}\text { Average glass temperature is } 1138^{\circ} \mathrm{C} \text {. Glass resistance is } 0.081 \Omega \text {. Current is } \\
1561 \mathrm{Amps} \text {. Glass density is } 2.26 \mathrm{~g} / \mathrm{cc} \text {. }\end{array}$ \\
\hline & & $4: 16$ & 38.3 & $\begin{array}{c}\text { Increased power from } 198 \text { to } 201 \mathrm{~kW} \text {. Average glass temperature is } 1138^{\circ} \mathrm{C} \text {. The } \\
\text { current is } 1560 \text { Amps. }\end{array}$ \\
\hline & & $4: 40$ & 38.7 & $\begin{array}{c}\text { Increased power from } 201 \text { to } 204 \mathrm{~kW} \text {. Average glass temperature is } 1132^{\circ} \mathrm{C} \text {. The } \\
\text { current is } 1550 \text { Amps. }\end{array}$ \\
\hline & & $4: 44$ & 38.7 & Changed T4 from 26 to 24 seconds. \\
\hline & & $4: 46$ & 38.8 & $\begin{array}{l}\text { Average glass temperature is } 1133^{\circ} \mathrm{C} \text {. Plenum temperature is } 475-481^{\circ} \mathrm{C} \text {. Glass } \\
\text { resistance is } 0.081 \Omega \text {. Current is } 1577 \text { Amps. Glass level is } 29.2 \mathrm{in} \text {. }\end{array}$ \\
\hline & & $4: 59$ & 39.0 & Changed $\mathrm{T} 4$ from 24 to 22 seconds. \\
\hline & & $5: 07$ & 39.1 & Plenum temperature is $477-458^{\circ} \mathrm{C}$. Average glass temperature is $1134^{\circ} \mathrm{C}$. \\
\hline & & $5: 33$ & 39.6 & Plenum temperature is $425-458^{\circ} \mathrm{C}$. \\
\hline & & $5: 49$ & 39.8 & Plenum temperature is $431-460^{\circ} \mathrm{C}$. \\
\hline & & $6: 06$ & 40.1 & Plenum temperature is $437-468^{\circ} \mathrm{C}$. \\
\hline
\end{tabular}


Table 3.2. Summary of Operational Events (continued).

\begin{tabular}{|c|c|c|c|c|}
\hline Test & Date & Time & $\begin{array}{l}\text { Run } \\
\text { Time } \\
\text { (hours) }\end{array}$ & Run time note \\
\hline \multirow{29}{*}{2} & \multirow{29}{*}{$1 / 24 / 13$} & $6: 51$ & 40.9 & $\begin{array}{c}\text { Decreased power from } 204 \text { to } 202 \mathrm{~kW} \text {, Average glass temperature is } 1145^{\circ} \mathrm{C} \text {. } \\
\text { Glass resistance is } 0.078 \Omega .\end{array}$ \\
\hline & & $7: 04$ & 41.1 & $\begin{array}{l}\text { Decreased power from } 202 \text { to } 200 \mathrm{~kW} \text {, current is } 1604 \text { Amps, voltage is } 124 \\
\text { volts. Average glass temperature is } 1145^{\circ} \mathrm{C} \text {. Glass resistance is } 0.077 \Omega \text {., Glass } \\
\text { level is } 29.5 \mathrm{in} \text {. }\end{array}$ \\
\hline & & $7: 38$ & 41.6 & Average glass temperature is $1145^{\circ} \mathrm{C}$. \\
\hline & & $7: 52$ & 41.9 & Average glass temperature is $1147^{\circ} \mathrm{C}$. \\
\hline & & 8:06 & 42.1 & $\begin{array}{l}\text { Average glass temperature is } 1144^{\circ} \mathrm{C} \text {. Plenum temperature is } 467-519^{\circ} \mathrm{C} \text {. Glass } \\
\text { resistance is } 0.077 \Omega \text {. Current is } 1577 \text { Amps. Glass level is } 29.2 \mathrm{in} \text {. Power is } 199 \\
\mathrm{~kW}, 124 \text { volts. Feed rate is } 164 \mathrm{~kg} / \mathrm{hr} \text {. }\end{array}$ \\
\hline & & $8: 20$ & 42.3 & $\begin{array}{l}\text { Average glass temperature is } 1146^{\circ} \mathrm{C} \text {. Plenum temperature is } 446-576^{\circ} \mathrm{C} \text {, glass } \\
\text { resistance is } 0.077 \Omega \text {. Current is } 1607 \text { Amps. Feed rate is } 167 \mathrm{~kg} / \mathrm{hr} \text {. Decreased } \\
\text { power from } 200 \text { to } 199 \mathrm{~kW} \text {. }\end{array}$ \\
\hline & & $8: 40$ & 42.7 & $\begin{array}{l}\text { Average glass temperature is } 1146^{\circ} \mathrm{C} \text {. Plenum temperature is } 497-513^{\circ} \mathrm{C} \text {, glass } \\
\text { resistance is } 0.077 \Omega \text {. Current is } 1601 \text { Amps. Power is } 198 \mathrm{~kW} \text {. }\end{array}$ \\
\hline & & $8: 52$ & 42.9 & $\begin{array}{c}\text { Decreased power from } 199 \text { to } 197 \mathrm{~kW} \text {. Average glass temperature is } 1147^{\circ} \mathrm{C} \\
\text { Current is } 1609 \text { Amps. }\end{array}$ \\
\hline & & $8: 55$ & 42.9 & $\begin{array}{l}\text { Average glass temperature is } 1144^{\circ} \mathrm{C} \text {, current is } 1593 \text { Amps. Power is } 195 \mathrm{~kW} . \\
\text { Feed rate is } \sim 172 \mathrm{~kg} / \mathrm{hr} \text {. }\end{array}$ \\
\hline & & $9: 35$ & 43.6 & $\begin{array}{c}\text { Average glass temperature is } 1143^{\circ} \mathrm{C} \text {, current is } 1590 \text { Amps. Power is } 195 \mathrm{~kW} . \\
\text { Glass level is } 29.9 \mathrm{in.}\end{array}$ \\
\hline & & 9:52 & 43.9 & Average glass temperature is $1141^{\circ} \mathrm{C}$, current is $1580 \mathrm{Amps}$. \\
\hline & & 10:10 & 44.2 & Average glass temperature is $1142^{\circ} \mathrm{C}$. \\
\hline & & $10: 39$ & 44.7 & Average glass temperature is $1145^{\circ} \mathrm{C}$. \\
\hline & & 10:56 & 44.9 & Average glass temperature is $1145^{\circ} \mathrm{C}$. \\
\hline & & 11:11 & 45.2 & Average glass temperature is $1144^{\circ} \mathrm{C}$. The current is $1582 \mathrm{Amps}$. \\
\hline & & 12:05 & 46.1 & $\begin{array}{c}\text { Average glass temperature is } 1137^{\circ} \mathrm{C} \text {. Melter pressure spike is due to shift of } \mathrm{CC} \\
\text { on the south east side of the melter. }\end{array}$ \\
\hline & & 12:14 & 46.2 & Increased power from 196 to $199 \mathrm{~kW}$. Average glass temperature is $1137^{\circ} \mathrm{C}$. \\
\hline & & $12: 20$ & 46.3 & Average glass temperature is $1137^{\circ} \mathrm{C}$. \\
\hline & & $12: 35$ & 46.6 & Average glass temperature is $1141^{\circ} \mathrm{C}$. \\
\hline & & $12: 47$ & 46.8 & Average glass temperature is $1140^{\circ} \mathrm{C}$. Feed rate is $\sim 183.9 \mathrm{~kg} / \mathrm{hr}$. \\
\hline & & 12:56 & 46.9 & Increased power from 199 to $200 \mathrm{~kW}$. Average glass temperature is $1137^{\circ} \mathrm{C}$. \\
\hline & & 13:08 & 47.1 & Average glass temperature is $1140^{\circ} \mathrm{C}$, the current is $1593 \mathrm{Amps}$. \\
\hline & & 13:37 & 47.6 & Average glass temperature is $1138^{\circ} \mathrm{C}$. \\
\hline & & 13:55 & 47.9 & Average glass temperature is $1136^{\circ} \mathrm{C}$. Current is 1567 Amps. \\
\hline & & 14:09 & 48.2 & Average glass temperature is $1139^{\circ} \mathrm{C}$ \\
\hline & & 14:13 & 48.2 & Performed film cooler rinse. \\
\hline & & $14: 56$ & 48.9 & Plenum temperature is $471-520^{\circ} \mathrm{C}$. \\
\hline & & 15:16 & 49.3 & Plenum temperature is $469-497^{\circ} \mathrm{C}$. \\
\hline & & 15:39 & 49.7 & Plenum temperature is $473-503^{\circ} \mathrm{C}$. \\
\hline
\end{tabular}


Table 3.2. Summary of Operational Events (continued).

\begin{tabular}{|c|c|c|c|c|}
\hline Test & Date & Time & $\begin{array}{l}\text { Run } \\
\text { Time } \\
\text { (hours) }\end{array}$ & Run time note \\
\hline \multirow{19}{*}{2} & \multirow{17}{*}{$1 / 24 / 13$} & $15: 58$ & 50.0 & $\begin{array}{c}\begin{array}{c}\text { Emergency off-gas is tripped when melter pressure went positive, immediately } \\
\text { reset. }\end{array}\end{array}$ \\
\hline & & $16: 40$ & 50.7 & LabVIEW reset and restarted. \\
\hline & & $16: 48$ & 50.8 & Plenum temperature is $456-492^{\circ} \mathrm{C}$. \\
\hline & & 17:00 & 51.0 & Secured feeding. End of Test 2. Performed water line flush. \\
\hline & & $17: 20$ & 51.3 & $\begin{array}{c}\text { Reduced power from } 202 \text { to } 190 \mathrm{~kW} \text {. Average glass temperature is } 1150^{\circ} \mathrm{C} \text {. } \\
\text { Current is } 1600 \mathrm{Amps}\end{array}$ \\
\hline & & $17: 21$ & 51.4 & $\begin{array}{c}\text { Removed feed from feed tank. Starting mass is } 1473.0 \mathrm{~kg} \text {. Ending mass is } 10.5 \\
\mathrm{~kg} \text {. Net removed feed mass is } 1462.5 \mathrm{~kg} \text {. }\end{array}$ \\
\hline & & $17: 31$ & 51.5 & $\begin{array}{l}\text { Reduced power from } 190 \text { to } 170 \mathrm{~kW} \text {. Average glass temperature } 1163^{\circ} \mathrm{C} \text {. Current } \\
\text { is } 1643 \text { Amps. }\end{array}$ \\
\hline & & $17: 48$ & 51.8 & Reduced power from 170 to $130 \mathrm{~kW}$. Average glass temperature is $1164^{\circ} \mathrm{C}$. \\
\hline & & 18:10 & 52.2 & Reduced power from 130 to $120 \mathrm{~kW}$. Average glass temperature is $1157^{\circ} \mathrm{C}$. \\
\hline & & $18: 12$ & 52.2 & Cold Cap is gone. \\
\hline & & 18:59 & 53.0 & $\begin{array}{l}\text { Reduced bubblers as follows: Lances L1 A\&B and Lances L2 A\&B from } 16 \text { to } \\
\qquad 10 \mathrm{lpm}, \mathrm{L} 3 \mathrm{~A} \& \mathrm{~B} \text { from } 8 \text { to } 5 \mathrm{lpm} \text {. }\end{array}$ \\
\hline & & 19:11 & 53.2 & Reduced bubblers Lances L1 A\&B, L2 A\&B and L3 A\&B to $1.5 \mathrm{lpm}$. \\
\hline & & 19:16 & 53.3 & Started melter and off-gas shut downs. \\
\hline & & 19:31 & 53.5 & Reduced power from 120 to $100 \mathrm{~kW}$. Average glass temperature is $1151^{\circ} \mathrm{C}$. \\
\hline & & 19:33 & 53.6 & $\begin{array}{l}\text { Reduced discharge power from } 18 \text { to } 12 \mathrm{~kW} \text {. Discharge temperature is } 1065 \text { - } \\
1086^{\circ} \mathrm{C} .\end{array}$ \\
\hline & & 19:37 & 53.6 & Reduced power from 100 to $80 \mathrm{~kW}$. Average glass temperature is $1156^{\circ} \mathrm{C}$ \\
\hline & & 20:06 & 54.1 & Reduced power from 80 to $75 \mathrm{~kW}$. Average glass temperature is $1155^{\circ} \mathrm{C}$ \\
\hline & \multirow{2}{*}{$1 / 25 / 13$} & 2:00 & - & Blow downs. \\
\hline & & $2: 10$ & - & Off gas shut down is now complete. \\
\hline \multirow{11}{*}{3} & \multirow{11}{*}{$1 / 29 / 13$} & $0: 30$ & - & $\begin{array}{l}\text { Transferred } 2923.5 \mathrm{~kg} \text { feed from mix tank to the feed tank. Plus } 496.91 \mathrm{~kg} \text { water } \\
\text { added (Recipe \#1). } 3410.4 \mathrm{~kg} \text { starting mass, out of recirculation. }\end{array}$ \\
\hline & & $7: 30$ & - & $\begin{array}{l}\text { Transferred } 518.5 \mathrm{~kg} \text { of Test \#2 Al-19 feed plus } 5 \mathrm{~kg} \text { flush water. Mass of feed at } \\
\text { start is } 3370.5 \mathrm{~kg} \text {, mass of feed after the transfer is } 3898.0 \mathrm{~kg} \text {. Net mass } \\
\text { transferred is } 527.5 \mathrm{~kg} \text {. }\end{array}$ \\
\hline & & $8: 00$ & - & Started melter and off gas system checks. \\
\hline & & $9: 30$ & 0.0 & Started feeding water at $0.8 \mathrm{l} / \mathrm{min}$. \\
\hline & & $9: 32$ & 0.0 & Rebooted the computer system. \\
\hline & & 9:45 & 0.3 & Rebooted the computer system. \\
\hline & & $9: 52$ & 0.4 & Increased water flow rate to $1.6 \mathrm{l} / \mathrm{min}$. \\
\hline & & 9:53 & 0.4 & Increased power from 135 to $145 \mathrm{~kW}$. \\
\hline & & 9:57 & 0.5 & Increased bubbling L1 and L2 7.0 lpm, L3 and L4 3.5 lpm. \\
\hline & & 10:08 & 0.6 & Increased power from 145 to $150 \mathrm{~kW}$. Average glass temperature is $1145^{\circ} \mathrm{C}$. \\
\hline & & $10: 10$ & 0.7 & Increased water from 1.6 to $2.41 / \mathrm{min}$. \\
\hline
\end{tabular}




\section{Table 3.2. Summary of Operational Events (continued).}

\begin{tabular}{|c|c|c|c|c|}
\hline Test & Date & Time & $\begin{array}{c}\text { Run } \\
\text { Time } \\
\text { (hours) }\end{array}$ & Run time note \\
\hline \multirow{32}{*}{3} & \multirow{32}{*}{$1 / 29 / 13$} & $10: 21$ & 0.9 & Turned on SBS booster pump. \\
\hline & & $10: 23$ & 0.9 & Increased power from 150 to $155 \mathrm{~kW}$. Average glass temperature is $1140^{\circ} \mathrm{C}$. \\
\hline & & $10: 30$ & 1.0 & Secured water flow and starting feeding slurry. \\
\hline & & $10: 34$ & 1.1 & Increased power from 155 to $160 \mathrm{~kW}$. Average glass temperature is $1134^{\circ} \mathrm{C}$. \\
\hline & & $10: 44$ & 1.2 & Increased power from 160 to $170 \mathrm{~kW}$. Average glass temperature is $1137^{\circ} \mathrm{C}$. \\
\hline & & $11: 18$ & 1.8 & Increased power from 170 to $175 \mathrm{~kW}$. Average glass temperature is $1146^{\circ} \mathrm{C}$. \\
\hline & & $11: 20$ & 1.8 & Average glass temperature is $1147^{\circ} \mathrm{C}$. \\
\hline & & 11:41 & 2.2 & Reduced power from 175 to $170 \mathrm{~kW}$. Average glass temperature is $1162^{\circ} \mathrm{C}$ \\
\hline & & 11:44 & 2.2 & Increased bubbling L1 and L2 to $8.0 \mathrm{lpm}$ and L3 and L4 to $4.0 \mathrm{lpm}$. \\
\hline & & 12:07 & 2.6 & Increased bubbling L1 and L2 to $10.0 \mathrm{lpm} \mathrm{L} 3$ and L4 to $5 \mathrm{lpm}$. \\
\hline & & $12: 20$ & 2.8 & Average glass temperature is $1167^{\circ} \mathrm{C}$. \\
\hline & & $12: 28$ & 3.0 & Reduced power from 170 to $160 \mathrm{~kW}$. Average glass temperature is $11712^{\circ} \mathrm{C}$. \\
\hline & & $12: 47$ & 3.3 & Average glass temperature is $1151^{\circ} \mathrm{C}$. Plenum temperature is $654-660^{\circ} \mathrm{C}$. \\
\hline & & 13:02 & 3.5 & Average glass temperature is $1145^{\circ} \mathrm{C}$. Increased power from 160 to $165 \mathrm{~kW}$. \\
\hline & & 13:17 & 3.8 & Average glass temperature is $1144^{\circ} \mathrm{C}$. Plenum temperature is $611-636^{\circ} \mathrm{C}$. \\
\hline & & 13:19 & 3.8 & Increased power from 165 to $170 \mathrm{~kW}$. Average glass temperature is $1144^{\circ} \mathrm{C}$. \\
\hline & & $13: 35$ & 4.1 & Average glass temperature is $1145^{\circ} \mathrm{C}$. \\
\hline & & 13:50 & 4.3 & Average glass temperature is $1145^{\circ} \mathrm{C}$. \\
\hline & & 14:11 & 4.7 & Average glass temperature is $1148^{\circ} \mathrm{C}$. \\
\hline & & $14: 29$ & 5.0 & Average glass temperature is $1129^{\circ} \mathrm{C}$. \\
\hline & & $14: 49$ & 5.3 & Average glass temperature is $1137^{\circ} \mathrm{C}$. Plenum temperature is $544-573^{\circ} \mathrm{C}$. \\
\hline & & 14:58 & 5.5 & $\begin{array}{l}\text { Increased bubbling as follows: L1 and L2 from } 12.0 \text { to } 14.0 \mathrm{lpm} \text { each and L3 and } \\
\text { L4 from } 6.0 \text { to } 7.0 \mathrm{lpm} \text { each. }\end{array}$ \\
\hline & & 15:04 & 5.6 & Plenum temperature is $538-565^{\circ} \mathrm{C}$. \\
\hline & & 15:07 & 5.6 & $\begin{array}{l}\text { Increased power from } 170 \text { to } 180 \mathrm{~kW} \text {. Average glass temperature is } 1138^{\circ} \mathrm{C} \text {, } \\
\text { current is } 1546 \text { Amps. }\end{array}$ \\
\hline & & 15:08 & 5.6 & $\begin{array}{l}\text { Melter pressure spiked and caused emergency off gas to trip. Increased speeds of } \\
\text { blower } 701 \text { from } 50 \text { to } 51 \mathrm{~Hz} \text {, blower } 801 \text { from } 20 \text { to } 23 \mathrm{~Hz} \text {. }\end{array}$ \\
\hline & & 15:19 & 5.8 & Plenum temperature is $530-553^{\circ} \mathrm{C}$. \\
\hline & & $15: 22$ & 5.9 & $\begin{array}{c}\text { Increased power from } 180 \text { to } 190 \mathrm{~kW} \text {. Average glass temperature is } 1129^{\circ} \mathrm{C}, \\
\text { current is } 1577 \text { Amps. }\end{array}$ \\
\hline & & $15: 34$ & 6.1 & Plenum temperature is $523-544^{\circ} \mathrm{C}$. Current is 1594 Amps. \\
\hline & & 15:51 & 6.4 & Current is 1605 Amps. Plenum temperature is $500-542^{\circ} \mathrm{C}$. \\
\hline & & $16: 05$ & 6.6 & Plenum temperature is $491-525^{\circ} \mathrm{C}$. \\
\hline & & $16: 19$ & 6.8 & Plenum temperature is $513-539^{\circ} \mathrm{C}$. \\
\hline & & $16: 21$ & 6.9 & $\begin{array}{c}\text { Increased bubbling on Lances: L1A from } 14.0 \text { to } 16.0 \mathrm{lpm} \text {, L2A from } 14.0 \text { to } 16.0 \\
\text { lpm, L3A from } 7.1 \text { to } 8.1 \mathrm{lpm}, \mathrm{L} 4 \mathrm{~A} \text { from } 7.1 \text { to } 8.1 \mathrm{lpm} \text {. }\end{array}$ \\
\hline
\end{tabular}


Table 3.2. Summary of Operational Events (continued).

\begin{tabular}{|c|c|c|c|c|}
\hline Test & Date & Time & $\begin{array}{l}\text { Run } \\
\text { Time } \\
\text { (hours) }\end{array}$ & Run time note \\
\hline \multirow{26}{*}{3} & \multirow{26}{*}{$1 / 29 / 13$} & $16: 34$ & 7.1 & Plenum temperature is $502-539^{\circ} \mathrm{C}$. \\
\hline & & $16: 45$ & 7.3 & Decreased power from 190 to $188 \mathrm{~kW}$ due to current limit. \\
\hline & & $16: 49$ & 7.3 & Plenum temperature is $490-529^{\circ} \mathrm{C}$. \\
\hline & & $16: 56$ & 7.4 & $\begin{array}{c}\text { Increased bubbling on Lances L1A and L1B from } 16.0 \text { to } 20.0 \mathrm{lpm} \text {, also } \\
\text { increased L3A and L4A from } 8.1 \text { to } 10.1 \mathrm{lpm} \text {. Total bubbling for } \\
\mathrm{L} 1 \mathrm{~A}+\mathrm{L} 1 \mathrm{~B}+\mathrm{L} 3 \mathrm{~A}+\mathrm{L} 4 \mathrm{~A} \text { is } 60.2 \mathrm{lpm} \text {. }\end{array}$ \\
\hline & & $17: 04$ & 7.6 & Plenum temperature is $498-527^{\circ} \mathrm{C}$. \\
\hline & & $17: 17$ & 7.8 & Plenum temperature is $486-529^{\circ} \mathrm{C}$. \\
\hline & & $17: 34$ & 8.1 & Plenum temperature is $453-533^{\circ} \mathrm{C}$. \\
\hline & & $17: 43$ & 8.2 & $\begin{array}{c}\text { Increased bubbling on Lances L1A and L2A from } 20.0 \text { to } 24.1 \mathrm{lpm} \text { also increased } \\
\text { L3A and L4A from } 10.1 \text { to } 12.1 \mathrm{lpm} \text { to increase openings in CC. }\end{array}$ \\
\hline & & 18:03 & 8.6 & $\begin{array}{l}\text { Increased power from } 188 \text { to } 195 \mathrm{~kW} \text {. Average glass temperature is } 1132^{\circ} \mathrm{C} \text {. } \\
\text { Since only secondary current is being monitored on LabVIEW, we will monitor } \\
\text { primary current locally. Power will be set by primary current not by LabVIEW } \\
\text { (secondary) current. Limit will be } 530 \text { Amps on primary current. }\end{array}$ \\
\hline & & 18:04 & 8.6 & Plenum temperature is $529-534^{\circ} \mathrm{C}$. \\
\hline & & $18: 10$ & 8.7 & $\begin{array}{l}\text { Increased power from } 195 \text { to } 200 \mathrm{~kW} \text {. Average glass temperature is } 1130^{\circ} \mathrm{C} \text {. } \\
\text { Primary current is at } 517 \mathrm{Amps} \text {. Secondary current is at } 1608 \mathrm{Amps} \text {. }\end{array}$ \\
\hline & & $18: 39$ & 9.2 & Plenum temperature is $514-519^{\circ} \mathrm{C}$. \\
\hline & & 18:49 & 9.3 & Plenum temperature is $485-522^{\circ} \mathrm{C}$. \\
\hline & & $18: 52$ & 9.4 & $\begin{array}{l}\text { Increased power from } 200 \text { to } 210 \mathrm{~kW} \text {, Average glass temperature is } 1129^{\circ} \mathrm{C} \\
\text { Primary current is } 524 \mathrm{Amps} \text {, and the secondary current is } 1625 \mathrm{Amps} \text {. }\end{array}$ \\
\hline & & 19:05 & 9.6 & Plenum temperature is $485-529^{\circ} \mathrm{C}$. \\
\hline & & 19:17 & 9.8 & Plenum temperature is $483-529^{\circ} \mathrm{C}$. \\
\hline & & 19:34 & 10.1 & $\begin{array}{c}\text { Average glass temperature is } 1139^{\circ} \mathrm{C} \text {. Plenum temperature is } 522-536^{\circ} \mathrm{C} . \\
\text { Secondary current is } 1662 \mathrm{Amps} \text {. }\end{array}$ \\
\hline & & 19:49 & 10.3 & $\begin{array}{l}\text { Plenum temperature is } 519-523^{\circ} \mathrm{C} \text {. Melter pressure went positive and tripped } \\
\text { emergency off gas. Changed T4 from } 27 \text { to } 26.7 \text { seconds. }\end{array}$ \\
\hline & & 19:50 & 10.3 & $\begin{array}{l}\text { Increased power from } 210 \text { to } 215 \mathrm{~kW} \text {. Average glass temperature is } 1138^{\circ} \mathrm{C} \text {. } \\
\text { Primary current is } 542 \mathrm{Amps} \text {, secondary current is } 1984 \text { Amps. }\end{array}$ \\
\hline & & 20:19 & 10.8 & $\begin{array}{c}\text { Reduced bubbling as follows: Lances L1 and L2 from } 24 \text { to } 22 \mathrm{lpm} \text { each, and L3 } \\
\text { and L4 from } 12 \text { to } 11 \mathrm{lpm} \text { each. Total is at } 66 \mathrm{lpm} \text {. }\end{array}$ \\
\hline & & 20:20 & 10.8 & $\begin{array}{l}\text { Average glass temperature is } 1145^{\circ} \mathrm{C} \text {. Plenum temperature is } 508-527^{\circ} \mathrm{C} \text {. } \\
\text { Primary current is } 550 \mathrm{Amps} \text {, and the secondary current is } 1705 \mathrm{Amps} \text {. }\end{array}$ \\
\hline & & $20: 33$ & 11.1 & Plenum temperature is $497-528^{\circ} \mathrm{C}$. \\
\hline & & $20: 53$ & 11.4 & $\begin{array}{c}\text { Average glass temperature is } 1141^{\circ} \mathrm{C} \text {. Plenum temperature is } 507-532^{\circ} \mathrm{C} \text {. Glass } \\
\text { resistance is } 0.073 \Omega \text {. }\end{array}$ \\
\hline & & 21:09 & 11.7 & Plenum temperature is $495-538^{\circ} \mathrm{C}$. Glass resistance is $0.073 \Omega$. \\
\hline & & $21: 19$ & 11.8 & Plenum temperature is $504-531^{\circ} \mathrm{C}$. \\
\hline & & $21: 37$ & 12.1 & Plenum temperature is $494-521^{\circ} \mathrm{C}$. \\
\hline
\end{tabular}




\section{Table 3.2. Summary of Operational Events (continued).}

\begin{tabular}{|c|c|c|c|c|}
\hline Test & Date & Time & $\begin{array}{l}\text { Run } \\
\text { Time } \\
\text { (hours) }\end{array}$ & Run time note \\
\hline \multirow{26}{*}{3} & \multirow{18}{*}{$1 / 29 / 13$} & $21: 49$ & 12.3 & Plenum temperature is $497-522^{\circ} \mathrm{C}$. \\
\hline & & $21: 54$ & 12.4 & $\begin{array}{l}\text { Performed film cooler rinse. Film cooler differential pressure was } 1.7 \text { in W.C. at } \\
\text { the end } 1.8 \text { in W.C. }\end{array}$ \\
\hline & & 22:05 & 12.6 & $\begin{array}{l}\text { Observed glass resistance change from } 0.073 \Omega \text { to } 0.076 \Omega \text { during glass } \\
\text { discharge. }\end{array}$ \\
\hline & & $22: 34$ & 13.1 & Experiencing high electrode temperatures and alarming. \\
\hline & & $22: 36$ & 13.1 & $\begin{array}{l}\text { Reduced electric power from } 215 \text { to } 212 \mathrm{~kW} \text {, east electrode alarm at } 1176^{\circ} \mathrm{C} \text {. } \\
\text { Average glass temperature is } 1145^{\circ} \mathrm{C} \text {. Plenum temperature is } 515-519^{\circ} \mathrm{C} \text {. Glass } \\
\text { resistance is } 0.071 \Omega \text {. }\end{array}$ \\
\hline & & $22: 50$ & 13.3 & $\begin{array}{c}\text { Average glass temperature is } 1145^{\circ} \mathrm{C} \text {. Plenum temperature is } 514-522^{\circ} \mathrm{C} \text {. Glass } \\
\text { resistance is } 0.071 \Omega \text {. }\end{array}$ \\
\hline & & 23:01 & 13.5 & $\begin{array}{l}\text { Plenum temperature is } 517-518^{\circ} \mathrm{C} \text {. Glass resistance is } 0.070 \Omega \text {. Glass level is } \\
29.7 \mathrm{in.}\end{array}$ \\
\hline & & 23:09 & 13.7 & $\begin{array}{l}\text { Reducing power from } 212 \text { to } 209 \mathrm{~kW} \text {. Average glass temperature is } 1152^{\circ} \mathrm{C} \text {. } \\
\text { Glass level is } 30.0 \mathrm{in} \text {, Secondary current is } 1711 \text { Amps. }\end{array}$ \\
\hline & & 23:13 & 13.7 & Changed T4 from 26.7 to 26.9 seconds. Feed rate is $181 \mathrm{~kg} / \mathrm{hr}$. \\
\hline & & 23:15 & 13.8 & PBS high $\mathrm{pH}$ alarm. Proved to be misaligned probe. \\
\hline & & 23:18 & 13.8 & Plenum temperature is $506-513^{\circ} \mathrm{C}$. \\
\hline & & 23:20 & 13.8 & $\begin{array}{l}\text { Reduced power from } 209 \text { to } 208 \mathrm{~kW} \text {. Thermowell \#2 temperatures are } 1154 \text { and } \\
1160^{\circ} \mathrm{C} \text {. }\end{array}$ \\
\hline & & $23: 22$ & 13.9 & Glass resistance is $0.069 \Omega$. Secondary current is 1727 Amps. \\
\hline & & $23: 27$ & 14.0 & Feed rate is rising. 10 Minutes average shows $187 \mathrm{~kg} / \mathrm{hr}$. \\
\hline & & $23: 35$ & 14.1 & $\begin{array}{l}\text { Average glass temperature is } 1149^{\circ} \mathrm{C} \text {. Plenum temperature is } 519-510^{\circ} \mathrm{C} \text {. } \\
\text { Secondary current is } 1692 \text { Amps. }\end{array}$ \\
\hline & & 23:42 & 14.2 & $\begin{array}{l}\text { Slowed feeding to maintain target feeding } 179 \mathrm{~kg} / \mathrm{hr} \text {. Currently averaging } 182 \\
\mathrm{~kg} / \mathrm{hr} \text {. Changed T4 from } 26.9 \text { to } 27.0 \text { seconds. }\end{array}$ \\
\hline & & 23:46 & 14.3 & Average glass temperature is $1148^{\circ} \mathrm{C}$. Plenum temperature is $507-521^{\circ} \mathrm{C}$. \\
\hline & & 23:56 & 14.4 & $\begin{array}{l}\text { Average glass temperature is } 1149^{\circ} \mathrm{C} \text {. Thermo well } \# 1 \text { temperatures are between } \\
1133 \text { and } 1149^{\circ} \mathrm{C} \text {, and thermo well } \# 2 \text { temperatures are } 1141-1153^{\circ} \mathrm{C} \text {. }\end{array}$ \\
\hline & \multirow{8}{*}{$1 / 30 / 13$} & 0:08 & 14.6 & $\begin{array}{l}2 \text { Minutes in the } 1 / 2 \text { hour feed cycle, feed rate started to drop. Adjusting T4 from } \\
28 \text { to } 27 \text { seconds. }\end{array}$ \\
\hline & & $0: 16$ & 14.8 & $\begin{array}{c}\text { Average glass temperature is } 1151^{\circ} \mathrm{C} \text {. Plenum temperature is } 514-524^{\circ} \mathrm{C} \text {. Glass } \\
\text { resistance is } 0.071 \Omega \text {. }\end{array}$ \\
\hline & & $0: 17$ & 14.8 & Average glass temperature is $1151^{\circ} \mathrm{C}$, glass density is $2.28 \mathrm{~g} / \mathrm{cc}$. \\
\hline & & $0: 19$ & 14.8 & Average glass temperature is $1145^{\circ} \mathrm{C}$. Plenum temperature is $515-519^{\circ} \mathrm{C}$. \\
\hline & & $0: 34$ & 15.1 & $\begin{array}{c}\text { Average glass temperature is } 1147^{\circ} \mathrm{C} \text {. Plenum temperature is } 518-523^{\circ} \mathrm{C} \text {. Glass } \\
\text { resistance is } 0.071 \Omega \text {. }\end{array}$ \\
\hline & & $0: 49$ & 15.3 & $\begin{array}{c}\text { Average glass temperature is } 1146^{\circ} \mathrm{C} \text {. Plenum temperature is } 519-525^{\circ} \mathrm{C} \text {. } \\
\text { Secondary current is } 1692 \mathrm{Amps} \text {, glass level is } 29.8 \mathrm{in} .\end{array}$ \\
\hline & & $1: 04$ & 15.6 & $\begin{array}{c}\text { Average glass temperature } 1146^{\circ} \mathrm{C} \text {. Plenum temperature is } 515-520^{\circ} \mathrm{C} \text {. Glass } \\
\text { resistance is } 0.071 \Omega \text {. Secondary current is } 1692 \mathrm{Amps} \text {. }\end{array}$ \\
\hline & & $1: 19$ & 15.8 & $\begin{array}{c}\text { Average glass temperature is } 1149^{\circ} \mathrm{C} \text {. Plenum temperature is } 518-519^{\circ} \mathrm{C} \text {. Glass } \\
\text { resistance is } 0.071 \Omega \text {. Glass level is } 30.1 \mathrm{in} .\end{array}$ \\
\hline
\end{tabular}


Table 3.2. Summary of Operational Events (continued).

\begin{tabular}{|c|c|c|c|c|}
\hline Test & Date & Time & $\begin{array}{c}\text { Run } \\
\text { Time } \\
\text { (hours) }\end{array}$ & Run time note \\
\hline \multirow{29}{*}{3} & \multirow{29}{*}{$1 / 30 / 13$} & $1: 32$ & 16.0 & Changing T4 from 27 to 26.5 seconds. \\
\hline & & $1: 34$ & 16.1 & Plenum temperature is $507-518^{\circ} \mathrm{C}$. Glass resistance is $0.072 \Omega$. \\
\hline & & $1: 49$ & 16.3 & $\begin{array}{c}\text { Plenum temperature is } 503-521^{\circ} \mathrm{C} \text {. Glass density is } 2.29 \mathrm{~g} / \mathrm{cc} \text { and secondary } \\
\text { current is } 1691 \text { Amps. }\end{array}$ \\
\hline & & $1: 50$ & 16.3 & $\begin{array}{l}\text { Last hour feed cycle high of } 183 \mathrm{~kg} / \mathrm{hr} \text {, low of } 174 \mathrm{~kg} / \mathrm{hr} \text {; changes to feed shot } \\
\text { frequency made. Watching and will monitor. }\end{array}$ \\
\hline & & 2:04 & 16.6 & Plenum temperature is $514-516^{\circ} \mathrm{C}$. Glass level is $30.0 \mathrm{in}$. \\
\hline & & 2:05 & 16.6 & $\begin{array}{c}\text { Increased power from } 206 \text { to } 209 \mathrm{~kW} \text {. Secondary current is } 1714 \text { Amps, power is } \\
121 \text { volts and glass resistance is } 0.071 \Omega \text {. Glass density is } 2.29 \mathrm{~g} / \mathrm{cc} \text {. }\end{array}$ \\
\hline & & $2: 19$ & 16.8 & Plenum temperature is $506-510^{\circ} \mathrm{C}$. Average glass temperature is $1148^{\circ} \mathrm{C}$. \\
\hline & & $2: 34$ & 17.1 & Plenum temperature is $499-508^{\circ} \mathrm{C}$. \\
\hline & & $2: 49$ & 17.3 & Plenum temperature is $507-509^{\circ} \mathrm{C}$. \\
\hline & & 3:04 & 17.6 & $\begin{array}{c}\text { Plenum temperature is } 508-511^{\circ} \mathrm{C} \text {. Secondary current is } 1714 \mathrm{Amps} \text {, Glass } \\
\text { resistance is } 0.071 \Omega \text {. Secondary current is } 1714 \text { Amps. }\end{array}$ \\
\hline & & $3: 20$ & 17.8 & Plenum temperature is $507^{\circ} \mathrm{C}$. Average glass temperature is $1150^{\circ} \mathrm{C}$ \\
\hline & & $3: 34$ & 18.1 & Plenum temperature is $505-506^{\circ} \mathrm{C}$. Glass temperature is $1158^{\circ} \mathrm{C}$. \\
\hline & & $3: 50$ & 18.3 & Reduced mixer speed from 30 to $25 \mathrm{~Hz}$. \\
\hline & & $3: 51$ & 18.4 & Plenum temperature is $504-506^{\circ} \mathrm{C}$. \\
\hline & & $4: 02$ & 18.5 & Changed T4 from 26.5 to 27.0 seconds. \\
\hline & & $4: 04$ & 18.6 & $\begin{array}{l}\text { Plenum temperature is } 502-514^{\circ} \mathrm{C} \text {, Glass resistance is } 0.070 \Omega \text {. Glass level is } \\
29.6 \mathrm{in} \text {. Glass temperature is } 1148^{\circ} \mathrm{C} \text {. }\end{array}$ \\
\hline & & $4: 21$ & 18.9 & $\begin{array}{l}\text { Plenum temperature is } 496-513^{\circ} \mathrm{C} \text {, Glass resistance is } 0.070 \Omega \text {. Glass level is } \\
29.7 \text { in. Glass temperature is } 1150^{\circ} \mathrm{C} \text {. Secondary current is } 1715 \mathrm{Amps} \text {. }\end{array}$ \\
\hline & & $4: 23$ & 18.9 & SBS outlet pressure indicator is fixed. Now it reads -37.5 in W.C. \\
\hline & & $4: 35$ & 19.1 & $\begin{array}{l}\text { Plenum temperature is } 500-508^{\circ} \mathrm{C} \text {. Glass resistance is } 0.070 \Omega \text {. Glass level is } \\
30.0 \mathrm{in} \text {. Glass temperature is } 1157^{\circ} \mathrm{C} \text {. Secondary current is } 1718 \mathrm{Amps} \text {. }\end{array}$ \\
\hline & & $4: 36$ & 19.1 & Changed T4 from 27 to 28 seconds. \\
\hline & & $4: 37$ & 19.1 & SBS outlet pressure indicator appears out of service. \\
\hline & & $4: 49$ & 19.3 & Plenum temperature is $506-508^{\circ} \mathrm{C}$. Glass density is $2.28 \mathrm{~g} / \mathrm{cc}$. \\
\hline & & $4: 57$ & 19.5 & Changed T4 from 28.0 to 26.5 seconds. \\
\hline & & $5: 02$ & 19.5 & $\begin{array}{l}\text { Increased power from } 209 \text { to } 212 \mathrm{~kW} \text {. Average Glass temperature is } 1129^{\circ} \mathrm{C} \text {. } \\
\text { Glass resistance is } 0.071 \Omega \text {. Secondary current is } 1718 \mathrm{Amps}, 122 \text { volts. }\end{array}$ \\
\hline & & $5: 06$ & 19.6 & Plenum temperature is $500-501^{\circ} \mathrm{C}$. \\
\hline & & $5: 19$ & 19.8 & Plenum temperature is $500-510^{\circ} \mathrm{C}$. \\
\hline & & $5: 21$ & 19.9 & $\begin{array}{c}\text { Reduced power from } 212 \text { to } 209 \mathrm{~kW} \text {, east electrode temperature is } 1179^{\circ} \mathrm{C} \text {. } \\
\text { Average Glass temperature is } 1142^{\circ} \mathrm{C}\end{array}$ \\
\hline & & $5: 32$ & 20.0 & $\begin{array}{l}\text { Reduced bubbling as follows: Lances L1 and L2 from } 22.1 \text { to } 20.1 \mathrm{lpm}, \mathrm{L} 3 \text { and } \\
\text { L4 from } 11.2 \text { to } 10.1 \mathrm{lpm} \text {. Total combined bubbling is } 60.4 \mathrm{lpm} \text {. }\end{array}$ \\
\hline & & $5: 34$ & 20.1 & Plenum temperature is $519-511^{\circ} \mathrm{C}$. \\
\hline
\end{tabular}


Table 3.2. Summary of Operational Events (continued).

\begin{tabular}{|c|c|c|c|c|}
\hline Test & Date & Time & $\begin{array}{l}\text { Run } \\
\text { Time } \\
\text { (hours) }\end{array}$ & Run time note \\
\hline \multirow{23}{*}{3} & \multirow{23}{*}{$1 / 30 / 13$} & $5: 52$ & 20.4 & Reduced power from 209 to $206 \mathrm{~kW}$. \\
\hline & & $5: 53$ & 20.4 & Plenum temperature is $510-512^{\circ} \mathrm{C}$. Average Glass temperature is $1154^{\circ} \mathrm{C}$. \\
\hline & & $5: 59$ & 20.5 & Reduced power from 206 to $203 \mathrm{~kW}$. Average Glass temperature is $1154^{\circ} \mathrm{C}$. \\
\hline & & $6: 20$ & 20.8 & Plenum temperature is $497-502^{\circ} \mathrm{C}$. \\
\hline & & $6: 34$ & 21.1 & $\begin{array}{l}\text { Plenum temperature is } 498-501^{\circ} \mathrm{C} \text {. Average Glass temperature is } 1153^{\circ} \mathrm{C} \text {. Glass } \\
\text { resistance is } 0.068 \Omega \text {. Thermowell } \# 1 \text { temperatures are between } 1123 \text { and } \\
1148^{\circ} \mathrm{C} \text {, and thermo well } \# 2 \text { temperatures are } 1157-1162^{\circ} \mathrm{C} \text {. }\end{array}$ \\
\hline & & $6: 45$ & 21.3 & $\begin{array}{c}\text { Transferred feed from mix tank. Starting mass is } 2986 \mathrm{~kg} \text {, ending mass is } 37.5 \mathrm{~kg} \text {. } \\
\text { Net mass transferred is } 2948.5 \mathrm{~kg} \text {. Also added } 491.0 \mathrm{~kg} \text { water. Total mass } \\
\text { transferred is } 3439.5 \mathrm{~kg} \text {. }\end{array}$ \\
\hline & & $7: 13$ & 21.7 & $\begin{array}{c}\text { Transferred } 275 \mathrm{~kg} \text { feed }+5 \mathrm{~kg} \text { water to feed tank. (Ref: residual feed from Test } \\
\text { \#2, VSL-1987-12 page } 72 \text { ). Feed tank mass in recirculation is } 3797.0 \mathrm{~kg} \text {, end } \\
\text { mass is } 4077.0 \mathrm{~kg} \text {. Net mass transferred is } 280.0 \mathrm{~kg} \text {. }\end{array}$ \\
\hline & & $7: 19$ & 21.8 & $\begin{array}{l}\text { Plenum temperature is } 476-480^{\circ} \mathrm{C} \text {. Glass resistance is } 0.069 \Omega \text {. Average glass } \\
\text { temperature is } 1153^{\circ} \mathrm{C} \text {. Secondary current is } 1715 \text { Amps. Power is } 201 \mathrm{~kW}, 118 \\
\text { volts. }\end{array}$ \\
\hline & & $7: 49$ & 22.3 & $\begin{array}{l}\text { Plenum temperature is } 476-485^{\circ} \mathrm{C} \text {. Glass resistance is } 0.069 \Omega \text {. Average glass } \\
\text { temperature is } 1149^{\circ} \mathrm{C} \text {. Secondary current is } 1705 \text { Amps. Power is } 202 \mathrm{~kW}, 118 \\
\text { volts. }\end{array}$ \\
\hline & & 8:04 & 22.6 & $\begin{array}{l}\text { Plenum temperature is } 484^{\circ} \mathrm{C} \text {. Glass resistance is } 0.069 \Omega \text {. Average glass } \\
\text { temperature is } 1156^{\circ} \mathrm{C} \text {. Secondary current is } 1706 \text { Amps. Power is } 201 \mathrm{~kW}, 118 \\
\text { volts. }\end{array}$ \\
\hline & & $8: 19$ & 22.8 & $\begin{array}{l}\text { Plenum temperature is } 474-497^{\circ} \mathrm{C} \text {. Glass resistance is } 0.069 \Omega \text {. Average glass } \\
\text { temperature is } 1152^{\circ} \mathrm{C} \text {. Secondary current is } 1713 \text { Amps. Power is } 201 \mathrm{~kW}, 118 \\
\text { volts. }\end{array}$ \\
\hline & & $8: 26$ & 22.9 & Emergency off gas is tripped due to CC shifting/collapsing. \\
\hline & & $8: 34$ & 23.1 & $\begin{array}{l}\text { Plenum temperature is } 479-484^{\circ} \mathrm{C} \text {. Glass resistance is } 0.070 \Omega \text {. Average glass } \\
\text { temperature is } 1151^{\circ} \mathrm{C} \text {. Secondary current is } 1706 \text { Amps. Power is } 201 \mathrm{~kW}, 118 \\
\text { volts. Feed rate is } 176 \mathrm{~kg} / \mathrm{hr} \text {. Total bubbling is } 60 \mathrm{lpm} \text {. }\end{array}$ \\
\hline & & $8: 50$ & 23.3 & $\begin{array}{l}\text { Plenum temperature is } 463-488^{\circ} \mathrm{C} \text {. Average glass temperature is } 1150^{\circ} \mathrm{C} \text {. } \\
\text { Secondary current is } 1710 \text { Amps. Power is } 203 \mathrm{~kW} \text {. }\end{array}$ \\
\hline & & 9:04 & 23.6 & Average glass temperature is $1145^{\circ} \mathrm{C}$. \\
\hline & & 9:19 & 23.8 & $\begin{array}{l}\text { Average glass temperature is } 1143^{\circ} \mathrm{C} \text {. Plenum temperature is } 463-487^{\circ} \mathrm{C} \text {. } \\
\text { Secondary current is } 1694 \mathrm{Amps} \text {. Power is } 201 \mathrm{~kW}, 118 \text { volts. }\end{array}$ \\
\hline & & $9: 49$ & 24.3 & Average glass temperature is $1145^{\circ} \mathrm{C}$. \\
\hline & & 10:04 & 24.6 & Average glass temperature $1142^{\circ} \mathrm{C}$. Plenum temperature is $464-479^{\circ} \mathrm{C}$ \\
\hline & & 10:19 & 24.8 & Average glass temperature is $1145^{\circ} \mathrm{C}$. \\
\hline & & $10: 36$ & 25.1 & Average glass temperature is $1147^{\circ} \mathrm{C}$. \\
\hline & & 11:04 & 25.6 & Average glass temperature is $1152^{\circ} \mathrm{C}$. \\
\hline & & 11:19 & 25.8 & Average glass temperature is $1150^{\circ} \mathrm{C}$. \\
\hline & & $11: 25$ & 25.9 & Melter pressure spike due to cold cap shift. \\
\hline
\end{tabular}


Table 3.2. Summary of Operational Events (continued).

\begin{tabular}{|c|c|c|c|c|}
\hline Test & Date & Time & $\begin{array}{l}\text { Run } \\
\text { Time } \\
\text { (hours) }\end{array}$ & Run time note \\
\hline \multirow{26}{*}{3} & \multirow{26}{*}{$1 / 30 / 13$} & $11: 30$ & 26.0 & $\begin{array}{l}\text { Transfer feed to mix tank. Starting mass } 37.5 \mathrm{~kg} \text {, ending mass is } 1518.5 \mathrm{~kg} \text {. Net } \\
\text { mass transferred is } 1481.0 \mathrm{~kg} \text { including } 10 \mathrm{~kg} \text { water. }\end{array}$ \\
\hline & & $11: 49$ & 26.3 & $\begin{array}{c}\text { Average glass temperature is } 1143^{\circ} \mathrm{C} \text {. Plenum temperature is } 457-492^{\circ} \mathrm{C} \text {. } \\
\text { Secondary current is } 1689 \text { Amps. Power is } 201 \mathrm{~kW}, 119 \text { volts. Glass resistance is } \\
0.071 \Omega \text { and feed rate is } 180 \mathrm{~kg} / \mathrm{hr} \text {. }\end{array}$ \\
\hline & & 12:04 & 26.6 & $\begin{array}{l}\text { Average glass is temperature } 1147^{\circ} \mathrm{C} \text {. Plenum temperature is } 447-498^{\circ} \mathrm{C} \text {. } \\
\text { Secondary current is } 1699 \text { Amps. Power is } 201 \mathrm{~kW}, 118 \text { volts. Glass resistance is } \\
0.070 \Omega \text {. }\end{array}$ \\
\hline & & $12: 34$ & 27.1 & $\begin{array}{c}\text { Average glass temperature is } 1145^{\circ} \mathrm{C} \text {. Plenum temperature is } 457-490^{\circ} \mathrm{C} \text {. } \\
\text { Secondary current is } 1628 \text { Amps and } 122 \text { volts. }\end{array}$ \\
\hline & & $12: 35$ & 27.1 & Emergency off gas is tripped due to large amount of feed entering glass pool. \\
\hline & & $12: 49$ & 27.3 & $\begin{array}{c}\text { Average glass temperature is } 1141^{\circ} \mathrm{C} \text {. Secondary current is } 1685 \text { Amps, } 119 \\
\text { volts. Glass resistance is } 0.070 \Omega .\end{array}$ \\
\hline & & $12: 50$ & 27.3 & $\begin{array}{l}\text { Paused feeding to clear feed tube. Manual water line flush, increased air purge } \\
\text { time from } 3 \text { to } 5 \text { seconds, switched from nozzle A purge air to B purge air. }\end{array}$ \\
\hline & & 13:03 & 27.6 & Decreased purge time from 5 to 3 seconds. \\
\hline & & 13:04 & 27.6 & $\begin{array}{c}\text { Average glass temperature is } 1152^{\circ} \mathrm{C} \text {. Plenum temperature is } 461-496^{\circ} \mathrm{C} \text {. } \\
\text { Secondary current is } 1711 \text { Amps. }\end{array}$ \\
\hline & & 13:08 & 27.6 & Switched back to A side of air purge. Line was clogged with feed. \\
\hline & & 13:19 & 27.8 & Average glass temperature is $1152^{\circ} \mathrm{C}$. Secondary current is 1713 Amps. \\
\hline & & 13:34 & 28.1 & Average glass temperature is $1142^{\circ} \mathrm{C}$. Secondary current is 1690 Amps. \\
\hline & & 14:04 & 28.6 & Average glass temperature is $1150^{\circ} \mathrm{C}$. \\
\hline & & $14: 39$ & 29.2 & Plenum temperature is $435-494^{\circ} \mathrm{C}$. \\
\hline & & $14: 51$ & 29.4 & Secured power to neutralization tank caustic pump. \\
\hline & & 14:54 & 29.4 & Plenum temperature is $417-490^{\circ} \mathrm{C}$. \\
\hline & & $14: 55$ & 29.4 & Performed pre-deluge and blow down of WESP. \\
\hline & & 15:04 & 29.6 & Performed post deluge blow down of the WESP. \\
\hline & & 15:06 & 29.6 & $\begin{array}{c}\text { Average glass temperature is } 1149^{\circ} \mathrm{C} \text {. Plenum temperature is } 445-484^{\circ} \mathrm{C} \text {. } \\
\text { Secondary current is } 1698 \text { Amps. }\end{array}$ \\
\hline & & 15:09 & 29.7 & Melter pressure spiked due to opening port on transition line for off gas sampling. \\
\hline & & $15: 19$ & 29.8 & $\begin{array}{c}\text { Plenum temperature is } 444-478^{\circ} \mathrm{C} \text {. Average glass temperature is } 1146^{\circ} \mathrm{C} \text {. } \\
\text { Secondary current is } 1706 \mathrm{Amps} \text {. }\end{array}$ \\
\hline & & $15: 32$ & 30.0 & Emergency off gas is tripped 3 times during the glass discharge. \\
\hline & & $15: 49$ & 30.3 & $\begin{array}{c}\text { Plenum temperature is } 442-472^{\circ} \mathrm{C} \text {. Average glass temperature is } 1148^{\circ} \mathrm{C} \text {. } \\
\text { Secondary current is } 1706 \mathrm{Amps} \text {. }\end{array}$ \\
\hline & & 16:04 & 30.6 & Plenum temperature is $398-473^{\circ} \mathrm{C}$. \\
\hline & & $16: 10$ & 30.7 & $\begin{array}{l}\text { Melter pressure is spiked due to the opening of the transition line. Off gas } \\
\text { sampling is completed. }\end{array}$ \\
\hline & & $16: 19$ & 30.8 & $\begin{array}{l}\text { Plenum temperature is } 420-476^{\circ} \mathrm{C} \text {. Average glass temperature is } 1146^{\circ} \mathrm{C} \text {. } \\
\text { Secondary current is } 1690 \mathrm{Amps} \text {. Glass resistance is } 0.071 \Omega \text {. }\end{array}$ \\
\hline
\end{tabular}


Table 3.2. Summary of Operational Events (continued).

\begin{tabular}{|c|c|c|c|c|}
\hline Test & Date & Time & $\begin{array}{c}\text { Run } \\
\text { Time } \\
\text { (hours) }\end{array}$ & Run time note \\
\hline \multirow{26}{*}{3} & \multirow{26}{*}{$1 / 30 / 13$} & $16: 34$ & 31.1 & $\begin{array}{c}\text { Plenum temperature is } 423-482^{\circ} \mathrm{C} \text {. Average glass temperature is } 1149^{\circ} \mathrm{C} \text {. } \\
\text { Secondary current is } 1690 \mathrm{Amps} \text {. Glass resistance is } 0.070 \Omega \text {. }\end{array}$ \\
\hline & & $16: 49$ & 31.3 & $\begin{array}{c}\text { Plenum temperature is } 449-482^{\circ} \mathrm{C} \text {. Average glass temperature is } 1151^{\circ} \mathrm{C} \text {. } \\
\text { Secondary current is } 1714 \mathrm{Amps} \text {. Glass resistance is } 0.068 \Omega \text {. }\end{array}$ \\
\hline & & $17: 04$ & 31.6 & $\begin{array}{l}\text { Plenum temperature is } 454-476^{\circ} \mathrm{C} \text {. Average glass temperature is } 1151^{\circ} \mathrm{C} \text {. } \\
\text { Secondary current is } 1711 \mathrm{Amps} \text {. Glass resistance is } 0.068 \Omega \text {. }\end{array}$ \\
\hline & & $17: 19$ & 31.8 & $\begin{array}{c}\text { Plenum temperature is } 458-474^{\circ} \mathrm{C} \text {. Average glass temperature is } 1152^{\circ} \mathrm{C} \text {. } \\
\text { Secondary current is } 1715 \mathrm{Amps} \text {. Glass resistance is } 0.069 \Omega \text {. }\end{array}$ \\
\hline & & $17: 34$ & 32.1 & $\begin{array}{c}\text { Plenum temperature is } 453-481^{\circ} \mathrm{C} \text {. Average glass temperature is } 1143^{\circ} \mathrm{C} \text {. } \\
\text { Secondary current is } 1632 \mathrm{Amps} \text {. Glass resistance is } 0.075 \Omega \text {. }\end{array}$ \\
\hline & & $17: 49$ & 32.3 & $\begin{array}{l}\text { Plenum temperature is } 446-490^{\circ} \mathrm{C} \text {. Also found SBS over flow tank top collapsed, } \\
\text { had to use pry bar to pop it out, all satisfactory now. }\end{array}$ \\
\hline & & 18:04 & 32.6 & $\begin{array}{l}\text { Plenum temperature is } 429-493^{\circ} \mathrm{C} \text {. Average glass temperature is } 1150^{\circ} \mathrm{C} \text {. } \\
\text { Secondary current is } 1704 \text { Amps. Glass resistance is } 0.070 \Omega \text {. }\end{array}$ \\
\hline & & $18: 22$ & 32.9 & $\begin{array}{c}\text { Melter pressure went positive } 3 \text { times during glass discharge, reset emergency off } \\
\text { gas each time. }\end{array}$ \\
\hline & & $18: 24$ & 32.9 & Plenum temperature is $452-501^{\circ} \mathrm{C}$. \\
\hline & & $18: 36$ & 33.1 & Plenum temperature is $438-504^{\circ} \mathrm{C}$. \\
\hline & & $18: 49$ & 33.3 & $\begin{array}{c}\text { Plenum temperature is } 463-503^{\circ} \mathrm{C} \text {. Average glass temperature is } 1151^{\circ} \mathrm{C} \text {. } \\
\text { Secondary current is } 1693 \mathrm{Amps} \text {. Glass resistance is } 0.070 \Omega \text {. }\end{array}$ \\
\hline & & 19:19 & 33.8 & Average glass temperature is $1150^{\circ} \mathrm{C}$. Secondary current is 1632 Amps. \\
\hline & & $19: 25$ & 33.9 & Started off gas sampling. \\
\hline & & $19: 34$ & 34.1 & $\begin{array}{c}\text { Plenum temperature is } 455-491^{\circ} \mathrm{C} \text {. Average glass temperature is } 1151^{\circ} \mathrm{C} \text {. } \\
\text { Secondary current is } 1691 \mathrm{Amps} \text {. Glass resistance is } 0.070 \Omega \text {. }\end{array}$ \\
\hline & & $19: 49$ & 34.3 & $\begin{array}{c}\text { Plenum temperature is } 462-477^{\circ} \mathrm{C} \text {. Average glass temperature is } 1153^{\circ} \mathrm{C} \text {. } \\
\text { Secondary current is } 1703 \mathrm{Amps} \text {. Glass resistance is } 0.069 \Omega \text {. }\end{array}$ \\
\hline & & $20: 14$ & 34.7 & Plenum temperature is $399-478^{\circ} \mathrm{C}$ \\
\hline & & 20:19 & 34.8 & Plenum temperature is $399-506^{\circ} \mathrm{C}$. \\
\hline & & $20: 28$ & 35.0 & Off gas sampling is completed. \\
\hline & & $20: 32$ & 35.0 & Paused feeding to collect a feed sample. \\
\hline & & $20: 47$ & 35.3 & $\begin{array}{c}\text { Transferred feed from mix tank to feed tank. Starting tank mass is } 1516.0 \mathrm{~kg} \text {, } \\
\text { ending tank mass is } 68.5 \mathrm{~kg} \text {. Net mass of feed transferred is } 1447.5 \mathrm{~kg} \text {. Plus } 246.0 \\
\mathrm{~kg} \text { water. Total mass transferred is } 1693.5 \mathrm{~kg} \text {. }\end{array}$ \\
\hline & & $20: 54$ & 35.4 & $\begin{array}{l}\text { Plenum temperature is } 474-479^{\circ} \mathrm{C} \text {. Average glass temperature is } 1146^{\circ} \mathrm{C} \text {. } \\
\text { Secondary current is } 1681 \mathrm{Amps} \text {. Glass resistance is } 0.071 \Omega \text {. }\end{array}$ \\
\hline & & 21:04 & 35.6 & $\begin{array}{c}\text { Plenum temperature is } 478-482^{\circ} \mathrm{C} \text {. Average glass temperature is } 1144^{\circ} \mathrm{C} \text {. } \\
\text { Secondary current is } 1684 \mathrm{Amps} \text {. Glass resistance is } 0.071 \Omega \text {. }\end{array}$ \\
\hline & & $21: 19$ & 35.8 & $\begin{array}{c}\text { Plenum temperature is } 406-467^{\circ} \mathrm{C} \text {. Average glass temperature is } 1147^{\circ} \mathrm{C} \text {. } \\
\text { Secondary current is } 1704 \text { Amps. Glass resistance is } 0.070 \Omega \text {. }\end{array}$ \\
\hline & & $21: 34$ & 36.1 & $\begin{array}{c}\text { Plenum temperature is } 443-475^{\circ} \mathrm{C} \text {. Average glass temperature is } 1140^{\circ} \mathrm{C} \text {. } \\
\text { Secondary current is } 1620 \mathrm{Amps} \text {. Glass resistance is } 0.077 \Omega \text {. }\end{array}$ \\
\hline & & $21: 44$ & 36.2 & $\begin{array}{l}\text { Performed a film cooler rinse. Film cooler differential pressure before and after } \\
\text { were }-1.4 \text { and }-1.3 \text { in W.C. respectively. }\end{array}$ \\
\hline & & $21: 49$ & 36.3 & $\begin{array}{c}\text { Plenum temperature is } 447-488^{\circ} \mathrm{C} \text {. Average glass temperature is } 1145^{\circ} \mathrm{C} \text {. } \\
\text { Secondary current is } 1680 \mathrm{Amps} \text {. Glass resistance is } 0.071 \Omega \text {. }\end{array}$ \\
\hline
\end{tabular}


Table 3.2. Summary of Operational Events (continued).

\begin{tabular}{|c|c|c|c|c|}
\hline Test & Date & Time & $\begin{array}{l}\text { Run } \\
\text { Time } \\
\text { (hours) }\end{array}$ & Run time note \\
\hline \multirow{26}{*}{3} & \multirow{12}{*}{$1 / 30 / 13$} & $22: 04$ & 36.6 & $\begin{array}{c}\text { Plenum temperature is } 453-500^{\circ} \mathrm{C} \text {. Average glass temperature is } 1147^{\circ} \mathrm{C} \text {. } \\
\text { Secondary current is } 1690 \mathrm{Amps} \text {. Glass resistance is } 0.071 \Omega \text {. }\end{array}$ \\
\hline & & $22: 19$ & 36.8 & $\begin{array}{l}\text { Plenum temperature is } 425-477^{\circ} \mathrm{C} \text {. Average glass temperature is } 1148^{\circ} \mathrm{C} \text {. } \\
\text { Secondary current is } 1712 \mathrm{Amps} \text {. Glass resistance is } 0.069 \Omega \text {. }\end{array}$ \\
\hline & & $22: 34$ & 37.1 & $\begin{array}{c}\text { Plenum temperature is } 463-473^{\circ} \mathrm{C} \text {. Average glass temperature is } 1141^{\circ} \mathrm{C} \text {. Glass } \\
\text { resistance is } 0.070 \Omega .\end{array}$ \\
\hline & & $22: 47$ & 37.3 & $\begin{array}{l}\text { Adjusting blower } 701 / 702 \text { from } 52 \text { to } 54 \mathrm{~Hz} \text { and } 801 \text { from } 22 \text { to } 20 \mathrm{~Hz} \text {. This } \\
\text { brings blower } 701 / 702 \text { outlet pressures from }-8.0 \text { to }-2.0 \text { in W.C. }\end{array}$ \\
\hline & & $22: 49$ & 37.3 & Plenum temperature is $460-470^{\circ} \mathrm{C}$. \\
\hline & & $22: 53$ & 37.4 & $\begin{array}{l}\text { Further adjusting blowers: blowers } 701 / 702 \text { are set to } 55 \mathrm{~Hz} \text { and blower } 801 \text { is set } \\
\text { to } 18 \mathrm{~Hz} \text {. }\end{array}$ \\
\hline & & 23:05 & 37.6 & Plenum temperature is $467-475^{\circ} \mathrm{C}$. \\
\hline & & 23:19 & 37.8 & Plenum temperature is $473-474^{\circ} \mathrm{C}$. \\
\hline & & $23: 35$ & 38.1 & Plenum temperature is $471-478^{\circ} \mathrm{C}$. Average glass temperature is $1147^{\circ} \mathrm{C}$. \\
\hline & & 23:50 & 38.3 & Plenum temperature is $470-478^{\circ} \mathrm{C}$. \\
\hline & & 23:54 & 38.4 & Melter emergency off gas is opened momentarily, did not see any collapse of CC. \\
\hline & & 23:56 & 38.4 & Plenum temperature is $466-483^{\circ} \mathrm{C}$. Average glass temperature is $1152^{\circ} \mathrm{C}$. \\
\hline & \multirow{14}{*}{$1 / 31 / 13$} & $0: 15$ & 38.8 & $\begin{array}{l}\text { SBS overflow tank implodes: top is no longer convex. SBS outlet pressure }-63.5 \\
\text { in W.C. }\end{array}$ \\
\hline & & $0: 21$ & 38.9 & $\begin{array}{c}\text { Plenum temperature is } 471-476^{\circ} \mathrm{C} \text {. Secondary current is } 1691 \text { Amps. Glass } \\
\text { resistance is } 0.070 \Omega \text {. Glass density is } 2.30 \mathrm{~g} / \mathrm{cc} \text {. }\end{array}$ \\
\hline & & $0: 34$ & 39.1 & Plenum temperature is $463-475^{\circ} \mathrm{C}$. \\
\hline & & $0: 50$ & 39.3 & Plenum temperature is $451-474^{\circ} \mathrm{C}$. \\
\hline & & $1: 04$ & 39.6 & Plenum temperature is $462-463^{\circ} \mathrm{C}$. \\
\hline & & $1: 13$ & 39.7 & $\begin{array}{l}\text { As previously noticed, feed tank AOD pump seemed to stopping a beat or so. } \\
\text { Increased AOD pump pressure } \sim 32 \text { psi and will continue to monitor. }\end{array}$ \\
\hline & & $1: 22$ & 39.9 & $\begin{array}{l}\text { Increased bubbling as follows: Lances } 1 \mathrm{~A} \text { from } 20.3 \text { to } 22.3 \mathrm{lpm} \text {, Lance } 2 \mathrm{~A} \text { from } \\
19.8 \text { to } 21.8 \mathrm{lpm} \text {, Lance } 3 \mathrm{~A} \text { from } 10.1 \text { to } 11.1 \mathrm{lpm} \text {, Lance } 4 \mathrm{~A} \text { from } 10.1 \text { to } \\
11.1 \mathrm{lpm} \text {. }\end{array}$ \\
\hline & & $1: 23$ & 39.9 & Emergency off gas tripped. \\
\hline & & $1: 26$ & 39.9 & $\begin{array}{l}\text { Plenum temperature is } 461-471^{\circ} \mathrm{C} \text {. Secondary current is } 1699 \text { Amps. Glass level } \\
\text { is } 29.7 \text { in. }\end{array}$ \\
\hline & & $1: 43$ & 40.2 & $\begin{array}{l}\text { Blower speeds; blower } 801 \text { is } 24 \mathrm{~Hz} \text {, blowers } 701 / 702 \text { is } 50 \mathrm{~Hz} \text {, during discharge } \\
\text { to avoid positive pressure spike returned to blower } 801 \text { to } 20 \mathrm{~Hz} \text { and blower } \\
701 / 702 \text { to } 50 \mathrm{~Hz} \text {. }\end{array}$ \\
\hline & & $1: 44$ & 40.2 & Plenum temperature is $479-499^{\circ} \mathrm{C}$ \\
\hline & & 2:03 & 40.6 & $\begin{array}{l}\text { Previously increased bubbling to increase plenum temperatures to help dissolve } \\
\text { the ridge. }\end{array}$ \\
\hline & & 2:04 & 40.6 & $\begin{array}{l}\text { Blower } 701 / 702 \text { speed changed from } 50 \text { to } 55 \mathrm{~Hz} \text {, blower } 801 \text { speed changed } \\
\text { from } 20 \text { to } 23 \mathrm{~Hz} \text {. Melter vacuum is }-2.11 \text { in W.C. and blower outlet is }-6.8 \text { in } \\
\text { W.C. }\end{array}$ \\
\hline & & $2: 10$ & 40.7 & Changed T4 from 25 to 24 seconds. \\
\hline
\end{tabular}


Table 3.2. Summary of Operational Events (continued).

\begin{tabular}{|c|c|c|c|c|}
\hline Test & Date & Time & $\begin{array}{l}\text { Run } \\
\text { Time } \\
\text { (hours) }\end{array}$ & Run time note \\
\hline \multirow{26}{*}{3} & \multirow{26}{*}{$1 / 31 / 13$} & $2: 19$ & 40.8 & Plenum temperature is $486-522^{\circ} \mathrm{C}$. Average glass temperature is $1147^{\circ} \mathrm{C}$. \\
\hline & & $2: 23$ & 40.9 & $\begin{array}{l}\text { Reduced bubbling as follows: Lance } 1 \mathrm{~A} \text { from } 22.3 \text { to } 20.3 \mathrm{lpm} \text {, Lance } 2 \mathrm{~A} \text { from } \\
21.8 \text { to } 19.8 \mathrm{lpm} \text {, Lance } 3 \mathrm{~A} \text { from } 11.1 \text { to } 10.1 \mathrm{lpm} \text { and } \mathrm{L} 4 \mathrm{~A} \text { from } 11.1 \text { to } 10.1 \\
\mathrm{lpm} \text {. }\end{array}$ \\
\hline & & $2: 26$ & 40.9 & Average glass temperature is $1147^{\circ} \mathrm{C}$. \\
\hline & & $2: 36$ & 41.1 & $\begin{array}{c}\text { Plenum temperature is } 482-532^{\circ} \mathrm{C} \text {. Average glass temperature is } 1144^{\circ} \mathrm{C} \text {. } \\
\text { Secondary current is } 1686 \text { Amps. }\end{array}$ \\
\hline & & $2: 52$ & 41.4 & $\begin{array}{l}\text { Plenum temperature is } 466-539^{\circ} \mathrm{C} \text {. Average glass temperature is } 1148^{\circ} \mathrm{C} \text {. Glass } \\
\text { density is } 2.30 \mathrm{~g} / \mathrm{cc} \text {. }\end{array}$ \\
\hline & & 3:01 & 41.5 & Changed from 24 to 24.5 seconds. \\
\hline & & $3: 19$ & 41.8 & Plenum temperature is $463-544^{\circ} \mathrm{C}$. \\
\hline & & $3: 36$ & 42.1 & $\begin{array}{c}\text { Increased power to } 206 \mathrm{~kW} \text {. Average glass temperature is } 1144^{\circ} \mathrm{C} \text {. Secondary } \\
\text { current is } 1688 \text { Amps. Glass resistance is } 0.071 \Omega \text {. }\end{array}$ \\
\hline & & $3: 37$ & 42.1 & $\begin{array}{l}\text { Exposed plenum thermocouple almost touching CC. Plenum temperature is } 467- \\
\qquad 494^{\circ} \mathrm{C} .\end{array}$ \\
\hline & & $3: 47$ & 42.3 & $\begin{array}{l}\text { Increased power from } 206 \text { to } 208 \mathrm{~kW} \text {. Average glass temperature is } 1146^{\circ} \mathrm{C} \text {. } \\
\text { Thermo well \#1 reading is } 1115-1147^{\circ} \mathrm{C} \text {. Thermowell } \# 2 \text { reading is } 1142- \\
1152^{\circ} \mathrm{C} \text {. }\end{array}$ \\
\hline & & $3: 50$ & 42.3 & Plenum temperature is $469-490^{\circ} \mathrm{C}$. \\
\hline & & $4: 05$ & 42.6 & $\begin{array}{l}\text { Power is } 208 \mathrm{~kW}, 120 \text { volts, secondary current is } 1717 \mathrm{Amps} \text {, primary current is } \\
551.7 \mathrm{Amps} \text {, and glass resistance is } 0.070 \Omega \text {. }\end{array}$ \\
\hline & & 4:07 & 42.6 & Plenum temperature is $445-495^{\circ} \mathrm{C}$. Average glass temperature is $1147^{\circ} \mathrm{C}$. \\
\hline & & $4: 20$ & 42.8 & $\begin{array}{c}\text { Plenum temperature is } 438-501{ }^{\circ} \mathrm{C} \text {. Secondary current is } 1729 \text { Amps. Glass } \\
\text { resistance is } 0.069 \Omega .\end{array}$ \\
\hline & & $4: 23$ & 42.9 & Decreased blower $701 / 702$ from 54 to $51 \mathrm{~Hz}$, blower 801 from 24 to $20 \mathrm{~Hz}$. \\
\hline & & $4: 34$ & 43.1 & $\begin{array}{c}\text { Plenum temperature is } 430-510^{\circ} \mathrm{C} \text {. Average glass temperature is } 1150^{\circ} \mathrm{C} \text {. Glass } \\
\text { level is } 29.8 \mathrm{in} .\end{array}$ \\
\hline & & $4: 37$ & 43.1 & $\begin{array}{c}\text { Decreased power from } 208 \text { to } 206 \mathrm{~kW} \text {. Primary current is } 558.3 \mathrm{Amps} \text {, secondary } \\
\text { current is } 1738 \mathrm{Amps} \text {. Average glass temperature is } 1152^{\circ} \mathrm{C} \text {. }\end{array}$ \\
\hline & & $4: 46$ & 43.3 & Plenum temperature is $428-506^{\circ} \mathrm{C}$. \\
\hline & & $4: 48$ & 43.3 & $\begin{array}{l}\text { Melter pressure went positive, returned blowers } 701 / 702 \text { from } 51 \text { to } 54 \mathrm{~Hz} \text { and } \\
\text { blower } 801 \text { from } 20 \text { to } 24 \mathrm{~Hz} \text {. }\end{array}$ \\
\hline & & $4: 53$ & 43.4 & Lowered melter power from 206 to $203 \mathrm{~kW}$. \\
\hline & & $5: 05$ & 43.6 & Plenum temperature is $436-489^{\circ} \mathrm{C}$. Average glass temperature is $1147^{\circ} \mathrm{C}$. \\
\hline & & $5: 14$ & 43.7 & $\begin{array}{l}\text { Currently bubbling as follows: L1 A and B at } 20 \mathrm{lpm}, \mathrm{L} 2 \mathrm{~A} \text { and B at } 20 \mathrm{lpm}, \mathrm{L} 3 \\
\text { and L4 at } 10 \mathrm{lpm} .\end{array}$ \\
\hline & & $5: 20$ & 43.8 & $\begin{array}{c}\text { Observing love controller registering temperature of } 1172^{\circ} \mathrm{C} \text {. LabVIEW is } \\
\text { showing } 1168^{\circ} \mathrm{C} \text {. Secondary current is } 1707 \mathrm{Amps} \text {, and Glass resistance is } 0.069 \\
\Omega \text {. }\end{array}$ \\
\hline & & $5: 22$ & 43.9 & Lowered electric power from 203 to $200 \mathrm{~kW}$. \\
\hline & & $5: 23$ & 43.9 & Plenum temperature is $446-485^{\circ} \mathrm{C}$. Average glass temperature is $1148^{\circ} \mathrm{C}$. \\
\hline & & $5: 37$ & 44.1 & Plenum temperature is $441-486^{\circ} \mathrm{C}$. \\
\hline
\end{tabular}


Table 3.2. Summary of Operational Events (continued).

\begin{tabular}{|c|c|c|c|c|}
\hline Test & Date & Time & $\begin{array}{l}\text { Run } \\
\text { Time } \\
\text { (hours) }\end{array}$ & Run time note \\
\hline \multirow{32}{*}{3} & \multirow{32}{*}{$1 / 31 / 13$} & $5: 55$ & 44.4 & Plenum temperature is $427-503^{\circ} \mathrm{C}$. \\
\hline & & $6: 00$ & 44.5 & Adjusted T4 from 25 to 25.3 seconds. Feed rate $182.0 \mathrm{~kg} / \mathrm{hr}$ for 2 readings. \\
\hline & & $6: 08$ & 44.6 & $\begin{array}{c}\text { Reduced power from } 200 \text { to } 199 \mathrm{~kW} \text {. Secondary current is } 1700 \text { Amps, and glass } \\
\text { resistance is } 0.068 \Omega \text {. }\end{array}$ \\
\hline & & $6: 09$ & 44.7 & Plenum temperature is $425-518^{\circ} \mathrm{C}$. \\
\hline & & $6: 49$ & 45.3 & Plenum temperature is $431-505^{\circ} \mathrm{C}$. Average glass temperature is $1142^{\circ} \mathrm{C}$. \\
\hline & & $7: 04$ & 45.6 & Average glass temperature is $1145^{\circ} \mathrm{C}$. \\
\hline & & $7: 19$ & 45.8 & Plenum temperature is $440-460^{\circ} \mathrm{C}$. Average glass temperature is $1146^{\circ} \mathrm{C}$. \\
\hline & & $7: 34$ & 46.1 & Plenum temperature is $447-448^{\circ} \mathrm{C}$. Average glass temperature is $1140^{\circ} \mathrm{C}$. \\
\hline & & $7: 35$ & 46.1 & Increased power from 199 to $201 \mathrm{~kW}$. Average glass temperature is $1140^{\circ} \mathrm{C}$. \\
\hline & & $7: 49$ & 46.3 & $\begin{array}{c}\text { Plenum temperature is } 437-443^{\circ} \mathrm{C} \text {. Average glass temperature is } 1146^{\circ} \mathrm{C} \text {. } \\
\text { Secondary current is } 1699 \text { Amps, } 118 \text { volts. }\end{array}$ \\
\hline & & $7: 56$ & 46.4 & $\begin{array}{c}\text { Increased power from } 201 \text { to } 202 \mathrm{~kW} \text {. Average glass temperature is } 1146^{\circ} \mathrm{C} \text {. } \\
\text { Secondary current is } 1702 \mathrm{Amps}, 118 \text { volts. }\end{array}$ \\
\hline & & $8: 19$ & 46.8 & Average glass temperature is $1145^{\circ} \mathrm{C}$. \\
\hline & & $8: 21$ & 46.9 & Switched purge air from A to B due to blockage on A. \\
\hline & & $8: 34$ & 47.1 & Average glass temperature is $1146^{\circ} \mathrm{C}$. \\
\hline & & $8: 49$ & 47.3 & Average glass temperature is $1150^{\circ} \mathrm{C}$. \\
\hline & & 9:04 & 47.6 & $\begin{array}{l}\text { Average glass temperature is } 1148^{\circ} \mathrm{C} \text {. Secondary current is } 1694 \text { Amps. Power is } \\
\text { set to } 202 \mathrm{~kW} \text {. }\end{array}$ \\
\hline & & $9: 16$ & 47.8 & Decreased AOD pump pressure from 30 to 25 psi to help to control feed rate. \\
\hline & & 9:19 & 47.8 & Average glass temperature is $1148^{\circ} \mathrm{C}$. \\
\hline & & $9: 34$ & 48.1 & Plenum temperature is $452-453^{\circ} \mathrm{C}$. Average glass temperature is $1151^{\circ} \mathrm{C}$. \\
\hline & & 9:49 & 48.3 & Average glass temperature is $1147^{\circ} \mathrm{C}$. \\
\hline & & 10:05 & 48.6 & Performed film cooler rinse. \\
\hline & & $10: 30$ & 49.0 & Stopped feeding. End of Test. \\
\hline & & $10: 42$ & 49.2 & Reduced power from 202 to $192 \mathrm{~kW}$. Average glass temperature is $1154^{\circ} \mathrm{C}$. \\
\hline & & 10:52 & 49.4 & Reduced power from 192 to $185 \mathrm{~kW}$. Average glass temperature is $1175^{\circ} \mathrm{C}$. \\
\hline & & 10:58 & 49.5 & $\begin{array}{l}\text { Removed feed from the feed tank. Starting mass is } 909 \mathrm{~kg} \text {. Feed sample mass is } \\
1.275 \mathrm{~kg} \text {. End mass is } 10 \mathrm{~kg} \text {. Net mass removed is } 897.72 \mathrm{~kg} \text {. Feed sample is } \\
\text { taken. }\end{array}$ \\
\hline & & 11:02 & 49.5 & $\begin{array}{l}\text { Reduced power to } 165 \mathrm{~kW} \text { and increased bubbling to } 5 \mathrm{lpm} \text { on all bubblers. High } \\
\text { electrode alarm came in. Average glass temperature is } 1156^{\circ} \mathrm{C} \text {. }\end{array}$ \\
\hline & & 11:05 & 49.6 & Moved 800 gallons of waste water from bottom tanks to effluent tank. \\
\hline & & 11:20 & 49.8 & Reduced power to $155 \mathrm{~kW}$. Average glass temperature is $1159^{\circ} \mathrm{C}$. \\
\hline & & $11: 37$ & 50.1 & Reduced power to $145 \mathrm{~kW}$. Average glass temperature is $1158^{\circ} \mathrm{C}$. \\
\hline & & 11:46 & 50.3 & Reduced power to $135 \mathrm{~kW}$. Average glass temperature is $1161^{\circ} \mathrm{C}$. \\
\hline & & 12:00 & 50.5 & Reduced power to $125 \mathrm{~kW}$. Average glass temperature is $1160^{\circ} \mathrm{C}$. \\
\hline & & $12: 10$ & 50.7 & Reduced power to $115 \mathrm{~kW}$. Average glass temperature is $1157^{\circ} \mathrm{C}$. \\
\hline
\end{tabular}


Table 3.2. Summary of Operational Events (continued).

\begin{tabular}{|c|c|c|c|c|}
\hline Test & Date & Time & $\begin{array}{l}\text { Run } \\
\text { Time } \\
\text { (hours) }\end{array}$ & Run time note \\
\hline \multirow{5}{*}{3} & \multirow{4}{*}{$1 / 31 / 13$} & 12:41 & 51.2 & $\begin{array}{c}\text { Reduced power to } 110 \mathrm{~kW} \text {. Average glass temperature is } 1156^{\circ} \mathrm{C} \text {. Also reduced } \\
\text { bubbling to } 2.5 \mathrm{lpm} \text { on all bubblers. }\end{array}$ \\
\hline & & 13:09 & 51.7 & Reduced power to $105 \mathrm{~kW}$. Average glass temperature is $1156^{\circ} \mathrm{C}$. \\
\hline & & 13:23 & 51.9 & Turned off power to discharge chamber for inspection/repair. \\
\hline & & 14:30 & 53.0 & Reduced power to $105 \mathrm{~kW}$. Average glass temperature is $1140^{\circ} \mathrm{C}$. \\
\hline & $2 / 2 / 13$ & $3: 58$ & - & $\begin{array}{l}\text { DM1200 discharge chamber was fixed. The glass discharge was pulled out and } \\
\text { sampled. This drum will be utilized at the beginning of Test } 4 \text {. }\end{array}$ \\
\hline \multirow{22}{*}{4} & \multirow{22}{*}{$2 / 6 / 13$} & $0: 30$ & - & Transferred feed to mix tank. $2957.0 \mathrm{~kg}$ that includes $10 \mathrm{~kg}$ water is transferred. \\
\hline & & $3: 42$ & - & $\begin{array}{l}\text { Transferred feed from mix tank to feed tank. Starting mass is } 2957.0 \mathrm{~kg} \text {, ending } \\
\text { mass is } 37.0 \mathrm{~kg} \text {. Net mass transferred is } 2920.0 \mathrm{~kg} \text { plus } 496.4 \mathrm{~kg} \text { water is } \\
\text { transferred. } 10 \mathrm{~kg} \text { of this water is added later as flush water. }\end{array}$ \\
\hline & & $4: 25$ & - & Secured mix tank mixer, energized feed tank mixer, and placed in recirculation. \\
\hline & & $6: 40$ & - & Decreased power from 97 to $96 \mathrm{~kW}$. Average glass temperature is $1169^{\circ} \mathrm{C}$. \\
\hline & & $7: 13$ & - & Decreased power from 96 to $95 \mathrm{~kW}$. Average glass temperature is $1171^{\circ} \mathrm{C}$. \\
\hline & & $7: 16$ & - & Increased discharge power from $13 \mathrm{~kW}$ to $14 \mathrm{~kW}$. Temperature is $794^{\circ} \mathrm{C}$. \\
\hline & & $7: 40$ & - & Decreased power from 95 to $94 \mathrm{~kW}$. Average glass temperature is $1171^{\circ} \mathrm{C}$. \\
\hline & & $8: 17$ & - & Increased discharge chamber power from 14.0 to $15.0 \mathrm{~kW}$. Temperature is $783^{\circ} \mathrm{C}$. \\
\hline & & $8: 40$ & - & $\begin{array}{l}\text { Added the remaining residual feed from Test \#2 (Ref. VSL 1987-12 Page 72). } \\
\text { Feed tank mass in recirculation is } 3370.0 \mathrm{~kg} \text {, end feed tank mass in recirculation } \\
\text { is } 4024.5 \mathrm{~kg} \text {. Mass transferred that includes } 10 \mathrm{~kg} \text { water is } 654.5 \mathrm{~kg} \text {. }\end{array}$ \\
\hline & & 9:01 & - & Increased discharge chamber power from 15 to $16 \mathrm{~kW}$. Temperature is $799^{\circ} \mathrm{C}$. \\
\hline & & 9:30 & - & Increased discharge chamber power from 16 to $17 \mathrm{~kW}$. Temperature is $820^{\circ} \mathrm{C}$. \\
\hline & & 9:58 & - & $\begin{array}{l}\text { Increased power to electrodes from } 94 \text { to } 97 \mathrm{~kW} \text {. Average glass temperature is } \\
\qquad 1162^{\circ} \mathrm{C} .\end{array}$ \\
\hline & & 10:00 & - & $\begin{array}{l}\text { Increased discharge chamber power from } 17 \text { to } 18 \mathrm{~kW} \text {. Temperature is } 841- \\
\qquad 863^{\circ} \mathrm{C} .\end{array}$ \\
\hline & & 10:02 & - & Adjusted Lances 1 and 2 to $6.0 \mathrm{lpm}$, and Lances 3 and 4 to $3.0 \mathrm{lpm}$. \\
\hline & & 11:47 & - & Increased power from 97 to $100 \mathrm{~kW}$. Average glass temperature is $1160^{\circ} \mathrm{C}$. \\
\hline & & $12: 00$ & 0.0 & Started feeding water at $0.8 \mathrm{l} / \mathrm{min}$. Average glass temperature is $1161^{\circ} \mathrm{C}$. \\
\hline & & $12: 10$ & 0.2 & Increased power from 100 to $105 \mathrm{~kW}$. Average glass temperature is $1159^{\circ} \mathrm{C}$. \\
\hline & & 12:10 & 0.2 & Increased water feed from 0.8 to $1.61 / \mathrm{min}$. \\
\hline & & $12: 25$ & 0.4 & Increased power from 105 to $110 \mathrm{~kW}$. Average glass temperature $1154^{\circ} \mathrm{C}$. \\
\hline & & $12: 30$ & 0.5 & Increased water feed from 1.6 to $2.41 / \mathrm{min}$. \\
\hline & & $12: 35$ & 0.6 & Increased power from 110 to $115 \mathrm{~kW}$. Average glass temperature is $1148^{\circ} \mathrm{C}$. \\
\hline & & $12: 43$ & 0.7 & Increased power from 115 to $125 \mathrm{~kW}$. Average glass temperature is $1140^{\circ} \mathrm{C}$. \\
\hline
\end{tabular}




\section{Table 3.2. Summary of Operational Events (continued).}

\begin{tabular}{|c|c|c|c|c|}
\hline Test & Date & Time & $\begin{array}{l}\text { Run } \\
\text { Time } \\
\text { (hours) }\end{array}$ & Run time note \\
\hline \multirow{27}{*}{4} & \multirow{27}{*}{$2 / 6 / 13$} & 12:46 & 0.8 & Increased water feed from 2.46 to $3.0 \mathrm{l} / \mathrm{min}$. Plenum temperature is $800-864^{\circ} \mathrm{C}$. \\
\hline & & $12: 53$ & 0.9 & $\begin{array}{c}\text { Increased power from } 125 \text { to } 130 \mathrm{~kW} \text {. Average glass temperature is } 1135^{\circ} \mathrm{C} \text {. } \\
\text { Plenum temperature is } 726-825^{\circ} \mathrm{C} \text {. }\end{array}$ \\
\hline & & 13:00 & 1.0 & Secured feeding water. Started feeding slurry. \\
\hline & & 13:17 & 1.3 & Increased power from 130 to $135 \mathrm{~kW}$. Average glass temperature is $1135^{\circ} \mathrm{C}$. \\
\hline & & 13:24 & 1.4 & Average glass temperature is $1141^{\circ} \mathrm{C}$. \\
\hline & & $13: 32$ & 1.5 & Increased power from 135 to $140 \mathrm{~kW}$. Average glass temperature is $1140^{\circ} \mathrm{C}$. \\
\hline & & $13: 35$ & 1.6 & Average glass temperature $1140^{\circ} \mathrm{C}$. \\
\hline & & 13:47 & 1.8 & Increased power from 140 to $145 \mathrm{~kW}$. Average glass temperature is $1145^{\circ} \mathrm{C}$. \\
\hline & & 14:02 & 2.0 & $\begin{array}{l}\text { Increased bubbling on Lances } 1 \mathrm{~A} \text { and } 2 \mathrm{~A} \text { from } 6.0 \text { to } 7.0 \mathrm{lpm} \text {, and Lances } 3 \mathrm{~A} \text { and } \\
\text { 4A from } 3.0 \text { to } 3.5 \mathrm{lpm} \text {. }\end{array}$ \\
\hline & & 14:23 & 2.4 & Average glass temperature is $1161^{\circ} \mathrm{C}$. Plenum temperature is $705-714^{\circ} \mathrm{C}$. \\
\hline & & $14: 26$ & 2.4 & Decreased power from 145 to $140 \mathrm{~kW}$. Average glass temperature is $1163^{\circ} \mathrm{C}$. \\
\hline & & 14:37 & 2.6 & Average glass temperature is $1163^{\circ} \mathrm{C}$. \\
\hline & & $14: 55$ & 2.9 & $\begin{array}{c}\text { Primary current is } 428 \text { Amps and secondary current is } 1344 \text { Amps. Average glass } \\
\text { temperature is } 1167^{\circ} \mathrm{C} \text {. }\end{array}$ \\
\hline & & 15:02 & 3.0 & $\begin{array}{l}\text { Increased bubbling on Lance } 1 \mathrm{~A} \text { from } 7.2 \text { to } 8.2 \mathrm{lpm} \text { to maintain } 8.0 \mathrm{lpm} \text {, Lance } \\
\text { 2A from } 7.0 \text { to } 8.0 \mathrm{lpm} \text { to maintain } 8.0 \mathrm{lpm} \text {, Lance } 3 \mathrm{~A} \text { from } 3.6 \text { to } 4.1 \mathrm{lpm} \text { to } \\
\text { maintain } 4.0 \mathrm{lpm} \text {, and Lance } 4 \mathrm{~A} \text { from } 3.6 \text { to } 4.1 \mathrm{lpm} \text { to maintain } 4.0 \mathrm{lpm} \text {. }\end{array}$ \\
\hline & & 15:04 & 3.1 & Decreased power from 140 to $137 \mathrm{~kW}$. Primary current is 426 Amps. \\
\hline & & $15: 11$ & 3.2 & $\begin{array}{c}\text { Primary current is } 427 \text { Amps and secondary current is } 1341 \text { Amps. Average glass } \\
\text { temperature is } 1172^{\circ} \mathrm{C}\end{array}$ \\
\hline & & $15: 13$ & 3.2 & Decrease power from 137 to $132 \mathrm{~kW}$. Primary current 420 Amps. \\
\hline & & $15: 20$ & 3.3 & $\begin{array}{c}\text { Primary current is } 420 \mathrm{Amps} \text { and secondary current is } 1335 \text { Amps. Average glass } \\
\text { temperature is } 1172^{\circ} \mathrm{C} \text {. }\end{array}$ \\
\hline & & $15: 32$ & 3.5 & Decrease power from 132 to $130 \mathrm{~kW}$. Primary current 419 Amps. \\
\hline & & $15: 36$ & 3.6 & Completed clearing SBS blow down lance. \\
\hline & & $15: 37$ & 3.6 & Decrease power from 130 to $125 \mathrm{~kW}$. Primary current 412 Amps. \\
\hline & & $15: 49$ & 3.8 & $\begin{array}{c}\text { Primary current is } 411 \text { Amps and secondary current is } 1315 \text { Amps. Average glass } \\
\text { temperature is } 1175^{\circ} \mathrm{C} \text {. }\end{array}$ \\
\hline & & $16: 00$ & 4.0 & $\begin{array}{l}\text { Increased bubbling on Lance } 1 \mathrm{~A} \text { from } 8.2 \text { to } 9.2 \mathrm{lpm} \text { to maintain } 9.0 \mathrm{lpm} \text {, Lance } \\
2 \mathrm{~A} \text { from } 8.0 \text { to } 9.0 \mathrm{lpm} \text { to maintain } 9.0 \mathrm{lpm} \text {, Lance } 3 \mathrm{~A} \text { from } 4.1 \text { to } 4.6 \mathrm{lpm} \text { to } \\
\text { maintain } 4.5 \mathrm{lpm} \text {, and Lance } 4 \mathrm{~A} \text { from } 4.1 \text { to } 4.6 \mathrm{lpm} \text { to maintain } 4.5 \mathrm{lpm} \text {. }\end{array}$ \\
\hline & & $16: 05$ & 4.1 & $\begin{array}{c}\text { Primary current is } 411 \mathrm{Amps} \text { and secondary current is } 1314 \text { Amps. Average glass } \\
\text { temperature is } 1173^{\circ} \mathrm{C} \text {. }\end{array}$ \\
\hline & & $16: 18$ & 4.3 & $\begin{array}{c}\text { Reduced power from } 125 \text { to } 120 \mathrm{~kW} \text {. Primary current } 403 \text { Amps. Average glass } \\
\text { temperature is } 1174^{\circ} \mathrm{C} \text {. }\end{array}$ \\
\hline & & $16: 20$ & 4.3 & $\begin{array}{c}\text { Primary current is } 403 \text { Amps and secondary current is } 1290 \text { Amps. Average glass } \\
\text { temperature is } 1172^{\circ} \mathrm{C} \text {. }\end{array}$ \\
\hline & & $16: 42$ & 4.7 & $\begin{array}{c}\text { Primary current is } 397 \text { Amps and secondary current is } 1269 \text { Amps. Average glass } \\
\text { temperature is } 1167^{\circ} \mathrm{C} \text {. }\end{array}$ \\
\hline
\end{tabular}


Table 3.2. Summary of Operational Events (continued).

\begin{tabular}{|c|c|c|c|c|}
\hline Test & Date & Time & $\begin{array}{l}\text { Run } \\
\text { Time } \\
\text { (hours) }\end{array}$ & Run time note \\
\hline \multirow{20}{*}{4} & \multirow{20}{*}{$2 / 6 / 13$} & 17:02 & 5.0 & $\begin{array}{l}\text { Changed bubbling on Lance } 1 \mathrm{~A} \text { from } 9.2 \text { to } 10.1 \mathrm{lpm} \text { to maintain } 10.0 \mathrm{lpm} \text {, Lance } \\
2 \mathrm{~A} \text { from } 9.0 \text { to } 10.0 \mathrm{lpm} \text { to maintain } 10.0 \mathrm{lpm} \text {, Lance } 3 \mathrm{~A} \text { from } 4.6 \text { to } 5.1 \mathrm{lpm} \text { to } \\
\text { maintain } 5.0 \mathrm{lpm} \text {, and Lance } 4 \mathrm{~A} \text { from } 4.6 \text { to } 5.1 \mathrm{lpm} \text { to maintain } 5.0 \mathrm{lpm} \text {. }\end{array}$ \\
\hline & & 17:19 & 5.3 & Plenum temperature is $601-604^{\circ} \mathrm{C}$. Average glass temperature is $1157^{\circ} \mathrm{C}$. \\
\hline & & 17:35 & 5.6 & $\begin{array}{l}\text { SBS blow down totalizer indicated } 27.20 \text { gallons at the start of blow down, when } \\
\text { it reached } 35.58 \text { gallons it "froze", continued pumping for about } 3 \text { minutes and } \\
\text { blow down tank appeared to rise by visual indication. }\end{array}$ \\
\hline & & 18:01 & 6.0 & $\begin{array}{l}\text { Changed bubbling on Lance } 1 \mathrm{~A} \text { from } 10.1 \text { to } 11.0 \mathrm{lpm} \text { to maintain } 11.0 \mathrm{lpm} \text {, } \\
\text { Lance } 2 \mathrm{~A} \text { from } 10.0 \text { to } 11.0 \mathrm{lpm} \text { to maintain } 11.0 \mathrm{lpm} \text {, Lance } 3 \mathrm{~A} \text { from } 5.1 \text { to } 5.5 \\
\text { lpm to maintain } 5.5 \mathrm{lpm} \text {, and Lance } 4 \mathrm{~A} \text { from } 5.1 \text { to } 5.5 \mathrm{lpm} \text { to maintain } 5.5 \mathrm{lpm} \text {. }\end{array}$ \\
\hline & & $18: 37$ & 6.6 & $\begin{array}{c}\text { Increased power from } 120 \text { to } 125 \mathrm{~kW} \text {. Average glass temperature is } 1139^{\circ} \mathrm{C} \text {. } \\
\text { Primary current is } 390 \text { Amps. }\end{array}$ \\
\hline & & $18: 40$ & 6.7 & $\begin{array}{c}\text { Increased power from } 125 \text { to } 130 \mathrm{~kW} \text {. Average glass temperature is } 1138^{\circ} \mathrm{C} \text {. } \\
\text { Primary current is } 398 \text { Amps. }\end{array}$ \\
\hline & & 18:42 & 6.7 & Average glass temperature is $1138^{\circ} \mathrm{C}$. Primary current is $406 \mathrm{Amps}$. \\
\hline & & 18:50 & 6.8 & $\begin{array}{c}\text { Increased power from } 130 \text { to } 135 \mathrm{~kW} \text {. Average glass temperature is } 1138^{\circ} \mathrm{C} \text {. } \\
\text { Primary current is } 406 \text { Amps. }\end{array}$ \\
\hline & & 19:00 & 7.0 & $\begin{array}{c}\text { Changed bubbling on Lance 1A from } 11.0 \text { to maintain } 12.0 \mathrm{lpm} \text {, Lance } 2 \mathrm{~A} \text { from } \\
11.0 \text { to maintain } 12.0 \mathrm{lpm} \text {, Lance } 3 \mathrm{~A} \text { from } 5.5 \text { to maintain } 6.0 \mathrm{lpm} \text {, and Lance } 4 \mathrm{~A} \\
\text { from } 5.5 \text { to maintain } 6.0 \mathrm{lpm} \text {. }\end{array}$ \\
\hline & & 19:04 & 7.1 & $\begin{array}{l}\text { Increased power from } 135 \text { to } 140 \mathrm{~kW} \text {. Average glass temperature is } 1140^{\circ} \mathrm{C} \text {. } \\
\text { Primary current is } 414 \text { Amps. Thermocouple } 03 \text { is not spiking any more. }\end{array}$ \\
\hline & & 19:09 & 7.2 & $\begin{array}{l}\text { Average glass temperature is } 1141^{\circ} \mathrm{C} \text {. Primary current is } 415 \mathrm{Amps} \text {. Secondary } \\
\text { current is } 1302 \mathrm{Amps} \text {. Plenum temperature is } 536-546^{\circ} \mathrm{C} \text {. }\end{array}$ \\
\hline & & 19:16 & 7.3 & $\begin{array}{l}\text { Increased power from } 140 \text { to } 150 \mathrm{~kW} \text {. Average glass temperature is } 1141^{\circ} \mathrm{C} \text {. } \\
\text { Primary current is } 430 \mathrm{Amps} \text {. Plenum temperature is } 532-544^{\circ} \mathrm{C} \text {. }\end{array}$ \\
\hline & & 19:19 & 7.3 & $\begin{array}{l}\text { Average glass temperature is } 1144^{\circ} \mathrm{C} \text {. Primary current is } 432 \mathrm{Amps} \text {. Plenum } \\
\text { temperature is } 524-547^{\circ} \mathrm{C} \text {. Glass resistance is } 0.084 \Omega \text {. }\end{array}$ \\
\hline & & 19:32 & 7.5 & Found process heater is not set to $68^{\circ} \mathrm{C}$, increased temperature set point to $68^{\circ} \mathrm{C}$. \\
\hline & & 19:47 & 7.8 & $\begin{array}{c}\text { Average glass temperature is } 1146^{\circ} \mathrm{C} \text {. Primary current is } 432 \text { Amps. Secondary } \\
\text { current is } 1349 \text { Amps. }\end{array}$ \\
\hline & & $19: 48$ & 7.8 & $\begin{array}{l}\text { Increased power from } 150 \text { to } 153 \mathrm{~kW} \text {. Average glass temperature is } 1146^{\circ} \mathrm{C} \text {. } \\
\text { Primary current is } 436 \mathrm{Amps} \text {. Glass resistance is } 0.083 \Omega \text {. }\end{array}$ \\
\hline & & 20:09 & 8.2 & $\begin{array}{c}\text { Average glass temperature is } 1153^{\circ} \mathrm{C} \text {. Primary current is } 440 \text { Amps. Secondary } \\
\text { current is } 1382 \text { Amps. }\end{array}$ \\
\hline & & 20:16 & 8.3 & $\begin{array}{l}\text { Changed bubbling on Lance } 1 \mathrm{~A} \text { from } 12.2 \text { to } 13.2 \mathrm{lpm} \text { to maintain } 13.0 \mathrm{lpm} \text {, } \\
\text { Lance } 2 \mathrm{~A} \text { from } 12.0 \text { to } 13.0 \mathrm{lpm} \text { to maintain } 13.0 \mathrm{lpm} \text {, Lance } 3 \mathrm{~A} \text { from } 6.1 \text { to } 6.6 \\
\text { lpm to maintain } 6.5 \mathrm{lpm} \text {, and Lance } 4 \mathrm{~A} \text { from } 6.1 \text { to } 6.6 \mathrm{lpm} \text { to maintain } 6.5 \mathrm{lpm} \text {. }\end{array}$ \\
\hline & & 20:23 & 8.4 & $\begin{array}{l}\text { Decreased power from } 153 \text { to } 150 \mathrm{~kW} \text {. Average glass temperature is } 1155^{\circ} \mathrm{C} \text {. } \\
\text { Primary current is } 438 \mathrm{Amps} \text {. Secondary current is } 1369 \mathrm{Amps} \text {. }\end{array}$ \\
\hline & & 20:26 & 8.4 & $\begin{array}{c}\text { Average glass temperature is } 1154^{\circ} \mathrm{C} \text {. Primary current is } 437 \text { Amps. Secondary } \\
\text { current is } 1372 \text { Amps. }\end{array}$ \\
\hline
\end{tabular}


Table 3.2. Summary of Operational Events (continued).

\begin{tabular}{|c|c|c|c|c|}
\hline Test & Date & Time & $\begin{array}{l}\text { Run } \\
\text { Time } \\
\text { (hours) }\end{array}$ & Run time note \\
\hline \multirow{23}{*}{4} & \multirow{23}{*}{$2 / 6 / 13$} & $20: 35$ & 8.6 & Paused feeding to collect feed sample. \\
\hline & & $20: 39$ & 8.7 & Resumed feeding. \\
\hline & & $20: 55$ & 8.9 & $\begin{array}{l}\text { Decreased power from } 150 \text { to } 145 \mathrm{~kW} \text {. Average glass temperature is } 1156^{\circ} \mathrm{C} \text {. } \\
\text { Primary current is } 435 \mathrm{Amps} \text {. Glass resistance is } 0.080 \Omega \text {. }\end{array}$ \\
\hline & & $21: 00$ & 9.0 & $\begin{array}{l}\text { Changed bubbling on Lance } 1 \mathrm{~A} \text { from } 13.2 \text { to } 14.2 \mathrm{lpm} \text { to maintain } 14.0 \mathrm{lpm} \text {, } \\
\text { Lance } 2 \mathrm{~A} \text { from } 13.0 \text { to } 14.0 \mathrm{lpm} \text { to maintain } 14.0 \mathrm{lpm} \text {, Lance } 3 \mathrm{~A} \text { from } 6.6 \text { to } 7.1 \\
\text { lpm to maintain } 7.0 \mathrm{lpm} \text {, and Lance } 4 \mathrm{~A} \text { from } 6.6 \text { to } 7.1 \mathrm{lpm} \text { to maintain } 7.0 \mathrm{lpm} \text {. }\end{array}$ \\
\hline & & 21:04 & 9.1 & $\begin{array}{l}\text { Average glass temperature is } 1159^{\circ} \mathrm{C} \text {. Primary current is } 435 \mathrm{Amps} \text {. Glass } \\
\text { resistance is } 0.079 \Omega \text {. Plenum temperature is } 487-505^{\circ} \mathrm{C} \text {. }\end{array}$ \\
\hline & & $21: 19$ & 9.3 & $\begin{array}{l}\text { Average glass temperature is } 1156^{\circ} \mathrm{C} \text {. Primary current is } 434 \mathrm{Amps} \text {. Glass } \\
\text { resistance is } 0.080 \Omega \text {. Plenum temperature is } 489-499^{\circ} \mathrm{C} \text {. }\end{array}$ \\
\hline & & $21: 24$ & 9.4 & $\begin{array}{l}\text { Average glass temperature is } 1160^{\circ} \mathrm{C} \text {. Secondary current is } 1365 \text { Amps. Glass } \\
\text { density is } 2.33 \mathrm{~g} / \mathrm{cc} \text {. Plenum temperature is } 484-503^{\circ} \mathrm{C} \text {. }\end{array}$ \\
\hline & & $21: 52$ & 9.9 & $\begin{array}{c}\text { Average glass temperature is } 1156^{\circ} \mathrm{C} \text {. Glass resistance is } 0.081 \Omega \text {. Plenum } \\
\text { temperature is } 445-501{ }^{\circ} \mathrm{C} \text {. Electrode voltage is } 109 \text { volts. }\end{array}$ \\
\hline & & $21: 59$ & 10.0 & $\begin{array}{l}\text { Increased bubbling on Lance 1A from } 14.2 \text { to } 15.2 \mathrm{lpm} \text {, Lance } 2 \mathrm{~A} \text { from } 14.0 \text { to } \\
15.0 \mathrm{lpm} \text {, Lance } 3 \mathrm{~A} \text { from } 7.1 \text { to } 7.6 \mathrm{lpm} \text {, and Lance } 4 \mathrm{~A} \text { from } 7.1 \text { to } 7.6 \mathrm{lpm} \text {. } \\
\text { Primary current is } 431 \text { Amps. }\end{array}$ \\
\hline & & $22: 05$ & 10.1 & $\begin{array}{c}\text { Average glass temperature is } 1114^{\circ} \mathrm{C} \text {. Glass density is } 2.34 \mathrm{~g} / \mathrm{cc} \text {. Plenum } \\
\text { temperature is } 460-494^{\circ} \mathrm{C} \text {. }\end{array}$ \\
\hline & & $22: 33$ & 10.5 & $\begin{array}{l}\text { Increased power from } 145 \text { to } 148 \mathrm{~kW} \text {. Average glass temperature is } 1146^{\circ} \mathrm{C} \text {. } \\
\text { Glass resistance is } 0.084 \Omega \text {. Primary current is } 430 \text { Amps, and secondary current } \\
\text { is } 1346 \text { Amps. }\end{array}$ \\
\hline & & $22: 34$ & 10.6 & $\begin{array}{l}\text { Average glass temperature is } 1148^{\circ} \mathrm{C} \text {. Secondary current is } 1348 \text { Amps. Glass } \\
\text { resistance is } 0.083 \Omega \text {. Plenum temperature is } 461-485^{\circ} \mathrm{C} \text {. }\end{array}$ \\
\hline & & $22: 42$ & 10.7 & $\begin{array}{l}\text { Measured primary current using Amp probe; 433Amps. Indicator shows } 431 \\
\text { Amps. }\end{array}$ \\
\hline & & $22: 49$ & 10.8 & Average glass temperature is $1150^{\circ} \mathrm{C}$. Plenum temperature is $465-477^{\circ} \mathrm{C}$. \\
\hline & & 22:59 & 11.0 & $\begin{array}{l}\text { Increased bubbling on Lance } 1 \mathrm{~A} \text { from } 15.2 \text { to } 16.2 \mathrm{lpm} \text {, Lance } 2 \mathrm{~A} \text { from } 15.0 \text { to } \\
16.0 \mathrm{lpm} \text {, Lance } 3 \mathrm{~A} \text { from } 7.6 \text { to } 8.1 \mathrm{lpm} \text {, and Lance } 4 \mathrm{~A} \text { from } 7.6 \text { to } 8.1 \mathrm{lpm} \text {. }\end{array}$ \\
\hline & & 23:05 & 11.1 & $\begin{array}{l}\text { Upon changing discharge can, found a 5"x3"x10" glass chunk hanging from } \\
\text { discharge chamber floor, was able to dislodge into a new can. Looked up, } \\
\text { appeared that glass flow was even and coming from the floor, will watch closely. }\end{array}$ \\
\hline & & $23: 18$ & 11.3 & $\begin{array}{l}\text { Increased power from } 148 \text { to } 151 \mathrm{~kW} \text {. Average glass temperature is } 1142^{\circ} \mathrm{C} . \\
\text { Voltage is } 114 \text { volts. }\end{array}$ \\
\hline & & 23:20 & 11.3 & Average glass temperature is $1139^{\circ} \mathrm{C}$. Plenum temperature is $449-478^{\circ} \mathrm{C}$. \\
\hline & & $23: 26$ & 11.4 & Performed film cooler rinse. \\
\hline & & 23:35 & 11.6 & $\begin{array}{c}\text { Average glass temperature is } 1145^{\circ} \mathrm{C} \text {. Plenum temperature is } 458-477^{\circ} \mathrm{C} \text {. Glass } \\
\text { level is } 28.9 \mathrm{in} .\end{array}$ \\
\hline & & 23:42 & 11.7 & $\begin{array}{c}\text { Increased power from } 151 \text { to } 154 \mathrm{~kW} \text {. Average glass temperature is } 1143^{\circ} \mathrm{C} \text {. Glass } \\
\text { resistance is } 0.086 \Omega \text {. Glass density is } 2.30 \mathrm{~g} / \mathrm{cc} \text {. }\end{array}$ \\
\hline & & 23:49 & 11.8 & Average glass temperature is $1143^{\circ} \mathrm{C}$. Plenum temperature is $447-473^{\circ} \mathrm{C}$. \\
\hline & & 23:51 & 11.9 & Increased power from 154 to $157 \mathrm{~kW}$. \\
\hline
\end{tabular}


Table 3.2. Summary of Operational Events (continued).

\begin{tabular}{|c|c|c|c|c|}
\hline Test & Date & Time & $\begin{array}{l}\text { Run } \\
\text { Time } \\
\text { (hours) }\end{array}$ & Run time note \\
\hline \multirow{27}{*}{4} & $2 / 6 / 13$ & 23:59 & 12.0 & $\begin{array}{l}\text { Increased bubbling on Lance } 1 \mathrm{~A} \text { from } 16.2 \text { to } 17.2 \mathrm{lpm} \text {, Lance } 2 \mathrm{~A} \text { from } 16.0 \text { to } \\
17.0 \mathrm{lpm} \text {, Lance } 3 \mathrm{~A} \text { from } 8.1 \text { to } 8.6 \mathrm{lpm} \text {, and Lance } 4 \mathrm{~A} \text { from } 8.1 \text { to } 8.6 \mathrm{lpm} \text {. }\end{array}$ \\
\hline & \multirow{26}{*}{$2 / 7 / 13$} & $0: 14$ & 12.2 & $\begin{array}{c}\text { Increased power } 157 \text { to } 160 \mathrm{~kW} \text {. Average glass temperature is } 1146^{\circ} \mathrm{C} \text {. Glass } \\
\text { resistance is } 0.085 \Omega \text {. Plenum temperature is } 433-457^{\circ} \mathrm{C} \text {. }\end{array}$ \\
\hline & & $0: 19$ & 12.3 & $\begin{array}{l}\text { Average glass temperature is } 1147^{\circ} \mathrm{C} \text {. Glass level is } 28.9 \mathrm{in} \text {. Plenum temperature } \\
\text { is } 433-453^{\circ} \mathrm{C} \text {. }\end{array}$ \\
\hline & & $0: 34$ & 12.6 & $\begin{array}{l}\text { Average glass temperature is } 1150^{\circ} \mathrm{C} \text {. Glass resistance is } 0.083 \Omega \text {. Plenum } \\
\text { temperature is } 431-452^{\circ} \mathrm{C} \text {. }\end{array}$ \\
\hline & & $0: 49$ & 12.8 & Plenum temperature is $423-448^{\circ} \mathrm{C}$. \\
\hline & & $0: 59$ & 13.0 & Increased power from 160 to $163 \mathrm{~kW}$. Average glass temperature is $1143^{\circ} \mathrm{C}$. \\
\hline & & $1: 05$ & 13.1 & $\begin{array}{l}\text { Increased bubbling on Lance 1A from } 17.2 \text { to } 18.2 \mathrm{lpm} \text {, Lance } 2 \mathrm{~A} \text { from } 17.0 \text { to } \\
18.0 \mathrm{lpm} \text {, Lance } 3 \mathrm{~A} \text { from } 8.6 \text { to } 9.1 \mathrm{lpm} \text {, and Lance } 4 \mathrm{~A} \text { from } 8.6 \text { to } 9.1 \mathrm{lpm} \text {. }\end{array}$ \\
\hline & & 1:08 & 13.1 & Plenum temperature is $415-450^{\circ} \mathrm{C}$. \\
\hline & & $1: 14$ & 13.2 & $\begin{array}{c}\text { Increased power from } 163 \text { to } 166 \mathrm{~kW} \text {. Average glass temperature is } 1145^{\circ} \mathrm{C} \text {. } \\
\text { Primary current is } 452 \text { Amps. }\end{array}$ \\
\hline & & $1: 21$ & 13.4 & Average glass temperature is $1137^{\circ} \mathrm{C}$. Plenum temperature is $420-456^{\circ} \mathrm{C}$. \\
\hline & & $1: 26$ & 13.4 & Increased power from 166 to $169 \mathrm{~kW}$. Primary current is $458 \mathrm{Amps}$. \\
\hline & & $1: 34$ & 13.6 & $\begin{array}{l}\text { Plenum temperature is } 426-458^{\circ} \mathrm{C} \text {. Glass resistance is } 0.084 \Omega \text {. Glass density is } \\
2.32 \mathrm{~g} / \mathrm{cc} \text {. }\end{array}$ \\
\hline & & $1: 49$ & 13.8 & Average glass temperature is $1152^{\circ} \mathrm{C}$. Plenum temperature is $429-457^{\circ} \mathrm{C}$. \\
\hline & & $1: 54$ & 13.9 & Found Lance $2 \mathrm{~A}$ set at $17.0 \mathrm{lpm}$ changed to $18.0 \mathrm{lpm}$. \\
\hline & & $1: 55$ & 13.9 & Physical review of thermo well temperatures performed. No problems noted. \\
\hline & & 2:05 & 14.1 & Average glass temperature is $1155^{\circ} \mathrm{C}$. Plenum temperature is $427-453^{\circ} \mathrm{C}$. \\
\hline & & 2:09 & 14.2 & $\begin{array}{l}\text { Increased bubbling on Lance 1A from } 18.2 \text { to } 19.2 \mathrm{lpm} \text {, Lance } 2 \mathrm{~A} \text { from } 18.0 \text { to } \\
19.0 \mathrm{lpm} \text {, Lance 3A from 9.1 to } 9.6 \mathrm{lpm} \text {, and Lance 4A from } 9.1 \text { to } 9.6 \mathrm{lpm} \text {. }\end{array}$ \\
\hline & & $2: 11$ & 14.2 & $\begin{array}{l}\text { Transfer of six drums of feed to mix tank. Energizing mixer blade to } 30 \mathrm{~Hz} \text {. Mass } \\
\text { is } 1515.0 \mathrm{~kg} \text {. }\end{array}$ \\
\hline & & $2: 20$ & 14.3 & $\begin{array}{c}\text { Average glass temperature is } 1152^{\circ} \mathrm{C} \text {. Plenum temperature is } 427-456^{\circ} \mathrm{C} \text {. Glass } \\
\text { resistance is } 0.083 \Omega \text {. Glass level is } 29.0 \mathrm{in} \text {. }\end{array}$ \\
\hline & & $2: 34$ & 14.6 & $\begin{array}{c}\text { Average glass temperature is } 1151^{\circ} \mathrm{C} \text {. Plenum temperature is } 423-460^{\circ} \mathrm{C} \text {. Glass } \\
\text { resistance is } 0.081 \Omega \text {. Secondary current is } 1454 \mathrm{Amps} \text {. }\end{array}$ \\
\hline & & $2: 51$ & 14.9 & Average glass temperature is $1153^{\circ} \mathrm{C}$. Plenum temperature is $439-461^{\circ} \mathrm{C}$. \\
\hline & & 3:02 & 15.0 & $\begin{array}{l}\text { Increased bubbling on Lance } 1 \mathrm{~A} \text { from } 19.2 \text { to } 20.2 \mathrm{lpm} \text {, Lance } 2 \mathrm{~A} \text { from } 19.0 \text { to } \\
20.0 \mathrm{lpm} \text {, Lance } 3 \mathrm{~A} \text { from } 9.6 \text { to } 10.1 \mathrm{lpm} \text {, and Lance } 4 \mathrm{~A} \text { from } 9.6 \text { to } 10.1 \mathrm{lpm} \text {. }\end{array}$ \\
\hline & & 3:05 & 15.1 & Average glass temperature is $1153^{\circ} \mathrm{C}$. Plenum temperature is $443-463^{\circ} \mathrm{C}$. \\
\hline & & $3: 17$ & 15.3 & Changed T4 from 34 to 33.5 seconds. \\
\hline & & $3: 19$ & 15.3 & $\begin{array}{c}\text { Average glass temperature is } 1157^{\circ} \mathrm{C} \text {. Plenum temperature is } 444-467^{\circ} \mathrm{C} \text {. Glass } \\
\text { level is } 29.5 \mathrm{in} .\end{array}$ \\
\hline & & $3: 35$ & 15.6 & $\begin{array}{l}\text { Average glass temperature is } 1150^{\circ} \mathrm{C} \text {. Plenum temperature is } 447-472^{\circ} \mathrm{C} \text {. Glass } \\
\text { resistance is } 0.083 \Omega \text {. Secondary current is } 1445 \text { Amps. Glass density is } 2.30 \\
\mathrm{~g} / \mathrm{cc} \text {. }\end{array}$ \\
\hline & & $3: 44$ & 15.7 & Average glass temperature is $1152^{\circ} \mathrm{C}$. Plenum temperature is $453-478^{\circ} \mathrm{C}$ \\
\hline
\end{tabular}


Table 3.2. Summary of Operational Events (continued).

\begin{tabular}{|c|c|c|c|c|}
\hline Test & Date & Time & $\begin{array}{l}\text { Run } \\
\text { Time } \\
\text { (hours) }\end{array}$ & Run time note \\
\hline \multirow{27}{*}{4} & \multirow{27}{*}{$2 / 7 / 13$} & 4:03 & 16.0 & $\begin{array}{c}\text { Increased bubbling on Lance } 1 \mathrm{~A} \text { from } 20.2 \text { to } 21.2 \mathrm{lpm} \text {, Lance } 2 \mathrm{~A} \text { from } 20.0 \text { to } \\
21.0 \mathrm{lpm} \text {, Lance } 3 \mathrm{~A} \text { from } 10.1 \text { to } 10.6 \mathrm{lpm} \text {, and Lance } 4 \mathrm{~A} \text { from } 10.1 \text { to } 10.6 \mathrm{lpm} \text {. } \\
\text { Average glass temperature is } 1155^{\circ} \mathrm{C} \text {. }\end{array}$ \\
\hline & & 4:07 & 16.1 & $\begin{array}{c}\text { Average glass temperature is } 1155^{\circ} \mathrm{C} \text {. Plenum temperature is } 454-476^{\circ} \mathrm{C} \text {. Glass } \\
\text { density is } 2.29 \mathrm{~g} / \mathrm{cc} \text {. }\end{array}$ \\
\hline & & $4: 19$ & 16.3 & $\begin{array}{c}\text { Average glass temperature is } 1154^{\circ} \mathrm{C} \text {. Plenum temperature is } 451-471^{\circ} \mathrm{C} \text {. } \\
\text { Secondary current is } 1442 \text { Amps. }\end{array}$ \\
\hline & & $4: 31$ & 16.5 & Changed T4 from 33.0 to 32.5 seconds. \\
\hline & & $4: 34$ & 16.6 & Average glass temperature is $1157^{\circ} \mathrm{C}$. Plenum temperature is $454-472^{\circ} \mathrm{C}$. \\
\hline & & $4: 49$ & 16.8 & Average glass temperature is $1152^{\circ} \mathrm{C}$. Plenum temperature is $457-471^{\circ} \mathrm{C}$. \\
\hline & & $5: 00$ & 17.0 & $\begin{array}{l}\text { Increased bubbling on Lance } 1 \mathrm{~A} \text { from } 21.2 \text { to } 22.2 \mathrm{lpm} \text {, Lance } 2 \mathrm{~A} \text { from } 21.0 \text { to } \\
22.0 \mathrm{lpm} \text {, Lance } 3 \mathrm{~A} \text { from } 10.6 \text { to } 11.1 \mathrm{lpm} \text {, and Lance } 4 \mathrm{~A} \text { from } 10.6 \text { to } 11.1 \mathrm{lpm} \text {. } \\
\text { Average glass temperature is } 1155^{\circ} \mathrm{C} \text {. }\end{array}$ \\
\hline & & $5: 04$ & 17.1 & Plenum temperature is $456-477^{\circ} \mathrm{C}$. \\
\hline & & $5: 28$ & 17.5 & Plenum temperature is $459-475^{\circ} \mathrm{C}$. \\
\hline & & $5: 42$ & 17.7 & Plenum temperature is $451-456^{\circ} \mathrm{C}$. \\
\hline & & $5: 48$ & 17.8 & Average glass temperature is $1146^{\circ} \mathrm{C}$. Plenum temperature is $454-458^{\circ} \mathrm{C}$. \\
\hline & & $6: 00$ & 18.0 & $\begin{array}{c}\text { Increased power from } 169 \text { to } 172 \mathrm{~kW} \text {. Average glass temperature is } 1147^{\circ} \mathrm{C} \text {. } \\
\text { Primary current is } 460 \text { Amps. }\end{array}$ \\
\hline & & $6: 03$ & 18.0 & $\begin{array}{l}\text { Increased bubbling on Lance } 1 \mathrm{~A} \text { from } 22.2 \text { to } 23.2 \mathrm{lpm} \text {, Lance } 2 \mathrm{~A} \text { from } 22.0 \text { to } \\
23.0 \mathrm{lpm} \text {, Lance } 3 \mathrm{~A} \text { from } 11.1 \text { to } 11.6 \mathrm{lpm} \text {, and Lance } 4 \mathrm{~A} \text { from } 11.0 \text { to } 11.6 \mathrm{lpm} \text {. }\end{array}$ \\
\hline & & $6: 04$ & 18.1 & Plenum temperature is $447-467^{\circ} \mathrm{C}$. \\
\hline & & $6: 10$ & 18.2 & $\begin{array}{c}\text { Increased power from } 172 \text { to } 175 \mathrm{~kW} \text {. Average glass temperature is } 1142^{\circ} \mathrm{C} \text {. } \\
\text { Primary current is } 465 \text { Amps. }\end{array}$ \\
\hline & & $6: 20$ & 18.3 & $\begin{array}{c}\text { Increased power from } 175 \text { to } 178 \mathrm{~kW} \text {. Average glass temperature is } 1136^{\circ} \mathrm{C} \text {. } \\
\text { Primary current is } 467 \text { Amps. }\end{array}$ \\
\hline & & $6: 31$ & 18.5 & $\begin{array}{c}\text { Increased power from } 178 \text { to } 181 \mathrm{~kW} \text {. Average glass temperature is } 1138^{\circ} \mathrm{C} \text {. } \\
\text { Primary current is } 473 \text { Amps. }\end{array}$ \\
\hline & & $6: 59$ & 19.0 & Average glass temperature is $1141^{\circ} \mathrm{C}$. \\
\hline & & $7: 03$ & 19.0 & $\begin{array}{l}\text { Increased bubbling on Lance } 1 \mathrm{~A} \text { from } 23.2 \text { to } 24.2 \mathrm{lpm} \text {, Lance } 2 \mathrm{~A} \text { from } 23.0 \text { to } \\
23.9 \mathrm{lpm} \text {, Lance } 3 \mathrm{~A} \text { from } 11.6 \text { to } 12.1 \mathrm{lpm} \text {, and Lance } 4 \mathrm{~A} \text { from } 11.6 \text { to } 12.1 \mathrm{lpm} \text {. }\end{array}$ \\
\hline & & $7: 13$ & 19.2 & $\begin{array}{c}\text { Increased power from } 181 \text { to } 184 \mathrm{~kW} \text {. Average glass temperature is } 1142^{\circ} \mathrm{C} \text {. } \\
\text { Primary current is } 478 \text { Amps. }\end{array}$ \\
\hline & & $7: 15$ & 19.3 & Average glass temperature is $1141^{\circ} \mathrm{C}$. Plenum temperature is $460-481^{\circ} \mathrm{C}$. \\
\hline & & $7: 32$ & 19.5 & $\begin{array}{c}\text { Increased power from } 184 \text { to } 187 \mathrm{~kW} \text {. Average glass temperature is } 1142^{\circ} \mathrm{C} \text {. } \\
\text { Primary current is } 483 \text { Amps. }\end{array}$ \\
\hline & & $7: 34$ & 19.6 & Plenum temperature is $472-483^{\circ} \mathrm{C}$. Primary current 484 Amps. \\
\hline & & $7: 44$ & 19.7 & $\begin{array}{c}\text { Increased power from } 187 \text { to } 192 \mathrm{~kW} \text {. Average glass temperature is } 1140^{\circ} \mathrm{C} \text {. } \\
\text { Primary current is } 491 \text { Amps. }\end{array}$ \\
\hline & & $7: 48$ & 19.8 & Average glass temperature is $1137^{\circ} \mathrm{C}$. Plenum temperature is $462-468^{\circ} \mathrm{C}$. \\
\hline & & $8: 02$ & 20.0 & $\begin{array}{l}\text { Increased bubbling on Lance } 1 \mathrm{~A} \text { from } 24.3 \text { to } 25.3 \mathrm{lpm} \text {, Lance } 2 \mathrm{~A} \text { from } 23.9 \text { to } \\
24.9 \mathrm{lpm} \text {, Lance } 3 \mathrm{~A} \text { from } 12.1 \text { to } 12.6 \mathrm{lpm} \text {, and Lance } 4 \mathrm{~A} \text { from } 12.1 \text { to } 12.6 \mathrm{lpm} \text {. }\end{array}$ \\
\hline & & $8: 03$ & 20.1 & $\begin{array}{l}\text { Average glass temperature is } 1144^{\circ} \mathrm{C} \text {. Plenum temperature is } 469-481^{\circ} \mathrm{C} \text {. } \\
\text { Primary current is } 498 \text { Amps. }\end{array}$ \\
\hline
\end{tabular}




\section{Table 3.2. Summary of Operational Events (continued).}

\begin{tabular}{|c|c|c|c|c|}
\hline Test & Date & Time & $\begin{array}{l}\text { Run } \\
\text { Time } \\
\text { (hours) }\end{array}$ & Run time note \\
\hline \multirow{28}{*}{4} & \multirow{28}{*}{$2 / 7 / 13$} & $8: 19$ & 20.3 & $\begin{array}{l}\text { Average glass temperature is } 1151^{\circ} \mathrm{C} \text {. Power is } 196 \mathrm{~kW} \text {. Primary current is } 498 \\
\text { Amps. Secondary current is } 1544 \text { Amps. Glass resistance is } 0.082 \Omega \text {. }\end{array}$ \\
\hline & & 9:03 & 21.0 & $\begin{array}{l}\text { Increased bubbling on Lance } 1 \mathrm{~A} \text { from } 25.3 \text { to } 26.3 \mathrm{lpm} \text {, Lance } 2 \mathrm{~A} \text { from } 24.9 \text { to } \\
25.9 \mathrm{lpm} \text {, Lance } 3 \mathrm{~A} \text { from } 12.6 \text { to } 13.1 \mathrm{lpm} \text {, and Lance } 4 \mathrm{~A} \text { from } 12.6 \text { to } 13.1 \mathrm{lpm} \text {. } \\
\text { Total bubbling is } 78.0 \mathrm{lpm} \text {. }\end{array}$ \\
\hline & & 9:04 & 21.1 & Average glass temperature is $1149^{\circ} \mathrm{C}$. \\
\hline & & $9: 19$ & 21.3 & $\begin{array}{c}\text { Now at maximum bubbling rate of } 78 \mathrm{lpm} \text {. Average glass temperature is } 1144^{\circ} \mathrm{C} \text {. } \\
\text { Primary current is } 494 \text { Amps. }\end{array}$ \\
\hline & & $9: 25$ & 21.4 & $\begin{array}{l}\text { Transferred } 887.5 \mathrm{~kg} \text { of Test } 3 \text { residual feed. Transferred feed from mix tank to } \\
\text { feed tank. Starting tank mass is } 1510.0 \mathrm{~kg} \text {, ending tank mass is } 36.0 \mathrm{~kg} \text {. Net mass } \\
\text { transferred is } 1474.0 \mathrm{~kg} \text {. Plus } 250 \mathrm{~kg} \text { water in addition to } 887.5 \mathrm{~kg} \text { (from Test } 3 \\
\text { residue) add up to total of } 2611.5 \mathrm{~kg} \text { net mass transfer. }\end{array}$ \\
\hline & & 9:35 & 21.6 & Average glass temperature is $1143^{\circ} \mathrm{C}$. \\
\hline & & $9: 48$ & 21.8 & $\begin{array}{c}\text { Decreased feed rate by changing T4 from } 26 \text { to } 30 \text { seconds. Feed rate increased } \\
\text { due to feed transfer. }\end{array}$ \\
\hline & & 9:50 & 21.8 & $\begin{array}{c}\text { Increased power from } 192 \text { to } 195 \mathrm{~kW} \text {. Average glass temperature is } 1141^{\circ} \mathrm{C} \text {. } \\
\text { Primary current is } 496 \text { Amps. }\end{array}$ \\
\hline & & $9: 52$ & 21.9 & Average glass temperature is $1139^{\circ} \mathrm{C}$. \\
\hline & & 10:06 & 22.1 & Increased power from 195 to $199 \mathrm{~kW}$. Average glass temperature is $1140^{\circ} \mathrm{C}$. \\
\hline & & $10: 21$ & 22.4 & Increased power from 199 to $201 \mathrm{~kW}$. Average glass temperature is $1138^{\circ} \mathrm{C}$. \\
\hline & & $10: 22$ & 22.4 & Average glass temperature is $1138^{\circ} \mathrm{C}$. \\
\hline & & $10: 35$ & 22.6 & Increased power from 204 to $209 \mathrm{~kW}$. Average glass temperature is $1139^{\circ} \mathrm{C}$. \\
\hline & & $10: 36$ & 22.6 & Average glass temperature is $1143^{\circ} \mathrm{C}$. \\
\hline & & 11:03 & 23.1 & Increased power from 209 to $215 \mathrm{~kW}$. Average glass temperature is $1140^{\circ} \mathrm{C}$. \\
\hline & & $11: 12$ & 23.2 & $\begin{array}{c}\text { Increased power from } 215 \text { to } 220 \mathrm{~kW} \text {. Average glass temperature is } 1142^{\circ} \mathrm{C} \text {. } \\
\text { Primary current is } 527 \text { Amps. }\end{array}$ \\
\hline & & $11: 49$ & 23.8 & Average glass temperature is $1145^{\circ} \mathrm{C}$. Primary current is 534 Amps. \\
\hline & & 12:12 & 24.2 & $\begin{array}{c}\text { Average glass temperature is } 1142^{\circ} \mathrm{C} \text {. Plenum temperature is } 432-456^{\circ} \mathrm{C} \text {. } \\
\text { Primary current is } 530 \text { Amps. }\end{array}$ \\
\hline & & $12: 32$ & 24.5 & Average glass temperature is $1148^{\circ} \mathrm{C}$. Plenum temperature is $444-472^{\circ} \mathrm{C}$. \\
\hline & & 13:14 & 25.1 & Increased power from 220 to $221 \mathrm{~kW}$. Average glass temperature is $1149^{\circ} \mathrm{C}$. \\
\hline & & $13: 34$ & 25.6 & $\begin{array}{c}\text { Average glass temperature is } 1154^{\circ} \mathrm{C} \text {. Plenum temperature is } 444-461^{\circ} \mathrm{C} \text {. } \\
\text { Primary current is } 540 \text { Amps. }\end{array}$ \\
\hline & & 13:49 & 25.8 & Average glass temperature is $1155^{\circ} \mathrm{C}$. \\
\hline & & 14:04 & 26.1 & Average glass temperature is $1155^{\circ} \mathrm{C}$. Primary current is $541 \mathrm{Amps}$. \\
\hline & & 14:19 & 26.3 & Average glass temperature is $1156^{\circ} \mathrm{C}$. \\
\hline & & $14: 40$ & 26.7 & Average glass temperature is $1151^{\circ} \mathrm{C}$ \\
\hline & & $14: 47$ & 26.8 & Performed WESP blow down, pre-deluge post deluge. \\
\hline & & 15:00 & 27.0 & Starting off gas sampling, observed melter pressure spike. \\
\hline & & $15: 19$ & 27.3 & $\begin{array}{l}\text { Average glass temperature is } 1157^{\circ} \mathrm{C} \text {. Plenum temperature is } 453-468^{\circ} \mathrm{C} \text {. } \\
\text { Primary current is } 546 \text { Amps. Glass resistance is } 0.081 \Omega \text {. }\end{array}$ \\
\hline
\end{tabular}


Table 3.2. Summary of Operational Events (continued).

\begin{tabular}{|c|c|c|c|c|}
\hline Test & Date & Time & $\begin{array}{l}\text { Run } \\
\text { Time } \\
\text { (hours) }\end{array}$ & Run time note \\
\hline \multirow{24}{*}{4} & \multirow{24}{*}{$2 / 7 / 13$} & 15:33 & 27.6 & $\begin{array}{l}\text { Average glass temperature is } 1159^{\circ} \mathrm{C} \text {. Plenum temperature is } 403-473^{\circ} \mathrm{C} \text {. } \\
\text { Primary current is } 548 \mathrm{Amps} \text {. Glass resistance is } 0.080 \Omega \text {. }\end{array}$ \\
\hline & & $15: 49$ & 27.8 & $\begin{array}{l}\text { Average glass temperature is } 1161^{\circ} \mathrm{C} \text {. Plenum temperature is } 452-472^{\circ} \mathrm{C} \text {. } \\
\text { Primary current is } 545 \text { Amps. Secondary current is } 1660 \text { Amps. Glass resistance } \\
\text { is } 0.081 \Omega \text {. }\end{array}$ \\
\hline & & $16: 01$ & 28.0 & Melter pressure spike due to off gas sampling in transition line. \\
\hline & & 16:04 & 28.1 & $\begin{array}{c}\text { Average glass temperature is } 1159^{\circ} \mathrm{C} \text {. Plenum temperature is } 457-473^{\circ} \mathrm{C} \text {. } \\
\text { Primary current is } 548 \text { Amps. }\end{array}$ \\
\hline & & $16: 19$ & 28.3 & $\begin{array}{l}\text { Average glass temperature is } 1159^{\circ} \mathrm{C} \text {. Plenum temperature is } 459-477^{\circ} \mathrm{C} \text {. } \\
\text { Primary current is } 548 \text { Amps. Secondary current is } 1667 \text { Amps. Glass resistance } \\
\text { is } 0.080 \Omega \text {. }\end{array}$ \\
\hline & & $16: 45$ & 28.7 & $\begin{array}{l}\text { Average glass temperature is } 1157^{\circ} \mathrm{C} \text {. Plenum temperature is } 409-473^{\circ} \mathrm{C} \text {. } \\
\text { Primary current is } 543 \text { Amps. Secondary current is } 1634 \text { Amps. Glass resistance } \\
\text { is } 0.082 \Omega \text {. }\end{array}$ \\
\hline & & $16: 46$ & 28.8 & Melter pressure went positive due to CC collapsing. \\
\hline & & $16: 49$ & 28.8 & $\begin{array}{l}\text { Average glass temperature is } 1156^{\circ} \mathrm{C} \text {. Plenum temperature is } 409-473^{\circ} \mathrm{C} \text {. } \\
\text { Primary current is } 546 \mathrm{Amps} \text {. Secondary current is } 1655 \mathrm{Amps} \text {. }\end{array}$ \\
\hline & & $17: 02$ & 29.0 & $\begin{array}{l}\text { Transferred feed to mix tank. Starting mass is } 34.5 \mathrm{~kg} \text {, ending mass is } 2726.5 \mathrm{~kg} \text {. } \\
\text { Net transferred feed mass is } 2692.0 \mathrm{~kg} \text {. Net mass includes } 10 \mathrm{~kg} \text { water. }\end{array}$ \\
\hline & & 17:04 & 29.1 & $\begin{array}{c}\text { Average glass temperature is } 1164^{\circ} \mathrm{C} \text {. Plenum temperature is } 464-477^{\circ} \mathrm{C} \text {. } \\
\text { Primary current is } 549 \text { Amps }\end{array}$ \\
\hline & & $17: 11$ & 29.2 & $\begin{array}{c}\text { Reduced power from } 221 \text { to } 218 \mathrm{~kW} \text {. Average glass temperature is } 1163^{\circ} \mathrm{C} \text {. } \\
\text { Primary current is } 545 \mathrm{Amps}\end{array}$ \\
\hline & & $17: 34$ & 29.6 & $\begin{array}{l}\text { Average glass temperature is } 1161^{\circ} \mathrm{C} \text {. Plenum temperature is } 460-484^{\circ} \mathrm{C} \text {. } \\
\text { Primary current is } 546 \mathrm{Amps} \text {. Secondary current is } 1661 \mathrm{Amps} \text {. }\end{array}$ \\
\hline & & $17: 40$ & 29.7 & $\begin{array}{l}\text { Reduced power from } 218 \text { to } 216 \mathrm{~kW} \text {. Average glass temperature is } 1161^{\circ} \mathrm{C} \text {. } \\
\text { Primary current is } 544 \mathrm{Amps} \text {. Secondary current is } 1667 \mathrm{Amps} \text {. }\end{array}$ \\
\hline & & $18: 19$ & 30.3 & $\begin{array}{l}\text { Average glass temperature is } 1159^{\circ} \mathrm{C} \text {. Plenum temperature is } 461-486^{\circ} \mathrm{C} \text {. } \\
\text { Primary current is } 539 \text { Amps. }\end{array}$ \\
\hline & & 18:34 & 30.6 & $\begin{array}{c}\text { Average glass temperature is } 1158^{\circ} \mathrm{C} \text {. Plenum temperature is } 464-495^{\circ} \mathrm{C} \text {. } \\
\text { Primary current is } 535 \text { Amps. }\end{array}$ \\
\hline & & 18:41 & 30.7 & Plenum temperature is $464-476^{\circ} \mathrm{C}$. Primary current is $533 \mathrm{Amps}$. \\
\hline & & 18:49 & 30.8 & $\begin{array}{l}\text { Average glass temperature is } 1152^{\circ} \mathrm{C} \text {. Plenum temperature is } 466-476^{\circ} \mathrm{C} \text {. } \\
\text { Primary current is } 535 \mathrm{Amps} \text {. }\end{array}$ \\
\hline & & 19:04 & 31.1 & $\begin{array}{l}\text { Average glass temperature is } 1153^{\circ} \mathrm{C} \text {. Plenum temperature is } 468-475^{\circ} \mathrm{C} \text {. } \\
\text { Primary current is } 534 \text { Amps. }\end{array}$ \\
\hline & & 19:19 & 31.3 & $\begin{array}{l}\text { Changed T4 from } 25.5 \text { to } 25 \text { seconds. Average glass temperature is } 1148^{\circ} \mathrm{C} \text {. } \\
\text { Plenum temperature is } 469-489^{\circ} \mathrm{C} \text {. Primary current is } 530 \text { Amps. }\end{array}$ \\
\hline & & 19:34 & 31.6 & $\begin{array}{l}\text { Average glass temperature is } 1156^{\circ} \mathrm{C} \text {. Plenum temperature is } 463-488^{\circ} \mathrm{C} \text {. } \\
\text { Primary current is } 531 \mathrm{Amps} \text {. Glass resistance is } 0.082 \Omega \text {. }\end{array}$ \\
\hline & & 19:49 & 31.8 & $\begin{array}{c}\text { Average glass temperature is } 1152^{\circ} \mathrm{C} \text {. Primary current is } 533 \text { Amps. Secondary } \\
\text { current is } 1152 \text { Amps. }\end{array}$ \\
\hline & & 20:02 & 32.0 & Collected a feed sample. \\
\hline & & 20:07 & 32.1 & $\begin{array}{l}\text { Average glass temperature is } 1153^{\circ} \mathrm{C} \text {. Plenum temperature is } 452-495^{\circ} \mathrm{C} \text {. } \\
\text { Primary current is } 537 \text { Amps. }\end{array}$ \\
\hline & & $20: 19$ & 32.3 & $\begin{array}{l}\text { Average glass temperature is } 1157^{\circ} \mathrm{C} \text {. Plenum temperature is } 454-499^{\circ} \mathrm{C} \text {. } \\
\text { Primary current is } 536 \mathrm{Amps} \text {. Glass resistance is } 0.080 \Omega \text {. }\end{array}$ \\
\hline
\end{tabular}




\section{Table 3.2. Summary of Operational Events (continued).}

\begin{tabular}{|c|c|c|c|c|}
\hline Test & Date & Time & $\begin{array}{l}\text { Run } \\
\text { Time } \\
\text { (hours) }\end{array}$ & Run time note \\
\hline \multirow{26}{*}{4} & \multirow{17}{*}{$2 / 7 / 13$} & 20:36 & 32.6 & $\begin{array}{l}\text { Average glass temperature is } 1154^{\circ} \mathrm{C} \text {. Plenum temperature is } 462-488^{\circ} \mathrm{C} \text {. } \\
\text { Secondary current is } 1627 \text { Amps. Glass level is } 29 \text {. in. }\end{array}$ \\
\hline & & $20: 49$ & 32.8 & Average glass temperature is $1157^{\circ} \mathrm{C}$. Plenum temperature is $467-489^{\circ} \mathrm{C}$. \\
\hline & & 21:04 & 33.1 & $\begin{array}{c}\text { Plenum temperature is } 468-479^{\circ} \mathrm{C} \text {. Primary current is } 532 \mathrm{Amps} \text {. Secondary } \\
\text { current is } 1627 \mathrm{Amps} \text {. Glass resistance is } 0.081 \Omega\end{array}$ \\
\hline & & 21:20 & 33.3 & $\begin{array}{c}\text { Average glass temperature is } 1158^{\circ} \mathrm{C} \text {. Primary current is } 533 \mathrm{Amps} \text {. Secondary } \\
\text { current is } 1623 \mathrm{Amps} \text {. Glass resistance is } 0.081 \Omega\end{array}$ \\
\hline & & $21: 28$ & 33.5 & Change Lance bubbler 1A from 26.3 to $26.4 \mathrm{lpm}$ to maintain flow. \\
\hline & & 21:34 & 33.6 & $\begin{array}{c}\text { Average glass temperature is } 1153^{\circ} \mathrm{C} \text {. Plenum temperature is } 468-481^{\circ} \mathrm{C} \\
\text { Secondary current is } 1620 \mathrm{Amps} \text {. }\end{array}$ \\
\hline & & $21: 49$ & 33.8 & $\begin{array}{l}\text { Average glass temperature is } 1149^{\circ} \mathrm{C} \text {. Plenum temperature is } 467-469^{\circ} \mathrm{C} \text {. } \\
\text { Primary current is } 531 \text { Amps. Secondary current is } 1618 \text { Amps. Glass density is } \\
2.27 \mathrm{~g} / \mathrm{cc} \text {. }\end{array}$ \\
\hline & & 22:05 & 34.1 & $\begin{array}{l}\text { Average glass temperature is } 1153^{\circ} \mathrm{C} \text {. Plenum temperature is } 462-460^{\circ} \mathrm{C} \text {. } \\
\text { Primary current is } 532 \text { Amps. Secondary current is } 1626 \text { Amps. Glass density is } \\
2.27 \mathrm{~g} / \mathrm{cc} \text {. }\end{array}$ \\
\hline & & 22:19 & 34.3 & Average glass temperature is $1154^{\circ} \mathrm{C}$. Plenum temperature is $452-472^{\circ} \mathrm{C}$. \\
\hline & & 22:25 & 34.4 & Changed T4 from 24 to 23.5 seconds. \\
\hline & & 22:34 & 34.6 & $\begin{array}{l}\text { Average glass temperature is } 1153^{\circ} \mathrm{C} \text {. Plenum temperature is } 465-469^{\circ} \mathrm{C} \text {. } \\
\text { Primary current is } 531 \text { Amps. Glass density is } 2.26 \mathrm{~g} / \mathrm{cc} \text {. }\end{array}$ \\
\hline & & 22:49 & 34.8 & Average glass temperature is $1150^{\circ} \mathrm{C}$. Plenum temperature is $460-466^{\circ} \mathrm{C}$. \\
\hline & & 23:04 & 35.1 & $\begin{array}{l}\text { Average glass temperature is } 1149^{\circ} \mathrm{C} \text {. Plenum temperature is } 457-473^{\circ} \mathrm{C} \text {. } \\
\text { Primary current is } 527 \text { Amps. Glass resistance is } 0.084 \Omega \text {. }\end{array}$ \\
\hline & & 23:05 & 35.1 & Performed a film cooler rinse. \\
\hline & & 23:16 & 35.3 & Plenum temperature is $458-469^{\circ} \mathrm{C}$ \\
\hline & & 23:34 & 35.6 & $\begin{array}{l}\text { Average glass temperature is } 1145^{\circ} \mathrm{C} \text {. Plenum temperature is } 445-456^{\circ} \mathrm{C} \text {. } \\
\text { Primary current is } 530 \text { Amps. Secondary current is } 1613 \text { Amps. Glass resistance } \\
\text { is } 0.082 \Omega \text {. }\end{array}$ \\
\hline & & 23:49 & 35.8 & Plenum temperature is $437-441^{\circ} \mathrm{C}$. Secondary current is $1601 \mathrm{Amps}$. \\
\hline & \multirow{9}{*}{$2 / 8 / 13$} & 0:04 & 36.1 & Changed T4 from 23.5 to 23.0 seconds. \\
\hline & & 0:05 & 36.1 & $\begin{array}{l}\text { Average glass temperature is } 1153^{\circ} \mathrm{C} \text {. Plenum temperature is } 438-440^{\circ} \mathrm{C} \text {. } \\
\text { Primary current is } 534 \mathrm{Amps} \text {. Glass resistance is } 0.081 \Omega \text {. }\end{array}$ \\
\hline & & $0: 11$ & 36.2 & $\begin{array}{l}\text { Reduced power from } 213 \text { to } 211 \mathrm{~kW} \text {. Average glass temperature is } 1169^{\circ} \mathrm{C} \text {. } \\
\text { Primary current is } 532 \mathrm{Amps} \text {. Secondary current is } 1620 \mathrm{Amps} \text {. }\end{array}$ \\
\hline & & $0: 19$ & 36.3 & $\begin{array}{l}\text { Average glass temperature is } 1154^{\circ} \mathrm{C} \text {. Plenum temperature is } 436-447^{\circ} \mathrm{C} \text {. } \\
\text { Secondary current is } 1628 \mathrm{Amps} \text {. Glass resistance is } 0.080 \Omega \text {. }\end{array}$ \\
\hline & & $0: 34$ & 36.6 & Average glass temperature is $1156^{\circ} \mathrm{C}$. Plenum temperature is $434-447^{\circ} \mathrm{C}$. \\
\hline & & $0: 49$ & 36.8 & Plenum temperature is $436-446^{\circ} \mathrm{C}$. \\
\hline & & $1: 03$ & 37.0 & $\begin{array}{l}\text { Plenum temperature is } 434-444^{\circ} \mathrm{C} \text {. Investigating SBS overflow tank vent } \\
\text { pressure. Discovered that rubber hose between the overflow tank and the gauge } \\
\text { has water, cleared and reinstalled. All is satisfactory now. }\end{array}$ \\
\hline & & $1: 19$ & 37.3 & $\begin{array}{l}\text { Average glass temperature is } 1151^{\circ} \mathrm{C} \text {. Plenum temperature is } 433-453^{\circ} \mathrm{C} \text {. } \\
\text { Primary current is } 530 \mathrm{Amps} \text {. Glass resistance is } 0.082 \Omega \text {. }\end{array}$ \\
\hline & & $1: 32$ & 37.5 & Begin transfer of feed from mix tank to feed tank. \\
\hline
\end{tabular}


Table 3.2. Summary of Operational Events (continued).

\begin{tabular}{|c|c|c|c|c|}
\hline Test & Date & Time & $\begin{array}{l}\text { Run } \\
\text { Time } \\
\text { (hours) }\end{array}$ & Run time note \\
\hline \multirow{25}{*}{4} & \multirow{25}{*}{$2 / 8 / 13$} & $1: 30$ & 37.5 & $\begin{array}{l}\text { Transferred feed to feed tank. Starting tank mass is } 2731.5 \mathrm{~kg} \text {. Ending mass tank } \\
\text { is } 33.0 \mathrm{~kg} \text {. Net feed mass transferred is } 2698.5 \mathrm{~kg} \text {. Plus } 458.5 \mathrm{~kg} \text { water is added. } \\
\text { Total mass transferred is } 3157.0 \mathrm{~kg} \text {. }\end{array}$ \\
\hline & & $1: 36$ & 37.6 & $\begin{array}{c}\text { Plenum temperature is } 439-457^{\circ} \mathrm{C} \text {. Secondary current is } 1620 \mathrm{Amps} \text {, and glass } \\
\text { level is } 30.0 \mathrm{in} .\end{array}$ \\
\hline & & $1: 41$ & 37.7 & Changed T4 from 23.0 to 25.0 seconds. \\
\hline & & $1: 43$ & 37.7 & Secured mix tank mixer blade. Feed transfer to feed tank is completed. \\
\hline & & $1: 49$ & 37.8 & $\begin{array}{l}\text { Average glass temperature is } 1149^{\circ} \mathrm{C} \text {. Plenum temperature is } 450-464^{\circ} \mathrm{C} \text {. } \\
\text { Primary current is } 531 \text { Amps. }\end{array}$ \\
\hline & & $2: 04$ & 38.1 & $\begin{array}{l}\text { Average glass temperature is } 1151^{\circ} \mathrm{C} \text {. Plenum temperature is } 449-476^{\circ} \mathrm{C} \text {. } \\
\text { Secondary current is } 1616 \text { Amps. }\end{array}$ \\
\hline & & $2: 19$ & 38.3 & $\begin{array}{c}\text { Average glass temperature is } 1137^{\circ} \mathrm{C} \text {. Plenum temperature is } 443-478^{\circ} \mathrm{C} \text {. } \\
\text { Primary current is } 526 \text { Amps. Glass resistance is } 0.083 \Omega \text {. Glass density is } 2.27 \\
\mathrm{~g} / \mathrm{cc} \text {. }\end{array}$ \\
\hline & & $2: 24$ & 38.4 & Increased power from 211 to $214 \mathrm{~kW}$. Average glass temperature is $1140^{\circ} \mathrm{C}$. \\
\hline & & $2: 31$ & 38.5 & Plenum temperature is $443-471^{\circ} \mathrm{C}$. \\
\hline & & $2: 49$ & 38.8 & Plenum temperature is $442-460^{\circ} \mathrm{C}$. \\
\hline & & $3: 04$ & 39.1 & $\begin{array}{l}\text { Average glass temperature is } 1144^{\circ} \mathrm{C} \text {. Plenum temperature is } 439-447^{\circ} \mathrm{C} \text {. } \\
\text { Primary current is } 531 \text { Amps. Glass resistance is } 0.082 \Omega \text {. Glass density is } 2.29 \\
\text { g/cc. }\end{array}$ \\
\hline & & $3: 16$ & 39.3 & $\begin{array}{c}\text { Increased power from } 214 \text { to } 217 \mathrm{~kW} \text {. Average glass temperature is } 1145^{\circ} \mathrm{C} \text {. } \\
\text { Primary current is } 536 \text { Amps. }\end{array}$ \\
\hline & & $3: 19$ & 39.3 & $\begin{array}{l}\text { Average glass temperature is } 1148^{\circ} \mathrm{C} \text {. Plenum temperature is } 441-450^{\circ} \mathrm{C} \text {. } \\
\text { Secondary current is } 1629 \text { Amps. Glass resistance is } 0.082 \Omega \text {. }\end{array}$ \\
\hline & & $3: 34$ & 39.6 & $\begin{array}{l}\text { Average glass temperature is } 1148^{\circ} \mathrm{C} \text {. Plenum temperature is } 448-449^{\circ} \mathrm{C} \text {. } \\
\text { Secondary current is } 1619 \mathrm{Amps} \text {. Glass resistance is } 0.082 \Omega \text {. }\end{array}$ \\
\hline & & $3: 48$ & 39.8 & $\begin{array}{l}\text { Secured all bottom effluent blow down tanks. Neutralization tank has } 510 \\
\text { gallons, caustic tank has } 525 \text { gallons and acid tank has } 525 \text { gallons fluids. }\end{array}$ \\
\hline & & $3: 49$ & 39.8 & Plenum temperature is $443-450^{\circ} \mathrm{C}$. \\
\hline & & 4:04 & 40.1 & $\begin{array}{l}\text { Average glass temperature is } 1151^{\circ} \mathrm{C} \text {. Plenum temperature is } 451-459^{\circ} \mathrm{C} \text {. } \\
\text { Secondary current is } 1638 \mathrm{Amps} \text {, voltage is } 133 \text { volts. }\end{array}$ \\
\hline & & $4: 12$ & 40.2 & $\begin{array}{c}\text { During the last SBS blow down totalizer changed } 29.19 \text { gallons, actual liquid } \\
\text { taken out was } 40 \text { gallons. }\end{array}$ \\
\hline & & $4: 34$ & 40.6 & Average glass temperature is $1148^{\circ} \mathrm{C}$. Plenum temperature is $449-469^{\circ} \mathrm{C}$. \\
\hline & & $4: 38$ & 40.6 & Changed T4 from 25.0 to 25.5 seconds. \\
\hline & & $4: 50$ & 40.8 & $\begin{array}{l}4 \text { drums ( } \sim 220 \text { gallons) of liquid are removed from the neutralization tank. } \\
\text { Current bottom tank status: Acid tank (secured) has } 525 \text { gallons, caustic tank } \\
\text { (secured) has } 525 \text { gallons, and neutralization tank (in service) is } 410 \text { gallons. }\end{array}$ \\
\hline & & $5: 12$ & 41.2 & Plenum temperature is $456-468^{\circ} \mathrm{C}$. \\
\hline & & $5: 39$ & 41.7 & Plenum temperature is $470-477^{\circ} \mathrm{C}$. \\
\hline & & $5: 41$ & 41.7 & LabVIEW is stopped. Restarted. \\
\hline & & $5: 54$ & 41.9 & $\begin{array}{c}\text { Reduced power from } 217 \text { to } 214 \mathrm{~kW} \text {. Average glass temperature is } 1154^{\circ} \mathrm{C} \text {. } \\
\text { Primary current is } 540 \text { Amps. }\end{array}$ \\
\hline
\end{tabular}


Table 3.2. Summary of Operational Events (continued).

\begin{tabular}{|c|c|c|c|c|}
\hline Test & Date & Time & $\begin{array}{l}\text { Run } \\
\text { Time } \\
\text { (hours) }\end{array}$ & Run time note \\
\hline \multirow{26}{*}{4} & \multirow{26}{*}{$2 / 8 / 13$} & $6: 08$ & 42.1 & $\begin{array}{c}\text { Reduced power from } 214 \text { to } 211 \mathrm{~kW} \text {. Average glass temperature is } 1163^{\circ} \mathrm{C} \text {. } \\
\text { Primary current is } 533 \text { Amps. }\end{array}$ \\
\hline & & $6: 12$ & 42.2 & Plenum temperature is $471-491^{\circ} \mathrm{C}$. \\
\hline & & $6: 26$ & 42.4 & $\begin{array}{c}\text { Reduced power from } 211 \text { to } 208 \mathrm{~kW} \text {. Average glass temperature is } 1162^{\circ} \mathrm{C} \text {. } \\
\text { Primary current is } 528 \text { Amps. }\end{array}$ \\
\hline & & $6: 49$ & 42.8 & $\begin{array}{c}\text { Feed rate is } \sim 190 \mathrm{~kg} / \mathrm{hr} \text {. Power is } 210 \mathrm{~kW} \text {. Primary current is } 521 \text { Amps. } \\
\text { Secondary current is } 1593 \text { Amps. Voltage is } 132 \text { volts. Glass resistance is } 0.082 \\
\Omega .\end{array}$ \\
\hline & & $7: 04$ & 43.1 & $\begin{array}{c}\text { Feed rate is } \sim 190 \mathrm{~kg} / \mathrm{hr} \text {. Power is } 209 \mathrm{~kW} \text {. Primary current is } 524 \text { Amps. } \\
\text { Secondary current is } 1602 \text { Amps. Voltage is } 131 \text { volts. Glass resistance is } 0.081 \\
\Omega .\end{array}$ \\
\hline & & $7: 21$ & 43.4 & Average glass temperature is $1162^{\circ} \mathrm{C}$. Plenum temperature is $470-478^{\circ} \mathrm{C}$. \\
\hline & & $7: 35$ & 43.6 & Increased AOD pump pressure to $30 \mathrm{psi}$. \\
\hline & & $7: 40$ & 43.7 & Average glass temperature is $1157^{\circ} \mathrm{C}$. \\
\hline & & $7: 55$ & 43.9 & Average glass temperature is $1161^{\circ} \mathrm{C}$. Plenum temperature is $468-476^{\circ} \mathrm{C}$. \\
\hline & & $8: 10$ & 44.2 & $\begin{array}{l}\text { Feed rate is } \sim 200 \mathrm{~kg} / \mathrm{hr} \text {. Power is } 209 \mathrm{~kW} \text {. Primary current is } 523 \text { Amps. } \\
\text { Secondary current is } 1587 \text { Amps. Voltage is } 132 \text { volts. Average glass temperature } \\
1157^{\circ} \mathrm{C} \text {. }\end{array}$ \\
\hline & & $9: 10$ & 45.2 & Average glass temperature is $1163^{\circ} \mathrm{C}$ \\
\hline & & $9: 35$ & 45.6 & $\begin{array}{c}\text { Average glass temperature is } 1156^{\circ} \mathrm{C} \text {. Plenum temperature is } 464-476^{\circ} \mathrm{C} \text {. Feed } \\
\text { rate is } 195 \mathrm{~kg} / \mathrm{hr} \text {. }\end{array}$ \\
\hline & & 10:09 & 46.2 & $\begin{array}{l}\text { Average glass temperature is } 1161^{\circ} \mathrm{C} . \text { Plenum temperature is } 430-480^{\circ} \mathrm{C} . \text { Feed } \\
\text { rate is } 194 \mathrm{~kg} / \mathrm{hr} \text {. }\end{array}$ \\
\hline & & $10: 33$ & 46.5 & Paused feeding to collect feed sample. \\
\hline & & $10: 49$ & 46.8 & $\begin{array}{c}\text { Power is } 210 \mathrm{~kW} \text {. Primary current is } 528 \text { Amps. Secondary current is } 1617 \mathrm{Amps} \text {. } \\
\text { Voltage is } 130 \text { volts. Average glass temperature } 1159^{\circ} \mathrm{C} .\end{array}$ \\
\hline & & 11:04 & 47.1 & $\begin{array}{l}\text { Power is } 210 \mathrm{~kW} \text {. Primary current is } 530 \text { Amps. Secondary current is } 1622 \text { Amps. } \\
\text { Voltage is } 130 \text { volts. Average glass temperature } 1165^{\circ} \mathrm{C} \text {. Glass resistance is } \\
0.080 \Omega \text {. }\end{array}$ \\
\hline & & $11: 32$ & 47.5 & LabVIEW rebooted. \\
\hline & & 12:34 & 48.6 & Average glass temperature is $1162^{\circ} \mathrm{C}$. \\
\hline & & $12: 49$ & 48.8 & Average glass temperature is $1155^{\circ} \mathrm{C}$. Plenum temperature is $470-500^{\circ} \mathrm{C}$. \\
\hline & & 13:04 & 49.1 & Average glass temperature is $1158^{\circ} \mathrm{C}$. \\
\hline & & 13:19 & 49.3 & $\begin{array}{l}\text { Power is } 209 \mathrm{~kW} \text {. Primary current is } 520 \text { Amps. Secondary current is } 1589 \text { Amps. } \\
\text { Voltage is } 132 \text { volts. Average glass temperature } 1158^{\circ} \mathrm{C} \text {. Glass resistance is } \\
0.084 \Omega \text {. }\end{array}$ \\
\hline & & 13:49 & 49.8 & Average glass temperature is $1156^{\circ} \mathrm{C}$. \\
\hline & & 14:04 & 50.1 & Average glass temperature is $1153^{\circ} \mathrm{C}$. \\
\hline & & 14:19 & 50.3 & Average glass temperature is $1159^{\circ} \mathrm{C}$. \\
\hline & & $14: 38$ & 50.6 & $\begin{array}{c}\text { Average glass temperature is } 1159^{\circ} \mathrm{C} \text {. Primary current is } 525 \mathrm{Amps} \text {. Secondary } \\
\text { current is } 1608 \text { Amps. }\end{array}$ \\
\hline & & $14: 41$ & 50.7 & All effluent tanks are emptied. \\
\hline
\end{tabular}


Table 3.2. Summary of Operational Events (continued).

\begin{tabular}{|c|c|c|c|c|}
\hline Test & Date & Time & $\begin{array}{l}\text { Run } \\
\text { Time } \\
\text { (hours) }\end{array}$ & Run time note \\
\hline \multirow{24}{*}{4} & \multirow{24}{*}{$2 / 8 / 13$} & 14:58 & 51.0 & $\begin{array}{c}\text { Average glass temperature is } 1142^{\circ} \mathrm{C} \text {. Primary current is } 519 \text { Amps. Secondary } \\
\text { current is } 1598 \text { Amps. }\end{array}$ \\
\hline & & 15:00 & 51.0 & $\begin{array}{l}\text { Increased power from } 208 \text { to } 211 \mathrm{~kW} \text {. Average glass temperature is } 1142^{\circ} \mathrm{C} \text {. } \\
\text { Possible discharge caused temperatures to drop. Primary current is } 513 \mathrm{Amps} \text {. }\end{array}$ \\
\hline & & 15:04 & 51.1 & Average glass temperature is $1147^{\circ} \mathrm{C}$. Primary current is $520 \mathrm{Amps}$. \\
\hline & & 15:19 & 51.3 & $\begin{array}{l}\text { Average glass temperature is } 1155^{\circ} \mathrm{C} \text {. Plenum temperature is } 435-488^{\circ} \mathrm{C} \\
\text { Primary current is } 520 \mathrm{Amps} \text {. Secondary current is } 1582 \mathrm{Amps} .\end{array}$ \\
\hline & & $15: 30$ & 51.5 & LabVIEW rebooted. \\
\hline & & 15:34 & 51.6 & $\begin{array}{c}\text { Average glass temperature is } 1154^{\circ} \mathrm{C} \text {. Primary current is } 522 \mathrm{Amps} \text {. Secondary } \\
\text { current is } 1598 \mathrm{Amps} .\end{array}$ \\
\hline & & 15:49 & 51.8 & $\begin{array}{l}\text { Average glass temperature is } 1152^{\circ} \mathrm{C} \text {. Primary current is } 524 \text { Amps. Secondary } \\
\text { current is } 1598 \text { Amps. }\end{array}$ \\
\hline & & $16: 03$ & 52.0 & $\begin{array}{l}\text { Average glass temperature is } 1151^{\circ} \mathrm{C} \text {. Primary current is } 520 \text { Amps. Secondary } \\
\text { current is } 1569 \text { Amps. }\end{array}$ \\
\hline & & $16: 20$ & 52.3 & $\begin{array}{l}\text { Average glass temperature is } 1152^{\circ} \mathrm{C} \text {. Primary current is } 521 \text { Amps. Secondary } \\
\text { current is } 1582 \text { Amps. Changed T4 from } 25 \text { to } 25.3 \text { seconds. }\end{array}$ \\
\hline & & $16: 34$ & 52.6 & $\begin{array}{c}\text { Average glass temperature is } 1147^{\circ} \mathrm{C} \text {. Primary current is } 516 \text { Amps. Secondary } \\
\text { current is } 1562 \text { Amps. }\end{array}$ \\
\hline & & $16: 46$ & 52.8 & $\begin{array}{l}\text { Increased power from } 211 \text { to } 214 \mathrm{~kW} \text {. Primary current is } 521 \text { Amps. Secondary } \\
\text { current is } 1579 \text { Amps. Average glass temperature is } 1147^{\circ} \mathrm{C} \text {. Glass resistance is } \\
0.086 \Omega \text {. }\end{array}$ \\
\hline & & $16: 49$ & 52.8 & $\begin{array}{l}\text { Primary current is } 522 \text { Amps. Secondary current is } 1579 \text { Amps. Average glass } \\
\text { temperature is } 1150^{\circ} \mathrm{C} \text {. Glass resistance is } 0.085 \Omega \text {. Plenum temperature is } 394- \\
443^{\circ} \mathrm{C}\end{array}$ \\
\hline & & 17:02 & 53.0 & Stopped feeding slurry. End of Test 4. \\
\hline & & $17: 12$ & 53.2 & $\begin{array}{l}\text { Reduced power from } 214 \mathrm{~kW} \text { to } 200 \mathrm{~kW} \text {. Average glass temperature is } 1163^{\circ} \mathrm{C} \text {. } \\
\text { Primary current is } 518 \mathrm{Amps} \text {. Secondary current is } 1590 \mathrm{Amps} \text {. }\end{array}$ \\
\hline & & $17: 15$ & 53.3 & $\begin{array}{l}\text { Reduced power from } 200 \text { to } 190 \mathrm{~kW} \text {. Average glass temperature is } 1167^{\circ} \mathrm{C} \text {. } \\
\text { Primary current is } 506 \text { Amps. Secondary current is } 1557 \mathrm{Amps} \text {. }\end{array}$ \\
\hline & & 17:20 & 53.3 & $\begin{array}{l}\text { Reduced power from } 190 \text { to } 180 \mathrm{~kW} \text {. Average glass temperature is } 1170^{\circ} \mathrm{C} \text {. } \\
\text { Primary current is } 494 \text { Amps. Secondary current is } 1531 \text { Amps. }\end{array}$ \\
\hline & & $17: 26$ & 53.4 & Average glass temperature is $1171^{\circ} \mathrm{C}$. Reduced power from 180 to $150 \mathrm{~kW}$. \\
\hline & & $17: 34$ & 53.6 & $\begin{array}{l}\text { Removed feed from the feed tank. Starting tank mass is } 784.0 \mathrm{~kg} \text {. Ending tank } \\
\text { mass is } 16.0 \mathrm{~kg} \text {. Net mass of feed removed is } 768.0 \mathrm{~kg} \text {. }\end{array}$ \\
\hline & & $18: 26$ & 54.4 & $\begin{array}{l}\text { Reduced bubbling as follows: Lance } 1 \mathrm{~A} \text { from } 26.4 \text { to } 15.4 \mathrm{lpm} \text {, Lance } 2 \mathrm{~A} \text { from } \\
24.9 \text { to } 14.9 \mathrm{lpm} \text {, Lance L3A from } 13.1 \text { to } 8.1 \mathrm{lpm} \text {, Lance } 4 \mathrm{~A} \text { from } 13.1 \text { to } 8.1 \\
\text { lpm. }\end{array}$ \\
\hline & & 18:29 & 54.5 & Reduced power from 150 to $120 \mathrm{~kW}$. Average glass temperature is $1160^{\circ} \mathrm{C}$. \\
\hline & & 18:39 & 54.7 & $\begin{array}{l}\text { Reduced bubbling as follows: Lance } 1 \mathrm{~A} \text { from } 15.4 \text { to } 8.4 \mathrm{lpm} \text {, Lance } 2 \mathrm{~A} \text { from } \\
14.9 \text { to } 8.9 \mathrm{lpm} \text {, Lance L3A from } 8.1 \text { to } 5.1 \mathrm{lpm} \text {, Lance } 4 \mathrm{~A} \text { from } 8.1 \text { to } 5.1 \mathrm{lpm} \text {. }\end{array}$ \\
\hline & & $18: 47$ & 54.8 & Reduced power from 120 to $100 \mathrm{~kW}$. Average glass temperature is $1162^{\circ} \mathrm{C}$. \\
\hline & & 19:02 & 55.0 & Reduced power from 100 to $90 \mathrm{~kW}$. Average glass temperature is $1162^{\circ} \mathrm{C}$. \\
\hline & & 19:27 & 55.5 & Cold cap is gone. \\
\hline
\end{tabular}




\section{Table 3.2. Summary of Operational Events (continued).}

\begin{tabular}{|c|c|c|c|c|}
\hline \multirow{2}{*}{ Test } & Date & Time & $\begin{array}{c}\text { Run } \\
\text { Time } \\
\text { (hours) }\end{array}$ & Run time note \\
\hline \multirow{4}{*}{4} & \multirow{3}{*}{$2 / 8 / 13$} & $19: 33$ & 55.5 & Reduced bubbling in all Lances to $1.5 \mathrm{lpm}$ and turned on the bypass rotameters. \\
\cline { 3 - 5 } & & $20: 30$ & 56.5 & All 4 set to $0.5 \mathrm{scfh}$. \\
\cline { 3 - 5 } & & $20: 58$ & 57.0 & Reduced power from 90 to $80 \mathrm{~kW}$. Average glass temperature is $1149^{\circ} \mathrm{C}$. \\
\cline { 3 - 5 } & & $21: 05$ & 57.1 & Performed WESP blow down. Performed deluge and post-deluge blow down. \\
\cline { 3 - 5 } & & $21: 45$ & 57.8 & Melter and off gas shutdown are completed. \\
\hline
\end{tabular}


Table 3.3. Cold Cap Observations.

\begin{tabular}{|c|c|c|c|c|}
\hline Test & Date & Time & $\begin{array}{l}\text { Run } \\
\text { Time } \\
\text { (hours) }\end{array}$ & Cold Cap Observations \\
\hline \multirow{33}{*}{1} & \multirow{33}{*}{$1 / 15 / 13$} & $10: 05$ & 0.0 & Start water feeding at $0.5 \mathrm{liter} / \mathrm{min}$. \\
\hline & & $10: 25$ & 0.3 & Water flow rate was raised to 1.0 liter $/ \mathrm{min}$. \\
\hline & & $10: 45$ & 0.7 & Water flow rate was raised to 2.0 liter $/ \mathrm{min}$. \\
\hline & & $11: 25$ & 1.3 & Cold cap is slightly foamy. Water flow rate was raised to 2.5 liter/min \\
\hline & & $11: 43$ & 1.6 & Water flow rate was raised to 3.0 liter $/ \mathrm{min}$. \\
\hline & & $11: 50$ & 1.7 & Secured feeding water. Started feeding HWI-Al-19 feed. \\
\hline & & $12: 20$ & 2.2 & $\sim 50 \%$, large openings on east and west sides. \\
\hline & & $12: 35$ & 2.5 & $\sim 60 \%$, openings on north east and south west corners. \\
\hline & & $12: 55$ & 2.8 & $\sim 70 \%$, openings on north east and south west corners. \\
\hline & & $13: 20$ & 3.2 & $\begin{array}{c}\sim 80 \% \text {, openings on north east and south west corners. CC is } \sim 3 \text { ” thick at } \\
\text { openings. Feed is boiling on surface. }\end{array}$ \\
\hline & & $13: 35$ & 3.5 & $\sim 80-85 \%$, openings on east and west sides with boiling feed on top of CC. \\
\hline & & $13: 50$ & 3.7 & $\begin{array}{l}\sim 85 \% \text {, openings are still visible on east and west sides. Feed is flowing into melt } \\
\text { pool. }\end{array}$ \\
\hline & & 14:05 & 4.0 & $\sim 85 \%$, same as last reading. \\
\hline & & $14: 20$ & 4.2 & $\sim 85 \%$, flat, openings on east and west sides. \\
\hline & & $14: 35$ & 4.5 & $\sim 85 \%$, flat and soft, visible openings and east and west sides. \\
\hline & & $14: 52$ & 4.8 & $\sim 90 \%$, east side opening is larger than the west side. CC is floating. \\
\hline & & $15: 15$ & 5.2 & $\begin{array}{c}\sim 90 \% \text {, openings on north west and south east corners. South east opening is } \\
\text { larger. Feed is boiling on surface between openings. }\end{array}$ \\
\hline & & $15: 32$ & 5.4 & $\sim 95 \%$, both openings are closing up with ridges. \\
\hline & & $15: 52$ & 5.8 & $\sim 95 \%$, cone shaped openings with thick CC is visible. \\
\hline & & 16:04 & 6.0 & $\sim 95 \%$, is about $4 "$ thick and flat. \\
\hline & & $16: 20$ & 6.2 & $\sim 90 \%$, the ridges are slightly lower than last observation. \\
\hline & & $16: 42$ & 6.6 & $\sim 90 \%$, very little to no change in $\mathrm{CC}$ conditions. \\
\hline & & $17: 18$ & 7.2 & $\sim 95 \%$, mounding around bubblers, pooling feed in the center. \\
\hline & & $17: 41$ & 7.6 & $\sim 98 \%$. \\
\hline & & 18:03 & 8.0 & $\sim 95 \%$. \\
\hline & & $18: 21$ & 8.3 & $\sim 95 \%$. \\
\hline & & $18: 40$ & 8.6 & $\begin{array}{l}\sim 95 \% \text {, lots of glass wool hanging near the north view port. Appears as two } \\
\text { volcanoes with lots of molten glass splashing over the entire plenum area. }\end{array}$ \\
\hline & & 19:02 & 9.0 & $\sim 95 \%$. CC is closing up but held open by bubblers. Still lots of splattered glass. \\
\hline & & 19:18 & 9.2 & $\begin{array}{l}\sim 95 \% \text {, large pool of feed liquid is visible in center. CC has shifted and middle } \\
\text { has started to reopen. }\end{array}$ \\
\hline & & 19:47 & 9.7 & $\sim 95 \%$, very foggy in plenum, large pooling and less spitting of molten glass. \\
\hline & & $20: 23$ & 10.3 & $\sim 95 \%$, not be able to see east side due to large ridge blocking. \\
\hline & & 20:40 & 10.6 & $\begin{array}{l}\sim 95 \% \text {, starting to see east side since glass discharge. Both openings still have } \\
\text { large ridges. }\end{array}$ \\
\hline & & 21:05 & 11.0 & $\sim 98 \%$, dark inside, spitting glass out like spider webs. \\
\hline
\end{tabular}


Table 3.3. Cold Cap Observations (continued).

\begin{tabular}{|c|c|c|c|c|}
\hline Test & Date & Time & $\begin{array}{c}\text { Run } \\
\text { Time } \\
\text { (hours) }\end{array}$ & Cold Cap Observations \\
\hline \multirow{29}{*}{1} & \multirow{12}{*}{$1 / 15 / 13$} & $21: 27$ & 11.4 & $\sim 90 \%$, flat, spider web look. \\
\hline & & $21: 37$ & 11.5 & $\sim 90 \%$, flat, two openings, spider web look is gone. \\
\hline & & $21: 52$ & 11.8 & $\sim 95 \%$, flat, $5 "$ thick one opening. \\
\hline & & 22:02 & 11.9 & $\sim 90 \%$. \\
\hline & & $22: 22$ & 12.3 & $\sim 90 \%$, flat. \\
\hline & & $22: 34$ & 12.5 & $\sim 90 \%$, two openings. \\
\hline & & 22:48 & 12.7 & $\sim 90 \%$, openings on east and west sides. Feed is boiling on surface. \\
\hline & & 23:00 & 12.9 & $\begin{array}{c}\sim 90 \% \text { with two openings visible from north view port. Slight ridges around } \\
\text { each opening. Bubbling on the surface exists. }\end{array}$ \\
\hline & & 23:16 & 13.2 & $\begin{array}{c}\sim 90 \% \text {, ridges around openings are starting to become more pronounced. Liquid } \\
\text { on the flat surface is boiling. }\end{array}$ \\
\hline & & $23: 38$ & 13.5 & $\sim 90$, two openings, east side more open than west side. \\
\hline & & $23: 53$ & 13.8 & CC collapses. Low pressure alarm. \\
\hline & & 23:55 & 13.8 & $\begin{array}{l}\text { Reduced bubbling from } 17.1 \mathrm{lpm} \text { to } 15.0 \mathrm{lpm} \text { on each bubbler attempting to } \\
\text { reduce ridges along CC and to increase openings. }\end{array}$ \\
\hline & \multirow{17}{*}{$1 / 16 / 13$} & 0:05 & 14.0 & $\sim 95 \%$, two openings on east and west, boiling liquid on surface. \\
\hline & & $0: 13$ & 14.1 & $\sim 95 \%$, east opening is not visible. \\
\hline & & $0: 31$ & 14.4 & $\sim 95 \%$, west side is slightly open, east side is open liquid on top of CC. \\
\hline & & $0: 40$ & 14.6 & East side opening is not visible and west side opening is increasing. \\
\hline & & 1:02 & 14.9 & $\sim 90 \%$, east opening is visible and west side opening expanding. \\
\hline & & $1: 28$ & 15.4 & $\sim 95 \%$, covered some liquid on top of CC. \\
\hline & & $1: 41$ & 15.6 & $\begin{array}{l}\sim 85-90 \%, \text { both openings are surrounded by ridges. Liquid boiling vigorously } \\
\text { on the surface. }\end{array}$ \\
\hline & & $1: 55$ & 15.8 & $\begin{array}{l}\sim 95 \% \text {, covered with thick wet surface, slight splashing. Opening on north west } \\
\text { side. }\end{array}$ \\
\hline & & $2: 15$ & 16.2 & $\sim 90 \%$, ridge at the opening persists. Vigorous liquid boiling on the surface. \\
\hline & & $2: 43$ & 16.6 & East and west sides are open, boiling liquid on top of CC. \\
\hline & & 3:01 & 16.9 & $\begin{array}{c}\text { Both east and west sides are open, ridge is visible after glass discharge. Boiling } \\
\text { liquid on top of CC. }\end{array}$ \\
\hline & & $3: 19$ & 17.2 & $\sim 85 \%$, opening on east and west sides, ridge exists behind east side. \\
\hline & & $3: 32$ & 17.4 & $\begin{array}{l}\sim 80-85 \% \text {, ridges at the opening starting to dissolve. Observed liquid rushing to } \\
\text { the opening. Similarly the east opening is visibly available. }\end{array}$ \\
\hline & & $3: 50$ & 17.7 & $\sim 80-85 \%$ \\
\hline & & $4: 11$ & 18.1 & $\begin{array}{l}\sim 85 \text {, openings of east and west sides and a small ridge around opening. Lots of } \\
\text { feed boiling on surface. }\end{array}$ \\
\hline & & $4: 24$ & 18.3 & $\begin{array}{c}\sim 85 \% \text {, evenly flat, ridges almost non-existent. Boiling on the surface, both } \\
\text { openings are visible. }\end{array}$ \\
\hline & & $5: 08$ & 19.0 & $\sim 85 \%$, openings on east and west sides. \\
\hline
\end{tabular}


Table 3.3. Cold Cap Observations (continued).

\begin{tabular}{|c|c|c|c|c|}
\hline Test & Date & Time & $\begin{array}{c}\text { Run } \\
\text { Time } \\
\text { (hours) }\end{array}$ & Cold Cap Observations \\
\hline \multirow{28}{*}{1} & \multirow{28}{*}{$1 / 16 / 13$} & $5: 19$ & 19.2 & $\sim 85 \%$, similar to last observation. \\
\hline & & $5: 34$ & 19.5 & $! 85 \%$, heavily boiling on flat surface. \\
\hline & & $5: 35$ & 19.5 & $\begin{array}{l}\text { Build up from feed spray on the instrument port observed, will continue to } \\
\text { monitor. No impact on data and feed shots. }\end{array}$ \\
\hline & & $5: 48$ & 19.7 & $\begin{array}{l}\sim 85 \% \text {, ridge building up on west side opening. Feed on surface is pooling and } \\
\text { boiling. }\end{array}$ \\
\hline & & 6:04 & 20.0 & $\sim 85 \%$, ridge on west side opening. Feed is flowing into east opening. \\
\hline & & $6: 19$ & 20.2 & $\begin{array}{l}\sim 80-85 \% \text {, still ridge on west side opening. East side opening has feed flowing } \\
\text { into it. }\end{array}$ \\
\hline & & $8: 20$ & 22.2 & $\begin{array}{c}\sim 85-90 \% \text {, openings are on east and west sides with slight mounds around each. } \\
\text { Boiling feed on surface. CC is moving. }\end{array}$ \\
\hline & & $8: 40$ & 22.6 & $\sim 85-90 \%$, conditions are the same as before. \\
\hline & & 9:00 & 22.9 & $\sim 85-90 \%$, conditions are unchanged from last observation. \\
\hline & & $9: 51$ & 23.8 & $\begin{array}{c}\sim 75-80 \% \text {, conditions unchanged from last observation. Openings are increased } \\
\text { due to drop of feed rate. }\end{array}$ \\
\hline & & 10:05 & 24.0 & $\begin{array}{c}\sim 85 \% \text {, looks flat with boiling feed on top. Openings on east and west sides are } \\
\text { visible. }\end{array}$ \\
\hline & & 10:20 & 24.2 & $\sim 85 \%$, openings on east and west sides are visible with feed boiling on top of CC. \\
\hline & & $10: 35$ & 24.5 & $\sim 85 \%$, unchanged since last reading. \\
\hline & & 10:50 & 24.7 & $\sim 85 \%$, unchanged since last reading. \\
\hline & & 11:05 & 25.0 & $\sim 85 \%$, openings on east and west sides are visible with feed flowing into opening. \\
\hline & & 11:49 & 25.7 & $\sim 85 \%$, openings on east and west sides are still visible. \\
\hline & & $12: 25$ & 26.3 & $\begin{array}{c}\sim 85-90 \% \text {, openings in north east and south west corners. CC is fairly flat with } \\
\text { feed boiling on surface. }\end{array}$ \\
\hline & & $12: 40$ & 26.6 & $\sim 85-90 \%$, conditions are unchanged from previous observation. \\
\hline & & 13:00 & 26.9 & $\begin{array}{c}\sim 85-90 \% \text {, openings in north east and south west corners. Slight ridge around both } \\
\text { openings. Feed is boiling on surface. }\end{array}$ \\
\hline & & 13:20 & 27.2 & $\sim 85-90 \%$, conditions are unchanged from previous observation. \\
\hline & & 13:40 & 27.6 & $\sim 85-90 \%$, conditions are unchanged from previous observation. \\
\hline & & $14: 23$ & 28.3 & $\sim 85-90 \%$, conditions are unchanged from previous observation. \\
\hline & & $14: 41$ & 28.6 & $\sim 90 \%$, two openings are flat on top. \\
\hline & & 15:05 & 29.0 & $\sim 85 \%, \mathrm{CC}$ opened up some; liquid feed is boiling. \\
\hline & & $15: 26$ & 29.3 & $\sim 85 \%$, conditions are unchanged from previous observation. \\
\hline & & 15:49 & 29.7 & $\sim 80 \%$, two large openings. \\
\hline & & $15: 58$ & 29.9 & $\sim 80 \%$, CC has been slowly opening up. \\
\hline & & 16:09 & 30.1 & $\sim 80 \%, \mathrm{CC}$ has thinned out some and ridges are smaller around openings. \\
\hline
\end{tabular}


Table 3.3. Cold Cap Observations (continued).

\begin{tabular}{|c|c|c|c|c|}
\hline Test & Date & Time & $\begin{array}{c}\text { Run } \\
\text { Time } \\
\text { (hours) }\end{array}$ & Cold Cap Observations \\
\hline \multirow{32}{*}{1} & \multirow{24}{*}{$1 / 16 / 13$} & $16: 19$ & 30.2 & $\sim 80 \%$ and $5 "$ thick and flat. \\
\hline & & $16: 34$ & 30.5 & $\sim 80 \%$, starting to close up somewhat. More feed is flowing into openings. \\
\hline & & $16: 49$ & 30.7 & $\sim 80 \%$, very little to no change for this observation. \\
\hline & & $17: 04$ & 31.0 & $\sim 80 \%, 5 \%$ thick and flat. \\
\hline & & $17: 22$ & 31.3 & $\sim 80 \%$, still the same. \\
\hline & & $17: 35$ & 31.5 & $\sim 80 \%$, still the same. \\
\hline & & $17: 49$ & 31.7 & $80 \%$, two openings with a flat surface. \\
\hline & & $18: 11$ & 32.1 & $\sim 80 \%$, flat, openings are in north west and south east corners. \\
\hline & & $18: 28$ & 32.4 & $\sim 85 \%$, flat two openings. Feed pooling on top. \\
\hline & & $18: 37$ & 32.5 & $\sim 80 \%$, same as above. \\
\hline & & $18: 52$ & 32.8 & $\sim 80 \%$, flat, two openings, feed is pooling on top of CC and boiling. \\
\hline & & 19:09 & 33.1 & $\begin{array}{c}\sim 85 \%, \text { CC has closed up slightly, CC is mostly level. The openings have feed } \\
\text { flowing in. }\end{array}$ \\
\hline & & $19: 45$ & 33.7 & $\sim 80 \%$, same as above. \\
\hline & & $20: 12$ & 34.1 & $\sim 80 \%$, ridges have grown somewhat. \\
\hline & & $20: 27$ & 34.4 & $\begin{array}{l}\sim 80 \% \text {, west side opening has shifted slightly to the north, closer to thermo well } \\
\qquad \# 2 \text {. }\end{array}$ \\
\hline & & $20: 53$ & 34.8 & $\sim 80 \%, \mathrm{CC}$ is thinner than last observation. \\
\hline & & 21:09 & 35.1 & $\begin{array}{l}\sim 80 \% \text {, east side has thinned out. Most of the liquid feed is flowing into the west } \\
\text { side opening. }\end{array}$ \\
\hline & & $21: 38$ & 35.5 & $\sim 60-65 \%$, east and west sides are wide open. \\
\hline & & 21:54 & 35.8 & $\begin{array}{l}\sim 70 \% \text {, east side is closing faster than the west side. Boiling liquid seen on top of } \\
\text { CC. }\end{array}$ \\
\hline & & $22: 26$ & 36.3 & $\begin{array}{l}\sim 75 \% \text {, east side opening migrates south and west side opening is receiving boiling } \\
\text { liquid. }\end{array}$ \\
\hline & & $22: 43$ & 36.6 & $\sim 80 \%$, east side closing up and west side still open. \\
\hline & & 23:03 & 37.0 & $\sim 80 \%$, east side opening migrates south, west side receives boiling liquid. \\
\hline & & 23:18 & 37.2 & $80 \%$, east side is open, west side is open. \\
\hline & & $23: 41$ & 37.6 & $\begin{array}{c}\sim 80 \% \text {, east side opening is larger than west side opening. Feed is flowing into } \\
\text { both openings. }\end{array}$ \\
\hline & \multirow{8}{*}{$1 / 17 / 13$} & 0:05 & 38.0 & $\sim 80 \%$, east side has soft cap, west side open and has feed flowing into it. \\
\hline & & $0: 19$ & 38.2 & $\sim 80 \%$, east side opening has hardened up, west side open with feed flowing in it. \\
\hline & & $0: 44$ & 38.6 & $\sim 85 \%$, east side is closing up and west side opening receiving liquid flow. \\
\hline & & 1:02 & 38.9 & $\sim 70 \%$. \\
\hline & & 1:04 & 39.0 & $\sim 70 \%$. \\
\hline & & $1: 19$ & 39.2 & $80 \%$, East portion of CC is floating, liquid flows to all openings. \\
\hline & & $1: 31$ & 39.4 & $80 \%$, less flexing in CC. \\
\hline & & $1: 42$ & 39.6 & $\sim 80 \%$, no changes from the previous observation. \\
\hline
\end{tabular}


Table 3.3. Cold Cap Observations (continued).

\begin{tabular}{|c|c|c|c|c|}
\hline Test & Date & Time & $\begin{array}{c}\text { Run } \\
\text { Time } \\
\text { (hours) }\end{array}$ & Cold Cap Observations \\
\hline \multirow{32}{*}{1} & \multirow{32}{*}{$1 / 17 / 13$} & $1: 52$ & 39.8 & $\sim 80 \%$, maintaining similar conditions. \\
\hline & & $2: 10$ & 40.1 & $85 \%$, east side opening is showing a ridge build up. \\
\hline & & $2: 37$ & 40.5 & $\sim 85 \%$, east side is steady, west side migrates south. \\
\hline & & $2: 51$ & 40.8 & $\begin{array}{c}\sim 80-85 \% \text {, west side opening much is greater than east opening, ridges are present } \\
\text { around the openings. }\end{array}$ \\
\hline & & 3:09 & 41.1 & $\sim 90 \%$, ridge on the east opening. \\
\hline & & $3: 25$ & 41.3 & $\begin{array}{c}\sim 90 \% \text {, similar to last observation. North east north west openings and ridge appear } \\
\text { the same with some boiling feed. }\end{array}$ \\
\hline & & $3: 41$ & 41.6 & $\sim 85 \%$, reduced bubbling on Lances $1 \mathrm{~A}, 2 \mathrm{~A}, 1 \mathrm{~B}$ and $2 \mathrm{~B}$ from $12 \mathrm{lpm}$ to $11 \mathrm{lpm}$. \\
\hline & & $3: 42$ & 41.6 & $\begin{array}{c}\sim 85 \% \text {, remains similar to previous observation, ridge reduced slightly and surface } \\
\text { appears wetter. }\end{array}$ \\
\hline & & 4:05 & 42.0 & $\sim 85 \%$, remains open on north east and north west and CC level is rising. \\
\hline & & $4: 22$ & 42.3 & $\sim 85 \%$, east side of CC holds boiling liquid, west side open. \\
\hline & & $4: 32$ & 42.4 & $\sim 90 \%$, east side appears to have closed. \\
\hline & & $4: 47$ & 42.7 & $\begin{array}{l}\sim 85 \% \text {, east side still seems closed west side still open. West center viewport nearly } \\
\text { shut and little build up upon exposed plenum. }\end{array}$ \\
\hline & & $5: 10$ & 43.1 & $\begin{array}{c}\sim 85-90 \% \text {, no major change except CC level is rising and appears wetter with } \\
\text { boiling feed. }\end{array}$ \\
\hline & & $5: 44$ & 43.6 & $\sim 90 \%$, one opening on west side, wet feed on surface. \\
\hline & & $5: 44$ & 43.6 & $\sim 90 \%$, one opening on west side, wet feed on the surface. \\
\hline & & $7: 06$ & 45.0 & $\sim 90 \%$, opening on northwest side, light is visible on south east side. \\
\hline & & $7: 21$ & 45.3 & $\begin{array}{c}\sim 90-95 \% \text {, opening on west side, light is visible on east side. CC is thick with feed } \\
\text { boiling on surface. }\end{array}$ \\
\hline & & $7: 36$ & 45.5 & $\sim 90-95 \%$, conditions unchanged from previous observation. \\
\hline & & $7: 51$ & 45.8 & $\sim 95 \%$, conditions unchanged from previous observation. \\
\hline & & 8:07 & 46.0 & $\sim 95 \%$, conditions are unchanged from the previous observation. \\
\hline & & $8: 21$ & 46.3 & $\begin{array}{c}\sim 95 \% \text {, opening on west side, light is visible on east side, ridge exists around } \\
\text { opening on west side. Feed is boiling on the surface. }\end{array}$ \\
\hline & & $8: 36$ & 46.5 & $\sim 95 \%$, opening on west side, light is visible on east side, feed is boiling on surface. \\
\hline & & $8: 51$ & 46.8 & $\sim 95 \%$, unchanged from previous observation, some light is visible on east. \\
\hline & & 9:08 & 47.0 & $\sim 95 \%$ with opening on west side, feed is boiling on surface with ridge at opening. \\
\hline & & $9: 31$ & 47.4 & $\sim 95 \%$, no change from previous observation. \\
\hline & & 9:46 & 47.7 & $\sim 95 \%$, opening on west mid side, light is visible on south east corner. \\
\hline & & 10:07 & 48.0 & $\sim 95 \%$, conditions are unchanged from previous condition. \\
\hline & & $10: 26$ & 48.3 & $\sim 95 \%$, conditions are unchanged from previous condition. \\
\hline & & $10: 53$ & 48.8 & $\sim 95 \%$, conditions are unchanged from previous condition. \\
\hline & & 11:04 & 49.0 & $\sim 95 \%$, conditions are unchanged from previous condition. \\
\hline & & $11: 19$ & 49.2 & $\sim 95 \%$ with feed pooled on top, light is visible in east view port. \\
\hline & & $11: 34$ & 49.5 & $\sim 95 \%$ with no change from previous conditions. \\
\hline
\end{tabular}


Table 3.3. Cold Cap Observations (continued).

\begin{tabular}{|c|c|c|c|c|}
\hline Test & Date & Time & $\begin{array}{c}\text { Run } \\
\text { Time } \\
\text { (hours) }\end{array}$ & Cold Cap Observations \\
\hline \multirow{5}{*}{1} & \multirow{5}{*}{$1 / 17 / 13$} & $11: 46$ & 49.7 & $\sim 95 \%$, flat, east side is closed west side is open. \\
\hline & & 12:04 & 50.0 & $\begin{array}{c}\sim 95 \% \text {, is no change from last observation, very faint light is visible in east view } \\
\text { port, }\end{array}$ \\
\hline & & 12:19 & 50.2 & $\sim 94 \%$, no change in east view port. \\
\hline & & $12: 30$ & 50.4 & Secured feeding, End of the test. No longer able to maintain feeding. \\
\hline & & $15: 30$ & 53.4 & $\mathrm{CC}$ is gone. \\
\hline \multirow{29}{*}{2} & \multirow{29}{*}{$1 / 22 / 13$} & $14: 00$ & 0.0 & Started feeding water at $0.7 \mathrm{l} / \mathrm{min}$. \\
\hline & & $14: 15$ & 0.3 & Increased water flow rate to $1.4 \mathrm{l} / \mathrm{min}$. \\
\hline & & $14: 42$ & 0.7 & Increased water flow rate to $2.8 \mathrm{l} / \mathrm{min}$. \\
\hline & & 15:00 & 1.0 & Secured water flow and starting feeding slurry. \\
\hline & & $15: 27$ & 1.5 & $\sim 60 \%$, mostly a hot cap. \\
\hline & & 15:44 & 1.7 & $\sim 75 \%$, thin and mostly liquid boiling on surface. \\
\hline & & 16:03 & 2.1 & $\sim 85 \%$, with openings and feed boiling on flat surface. \\
\hline & & $16: 23$ & 2.4 & $\sim 90 \%, 2$ openings, feed is boiling on surface. \\
\hline & & $16: 49$ & 2.8 & $\sim 80 \%$, flat two openings exist. \\
\hline & & $17: 12$ & 3.2 & $\sim 75 \%$, flat three openings exist. \\
\hline & & $17: 24$ & 3.4 & $\sim 80 \%$, very thick shelf. \\
\hline & & $17: 38$ & 3.6 & $\sim 80 \%, 4$ " thick shelf and flat. \\
\hline & & $17: 58$ & 4.0 & $\sim 80 \%$, same as above. \\
\hline & & 18:05 & 4.1 & $\begin{array}{l}\sim 85 \% \text {, very strong boiling below feed tube, bubbler ports open and reveal a } \\
\text { thick cap. }\end{array}$ \\
\hline & & $18: 19$ & 4.3 & $\begin{array}{l}\sim 85 \% \text {, bubbler below feed tube is starting to open. Vigorous bubbling and very } \\
\text { wet cap in places. }\end{array}$ \\
\hline & & $18: 33$ & 4.6 & $\sim 85 \%$, glass is splashing over edge of bubbler holes, very vigorous splashing. \\
\hline & & $18: 50$ & 4.8 & $\sim 80 \%$, opening closest to west wall has reopened. \\
\hline & & 19:04 & 5.1 & $\sim 80 \%$, same as above. \\
\hline & & 19:26 & 5.4 & $\sim 80 \%, \mathrm{CC}$ is thin but has ridges around the openings \\
\hline & & 19:34 & 5.6 & $\begin{array}{c}\sim 75 \%, \text { CC has opened up due to taking feed sample. The ridges are gone and } \\
\text { feed is flowing into the opening. }\end{array}$ \\
\hline & & 19:48 & 5.8 & $\begin{array}{l}\sim 75 \% \text {, openings at north east and southwest corners, feed is boiling on the } \\
\text { surface between the openings, feed is flowing into the openings. }\end{array}$ \\
\hline & & 20:20 & 6.3 & $\begin{array}{c}\sim 80 \% \text {, feed is boiling on surface, flowing into north east and southwest corner } \\
\text { openings. }\end{array}$ \\
\hline & & $20: 35$ & 6.6 & $\sim 80 \%$, there is more liquid feed on $\mathrm{CC}$. \\
\hline & & $20: 55$ & 6.9 & $\sim 80 \%$, one small and one large opening exist, feed is boiling on surface. \\
\hline & & 21:09 & 7.2 & $\sim 80 \%$, same as above. \\
\hline & & $21: 24$ & 7.4 & $\sim 80 \%$, feed is boiling on top of surface. \\
\hline & & $21: 49$ & 7.8 & $\sim 80 \%$, east and west sides are open, migrating north. \\
\hline & & $21: 54$ & 7.9 & Emergency off gas alarm is tripped, possibly due to CC circumstance. \\
\hline & & 22:04 & 8.1 & $\begin{array}{l}\sim 85 \% \text {, east side opening closing, west side maintains boiling and flowing } \\
\text { liquid. }\end{array}$ \\
\hline
\end{tabular}


Table 3.3. Cold Cap Observations (continued).

\begin{tabular}{|c|c|c|c|c|}
\hline Test & Date & Time & $\begin{array}{l}\text { Run } \\
\text { Time } \\
\text { (hours) }\end{array}$ & Cold Cap Observations \\
\hline & \multirow{5}{*}{$1 / 22 / 13$} & 22:36 & 8.6 & $\sim 80 \%$, same as above. \\
\hline & & 22:58 & 9.0 & $\begin{array}{l}\sim 75-80 \%, \text { north east and north west corners both have lots of boiling feed on } \\
\text { surface with openings. }\end{array}$ \\
\hline & & 23:11 & 9.2 & $\begin{array}{l}\sim 85 \% \text {, openings are on east and west sides, both openings have small ridges built } \\
\text { around them. Wet feed is boiling on surface. }\end{array}$ \\
\hline & & 23:26 & 9.4 & $\begin{array}{c}\sim 85 \% \text {, heavy boiling is observed, } \mathrm{CC} \text { is evenly flat, east and west openings are } \\
\text { visible. }\end{array}$ \\
\hline & & $23: 41$ & 9.7 & $\sim 85 \%$, openings on west and east sides. \\
\hline & \multirow{22}{*}{$1 / 23 / 13$} & 0:05 & 10.1 & $\sim 80 \%$, ridges on east and west sides. Boiling liquid is flowing north. \\
\hline & & $0: 21$ & 10.4 & $\sim 80 \%$, ridge on west side dissipates. Liquid flows to the west side opening. \\
\hline & & $0: 39$ & 10.7 & $\begin{array}{l}\sim 80 \% \text {, ridge reforms on east edge of the CC, boiling liquid flows west. West side } \\
\text { opens towards north side. }\end{array}$ \\
\hline & & $0: 52$ & 10.9 & $\begin{array}{l}\sim 80 \% \text {, ridge on east side remains with wet boiling feed flowing toward north east } \\
\text { corner. }\end{array}$ \\
\hline & & 1:06 & 11.1 & $\begin{array}{l}\sim 80 \% \text {, east side opening is larger than the west side opening. Feed is boiling on } \\
\text { surface. }\end{array}$ \\
\hline & & $1: 21$ & 11.4 & $\sim 80-85 \%$, evenly flat with heavy boiling, slight ridge at the opening. \\
\hline & & $1: 43$ & 11.7 & $\sim 80-85 \%$, east side opening is smaller than the west side. \\
\hline & & $2: 04$ & 12.1 & $\sim 90 \%$, east side opening slightly larger than west side. \\
\hline & & $2: 20$ & 12.3 & $\begin{array}{l}\sim 85-90 \% \text {, east side opening migrates north, narrows. West side is open receiving } \\
\text { liquid flow. }\end{array}$ \\
\hline & & $2: 37$ & 12.6 & East side opening dwindles, west side is open, boiling liquid sits on top of CC. \\
\hline & & $2: 52$ & 12.9 & $\begin{array}{c}\sim 85-90 \% \text {, openings on east and west sides. Deep pool of liquid feed is visible } \\
\text { between openings. }\end{array}$ \\
\hline & & $3: 17$ & 13.3 & $\begin{array}{l}\sim 85 \% \text {, openings on east and west sides, both openings have a small ridge around } \\
\text { them. Large pool of liquid feed is boiling between openings. }\end{array}$ \\
\hline & & $3: 25$ & 13.4 & $\begin{array}{l}\sim 85 \% \text {, similar to previous observation, large boiling feed remains as well. West } \\
\text { side opening appears slightly more open. }\end{array}$ \\
\hline & & $3: 41$ & 13.7 & $\sim 85 \%$, ridge building up on east side opening, west side opening is the same. \\
\hline & & 4:03 & 14.1 & $\begin{array}{l}\sim 85 \% \text {, ridge has developed on east side, west side opening is larger. Boiling liquid } \\
\text { sits on top of CC which is moving to the north west corner. }\end{array}$ \\
\hline & & $4: 19$ & 14.3 & $\begin{array}{c}\sim 85 \% \text {, east side migrates south, ridge is gone, and west side is open receiving } \\
\text { flow. }\end{array}$ \\
\hline & & $4: 36$ & 14.6 & $\begin{array}{l}\text { Flow of boiling liquid exist to east side, ridge is formed on the west side, opens } \\
\text { north. }\end{array}$ \\
\hline & & $4: 51$ & 14.9 & $\sim 80 \%$, east side is closing, west side is open. \\
\hline & & $5: 15$ & 15.3 & $\begin{array}{l}\sim 80 \% \text {, east side remains slightly closed with heavy boiling, feed is rushing into } \\
\text { west side which remains roughly the same with lots of wet boiling feed on CC } \\
\text { surface with slight ridge between east and west sides. }\end{array}$ \\
\hline & & $5: 32$ & 15.5 & $\sim 80 \%$, same conditions as before. \\
\hline & & $5: 54$ & 15.9 & $\begin{array}{l}\sim 80 \% \text {, lots of wet boiling feed splashing and rushing in openings on east and west } \\
\text { sides. West opening appears slightly smaller and east appears the same. }\end{array}$ \\
\hline & & 7:02 & 17.0 & $\begin{array}{l}\sim 90 \% \text { with opening in north west and north east corners. Feed is boiling on } \\
\text { surface. }\end{array}$ \\
\hline
\end{tabular}


Table 3.3. Cold Cap Observations (continued).

\begin{tabular}{|c|c|c|c|c|}
\hline Test & Date & Time & $\begin{array}{c}\text { Run } \\
\text { Time } \\
\text { (hours) }\end{array}$ & Cold Cap Observations \\
\hline \multirow{28}{*}{2} & \multirow{28}{*}{$1 / 23 / 13$} & $7: 16$ & 17.3 & $\begin{array}{l}\sim 90 \% \text {, opening in north east and north west corners. Light is visible from south } \\
\text { east corner. Feed is boiling on surface and flowing into openings. }\end{array}$ \\
\hline & & $7: 33$ & 17.6 & $\begin{array}{l}\sim 85 \% \text {, opening in north east and north west corners. Light is visible in south east } \\
\text { corner. CC is thinner with feed boiling on surface and flowing into openings. }\end{array}$ \\
\hline & & 8:06 & 18.1 & $\begin{array}{l}\sim 85-90 \% \text {, feed is boiling on surface, vigorous bubbling is present with a thick } \\
\text { CC. }\end{array}$ \\
\hline & & $8: 54$ & 18.9 & $\begin{array}{c}\sim 95 \% \text {, lots of boiling feed on top, very vigorous bubbling, splashing up through a } \\
\text { thick CC. }\end{array}$ \\
\hline & & $9: 30$ & 19.5 & $\begin{array}{l}\sim 90 \% \text {, openings exist in north east and north west corners, light is visible in south } \\
\text { east corner, large ridges exist around each opening. }\end{array}$ \\
\hline & & $9: 41$ & 19.7 & $\begin{array}{c}\sim 90-95 \% \text {, Opening in north east and north west corners with } 4 \text { inch ridges around } \\
\text { the openings. Feed is boiling in center on surface. }\end{array}$ \\
\hline & & 9:55 & 19.9 & $\sim 90-95 \%$, visual observations are unchanged from previous observation. \\
\hline & & 10:05 & 20.1 & $\begin{array}{c}\sim 95 \% \text {, openings on north east and northwest corners, light is visible in south east } \\
\text { corner, feed is boiling in center on top of CC. }\end{array}$ \\
\hline & & $10: 20$ & 20.3 & $\sim 90-95 \%$, conditions are unchanged from previous observation. \\
\hline & & $10: 35$ & 20.6 & $\sim 95 \%$, feed is boiling on the surface, hole is visible on west side of view port. \\
\hline & & $11: 20$ & 21.3 & $\begin{array}{l}\sim 90-95 \% \text {, looks about } 6 \text { in thick with boiling feed on top, opening on east and } \\
\text { west sides are still visible. }\end{array}$ \\
\hline & & $11: 35$ & 21.6 & $\sim 95 \%$, west side is visible with boiling feed on CC. \\
\hline & & $11: 50$ & 21.8 & $\sim 95 \%$, same as the last observation. \\
\hline & & 12:04 & 22.1 & $\begin{array}{c}\sim 95 \% \text { with feed boiling on top, openings in north east and north west corners with } \\
\text { ridges. }\end{array}$ \\
\hline & & $12: 25$ & 22.4 & $\sim 90-95 \%$, openings are visible in north west and south east corners. \\
\hline & & $12: 40$ & 22.7 & $\sim 90-95 \%$, conditions are unchanged from previous observation. \\
\hline & & $12: 55$ & 22.9 & $\sim 90-95 \%$, conditions are unchanged. \\
\hline & & $13: 15$ & 23.3 & $\begin{array}{l}\sim 90-95 \% \text {, opening is visible in northwest corner with light visible from north east } \\
\text { and south east corners. Feed is boiling on surface. }\end{array}$ \\
\hline & & $13: 39$ & 23.7 & $\begin{array}{l}\sim 90-95 \%, \sim 6 " \text { thick, single opening in north west corner, feed is boiling on } \\
\text { surface and flowing into opening. }\end{array}$ \\
\hline & & $13: 54$ & 23.9 & $\sim 90-95 \%$, opening in north west corner, light is visible on surface. \\
\hline & & $14: 15$ & 24.3 & $\sim 90-95 \%$, conditions are unchanged from previous observation \\
\hline & & $14: 35$ & 24.6 & $\sim 95 \%$, opening on west side still visible with ridge, visible light on east side. \\
\hline & & 15:04 & 25.1 & $\sim 85 \%$, not able to see east side opening. There is large ridge on the west side. \\
\hline & & $15: 19$ & 25.3 & $\sim 85 \%$, no visible changes for this observation. \\
\hline & & $15: 39$ & 25.7 & $\sim 85 \%$, west side ridge has melted down and closed the CC somewhat. \\
\hline & & $15: 53$ & 25.9 & $\sim 90 \%, \sim 8$ " thick, not able to see feed flowing into single opening on west side. \\
\hline & & 16:09 & 26.2 & Unchanged from previous observation. \\
\hline & & $16: 49$ & 26.8 & $\sim 90 \%$, feed is flowing into the west opening. \\
\hline
\end{tabular}


Table 3.3. Cold Cap Observations (continued).

\begin{tabular}{|c|c|c|c|c|}
\hline Test & Date & Time & $\begin{array}{c}\text { Run } \\
\text { Time } \\
\text { (hours) }\end{array}$ & Cold Cap Observations \\
\hline \multirow{31}{*}{2} & \multirow{26}{*}{$1 / 23 / 13$} & $17: 21$ & 27.4 & $\begin{array}{l}\sim 85 \% \text {, east side ridge has broken down some, now able to see the opening on that } \\
\text { side. Feed is flowing into both openings that can be seen. }\end{array}$ \\
\hline & & $17: 39$ & 27.7 & $\begin{array}{l}\sim 90 \%, \sim 6 " \text { thick, feed is flowing into single west side opening through ridge } \\
\text { breakdown. }\end{array}$ \\
\hline & & $17: 50$ & 27.8 & $\sim 85 \%$, two openings. \\
\hline & & $18: 04$ & 28.1 & $\sim 85 \%$, large amount of feed is flowing into the openings. \\
\hline & & $18: 20$ & 28.3 & $\sim 90 \%$, same as above. \\
\hline & & $18: 27$ & 28.5 & Emergency off gas is tripped, $\mathrm{CC}$ is shifted. \\
\hline & & $18: 39$ & 28.7 & $\begin{array}{c}\sim 85 \% \text {, has dropped down. Able to see east side through north view port. Feed is } \\
\text { flowing heavily into the openings. }\end{array}$ \\
\hline & & $18: 49$ & 28.8 & $\sim 85 \%$, conditions unchanged. \\
\hline & & 19:04 & 29.1 & $\sim 85 \%$, no visible changes for this observation. \\
\hline & & $19: 20$ & 29.3 & $\sim 85 \%$, two openings, feed is boiling on top. \\
\hline & & $19: 39$ & 29.7 & $\sim 80 \%$, it has opened up due to feed sample being collected. \\
\hline & & 19:54 & 29.9 & $\sim 85 \%$, there are small ridges around east and west openings. \\
\hline & & $20: 12$ & 30.2 & $\begin{array}{c}\sim 85 \% \text {, openings on west side and north east corner, feed boiling on surface } \\
\text { between the openings. }\end{array}$ \\
\hline & & $20: 26$ & 30.4 & Off gas sampling has started. \\
\hline & & $20: 49$ & 30.8 & $\sim 85 \%$, small ridges around both openings with feed flowing into each opening. \\
\hline & & 21:00 & 31.0 & End of off gas testing, melter pressure spiked. \\
\hline & & 21:04 & 31.1 & $\sim 85 \%$, no visible changes for this observation. \\
\hline & & $21: 25$ & 31.4 & $\sim 85 \%$, two openings exist. \\
\hline & & $21: 39$ & 31.7 & $\sim 85 \%$, two openings, flat about 5 " thick CC. \\
\hline & & 21:54 & 31.9 & $\sim 85 \%$, same as before. \\
\hline & & 22:08 & 32.1 & $\sim 85 \%$, flat, feed is boiling on top. \\
\hline & & $22: 24$ & 32.4 & $\sim 85 \%$, same as above. \\
\hline & & $22: 48$ & 32.8 & $\begin{array}{c}\sim 80-85 \% \text {, heavy boiling on the surface, observed liquid freely flowing from the } \\
\text { surface to both east and west openings. }\end{array}$ \\
\hline & & 23:08 & 33.1 & $\begin{array}{c}\sim 80-85 \% \text {, ridge on west side near opening and feed is easily rushing in on east } \\
\text { side, heavy boiling on surface. }\end{array}$ \\
\hline & & $23: 38$ & 33.6 & $\begin{array}{c}\sim 80 \% \text {, ridge along west edge of CC. Liquid flow in east side, east opening } \\
\text { expands. }\end{array}$ \\
\hline & & $23: 50$ & 33.8 & $\begin{array}{l}\sim 85 \% \text {, lots of boiling feed, ridge remains on west side with boiling feed between } \\
\text { east and west openings. Thick CC with some splashing. }\end{array}$ \\
\hline & \multirow{5}{*}{$1 / 24 / 13$} & 0:08 & 34.1 & $\sim 80 \%$, east opening grows, west is steady, liquid flow on east side. \\
\hline & & $0: 20$ & 34.3 & $\sim 80 \%$, same as prior observation. \\
\hline & & $0: 37$ & 34.6 & $\sim 80 \%$. \\
\hline & & $0: 43$ & 34.7 & $\begin{array}{c}\sim 85 \% \text {, opening on east side shrunk slightly, heavy boiling of feed on surface with } \\
\text { splashing. }\end{array}$ \\
\hline & & $0: 54$ & 34.9 & $\sim 85 \%$, similar to prior conditions. \\
\hline
\end{tabular}


Table 3.3. Cold Cap Observations (continued).

\begin{tabular}{|c|c|c|c|c|}
\hline Test & Date & Time & $\begin{array}{c}\text { Run } \\
\text { Time } \\
\text { (hours) }\end{array}$ & Cold Cap Observations \\
\hline \multirow{26}{*}{2} & \multirow{26}{*}{$1 / 24 / 13$} & 1:04 & 35.1 & $\sim 80-85 \%$, consistently the same from the last observation. \\
\hline & & $1: 19$ & 35.3 & $\begin{array}{l}\sim 80 \% \text {, ridge along west side of CC forces boiling liquid to east side, opening } \\
\text { which has been reduced, west side is open and enlarging. }\end{array}$ \\
\hline & & $1: 45$ & 35.8 & $\begin{array}{l}\text { East side opening diminishes, west side closing also. CC appears thick on the east } \\
\text { side, softer on west side. }\end{array}$ \\
\hline & & 2:06 & 36.1 & $\sim 80 \%$, east side opening holding steady, west side opening migrates south. \\
\hline & & $2: 34$ & 36.6 & $\begin{array}{c}\sim 85 \% \text {, ridge on east side, west side of CC grows and is fixed, not moving, boiling } \\
\text { liquid on surface. }\end{array}$ \\
\hline & & $2: 52$ & 36.9 & $\begin{array}{c}\sim 80 \% \text {, ridges on east and west edges of CC, boiling liquid pools on top of CC. } \\
\text { West side is more open. }\end{array}$ \\
\hline & & 3:09 & 37.2 & $\begin{array}{c}\sim 75-80 \% \text {, ridges still exist on east and west edge. Losing north edge of CC as east } \\
\text { side migrates south. Boiling liquid on top of CC. }\end{array}$ \\
\hline & & $3: 25$ & 37.4 & $\begin{array}{l}\sim 80 \% \text {, east side opening dwindles, west side has ridge, still receives boiling flow. } \\
\text { CC is thick not spreading southward. }\end{array}$ \\
\hline & & $3: 52$ & 37.9 & $\begin{array}{c}\sim 85 \% \text {, west side closes, east side narrows. Ridge on west side causes boiling } \\
\text { liquid to flow north west corner. }\end{array}$ \\
\hline & & $4: 10$ & 38.2 & $\begin{array}{c}\sim 85 \% \text {, no major change in CC, west side edge still has ridge between west and } \\
\text { east openings. Lots of boiling feed. Feed flows towards north west. }\end{array}$ \\
\hline & & $4: 46$ & 38.8 & $\begin{array}{l}\sim 90 \% \text {, east side opening is barely seen, west side cap grows, west side liquid } \\
\text { boils on top of cap. Fibers are seen dangling from interior roof. }\end{array}$ \\
\hline & & 5:07 & 39.1 & $\begin{array}{c}\sim 90 \% \text {, east and west opening have small ridges around them. Feed is boiling on } \\
\text { surface. }\end{array}$ \\
\hline & & $5: 33$ & 39.6 & $\sim 90 \%$, east side closed up west side is open, lots of feed on the surface. \\
\hline & & $5: 49$ & 39.8 & $\begin{array}{l}\sim 90 \% \text {, east side is closed, west side is open with a small ridge around it. Lots of } \\
\text { feed boiling on surface. }\end{array}$ \\
\hline & & 6:06 & 40.1 & $\sim 90 \%$, one opening on the west side, feed is boiling on surface. \\
\hline & & $6: 35$ & 40.6 & $\sim 90 \%$, same, one opening on west side, feed is boiling on surface. CC is thick. \\
\hline & & 7:02 & 41.0 & $\begin{array}{c}\sim 95 \% \text { with opening at north west corner, feed is boiling on surface with ridge } \\
\text { opening. }\end{array}$ \\
\hline & & $7: 18$ & 41.3 & $\sim 95 \%$, conditions are same as the previous observation. \\
\hline & & $7: 38$ & 41.6 & $\sim 95 \%$, feed is boiling on surface, opening in north west corner with a ridge. \\
\hline & & $7: 52$ & 41.9 & $\begin{array}{l}\sim 95 \% \text { with small openings in northwest and north west corners. Feed is boiling on } \\
\text { surface, ridges exist at openings. }\end{array}$ \\
\hline & & 8:06 & 42.1 & $\begin{array}{c}\sim 95 \% \text {, with openings on north east and north west corners with ridges around } \\
\text { openings. Feed is boiling on surface. }\end{array}$ \\
\hline & & $8: 20$ & 42.3 & $\begin{array}{l}\sim 95 \% \text { with a small opening on north east corner, Two small openings on north } \\
\text { west side. Light is visible from south east corner. Feed is boiling on surface. }\end{array}$ \\
\hline & & $8: 40$ & 42.7 & $\sim 95 \%$, conditions are unchanged from previous observation. \\
\hline & & $8: 55$ & 42.9 & $\begin{array}{c}\sim 95 \% \text {, openings on east and west sides visible via north view port. Light is visible } \\
\text { on south east corner via south port. }\end{array}$ \\
\hline & & $9: 14$ & 43.2 & $\begin{array}{l}\sim 95 \% \text {, openings in north east and north west corners with ridges. Feed is boiling } \\
\text { on surface. }\end{array}$ \\
\hline & & $9: 35$ & 43.6 & $\begin{array}{l}\sim 95 \% \text { with openings in north east and north west corners. Feed is boiling on } \\
\text { surface. }\end{array}$ \\
\hline
\end{tabular}


Table 3.3. Cold Cap Observations (continued).

\begin{tabular}{|c|c|c|c|c|}
\hline Test & Date & Time & $\begin{array}{l}\text { Run } \\
\text { Time } \\
\text { (hours) }\end{array}$ & Cold Cap Observations \\
\hline \multirow{22}{*}{2} & \multirow{22}{*}{$1 / 24 / 13$} & $9: 52$ & 43.9 & $\sim 95 \%$, feed is boiling on surface, openings in north east and north west corners. \\
\hline & & $10: 10$ & 44.2 & $\begin{array}{l}\sim 95 \% \text {, feed is boiling on surface opening at north east and north west corners. } \\
\text { Feed is flowing into melt pool through the hole in ridge. }\end{array}$ \\
\hline & & $10: 39$ & 44.7 & $\begin{array}{l}\sim 95 \% \text {, east side and west side openings are still visible, west side has small ridge } \\
\text { with boiling feed on top. }\end{array}$ \\
\hline & & $10: 56$ & 44.9 & $\begin{array}{c}\sim 95 \% \text {, two openings in north east and north west corners with no ridges. Feed is } \\
\text { flowing into openings. }\end{array}$ \\
\hline & & 11:11 & 45.2 & $\begin{array}{l}\sim 95 \% \text { with openings in north east and north west corners. Feed is boiling on } \\
\text { surface. }\end{array}$ \\
\hline & & $11: 32$ & 45.5 & $\sim 95 \%$, no changes from the previous reading. \\
\hline & & $11: 49$ & 45.8 & $\begin{array}{l}\sim 95 \% \text { with openings in north east and north west corners. Feed is boiling on } \\
\text { surface. Ridges exist at openings. }\end{array}$ \\
\hline & & 12:05 & 46.1 & $\sim 85 \%$, with visible opening on east and west sides, still have boiling feed on CC. \\
\hline & & $12: 20$ & 46.3 & $\sim 95 \%$, no visible changes since last reading. \\
\hline & & $12: 35$ & 46.6 & $\begin{array}{l}\sim 95 \% \text { with visible openings on east and west sides of the melter CC. Surface is } \\
\text { flat with ridge on west side, feed is flowing into both openings. }\end{array}$ \\
\hline & & $12: 47$ & 46.8 & $\begin{array}{l}\sim 95 \% \text {, with openings at north east and north west corners. Feed is boiling on } \\
\text { surface. }\end{array}$ \\
\hline & & 13:08 & 47.1 & $\sim 95 \%$, feed is boiling on surface and flowing into north west corner opening. \\
\hline & & $13: 23$ & 47.4 & $\sim 95 \%$, same as previous reading. \\
\hline & & $13: 37$ & 47.6 & $\begin{array}{c}\sim 95 \% \text { with openings at north east and north west corners. Feed is boiling on } \\
\text { surface and flowing into melt pool via northwest opening. }\end{array}$ \\
\hline & & 13:55 & 47.9 & $\sim 95 \%$, openings in north east and north west corners, feed is boiling on surface. \\
\hline & & 14:09 & 48.2 & $\begin{array}{l}\sim 95 \% \text { with north west opening smaller and north east opening is opening up } \\
\text { more. Feed is boiling on surface. }\end{array}$ \\
\hline & & $14: 56$ & 48.9 & $\sim 95 \%$, two small openings. \\
\hline & & $15: 16$ & 49.3 & $\sim 95 \%$, still the same as above. \\
\hline & & 15:39 & 49.7 & $\begin{array}{l}\sim 95 \% \text {, small openings in north east and south west corners, feed is boiling on } \\
\text { surface between openings. }\end{array}$ \\
\hline & & $16: 10$ & 50.2 & $\begin{array}{c}\sim 90 \% \text {, difficulty to see past view ports but light is visible in background } \\
\text { indicating larger openings. }\end{array}$ \\
\hline & & $16: 35$ & 50.6 & $\begin{array}{l}\sim 95 \% \text {, light is visible in background, no openings are visible at west wall below } \\
\text { the view ports. Vigorous bubbling, stalactite hanging from thermocouple. }\end{array}$ \\
\hline & & $17: 00$ & 51.0 & Secured feeding. End of the Test 2. \\
\hline \multirow{6}{*}{3} & \multirow{6}{*}{$1 / 29 / 13$} & $9: 30$ & 0.0 & Started feeding water at $0.8 \mathrm{l} / \mathrm{min}$. \\
\hline & & $9: 52$ & 0.4 & Increased water flow rate to $1.6 \mathrm{l} / \mathrm{min}$. \\
\hline & & $10: 10$ & 0.7 & Increased water from 1.6 to $2.41 / \mathrm{min}$. \\
\hline & & $10: 30$ & 1.0 & Secured water flow and starting feeding slurry \\
\hline & & $10: 35$ & 1.1 & $\sim 60 \%$, flat and soft. \\
\hline & & 11:05 & 1.6 & $\sim 80 \%$, visible opening on east and west sides. $\mathrm{CC}$ is flat. \\
\hline
\end{tabular}


Table 3.3. Cold Cap Observations (continued).

\begin{tabular}{|c|c|c|c|c|}
\hline Test & Date & Time & $\begin{array}{c}\text { Run } \\
\text { Time } \\
\text { (hours) }\end{array}$ & Cold Cap Observations \\
\hline \multirow{26}{*}{3} & \multirow{26}{*}{$1 / 29 / 13$} & $11: 20$ & 1.8 & $\sim 85-90 \%, \mathrm{CC}$ is still flat. \\
\hline & & $11: 35$ & 2.1 & $\sim 85-90 \%$, no visible change. \\
\hline & & $11: 54$ & 2.4 & $\sim 85-90 \%$, looks soft and opening on west side and east sides. \\
\hline & & $12: 20$ & 2.8 & $\begin{array}{c}\sim 85-90 \% \text {, small ridge on east side with opening about } 12 \text { ", visible opening on } \\
\text { east side with feed flowing to melt pool. }\end{array}$ \\
\hline & & $12: 47$ & 3.3 & $\begin{array}{c}\sim 95 \% \text { with opening on north east and mid north west sides. Feed is boiling on } \\
\text { surface with ridges at openings. }\end{array}$ \\
\hline & & 13:02 & 3.5 & $\sim 95 \%$, no changes from previous reading. \\
\hline & & 13:17 & 3.8 & $\begin{array}{l}\sim 95 \% \text { with openings on north east corner and north west-mid melter. Feed is } \\
\text { flowing into melt pool through north west opening. }\end{array}$ \\
\hline & & $13: 35$ & 4.1 & $\begin{array}{c}\sim 95 \% \text {, large ridge on west side opening and east side opening has small ridge } \\
\text { with feed flowing to opening. }\end{array}$ \\
\hline & & 13:50 & 4.3 & $\sim 95 \%$, no visible changes from last reading. \\
\hline & & 14:11 & 4.7 & $\begin{array}{c}\sim 95 \% \text {, two openings at north east and north west-mid melter with ridges at } \\
\text { openings. }\end{array}$ \\
\hline & & $14: 29$ & 5.0 & $\begin{array}{c}\sim 95 \% \text {, opening on east and west sides, visible both openings have a ridge at east } \\
\text { opening side, feed is flowing through ridge. }\end{array}$ \\
\hline & & $14: 49$ & 5.3 & $\begin{array}{l}\sim 95 \% \text { is very thick. The two openings are visible and small. The openings are } \\
10 "-12 \text { " thick in CC. }\end{array}$ \\
\hline & & 15:04 & 5.6 & $\sim 95 \%$, no visible changes yet since increasing bubbling. \\
\hline & & 15:08 & 5.6 & $\begin{array}{l}\text { CC is shifted since increasing bubbling. In turn caused liquid feed to flow into } \\
\text { the opening. }\end{array}$ \\
\hline & & $15: 19$ & 5.8 & $\begin{array}{c}\sim 95 \% \text {, is not as thick now due to lower liquid level. Also the two ridges that are } \\
\text { visible look taller now. }\end{array}$ \\
\hline & & $15: 34$ & 6.1 & $\sim 95 \%$, the surface has more liquid boiling on it. \\
\hline & & $15: 51$ & 6.4 & $\begin{array}{c}\sim 95 \% \text {, very thick CC is visible at bubbler openings. Spitting glass at openings, } \\
\text { no liquid is visible. }\end{array}$ \\
\hline & & 16:05 & 6.6 & $\sim 95 \%$, lots of spitting, bubbler holes might be closing on CC surface. \\
\hline & & $16: 19$ & 6.8 & $\sim 95 \%$, spitting glass, darker, $\mathrm{CC}$ bubbler holes are tighter. \\
\hline & & $16: 34$ & 7.1 & $\sim 95 \%$, openings appear to be holding the same sizes. \\
\hline & & $16: 49$ & 7.3 & $\sim 95 \%$, two small openings. \\
\hline & & 17:04 & 7.6 & $\sim 95 \%$, very thick two openings. \\
\hline & & $17: 17$ & 7.8 & $\sim 95 \%$, one very small and one larger opening dark inside, also very thick. \\
\hline & & $17: 34$ & 8.1 & $\sim 95 \%$, one opening. \\
\hline & & $17: 48$ & 8.3 & $\begin{array}{l}\sim 95 \% \text {, saw hot glass spilling over from the east side, but could not see on east } \\
\text { opening. Also saw second small opening beginning to form on north west side. }\end{array}$ \\
\hline & & $18: 04$ & 8.6 & $\begin{array}{l}\sim 95 \% \text {, not able to see east side at this time. Able to see west side, with three } \\
\text { openings running along the wall, two of the openings are about to merge. }\end{array}$ \\
\hline
\end{tabular}


Table 3.3. Cold Cap Observations (continued).

\begin{tabular}{|c|c|c|c|c|}
\hline Test & Date & Time & $\begin{array}{l}\text { Run } \\
\text { Time } \\
\text { (hours) }\end{array}$ & Cold Cap Observations \\
\hline \multirow{29}{*}{3} & \multirow{21}{*}{$1 / 29 / 13$} & 18:16 & 8.8 & $\begin{array}{c}\sim 95 \% \text {, two west side openings have merged into single opening. CC is } 5 " \text { thick } \\
\text { and no opening is visible on east side. }\end{array}$ \\
\hline & & $18: 39$ & 9.2 & $\sim 90 \%$. \\
\hline & & $18: 49$ & 9.3 & $\sim 90 \%$, no change from the previous observation. \\
\hline & & 19:05 & 9.6 & $\begin{array}{l}\sim 90 \% \text {, there is a ridge on the north to east side about } 8 \text { in. thick and going to the } \\
\text { west. }\end{array}$ \\
\hline & & 19:17 & 9.8 & $\sim 90 \%$, west wall opening, large ridge in center, significant light is visible beyond. \\
\hline & & 19:34 & 10.1 & $\begin{array}{l}\sim 90 \%, \text { able to see a small portion of the east side openings, very little to no } \\
\text { change on west side. }\end{array}$ \\
\hline & & 19:49 & 10.3 & $\sim 85 \%$ opened up since feed rate is a little low, $172 \mathrm{~kg} / \mathrm{hr}$. \\
\hline & & 20:04 & 10.6 & $\sim 85$, able to see more of the east opening. \\
\hline & & $20: 20$ & 10.8 & $\sim 85 \%$, reduced bubbling to close the CC up. \\
\hline & & $20: 33$ & 11.1 & $\sim 85 \%$, still separated from melt pool. \\
\hline & & $20: 53$ & 11.4 & $\sim 85 \%$, west side is more open than the east side, flows to south west side. \\
\hline & & 21:09 & 11.7 & $\sim 90 \%$, opening on north east, large pool of liquid in center is boiling. \\
\hline & & $21: 19$ & 11.8 & $\sim 85 \%$, feed is boiling on top, two large openings exist. \\
\hline & & $21: 37$ & 12.1 & $\sim 85 \%$, heavy boiling and steam exist, openings are larger. \\
\hline & & $21: 49$ & 12.3 & $\sim 85 \%$, feed is boiling on top. \\
\hline & & 22:34 & 13.1 & $\sim 85-90 \%$, observed buildups on east side, west side opening is slightly ridged. \\
\hline & & 22:50 & 13.3 & $\sim 90 \%$, flow of hot liquid moving eastward observed. West side is open. \\
\hline & & 23:01 & 13.5 & $\begin{array}{l}\sim 90 \% \text {, hot boiling feed on CC flowing to north east corner with west side opening } \\
\text { and thick CC is apparent. }\end{array}$ \\
\hline & & 23:18 & 13.8 & $\begin{array}{c}\sim 95 \% \text {, thick CC with heavy splashing, build up on east plenum increasing } \\
\text { slightly. }\end{array}$ \\
\hline & & 23:35 & 14.1 & $\sim 85-90 \%$, east side opens slightly, west side opening grows. \\
\hline & & 23:46 & 14.3 & $\begin{array}{l}\sim 90 \% \text {, heavy boiling present. Ridges around the openings, east opening is slightly } \\
\text { visible in between bubbling, build up exist on the exposed plenum thermocouple, } \\
\text { visible from the south port. }\end{array}$ \\
\hline & \multirow{8}{*}{$1 / 30 / 13$} & $0: 16$ & 14.8 & $\sim 90 \%$ remains unchanged. \\
\hline & & $0: 19$ & 14.8 & $\sim 90 \%$, west opening is slightly larger. \\
\hline & & $0: 34$ & 15.1 & $\begin{array}{c}\sim 85-90 \% \text {, west side opening grows. East side is barely visible due to dangling } \\
\text { filaments of glass. }\end{array}$ \\
\hline & & $0: 49$ & 15.3 & $\begin{array}{c}\sim 80-85 \% \text {, west edge of CC recedes eastward, east side is opening is minimal, } \\
\text { melt pool is visible south and west edges. }\end{array}$ \\
\hline & & $1: 04$ & 15.6 & $\begin{array}{c}\sim 80-85 \% \text {, east side opens slightly, west side edge of CC forms as a shelf. South } \\
\text { end of melt pool is opening. }\end{array}$ \\
\hline & & $1: 19$ & 15.8 & $\begin{array}{l}85 \% \text {, west and south portions of the CC contracted, east side } 85 \% \text { opening } \\
\text { remains constant, splashing hot liquid flows east and south. }\end{array}$ \\
\hline & & $1: 34$ & 16.1 & $85 \%$, east side opening is slightly greater. CC grows west and south. \\
\hline & & $1: 49$ & 16.3 & East side opening grows north, west side closes. \\
\hline
\end{tabular}


Table 3.3. Cold Cap Observations (continued).

\begin{tabular}{|c|c|c|c|c|}
\hline Test & Date & Time & $\begin{array}{l}\text { Run } \\
\text { Time } \\
\text { (hours) }\end{array}$ & Cold Cap Observations \\
\hline \multirow{26}{*}{3} & \multirow{26}{*}{$1 / 30 / 13$} & 2:04 & 16.6 & $\begin{array}{c}\sim 85 \% \text {, east opening is the same, west closes slightly; splashing, boiling liquid } \\
\text { flows west. }\end{array}$ \\
\hline & & $2: 19$ & 16.8 & $\begin{array}{c}\sim 90 \% \text {, ridge at west opening is slightly reduced; liquid from the surface is } \\
\text { flowing into opening. }\end{array}$ \\
\hline & & $2: 49$ & 17.3 & $\sim 90 \%$, no change. \\
\hline & & 3:04 & 17.6 & East opening migrates south, ridge on west edge sends boiling liquid east. \\
\hline & & $3: 20$ & 17.8 & $\begin{array}{c}\sim 90 \% \text {, openings on east and west sides, both openings have a small ridge } \\
\text { around them. Feed is boiling on CC. }\end{array}$ \\
\hline & & $3: 51$ & 18.4 & $\sim 90 \%$, unchanged. \\
\hline & & 4:04 & 18.6 & $\begin{array}{l}\sim 90 \% \text {, ridges exits on both east and west edges of CC. Boiling liquid flows } \\
\text { south. }\end{array}$ \\
\hline & & $4: 21$ & 18.9 & $\begin{array}{l}\sim 85 \% \text {, ridges remain, } \mathrm{CC} \text { dwindles on west edge boiling flows goes south. } \\
\text { Liquid sits atop of CC. }\end{array}$ \\
\hline & & $4: 35$ & 19.1 & $\sim 85 \%$, west edge receded eastward, ridge remains. \\
\hline & & $4: 49$ & 19.3 & $\begin{array}{l}\sim 80-85 \% \text {, mostly unchanged, boiling liquid flows south. Due to large discharge } \\
\text { a shelf exists. }\end{array}$ \\
\hline & & 5:06 & 19.6 & $\begin{array}{c}\sim 85 \% \text {, east opening is larger than west opening. Feed is boiling between } \\
\text { openings. }\end{array}$ \\
\hline & & $5: 19$ & 19.8 & $\begin{array}{l}\sim 85 \% \text {, feed is flowing into east opening, west opening has slight ridge, build up } \\
\text { around it. Feed is boiling on CC surface. }\end{array}$ \\
\hline & & $5: 34$ & 20.1 & $\begin{array}{c}\sim 85 \% \text {, heavy boiling on the CC surface, two openings exists on east and west } \\
\text { sides. }\end{array}$ \\
\hline & & $5: 53$ & 20.4 & $\sim 85-90 \%$. \\
\hline & & $6: 20$ & 20.8 & $\sim 85 \%$, heavy boiling on the surface. \\
\hline & & $6: 34$ & 21.1 & $\sim 85 \%$, no change from last observation. \\
\hline & & $7: 19$ & 21.8 & $\begin{array}{l}\sim 95 \% \text {, openings with ridges visible via north west \& center view ports. Light is } \\
\text { visible via south west view port. Feed collecting on top of cap and boiling. }\end{array}$ \\
\hline & & $7: 34$ & 22.1 & $\sim 95 \%$ with feed boiling on top, ridges and stalactites viable in the view ports. \\
\hline & & $7: 49$ & 22.3 & $\begin{array}{c}\sim 90 \% \text {, openings are visible via north west and center view ports. Light is } \\
\text { visible via south west view port. Ridges have decreased in size. Feed is boiling } \\
\text { on surface. }\end{array}$ \\
\hline & & $8: 04$ & 22.6 & $\sim 90 \%$, visual observations unchanged from previous observation. \\
\hline & & $8: 19$ & 22.8 & $\begin{array}{l}\sim 90 \% \text {, opening is visible via north and center view ports. Light is visible on } \\
\text { south east corner via south port. Ridges have reduced in size allowing feed to } \\
\text { flow into openings. Feed is boiling on surface. }\end{array}$ \\
\hline & & $8: 26$ & 22.9 & Emergency off gas is tripped due to CC shifting/collapsing. \\
\hline & & $8: 34$ & 23.1 & $\sim 90 \%$, visual observations are unchanged from previous observation. \\
\hline & & $8: 50$ & 23.3 & $\sim 95 \%$, no visual change. \\
\hline & & 9:04 & 23.6 & $\begin{array}{l}\sim 95 \% \text { with two openings at north east corner that is barely visible and at north } \\
\text { west corner. Feed is boiling on surface. }\end{array}$ \\
\hline & & $9: 19$ & 23.8 & $\sim 95 \%$ with no visual changes. \\
\hline
\end{tabular}


Table 3.3. Cold Cap Observations (continued).

\begin{tabular}{|c|c|c|c|c|}
\hline Test & Date & Time & $\begin{array}{l}\text { Run } \\
\text { Time } \\
\text { (hours) }\end{array}$ & Cold Cap Observations \\
\hline \multirow{27}{*}{3} & \multirow{27}{*}{$1 / 30 / 13$} & $9: 36$ & 24.1 & $\sim 95 \%$ with ridges around the bubbler openings and feed is boiling on surface. \\
\hline & & $9: 49$ & 24.3 & $\begin{array}{l}\sim 95 \% \text {, openings on east and west sides are visible with feed flowing to melt } \\
\text { pool. }\end{array}$ \\
\hline & & 10:04 & 24.6 & $\sim 95 \%$, both east and west side openings are still visible. \\
\hline & & 10:19 & 24.8 & $\sim 95 \%$, no visible changes. \\
\hline & & $10: 36$ & 25.1 & $\begin{array}{l}\sim 95 \% \text {, large ridge on west side is visible; east side is still visible with feed } \\
\text { flowing to melt pool. }\end{array}$ \\
\hline & & 10:49 & 25.3 & $95 \%$, no visible changes at this time. \\
\hline & & 11:04 & 25.6 & $\begin{array}{l}\sim 95 \% \text {, still large ridge/build up on west side, east side is still visible with } \\
\text { boiling feed on cap. }\end{array}$ \\
\hline & & 11:19 & 25.8 & $\sim 95 \%$, no visible changes. \\
\hline & & $11: 25$ & 25.9 & Melter pressure spike due to cold cap shift. \\
\hline & & $11: 34$ & 26.1 & $\begin{array}{l}\sim 90 \% \text {, openings on north east and north west corners are visible via north view } \\
\text { port. Openings have small ridges around them. Opening on west side center is } \\
\text { visible via center view port. Light is visible in south east corner via south view } \\
\text { port. }\end{array}$ \\
\hline & & 11:49 & 26.3 & $\begin{array}{l}\sim 90-95 \% \text {, openings in north east and west corners with small ridge. Opening on } \\
\text { west side center. Light is visible in south east corner. }\end{array}$ \\
\hline & & 12:04 & 26.6 & $\sim 90-95 \%$, visual observations unchanged from previous observation. \\
\hline & & 12:19 & 26.8 & $\begin{array}{c}\sim 95 \% \text {, north east corner opening is barely visible; feed is boiling on surface and } \\
\text { flowing into melt pool via north west opening. }\end{array}$ \\
\hline & & $12: 34$ & 27.1 & $\sim 90-95 \%$, no visual change from previous reading. \\
\hline & & $12: 49$ & 27.3 & $\begin{array}{l}\sim 95 \% \text {, north east opening is no longer visible. Ridge around north west } \\
\text { opening, feed is boiling on surface. }\end{array}$ \\
\hline & & 13:04 & 27.6 & $\sim 95 \%$, no visual change from previous reading. \\
\hline & & 13:19 & 27.8 & $\begin{array}{l}\sim 95 \% \text {, ridge around north west opening with feed boiling on surface. Light is } \\
\text { visible in north east and south east corners. }\end{array}$ \\
\hline & & 13:34 & 28.1 & $\sim 95 \%$, no visible changes from previous reading. \\
\hline & & 13:49 & 28.3 & $\begin{array}{l}\sim 95 \% \text { with opening in north west corner, light is visible in south east corner. } \\
\text { Feed is boiling on surface. }\end{array}$ \\
\hline & & 14:04 & 28.6 & $\begin{array}{l}\sim 95 \% \text { with opening in north west corner. Light is visible in south east and north } \\
\text { east corners. }\end{array}$ \\
\hline & & 14:39 & 29.2 & $\sim 95 \%$, opening is near view port at north west side, dark inside, very thick CC. \\
\hline & & 14:54 & 29.4 & $\sim 95 \%$, very thick, single opening in north west corner. \\
\hline & & 15:06 & 29.6 & $\begin{array}{l}\sim 95 \% \text {, able to see light emitting from the east side. West side has a small } \\
\text { opening that is spitting glass out. }\end{array}$ \\
\hline & & $15: 19$ & 29.8 & $\sim 95 \%$, no visible changes at this time. \\
\hline & & $15: 49$ & 30.3 & $\sim 95 \%$, not able to see any changes in CC conditions. \\
\hline & & 16:04 & 30.6 & $\sim 95 \%$, thick, opening on north west corner. \\
\hline & & 16:19 & 30.8 & $\sim 95 \%$, thick and dark inside. \\
\hline
\end{tabular}


Table 3.3. Cold Cap Observations (continued).

\begin{tabular}{|c|c|c|c|c|}
\hline Test & Date & Time & $\begin{array}{l}\text { Run Time } \\
\text { (hours) }\end{array}$ & Cold Cap Observations \\
\hline \multirow{33}{*}{3} & \multirow{28}{*}{$1 / 30 / 13$} & $16: 34$ & 31.1 & $\sim 95 \%$, same as before. \\
\hline & & $16: 49$ & 31.3 & $\begin{array}{l}\sim 95 \% \text {, west side opening is slightly larger with a cone shape mound. The face } \\
\text { of the mound is glazed (shiny). }\end{array}$ \\
\hline & & 17:04 & 31.6 & $\sim 95 \%$, no visible changes for this observation. \\
\hline & & $17: 19$ & 31.8 & $\sim 95 \%$, west side opening has opened up more to the south. \\
\hline & & $17: 34$ & 32.1 & $\begin{array}{l}\sim 95 \% \text {, west side is slowly closing back up. The cone shaped mound is more of } \\
\text { a ridge and it looks dry at this time. }\end{array}$ \\
\hline & & $17: 49$ & 32.3 & $\sim 90 \%$ and looks flat. \\
\hline & & 18:04 & 32.6 & $\sim 90 \%$, it is flat, 8 " thick, feed is boiling on surface. \\
\hline & & $18: 24$ & 32.9 & $\sim 90 \%$, very thick and single opening exist in west side. \\
\hline & & $18: 36$ & 33.1 & $\sim 90 \%$, unchanged from the last observation. \\
\hline & & $18: 49$ & 33.3 & $\sim 95 \%$, no visual of the east side, west side has closed up a little more. \\
\hline & & 19:19 & 33.8 & $\sim 95 \%$. \\
\hline & & $19: 34$ & 34.1 & $\begin{array}{l}\sim 90 \% \text {, still not able to see east side opening. Large amount of liquid that boils } \\
\text { between shots. Able to see liquid feed flowing into west side opening. }\end{array}$ \\
\hline & & $20: 14$ & 34.7 & $\sim 90 \%$, single opening exist in west side, very thick CC. \\
\hline & & $20: 19$ & 34.8 & $\begin{array}{l}\sim 90 \% \text {, single opening exists in west side, has opened up slightly since last } \\
\text { observation. }\end{array}$ \\
\hline & & $20: 54$ & 35.4 & $\sim 90 \%$, no visual of east side, west side opening ridge is melting down some. \\
\hline & & 21:04 & 35.6 & $\sim 90 \%$, no visible changes. \\
\hline & & $21: 19$ & 35.8 & $\sim 90 \%$, thick, flat, feed is boiling on top. \\
\hline & & $21: 34$ & 36.1 & $\sim 90 \%$, unchanged for this observation. \\
\hline & & 21:49 & 36.3 & $\sim 90 \%, 8 "$ thick, glazed on opening. \\
\hline & & 22:04 & 36.6 & $\sim 90 \%$, same as above. \\
\hline & & 22:19 & 36.8 & $\sim 90 \%$, thick, glazed, feed is boiling on surface. \\
\hline & & $22: 34$ & 37.1 & $\sim 90 \%$, only west opening is visible. Feed overflows to the opening. \\
\hline & & $22: 49$ & 37.3 & $\sim 90 \%$, glazed wall/ridge at west opening and heavy boiling. \\
\hline & & 23:05 & 37.6 & $\begin{array}{c}\sim 90 \% \text {, very thick, west side has single opening with heavy boiling and some } \\
\text { splashing. }\end{array}$ \\
\hline & & 23:19 & 37.8 & $\sim 90 \%$, ridge at west opening appears to be shifting towards the melt pool. \\
\hline & & $23: 35$ & 38.1 & $\sim 90 \%$, one opening on west side, thick around opening. \\
\hline & & $23: 50$ & 38.3 & $\sim 90 \%$, opening on west side, $\mathrm{CC}$ looks about 12 in thick. \\
\hline & & 23:56 & 38.4 & $\sim 90 \%$, can only see west side opening. \\
\hline & \multirow{5}{*}{$1 / 31 / 13$} & $0: 21$ & 38.9 & $\sim 90 \%$, west side is open; east side is not visible, thick CC. \\
\hline & & $0: 34$ & 39.1 & $\sim 90 \%$, thick cap and lots of boiling feed. \\
\hline & & $0: 50$ & 39.3 & $\sim 90 \%$ no appreciable change in CC. \\
\hline & & 1:04 & 39.6 & $\sim 90 \%$, west wall ridge persist to exist. \\
\hline & & $1: 26$ & 39.9 & $\sim 85 \%$, very thick, edge of CC is seen on west side extending south. \\
\hline
\end{tabular}


Table 3.3. Cold Cap Observations (continued).

\begin{tabular}{|c|c|c|c|c|}
\hline Test & Date & Time & $\begin{array}{l}\text { Run Time } \\
\text { (hours) }\end{array}$ & Cold Cap Observations \\
\hline \multirow{29}{*}{3} & \multirow{29}{*}{$1 / 31 / 13$} & $1: 44$ & 40.2 & $\sim 85 \%$, west side opening grows towards east side, flow goes south. \\
\hline & & 2:03 & 40.6 & $\begin{array}{l}\sim 90 \% \text {, wall ridge persists. Previously increased bubbling to increase plenum } \\
\text { temperatures to help dissolve the ridge. }\end{array}$ \\
\hline & & $2: 19$ & 40.8 & $\begin{array}{c}\sim 80 \% \text {, opening on west side, grows south, very thick. Ridge on west edge, flow } \\
\text { of boiling liquid goes south. }\end{array}$ \\
\hline & & $2: 36$ & 41.1 & $\sim 80 \%$, remains the same as before. \\
\hline & & $2: 52$ & 41.4 & $\begin{array}{c}\sim 80-85 \%, \text { ridge on west of CC sends boiling liquid south, south end is open, } \\
\text { collection of feed is seen on bubblers. }\end{array}$ \\
\hline & & $3: 19$ & 41.8 & $\sim 85 \%$, ridge built up around west opening. \\
\hline & & $3: 37$ & 42.1 & $\sim 90-95 \%$, opening remains on west side with splashing feed boiling. \\
\hline & & $3: 50$ & 42.3 & $\sim 90-95 \%$, no appreciable change in CC. \\
\hline & & $4: 07$ & 42.6 & $\sim 90-85 \%$, ridge on west edge diminished. North edge grows. \\
\hline & & $4: 20$ & 42.8 & $\begin{array}{l}\sim 90-95 \% \text {, opening moving south, ridge on west edge of CC flows liquid to } \\
\text { south. }\end{array}$ \\
\hline & & $4: 34$ & 43.1 & $\sim 90-95 \%$, mostly unchanged, splashing causes build up of feed on bubblers. \\
\hline & & $4: 46$ & 43.3 & $\sim 90-95 \%$, remains the same. \\
\hline & & $5: 05$ & 43.6 & $\begin{array}{l}\sim 90 \% \text {, heavy boiling at surface, feed overflows to west opening in between } \\
\text { shots. }\end{array}$ \\
\hline & & $5: 23$ & 43.9 & $\sim 90 \%$, same as last observation. \\
\hline & & $5: 37$ & 44.1 & $\sim 90 \%$, opening on west side has ridge around it. \\
\hline & & $5: 55$ & 44.4 & $\sim 90-95 \%$, unchanged. \\
\hline & & 6:09 & 44.7 & $\sim 90-95 \%$, buildup on C2 increased slightly along with the south side. \\
\hline & & $6: 49$ & 45.3 & $\sim 95 \%$, unchanged. Feed is boiling on surface. \\
\hline & & $7: 04$ & 45.6 & $\begin{array}{c}\sim 90-95 \% \text {, feed is boiling on surface and flowing into glass pool via north west } \\
\text { opening. Light is visible in south east corner. }\end{array}$ \\
\hline & & $7: 19$ & 45.8 & $\begin{array}{l}\sim 90 \% \text { with visible opening in north east corner. Ridge is around opening in } \\
\text { north west. Feed is boiling on surface. }\end{array}$ \\
\hline & & $7: 34$ & 46.1 & $\sim 90 \%$ with no visible changes from previous observation. \\
\hline & & $7: 49$ & 46.3 & $\begin{array}{l}\sim 90 \% \text { with openings in north east and north west corners. Feed is boiling on } \\
\text { surface and flowing into north east opening. }\end{array}$ \\
\hline & & 8:04 & 46.6 & $\sim 90 \%$, no significant change since the last reading. \\
\hline & & $8: 19$ & 46.8 & $\sim 90 \%$, only one opening is visible in view in northeast port. \\
\hline & & $8: 34$ & 47.1 & $\begin{array}{l}\sim 90 \%, \text { feed is boiling on surface with openings in north east and north west } \\
\text { corners. }\end{array}$ \\
\hline & & $8: 49$ & 47.3 & $\begin{array}{c}\sim 90 \% \text {, feed is boiling on surface and flowing into melt pool via north west } \\
\text { opening. }\end{array}$ \\
\hline & & 9:04 & 47.6 & $\begin{array}{l}\sim 90 \% \text { with openings in north east and north west corners. Light is visible in } \\
\text { south east corner. Ridges at openings. }\end{array}$ \\
\hline & & $9: 19$ & 47.8 & $\sim 90 \%$ with no visual change. \\
\hline & & $9: 34$ & 48.1 & $\begin{array}{l}\sim 90 \% \text { with feed boiling on surface. Openings in north west and north west } \\
\text { corners. }\end{array}$ \\
\hline
\end{tabular}


Table 3.3. Cold Cap Observations (continued).

\begin{tabular}{|c|c|c|c|c|}
\hline Test & Date & Time & $\begin{array}{l}\text { Run Time } \\
\text { (hours) }\end{array}$ & Cold Cap Observations \\
\hline \multirow{3}{*}{3} & \multirow{3}{*}{$1 / 31 / 13$} & $9: 49$ & 48.3 & $\begin{array}{c}\sim 95 \% \text {, both east and west side openings are visible, west side has visible ridge } \\
\text { boiling feed on top. }\end{array}$ \\
\hline & & 10:04 & 48.6 & $\begin{array}{l}\sim 90 \% \text {, openings are visible on north east and north west via first view port. } \\
\text { Faint light is visible in second view port. Thick CC is visible. }\end{array}$ \\
\hline & & $10: 30$ & 49.0 & Stopped feeding. End of Test. \\
\hline \multirow{27}{*}{4} & \multirow{27}{*}{$2 / 6 / 13$} & 12:00 & 0.0 & Started feeding water at $0.8 \mathrm{l} / \mathrm{min}$. \\
\hline & & 12:10 & 0.2 & Increased water feed from 0.8 to $1.61 / \mathrm{min}$. \\
\hline & & $12: 30$ & 0.5 & Increased water feed from 1.6 to $2.41 / \mathrm{min}$. \\
\hline & & $12: 46$ & 0.8 & Increased water feed from 2.46 to $3.0 \mathrm{l} / \mathrm{min}$. \\
\hline & & 13:00 & 1.0 & Secured feeding water. Started feeding slurry. \\
\hline & & 13:05 & 1.1 & $\begin{array}{l}\sim 75-80 \% \text {, openings are visible in north west corner and north west side and wst } \\
\text { side center. Light is visible from south east and south west corners. CC is flat } \\
\text { and fluid feed is boiling on surface. }\end{array}$ \\
\hline & & 13:24 & 1.4 & $\sim 80 \%$, openings in north east corner and west side with feed boiling on surface. \\
\hline & & 13:35 & 1.6 & $\begin{array}{l}\sim 80-85 \% \text { with openings in north east corner and west side. Feed is boiling on } \\
\text { surface and flowing into melt pool in both openings. }\end{array}$ \\
\hline & & 13:49 & 1.8 & $\sim 85-90 \%$. Feed is boiling on top with feed boiling into the melt pool. \\
\hline & & 14:08 & 2.1 & $\begin{array}{l}\sim 90-95 \% \text { with openings smaller on north east corner and west side. Feed is } \\
\text { boiling on surface and flowing into melt pool via west opening. }\end{array}$ \\
\hline & & 14:23 & 2.4 & $\begin{array}{l}\sim 90-95 \% \text { with feed flowing into north east opening, small opening on west } \\
\text { side. }\end{array}$ \\
\hline & & $14: 37$ & 2.6 & $\begin{array}{l}\sim 95 \% \text { with two openings at north west corner and west side with feed flowing } \\
\text { into the melt pool via north east corner. }\end{array}$ \\
\hline & & $14: 55$ & 2.9 & $\sim 95 \%$, dry in some spots. Liquid is boiling in the center underneath feed tube. \\
\hline & & $15: 11$ & 3.2 & $\sim 95 \%$, small area of boiling, bubbling is steady, not vigorous. \\
\hline & & 15:20 & 3.3 & $\sim 95 \%$, is visibly heaving up and down. \\
\hline & & $15: 49$ & 3.8 & $\sim 95 \%$ and very wet. \\
\hline & & $16: 05$ & 4.1 & $\sim 95 \%$, very wet and having small splatter. \\
\hline & & $16: 20$ & 4.3 & $\% 95 \%$ and no change from last observation. \\
\hline & & $16: 42$ & 4.7 & $95 \%$, north west opening is starting to close. \\
\hline & & $16: 59$ & 5.0 & $\sim 95 \%$, very wet. \\
\hline & & 17:19 & 5.3 & $\sim 90 \%$, there is more feed boiling on surface. Glass is splashing out of openings. \\
\hline & & $17: 52$ & 5.9 & $\sim 95 \%$, north west opening is closed slightly, west side is opening larger. \\
\hline & & 18:42 & 6.7 & $\sim 95 \%$, openings are at north east and south west corners. \\
\hline & & 19:09 & 7.2 & $\begin{array}{l}\sim 90 \%, \text { much darker and fixed (not floating). The openings have ridges that are } \\
\text { higher than before. }\end{array}$ \\
\hline & & 19:19 & 7.3 & $\sim 90 \%$, no visible changes. \\
\hline & & 19:47 & 7.8 & $\sim 95 \%$, deep wells for each bubbler port. \\
\hline & & 20:09 & 8.2 & $\sim 95 \%$, bubbler opening at west has very thick ridge, barely open. \\
\hline
\end{tabular}


Table 3.3. Cold Cap Observations (continued).

\begin{tabular}{|c|c|c|c|c|}
\hline Test & Date & Time & $\begin{array}{c}\text { Run Time } \\
\text { (hours) }\end{array}$ & Cold Cap Observations \\
\hline \multirow{26}{*}{4} & \multirow{12}{*}{$2 / 6 / 13$} & 20:26 & 8.4 & $\sim 95 \%$ with violent splashing of glass, visible liquid spills into bubbler well. \\
\hline & & 21:04 & 9.1 & $\begin{array}{l}\sim 90 \% \text {, is fixed to the walls. Ridges around openings are } 3.4 " \text { taller than the cap. } \\
\text { Liquid is flowing over ridge. }\end{array}$ \\
\hline & & $21: 19$ & 9.3 & $\sim 90-95 \%$, no visible changes. \\
\hline & & $21: 34$ & 9.6 & $\sim 95 \%$, ridges hold boiling liquid atop CC. Openings at east and west sides. \\
\hline & & $21: 52$ & 9.9 & $\sim 95 \%$, west side opening is reduced, east side remains constant. \\
\hline & & 22:05 & 10.1 & $\begin{array}{c}\sim 95 \% \text {, large pool of boiling liquid tops cap. Ridges exits on east and west } \\
\text { edges. }\end{array}$ \\
\hline & & 22:34 & 10.6 & $\begin{array}{c}\sim 95 \% \text {, ridges persist. Boiling liquid flows towards east side opening. West side } \\
\text { opening grows slightly. }\end{array}$ \\
\hline & & 22:49 & 10.8 & $\begin{array}{l}\sim 95 \% \text {, ridge remains on west edge opening. East opening receives boiling } \\
\text { liquid, grows towards CC center. Ridge on east side opening is reduced. }\end{array}$ \\
\hline & & 23:04 & 11.1 & $\sim 95 \%$, west side opening grows, east side opening remains the same. \\
\hline & & 23:20 & 11.3 & $\begin{array}{l}\sim 95 \% \text {, boiling liquid is no longer on top of CC. CC is fixed, east side is } \\
\text { consistent, west side is reduced. Ridge is still on west side. }\end{array}$ \\
\hline & & 23:35 & 11.6 & $\begin{array}{l}\sim 95 \% \text {, prominent ridge on west side edge of the CC. Boiling liquid starts to } \\
\text { pool: east side opening is the same, west side opening is reduced. }\end{array}$ \\
\hline & & 23:49 & 11.8 & $\begin{array}{c}\sim 95 \% \text {, ridges make CC appear very thick, pooling liquid flows east, west } \\
\text { opening shrinks. }\end{array}$ \\
\hline & \multirow{14}{*}{$2 / 7 / 13$} & 0:04 & 12.1 & $\begin{array}{l}\sim 95 \% \text {, increase of bubbling results in build-up of ridges at east and west } \\
\text { openings in the CC. Boiling liquid pools between ridges. }\end{array}$ \\
\hline & & $0: 19$ & 12.3 & $\sim 95 \%$ unchanged from last observation. \\
\hline & & $0: 34$ & 12.6 & $\begin{array}{l}\sim 95 \% \text {, east side opening remains consistent, west side ridge diverts flow west } \\
\text { and south, west side opening migrates south. }\end{array}$ \\
\hline & & $0: 49$ & 12.8 & $\begin{array}{l}\sim 95 \text {, east side is open, west side is closing up slightly with ridge around it. CC } \\
\text { is thick with feed boiling on surface. }\end{array}$ \\
\hline & & $1: 08$ & 13.1 & $\sim 95 \%$, thick and east side is still open, west side opening is closing up. \\
\hline & & $1: 21$ & 13.4 & $\begin{array}{c}\sim 95 \% \text {, ridges persist. West side opening migrates south. East side opening } \\
\text { remains constant. }\end{array}$ \\
\hline & & $1: 34$ & 13.6 & $\begin{array}{l}\sim 95 \% \text {, east side is constant, west side opening ridge causes pooling of liquid } \\
\text { feed from the middle of the cap to north end. }\end{array}$ \\
\hline & & $1: 49$ & 13.8 & $\begin{array}{l}\sim 95 \% \text {, west side opening grows, east side remains. Large pool of liquid } \\
\text { between ridges. }\end{array}$ \\
\hline & & 2:05 & 14.1 & $\begin{array}{l}\sim 95 \% \text {, west side opening gains towards south side. Boiling liquid still pools } \\
\text { between ridges. East side remains steady. }\end{array}$ \\
\hline & & $2: 20$ & 14.3 & $\sim 95 \%$, east side opening is constant, west side opening is steady. \\
\hline & & $2: 34$ & 14.6 & $\begin{array}{l}\sim 95 \% \text {, ridge plus dangling fibers obscure east opening visually. West side } \\
\text { opening is steady. Liquid pools between the ridges. }\end{array}$ \\
\hline & & $2: 51$ & 14.9 & $\begin{array}{c}\sim 90-95 \% \text {, west side opening increases towards south end, east side remains } \\
\text { open. }\end{array}$ \\
\hline & & $3: 05$ & 15.1 & $\begin{array}{c}\sim 90 \% \text {, west side opening grows south east, CC reduces to } 90 \% \text {. East end of CC } \\
\text { unchanged. CC is thick and has boiling liquid on top. }\end{array}$ \\
\hline & & $3: 19$ & 15.3 & $\begin{array}{l}\sim 85-90 \% \text {, east side opening exists, but it is difficult to view as the east side } \\
\text { ridge is high. West side opening grows towards south east. }\end{array}$ \\
\hline
\end{tabular}


Table 3.3. Cold Cap Observations (continued).

\begin{tabular}{|c|c|c|c|c|}
\hline Test & Date & Time & $\begin{array}{l}\text { Run Time } \\
\text { (hours) }\end{array}$ & Cold Cap Observations \\
\hline \multirow{24}{*}{4} & \multirow{24}{*}{$2 / 7 / 13$} & $3: 35$ & 15.6 & $\begin{array}{l}\sim 85-90 \% \text {, after glass discharge boiling liquid flowed into west side opening, } \\
\text { east side opening appears the same size. West side opening increases. }\end{array}$ \\
\hline & & $3: 44$ & 15.7 & $\begin{array}{c}\sim 90-95 \text {, east and west sides are open. Both have ridges, feed is boiling on } \\
\text { surface between openings. }\end{array}$ \\
\hline & & 4:07 & 16.1 & $\begin{array}{l}\text { East side unchanged, west side is open on west and south edges. Strong } \\
\text { bubbling builds a ridge on west side of CC. Boiling liquid pools on top of CC. }\end{array}$ \\
\hline & & $4: 19$ & 16.3 & $\begin{array}{l}\sim 85-90 \% \text {, south side is open, north side is fixed to melter wall. East side } \\
\text { opening remains constant and west side is visible to south. }\end{array}$ \\
\hline & & $4: 34$ & 16.6 & $\begin{array}{l}\sim 90 \% \text {, east side opening unchanged, west side edge of CC is seen towards } \\
\text { south side. Ridges are prominent. }\end{array}$ \\
\hline & & $4: 49$ & 16.8 & $\begin{array}{l}\sim 85-90 \% \text {, east side opening grows, ridges persist, opening on west side } \\
\text { enlarges. }\end{array}$ \\
\hline & & 5:04 & 17.1 & $\sim 85-90 \%$, no appreciable change. \\
\hline & & $5: 28$ & 17.5 & $\sim 85 \%$, openings are getting larger. Ridges are increased. \\
\hline & & $5: 42$ & 17.7 & $\sim 85 \%$, no change. \\
\hline & & $5: 48$ & 17.8 & $\begin{array}{l}\sim 85-90 \% \text {, openings on east and west sides. Large ridge on east side, feed is } \\
\text { boiling on surface. }\end{array}$ \\
\hline & & $6: 04$ & 18.1 & $\begin{array}{l}\sim 85-90 \% \text {, east and west side openings are divided by a large ridge that keeps } \\
\text { growing in height with feed splashing into melt pool from boiling on CC } \\
\text { surface. }\end{array}$ \\
\hline & & $6: 59$ & 19.0 & $\begin{array}{l}\sim 95 \% \text { with opening in north east and north west corners, feed is boiling on } \\
\text { surface. }\end{array}$ \\
\hline & & $7: 15$ & 19.3 & $\begin{array}{l}\sim 95 \% \text { with no visible ridges around north east and north west corner openings. } \\
\text { Feed is boiling on surface. }\end{array}$ \\
\hline & & $7: 34$ & 19.6 & $\sim 95 \%$ with no visible changes from previous observation. \\
\hline & & $7: 48$ & 19.8 & $\begin{array}{l}\sim 90 \% \text { with north east corner opening up and allowing feed into melt pool, } \\
\text { slight ridge around north west opening. }\end{array}$ \\
\hline & & $8: 19$ & 20.3 & $\begin{array}{l}\sim 90 \% \text {, openings are visible via north view port in north east and north west } \\
\text { corners. Opening is visible via center view port on west center and light is } \\
\text { visible in south east and south west corners via south view port. Feed is boiling } \\
\text { on surface. }\end{array}$ \\
\hline & & $8: 34$ & 20.6 & $\begin{array}{l}\sim 90 \% \text {, feed is visible, boiling on top and pouring into both open holes into the } \\
\text { melt pool. }\end{array}$ \\
\hline & & $8: 49$ & 20.8 & $\begin{array}{c}\sim 90-95 \% \text {, a single ridge is starting to develop around the cold cap opens up } \\
\text { boiling feed which is pooling on top of CC. }\end{array}$ \\
\hline & & 9:04 & 21.1 & $\begin{array}{c}\sim 90 \% \text {, openings are visible in north east and north west corners with ridges. } \\
\text { Feed is boiling on surface. Light is visible in south east corner via south view } \\
\text { port. }\end{array}$ \\
\hline & & $9: 19$ & 21.3 & $\begin{array}{l}\begin{array}{l}\sim 90 \% \text {, no visual changes from last observation. Now at maximum bubbling rate } \\
\text { of } 78 \mathrm{lpm} .\end{array} \\
\end{array}$ \\
\hline & & $9: 35$ & 21.6 & $\begin{array}{l}\sim 90 \% \text { with no visible ridges on north east corner allowing feed to flow into } \\
\text { melt pool. Open in north west with ridges. }\end{array}$ \\
\hline & & $9: 52$ & 21.9 & $\begin{array}{l}\sim 90-95 \% \text { with visible openings in north east and north west corners. Ridges are } \\
\text { visible around both openings. Feed is boiling on surface. }\end{array}$ \\
\hline & & $10: 06$ & 22.1 & $\begin{array}{c}\sim 90 \% \text {, west side opening has a ridge east side opening is visible with boiling } \\
\text { feed on cap. }\end{array}$ \\
\hline & & $10: 22$ & 22.4 & $\sim 90 \%$, no visible changes. \\
\hline
\end{tabular}


Table 3.3. Cold Cap Observations (continued).

\begin{tabular}{|c|c|c|c|c|}
\hline Test & Date & Time & $\begin{array}{l}\text { Run Time } \\
\text { (hours) }\end{array}$ & Cold Cap Observations \\
\hline \multirow{27}{*}{4} & \multirow{27}{*}{$2 / 7 / 13$} & $10: 36$ & 22.6 & $\begin{array}{c}\sim 90 \% \text {, openings on east and west side of the melter, visible both openings have } \\
\text { ridges with boiling feed on top of cap. }\end{array}$ \\
\hline & & 11:04 & 23.1 & $\begin{array}{l}\sim 90-95 \% \text {, feed is boiling on top with low ridge around the east bubbler are with } \\
\text { some feed entering the hole plus a high ridge around the west hole. }\end{array}$ \\
\hline & & 11:19 & 23.3 & $\begin{array}{l}79-95 \% \text {, north east hole opened slightly, north west hole is unchanged. Feed is } \\
\text { boiling on top with slight increase in the flow going into the north east hole. }\end{array}$ \\
\hline & & $11: 34$ & 23.6 & $\sim 90 \%$, north east hole has feed flowing into it, north east hole is unchanged. \\
\hline & & $11: 49$ & 23.8 & $\begin{array}{c}\sim 90-95 \% \text { with ridges around north east and north west openings. Feed is } \\
\text { boiling on surface. }\end{array}$ \\
\hline & & 12:12 & 24.2 & $\begin{array}{l}\sim 95 \% \text { with } 10 " \text { ridge around north west, smaller ridge around north east corner. } \\
\text { Feed is boiling on surface. }\end{array}$ \\
\hline & & $12: 32$ & 24.5 & $\begin{array}{l}\sim 95 \% \text { with large ridges above both openings. North east opening is barely } \\
\text { visible from view port. Feed is boiling on surface. }\end{array}$ \\
\hline & & $12: 49$ & 24.8 & $\begin{array}{l}\sim 95 \% \text { with ridges around both openings. North east opening is barely visible } \\
\text { from openings. Feed is boiling on top of the cold cap. }\end{array}$ \\
\hline & & 13:04 & 25.1 & $\begin{array}{l}\sim 95 \% \text {, north east opening is no longer visible in first view port. The visible } \\
\text { opening has a ridge and feed is boiling on top of the cap. }\end{array}$ \\
\hline & & 13:34 & 25.6 & $\begin{array}{l}\sim 95 \%, \text { north east opening is barely visible, large ridge around north west } \\
\text { opening making feed and surface not visible. }\end{array}$ \\
\hline & & 13:49 & 25.8 & $\begin{array}{l}\sim 95 \% \text { with feed no visible boiling on surface, north east opening is still barely } \\
\text { visible. Large ridge around north west opening with glass bubbling out. }\end{array}$ \\
\hline & & 14:04 & 26.1 & $\sim 95 \%$ with no visible change from previous observation. \\
\hline & & 14:19 & 26.3 & $\begin{array}{c}\sim 90-95 \% \text { with opening in north east and north west corners. North east corner } \\
\text { is barely visible. North west opening is visually larger than before with glass } \\
\text { bubbling out of melt pool. }\end{array}$ \\
\hline & & 14:40 & 26.7 & $\begin{array}{l}\sim 95 \% \text {, north east opening is no longer visible from view port. North west } \\
\text { corner opening still has ridge around opening. Feed is boiling on surface. }\end{array}$ \\
\hline & & 15:04 & 27.1 & $\sim 95 \%$, no visual of the east side, west side opening has a large ridge around it. \\
\hline & & 15:19 & 27.3 & $\sim 95 \%$, same as last observation. \\
\hline & & $15: 33$ & 27.6 & $\sim 95 \%$, flat with large opening. \\
\hline & & $15: 49$ & 27.8 & $\sim 95-97 \%$, north east side opening is very small. \\
\hline & & 16:04 & 28.1 & $\begin{array}{l}\sim 95 \% \text {, west side has opened up slightly to a cone shaped opening. Glass is } \\
\text { spitting out. Feed is boiling briefly between the shots. }\end{array}$ \\
\hline & & 16:19 & 28.3 & $\sim 95 \%$, unchanged for this observation. \\
\hline & & $16: 45$ & 28.7 & $\sim 95 \%$. \\
\hline & & $16: 49$ & 28.8 & $\sim 95 \%$, now can see inside after CC collapsed. \\
\hline & & 17:04 & 29.1 & $\sim 95 \%$, opened up on the west side. \\
\hline & & $17: 19$ & 29.3 & $\begin{array}{c}\sim 95 \% \text {, thick and feed is boiling on top and there are ridges around the west } \\
\text { opening, west opening is not visible. }\end{array}$ \\
\hline & & $17: 34$ & 29.6 & $\begin{array}{l}\sim 95 \% \text {, able to see east side at this time. The ridge has melted down somewhat, } \\
\text { west side is slightly opened up. }\end{array}$ \\
\hline & & $17: 49$ & 29.8 & $\begin{array}{l}\sim 90 \% \text {, north east opening is visible in the first view port. Feed is visible going } \\
\text { into north west opening via second view port. }\end{array}$ \\
\hline & & 18:04 & 30.1 & $\begin{array}{c}\sim 90 \%, \text { north west pool no longer is visible in second view port. All else } \\
\text { unchanged. }\end{array}$ \\
\hline
\end{tabular}


Table 3.3. Cold Cap Observations (continued).

\begin{tabular}{|c|c|c|c|c|}
\hline Test & Date & Time & $\begin{array}{l}\text { Run Time } \\
\text { (hours) }\end{array}$ & Cold Cap Observations \\
\hline \multirow{26}{*}{4} & \multirow{22}{*}{$2 / 7 / 13$} & $18: 19$ & 30.3 & $\sim 90 \%$, still able to see east side opening. Ridges are much lower. \\
\hline & & $18: 41$ & 30.7 & $\sim 90 \%$, melter pressure went positive due to CC collapsing. \\
\hline & & $18: 49$ & 30.8 & $\sim 90 \%$, level looks lower since CC collapsed. \\
\hline & & 19:04 & 31.1 & $\sim 90 \%$, no changes. \\
\hline & & 19:19 & 31.3 & $\sim 90 \%$, has opened up somewhat. \\
\hline & & $19: 34$ & 31.6 & $\begin{array}{l}\sim 85 \% \text {, east side has a new hole is opening slightly south of the other opening. } \\
\text { No other changes. }\end{array}$ \\
\hline & & 19:49 & 31.8 & $\begin{array}{l}\sim 90 \% \text {, large liquid basin between openings, lots of visible light but openings } \\
\text { appear small. Large stalactite/stalagmite hanging from plenum thermocouple in } \\
\text { south view port. }\end{array}$ \\
\hline & & $20: 19$ & 32.3 & $\sim 90 \%$. \\
\hline & & $20: 36$ & 32.6 & $\begin{array}{l}\sim 90 \% \text {, ridges at east and west openings. Liquid is pooling on top of CC and } \\
\text { flowing west. West edge of CC is visible through view port. }\end{array}$ \\
\hline & & 20:49 & 32.8 & $\begin{array}{c}\sim 85 \% \text {, west edge of CC extends south. Pronounced ridge on east and west } \\
\text { openings. }\end{array}$ \\
\hline & & 21:04 & 33.1 & $\begin{array}{l}\sim 85-90 \% \text {, boiling liquid pools between ridges flows into melt pool on south } \\
\text { west edge opening. }\end{array}$ \\
\hline & & $21: 20$ & 33.3 & $\sim 90 \%$, molten glass is erupting into boiling pool of liquid feed. \\
\hline & & $21: 34$ & 33.6 & $\begin{array}{l}\sim 85-90 \% \text {, openings increase towards north edge of CC. Ridges block flow of } \\
\text { boiling liquid. Pronounced ridge on west edge opening. }\end{array}$ \\
\hline & & $21: 49$ & 33.8 & $\begin{array}{l}\sim 85 \% \text {, boiling liquid remains on top of cap. North side is opening up. Liquid is } \\
\text { coming from south east corner, ridges remain along openings. }\end{array}$ \\
\hline & & 22:05 & 34.1 & $\begin{array}{c}\sim 85-90 \% \text {, breech in ridge along east opening sends boiling liquid into east side } \\
\text { opening. West edge is seen. }\end{array}$ \\
\hline & & $22: 19$ & 34.3 & $\begin{array}{c}\sim 85 \% \text {, west edge of CC proceeds eastward north. Boiling flow is sent to east } \\
\text { opening. }\end{array}$ \\
\hline & & $22: 34$ & 34.6 & $\begin{array}{c}\sim 85-90 \%, \text { liquid pool on top of cap. Ridges around both openings, more light } \\
\text { from north edge than south edge of cap. }\end{array}$ \\
\hline & & $22: 49$ & 34.8 & $\begin{array}{l}\sim 85 \% \text {, bubbling allows a breech in east edge ridge: boiling liquid flow to south } \\
\text { east side of cap extending east opening southward. West side has a large ridge, } \\
\text { dangling fibers are seen. }\end{array}$ \\
\hline & & 23:04 & 35.1 & $\sim 85-90 \%$, mostly unchanged. \\
\hline & & $23: 16$ & 35.3 & $\sim 90 \%$, appears mostly unchanged with a lot of liquid feed on cap surface. \\
\hline & & $23: 34$ & 35.6 & $\begin{array}{l}\sim 90 \% \text {, east edge loses ridge boiling flow goes east. Space between east edge of } \\
\text { cap and west edge of CC narrows. CC appears thinner than before. }\end{array}$ \\
\hline & & $23: 49$ & 35.8 & $\begin{array}{l}\sim 85 \% \text { with glass level reduced during discharge. } \mathrm{CC} \text { is thick holds boiling } \\
\text { liquid which flows into the east opening which is migrating north. A high ridge } \\
\text { exists on west of cap. }\end{array}$ \\
\hline & \multirow{4}{*}{$2 / 8 / 13$} & $0: 05$ & 36.1 & $\begin{array}{c}\sim 80-85 \% \text {, between east and west opening narrows. Pool of boiling liquid } \\
\text { remains. }\end{array}$ \\
\hline & & $0: 19$ & 36.3 & $\begin{array}{c}\sim 80-85 \% \text {, boiling liquid pools atop cap. Ridges east and west edges of cap } \\
\text { openings migrate to north wall. West edge is seen. }\end{array}$ \\
\hline & & $0: 34$ & 36.6 & $\begin{array}{l}\sim 85 \% \text { increases towards west wall. High ridges exist around east and west } \\
\text { openings. Liquid pools. }\end{array}$ \\
\hline & & $0: 49$ & 36.8 & $\begin{array}{c}\sim 85 \% \text {, ridges on east and west openings persist with lots of feed pooled } \\
\text { between them. }\end{array}$ \\
\hline
\end{tabular}


Table 3.3. Cold Cap Observations (continued).

\begin{tabular}{|c|c|c|c|c|}
\hline Test & Date & Time & $\begin{array}{c}\text { Run Time } \\
\text { (hours) }\end{array}$ & Cold Cap Observations \\
\hline \multirow{24}{*}{4} & \multirow{24}{*}{$2 / 8 / 13$} & $1: 03$ & 37.0 & $\sim 85 \%$, no apparent change. \\
\hline & & $1: 19$ & 37.3 & $\begin{array}{l}\sim 90-95 \%, \text { north edge attached to wall, east opening reduced, hanging fibers and } \\
\text { ridges persist. Pooling liquid flows westerly. }\end{array}$ \\
\hline & & $1: 36$ & 37.6 & $\begin{array}{l}\sim 90 \% \text {, splashing and bubbling cause ridge build up around east and west } \\
\text { openings. Liquid is boiling on surface. }\end{array}$ \\
\hline & & $1: 49$ & 37.8 & $\begin{array}{l}\sim 90 \% \text {, high ridges make it difficult to see top of CC. No boiling liquid is seen } \\
\text { on top CC. Dangling fibers over east opening. }\end{array}$ \\
\hline & & 2:04 & 38.1 & $\begin{array}{l}\text { West edge ridge has grown onto the D1 bubbler pipe. East and west openings } \\
\text { diminish. }\end{array}$ \\
\hline & & $2: 19$ & 38.3 & $\begin{array}{c}\sim 90-95 \% \text {, ridge blocks view of east opening. Light is seen in south east corner } \\
\text { of melt pool. West edge opening diminishes. }\end{array}$ \\
\hline & & $2: 28$ & 38.5 & $\begin{array}{l}\sim 90 \% \text {, visual obstruction on the north view port. Large accumulation hangs on } \\
\text { the Lance bubbler. }\end{array}$ \\
\hline & & $2: 31$ & 38.5 & $\begin{array}{l}\sim 90 \% \text {, east opening is partially visible, heavy boiling on the surface, although } \\
\text { not visible west opening exists. }\end{array}$ \\
\hline & & $2: 49$ & 38.8 & $\begin{array}{l}\sim 90 \%, \text { mid-port is totally obstructed, no visual is available. South view port } \\
\text { shows D3 bubbler, also coated by solid build up. }\end{array}$ \\
\hline & & $3: 04$ & 39.1 & $\begin{array}{l}\sim 90-95 \% \text {, east opening is difficult to see, west side opening extends southward. } \\
\text { Light is seen in south east corner of melt pool. }\end{array}$ \\
\hline & & $3: 19$ & 39.3 & $\sim 90-95 \%$, large ridge is on the west. \\
\hline & & $3: 34$ & 39.6 & $\begin{array}{c}\sim 90-95 \% \text {, unable to see into east opening due to ridge and dangling fibers, can } \\
\text { see emitted light. West opening migrates south. }\end{array}$ \\
\hline & & $3: 49$ & 39.8 & $\begin{array}{l}\sim 90-95 \% \text {, east opening is mostly obscured by ridge and openings remain } \\
\text { mostly unchanged. }\end{array}$ \\
\hline & & 4:04 & 40.1 & $\sim 90-95 \%$, east opening emits light, west opening migrates south. \\
\hline & & $4: 34$ & 40.6 & $\begin{array}{l}\text { Unable to see into east side opening. West opening is narrow, moving southerly } \\
\text { and easterly. Light from south east corner of melt pool covers } 90-95 \% \text { melt } \\
\text { pool. }\end{array}$ \\
\hline & & $5: 12$ & 41.2 & $\begin{array}{l}\sim 95 \% \text {, east opening is not visible due to ridge. West side has feed dripping into } \\
\text { melt pool. Cannot see CC surface due to ridge }\end{array}$ \\
\hline & & $5: 39$ & 41.7 & $\sim 95 \%$, west side is open surrounded by a large ridge unable to see east side. \\
\hline & & $6: 12$ & 42.2 & $\begin{array}{c}\sim 95 \% \text {, east side is open with a large ridge around it, unable to see west side of } \\
\text { melter. }\end{array}$ \\
\hline & & $6: 49$ & 42.8 & $\begin{array}{l}\sim>95 \% \text {, small opening is visible via north view port. Light is visible via center } \\
\text { and south ports. Large mound running length of melter from north to south. } \\
\text { Feed is boiling on surface. }\end{array}$ \\
\hline & & 7:04 & 43.1 & $\sim>95 \%$, conditions are unchanged from the last observation. \\
\hline & & $7: 21$ & 43.4 & $\begin{array}{l}\sim 95 \% \text {, small opening on west side via north view port. Light is visible through } \\
\text { south view port. Feed is boiling on surface. }\end{array}$ \\
\hline & & $7: 40$ & 43.7 & $\sim 95 \%$, no visual change from the previous observation. \\
\hline & & $7: 55$ & 43.9 & $\begin{array}{l}\sim 95 \% \text {, feed is visibly flowing into the north west opening and boiling on } \\
\text { surface. Light is visible from south view port. }\end{array}$ \\
\hline & & $8: 10$ & 44.2 & $\begin{array}{l}\sim 95 \% \text {, opening is visible via north view port. Light is visible via center and } \\
\text { south view ports. Ridge at center of melt pool has decreased in size. Feed is } \\
\text { boiling on surface. }\end{array}$ \\
\hline
\end{tabular}


Table 3.3. Cold Cap Observations (continued).

\begin{tabular}{|c|c|c|c|c|}
\hline Test & Date & Time & $\begin{array}{l}\text { Run Time } \\
\text { (hours) }\end{array}$ & Cold Cap Observations \\
\hline \multirow{26}{*}{4} & \multirow{26}{*}{$2 / 8 / 13$} & $8: 19$ & 44.3 & $\begin{array}{l}\sim 95 \% \text { with feed is boiling on top, a small amount of light is visible in the center } \\
\text { view port. CC is still thick but the ridges are surrounding. The west side } \\
\text { opening is not too pronounced. }\end{array}$ \\
\hline & & $8: 34$ & 44.6 & $\begin{array}{c}\sim 95 \% \text { with feed boiling on surface, feed is moving it over the rim of the } \\
\text { opening into melter. }\end{array}$ \\
\hline & & $8: 49$ & 44.8 & $\begin{array}{l}\sim 95 \% \text {, feed is bale to build up and roll into the melt pool. Feed is bubbling on } \\
\text { top of the CC. }\end{array}$ \\
\hline & & $9: 10$ & 45.2 & $\begin{array}{l}\sim 95 \% \text {, feed is boiling on surface and flowing into melt pool through north west } \\
\text { opening. }\end{array}$ \\
\hline & & $9: 20$ & 45.3 & $\begin{array}{c}\sim 90 \% \text {, opening on west side is visible from north/center viewport. Light is } \\
\text { visible from south view port. }\end{array}$ \\
\hline & & $9: 35$ & 45.6 & $\begin{array}{l}\sim 95 \% \text {, feed is boiling surface, small ridge around north west opening with glass } \\
\text { bubbling up out of hole. }\end{array}$ \\
\hline & & $9: 49$ & 45.8 & $\sim 95 \%$, no significant changes from the last time. \\
\hline & & 10:09 & 46.2 & $\sim 95 \%$, openings on west side and light are visible from east side. \\
\hline & & $10: 27$ & 46.5 & $\begin{array}{l}\sim 95 \% \text {, opening on west side is visible with ridge, opening on east side is visible } \\
\text { a little boiling feed on cap. }\end{array}$ \\
\hline & & $10: 49$ & 46.8 & $\begin{array}{c}\sim 95 \% \text { with boiling feed on the surface. Openings in south east and north west } \\
\text { corners are visible via north view port. Small opening is visible on west-mid } \\
\text { side via center view port. Light is visible via south view port. }\end{array}$ \\
\hline & & 11:04 & 47.1 & $\sim 95 \%$, conditions are unchanged from last observation. \\
\hline & & $11: 20$ & 47.3 & $\begin{array}{l}\sim 95 \% \text {, openings in south east and north west corners are visible via north } \\
\text { viewport. Small opening on west-center side is visible via center view port. } \\
\text { Light is visible through south port. }\end{array}$ \\
\hline & & $11: 34$ & 47.6 & $\sim 95 \%$, conditions are unchanged from last observation. \\
\hline & & $11: 49$ & 47.8 & $\sim 95 \%$, conditions are unchanged from last observation. \\
\hline & & $12: 19$ & 48.3 & $\begin{array}{c}\sim 95 \% \text {, opening is visible in north west corner, west side and center light is } \\
\text { visible on east side. }\end{array}$ \\
\hline & & $12: 34$ & 48.6 & $\begin{array}{c}\sim 95 \% \text {, feed is boiling on surface and flowing into the melt pool via north west } \\
\text { corner. Light is still visible on east side. }\end{array}$ \\
\hline & & $12: 49$ & 48.8 & $\sim 95 \%$ with no visual changes from the last observation. \\
\hline & & 13:04 & 49.1 & $\begin{array}{l}\sim 95 \% \text { with feed boiling on surface. Glass is bubbling out from the opening onto } \\
\text { top of CC. }\end{array}$ \\
\hline & & $13: 19$ & 49.3 & $\begin{array}{l}\sim 95 \% \text {, feed is boiling on surface and flowing into openings. Openings are in } \\
\text { north west corner and west side center. Light is visible from south view port. }\end{array}$ \\
\hline & & $13: 34$ & 49.6 & $\sim 95 \%$, north west is visible, no change at this time. \\
\hline & & $13: 49$ & 49.8 & $\begin{array}{l}\sim 95 \% \text {, west side is visible; opening with ridge on east side is not visible at this } \\
\text { time. }\end{array}$ \\
\hline & & 14:04 & 50.1 & $\sim 95 \%$, no visible change. \\
\hline & & $14: 19$ & 50.3 & $\begin{array}{c}\sim 95 \% \text {, west side opening is still visible with a ridge, east side has a visible } \\
\text { light. }\end{array}$ \\
\hline & & $14: 38$ & 50.6 & $\begin{array}{c}\sim 95 \% \text {, very deep crevice is seen in north view port. No light is visible in mid } \\
\text { view port, light is visible beyond. }\end{array}$ \\
\hline & & $14: 58$ & 51.0 & $\begin{array}{l}\sim 95 \% \text {, opening has completely closed via north view port. Very little light is } \\
\text { visible. }\end{array}$ \\
\hline & & 15:04 & 51.1 & $\sim 95 \%$, small opening at west side, little light is visible. \\
\hline
\end{tabular}


Table 3.3. Cold Cap Observations (continued).

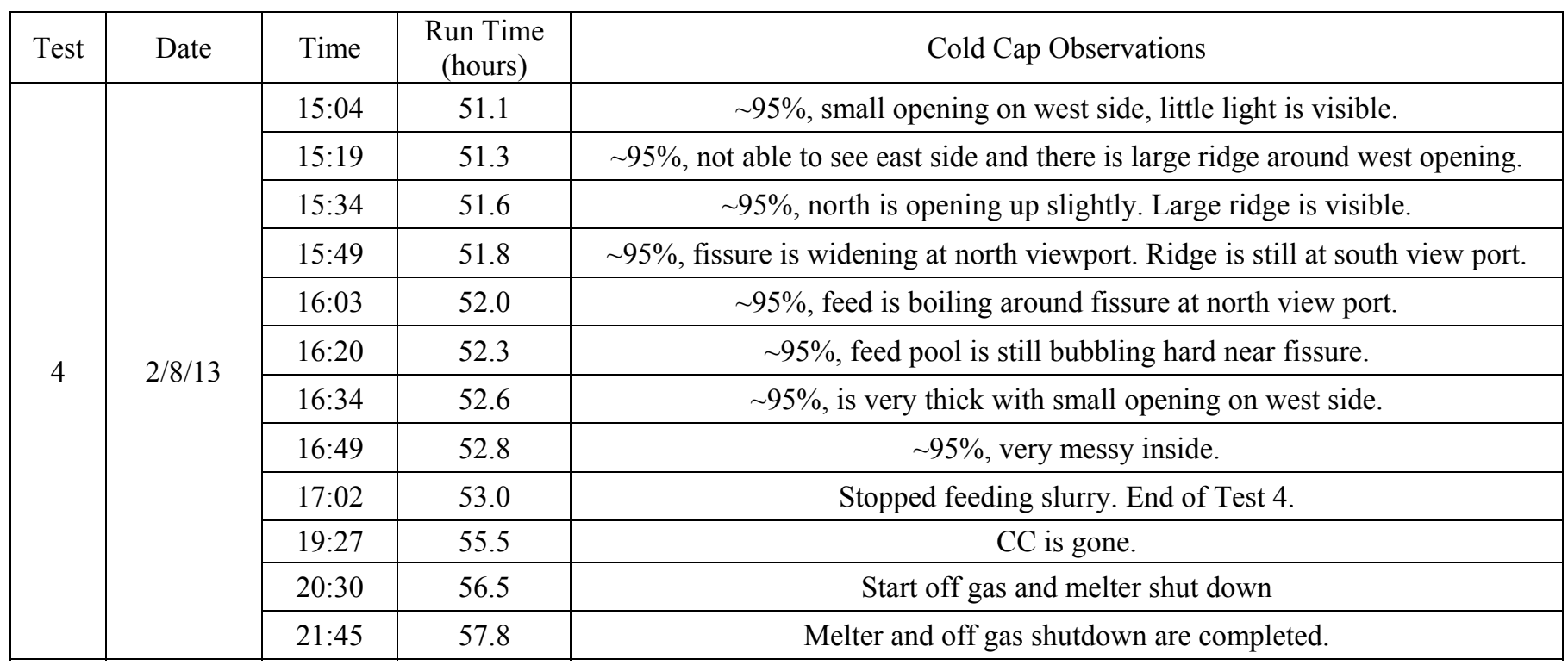


Table 3.4. DM1200 Melter System Measured Parameters.

\begin{tabular}{|c|c|c|c|c|c|c|}
\hline \multirow{2}{*}{\multicolumn{3}{|c|}{ TEST 1}} & \multirow{3}{*}{$\begin{array}{c}\text { Steady } \\
\text { State }\end{array}$} & \multicolumn{3}{|c|}{ Overall } \\
\hline & & & & avg & $\min$ & $\max$ \\
\hline \multirow{22}{*}{ 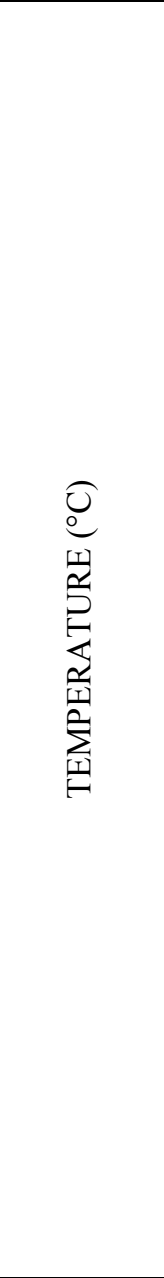 } & \multirow{8}{*}{ Glass } & 13" from floor E & & 1134 & 1080 & 1158 \\
\hline & & $15.5 "$ from floor $\mathrm{E}$ & 1131 & 1130 & 1075 & 1158 \\
\hline & & $18 "$ from floor $\mathrm{E}$ & 1130 & 1129 & 1071 & 1161 \\
\hline & & $27 "$ from floor E & 1111 & 1104 & 952 & 1142 \\
\hline & & 13" from floor $\mathrm{W}$ & 1154 & 1157 & 1133 & 1177 \\
\hline & & 15. " from floor $\mathrm{W}^{\prime}$ & 1155 & 1158 & 1135 & 1179 \\
\hline & & 18 " from floor $\mathrm{W}$ & 1155 & 1158 & 1131 & 1175 \\
\hline & & 27 " from floor $\mathrm{W}$ & 1142 & 1130 & 855 & 1161 \\
\hline & \multirow{6}{*}{ Plenum } & $\begin{array}{l}\text { T/W Plenum TC 8" } \\
\text { below lid (A2) }\end{array}$ & 517 & 510 & 337 & 848 \\
\hline & & $\begin{array}{c}\text { Exp. Plenum TC 17" } \\
\text { below lid (B2) }\end{array}$ & 628 & 580 & 413 & 851 \\
\hline & & $\begin{array}{l}\text { Exp. Plenum TC 17" } \\
\text { below lid (B3) }\end{array}$ & 532 & 504 & 286 & 847 \\
\hline & & $\begin{array}{c}\text { Exp. Plenum TC 17" } \\
\text { below lid (C1) }\end{array}$ & 557 & 527 & 323 & 833 \\
\hline & & $\begin{array}{c}\text { Exp. Plenum TC 17" } \\
\text { below lid (C2) }\end{array}$ & 556 & 525 & 341 & 840 \\
\hline & & $\begin{array}{c}\text { T/W Plenum TC 17" } \\
\text { below lid (D2) }\end{array}$ & 547 & 522 & 356 & 834 \\
\hline & \multirow{3}{*}{ Discharge } & TC 1 & 1038 & 1017 & 906 & 1070 \\
\hline & & TC 2 & 1071 & 1049 & 937 & 1093 \\
\hline & & Air Flow & 268 & 262 & 216 & 283 \\
\hline & \multirow{3}{*}{ Electrode } & East & 1132 & 1128 & 1054 & 1154 \\
\hline & & West & 1129 & 1130 & 1078 & 1155 \\
\hline & & Bottom & 1049 & 1039 & 949 & 1065 \\
\hline & \multirow{2}{*}{ Film Cooler } & Added Air & 76 & 76 & 74 & 78 \\
\hline & & Outlet & 367 & 353 & 201 & 493 \\
\hline \multicolumn{3}{|c|}{ Glass Resistance (ohms) } & 0.089 & 0.087 & 0.078 & 0.099 \\
\hline \multicolumn{3}{|c|}{ Glass Density $(\mathrm{g} / \mathrm{ml})$} & 2.26 & 2.29 & 2.20 & 2.48 \\
\hline \multicolumn{3}{|c|}{ Glass Pool Depth (inches) } & 29.4 & 29.2 & 27.4 & 30.4 \\
\hline \multirow{3}{*}{ Electrodes } & & urrent (A) & 1448 & 1392 & 1139 & 1519 \\
\hline & & oltage (V) & 129 & 121 & 103 & 131 \\
\hline & & ower $(\mathrm{kW})$ & 187 & 169 & 124 & 191 \\
\hline \multirow{3}{*}{$\begin{array}{l}\text { Lance } \\
\text { Bubblers }\end{array}$} & 1 & Rate (lpm) & 38.5 & 30.2 & 2.0 & 51.2 \\
\hline & 2 & Rate (lpm) & 39.6 & 31.0 & 2.1 & 40.6 \\
\hline & \multicolumn{2}{|c|}{ Total Bubbling (lpm) } & 79.3 & 62.5 & 5.3 & 81.0 \\
\hline
\end{tabular}


Table 3.4. DM1200 Melter System Measured Parameters (continued).

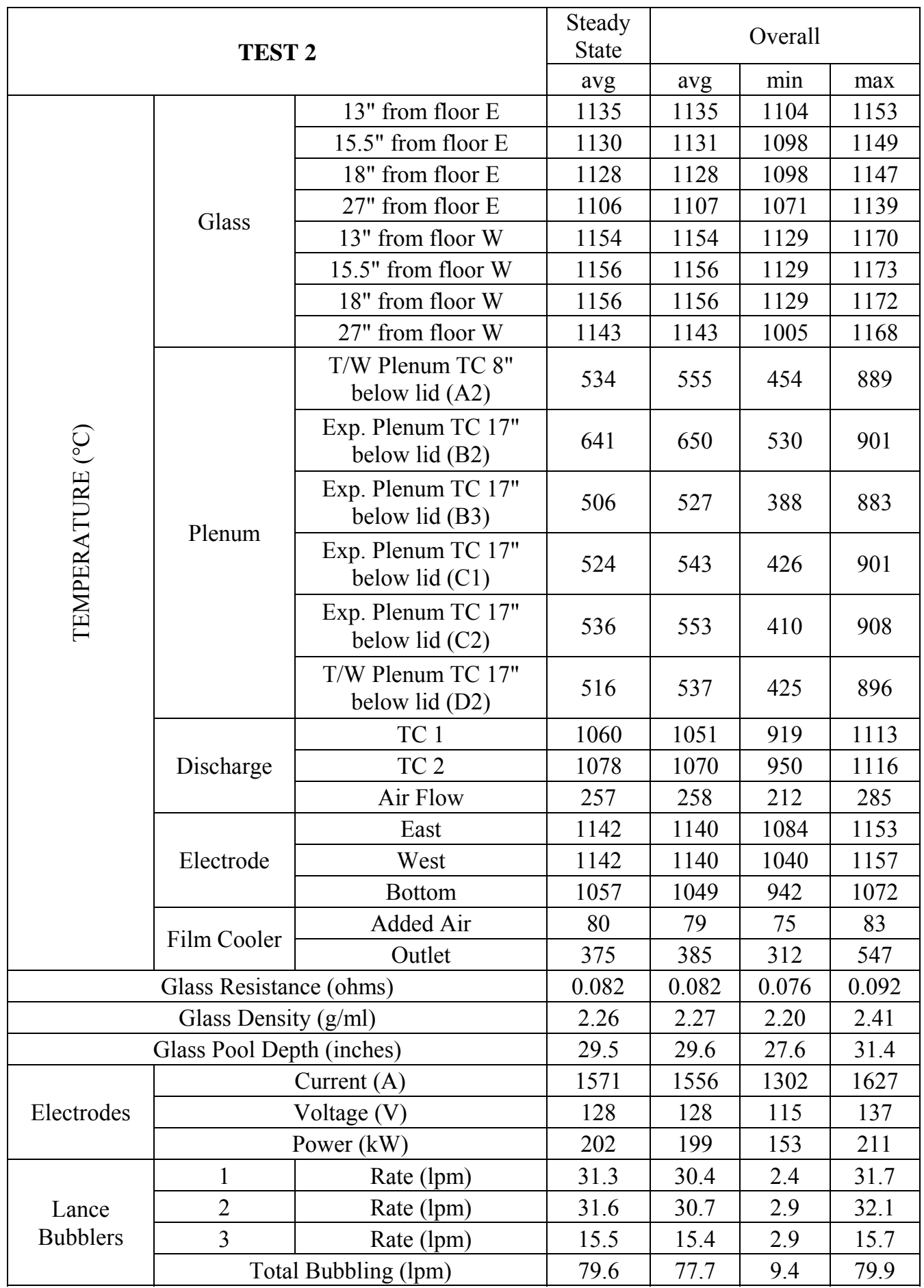


Table 3.4. DM1200 Melter System Measured Parameters (continued).

\begin{tabular}{|c|c|c|c|c|c|c|}
\hline \multirow{2}{*}{\multicolumn{3}{|c|}{ TEST 3}} & \multirow{3}{*}{$\begin{array}{c}\text { Steady } \\
\text { State }\end{array}$} & \multicolumn{3}{|c|}{ Overall } \\
\hline & & & & avg & $\min$ & $\max$ \\
\hline \multirow{22}{*}{ 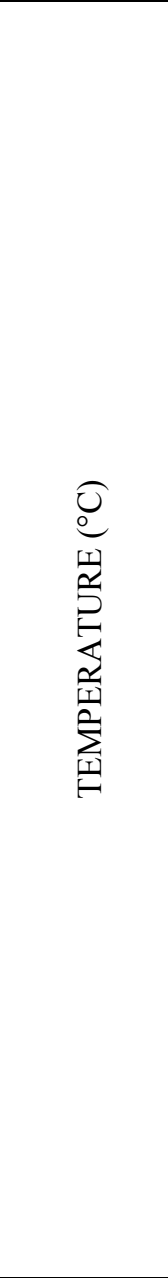 } & \multirow{8}{*}{ Glass } & $13 "$ from floor $\mathrm{E}$ & & 1143 & 1117 & 1173 \\
\hline & & $15.5 "$ from floor $\mathrm{E}$ & 1140 & 1137 & 1111 & 1168 \\
\hline & & $18 "$ from floor $\mathrm{E}$ & 1137 & 1135 & 1108 & 1164 \\
\hline & & $27 "$ from floor E & 1117 & 1110 & 1021 & 1146 \\
\hline & & 13 " from floor $\mathrm{W}$ & 1155 & 1154 & 1132 & 1179 \\
\hline & & $15.5 "$ from floor $\mathrm{W}$ & 1155 & 1154 & 1132 & 1179 \\
\hline & & 18 " from floor $\mathrm{W}$ & 1155 & 1154 & 1125 & 1178 \\
\hline & & 27 " from floor $\mathrm{W}$ & 1137 & 1129 & 942 & 1161 \\
\hline & \multirow{6}{*}{ Plenum } & $\begin{array}{c}\text { T/W Plenum Tc } 8 " \\
\text { below lid (A2) }\end{array}$ & 473 & 507 & 424 & 858 \\
\hline & & $\begin{array}{l}\text { Exp. Plenum TC 17" } \\
\text { below lid (B2) }\end{array}$ & 557 & 562 & 461 & 841 \\
\hline & & $\begin{array}{c}\text { Exp. Plenum TC 17" } \\
\text { below lid (B3) }\end{array}$ & 437 & 477 & 374 & 838 \\
\hline & & $\begin{array}{l}\text { Exp. Plenum TC 17" } \\
\text { below lid (C1) }\end{array}$ & 433 & 474 & 394 & 864 \\
\hline & & $\begin{array}{c}\text { Exp. Plenum TC 17" } \\
\text { below lid (C2) }\end{array}$ & 453 & 489 & 391 & 867 \\
\hline & & $\begin{array}{l}\text { T/W Plenum TC 17" } \\
\text { below lid (D2) }\end{array}$ & 473 & 504 & 436 & 871 \\
\hline & \multirow{3}{*}{ Discharge } & TC 1 & 941 & 942 & 850 & 1057 \\
\hline & & TC 2 & 1015 & 1010 & 948 & 1094 \\
\hline & & Air Flow & 238 & 240 & 210 & 272 \\
\hline & \multirow{3}{*}{ Electrode } & East & 1164 & 1161 & 1077 & 1177 \\
\hline & & West & 1139 & 1139 & 1048 & 1165 \\
\hline & & Bottom & 1053 & 1036 & 917 & 1057 \\
\hline & \multirow{2}{*}{ Film Cooler } & Added Air & 75 & 76 & 74 & 78 \\
\hline & & Outlet & 353 & 374 & 295 & 628 \\
\hline \multicolumn{3}{|c|}{ Glass Resistance (ohms) } & 0.070 & 0.071 & 0.067 & 0.088 \\
\hline \multicolumn{3}{|c|}{ Glass Density $(\mathrm{g} / \mathrm{ml})$} & 2.29 & 2.29 & 2.22 & 2.40 \\
\hline \multicolumn{3}{|c|}{ Glass Pool Depth (inches) } & 29.5 & 29.5 & 27.6 & 30.8 \\
\hline \multirow{3}{*}{ Electrodes } & & urrent $(\mathrm{A})$ & 1697 & 1670 & 1318 & 1937 \\
\hline & & oltage (V) & 119 & 119 & 105 & 139 \\
\hline & & ower $(\mathrm{kW})$ & 201 & 199 & 153 & 270 \\
\hline \multirow{5}{*}{$\begin{array}{l}\text { Lance } \\
\text { Bubblers }\end{array}$} & 1 & Rate (lpm) & 20.1 & 19.4 & 6.8 & 23.9 \\
\hline & 2 & Rate (lpm) & 20.0 & 19.6 & 6.9 & 24.4 \\
\hline & 3 & Rate (lpm) & 10.1 & 9.8 & 3.4 & 12.2 \\
\hline & 4 & Rate (lpm) & 10.1 & 9.8 & 3.3 & 12.2 \\
\hline & \multicolumn{2}{|c|}{ Total Bubbling (lpm) } & 61.5 & 59.7 & 21.8 & 73.6 \\
\hline
\end{tabular}


Table 3.4. DM1200 Melter System Measured Parameters (continued).

\begin{tabular}{|c|c|c|c|c|c|c|}
\hline \multirow{2}{*}{\multicolumn{3}{|c|}{ TEST 4}} & \multirow{2}{*}{$\begin{array}{c}\text { Steady } \\
\text { State } \\
\text { avg }\end{array}$} & \multicolumn{3}{|c|}{ Overall } \\
\hline & & & & avg & $\min$ & $\max$ \\
\hline \multirow{22}{*}{ 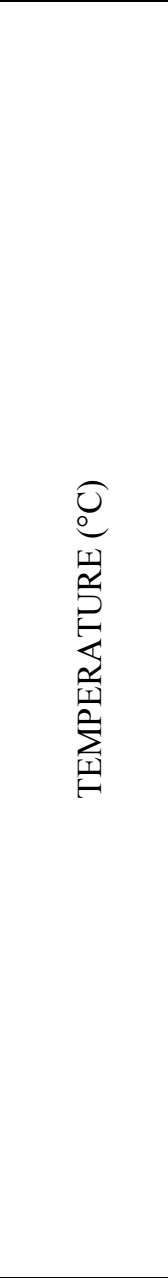 } & \multirow{8}{*}{ Glass } & $13 "$ from floor $\mathrm{E}$ & 1162 & 1163 & 1130 & 1200 \\
\hline & & $15 . \mathrm{s}^{\mathrm{N}}$ from floor $\mathrm{E}$ & 1166 & 1165 & 1138 & 1195 \\
\hline & & $18 "$ from floor $\mathrm{E}$ & 1167 & 1164 & 1053 & 1186 \\
\hline & & $27 "$ from floor E & 1133 & 1117 & 940 & 1164 \\
\hline & & 13 " from floor $\mathrm{W}$ & 1145 & 1142 & 1118 & 1162 \\
\hline & & $15.5 "$ from floor $\mathrm{W}$ & 1145 & 1142 & 1112 & 1163 \\
\hline & & 18 " from floor $\mathrm{W}$ & 1145 & 1142 & 1109 & 1162 \\
\hline & & 27 " from floor $\mathrm{W}$ & 1124 & 1115 & 887 & 1162 \\
\hline & \multirow{6}{*}{ Plenum } & $\begin{array}{c}\text { T/W Plenum TC } 8 " \\
\text { below lid (A2) }\end{array}$ & 467 & 494 & 440 & 794 \\
\hline & & $\begin{array}{c}\text { Exp. Plenum TC 17" } \\
\text { below lid (B2) }\end{array}$ & 527 & 527 & 418 & 771 \\
\hline & & $\begin{array}{l}\text { Exp. Plenum TC 17" } \\
\text { below lid (B3) }\end{array}$ & 420 & 458 & 356 & 768 \\
\hline & & $\begin{array}{c}\text { Exp. Plenum TC 17" } \\
\text { below lid (C1) }\end{array}$ & 415 & 450 & 361 & 795 \\
\hline & & $\begin{array}{l}\text { Exp. Plenum TC 17" } \\
\text { below lid (C2) }\end{array}$ & 426 & 460 & 345 & 804 \\
\hline & & $\begin{array}{c}\text { T/W Plenum TC 17" } \\
\text { below lid (D2) }\end{array}$ & 461 & 484 & 414 & 819 \\
\hline & \multirow{3}{*}{ Discharge } & TC 1 & 1088 & 1060 & 897 & 1127 \\
\hline & & TC 2 & 1086 & 1061 & 911 & 1123 \\
\hline & & Air Flow & 248 & 244 & 193 & 262 \\
\hline & \multirow{3}{*}{ Electrode } & East & 1157 & 1149 & 1077 & 1171 \\
\hline & & West & 1138 & 1131 & 1074 & 1152 \\
\hline & & Bottom & 1062 & 1036 & 950 & 1074 \\
\hline & \multirow{2}{*}{ Film Cooler } & Added Air & 77 & 76 & 73 & 84 \\
\hline & & Outlet & 336 & 344 & 69 & 582 \\
\hline \multicolumn{3}{|c|}{ Glass Resistance (ohms) } & 0.082 & 0.082 & 0.073 & 0.094 \\
\hline \multicolumn{3}{|c|}{ Glass Density $(\mathrm{g} / \mathrm{ml})$} & 2.26 & 2.28 & 2.22 & 2.41 \\
\hline \multicolumn{3}{|c|}{ Glass Pool Depth (inches) } & 29.2 & 29.2 & 27,7 & 30.2 \\
\hline \multirow{3}{*}{ Electrodes } & \multicolumn{2}{|c|}{ Current (A) } & 1619 & 1521 & 1176 & 1679 \\
\hline & \multicolumn{2}{|c|}{ Voltage (V) } & 132 & 125 & 95 & 138 \\
\hline & \multicolumn{2}{|c|}{ Power $(\mathrm{kW})$} & 214 & 191 & 122 & 223 \\
\hline \multirow{5}{*}{$\begin{array}{l}\text { Lance } \\
\text { Bubblers }\end{array}$} & 1 & Rate (lpm) & 26.0 & 21.9 & 6.0 & 26.3 \\
\hline & 2 & Rate (lpm) & 26.1 & 22.0 & 6.0 & 26.3 \\
\hline & 3 & Rate (lpm) & 13.1 & 11.0 & 3.0 & 13.2 \\
\hline & 4 & Rate (lpm) & 13.1 & 11.0 & 3.0 & 13.3 \\
\hline & \multicolumn{2}{|c|}{ Total Bubbling (lpm) } & 79.4 & 67.2 & 19.2 & 79.8 \\
\hline
\end{tabular}


Table 4.1. Measured DM1200 Off-Gas System Parameters.

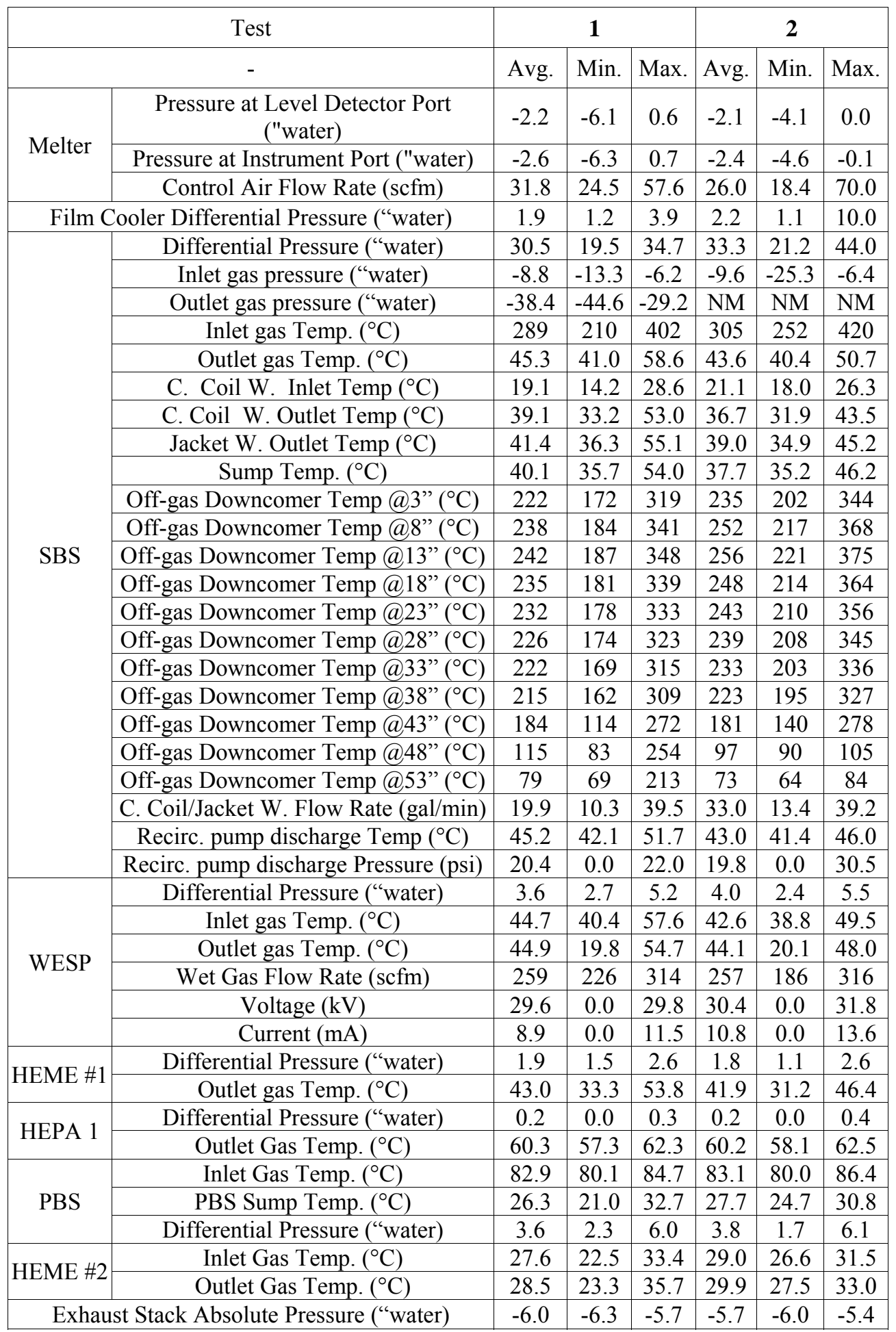

NM: not measured 
Table 4.1. Measured DM1200 Off-Gas System Parameters (continued).

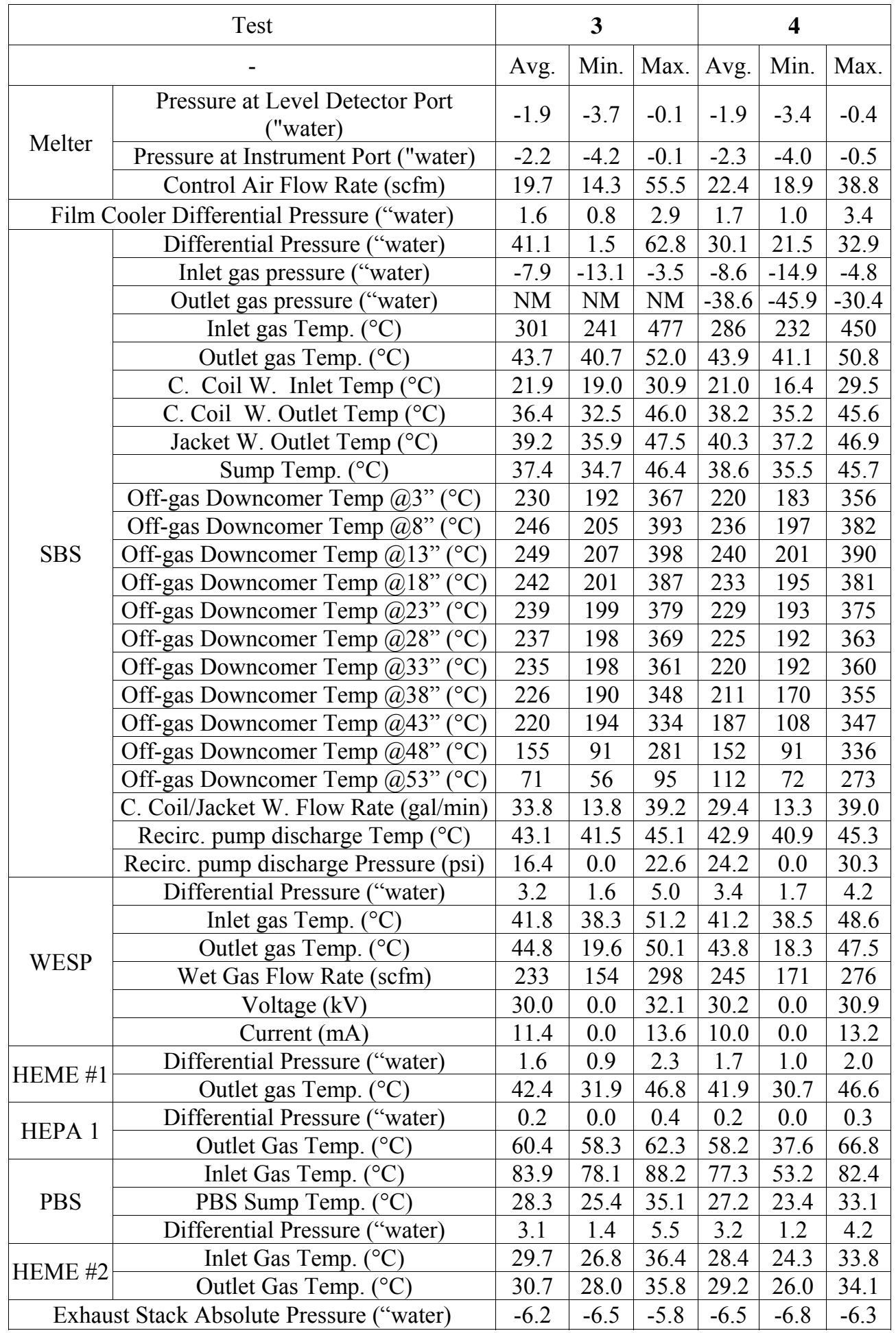

NM: not measured 
Table 4.2. Off-Gas Fluid Volumes.

\begin{tabular}{|c|c|c|c|}
\hline & Type of Sample & $\begin{array}{c}\text { Number of } \\
\text { Blow-downs }\end{array}$ & $\begin{array}{c}\text { Total Blow-down Volume } \\
\text { (gal) }\end{array}$ \\
\hline \multirow{5}{*}{ Test 1} & SBS & 12 & 481 \\
\hline & WESP & 5 & 122 \\
\hline & HEME 1 & 1 & NM \\
\hline & HEME 2 & 2 & 11 \\
\hline & PBS & 7 & 220 \\
\hline \multirow{5}{*}{ Test 2} & SBS & 17 & 690 \\
\hline & WESP & 4 & 81 \\
\hline & HEME 1 & 1 & $\mathrm{NM}$ \\
\hline & HEME 2 & 2 & 46 \\
\hline & PBS & 7 & 194 \\
\hline \multirow{5}{*}{ Test 3} & SBS & 17 & 661 \\
\hline & WESP & 3 & 127 \\
\hline & HEME 1 & 1 & NM \\
\hline & HEME 2 & 1 & 54 \\
\hline & PBS & 6 & 157 \\
\hline \multirow{5}{*}{ Test 4} & SBS & 25 & 1000 \\
\hline & WESP & 4 & 106 \\
\hline & HEME 1 & 1 & NM \\
\hline & HEME 2 & 2 & 26 \\
\hline & PBS & 8 & 203 \\
\hline
\end{tabular}

NM: not measured 
The Catholic University of America Vitreous State Laboratory
Testing of Optimized Bubbler Configuration for HLW Melter Final Report, VSL-13R2950-1, Rev. 0

Table 5.1. Measured Feed Sample Properties.

\begin{tabular}{|c|c|c|c|c|c|c|c|c|c|}
\hline \multirow{3}{*}{ Source } & \multirow{3}{*}{ Date } & \multirow{3}{*}{ Name } & \multirow{3}{*}{ \% Water } & \multirow{3}{*}{$\mathbf{p H}$} & \multirow{3}{*}{$\begin{array}{c}\text { Density } \\
\text { (g/ml) }\end{array}$} & \multicolumn{4}{|c|}{ Glass Yield } \\
\hline & & & & & & \multirow{2}{*}{$(g / l)$} & \multirow{2}{*}{$\begin{array}{c}\text { Measured } \\
\text { (kg/kg) } \\
\end{array}$} & \multirow{2}{*}{$\begin{array}{c}\text { Target } \\
\text { (kg/kg) }\end{array}$} & \multirow{2}{*}{ \%Dev. } \\
\hline & & & & & & & & & \\
\hline $\begin{array}{c}\text { As Received } \\
\text { Lot \# } 1.3 \\
\end{array}$ & $1 / 7 / 13$ & F-12U-44A & 50.75 & 8.49 & 1.54 & 673 & 0.437 & $\mathrm{NC}$ & $\mathrm{NC}$ \\
\hline $\begin{array}{c}\text { As Received } \\
\text { Lot \# } 1.5\end{array}$ & $1 / 10 / 13$ & F-12U-44B & 56.48 & 8.77 & 1.41 & 547 & 0.388 & $\mathrm{NC}$ & $\mathrm{NC}$ \\
\hline $\begin{array}{c}\text { As Received } \\
\text { Lot \# } 1.1 \\
\end{array}$ & $1 / 10 / 13$ & F-12U-49A & 52.92 & 8.39 & 1.48 & 616 & 0.416 & $\mathrm{NC}$ & $\mathrm{NC}$ \\
\hline $\begin{array}{c}\text { As Received } \\
\text { Lot \# } 1.2 \\
\end{array}$ & $1 / 10 / 13$ & F-12U-49B & 50.22 & 8.14 & 1.50 & 666 & 0.444 & $\mathrm{NC}$ & $\mathrm{NC}$ \\
\hline $\begin{array}{c}\text { As Received } \\
\text { Lot \# } 1.3\end{array}$ & $1 / 10 / 13$ & F-12U-49C & 50.88 & 8.44 & 1.52 & 614 & 0.404 & $\mathrm{NC}$ & $\mathrm{NC}$ \\
\hline $\begin{array}{c}\text { As Received } \\
\text { Lot \# } 1.4\end{array}$ & $1 / 10 / 13$ & F-12U-49D & 51.08 & 8.13 & 1.49 & 637 & 0.428 & $\mathrm{NC}$ & $\mathrm{NC}$ \\
\hline $\begin{array}{c}\text { As Received } \\
\text { Lot \# } 1.5\end{array}$ & $1 / 10 / 13$ & F-12U-49E & 56.18 & 8.74 & 1.39 & 530 & 0.381 & $\mathrm{NC}$ & $\mathrm{NC}$ \\
\hline $\begin{array}{c}\text { As Received } \\
\text { Lot \# } 1.6\end{array}$ & $1 / 10 / 13$ & $\mathrm{~F}-12 \mathrm{U}-49 \mathrm{~F}$ & 50.86 & 8.11 & 1.49 & 644 & 0.432 & $\mathrm{NC}$ & $\mathrm{NC}$ \\
\hline \multirow{2}{*}{ Test 1} & $1 / 16 / 13$ & F-12U-94A & 59.6 & 8.76 & 1.32 & 469 & 0.356 & 0.36 & -1.22 \\
\hline & $1 / 17 / 13$ & $\mathrm{~F}-12 \mathrm{U}-125 \mathrm{~A}$ & 71.72 & 9.23 & 1.23 & 308 & 0.251 & 0.36 & -30.33 \\
\hline \multirow{3}{*}{ Test 2} & $1 / 22 / 13$ & F-12V-10A & 58.42 & 8.67 & 1.37 & 501 & 0.366 & 0.36 & 1.67 \\
\hline & $1 / 23 / 13$ & F-12V-42A & 59.76 & 8.78 & 1.36 & 483 & 0.355 & 0.36 & -1.28 \\
\hline & $1 / 24 / 13$ & $\mathrm{~F}-12 \mathrm{~V}-60 \mathrm{~A}$ & 57.97 & 8.69 & 1.38 & 500 & 0.362 & 0.36 & 0.67 \\
\hline \multirow{3}{*}{ Test 3} & $1 / 29 / 13$ & F-12W-34A & 59.57 & 8.76 & 1.37 & 489 & 0.357 & 0.36 & -0.81 \\
\hline & $1 / 30 / 13$ & F-12W-63A & 60.78 & 8.76 & 1.33 & 453 & 0.341 & 0.36 & -5.33 \\
\hline & $1 / 31 / 13$ & $\mathrm{~F}-12 \mathrm{~W}-82 \mathrm{~A}$ & 60.71 & 8.89 & 1.34 & 461 & 0.344 & 0.36 & -4.36 \\
\hline \multirow{3}{*}{ Test 4} & $2 / 7 / 13$ & $\mathrm{~F}-12 \mathrm{~W}-115 \mathrm{~A}$ & 60.32 & 8.70 & 1.37 & 479 & 0.350 & 0.36 & -2.81 \\
\hline & $2 / 7 / 13$ & F-12X-6A & 60.71 & 8.80 & 1.33 & 455 & 0.342 & 0.36 & -4.89 \\
\hline & $2 / 8 / 13$ & F-12X-35A & 60.24 & 8.74 & 1.34 & 475 & 0.355 & 0.36 & -1.47 \\
\hline
\end{tabular}

$\mathrm{NC}-$ Not calculated 
Table 5.2. XRF Analyzed Compositions for As-Received Vitrified Melter Feed Samples; HWI-Al-19 Composition (wt\%).

\begin{tabular}{|c|c|c|c|c|c|c|c|}
\hline Constituent & Target & F-12U-44A & F-12U-44B & F-12U-49A & F-12U-49B & F-12U-49C & F-12U-49D \\
\hline $\mathrm{Al}_{2} \mathrm{O}_{3}$ & 23.97 & 23.33 & 23.54 & 23.18 & 23.31 & 23.41 & 23.44 \\
\hline $\mathrm{B}_{2} \mathrm{O}_{3}{ }^{*}$ & 19.19 & 18.26 & 18.19 & 18.18 & 18.21 & 18.23 & 18.31 \\
\hline $\mathrm{BaO}$ & 0.05 & 0.05 & 0.08 & 0.09 & 0.09 & 0.08 & 0.09 \\
\hline $\mathrm{Bi}_{2} \mathrm{O}_{3}$ & 1.14 & 1.22 & 1.26 & 1.35 & 1.26 & 1.29 & 1.23 \\
\hline $\mathrm{CaO}$ & 5.58 & 5.58 & 5.53 & 5.71 & 5.71 & 5.68 & 5.52 \\
\hline $\mathrm{CdO}$ & 0.02 & 0.04 & 0.04 & 0.03 & 0.03 & 0.04 & 0.03 \\
\hline $\mathrm{Cr}_{2} \mathrm{O}_{3}$ & 0.52 & 0.50 & 0.52 & 0.52 & 0.50 & 0.54 & 0.52 \\
\hline $\mathrm{F}^{S}$ & 0.67 & 0.30 & 0.30 & 0.30 & 0.30 & 0.30 & 0.30 \\
\hline $\mathrm{Fe}_{2} \mathrm{O}_{3}$ & 5.90 & 6.05 & 6.28 & 6.42 & 6.18 & 6.24 & 6.12 \\
\hline $\mathrm{K}_{2} \mathrm{O}$ & 0.14 & 0.22 & 0.19 & 0.12 & 0.16 & 0.12 & 0.15 \\
\hline $\mathrm{Li}_{2} \mathrm{O}^{*}$ & 3.57 & 3.50 & 3.45 & 3.58 & 3.57 & 3.58 & 3.53 \\
\hline $\mathrm{MgO}$ & 0.12 & 0.24 & 0.18 & 0.23 & 0.22 & 0.22 & 0.24 \\
\hline $\mathrm{MnO}$ & $\S$ & 0.03 & 0.02 & 0.02 & 0.03 & 0.02 & 0.02 \\
\hline $\mathrm{Na}_{2} \mathrm{O}$ & 9.58 & 9.99 & 9.28 & 9.32 & 8.97 & 9.48 & 9.57 \\
\hline $\mathrm{NiO}$ & 0.40 & 0.45 & 0.46 & 0.46 & 0.44 & 0.45 & 0.44 \\
\hline $\mathrm{P}_{2} \mathrm{O}_{5}$ & 1.05 & 1.08 & 1.08 & 1.15 & 1.12 & 1.12 & 1.10 \\
\hline $\mathrm{PbO}$ & 0.41 & 0.42 & 0.46 & 0.45 & 0.39 & 0.45 & 0.40 \\
\hline $\mathrm{SiO}_{2}$ & 27.00 & 28.02 & 28.43 & 28.15 & 28.77 & 28.02 & 28.25 \\
\hline $\mathrm{SO}_{3}$ & 0.20 & 0.20 & 0.21 & 0.21 & 0.19 & 0.20 & 0.19 \\
\hline $\mathrm{TiO}_{2}$ & 0.01 & 0.06 & 0.05 & 0.06 & 0.06 & 0.05 & 0.06 \\
\hline $\mathrm{ZnO}$ & 0.08 & 0.08 & 0.08 & 0.09 & 0.09 & 0.08 & 0.08 \\
\hline $\mathrm{ZrO}_{2}$ & 0.39 & 0.38 & 0.39 & 0.40 & 0.40 & 0.40 & 0.39 \\
\hline Sum & 100.00 & 100.00 & 100.00 & 100.00 & 100.00 & 100.00 & 100.00 \\
\hline
\end{tabular}

$\S$ - Not a target constituent

* DCP-AES measured values

$\$$ - F value estimated from XRF measurements on discharge glasses. 
Table 5.2. XRF Analyzed Compositions for As-Received Vitrified Melter Feed Samples; HWI-Al-19 Composition (wt\%) (continued).

\begin{tabular}{|c|c|c|c|c|c|}
\hline Constituent & Target & F-12U-49E & F-12U-49F & Average & $\%$ Dev. \\
\hline $\mathrm{Al}_{2} \mathrm{O}_{3}$ & 23.97 & 23.37 & 23.39 & 23.37 & -2.50 \\
\hline $\mathrm{B}_{2} \mathrm{O}_{3}{ }^{*}$ & 19.19 & 18.28 & 18.45 & 18.26 & -4.84 \\
\hline $\mathrm{BaO}$ & 0.05 & 0.09 & 0.08 & 0.08 & $\mathrm{NC}$ \\
\hline $\mathrm{Bi}_{2} \mathrm{O}_{3}$ & 1.14 & 1.24 & 1.28 & 1.27 & 11.0 \\
\hline $\mathrm{CaO}$ & 5.58 & 5.59 & 5.55 & 5.61 & 0.50 \\
\hline $\mathrm{CdO}$ & 0.02 & 0.04 & 0.04 & 0.04 & $\mathrm{NC}$ \\
\hline $\mathrm{Cr}_{2} \mathrm{O}_{3}$ & 0.52 & 0.50 & 0.50 & 0.51 & $\mathrm{NC}$ \\
\hline $\mathrm{F}^{\$}$ & 0.67 & 0.30 & 0.30 & 0.30 & $\mathrm{NC}$ \\
\hline $\mathrm{Fe}_{2} \mathrm{O}_{3}$ & 5.90 & 6.36 & 6.08 & 6.22 & 5.35 \\
\hline $\mathrm{K}_{2} \mathrm{O}$ & 0.14 & 0.11 & $<0.01$ & 0.13 & $\mathrm{NC}$ \\
\hline $\mathrm{Li}_{2} \mathrm{O}^{*}$ & 3.57 & 3.54 & 3.55 & 3.54 & -0.92 \\
\hline $\mathrm{MgO}$ & 0.12 & 0.22 & 0.18 & 0.22 & $\mathrm{NC}$ \\
\hline $\mathrm{MnO}$ & $\S$ & 0.03 & 0.02 & 0.02 & $\mathrm{NC}$ \\
\hline $\mathrm{Na}_{2} \mathrm{O}$ & 9.58 & 9.35 & 9.39 & 9.42 & -1.71 \\
\hline $\mathrm{NiO}$ & 0.40 & 0.43 & 0.45 & 0.45 & $\mathrm{NC}$ \\
\hline $\mathrm{P}_{2} \mathrm{O}_{5}$ & 1.05 & 1.07 & 1.13 & 1.11 & 5.28 \\
\hline $\mathrm{PbO}$ & 0.41 & 0.44 & 0.44 & 0.43 & $\mathrm{NC}$ \\
\hline $\mathrm{SiO}_{2}$ & 27.00 & 28.34 & 28.43 & 28.30 & 4.81 \\
\hline $\mathrm{SO}_{3}$ & 0.20 & 0.20 & 0.19 & 0.20 & $\mathrm{NC}$ \\
\hline $\mathrm{TiO}_{2}$ & 0.01 & 0.05 & 0.06 & 0.06 & $\mathrm{NC}$ \\
\hline $\mathrm{ZnO}$ & 0.08 & 0.08 & 0.08 & 0.08 & $\mathrm{NC}$ \\
\hline $\mathrm{ZrO}_{2}$ & 0.39 & 0.38 & 0.39 & 0.39 & $\mathrm{NC}$ \\
\hline Sum & 100.00 & 100.00 & 100.00 & 100.00 & $\mathrm{NC}$ \\
\hline
\end{tabular}

$\S$ - Not a target constituent

* DCP-AES measured values

$\$$ - F value estimated from XRF measurements on discharge glasses.

$\mathrm{NC}-$ Not calculated 
Table 5.3. XRF Analyzed Compositions of Vitrified Melter Feed Sampled During DM1200 Tests (wt\%).

\begin{tabular}{|c|c|c|c|c|c|c|c|}
\hline \multicolumn{2}{|c|}{ Test } & \multicolumn{2}{|c|}{1} & \multicolumn{3}{|c|}{2} & 3 \\
\hline Constituent & Target & F-12U-94A & F-12U-125A & F-12V-10A & $\mathrm{F}-12 \mathrm{~V}-42 \mathrm{~A}$ & $\mathrm{~F}-12 \mathrm{~V}-60 \mathrm{~A}$ & F-12W-34A \\
\hline $\mathrm{Al}_{2} \mathrm{O}_{3}$ & 23.97 & 23.68 & 24.23 & 23.19 & 23.57 & 22.96 & 23.22 \\
\hline $\mathrm{B}_{2} \mathrm{O}_{3}{ }^{*}$ & 19.19 & 18.14 & 17.21 & 18.38 & 18.20 & 18.10 & 18.62 \\
\hline $\mathrm{BaO}$ & 0.05 & 0.06 & 0.09 & 0.09 & 0.09 & 0.08 & 0.08 \\
\hline $\mathrm{Bi}_{2} \mathrm{O}_{3}$ & 1.14 & 1.26 & 1.31 & 1.25 & 1.28 & 1.25 & 1.24 \\
\hline $\mathrm{CaO}$ & 5.58 & 5.60 & 5.71 & 5.45 & 5.63 & 5.74 & 5.48 \\
\hline $\mathrm{CdO}$ & 0.02 & 0.03 & 0.04 & 0.04 & 0.03 & 0.03 & 0.03 \\
\hline $\mathrm{Cr}_{2} \mathrm{O}_{3}$ & 0.52 & 0.50 & 0.52 & 0.47 & 0.47 & 0.47 & 0.51 \\
\hline $\mathrm{F}^{\$}$ & 0.67 & 0.30 & 0.30 & 0.30 & 0.30 & 0.30 & 0.30 \\
\hline $\mathrm{Fe}_{2} \mathrm{O}_{3}$ & 5.90 & 6.37 & 6.49 & 5.93 & 6.13 & 6.03 & 5.79 \\
\hline $\mathrm{K}_{2} \mathrm{O}$ & 0.14 & 0.16 & 0.12 & 0.20 & 0.03 & 0.17 & 0.03 \\
\hline $\mathrm{Li}_{2} \mathrm{O}^{*}$ & 3.57 & 3.54 & 3.52 & 3.54 & 3.53 & 4.28 & 3.59 \\
\hline $\mathrm{MgO}$ & 0.12 & 0.22 & 0.23 & 0.23 & 0.22 & 0.22 & 0.27 \\
\hline $\mathrm{MnO}$ & $\S$ & 0.02 & 0.04 & 0.03 & 0.03 & 0.03 & 0.03 \\
\hline $\mathrm{Na}_{2} \mathrm{O}$ & 9.58 & 9.36 & 8.88 & 9.78 & 9.37 & 9.61 & 10.10 \\
\hline $\mathrm{NiO}$ & 0.40 & 0.45 & 0.46 & 0.44 & 0.45 & 0.45 & 0.45 \\
\hline $\mathrm{P}_{2} \mathrm{O}_{5}$ & 1.05 & 1.09 & 1.11 & 1.14 & 1.12 & 1.10 & 1.01 \\
\hline $\mathrm{PbO}$ & 0.41 & 0.41 & 0.53 & 0.41 & 0.41 & 0.43 & 0.42 \\
\hline $\mathrm{SiO}_{2}$ & 27.00 & 28.08 & 28.52 & 28.44 & 28.44 & 28.07 & 28.11 \\
\hline $\mathrm{SO}_{3}$ & 0.20 & 0.18 & 0.17 & 0.18 & 0.18 & 0.16 & 0.19 \\
\hline $\mathrm{TiO}_{2}$ & 0.01 & 0.06 & 0.04 & 0.06 & 0.05 & 0.05 & 0.05 \\
\hline $\mathrm{ZnO}$ & 0.08 & 0.09 & 0.08 & 0.07 & 0.08 & 0.09 & 0.09 \\
\hline $\mathrm{ZrO}_{2}$ & 0.39 & 0.40 & 0.39 & 0.38 & 0.39 & 0.38 & 0.38 \\
\hline Sum & 100.00 & 100.00 & 100.00 & 100.00 & 100.00 & 100.00 & 100.00 \\
\hline
\end{tabular}

$\S_{*}$ - Not a target constituent

* - DCP-AES measured values

$\$$ - F value estimated from XRF measurements on discharge glasses. 
Table 5.3. XRF Analyzed Compositions of Vitrified Melter Feed Sampled During DM1200 Tests, (wt \%) (continued).

\begin{tabular}{|c|c|c|c|c|c|c|c|c|}
\hline \multicolumn{2}{|c|}{ Test } & \multicolumn{2}{|c|}{3} & \multicolumn{3}{|c|}{4} & \multirow{2}{*}{ Average } & \multirow{2}{*}{$\%$ Dev. } \\
\hline Constituent & Target & F-12W-63A & F-12W-82A & F-12W-115A & F-12X-6A & F-12X-35A & & \\
\hline$\overline{\mathrm{Al}_{2} \mathrm{O}_{3}}$ & 23.97 & 23.07 & 23.24 & 23.06 & 23.13 & 23.01 & 23.30 & -2.79 \\
\hline $\mathrm{B}_{2} \mathrm{O}_{3}{ }^{*}$ & 19.19 & 18.44 & 18.35 & 18.46 & 18.50 & 18.35 & 18.25 & -4.91 \\
\hline $\mathrm{BaO}$ & 0.05 & 0.09 & 0.09 & 0.09 & 0.08 & 0.09 & 0.08 & $\mathrm{NC}$ \\
\hline $\mathrm{Bi}_{2} \mathrm{O}_{3}$ & 1.14 & 1.28 & 1.28 & 1.25 & 1.22 & 1.29 & 1.26 & 10.9 \\
\hline $\mathrm{CaO}$ & 5.58 & 5.64 & 5.65 & 5.68 & 5.59 & 5.69 & 5.62 & 0.78 \\
\hline $\mathrm{CdO}$ & 0.02 & 0.03 & 0.03 & 0.03 & 0.03 & 0.03 & 0.03 & $\mathrm{NC}$ \\
\hline $\mathrm{Cr}_{2} \mathrm{O}_{3}$ & 0.52 & 0.50 & 0.52 & 0.52 & 0.50 & 0.50 & 0.50 & $\mathrm{NC}$ \\
\hline $\mathrm{F}^{\$}$ & 0.67 & 0.30 & 0.30 & 0.30 & 0.30 & 0.30 & 0.30 & $\mathrm{NC}$ \\
\hline $\mathrm{Fe}_{2} \mathrm{O}_{3}$ & 5.90 & 5.94 & 5.95 & 5.81 & 5.83 & 6.00 & 6.02 & 2.09 \\
\hline $\mathrm{K}_{2} \mathrm{O}$ & 0.14 & 0.14 & 0.15 & 0.03 & 0.19 & 0.14 & 0.12 & $\mathrm{NC}$ \\
\hline $\mathrm{Li}_{2} \mathrm{O}^{*}$ & 3.57 & 3.48 & 3.31 & 3.52 & 3.54 & 3.50 & 3.58 & 0.19 \\
\hline $\mathrm{MgO}$ & 0.12 & 0.25 & 0.26 & 0.26 & 0.24 & 0.25 & 0.24 & $\mathrm{NC}$ \\
\hline $\mathrm{MnO}$ & $\S$ & 0.03 & 0.03 & 0.03 & 0.04 & 0.03 & 0.03 & $\mathrm{NC}$ \\
\hline $\mathrm{Na}_{2} \mathrm{O}$ & 9.58 & 9.85 & 9.83 & 9.91 & 9.88 & 9.76 & 9.67 & 0.90 \\
\hline $\mathrm{NiO}$ & 0.40 & 0.45 & 0.45 & 0.43 & 0.45 & 0.46 & 0.45 & $\mathrm{NC}$ \\
\hline $\mathrm{P}_{2} \mathrm{O}_{5}$ & 1.05 & 1.08 & 1.06 & 1.08 & 1.09 & 1.04 & 1.08 & 3.22 \\
\hline $\mathrm{PbO}$ & 0.41 & 0.40 & 0.41 & 0.42 & 0.40 & 0.42 & 0.42 & $\mathrm{NC}$ \\
\hline $\mathrm{SiO}_{2}$ & 27.00 & 28.30 & 28.35 & 28.38 & 28.26 & 28.41 & 28.30 & 4.82 \\
\hline $\mathrm{SO}_{3}$ & 0.20 & 0.22 & 0.22 & 0.20 & 0.22 & $\begin{array}{l}0.21 \\
\end{array}$ & $\begin{array}{l}0.19 \\
\end{array}$ & $\mathrm{NC}$ \\
\hline $\mathrm{TiO}_{2}$ & 0.01 & 0.05 & 0.05 & 0.06 & 0.05 & 0.04 & 0.05 & $\mathrm{NC}$ \\
\hline $\mathrm{ZnO}$ & 0.08 & 0.08 & 0.07 & 0.09 & 0.08 & 0.09 & 0.08 & $\mathrm{NC}$ \\
\hline $\mathrm{ZrO}_{2}$ & 0.39 & 0.40 & 0.38 & 0.39 & 0.37 & 0.39 & 0.39 & $\mathrm{NC}$ \\
\hline Sum & 100.00 & 100.00 & 100.00 & 100.00 & 100.00 & 100.00 & 100.00 & $\mathrm{NC}$ \\
\hline
\end{tabular}

$\S$ - Not a target constituent

* - DCP-AES measured values

$\$$ - F value estimated from XRF measurements on discharge glasses.

$\mathrm{NC}-$ Not calculated 
Table 5.4. Listing of Glass and Discharged Masses during DM1200 Tests.

\begin{tabular}{|c|c|c|c|c|}
\hline Test & Date & Name & $\begin{array}{c}\text { Mass } \\
(\mathrm{kg})\end{array}$ & $\begin{array}{c}\text { Cumulative Mass } \\
(\mathrm{kg})\end{array}$ \\
\hline \multirow{28}{*}{1} & \multirow{6}{*}{$1 / 15 / 2013$} & G-12U-67A & \multirow{7}{*}{484.5} & \multirow{7}{*}{484.5} \\
\hline & & G-12U-67B & & \\
\hline & & G-12U-68A & & \\
\hline & & G-12U-68B & & \\
\hline & & G-12U-78A & & \\
\hline & & G-12U-80A & & \\
\hline & \multirow{15}{*}{$1 / 16 / 2013$} & G-12U-81A & & \\
\hline & & G-12U-81B & \multirow{4}{*}{426.5} & \multirow{4}{*}{911.0} \\
\hline & & G-12U-83A & & \\
\hline & & G-12U-83B & & \\
\hline & & G-12U-84A & & \\
\hline & & G-12U-85A & \multirow{5}{*}{484.0} & \multirow{5}{*}{1395.0} \\
\hline & & G-12U-85B & & \\
\hline & & G-12U-94A & & \\
\hline & & G-12U-95A & & \\
\hline & & G-12U-95B & & \\
\hline & & G-12U-96A & \multirow{6}{*}{477.0} & \multirow{6}{*}{1872.0} \\
\hline & & G-12U-97A & & \\
\hline & & G-12U-97B & & \\
\hline & & G-12U-99A & & \\
\hline & & G-12U-110A & & \\
\hline & \multirow{7}{*}{$1 / 17 / 2013$} & G-12U-112A & & \\
\hline & & G-12U-112B & \multirow{6}{*}{494.0} & \multirow{6}{*}{2366.0} \\
\hline & & G-12U-113A & & \\
\hline & & G-12U-114A & & \\
\hline & & G-12U-114B & & \\
\hline & & G-12U-124A & & \\
\hline & & G-12U-125A & & \\
\hline \multirow{11}{*}{2} & \multirow{7}{*}{$1 / 22 / 2013$} & G-12U-151A & \multirow{5}{*}{467.5} & \multirow{5}{*}{2833.5} \\
\hline & & G-12U-151B & & \\
\hline & & G-12V-9A & & \\
\hline & & G-12V-10A & & \\
\hline & & G-12V-10B & & \\
\hline & & G-12V-11A & \multirow{6}{*}{482.5} & \multirow{6}{*}{3316.0} \\
\hline & & G-12V-12A & & \\
\hline & \multirow{4}{*}{$1 / 23 / 2013$} & G-12V-13A & & \\
\hline & & G-12V-14A & & \\
\hline & & G-12V-14B & & \\
\hline & & G-12V-23A & & \\
\hline
\end{tabular}


Table 5.4. Listing of Glass and Discharged Masses during DM1200 Tests (continued).

\begin{tabular}{|c|c|c|c|c|}
\hline Test & Date & Name & $\begin{array}{c}\text { Mass } \\
(\mathrm{kg})\end{array}$ & $\begin{array}{c}\text { Cumulative Mass } \\
(\mathrm{kg})\end{array}$ \\
\hline \multirow{33}{*}{2} & \multirow{14}{*}{$1 / 23 / 2013$} & G-12V-24A & \multirow{5}{*}{473.5} & \multirow{5}{*}{3789.5} \\
\hline & & G-12V-24B & & \\
\hline & & G-12V-25A & & \\
\hline & & $\mathrm{G}-12 \mathrm{~V}-27 \mathrm{~A}$ & & \\
\hline & & G-12V-28A & & \\
\hline & & G-12V-28B & \multirow{6}{*}{501.0} & \multirow{6}{*}{4290.5} \\
\hline & & G-12V-29A & & \\
\hline & & G-12V-39A & & \\
\hline & & G-12V-39B & & \\
\hline & & G-12V-41A & & \\
\hline & & G-12V-42A & & \\
\hline & & G-12V-43A & \multirow{7}{*}{490.0} & \multirow{7}{*}{4780.5} \\
\hline & & G-12V-43B & & \\
\hline & & G-12V-45A & & \\
\hline & \multirow{19}{*}{$1 / 24 / 2013$} & G-12V-46A & & \\
\hline & & G-12V-47A & & \\
\hline & & G-12V-56A & & \\
\hline & & G-12V-56B & & \\
\hline & & G-12V-57A & \multirow{9}{*}{435.0} & \multirow{9}{*}{5215.5} \\
\hline & & G-12V-57B & & \\
\hline & & G-12V-57C & & \\
\hline & & G-12V-59A & & \\
\hline & & G-12V-59B & & \\
\hline & & G-12V-60A & & \\
\hline & & G-12V-60B & & \\
\hline & & G-12V-61A & & \\
\hline & & G-12V-61B & & \\
\hline & & G-12V-61C & \multirow{6}{*}{405.5} & \multirow{6}{*}{5621.0} \\
\hline & & G-12V-63A & & \\
\hline & & G-12V-63B & & \\
\hline & & $\mathrm{G}-12 \mathrm{~V}-63 \mathrm{C}$ & & \\
\hline & & G-12V-72A & & \\
\hline & & G-12V-73A & & \\
\hline \multirow{10}{*}{3} & \multirow{10}{*}{$1 / 29 / 2013$} & G-12W-29A & \multirow{6}{*}{482.5} & \multirow{6}{*}{6103.5} \\
\hline & & G-12W-30A & & \\
\hline & & G-12W-31A & & \\
\hline & & G-12W-32A & & \\
\hline & & G-12W-32B & & \\
\hline & & G-12W-33A & & \\
\hline & & G-12W-34A & & \\
\hline & & G-12W-35A & & \\
\hline & & G-12W-35B & & \\
\hline & & G-12W-36A & & \\
\hline
\end{tabular}


Table 5.4. Listing of Glass and Discharged Masses during DM1200 Tests (continued).

\begin{tabular}{|c|c|c|c|c|}
\hline Test & Date & Name & $\begin{array}{c}\text { Mass } \\
(\mathrm{kg})\end{array}$ & $\begin{array}{c}\text { Cumulative Mass } \\
(\mathrm{kg})\end{array}$ \\
\hline \multirow{41}{*}{3} & \multirow{25}{*}{$1 / 30 / 2013$} & G-12W-37A & \multirow{4}{*}{495.5} & \multirow{4}{*}{6599.0} \\
\hline & & G-12W-38A & & \\
\hline & & G-12W-38B & & \\
\hline & & G-12W-39A & & \\
\hline & & G-12W-39B & \multirow{7}{*}{439.0} & \multirow{7}{*}{7038.0} \\
\hline & & G-12W-40A & & \\
\hline & & G-12W-43A & & \\
\hline & & $\mathrm{G}-12 \mathrm{~W}-43 \mathrm{~B}$ & & \\
\hline & & G-12W-44A & & \\
\hline & & G-12W-44B & & \\
\hline & & G-12W-47A & & \\
\hline & & G-12W-47B & \multirow{9}{*}{496.0} & \multirow{9}{*}{7534.0} \\
\hline & & G-12W-57A & & \\
\hline & & G-12W-57B & & \\
\hline & & G-12W-58A & & \\
\hline & & G-12W-58B & & \\
\hline & & G-12W-59A & & \\
\hline & & G-12W-59B & & \\
\hline & & G-12W-61A & & \\
\hline & & G-12W-61B & & \\
\hline & & $\mathrm{G}-12 \mathrm{~W}-62 \mathrm{~A}$ & \multirow{9}{*}{484.0} & \multirow{9}{*}{8018.0} \\
\hline & & G-12W-63A & & \\
\hline & & G-12W-63B & & \\
\hline & & G-12W-64A & & \\
\hline & & G-12W-64B & & \\
\hline & \multirow{16}{*}{$1 / 31 / 2013$} & G-12W-65A & & \\
\hline & & G-12W-65B & & \\
\hline & & G-12W-66A & & \\
\hline & & G-12W-76A & & \\
\hline & & $\mathrm{G}-12 \mathrm{~W}-76 \mathrm{~B}$ & \multirow{10}{*}{497.5} & \multirow{10}{*}{8515.5} \\
\hline & & G-12W-78A & & \\
\hline & & G-12W-79A & & \\
\hline & & G-12W-79B & & \\
\hline & & G-12W-80A & & \\
\hline & & G-12W-80B & & \\
\hline & & G-12W-81A & & \\
\hline & & G-12W-81B & & \\
\hline & & $\mathrm{G}-12 \mathrm{~W}-82 \mathrm{~A}$ & & \\
\hline & & $\mathrm{G}-12 \mathrm{~W}-82 \mathrm{~B}$ & & \\
\hline & & G-12W-91A & \multirow{2}{*}{116.0} & \multirow{2}{*}{8631.5} \\
\hline & & G-12W-91B & & \\
\hline
\end{tabular}


Table 5.4. Listing of Glass and Discharged Masses during DM1200 Tests (continued).

\begin{tabular}{|c|c|c|c|c|}
\hline Test & Date & Name & $\begin{array}{c}\text { Mass } \\
(\mathrm{kg})\end{array}$ & $\begin{array}{c}\text { Cumulative Mass } \\
(\mathrm{kg})\end{array}$ \\
\hline \multirow{43}{*}{4} & \multirow{8}{*}{$2 / 6 / 2013$} & G-12W-111A & \multirow{7}{*}{368.5} & \multirow{7}{*}{9000.0} \\
\hline & & G-12W-113A & & \\
\hline & & G-12W-114A & & \\
\hline & & G-12W-115A & & \\
\hline & & G-12W-115B & & \\
\hline & & G-12W-116A & & \\
\hline & & G-12W-125A & & \\
\hline & & $\mathrm{G}-12 \mathrm{~W}-126 \mathrm{~A}$ & \multirow{10}{*}{443.0} & \multirow{10}{*}{9443.0} \\
\hline & \multirow{28}{*}{$2 / 7 / 2013$} & $\mathrm{G}-12 \mathrm{~W}-126 \mathrm{~B}$ & & \\
\hline & & G-12W-127A & & \\
\hline & & G-12W-128A & & \\
\hline & & G-12W-128B & & \\
\hline & & G-12W-137A & & \\
\hline & & G-12W-138A & & \\
\hline & & G-12W-138B & & \\
\hline & & G-12W-140A & & \\
\hline & & G-12W-140B & & \\
\hline & & G-12W-142A & \multirow{8}{*}{494.5} & \multirow{8}{*}{9937.5} \\
\hline & & G-12W-143A & & \\
\hline & & G-12W-143B & & \\
\hline & & G-12W-145A & & \\
\hline & & G-12W-146A & & \\
\hline & & G-12W-146B & & \\
\hline & & G-12W-146C & & \\
\hline & & G-12W-147A & & \\
\hline & & G-12W-147B & \multirow{8}{*}{479.0} & \multirow{8}{*}{10416.5} \\
\hline & & G-12W-149A & & \\
\hline & & G-12W-151A & & \\
\hline & & G-12W-151B & & \\
\hline & & G-12X-6A & & \\
\hline & & G-12X-6B & & \\
\hline & & G-12X-7A & & \\
\hline & & G-12X-7B & & \\
\hline & & G-12X-11A & \multirow{10}{*}{468.5} & \multirow{10}{*}{10885.0} \\
\hline & & G-12X-11B & & \\
\hline & & G-12X-20A & & \\
\hline & \multirow{7}{*}{$2 / 8 / 2013$} & G-12X-20B & & \\
\hline & & G-12X-21A & & \\
\hline & & G-12X-30A & & \\
\hline & & G-12X-30B & & \\
\hline & & G-12X-31A & & \\
\hline & & G-12X-31B & & \\
\hline & & G-12X-32A & & \\
\hline
\end{tabular}


Table 5.4. Listing of Glass and Discharged Masses during DM1200 Tests (continued).

\begin{tabular}{|c|c|c|c|c|}
\hline Test & Date & Name & $\begin{array}{c}\text { Mass } \\
(\mathrm{kg})\end{array}$ & $\begin{array}{l}\text { Cumulative Mass } \\
(\mathrm{kg})\end{array}$ \\
\hline \multirow{16}{*}{4} & \multirow{16}{*}{$2 / 8 / 2013$} & G-12X-32B & \multirow{9}{*}{456.0} & \multirow{9}{*}{11341.0} \\
\hline & & G-12X-33A & & \\
\hline & & G-12X-33B & & \\
\hline & & G-12X-33C & & \\
\hline & & G-12X-34A & & \\
\hline & & G-12X-34B & & \\
\hline & & G-12X-35A & & \\
\hline & & G-12X-35B & & \\
\hline & & G-12X-35C & & \\
\hline & & G-12X-36A & \multirow{7}{*}{479.0} & \multirow{7}{*}{11820.0} \\
\hline & & G-12X-36B & & \\
\hline & & G-12X-38A & & \\
\hline & & G-12X-38B & & \\
\hline & & G-12X-39A & & \\
\hline & & G-12X-39B & & \\
\hline & & G-12X-41A & & \\
\hline
\end{tabular}


Table 5.5. XRF Analyzed Compositions for Glass Discharged During DM1200 Tests (wt\%).

\begin{tabular}{|c|c|c|c|c|c|c|c|c|}
\hline Test & \multicolumn{6}{|c|}{1} & \multicolumn{2}{|c|}{2} \\
\hline Mass (kg) & Target & 484.5 & 911.0 & 1395.0 & 1872.00 & 2366.0 & 2833.50 & 3316.0 \\
\hline Constituents & & $\begin{array}{c}\text { G-12U- } \\
80 \mathrm{~A}\end{array}$ & $\begin{array}{c}\text { G-12U- } \\
84 \mathrm{~A}\end{array}$ & $\begin{array}{c}\text { G-12U- } \\
95 \mathrm{~B}\end{array}$ & $\begin{array}{c}\text { G-12U- } \\
112 \mathrm{~A}\end{array}$ & $\begin{array}{c}\text { G-12U- } \\
125 \mathrm{~A}\end{array}$ & $\begin{array}{c}\text { G-12V- } \\
10 \mathrm{~B}\end{array}$ & $\begin{array}{c}\mathrm{G}-12 \mathrm{~V}- \\
23 \mathrm{~A}\end{array}$ \\
\hline $\mathrm{Al}_{2} \mathrm{O}_{3}$ & 23.97 & 23.69 & 23.79 & 23.61 & 23.68 & 23.65 & 23.68 & 23.85 \\
\hline $\mathrm{B}_{2} \mathrm{O}_{3}{ }^{*}$ & 19.19 & 17.21 & 17.43 & 17.62 & 17.77 & 17.90 & 17.99 & 18.06 \\
\hline $\mathrm{BaO}$ & 0.05 & 0.11 & 0.08 & 0.07 & 0.07 & 0.09 & 0.08 & 0.09 \\
\hline $\mathrm{Bi}_{2} \mathrm{O}_{3}$ & 1.14 & 1.17 & 1.22 & 1.26 & 1.24 & 1.20 & 1.28 & 1.22 \\
\hline $\mathrm{CaO}$ & 5.58 & 5.19 & 5.34 & 5.39 & 5.37 & 5.49 & 5.44 & 5.39 \\
\hline $\mathrm{CdO}$ & 0.02 & 0.04 & 0.04 & 0.04 & 0.03 & 0.04 & 0.03 & 0.03 \\
\hline $\mathrm{Cr}_{2} \mathrm{O}_{3}$ & 0.52 & 0.29 & 0.32 & 0.36 & 0.38 & 0.41 & 0.45 & 0.43 \\
\hline $\mathrm{F}$ & 0.67 & $0.13^{\#}$ & 0.17 & $0.23^{\#}$ & 0.25 & $0.26^{\#}$ & $0.23^{\#}$ & 0.25 \\
\hline $\mathrm{Fe}_{2} \mathrm{O}_{3}$ & 5.90 & 5.99 & 6.16 & 6.18 & 6.11 & 6.15 & 6.09 & 5.94 \\
\hline $\mathrm{K}_{2} \mathrm{O}$ & 0.14 & 0.18 & 0.13 & 0.16 & 0.13 & 0.06 & 0.03 & 0.12 \\
\hline $\mathrm{Li}_{2} \mathrm{O}^{*}$ & 3.57 & 3.39 & 3.42 & 3.45 & 3.48 & 3.50 & 3.51 & 3.52 \\
\hline $\mathrm{MgO}$ & 0.12 & 0.27 & 0.23 & 0.20 & 0.25 & 0.23 & 0.21 & 0.21 \\
\hline $\mathrm{MnO}$ & $\S$ & 0.05 & 0.04 & 0.04 & 0.04 & 0.04 & 0.03 & 0.03 \\
\hline $\mathrm{Na}_{2} \mathrm{O}$ & 9.58 & 10.10 & 9.87 & 9.80 & 9.74 & 9.45 & 9.58 & 9.53 \\
\hline $\mathrm{NiO}$ & 0.40 & 0.26 & 0.31 & 0.34 & 0.34 & 0.38 & 0.34 & 0.35 \\
\hline $\mathrm{P}_{2} \mathrm{O}_{5}$ & 1.05 & 1.10 & 1.10 & 1.10 & 1.14 & 1.08 & 1.12 & 1.11 \\
\hline $\mathrm{PbO}$ & 0.41 & 0.36 & 0.39 & 0.40 & 0.39 & 0.40 & 0.42 & 0.42 \\
\hline $\mathrm{SiO}_{2}$ & 27.00 & 29.57 & 28.99 & 28.84 & 28.76 & 28.85 & 28.73 & 28.71 \\
\hline $\mathrm{SO}_{3}$ & 0.20 & 0.09 & 0.13 & 0.13 & 0.13 & 0.16 & 0.12 & 0.13 \\
\hline $\mathrm{TiO}_{2}$ & 0.01 & 0.12 & 0.11 & 0.10 & 0.09 & 0.08 & 0.07 & 0.07 \\
\hline $\mathrm{ZnO}$ & 0.08 & 0.16 & 0.15 & 0.13 & 0.12 & 0.11 & 0.11 & 0.10 \\
\hline $\mathrm{ZrO}_{2}$ & 0.39 & 0.56 & 0.55 & 0.51 & 0.47 & 0.47 & 0.45 & 0.43 \\
\hline Sum & 100.00 & 100.00 & 100.00 & 100.00 & 100.00 & 100.00 & 100.00 & 100.00 \\
\hline
\end{tabular}

* Values calculated from $\mathrm{B}_{2} \mathrm{O}_{3}$ and $\mathrm{Li}_{2} \mathrm{O}$ analysis by DCP-AES on the last discharged glass sample from previous test and average feed sample analysis using a simple well stirred tank model

$\S$ - Not a target constituent

\# - F was measured by XRF, values for other samples calculated by interpolation 
Table 5.5. XRF Analyzed Compositions for Glass Discharged During DM1200 Tests (wt\%) (continued).

\begin{tabular}{|c|c|c|c|c|c|c|c|c|}
\hline Test & \multicolumn{6}{|c|}{2} & \multicolumn{2}{|c|}{3} \\
\hline Mass $(\mathrm{kg})$ & \multirow[b]{2}{*}{ Target } & 3789.5 & 4290.50 & 4780.5 & 5215.50 & 5621.0 & 6103.5 & 6599.0 \\
\hline Constituents & & $\begin{array}{c}\text { G-12V- } \\
28 \mathrm{~A}\end{array}$ & $\begin{array}{c}\mathrm{G}-12 \mathrm{~V}- \\
42 \mathrm{~A}\end{array}$ & $\begin{array}{c}\mathrm{G}-12 \mathrm{~V}- \\
56 \mathrm{~B}\end{array}$ & $\begin{array}{c}\mathrm{G}-12 \mathrm{~V}- \\
61 \mathrm{~B}\end{array}$ & $\begin{array}{c}\mathrm{G}-12 \mathrm{~V}- \\
73 \mathrm{~A}\end{array}$ & $\begin{array}{c}\text { G-12W- } \\
33 \mathrm{~A}\end{array}$ & $\begin{array}{c}\mathrm{G}-12 \mathrm{~W} \\
39 \mathrm{~A}\end{array}$ \\
\hline $\mathrm{Al}_{2} \mathrm{O}_{3}$ & 23.97 & 23.77 & 23.59 & 23.51 & 23.66 & 23.58 & 23.57 & 23.28 \\
\hline $\mathrm{B}_{2} \mathrm{O}_{3}{ }^{*}$ & 19.19 & 18.11 & 18.16 & 18.19 & 18.22 & 18.24 & 18.25 & 18.27 \\
\hline $\mathrm{BaO}$ & 0.05 & 0.10 & 0.11 & 0.07 & 0.10 & 0.07 & 0.08 & 0.07 \\
\hline $\mathrm{Bi}_{2} \mathrm{O}_{3}$ & 1.14 & 1.20 & 1.19 & 1.23 & 1.28 & 1.26 & 1.25 & 1.27 \\
\hline $\mathrm{CaO}$ & 5.58 & 5.42 & 5.46 & 5.50 & 5.57 & 5.55 & 5.56 & 5.68 \\
\hline $\mathrm{CdO}$ & 0.02 & 0.03 & 0.04 & 0.03 & 0.03 & 0.03 & 0.03 & 0.03 \\
\hline $\mathrm{Cr}_{2} \mathrm{O}_{3}$ & 0.52 & 0.42 & 0.41 & 0.43 & 0.45 & 0.43 & 0.47 & 0.51 \\
\hline $\mathrm{F}$ & 0.67 & $0.28^{\#}$ & 0.28 & $0.29^{\#}$ & 0.28 & $0.28^{\#}$ & $0.27^{\#}$ & 0.29 \\
\hline $\mathrm{Fe}_{2} \mathrm{O}_{3}$ & 5.90 & 6.01 & 5.82 & 5.98 & 5.98 & 5.95 & 5.65 & 5.90 \\
\hline $\mathrm{K}_{2} \mathrm{O}$ & 0.14 & 0.15 & 0.15 & 0.21 & 0.19 & 0.16 & 0.11 & 0.04 \\
\hline $\mathrm{Li}_{2} \mathrm{O}^{*}$ & 3.57 & 3.53 & 3.54 & 3.54 & 3.55 & 3.55 & 3.55 & 3.56 \\
\hline $\mathrm{MgO}$ & 0.12 & 0.22 & 0.23 & 0.17 & 0.21 & 0.22 & 0.26 & 0.26 \\
\hline $\mathrm{MnO}$ & $\S$ & 0.03 & 0.04 & 0.03 & 0.02 & 0.02 & 0.03 & 0.03 \\
\hline $\mathrm{Na}_{2} \mathrm{O}$ & 9.58 & 9.55 & 9.63 & 9.63 & 9.33 & 9.56 & 10.03 & 9.76 \\
\hline $\mathrm{NiO}$ & 0.40 & 0.35 & 0.33 & 0.38 & 0.37 & 0.36 & 0.33 & 0.38 \\
\hline $\mathrm{P}_{2} \mathrm{O}_{5}$ & 1.05 & 1.09 & 1.11 & 1.11 & 1.12 & 1.12 & 1.06 & 1.14 \\
\hline $\mathrm{PbO}$ & 0.41 & 0.37 & 0.40 & 0.40 & 0.41 & 0.40 & 0.40 & 0.41 \\
\hline $\mathrm{SiO}_{2}$ & 27.00 & 28.67 & 28.82 & 28.61 & 28.51 & 28.48 & 28.39 & 28.41 \\
\hline $\mathrm{SO}_{3}$ & 0.20 & 0.13 & 0.14 & 0.13 & 0.14 & 0.16 & 0.14 & 0.16 \\
\hline $\mathrm{TiO}_{2}$ & 0.01 & 0.07 & 0.06 & 0.05 & 0.07 & 0.07 & 0.05 & 0.06 \\
\hline $\mathrm{ZnO}$ & 0.08 & 0.09 & 0.09 & 0.09 & 0.09 & 0.09 & 0.10 & 0.09 \\
\hline $\mathrm{ZrO}_{2}$ & 0.39 & 0.42 & 0.41 & 0.41 & 0.41 & 0.41 & 0.41 & 0.42 \\
\hline Sum & 100.00 & 100.00 & 100.00 & 100.00 & 100.00 & 100.00 & 100.00 & 100.00 \\
\hline
\end{tabular}

* Values calculated from $\mathrm{B}_{2} \mathrm{O}_{3}$ and $\mathrm{Li}_{2} \mathrm{O}$ analysis by DCP-AES on the last discharged glass sample from previous test and average feed sample analysis using a simple well stirred tank model

$\S$ - Not a target constituent

\# - F was measured by XRF, values for other samples calculated by interpolation 
Table 5.5. XRF Analyzed Compositions for Glass Discharged During DM1200 Tests (wt\%) (continued).

\begin{tabular}{|c|c|c|c|c|c|c|c|c|}
\hline \multirow{3}{*}{\begin{tabular}{|c|} 
Test \\
Mass (kg) \\
Constituents \\
\end{tabular}} & \multicolumn{6}{|c|}{3} & \multicolumn{2}{|c|}{4} \\
\hline & \multirow[b]{2}{*}{ Target } & 7038.0 & 7534.0 & 8018.0 & 8515.5 & 8631.5 & 9000.0 & 9443.0 \\
\hline & & $\begin{array}{c}\mathrm{G}-12 \mathrm{~W}- \\
47 \mathrm{~A}\end{array}$ & $\begin{array}{c}\mathrm{G}-12 \mathrm{~W}- \\
61 \mathrm{~B}\end{array}$ & $\begin{array}{c}\text { G-12W- } \\
76 \mathrm{~A}\end{array}$ & $\begin{array}{c}\text { G-12W } \\
82 B\end{array}$ & $\begin{array}{c}\text { G-12W- } \\
91 B\end{array}$ & $\begin{array}{c}\text { G-12W- } \\
125 \mathrm{~A}\end{array}$ & $\begin{array}{c}\text { G-12W- } \\
140 B\end{array}$ \\
\hline $\mathrm{Al}_{2} \mathrm{O}_{3}$ & 23.97 & 23.19 & 23.31 & 23.54 & 23.57 & 23.27 & 23.19 & 23.28 \\
\hline $\mathrm{B}_{2} \mathrm{O}_{3}{ }^{*}$ & 19.19 & 18.28 & 18.29 & 18.29 & 18.30 & 18.30 & 18.30 & 18.30 \\
\hline $\mathrm{BaO}$ & 0.05 & 0.10 & 0.06 & 0.07 & 0.09 & 0.08 & 0.08 & 0.12 \\
\hline $\mathrm{Bi}_{2} \mathrm{O}_{3}$ & 1.14 & 1.24 & 1.26 & 1.20 & 1.15 & 1.24 & 1.25 & 1.28 \\
\hline $\mathrm{CaO}$ & 5.58 & 5.64 & 5.72 & 5.47 & 5.45 & 5.69 & 5.65 & 5.62 \\
\hline $\mathrm{CdO}$ & 0.02 & 0.03 & 0.03 & 0.02 & 0.03 & 0.03 & 0.03 & 0.03 \\
\hline $\mathrm{Cr}_{2} \mathrm{O}_{3}$ & 0.52 & 0.47 & 0.46 & 0.46 & 0.46 & 0.46 & 0.44 & 0.45 \\
\hline $\mathrm{F}$ & 0.67 & $0.30^{\#}$ & 0.29 & $0.29^{\#}$ & 0.35 & $0.37^{\#}$ & $0.25^{\#}$ & 0.25 \\
\hline $\mathrm{Fe}_{2} \mathrm{O}_{3}$ & 5.90 & 5.83 & 5.81 & 5.50 & 5.35 & 5.74 & 5.81 & 5.70 \\
\hline $\mathrm{K}_{2} \mathrm{O}$ & 0.14 & 0.15 & 0.03 & 0.17 & 0.14 & 0.12 & 0.16 & 0.13 \\
\hline $\mathrm{Li}_{2} \mathrm{O}^{*}$ & 3.57 & 3.56 & 3.56 & 3.56 & 3.56 & 3.56 & 3.56 & 3.56 \\
\hline $\mathrm{MgO}$ & 0.12 & 0.26 & 0.25 & 0.18 & 0.26 & 0.26 & 0.25 & 0.25 \\
\hline $\mathrm{MnO}$ & $\S$ & 0.03 & 0.03 & 0.04 & 0.02 & 0.02 & 0.03 & 0.03 \\
\hline $\mathrm{Na}_{2} \mathrm{O}$ & 9.58 & 10.01 & 9.86 & 10.17 & 10.20 & 9.64 & 9.96 & 9.91 \\
\hline $\mathrm{NiO}$ & 0.40 & 0.40 & 0.39 & 0.38 & 0.34 & 0.41 & 0.40 & 0.37 \\
\hline $\mathrm{P}_{2} \mathrm{O}_{5}$ & 1.05 & 1.08 & 1.07 & 1.12 & 1.07 & 1.09 & 1.09 & 1.04 \\
\hline $\mathrm{PbO}$ & 0.41 & 0.42 & 0.41 & 0.40 & 0.38 & 0.40 & 0.39 & 0.41 \\
\hline $\mathrm{SiO}_{2}$ & 27.00 & 28.31 & 28.44 & 28.45 & 28.63 & 28.62 & 28.47 & 28.60 \\
\hline $\mathrm{SO}_{3}$ & 0.20 & 0.15 & 0.16 & 0.16 & 0.16 & 0.16 & 0.14 & 0.14 \\
\hline $\mathrm{TiO}_{2}$ & 0.01 & 0.06 & 0.05 & 0.05 & 0.04 & 0.06 & 0.06 & 0.05 \\
\hline $\mathrm{ZnO}$ & 0.08 & 0.10 & 0.09 & 0.09 & 0.08 & 0.09 & 0.08 & 0.08 \\
\hline $\mathrm{ZrO}_{2}$ & 0.39 & 0.40 & 0.42 & 0.39 & 0.37 & 0.40 & 0.39 & 0.40 \\
\hline Sum & 100.00 & 100.00 & 100.00 & 100.00 & 100.00 & 100.00 & 100.00 & 100.00 \\
\hline
\end{tabular}

* Values calculated from $\mathrm{B}_{2} \mathrm{O}_{3}$ and $\mathrm{Li}_{2} \mathrm{O}$ analysis by DCP-AES on the last discharged glass sample from previous test and average feed sample analysis using a simple well stirred tank model

$\S$ - Not a target constituent

\# - F was measured by XRF, values for other samples calculated by interpolation 
Table 5.5. XRF Analyzed Compositions for Glass Discharged During DM1200 Tests (wt\%) (continued).

\begin{tabular}{|c|c|c|c|c|c|c|c|c|}
\hline Test & & & & 4 & & & & \\
\hline Mass (kg) & & \begin{tabular}{|l|}
9937.5 \\
\end{tabular} & 10416.5 & \begin{tabular}{|l|}
10885.0 \\
\end{tabular} & 11341.0 & 11820.0 & $A_{\text {yeroge }}$ & 0 \\
\hline Constituents & Target & $\begin{array}{c}\mathrm{G}-12 \mathrm{~W}- \\
147 \mathrm{~A}\end{array}$ & $\begin{array}{c}\text { G-12X- } \\
7 \mathrm{~B}\end{array}$ & $\begin{array}{c}\text { G-12X- } \\
32 \mathrm{~A}\end{array}$ & $\begin{array}{c}\text { G-12X- } \\
35 \mathrm{C}\end{array}$ & $\begin{array}{c}\text { G-12X- } \\
41 \mathrm{~A}\end{array}$ & Average & Dev. \\
\hline $\mathrm{Al}_{2} \mathrm{O}_{3}$ & 23.97 & 23.23 & 23.26 & 23.40 & 23.15 & 23.04 & 23.47 & -2.08 \\
\hline $\mathrm{B}_{2} \mathrm{O}_{3}{ }^{*}$ & 19.19 & 18.31 & 18.31 & 18.31 & 18.31 & 18.31 & 18.12 & $\mathrm{NC}$ \\
\hline $\mathrm{BaO}$ & 0.05 & & & 0.10 & 0.09 & 0.10 & 0.09 & $\mathrm{NC}$ \\
\hline $\mathrm{Bi}_{2} \mathrm{O}_{3}$ & 14 & 5 & 24 & 24 & 30 & 27 & 24 & 8.57 \\
\hline $\mathrm{CaO}$ & 5.58 & 5.68 & 5.63 & 55 & .71 & 69 & 53 & -0.86 \\
\hline $\mathrm{CdO}$ & 0.02 & 0.03 & 0.02 & 3 & 0 & 03 & 03 & $\mathrm{NC}$ \\
\hline $\mathrm{Cr}_{2} \mathrm{O}_{3}$ & 0.52 & 0.45 & 0.46 & 0.46 & 0.44 & 47 & 43 & $\mathrm{NC}$ \\
\hline $\mathrm{F}$ & 0.67 & $0.26^{\#}$ & 0.28 & $0.29^{\#}$ & 0.30 & $0.31^{\#}$ & 0.27 & $\mathrm{NC}$ \\
\hline $\mathrm{Fe}_{2} \mathrm{O}_{3}$ & 5.90 & 72 & 5.59 & 5.68 & 5.8 & 5.89 & 5.86 & -0.68 \\
\hline $\mathrm{K}_{2} \mathrm{O}$ & 0.14 & 15 & 0.16 & 14 & 0.17 & 18 & 0.13 & $\mathrm{NC}$ \\
\hline $\mathrm{Li}_{2} \mathrm{O}^{*}$ & 3.57 & 3.56 & 3.56 & 3.56 & 3.56 & 56 & .53 & $\mathrm{NC}$ \\
\hline $\mathrm{MgO}$ & 0.12 & & & & & & & $\mathrm{NC}$ \\
\hline $\mathrm{MnO}$ & 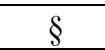 & & & & 0. & 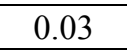 & 03 & $\mathrm{NC}$ \\
\hline $\mathrm{Na}_{2} \mathrm{O}$ & 9.58 & 10.09 & 10.08 & 10.11 & 9.6 & 10.04 & 9.82 & 2.47 \\
\hline $\mathrm{NiO}$ & 0.40 & 035 & 0.39 & & 0.3 & 0.40 & $02+2$ & $\mathrm{NC}$ \\
\hline $\mathrm{P}_{2} \mathrm{O}_{5}$ & 1.05 & 1.07 & 1.11 & 1.06 & 1.08 & 06 & 1.09 & 4.09 \\
\hline $\mathrm{PbO}$ & 0.41 & 0.41 & 0.40 & 0.40 & 0.43 & 0.42 & 0.40 & $\mathrm{NC}$ \\
\hline $\mathrm{SiO}_{2}$ & 27.00 & 28.40 & 28.44 & 28.40 & 28.58 & 28.25 & 28.61 & 5.96 \\
\hline $\mathrm{SO}_{3}$ & 0.20 & & & & 0.17 & 18 & & $\mathrm{NC}$ \\
\hline $\mathrm{TiO}_{2}$ & 0.01 & 0.06 & 0.07 & 0.05 & 0.07 & 0.05 & 0.07 & $\mathrm{NC}$ \\
\hline $\mathrm{ZnO}$ & 0.08 & 0.08 & 0.09 & 0.09 & 0.08 & 0.09 & 0.10 & $\mathrm{NC}$ \\
\hline $\mathrm{ZrO}_{2}$ & 0.39 & 0.39 & 0.39 & 0.39 & 0.40 & 0.41 & 0.43 & $\mathrm{NC}$ \\
\hline Sum & 100.00 & 100.00 & 100.00 & 100.00 & 100.00 & 100.00 & 100.00 & $\mathrm{NC}$ \\
\hline
\end{tabular}

* Values calculated from $\mathrm{B}_{2} \mathrm{O}_{3}$ and $\mathrm{Li}_{2} \mathrm{O}$ analysis by DCP-AES on the last discharged glass sample from previous test and average feed sample analysis using a simple well stirred tank model $\S$ - Not a target constituent

\# - F was measured by XRF, values for other samples calculated by interpolation 
Table 6.1. Results from Melter Off-Gas Emission Samples.

\begin{tabular}{|c|c|c|c|c|c|c|c|c|c|}
\hline & \multicolumn{4}{|c|}{$\begin{array}{c}\text { Test } 1 \\
\text { 1/16/2013 18:21 - 19:21 } \\
\text { 26.3\% Moisture, } 99.6 \% \text { Isokinetic }\end{array}$} & \multicolumn{4}{|c|}{$\begin{array}{c}\text { Test } 2 \\
\text { 1/23/2013 20:01 - 21:01 } \\
29.2 \% \text { Moisture, } 101 \% \text { Isokinetic }\end{array}$} \\
\hline & & $\begin{array}{c}\text { Feed }^{\#} \\
(\mathrm{mg} / \mathrm{min})\end{array}$ & $\begin{array}{c}\text { Output } \\
\text { (mg/min) }\end{array}$ & $\%$ Emitted & $\mathrm{DF}$ & $\begin{array}{c}\text { Feed }^{\#} \\
(\mathrm{mg} / \mathrm{min})\end{array}$ & $\begin{array}{c}\text { Output } \\
\text { (mg/min) }\end{array}$ & $\begin{array}{c}\% \\
\text { Emitted }\end{array}$ & $\mathrm{DF}$ \\
\hline \multirow{22}{*}{ لِّ } & Total $^{\$}$ & 2458333 & 3160 & 0.13 & 778 & 2983796 & 4173 & 0.14 & 715 \\
\hline & $\mathrm{Al}$ & 112223 & 87.9 & 0.08 & 1277 & 136211 & 130 & 0.10 & 1045 \\
\hline & B & 52706 & 252 & 0.48 & 209 & 63972 & 369 & 0.58 & 173 \\
\hline & $\mathrm{Ba}$ & 396 & 0.97 & 0.24 & 410 & 481 & 1.27 & 0.26 & 379 \\
\hline & $\mathrm{Bi}$ & 9050 & 46.0 & 0.51 & 197 & 10984 & 57.7 & 0.52 & 191 \\
\hline & $\mathrm{Ca}$ & 35299 & 57.6 & 0.16 & 613 & 42844 & 70.9 & 0.17 & 605 \\
\hline & $\mathrm{Cd}$ & 158 & 1.08 & 0.68 & 147 & 192 & 1.40 & 0.73 & 137 \\
\hline & $\mathrm{Cr}$ & 3149 & 26.1 & 0.83 & 121 & 3822 & 32.0 & 0.84 & 119 \\
\hline & $\mathrm{F}^{*}$ & 5930 & 845 & 14.2 & 7.02 & 7197 & 1156 & 16.1 & 6.22 \\
\hline & $\mathrm{Fe}$ & 36511 & 103 & 0.28 & 355 & 44315 & 129 & 0.29 & 342 \\
\hline & $\mathrm{K}$ & 1029 & 9.29 & 0.90 & 111 & 1248 & 12.1 & 0.97 & 103 \\
\hline & $\mathrm{Li}$ & 14676 & 48.3 & 0.33 & 304 & 17813 & 64.7 & 0.36 & 275 \\
\hline & $\mathrm{Mg}$ & 640 & 3.66 & 0.57 & 175 & 777 & 4.56 & 0.59 & 171 \\
\hline & $\mathrm{Na}$ & 62904 & 259 & 0.41 & 243 & 76349 & 326 & 0.43 & 234 \\
\hline & $\mathrm{Ni}$ & 2782 & 6.08 & 0.22 & 458 & 3376 & 7.42 & 0.22 & 455 \\
\hline & $\mathrm{P}$ & 4057 & 1.04 & 0.03 & 3893 & 4925 & 1.26 & 0.03 & 3910 \\
\hline & $\mathrm{Pb}$ & 3368 & 19.6 & 0.58 & 172 & 4088 & 27.6 & 0.68 & 148 \\
\hline & $\mathrm{S}^{*}$ & 709 & 29.4 & 4.14 & 187 & 861 & 114 & 13.2 & 7.58 \\
\hline & $\mathrm{Si}$ & 111701 & 150 & 0.13 & 747 & 135577 & 164 & 0.12 & 825 \\
\hline & $\mathrm{Ti}$ & 53 & 1.17 & 2.21 & 45.2 & 64 & 1.33 & 2.06 & 48.6 \\
\hline & $\mathrm{Zn}$ & 569 & 1.60 & 0.28 & 354 & 690 & 1.37 & 0.20 & 503 \\
\hline & $\mathrm{Zr}$ & 2555 & 2.75 & 0.11 & 930 & 3101 & 3.25 & 0.10 & 954 \\
\hline \multirow{3}{*}{ שే } & $\mathrm{B}$ & 52706 & 309 & 0.59 & 171 & 63972 & 386 & 0.60 & 166 \\
\hline & $\mathrm{F}$ & 5930 & 545 & 9.20 & 10.9 & 7197 & 764 & 10.6 & 9.42 \\
\hline & $\mathrm{S}$ & 709 & 181 & 25.5 & 3.93 & 861 & 310 & 36.1 & 2.77 \\
\hline
\end{tabular}

$\$$ - From gravimetric analysis of filters and particulate nitric acid rinses

\# - Feed rate calculated from target composition and total glass production rate

* - Calculated from analysis of water dissolution of filter particulate and direct analysis of nitric acid rinses 


\section{Table 6.1. Results from Melter Off-Gas Emission Samples (continued).}

\begin{tabular}{|c|c|c|c|c|c|c|c|c|c|}
\hline & \multicolumn{4}{|c|}{$\begin{array}{c}\text { Test } 3 \\
\text { 1/29/2013 15:10 - 16:10 } \\
35.8 \% \text { Moisture, } 105 \% \text { Isokinetic }\end{array}$} & \multicolumn{4}{|c|}{$\begin{array}{c}\text { Test } 4 \\
\text { 2/7/13 15:01 - 16:01 } \\
\text { 36.6\% Moisture, } 92.9 \% \text { Isokinetic }\end{array}$} \\
\hline & & $\begin{array}{c}\text { Feed }^{\#} \\
(\mathrm{mg} / \mathrm{min})\end{array}$ & $\begin{array}{c}\text { Output } \\
\text { (mg/min) }\end{array}$ & $\begin{array}{c}\% \\
\text { Emitted }\end{array}$ & $\mathrm{DF}$ & $\begin{array}{c}\text { Feed }^{\#} \\
(\mathrm{mg} / \mathrm{min})\end{array}$ & $\begin{array}{c}\text { Output } \\
\text { (mg/min) }\end{array}$ & $\begin{array}{c}\% \\
\text { Emitted } \\
\end{array}$ & $\mathrm{DF}$ \\
\hline \multirow{22}{*}{$\frac{\stackrel{0}{\tilde{U}}}{\overbrace{0}^{0}}$} & Total $^{\$}$ & 2978571 & 5267 & 0.18 & 566 & 3328571 & 5652 & 0.17 & 589 \\
\hline & $\mathrm{Al}$ & 132195 & 152 & 0.11 & 871 & 147729 & 212 & 0.14 & 697 \\
\hline & $\mathrm{B}$ & 62086 & 611 & 0.98 & 102 & 69382 & 306 & 0.44 & 227 \\
\hline & $\mathrm{Ba}$ & 467 & 1.22 & 0.26 & 384 & 522 & 2.16 & 0.41 & 242 \\
\hline & $\mathrm{Bi}$ & 10660 & 63.4 & 0.59 & 168 & 11913 & 93.9 & 0.79 & 127 \\
\hline & $\mathrm{Ca}$ & 41581 & 83.9 & 0.20 & 496 & 46467 & 125 & 0.27 & 372 \\
\hline & $\mathrm{Cd}$ & 187 & 2.48 & 1.33 & 75.3 & 209 & 3.91 & 1.87 & 53.4 \\
\hline & $\mathrm{Cr}$ & 3709 & 33.5 & 0.90 & 111 & 4145 & 55.3 & 1.34 & 74.9 \\
\hline & $\mathrm{F}^{*}$ & 6985 & 1514 & 21.7 & 4.61 & 7806 & 849 & 10.9 & 9.20 \\
\hline & $\mathrm{Fe}$ & 43009 & 149 & 0.35 & 289 & 48063 & 220 & 0.46 & 218 \\
\hline & $\mathrm{K}$ & 1212 & 11.9 & 0.98 & 102 & 1354 & 20.8 & 1.53 & 65.2 \\
\hline & $\mathrm{Li}$ & 17288 & 68.4 & 0.40 & 253 & 19320 & 109 & 0.56 & 177 \\
\hline & $\mathrm{Mg}$ & 754 & 4.76 & 0.63 & 159 & 843 & 8.27 & 0.98 & 102 \\
\hline & $\mathrm{Na}$ & 74098 & 354 & 0.48 & 209 & 82805 & 575 & 0.69 & 144 \\
\hline & $\mathrm{Ni}$ & 3277 & 9.44 & 0.29 & 347 & 3662 & 13.7 & 0.37 & 268 \\
\hline & $\mathrm{P}$ & 4779 & 17.3 & 0.36 & 276 & 5341 & 13.4 & 0.25 & 400 \\
\hline & $\mathrm{Pb}$ & 3968 & 27.8 & 0.70 & 143 & 4434 & 46.1 & 1.04 & 96.1 \\
\hline & $\mathrm{S}^{*}$ & 836 & 118 & 14.2 & 7.07 & 934 & 175 & 18.8 & 5.32 \\
\hline & $\mathrm{Si}$ & 131580 & 213 & 0.16 & 617 & 147041 & 324 & 0.22 & 454 \\
\hline & $\mathrm{Ti}$ & 62 & 0.85 & 1.36 & 73.8 & 70 & 1.82 & 2.61 & 38.4 \\
\hline & $\mathrm{Zn}$ & 670 & 1.76 & 0.26 & 381 & 749 & 2.90 & 0.39 & 258 \\
\hline & $\mathrm{Zr}$ & 3010 & 3.99 & 0.13 & 754 & 3364 & 6.29 & 0.19 & 535 \\
\hline \multirow{3}{*}{$\stackrel{\tilde{J}}{0}$} & $\mathrm{~B}$ & 62086 & 223 & 0.36 & 278 & 69382 & 983 & 1.42 & 70.6 \\
\hline & $\mathrm{F}$ & 6985 & 316 & 4.53 & 22.1 & 7806 & 1786 & 22.9 & 4.37 \\
\hline & $\mathrm{S}$ & 836 & 247 & 29.5 & 3.39 & 934 & 469 & 50.2 & 1.99 \\
\hline
\end{tabular}

$\$$ - From gravimetric analysis of filters and particulate nitric acid rinses

\# - Feed rate calculated from target composition and total glass production rate

* - Calculated from analysis of water dissolution of filter particulate and direct analysis of nitric acid rinses 
Table 6.2. Concentrations [ppmv] of Selected Species in Off-Gas Measured by FTIR Spectroscopy, Test 1.

\begin{tabular}{|c|c|c|c|c|c|c|c|c|c|}
\hline & \multicolumn{3}{|c|}{ Melter outlet } & \multicolumn{3}{c|}{ SBS outlet } & \multicolumn{3}{c|}{ WESP outlet } \\
\cline { 2 - 11 } & Aver. & Min. & Max. & Aver. & Min. & Max. & Aver. & Min. & Max. \\
\hline $\mathrm{N}_{2} \mathrm{O}$ & 1.8 & 1.3 & 2.3 & 2.1 & 1.2 & 5.1 & 2.0 & $<1.0$ & 4.5 \\
\hline $\mathrm{NO}$ & 400 & 292 & 553 & 444 & 252 & 1044 & 397 & 2.8 & 772 \\
\hline $\mathrm{NO}_{2}$ & 63.4 & 45.5 & 89.2 & 66.0 & 29.1 & 209 & 76.1 & $<1.0$ & 187 \\
\hline $\mathrm{NH}_{3}$ & 1.9 & $<1.0$ & 4.0 & $<1.0$ & $<1.0$ & 2.0 & $<1.0$ & $<1.0$ & $<1.0$ \\
\hline $\mathrm{H}_{2} \mathrm{O} \%$ & 26.7 & 18.8 & 37.3 & 10.2 & 8.1 & 16.1 & 8.7 & 4.9 & 15.9 \\
\hline $\mathrm{CO}_{2} \%$ & 0.59 & 0.46 & 0.87 & 0.63 & 0.41 & 1.39 & 0.59 & 0.06 & 1.21 \\
\hline $\mathrm{Nitrous} \mathrm{Acid}^{2}$ & 1.7 & 1.1 & 3.2 & 1.1 & $<1.0$ & 3.4 & 1.7 & $<1.0$ & 3.9 \\
\hline Nitric Acid & $<1.0$ & $<1.0$ & 1.1 & $<1.0$ & $<1.0$ & 14.2 & $<1.0$ & $<1.0$ & 1.7 \\
\hline $\mathrm{HCN}$ & $<1.0$ & $<1.0$ & 2.3 & $<1.0$ & $<1.0$ & $<1.0$ & $<1.0$ & $<1.0$ & $<1.0$ \\
\hline $\mathrm{CO}$ & 2.9 & 1.7 & 4.8 & 3.5 & $<1.0$ & 11.3 & 3.1 & $<1.0$ & 8.6 \\
\hline $\mathrm{HCl}$ & $<1.0$ & $<1.0$ & 1.0 & $<1.0$ & $<1.0$ & 8.1 & $<1.0$ & $<1.0$ & $<1.0$ \\
\hline $\mathrm{HF}$ & 16.5 & 7.0 & 28.9 & 9.8 & 4.2 & 25.4 & 4.4 & 2.4 & 10.5 \\
\hline
\end{tabular}


Table 6.3. Concentrations [ppmv] of Selected Species in Off-Gas Measured by FTIR Spectroscopy, Test 2.

\begin{tabular}{|c|c|c|c|c|c|c|c|c|c|c|c|c|}
\hline & \multicolumn{3}{|c|}{ Melter outlet } & \multicolumn{3}{|c|}{ SBS outlet } & \multicolumn{3}{|c|}{ WESP outlet } & \multicolumn{3}{|c|}{ HEPA outlet } \\
\hline & Aver. & Min. & Max. & Aver. & Min. & Max. & Aver. & Min. & Max. & Aver. & Min. & Max. \\
\hline $\mathrm{N}_{2} \mathrm{O}$ & 1.5 & $<1.0$ & 2.2 & 2.6 & 1.0 & 3.7 & 2.5 & $<1.0$ & 5.4 & 2.8 & 1.9 & 5.2 \\
\hline $\mathrm{NO}$ & 333 & 155 & 540 & 586 & 160 & 835 & 500 & 1.6 & 1102 & 532 & 402 & 903 \\
\hline $\mathrm{NO}_{2}$ & 48.9 & 25.6 & 90.9 & 93.4 & 26.3 & 153 & 92.5 & $<1.0$ & 266 & 102 & 70.8 & 229 \\
\hline $\mathrm{NH}_{3}$ & 6.4 & 4.6 & 10.0 & 4.7 & 4.4 & 5.9 & 4.6 & 3.0 & 6.7 & 5.1 & 4.5 & 5.5 \\
\hline $\mathrm{H}_{2} \mathrm{O} \%$ & 20.5 & 11.2 & 30.7 & 8.8 & 6.4 & 11.0 & 7.9 & 4.3 & 12.7 & 7.4 & 6.5 & 8.5 \\
\hline $\mathrm{CO}_{2} \%$ & 0.49 & 0.26 & 0.75 & 0.84 & 0.29 & 1.17 & 0.77 & 0.05 & 2.02 & 0.84 & 0.62 & 1.65 \\
\hline Nitrous Acid & 1.2 & $<1.0$ & 2.5 & 1.4 & $<1.0$ & 1.8 & 2.0 & $<1.0$ & 6.2 & 3.2 & 2.4 & 6.3 \\
\hline Nitric Acid & 4.5 & $<1.0$ & 19.2 & 1.7 & $<1.0$ & 22.7 & $<1.0$ & $<1.0$ & 2.4 & 1.0 & $<1.0$ & 1.8 \\
\hline $\mathrm{HCN}$ & $<1.0$ & $<1.0$ & 1.6 & $<1.0$ & $<1.0$ & $<1.0$ & $<1.0$ & $<1.0$ & $<1.0$ & $<1.0$ & $<1.0$ & $<1.0$ \\
\hline $\mathrm{CO}$ & 2.2 & $<1.0$ & 4.4 & 4.9 & 1.2 & 9.3 & 4.0 & $<1.0$ & 10.7 & 3.8 & 1.8 & 8.7 \\
\hline $\mathrm{HCl}$ & 3.2 & $<1.0$ & 12.5 & 1.0 & $<1.0$ & 8.3 & $<1.0$ & $<1.0$ & $<1.0$ & $<1.0$ & $<1.0$ & $<1.0$ \\
\hline $\mathrm{HF}$ & 27.5 & 6.3 & 67.6 & 14.8 & 5.3 & 22.6 & 4.1 & 2.2 & 16.6 & 2.7 & 2.5 & 3.0 \\
\hline
\end{tabular}


Table 6.4. Concentrations [ppmv] of Selected Species in Off-Gas Measured by FTIR Spectroscopy, Test 3.

\begin{tabular}{|c|c|c|c|c|c|c|c|c|c|c|c|c|}
\hline & \multicolumn{1}{|c|}{ Melter outlet } & \multicolumn{1}{c|}{ SBS outlet } & \multicolumn{4}{c|}{ WESP outlet } \\
\cline { 2 - 16 } & Aver. & Min. & Max. & Aver. & Min. & Max. & Aver. & Min. & Max. & Aver. & Min. & Max. \\
\hline $\mathrm{N}_{2} \mathrm{O}$ & 2.3 & 1.8 & 2.9 & 3.1 & 2.2 & 6.0 & 3.1 & 2.0 & 5.9 & 2.6 & $<1.0$ & 5.6 \\
\hline $\mathrm{NO}$ & 527 & 426 & 692 & 646 & 504 & 1087 & 582 & 398 & 993 & 467 & 1.6 & 885 \\
\hline $\mathrm{NO}_{2}$ & 61.7 & 47.3 & 90.3 & 93.1 & 61.7 & 224 & 104 & 56.5 & 254 & 93.0 & 1.3 & 234 \\
\hline $\mathrm{NH}_{3}$ & 7.9 & 3.3 & 16.2 & 4.7 & 3.9 & 10.0 & 3.1 & 2.3 & 4.2 & 1.8 & $<1.0$ & 3.9 \\
\hline $\mathrm{H}_{2} \mathrm{O} \%$ & 34.3 & 26.0 & 48.5 & 10.1 & 9.1 & 13.1 & 8.7 & 6.3 & 13.4 & 8.1 & 6.5 & 15.1 \\
\hline $\mathrm{CO}_{2} \%$ & 0.77 & 0.62 & 1.04 & 0.92 & 0.75 & 1.82 & 0.88 & 0.59 & 1.79 & 0.75 & 0.06 & 1.67 \\
\hline $\mathrm{Nitrous} \mathrm{Acid}$ & 1.7 & 1.1 & 3.3 & 1.2 & $<1.0$ & 4.7 & 2.3 & 1.3 & 5.5 & 2.8 & $<1.0$ & 6.2 \\
\hline Nitric Acid & 3.1 & $<1.0$ & 9.6 & 2.1 & $<1.0$ & 82.2 & $<1.0$ & $<1.0$ & 2.0 & 1.2 & $<1.0$ & 2.3 \\
\hline $\mathrm{HCN}$ & 1.2 & $<1.0$ & 2.1 & $<1.0$ & $<1.0$ & $<1.0$ & $<1.0$ & $<1.0$ & $<1.0$ & $<1.0$ & $<1.0$ & $<1.0$ \\
\hline $\mathrm{CO}$ & 2.0 & $<1.0$ & 3.8 & 3.7 & 1.7 & 10.9 & 3.4 & $<1.0$ & 10.3 & 3.3 & $<1.0$ & 10.7 \\
\hline $\mathrm{HCl}$ & 2.1 & $<1.0$ & 5.2 & 1.4 & $<1.0$ & 25.9 & $<1.0$ & $<1.0$ & $<1.0$ & $<1.0$ & $<1.0$ & $<1.0$ \\
\hline $\mathrm{HF}$ & 29.0 & 5.3 & 57.7 & 22.7 & 12.7 & 79.6 & 6.2 & 3.9 & 12.3 & 2.9 & 2.0 & 8.4 \\
\hline
\end{tabular}


Table 6.5. Concentrations [ppmv] of Selected Species in Off-Gas Measured by FTIR Spectroscopy, Test 4.

\begin{tabular}{|c|c|c|c|c|c|c|c|c|c|c|c|c|}
\hline & \multicolumn{1}{|c|}{ Melter outlet } & \multicolumn{1}{|c|}{ SBS outlet } & \multicolumn{4}{c|}{ WESP outlet } & \multicolumn{4}{c|}{ HEPA outlet } \\
\cline { 2 - 16 } & Aver. & Min. & Max. & Aver. & Min. & Max. & Aver. & Min. & Max. & Aver. & Min. & Max. \\
\hline $\mathrm{N}_{2} \mathrm{O}$ & 2.5 & 1.9 & 3.4 & 3.4 & 2.4 & 4.5 & 2.7 & $<1.0$ & 6.0 & 3.0 & 2.4 & 3.9 \\
\hline $\mathrm{NO}$ & 542 & 460 & 734 & 703 & 372 & 914 & 501 & 3.6 & 995 & 555 & 441 & 728 \\
\hline $\mathrm{NO}_{2}$ & 68.4 & 31.7 & 112 & 104 & 40.7 & 151 & 91.4 & $<1.0$ & 264 & 111 & 75.6 & 259 \\
\hline $\mathrm{NH}_{3}$ & 5.5 & 2.3 & 10.5 & 2.1 & $<1.0$ & 5.9 & 1.8 & $<1.0$ & 3.4 & 3.3 & 2.8 & 3.8 \\
\hline $\mathrm{H}_{2} \mathrm{O} \%$ & 34.7 & 27.2 & 50.3 & 9.0 & 7.1 & 11.8 & 8.4 & 7.0 & 13.7 & 7.7 & 6.6 & 9.5 \\
\hline $\mathrm{CO}_{2} \%$ & 0.76 & 0.64 & 0.97 & 0.99 & 0.57 & 1.37 & 0.76 & 0.06 & 1.63 & 0.83 & 0.64 & 1.01 \\
\hline $\mathrm{Nitrous} \mathrm{Acid}^{2}$ & 1.4 & $<1.0$ & 2.9 & 1.3 & $<1.0$ & 2.6 & 1.8 & $<1.0$ & 5.1 & 2.9 & 1.3 & 4.0 \\
\hline Nitric Acid & 7.2 & $<1.0$ & 62.7 & 4.7 & $<1.0$ & 52.1 & $<1.0$ & $<1.0$ & 1.8 & $<1.0$ & $<1.0$ & 1.0 \\
\hline $\mathrm{HCN}$ & $<1.0$ & $<1.0$ & 1.6 & $<1.0$ & $<1.0$ & $<1.0$ & $<1.0$ & $<1.0$ & $<1.0$ & $<1.0$ & $<1.0$ & $<1.0$ \\
\hline $\mathrm{CO}$ & 2.8 & 1.6 & 5.5 & 4.9 & 2.3 & 8.3 & 3.6 & $<1.0$ & 12.1 & 4.2 & 2.4 & 7.0 \\
\hline $\mathrm{HCl}$ & 3.3 & $<1.0$ & 22.9 & 9.1 & $<1.0$ & 26.8 & $<1.0$ & $<1.0$ & $<1.0$ & $<1.0$ & $<1.0$ & $<1.0$ \\
\hline $\mathrm{HF}$ & 49.6 & 8.9 & 142 & 21.1 & 3.5 & 87.3 & 4.2 & 2.7 & 12.7 & 10.8 & 8.0 & 20.2 \\
\hline
\end{tabular}


The Catholic University of America Vitreous State Laboratory
Testing of Optimized Bubbler Configuration for HLW Melter Final Report, VSL-13R2950-1, Rev. 0

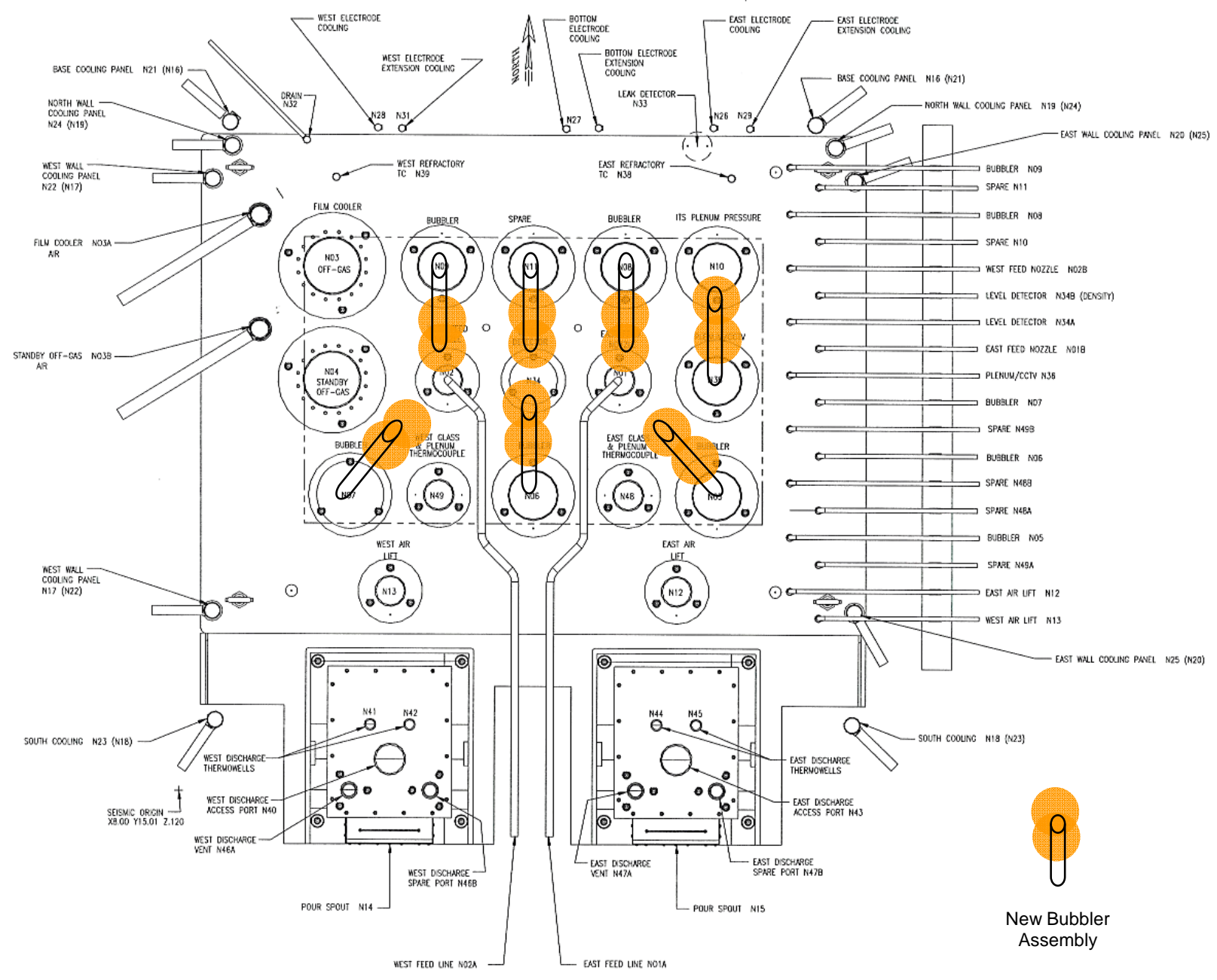

Figure 1.1. Lid diagram for WTP HLW melter. 
The Catholic University of America Vitreous State Laboratory
Testing of Optimized Bubbler Configuration for HLW Melter Final Report, VSL-13R2950-1, Rev. 0

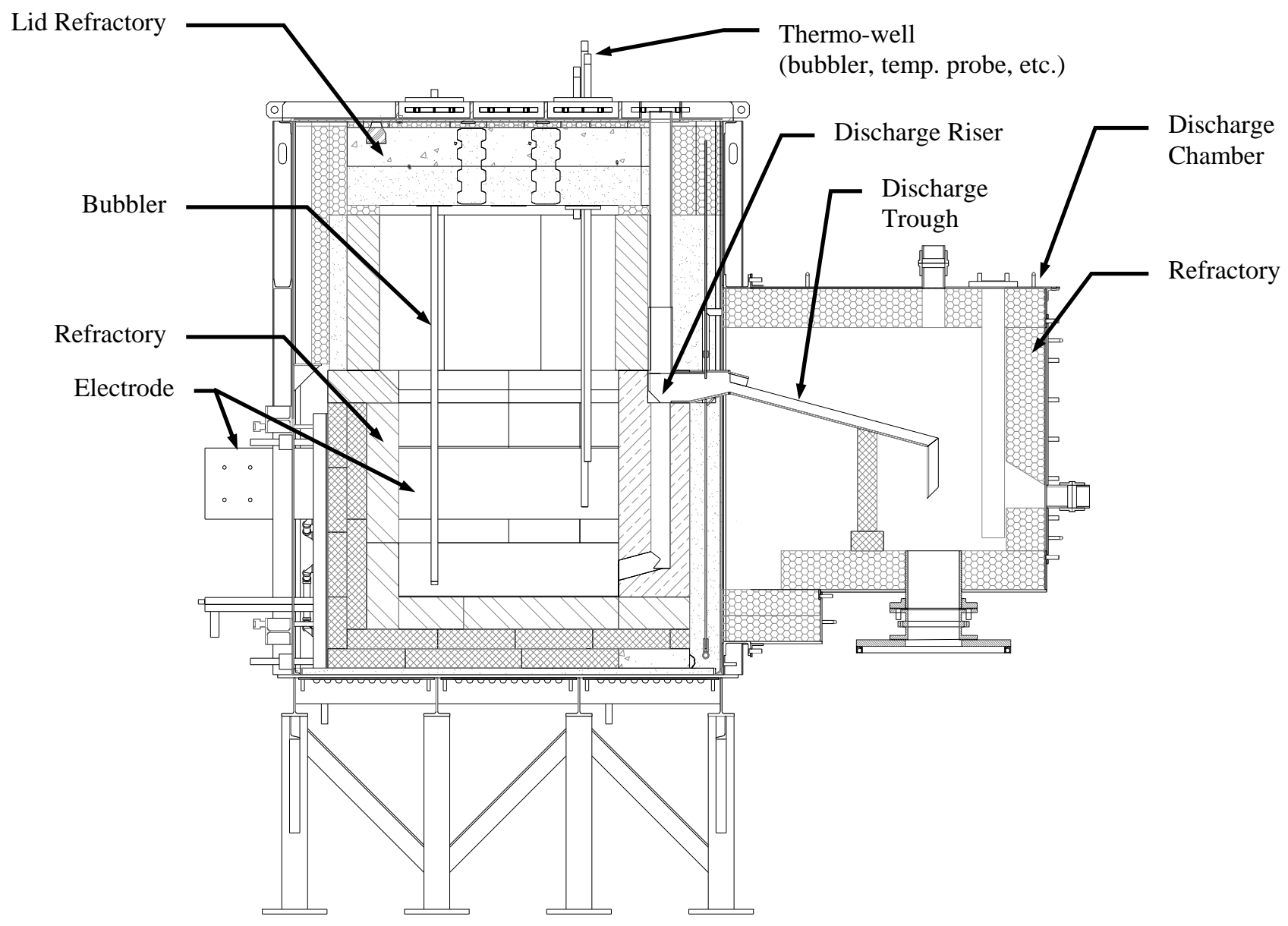

Figure 1.2. Cross-section of the DM1200 melter through the discharge chamber. 
The Catholic University of America Vitreous State Laboratory
Testing of Optimized Bubbler Configuration for HLW Melter

Final Report, VSL-13R2950-1, Rev. 0

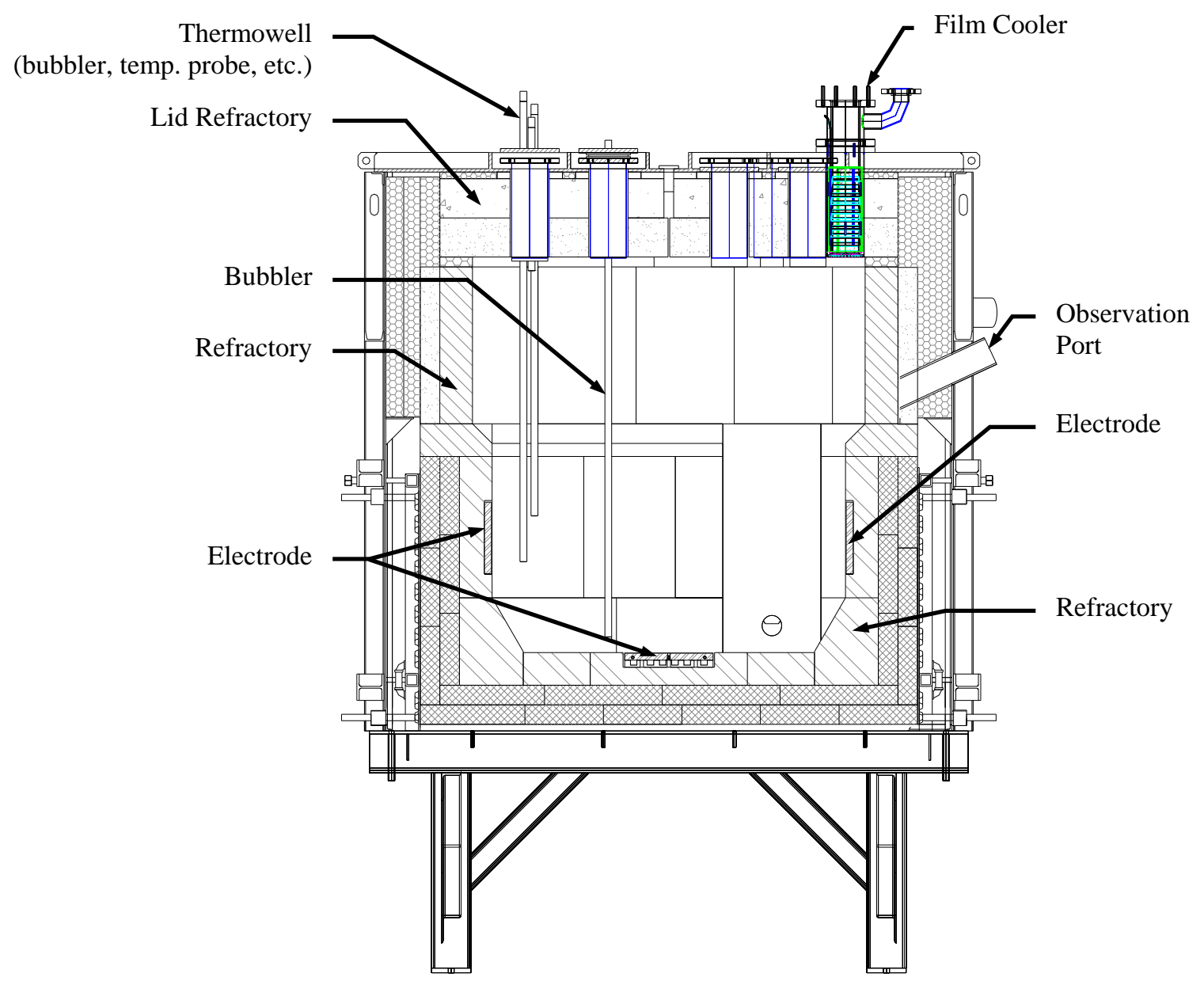

Figure 1.3. Cross-section through the DM1200 melter showing electrodes. 
The Catholic University of America Vitreous State Laboratory
Testing of Optimized Bubbler Configuration for HLW Melter

Final Report, VSL-13R2950-1, Rev. 0

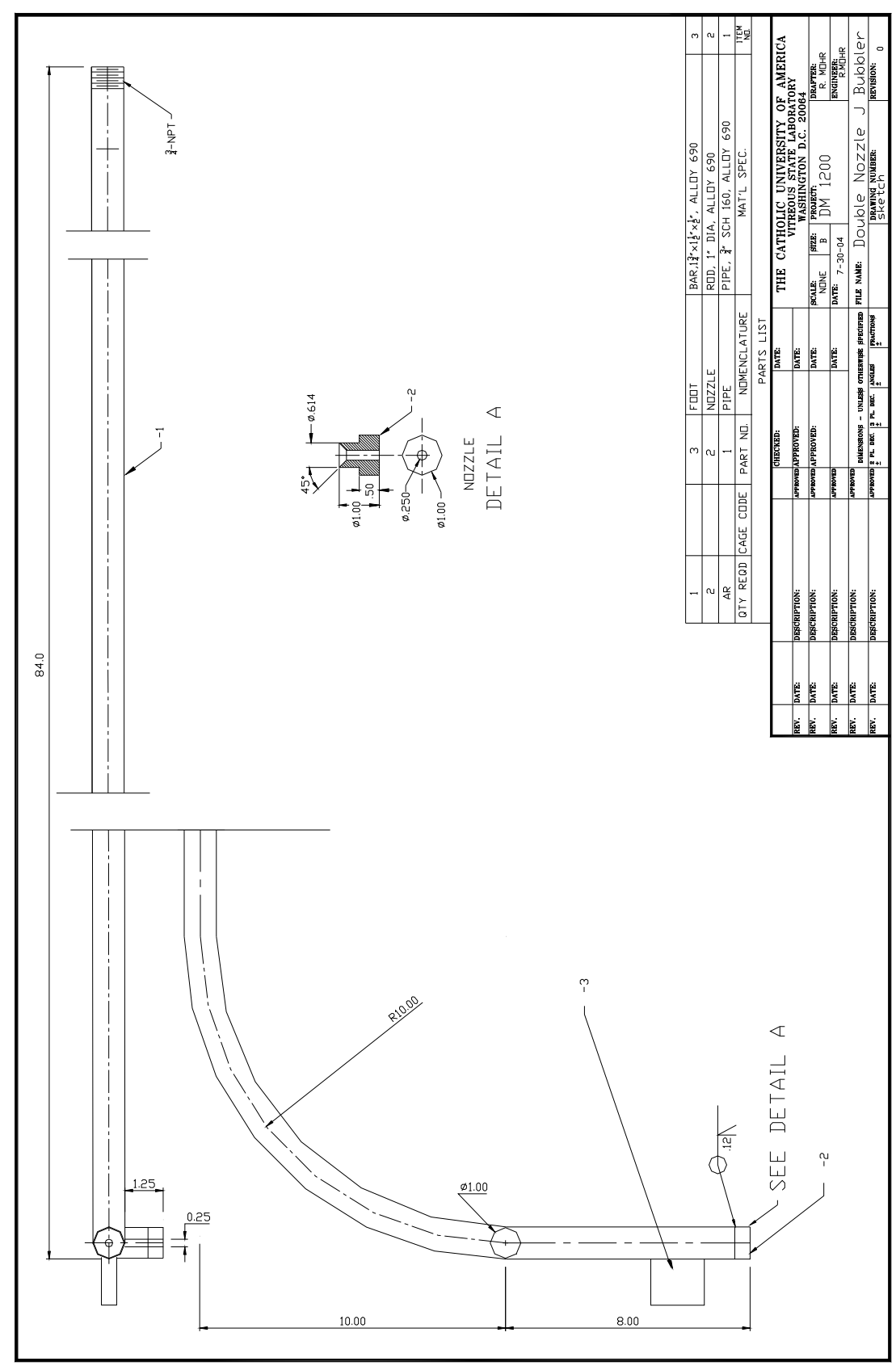

Figure 1.4. Specifications of Double-Outlet “J” Bubbler. 
The Catholic University of America Vitreous State Laboratory
Testing of Optimized Bubbler Configuration for HLW Melter Final Report, VSL-13R2950-1, Rev. 0

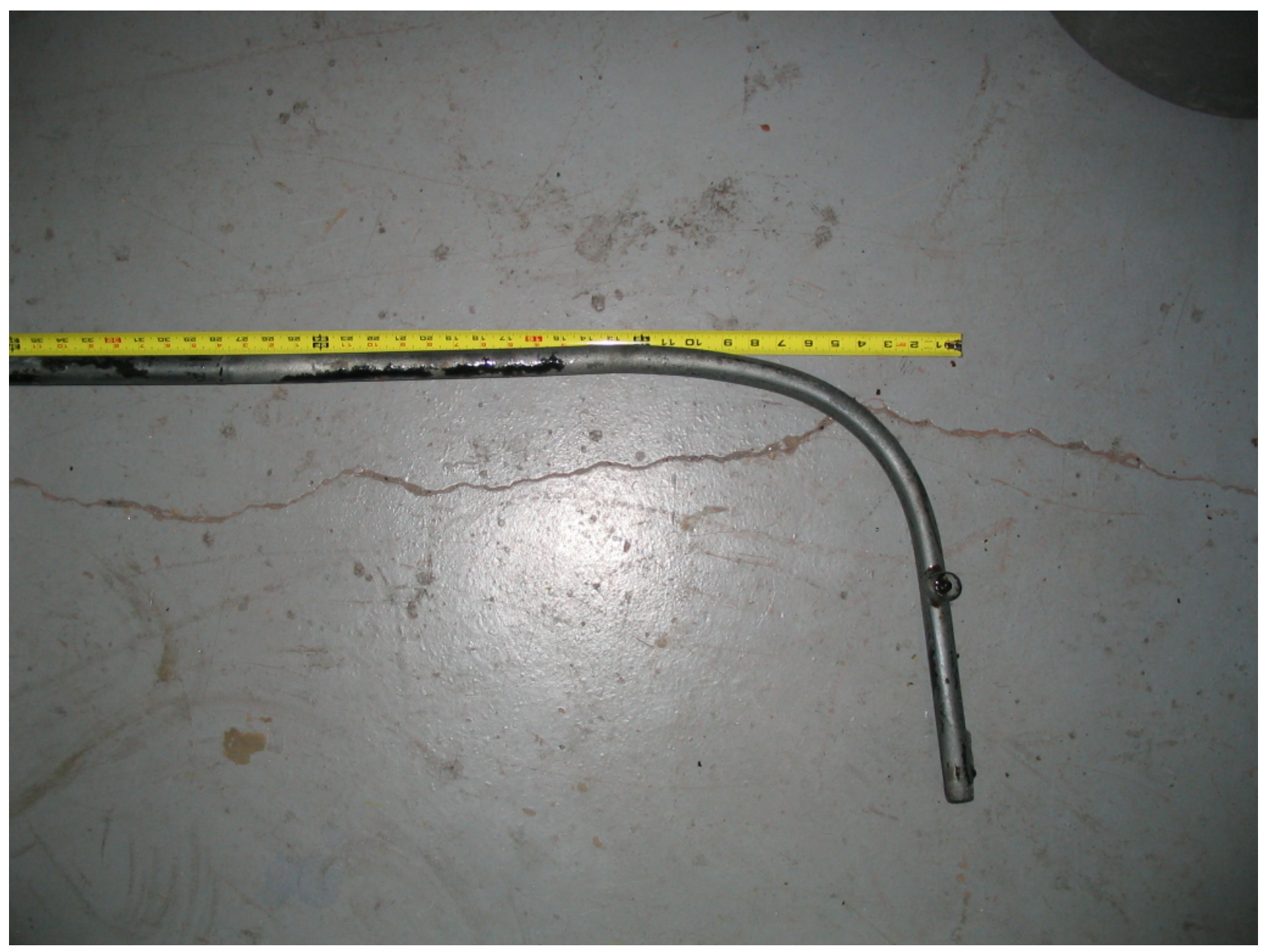

Figure 1.5. Double-Outlet “J” Bubbler. 
The Catholic University of America Vitreous State Laboratory
Testing of Optimized Bubbler Configuration for HLW Melter Final Report, VSL-13R2950-1, Rev. 0

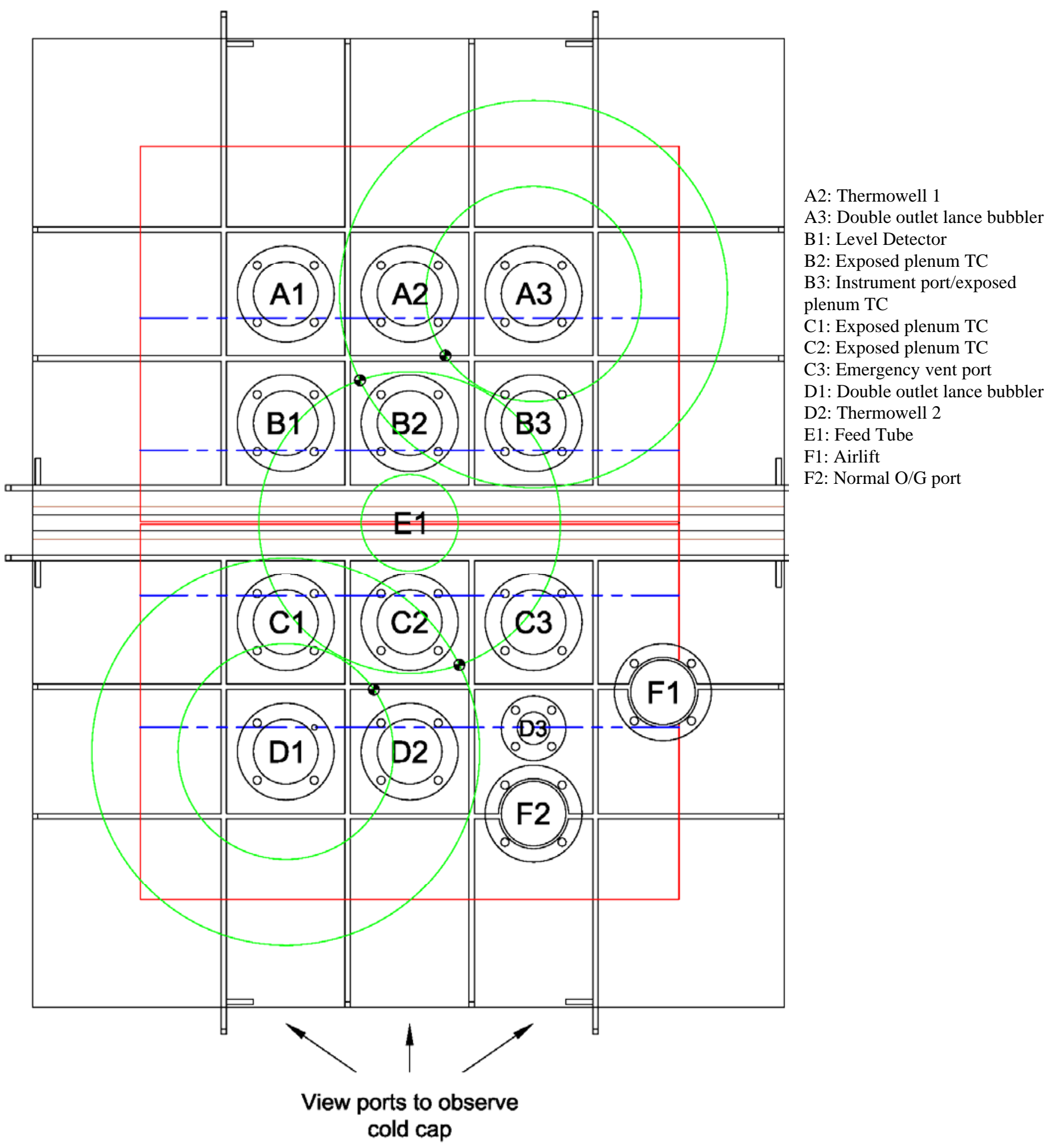

Figure 1.6. Placement of bubblers in the DM1200 melter, Nominal Configuration - 4 bubbling outlets. Note: Solid circles represent location of bubbler outlet. 
The Catholic University of America Vitreous State Laboratory
Testing of Optimized Bubbler Configuration for HLW Melter Final Report, VSL-13R2950-1, Rev. 0

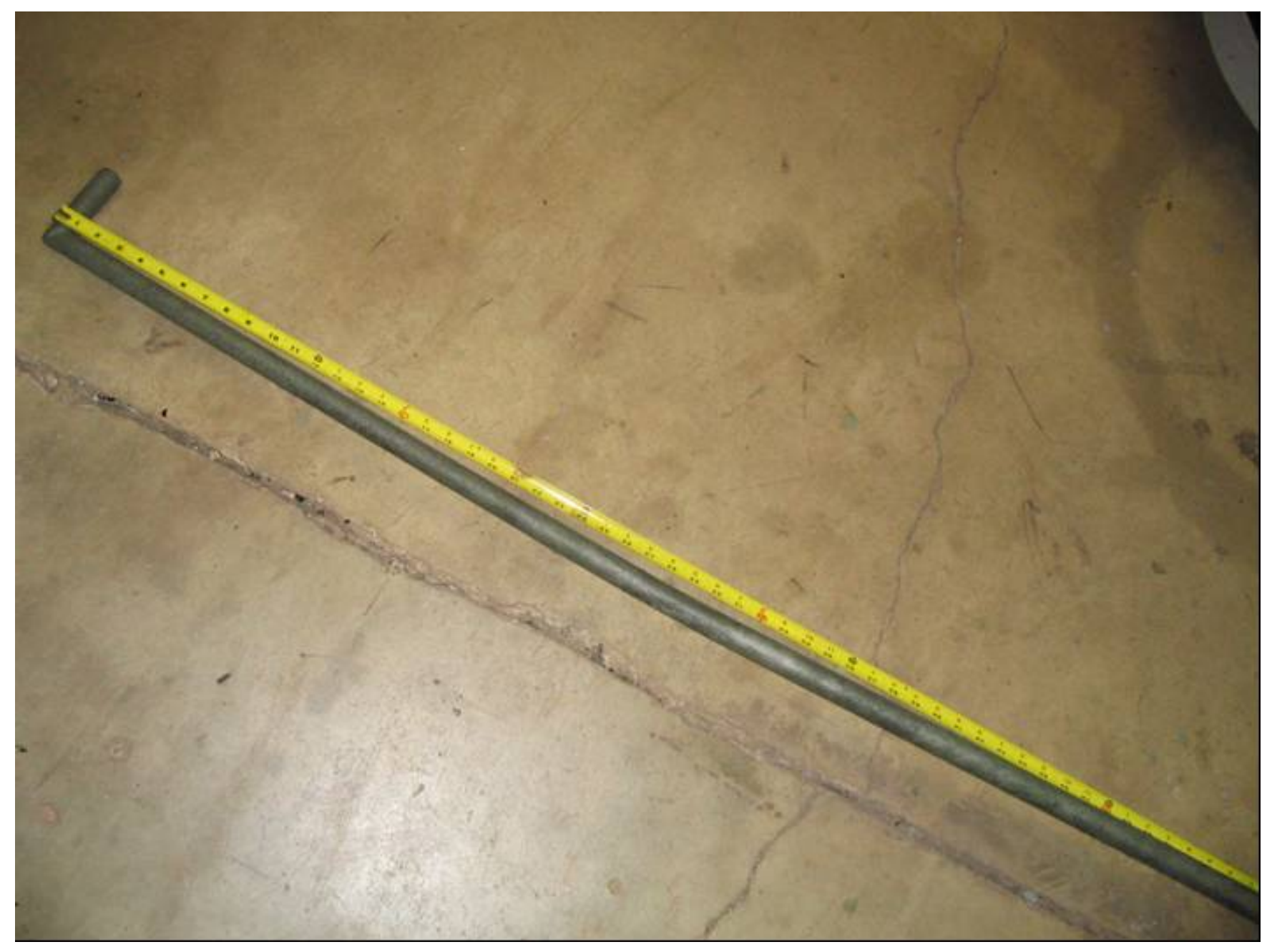

Figure 1.7. Single-Outlet “L” Bubbler. 


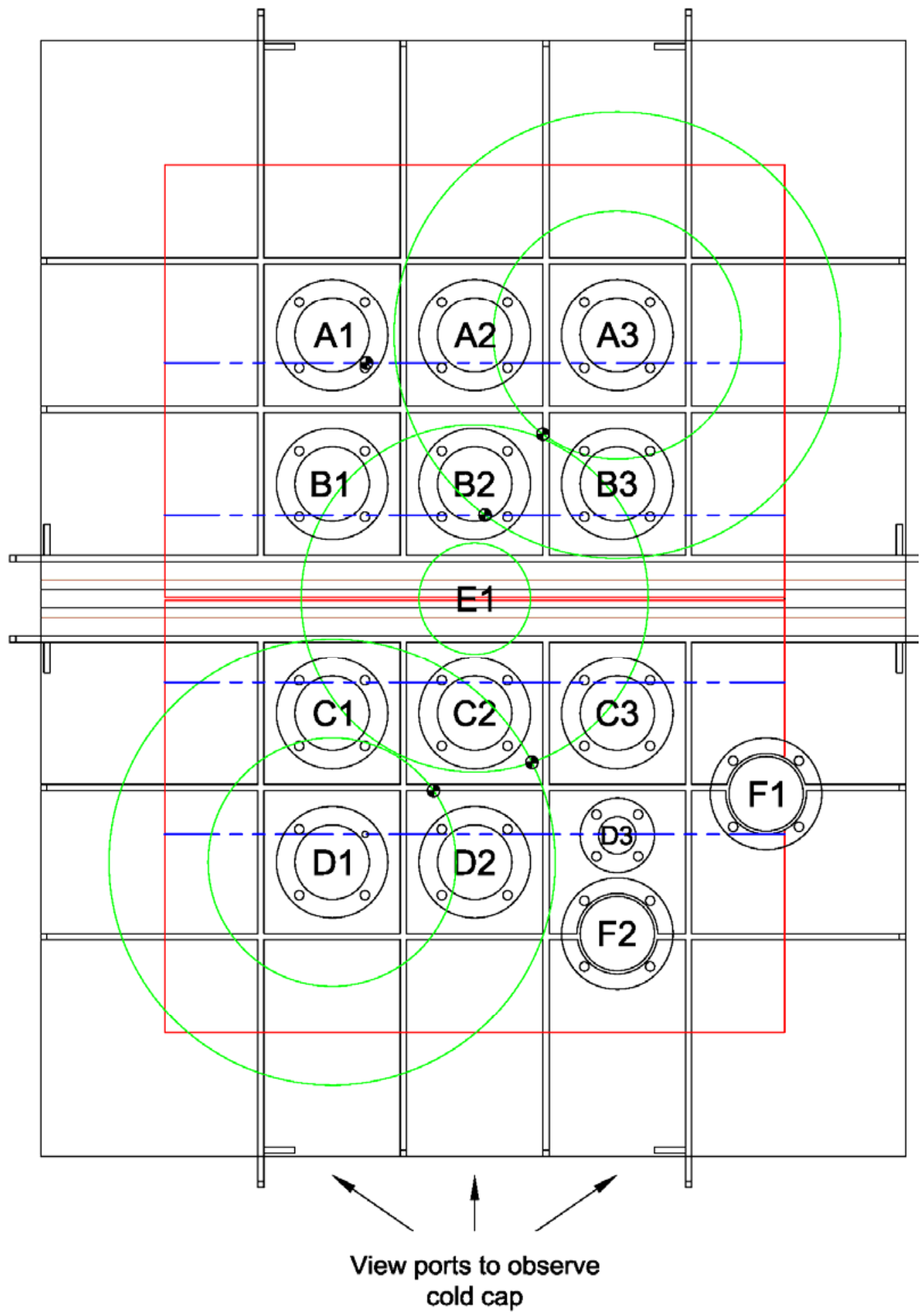

A1: Single outlet bubbler A2: Thermowell 1 A3: Double outlet lance bubbler B1: Level Detector B2: Exposed plenum TC B3: Instrument port/exposed plenum TC

C1: Exposed plenum TC

C2: Exposed plenum TC

C3: Emergency vent port

D1: Double outlet lance bubbler

D2: Thermowell 2

E1: Feed Tube

F1: Airlift

F2: Normal O/G port

Figure 1.8. Placement of bubblers in the DM1200 melter, 5 bubbling outlets. Note: Solid circles represent location of bubbler outlet. 
The Catholic University of America Vitreous State Laboratory
Testing of Optimized Bubbler Configuration for HLW Melter

Final Report, VSL-13R2950-1, Rev. 0

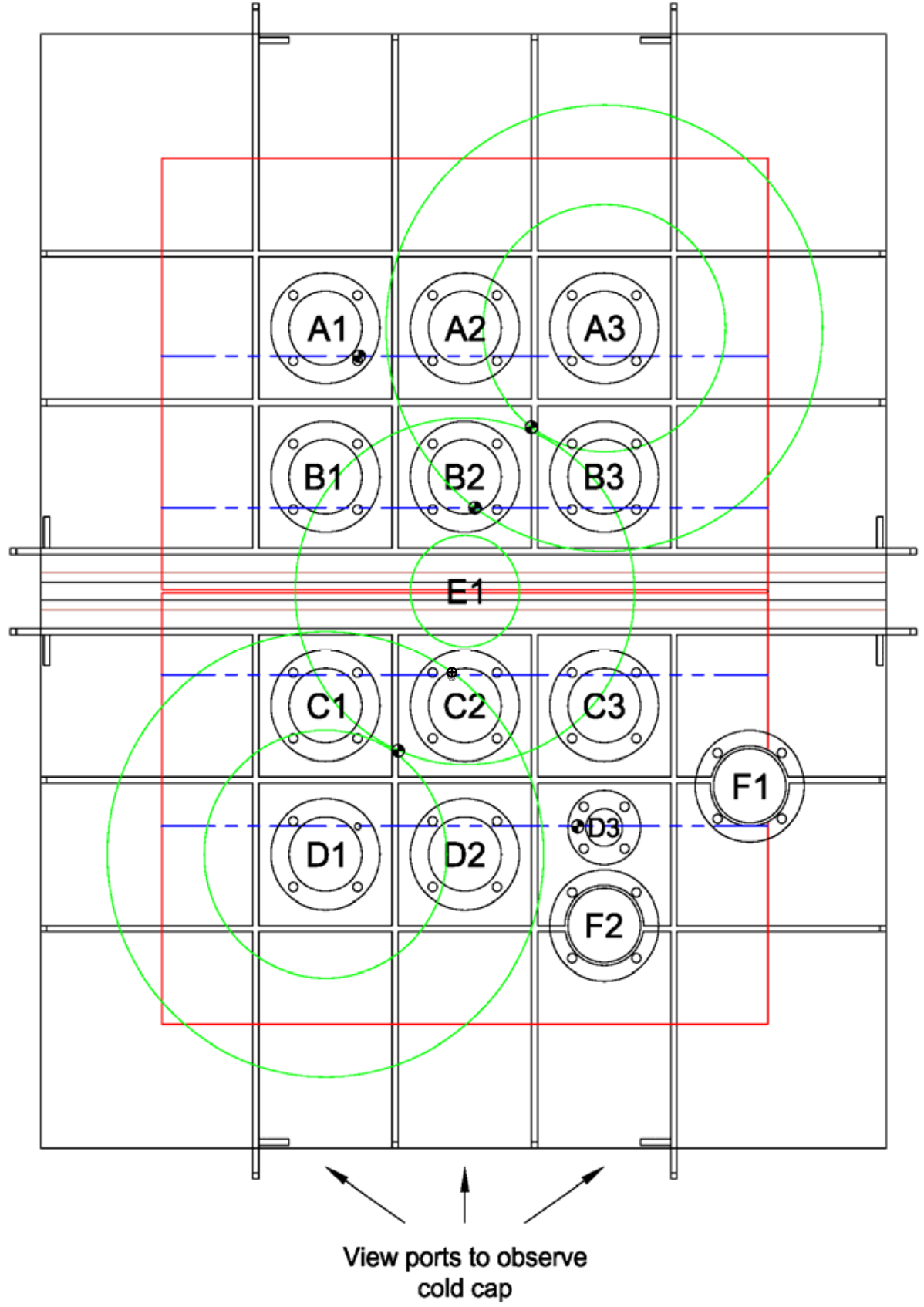

A1: Single outlet bubbler A2: Thermowell 1 A3: Double outlet lance bubbler B1: Level Detector B2: Exposed plenum TC B3: Instrument port/exposed plenum TC

C1: Exposed plenum TC C2: Exposed plenum TC C3: Emergency vent port D1: Double outlet lance bubbler D2: Thermowell 2

D3: Single outlet bubbler E1: Feed Tube F1: Airlift

F2: Normal O/G port

Figure 1.9. Placement of bubblers in the DM1200 melter, 6 bubbling outlets. Note: Solid circles represent location of bubbler outlet. 

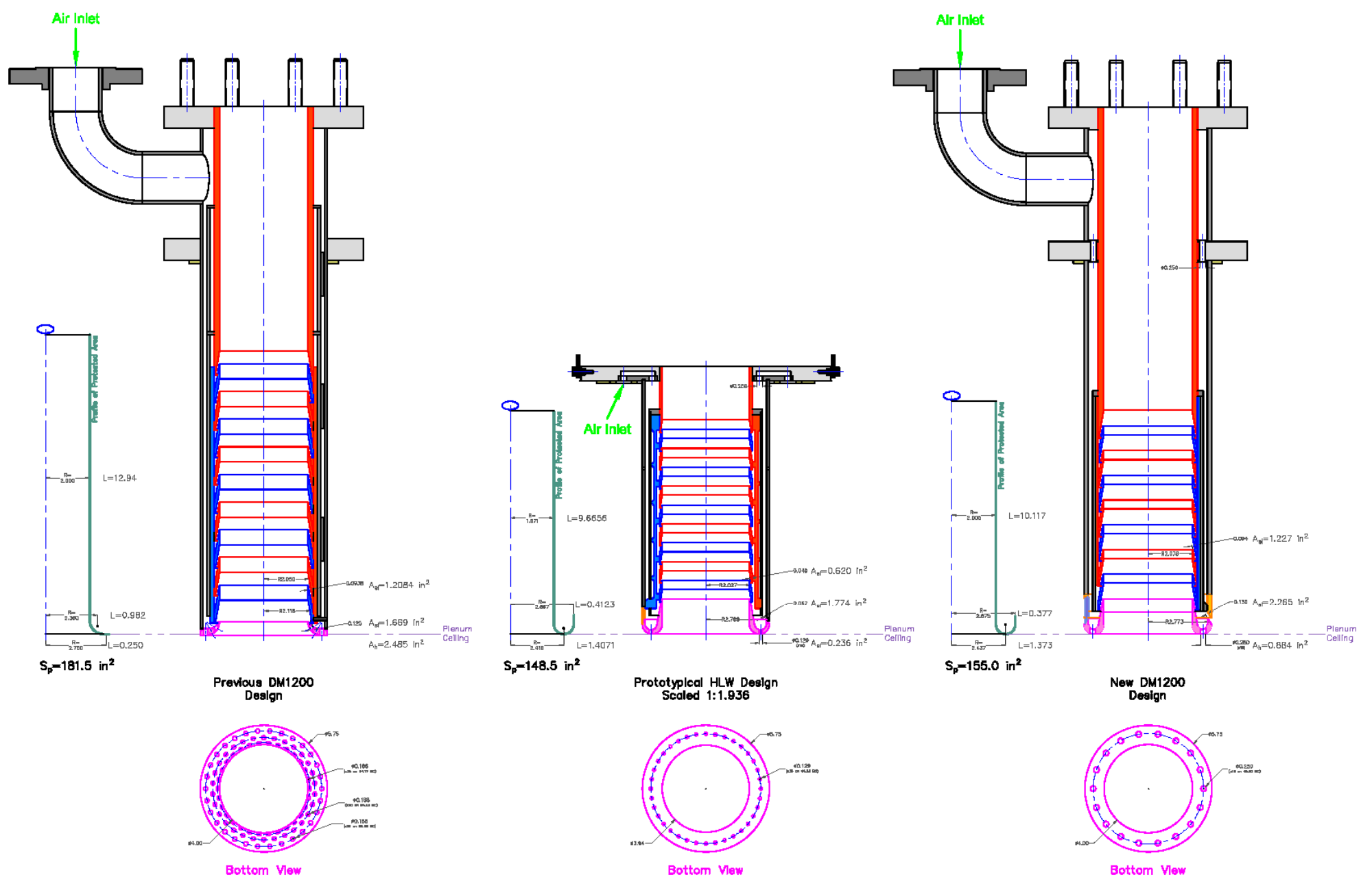

Figure 1.10. Comparison of original (left) and new (right) DM1200 film cooler designs with a scaled-down version of the WTP HLW design. 


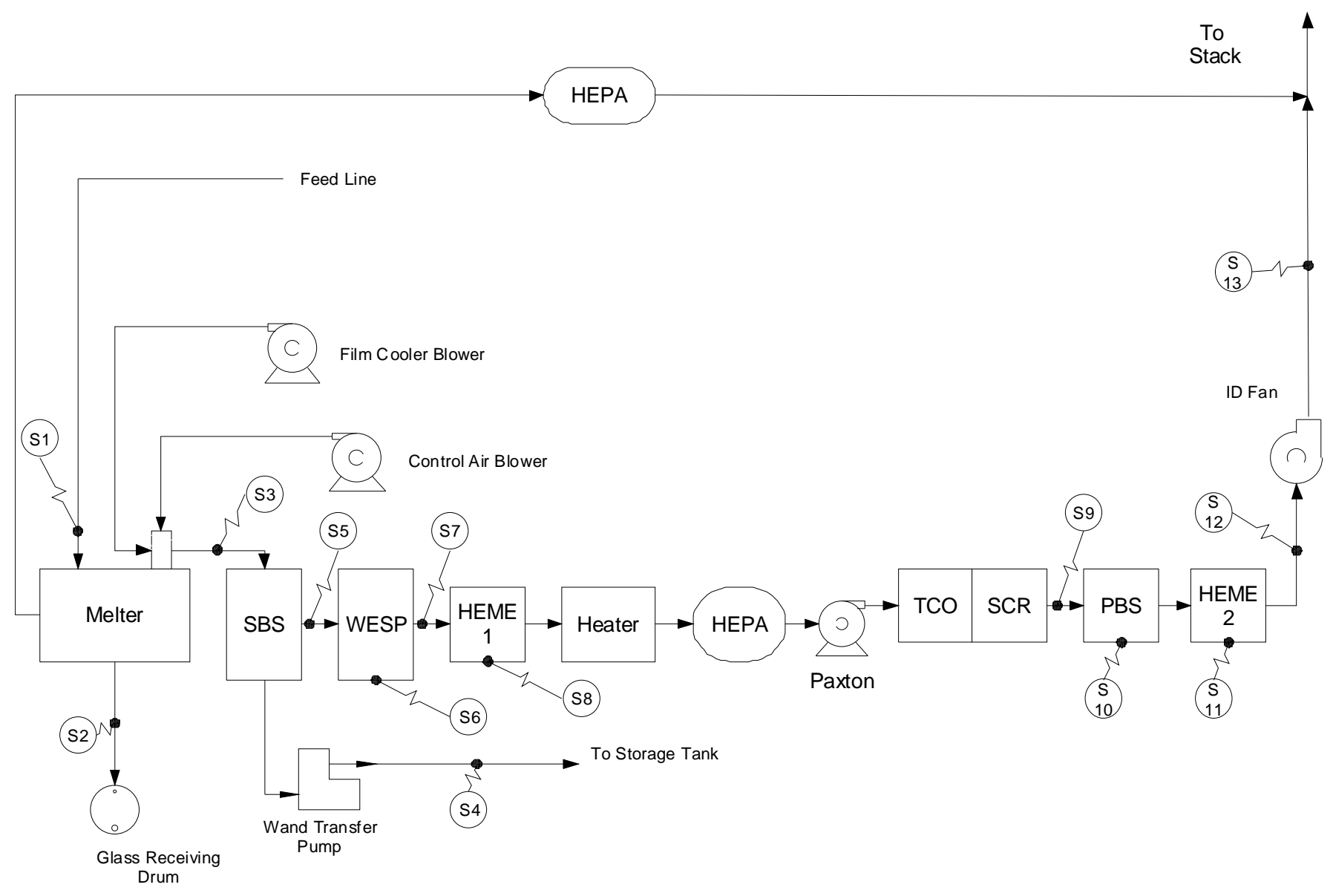

Figure 1.11. Schematic diagram of DM1200 off-gas system. “Sx" indicates sampling point 

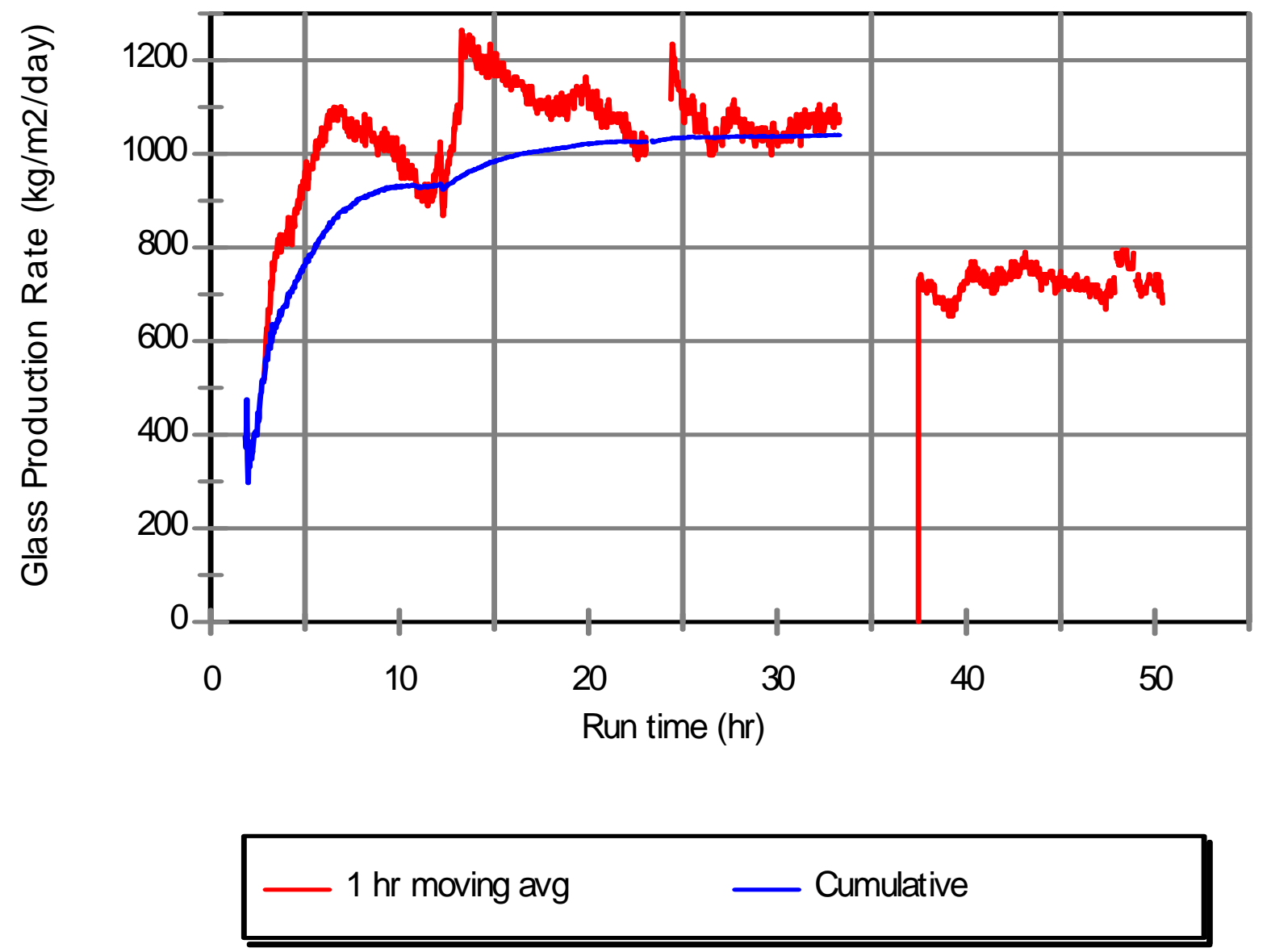

Figure 3.1.a. Production rates while processing with two double-outlet bubblers, Test 1. 

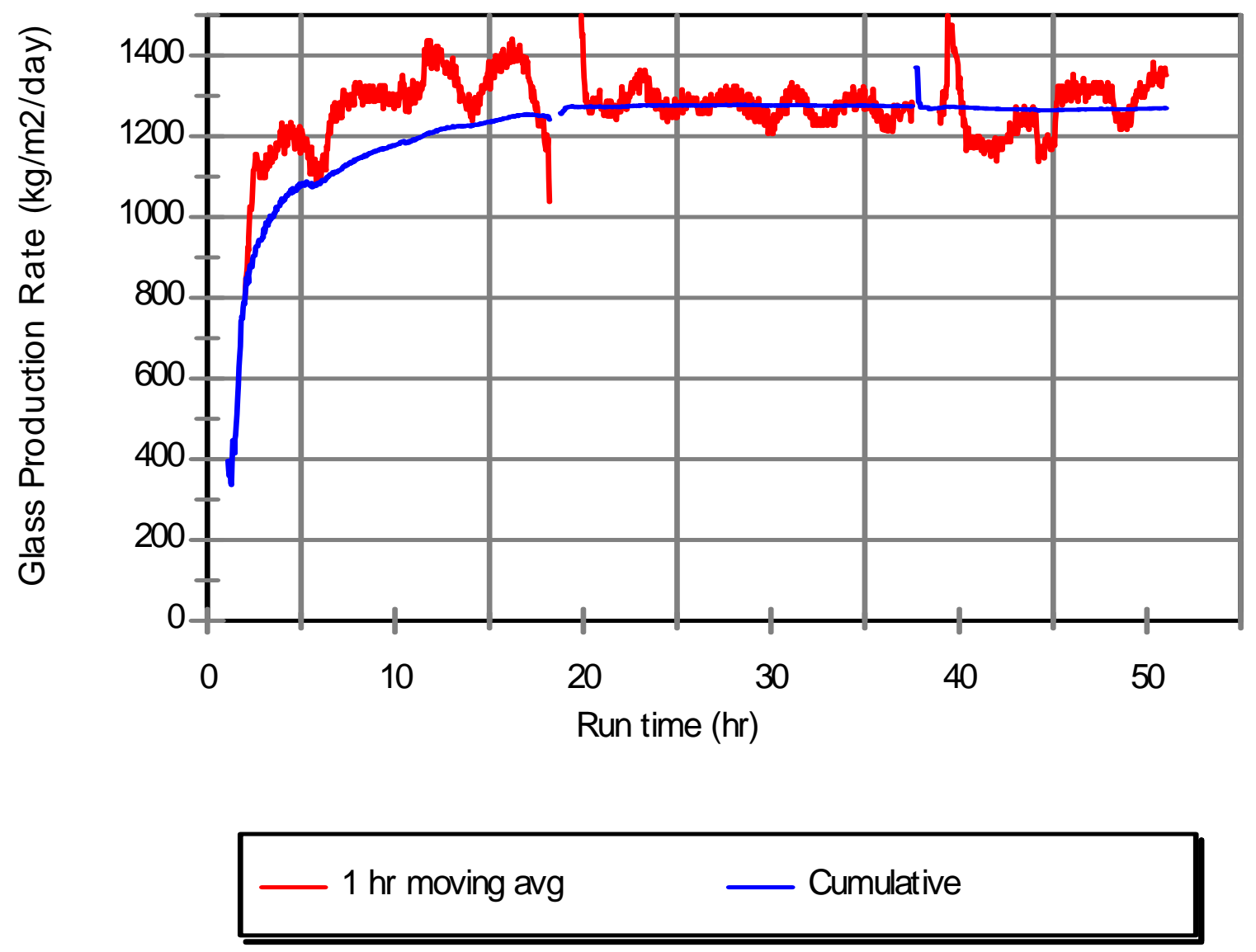

Figure 3.1.b. Production rates while processing with two double-outlet and one single-outlet bubblers, Test 2. 


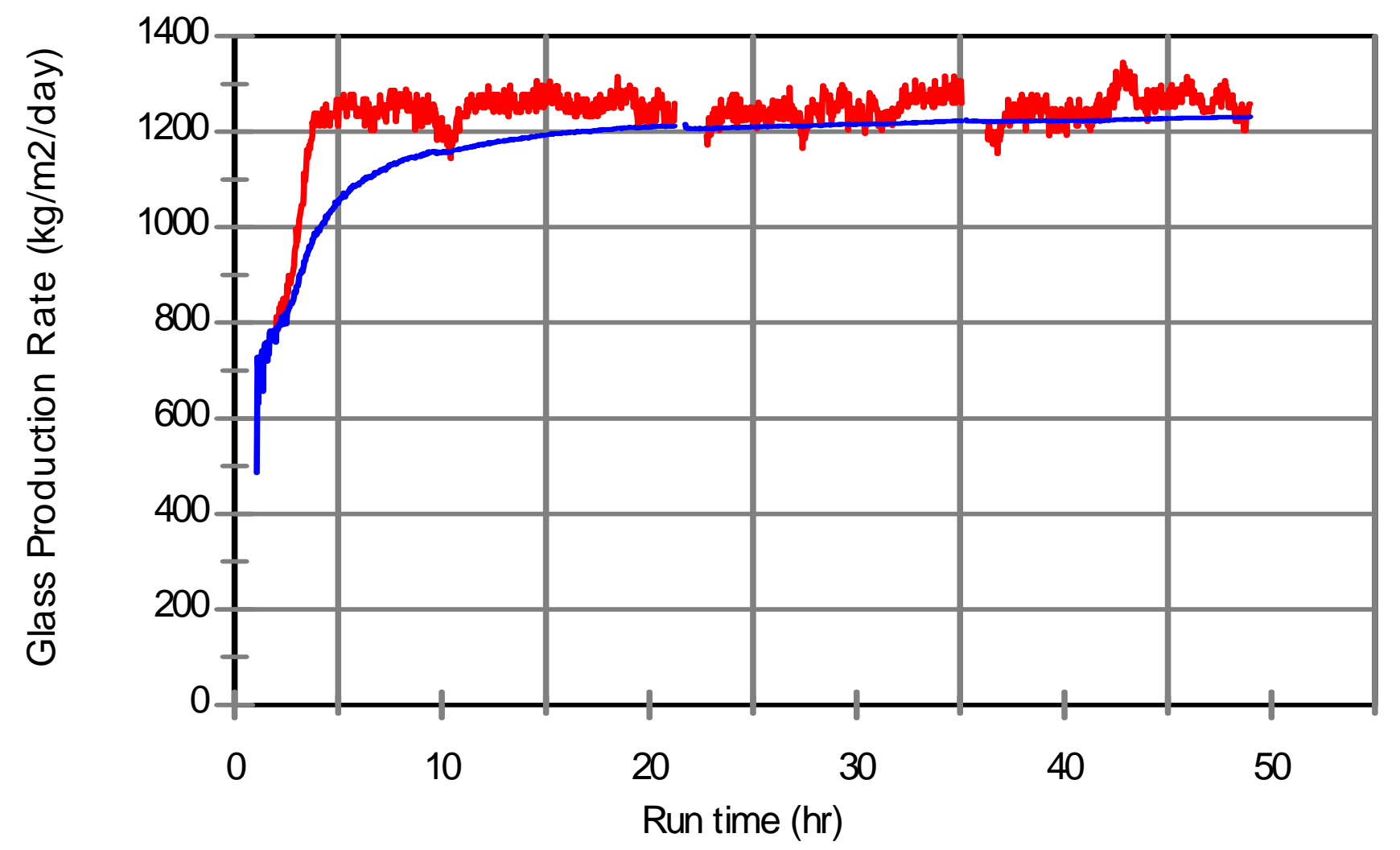

Figure 3.1.c. Production rates while processing with two double-outlet and two single-outlet bubblers, Test 3. 


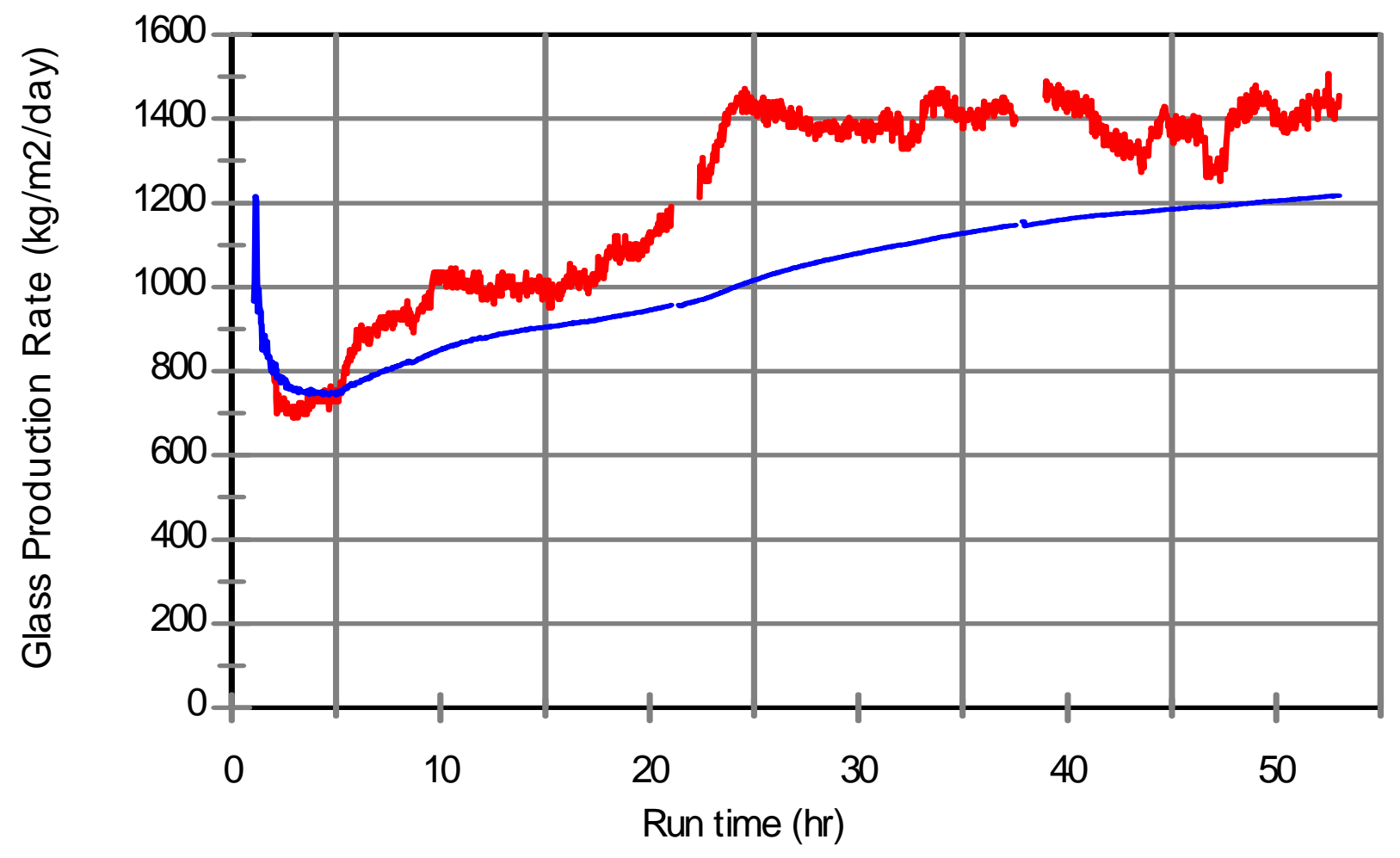

$1 \mathrm{hr}$ moving avg

Cumulative

Figure 3.1.d. Production rates while processing with two double-outlet and two single-outlet bubblers, Test 4. 


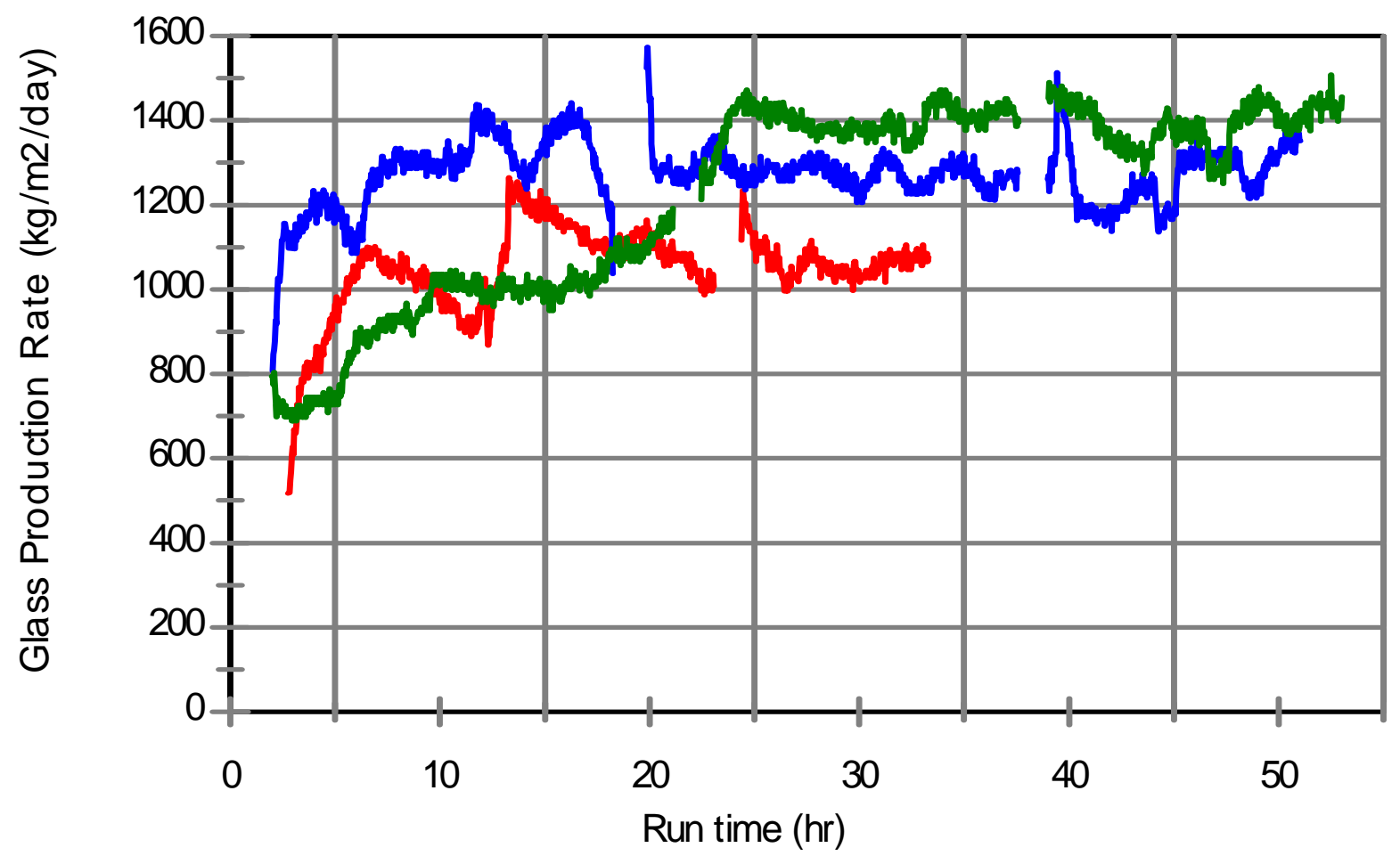

4 Outlets

5 Outlets

- 6 Outlets

Figure 3.2. Glass production rates while bubbling a total of $78 \mathrm{lpm}$. 


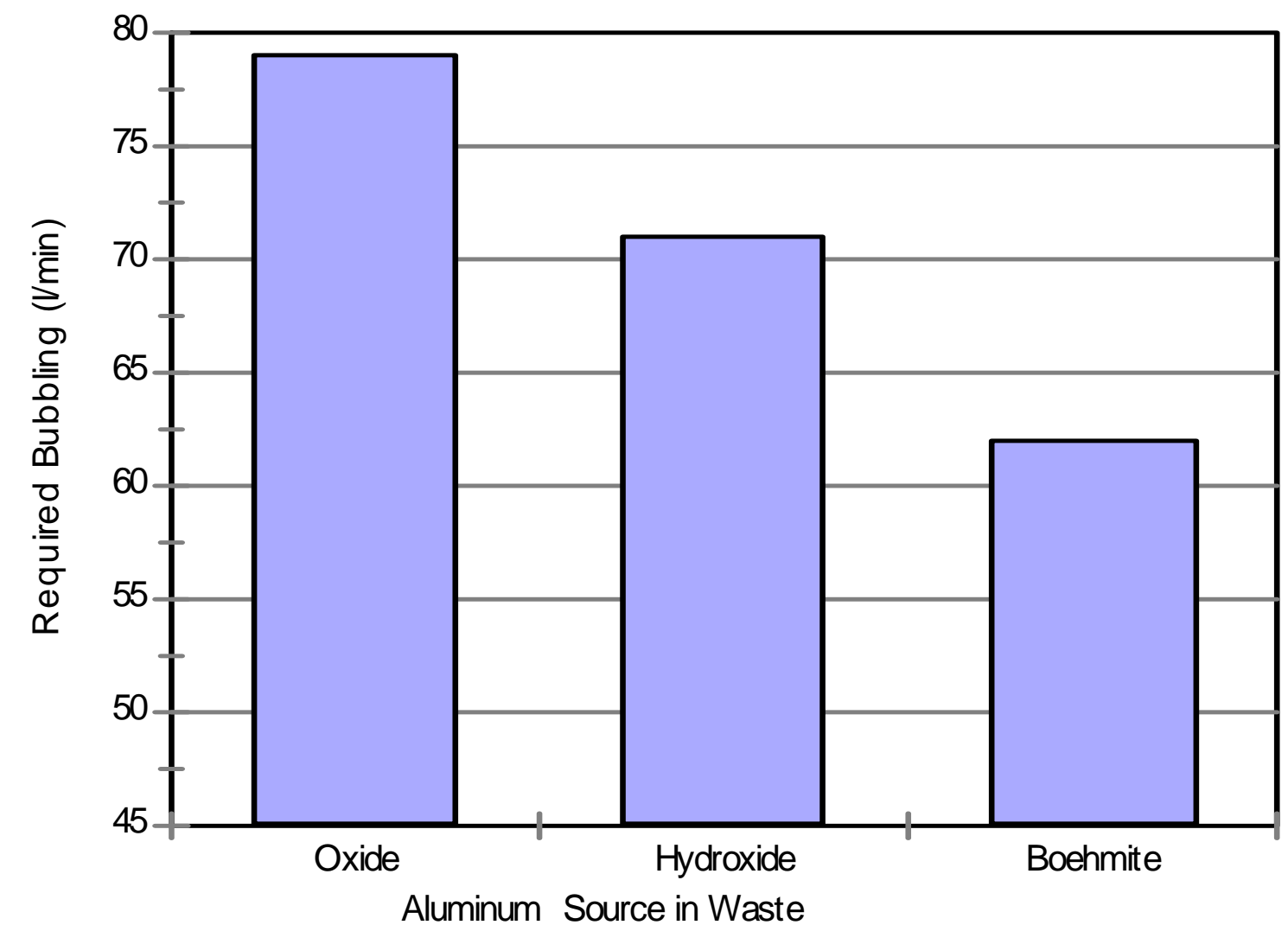

Figure 3.3. Bubbling rates used to process high aluminum waste at $1050 \mathrm{~kg} / \mathrm{m}^{2} / \mathrm{day}$ with four bubbler outlet nominal configuration. 


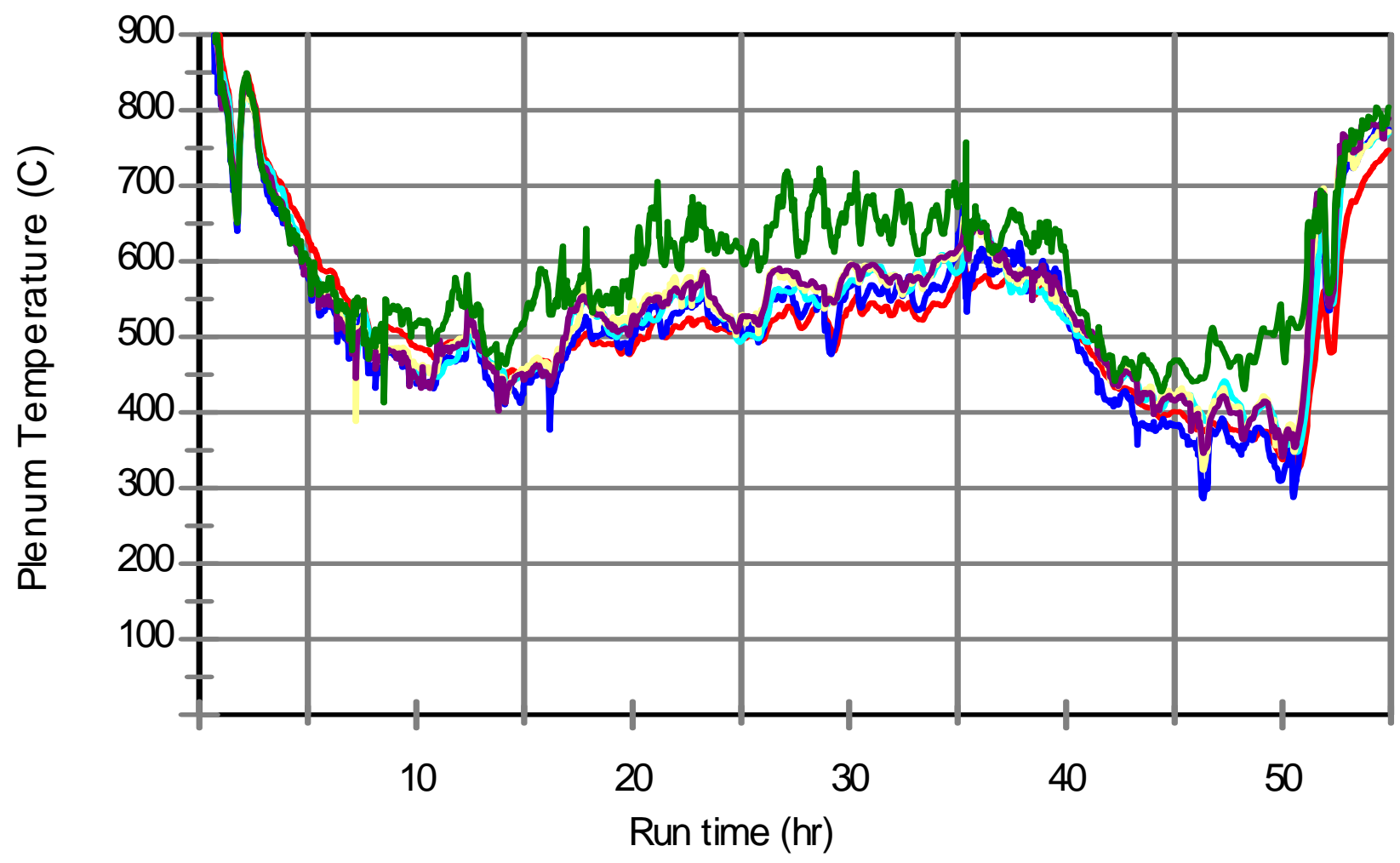

Figure 3.4.a. Plenum temperatures while processing with two double-outlet bubblers, Test 1. 


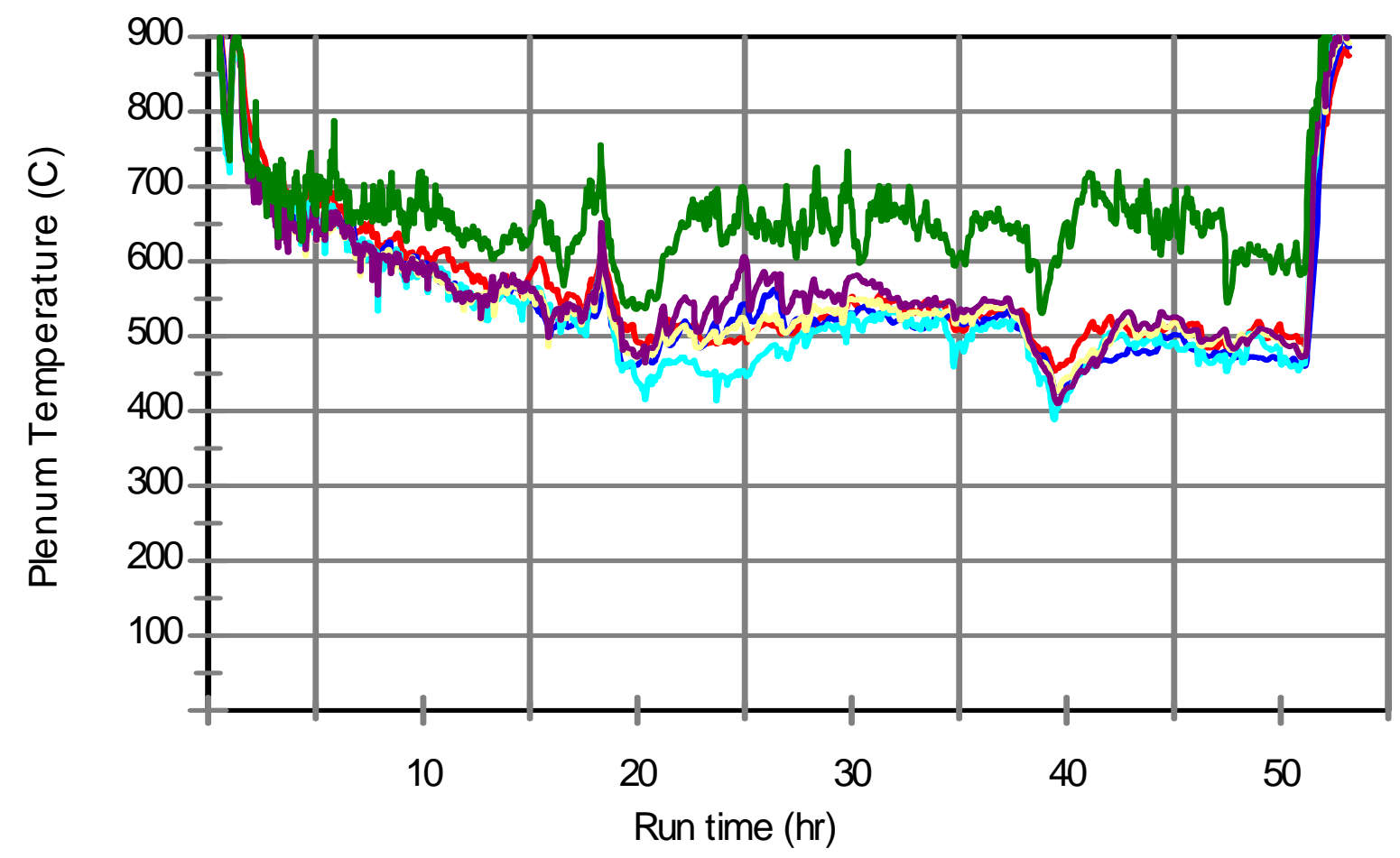

Figure 3.4.b. Plenum temperatures while processing with two double-outlet and one single-outlet bubblers, Test 2. 


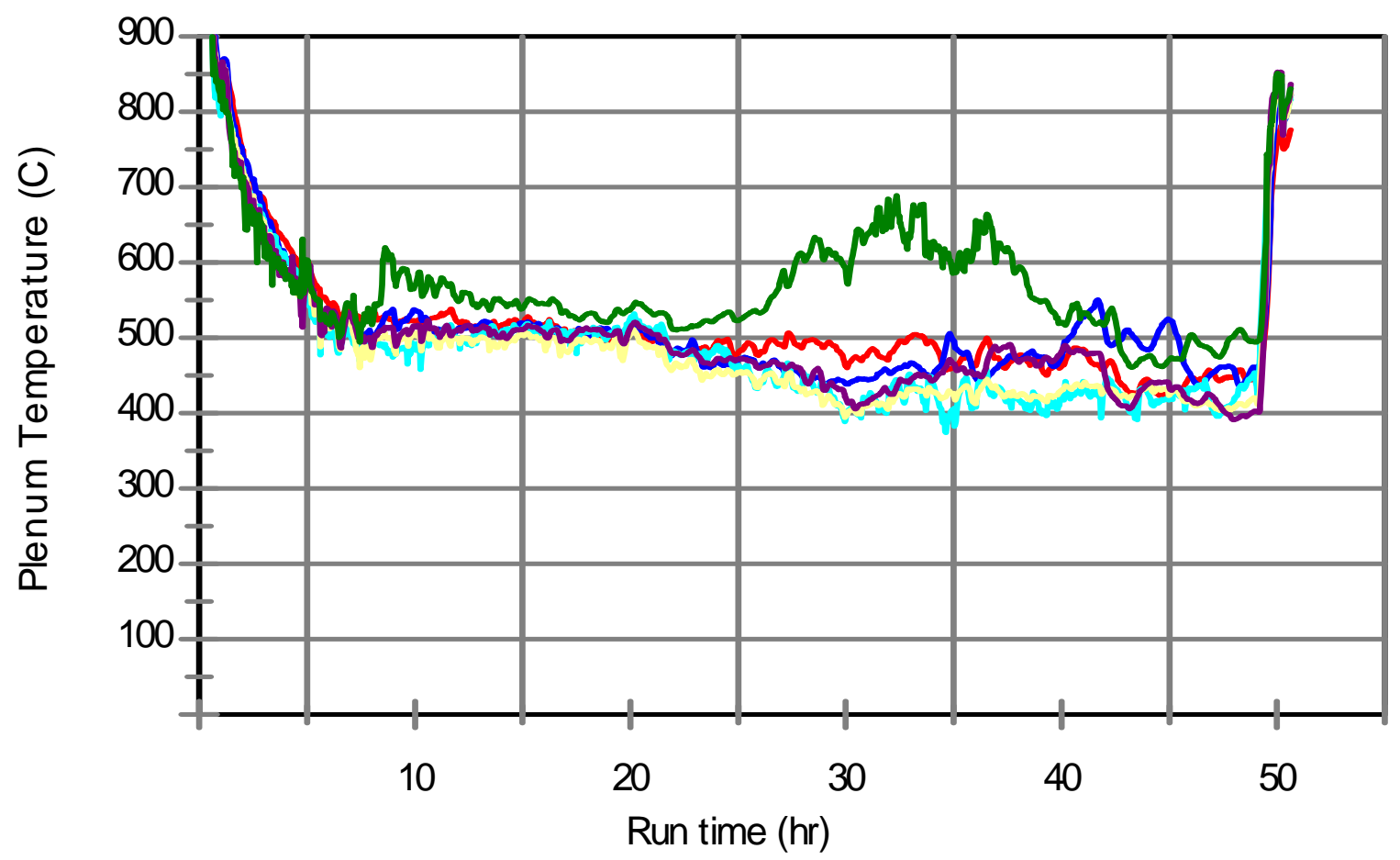

Figure 3.4.c. Plenum temperatures while processing with two double-outlet and two single-outlet bubblers, Test 3. 


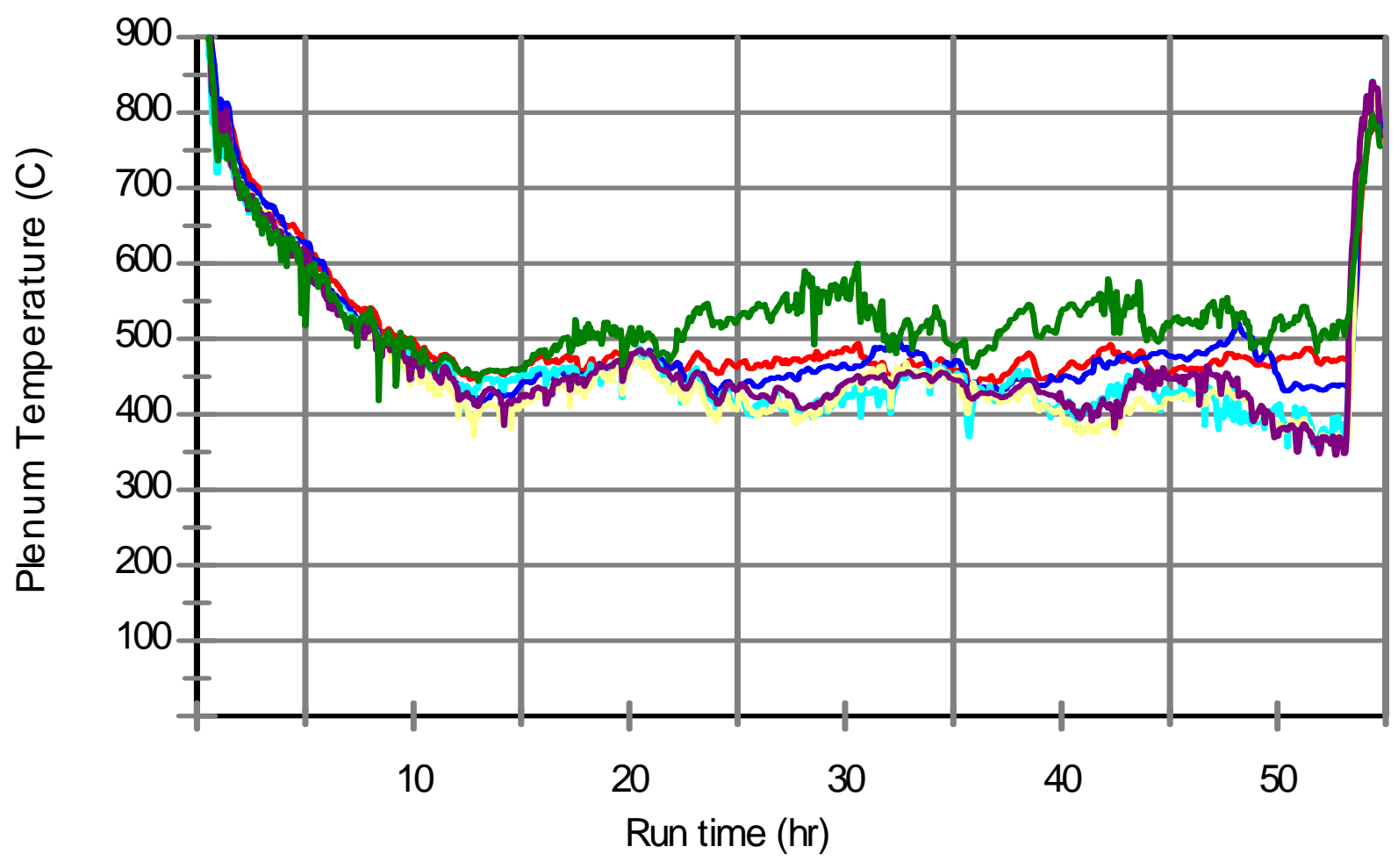

Figure 3.4.d. Plenum temperatures while processing with two double-outlet and two single-outlet bubblers, Test 4 . 
The Catholic University of America

Vitreous State Laboratory
Testing of Optimized Bubbler Configuration for HLW Melter Final Report, VSL-13R2950-1, Rev. 0

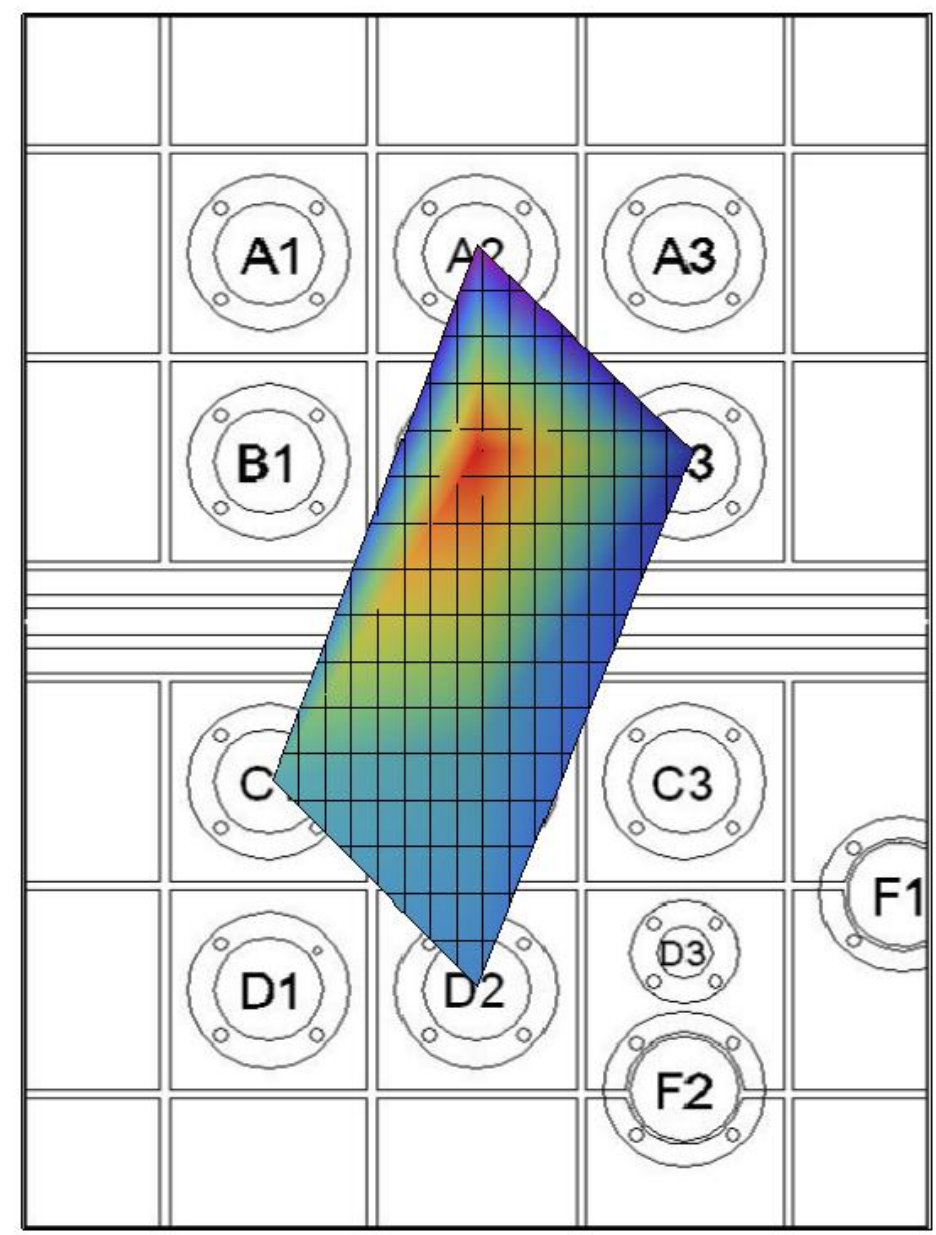

628

A2: Thermowell 1

A3: Double outlet lance bubbler

B1: Level Detector

B2: Exposed plenum TC

B3: Instrument port/exposed plenum TC

C1: Exposed plenum TC

C2: Exposed plenum TC

C3: Emergency vent port

D1: Double outlet lance bubbler

D2: Thermowell 2

E1: Feed Tube

F1: Airlift

517

Figure 3.5.a. Average steady state plenum temperatures $\left({ }^{\circ} \mathrm{C}\right)$ at monitoring locations; two double-outlet bubblers, Test 1. 
Testing of Optimized Bubbler Configuration for HLW Melter Final Report, VSL-13R2950-1, Rev. 0
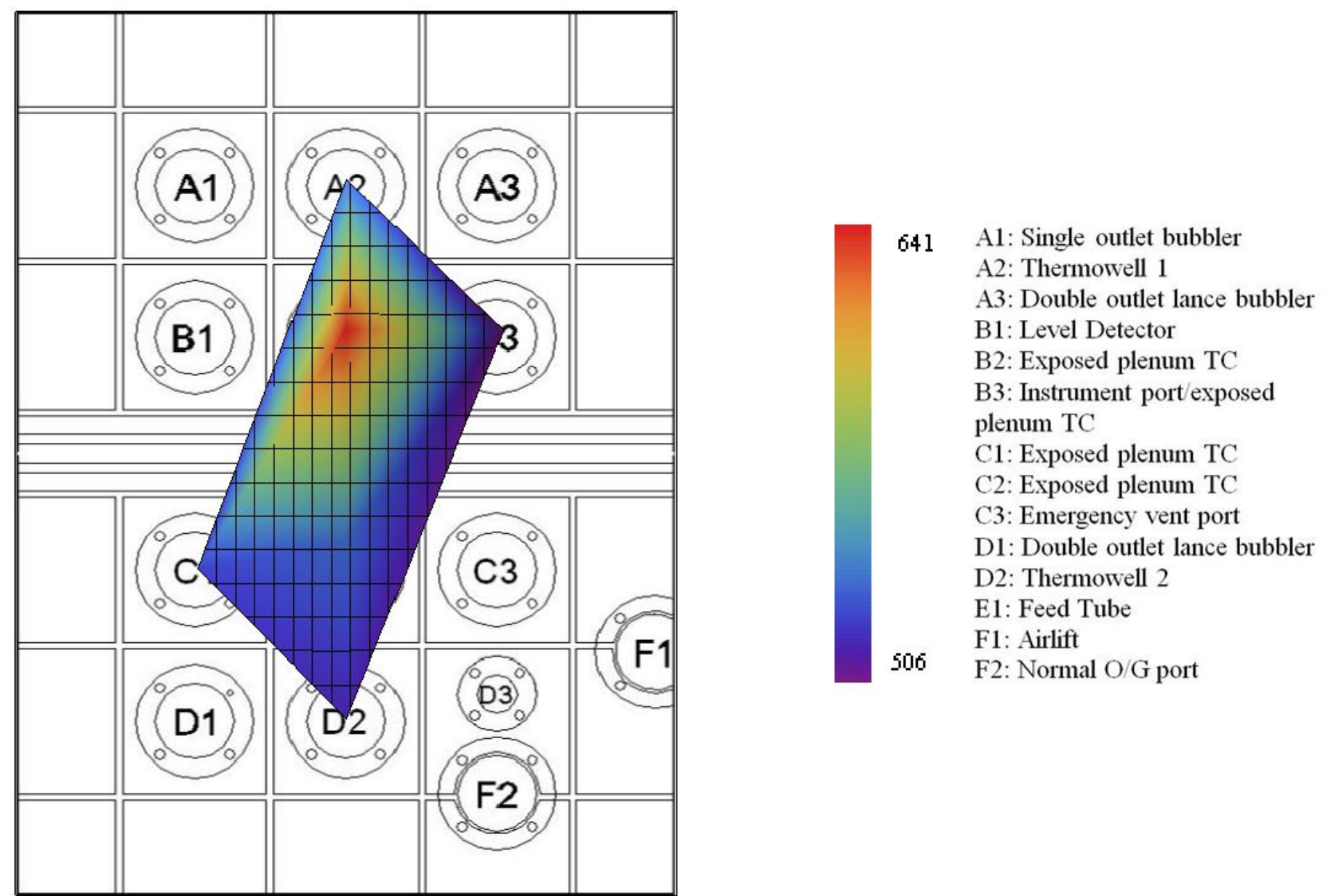

Figure 3.5.b. Average steady state plenum temperatures $\left({ }^{\circ} \mathrm{C}\right)$ at monitoring locations; two double-outlet and one single-outlet bubblers, Test 2. 
Testing of Optimized Bubbler Configuration for HLW Melter Final Report, VSL-13R2950-1, Rev. 0
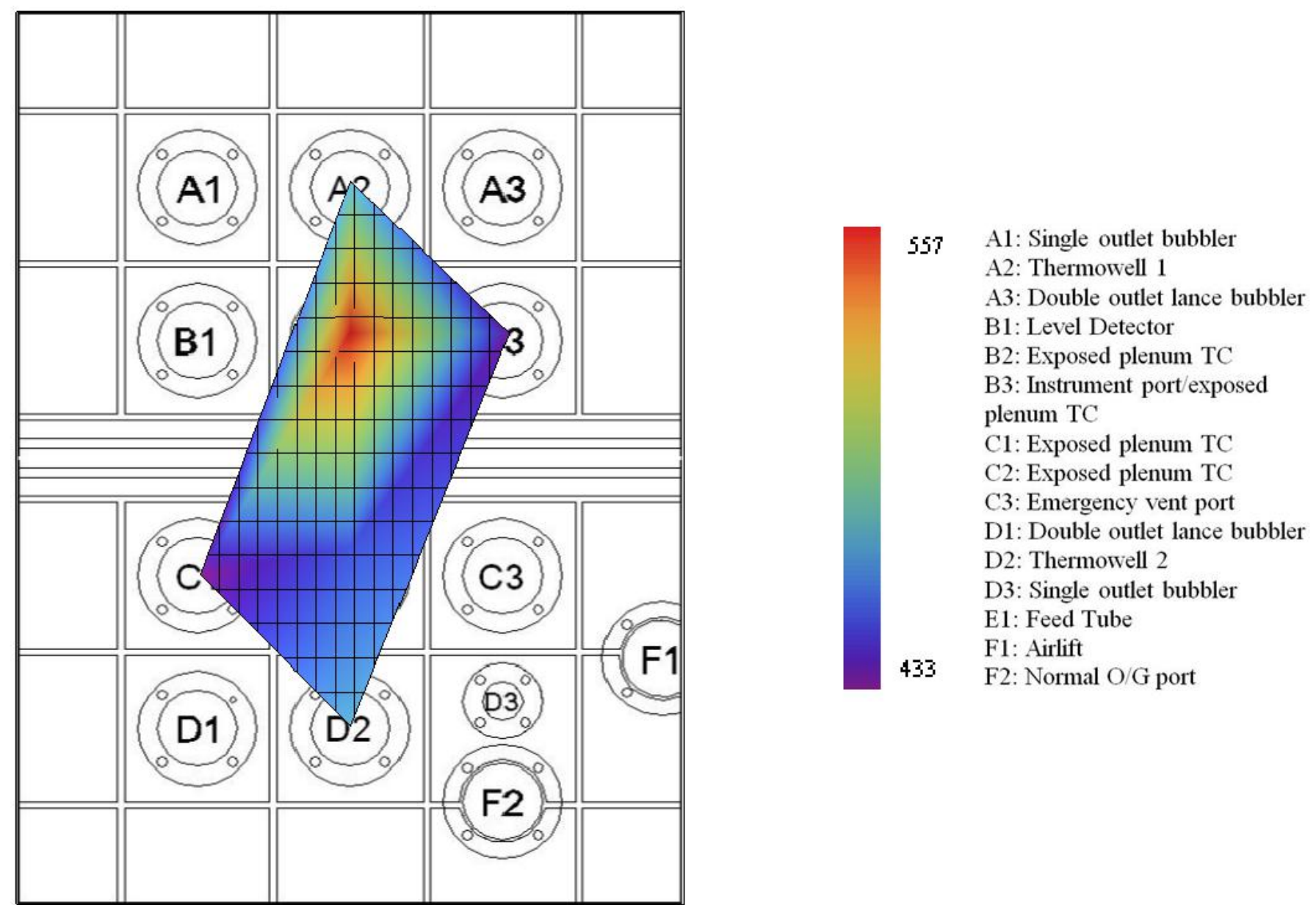

Figure 3.5.c. Average steady state plenum temperatures $\left({ }^{\circ} \mathrm{C}\right)$ at monitoring locations; two double-outlet and two single-outlet bubblers, Test 3 . 
Testing of Optimized Bubbler Configuration for HLW Melter Final Report, VSL-13R2950-1, Rev. 0
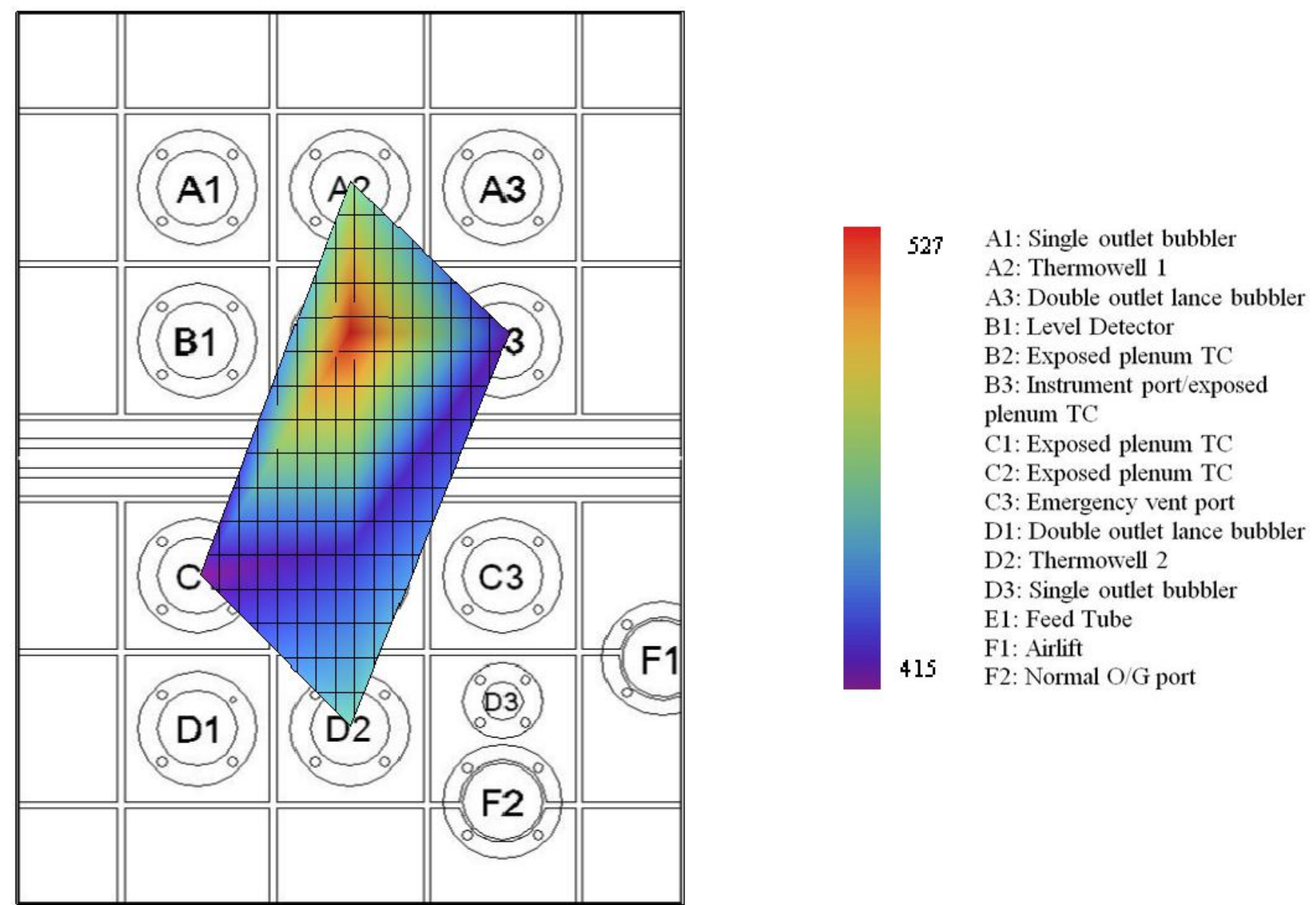

Figure 3.5.d. Average steady state plenum temperatures $\left({ }^{\circ} \mathrm{C}\right)$ at monitoring locations; two double-outlet and two single-outlet bubblers, Test 4. 

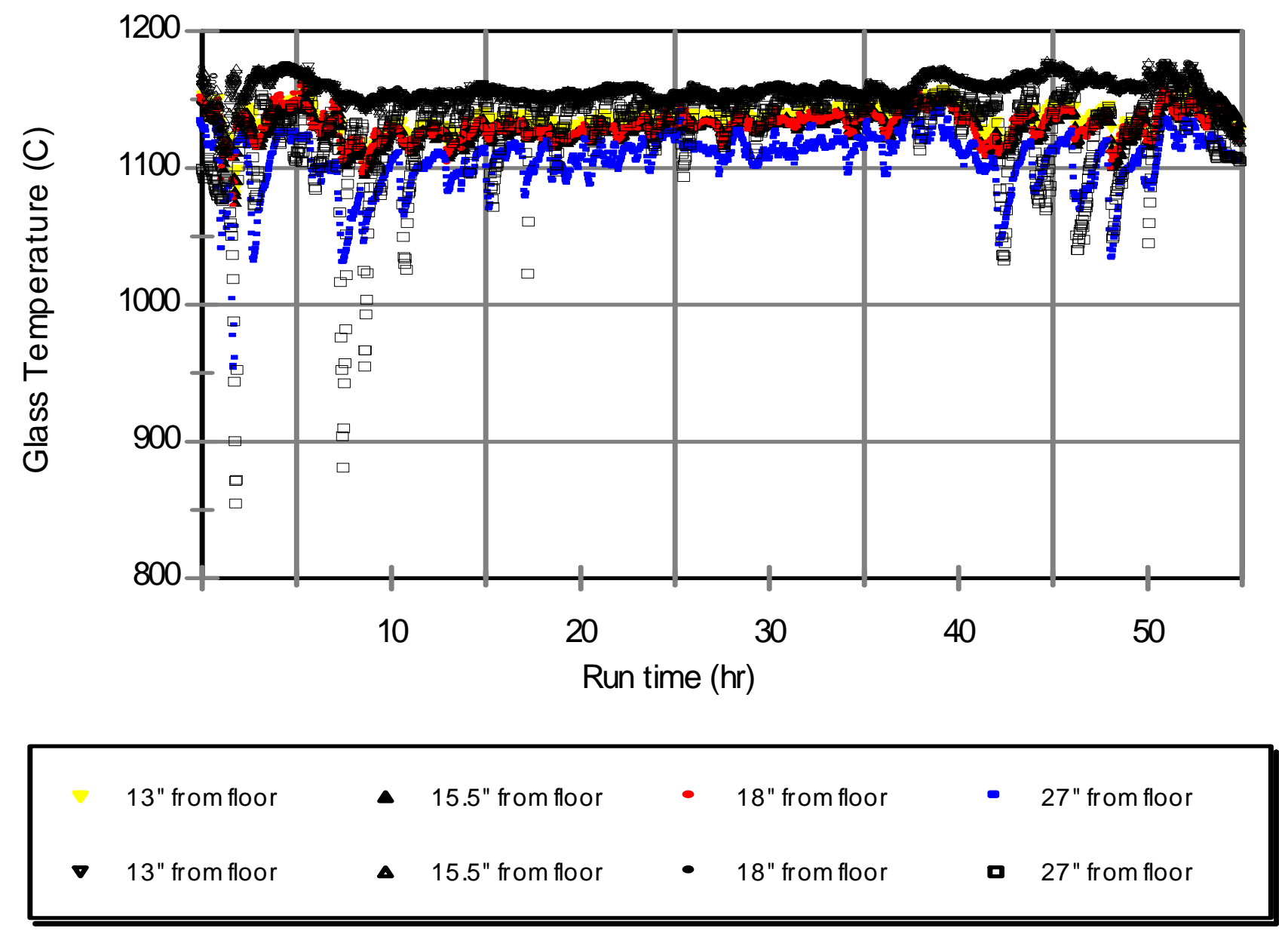

Figure 3.6.a. Glass temperatures while processing with two double-outlet bubblers, Test 1. 


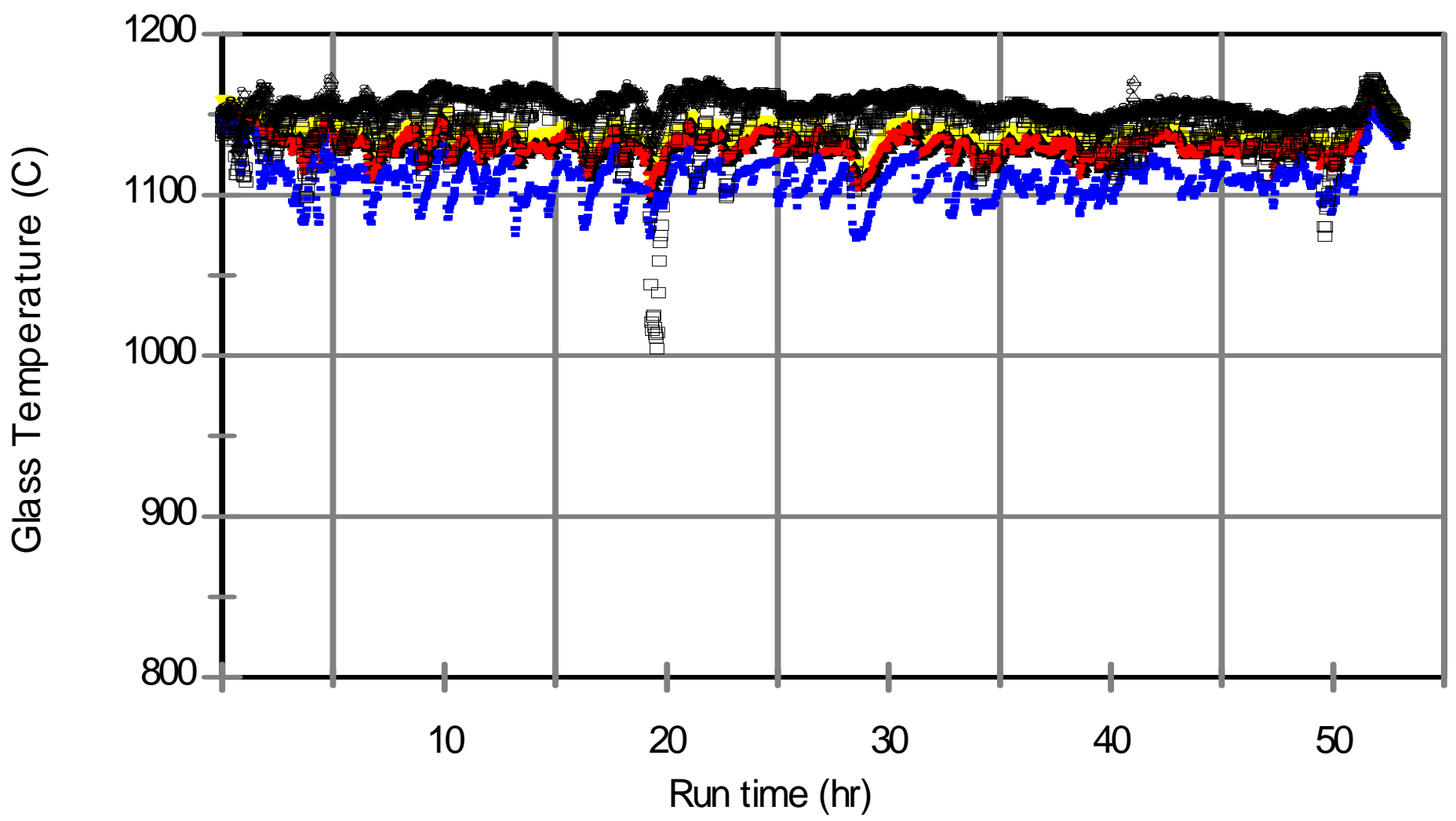

13 " from floor

- 13" from floor

ه 15.5" from floor
- 18 " from floor

- $\quad 18$ " from floor
- $\quad 27$ " from floor

- 27 "from floor

Figure 3.6.b. Glass temperatures while processing with two double-outlet and one single-outlet bubblers, Test 2. 

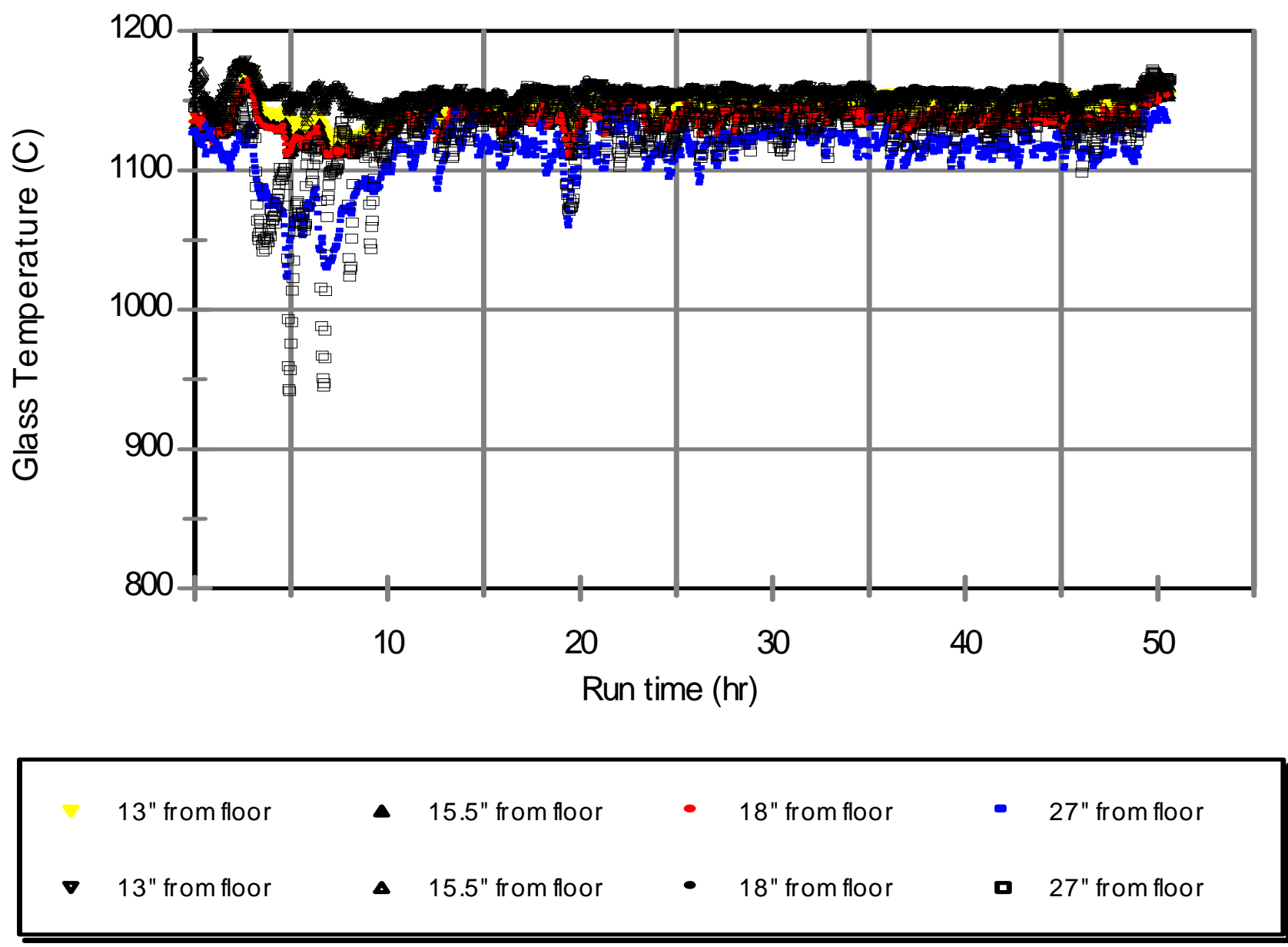

Figure 3.6.c. Glass temperatures while processing with two double-outlet and two single-outlet bubblers, Test 3. 

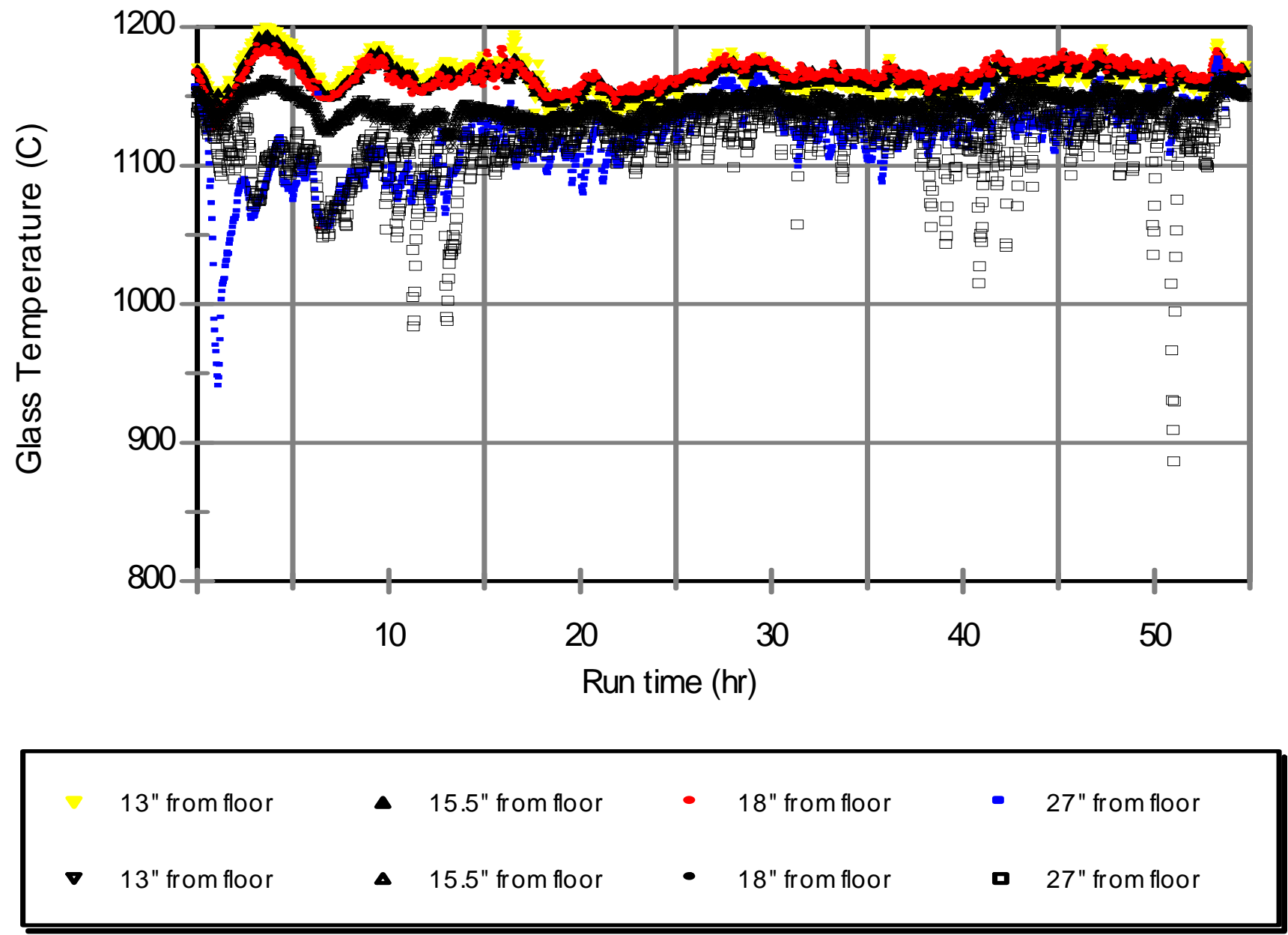

Figure 3.6.d. Glass temperatures while processing with two double-outlet and two single-outlet bubblers, Test 4 . 


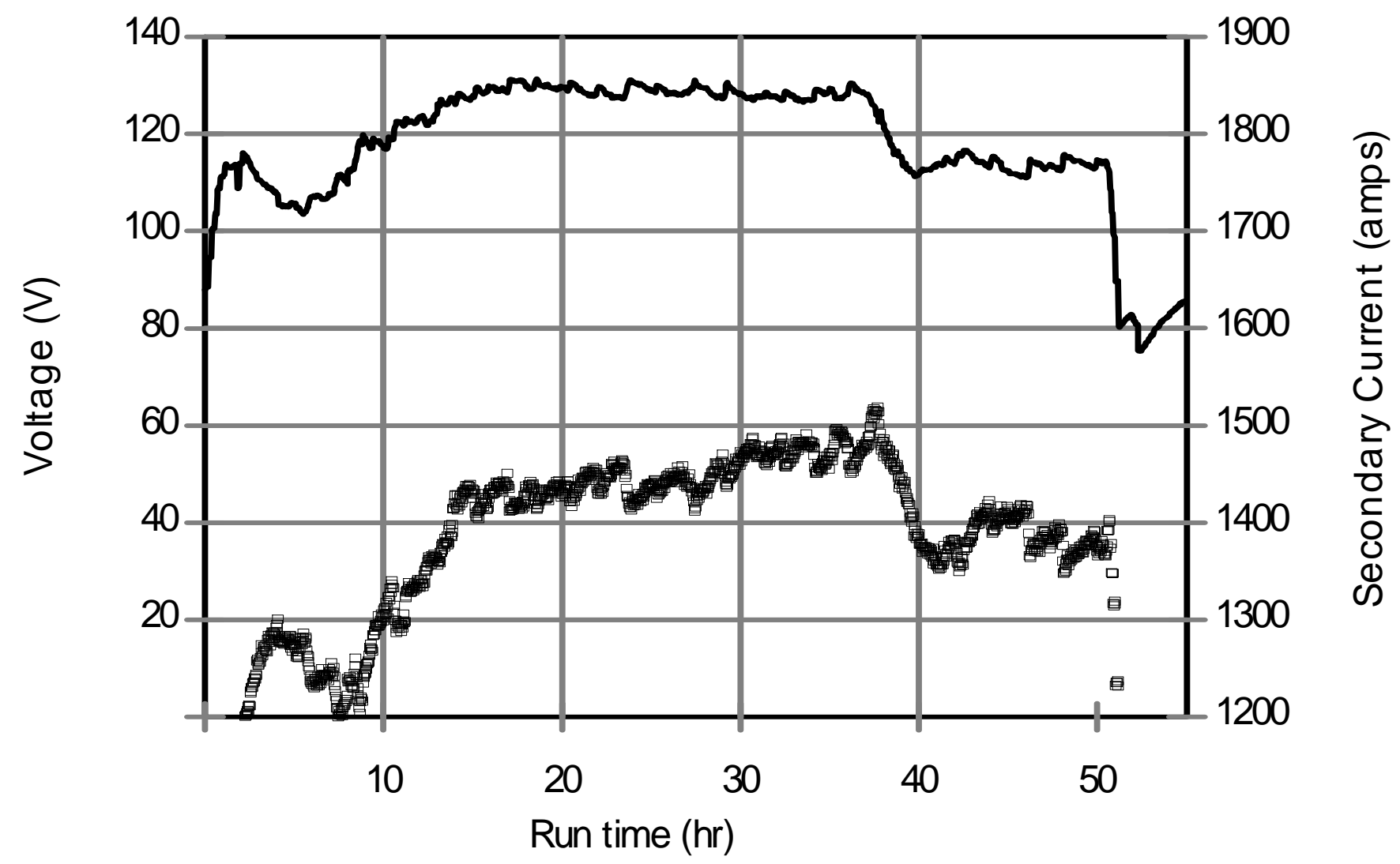

Figure 3.7.a. Electrode voltage and current while processing with two double-outlet bubblers, Test 1. 


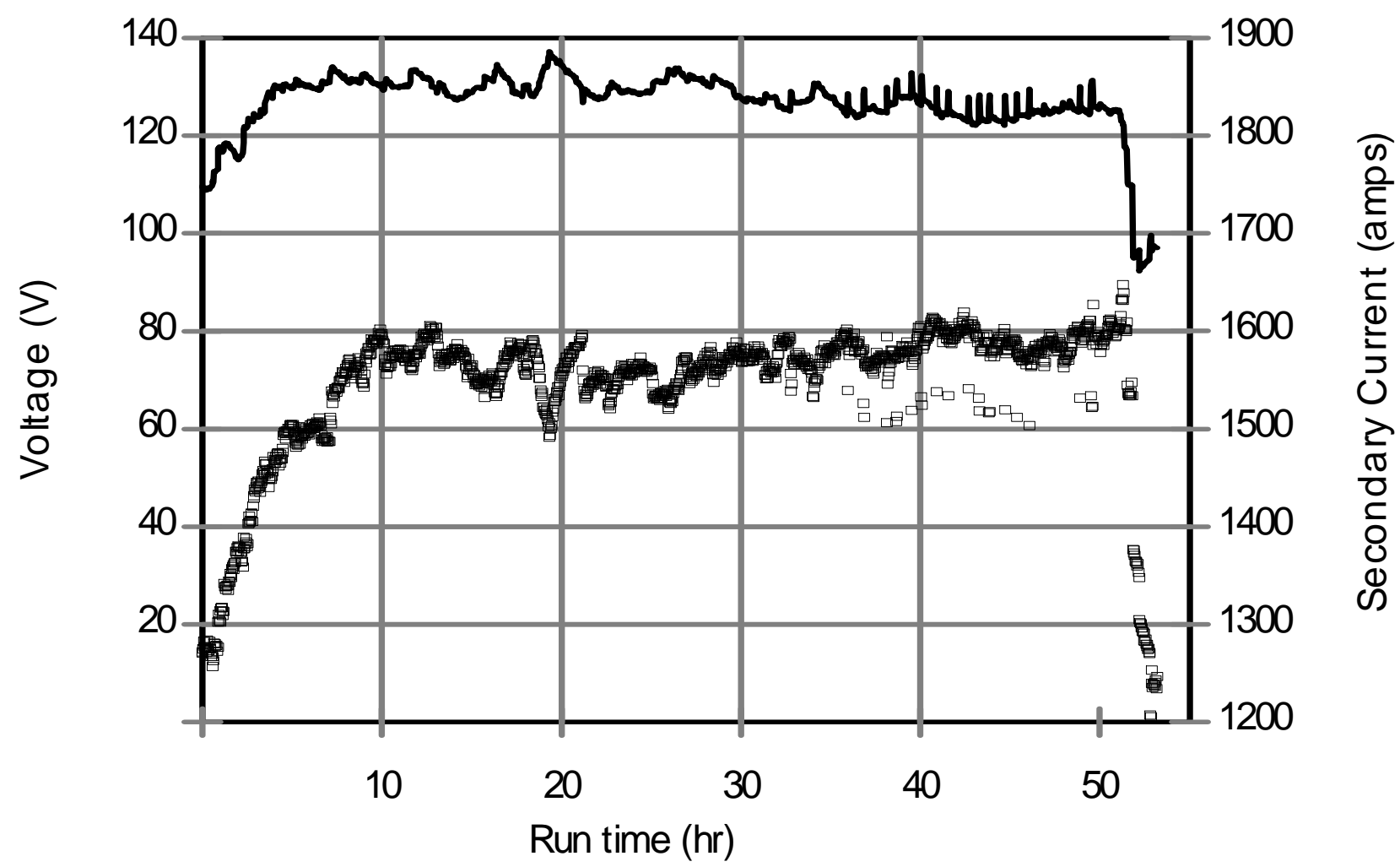

Figure 3.7.b. Electrode voltage and current while processing with two double-outlet and one single-outlet bubblers, Test 2 . 


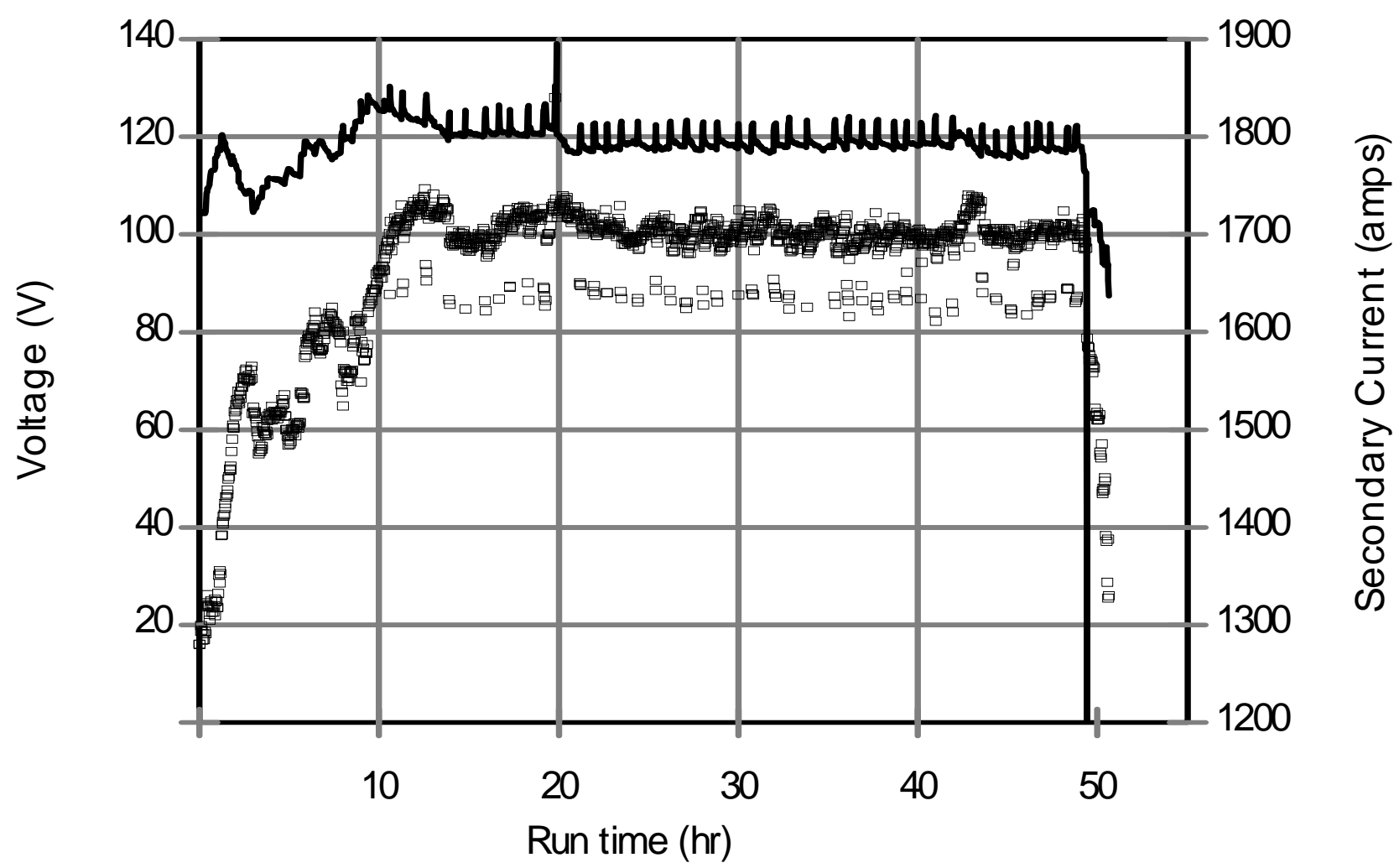

Voltage

- Current

Figure 3.7.c. Electrode voltage and current while processing with two double-outlet and two single-outlet bubblers, Test 3. 


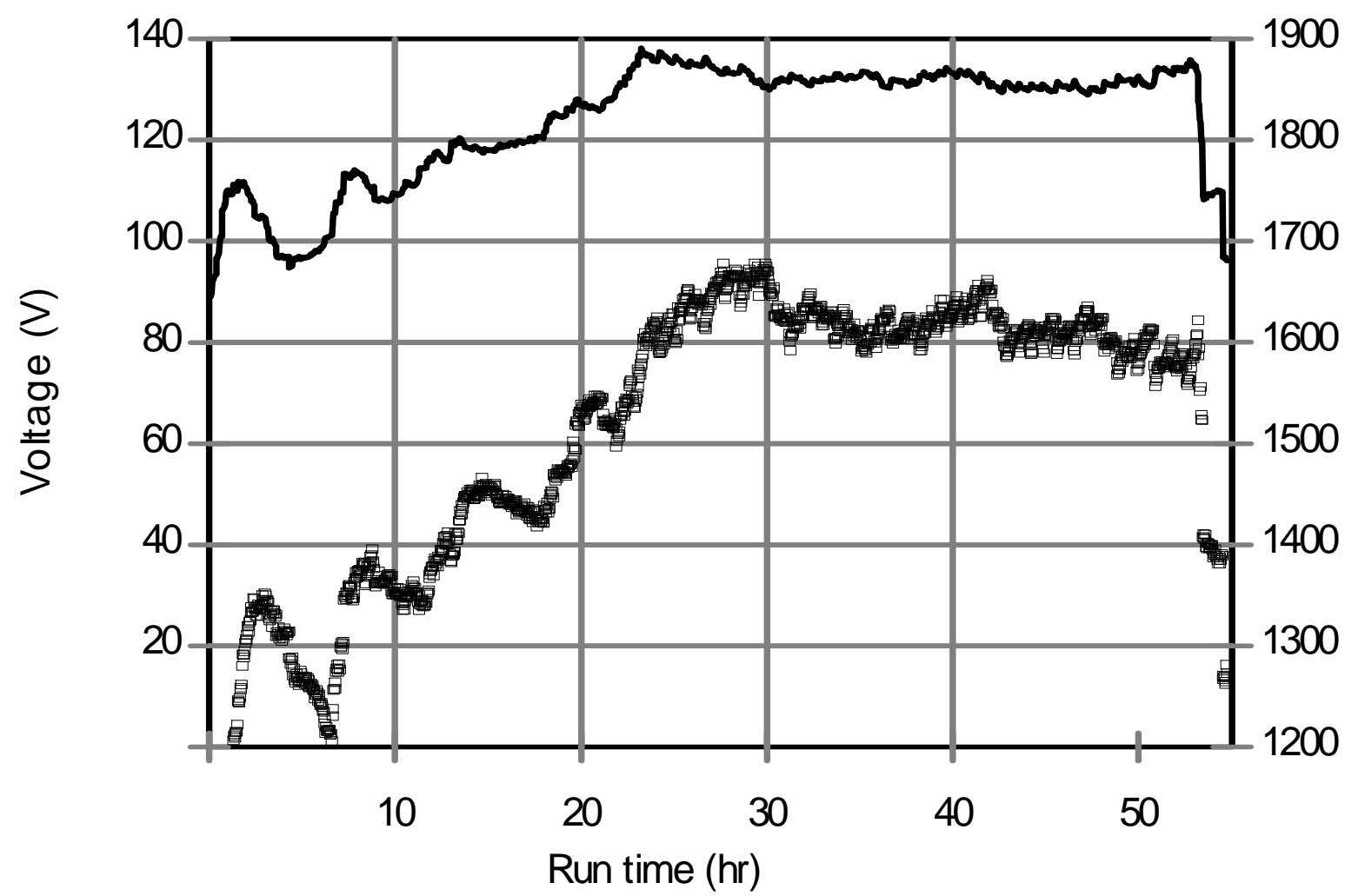

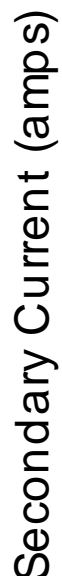

Figure 3.7.d. Electrode voltage and current while processing with two double-outlet and two single-outlet bubblers, Test 4. 


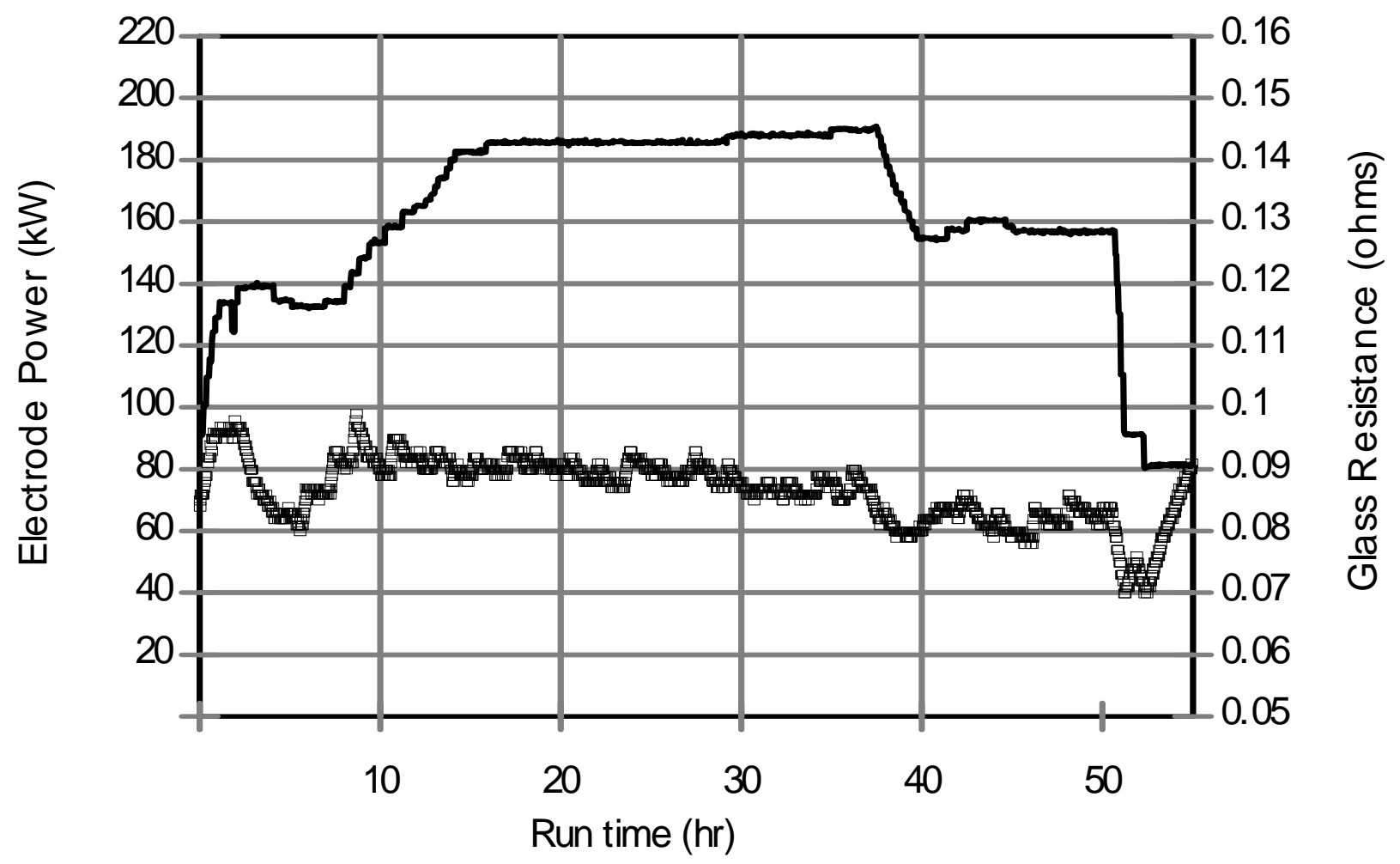

Figure 3.8.a. Electrode power and glass resistance while processing with two double-outlet bubblers, Test 1. 


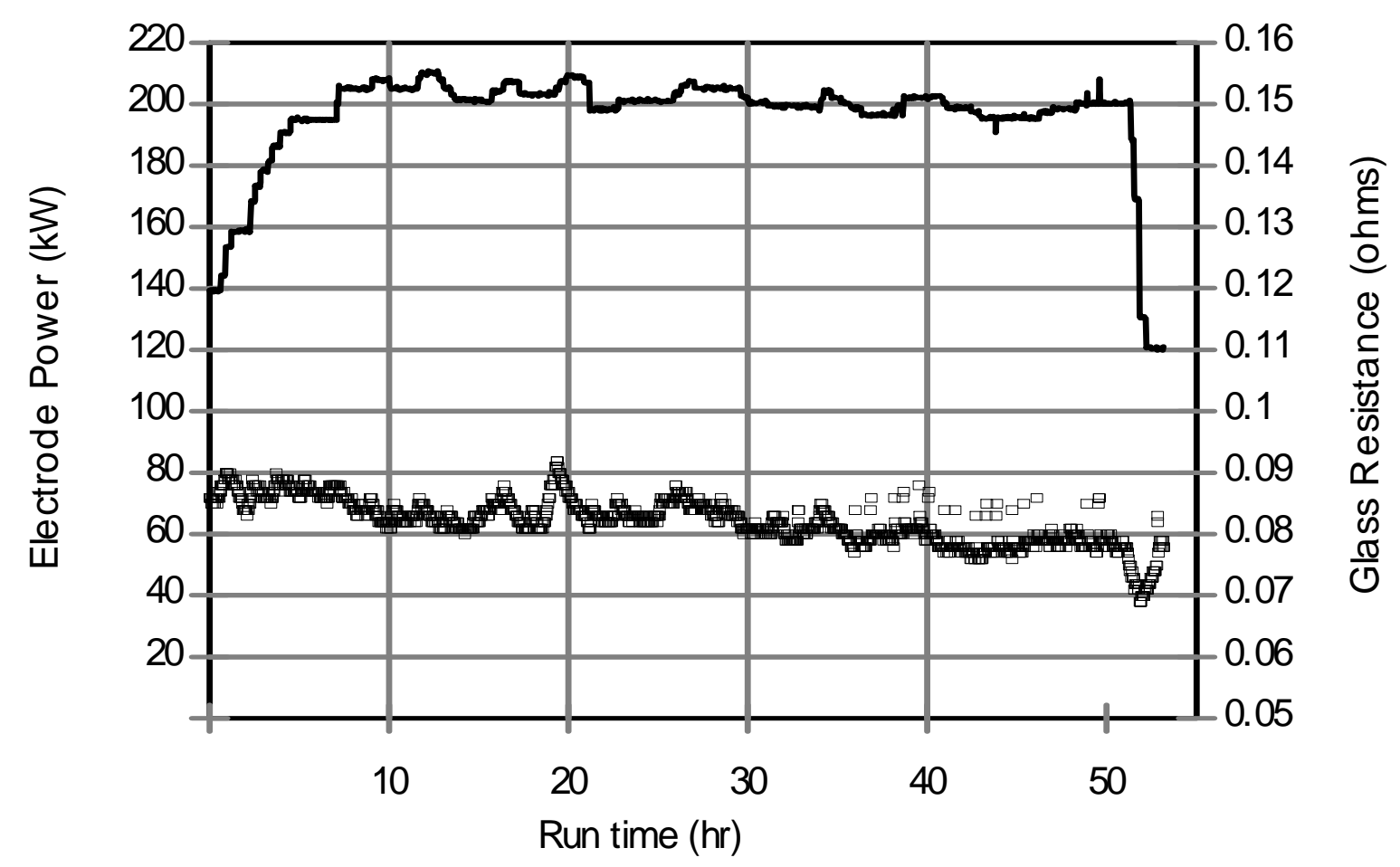

Power

- Resistance

Figure 3.8.b. Electrode power and glass resistance while processing with two double-outlet and one single-outlet bubblers, Test 2. 


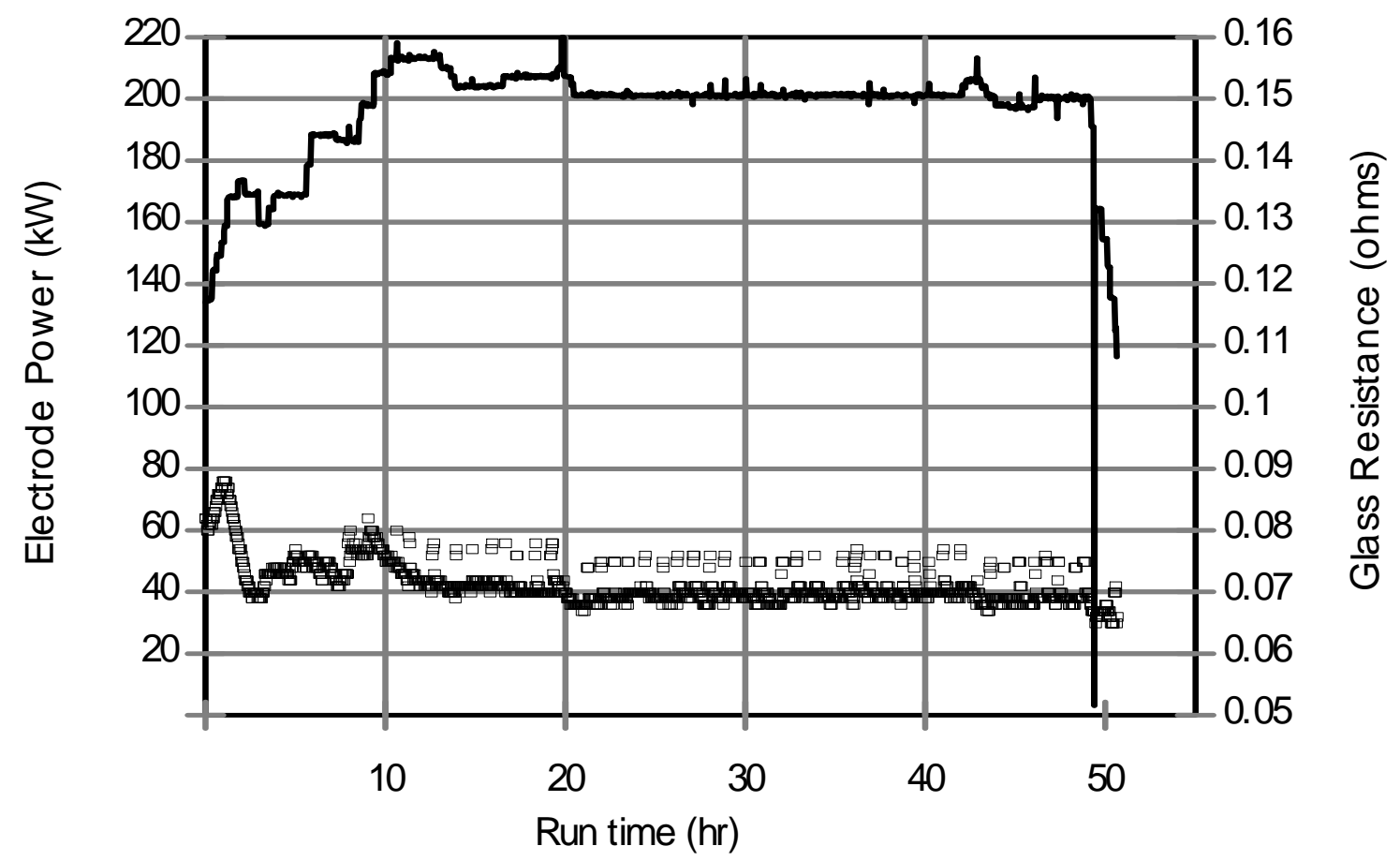

- Resistance

Figure 3.8.c. Electrode power and glass resistance while processing with two double-outlet and two single-outlet bubblers, Test 3. 

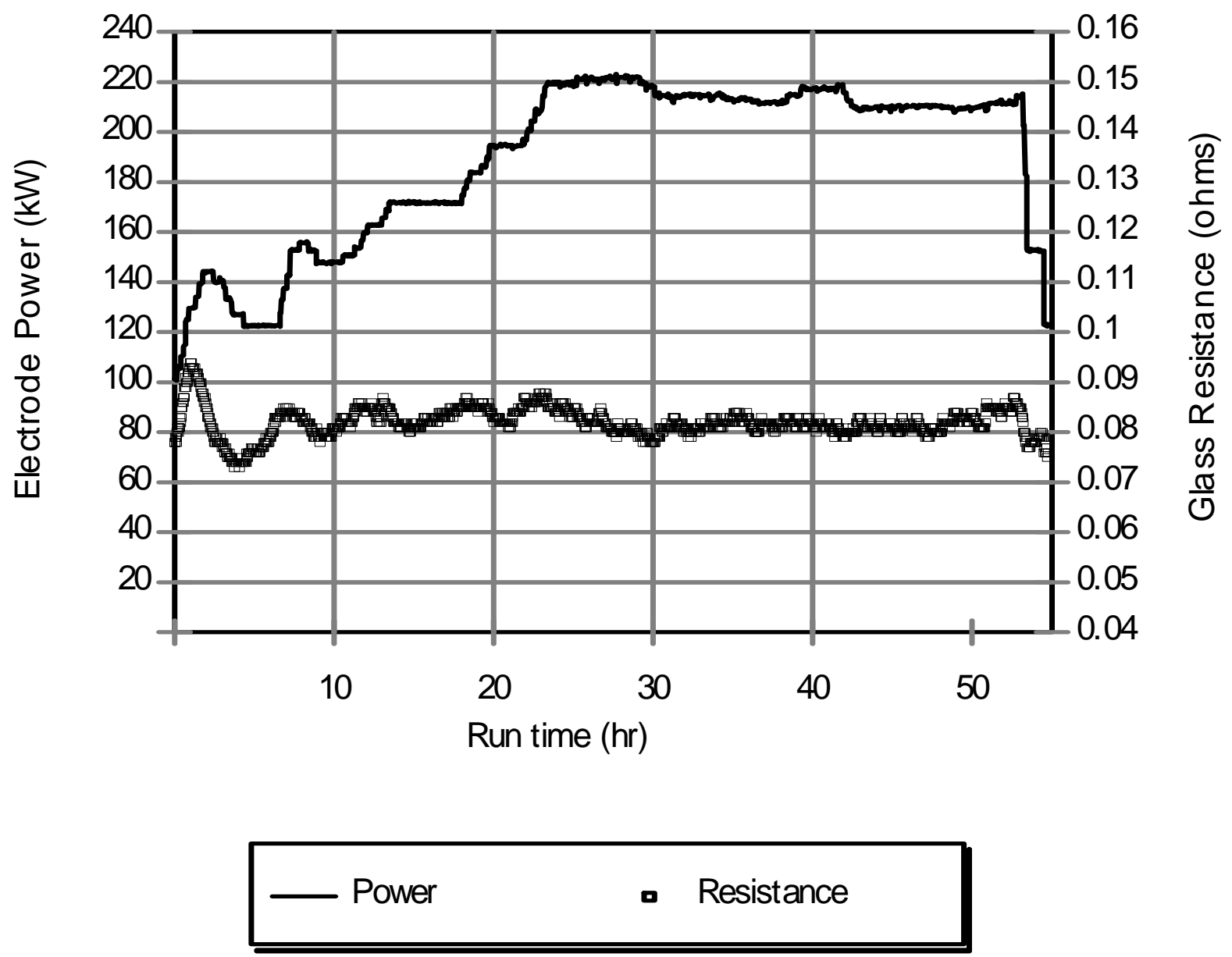

Figure 3.8.d. Electrode power and glass resistance while processing with two double-outlet and two single-outlet bubblers, Test 4 . 


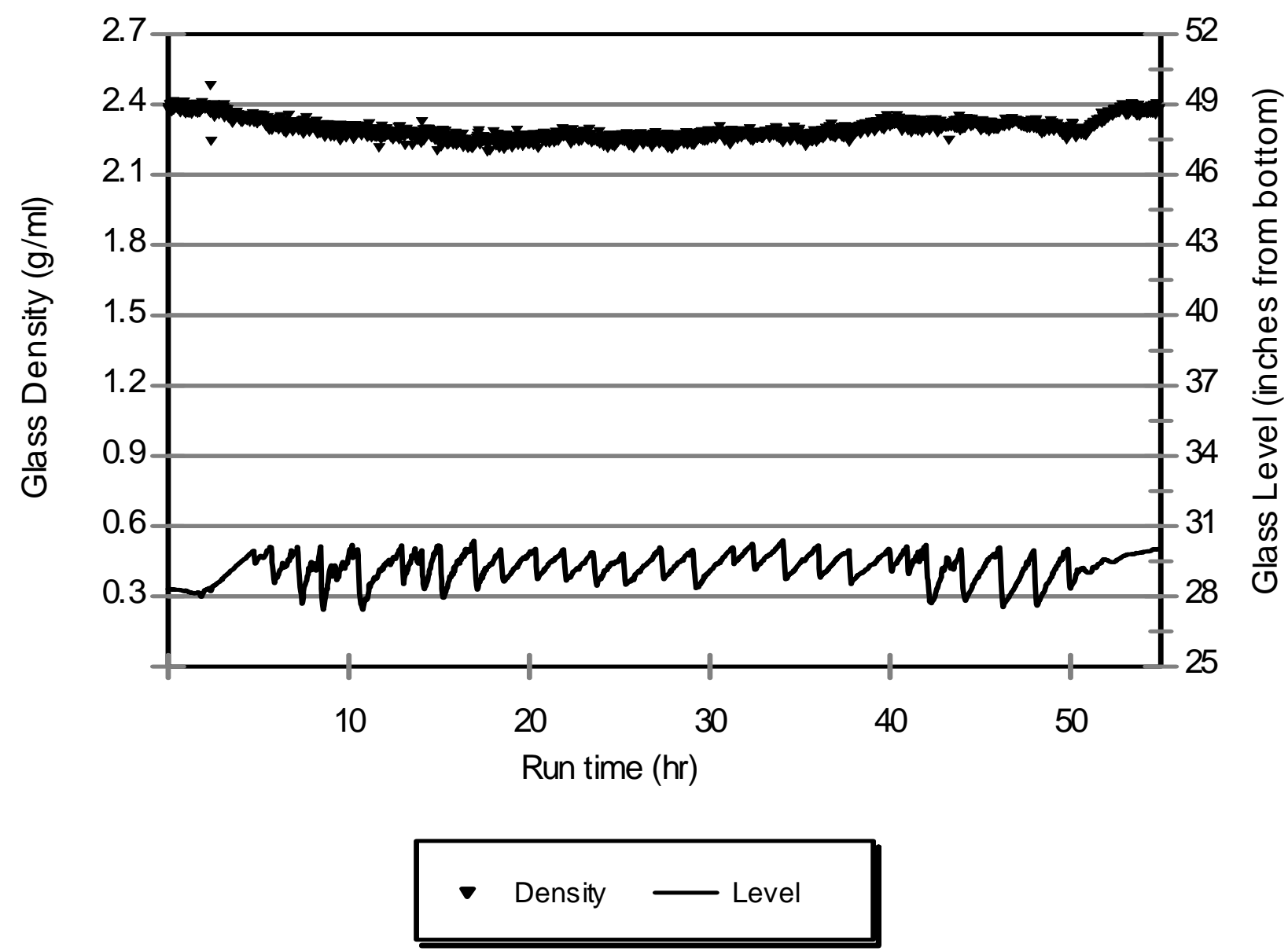

Figure 3.9.a. Glass density and level while processing with two double-outlet bubblers, Test 1. 


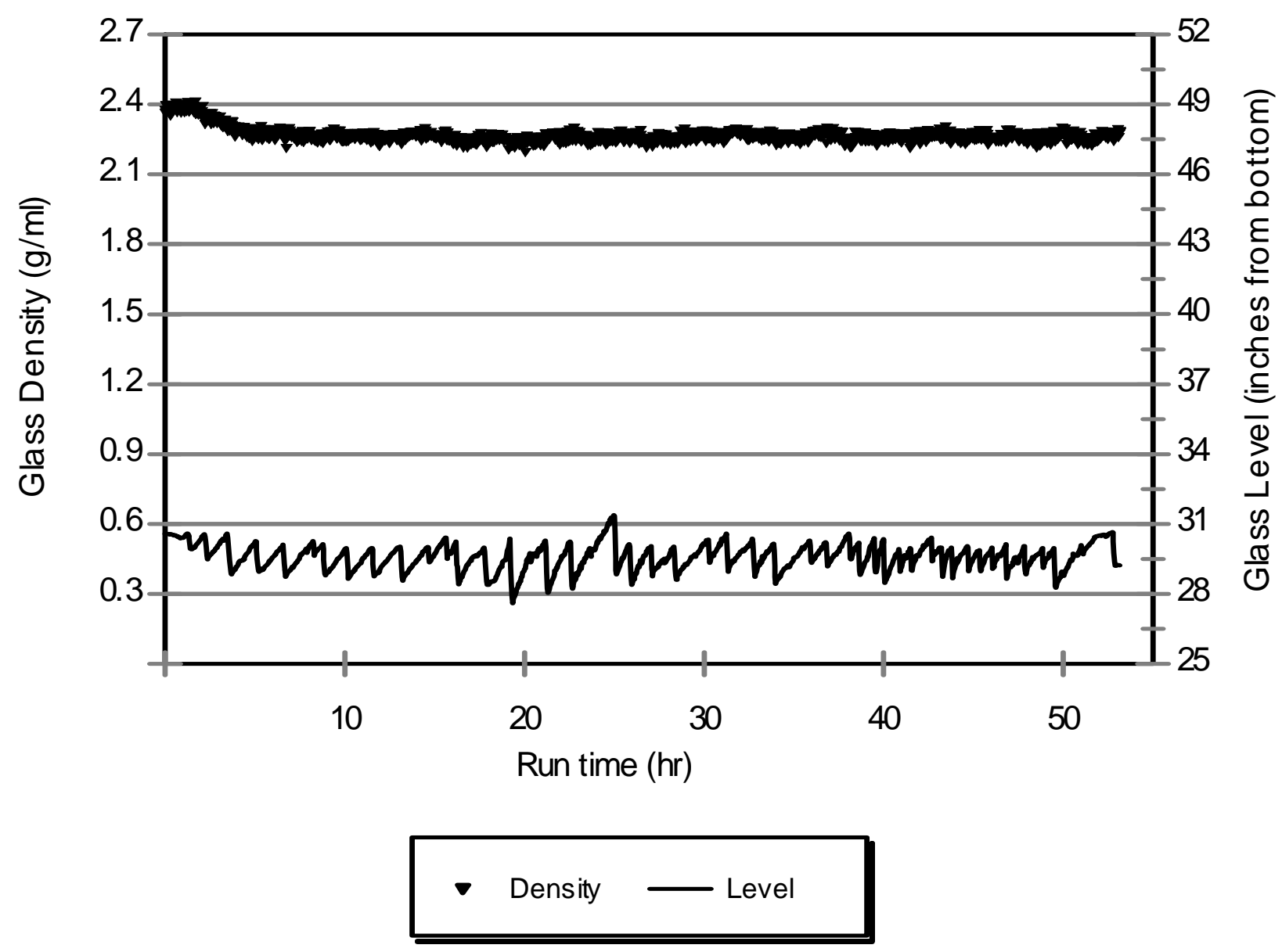

Figure 3.9.b. Glass density and level while processing with two double-outlet and one single-outlet bubblers, Test 2. 


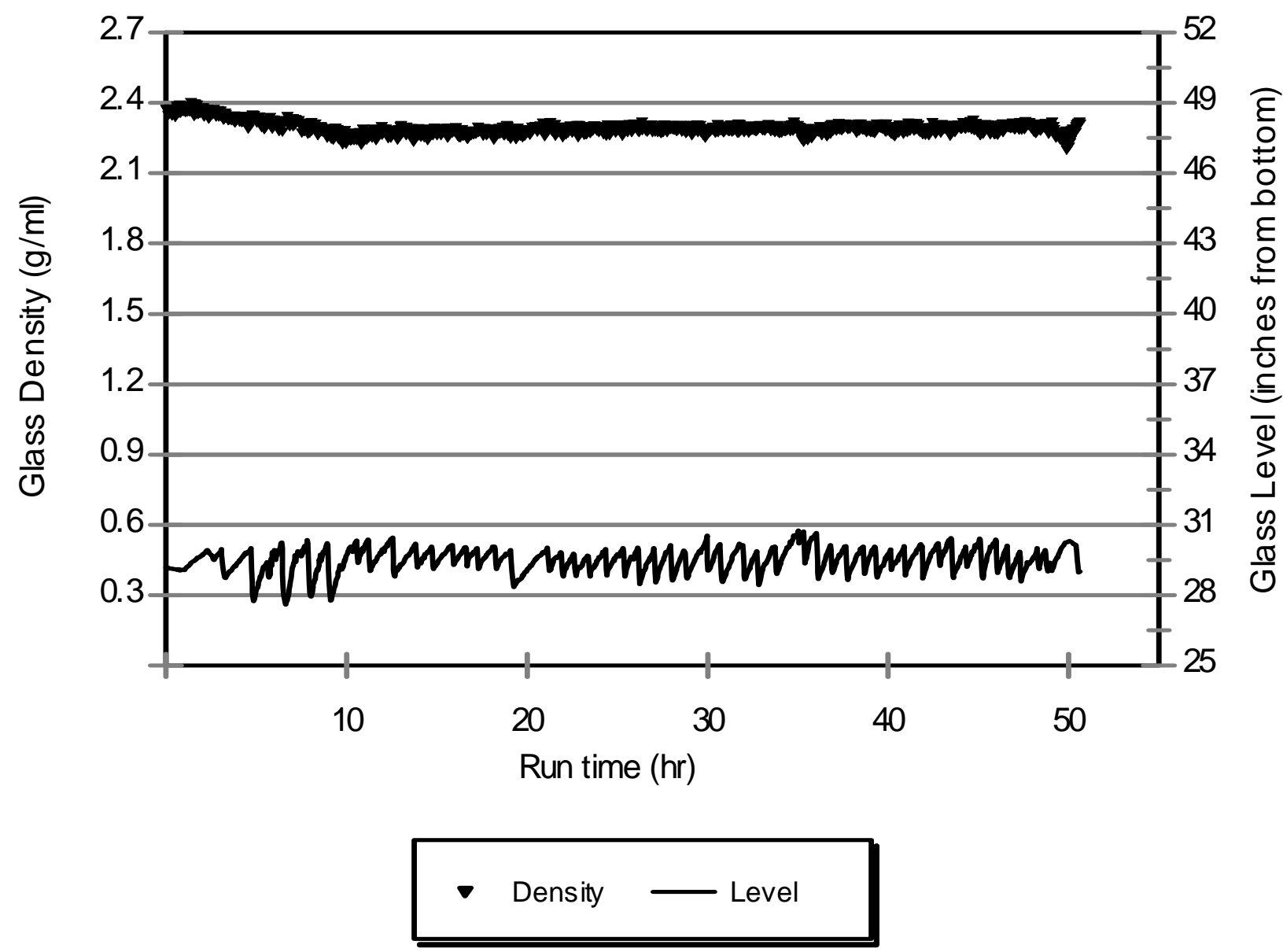

Figure 3.9.c. Glass density and level while processing with two double-outlet and two single-outlet bubblers, Test 3. 


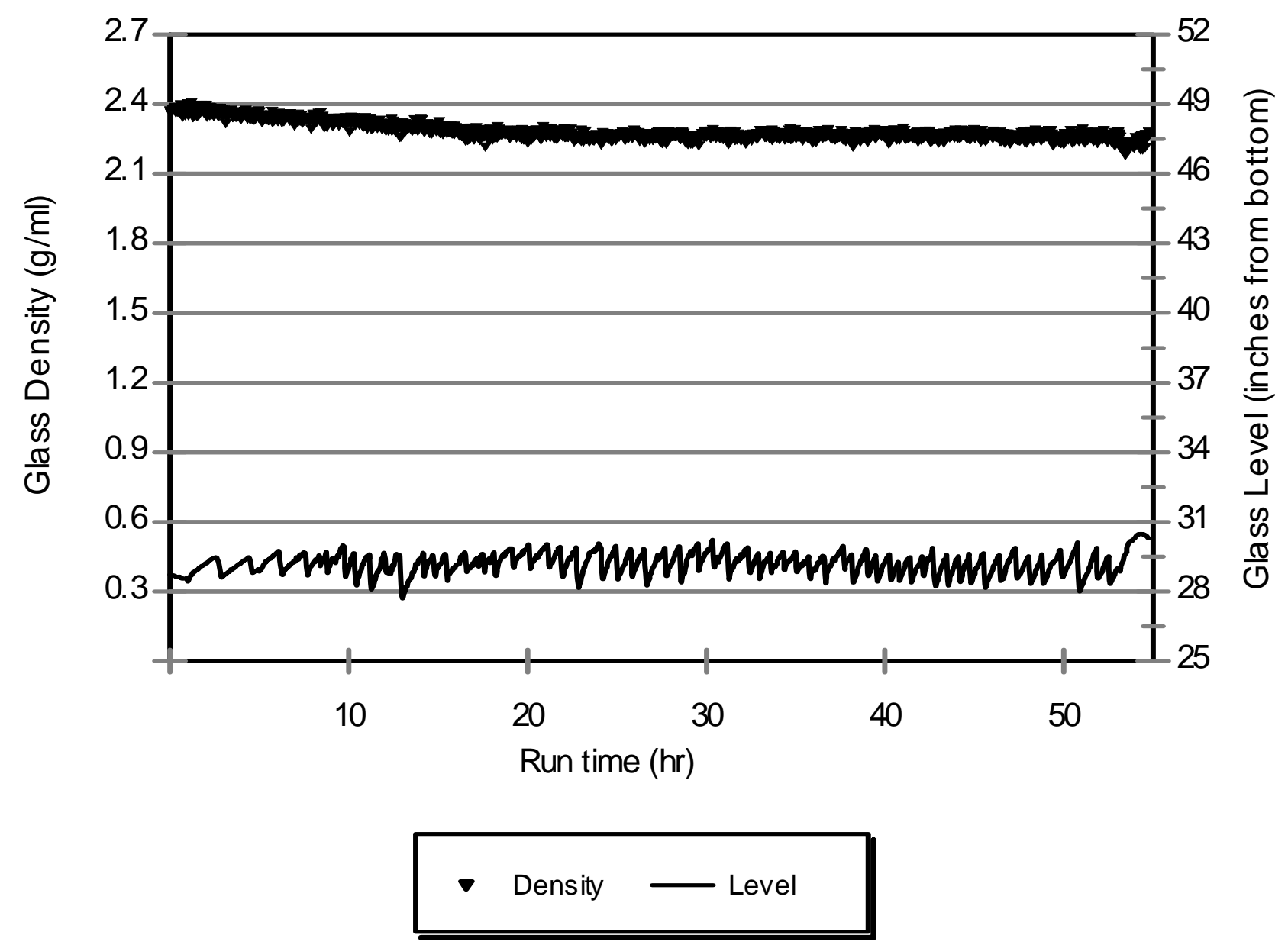

Figure 3.9.d. Glass density and level while processing with two double-outlet and two single-outlet bubblers, Test 4. 


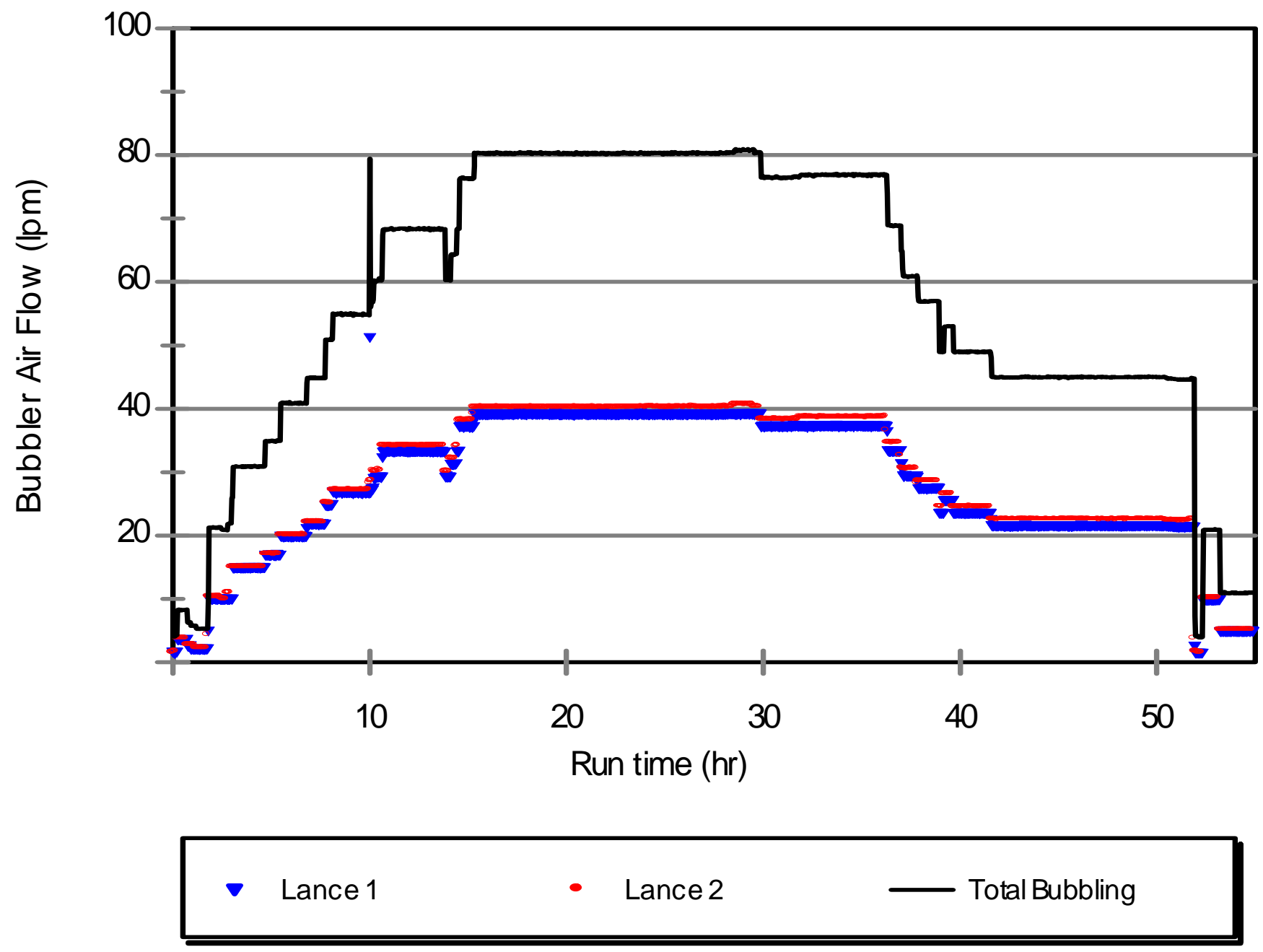

Figure 3.10.a. Glass pool bubbling while processing with two double-outlet bubblers, Test 1. 


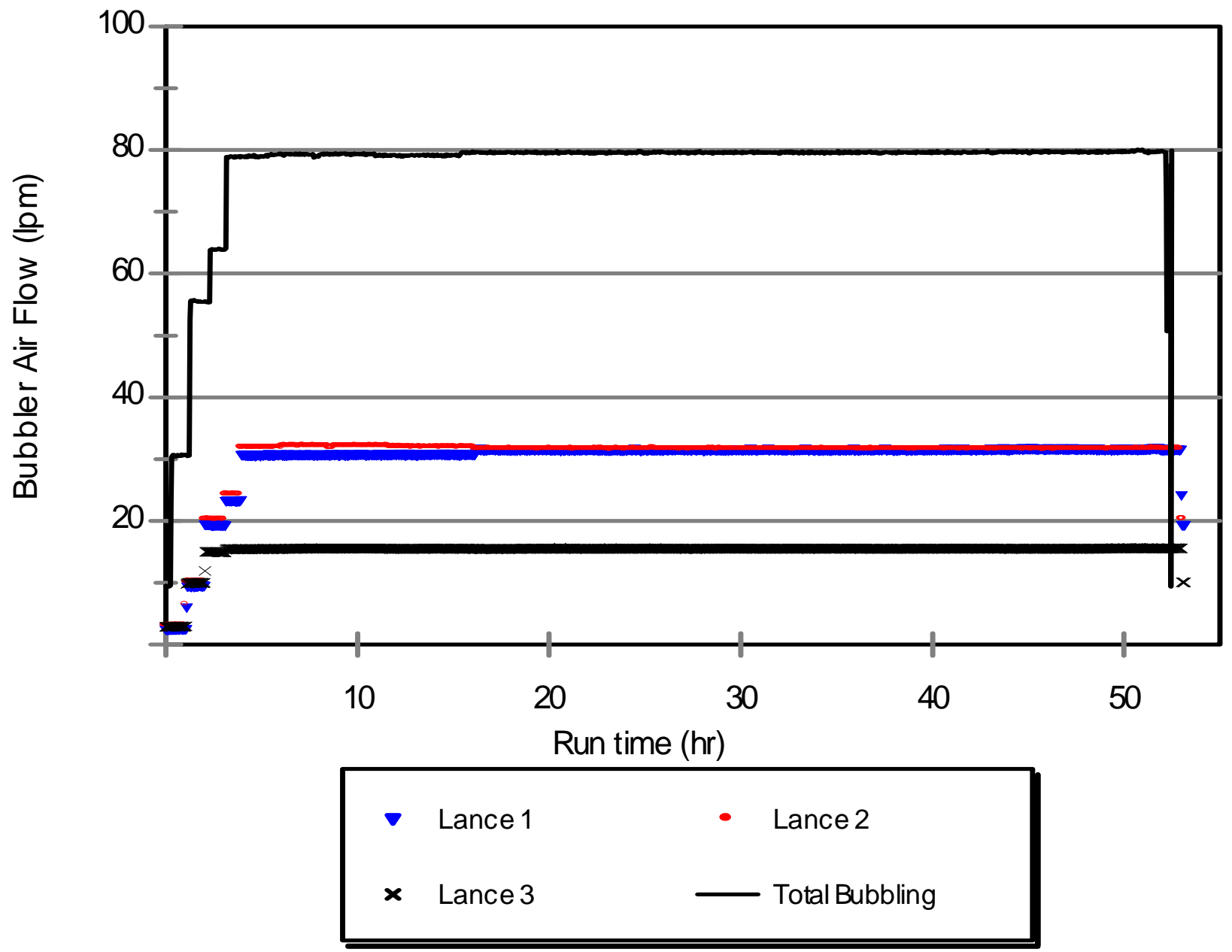

Figure 3.10.b. Glass pool bubbling while processing with two double-outlet and one single-outlet bubblers, Test 2. 


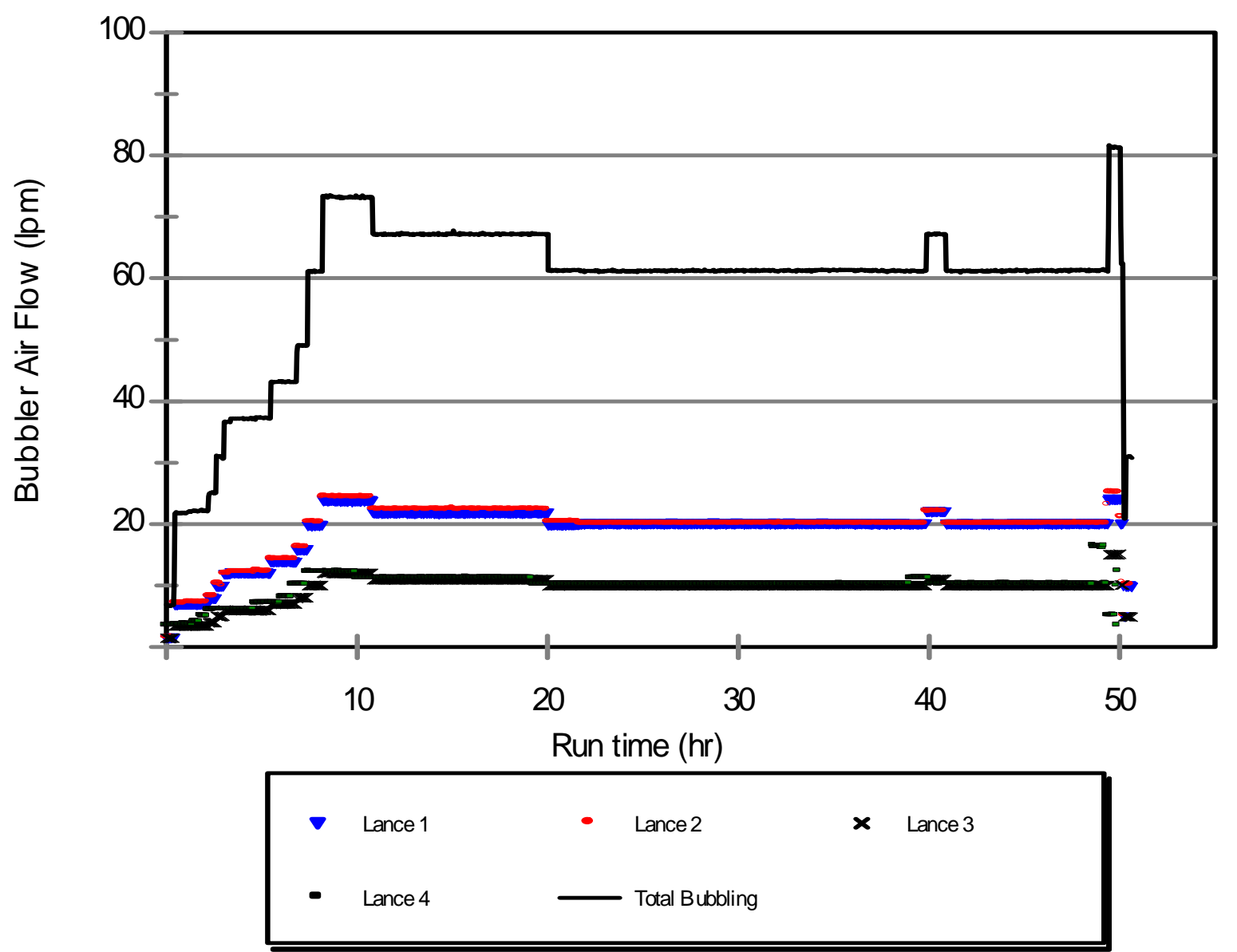

Figure 3.10.c. Glass pool bubbling while processing with two double-outlet and two single-outlet bubblers, Test 3. 


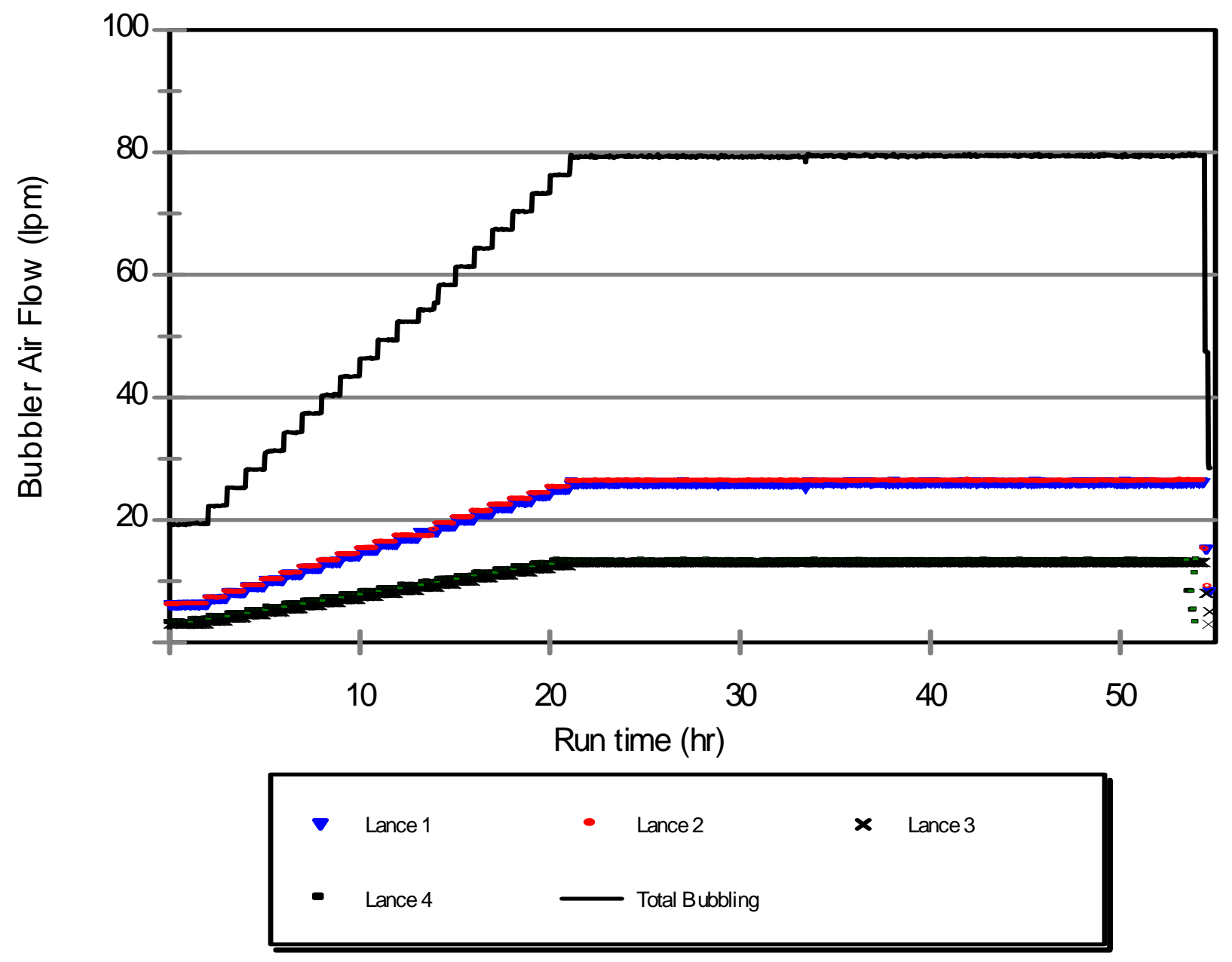

Figure 3.10.d. Glass pool bubbling while processing with two double-outlet and two single-outlet bubblers, Test 4. 


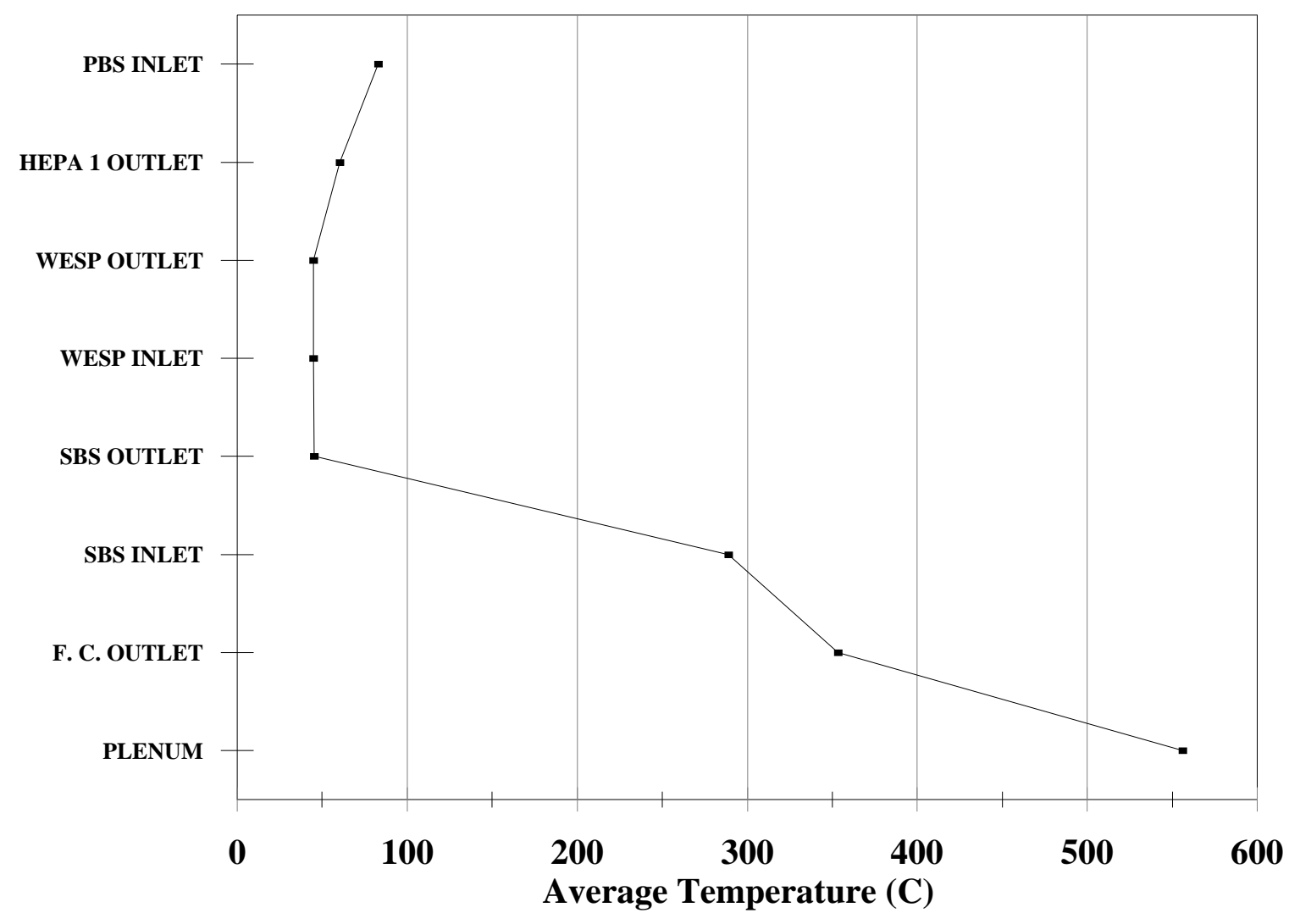

Figure 4.1. Average gas temperatures along the DM1200 off-gas train while processing with two double-outlet bubblers, Test 1. 


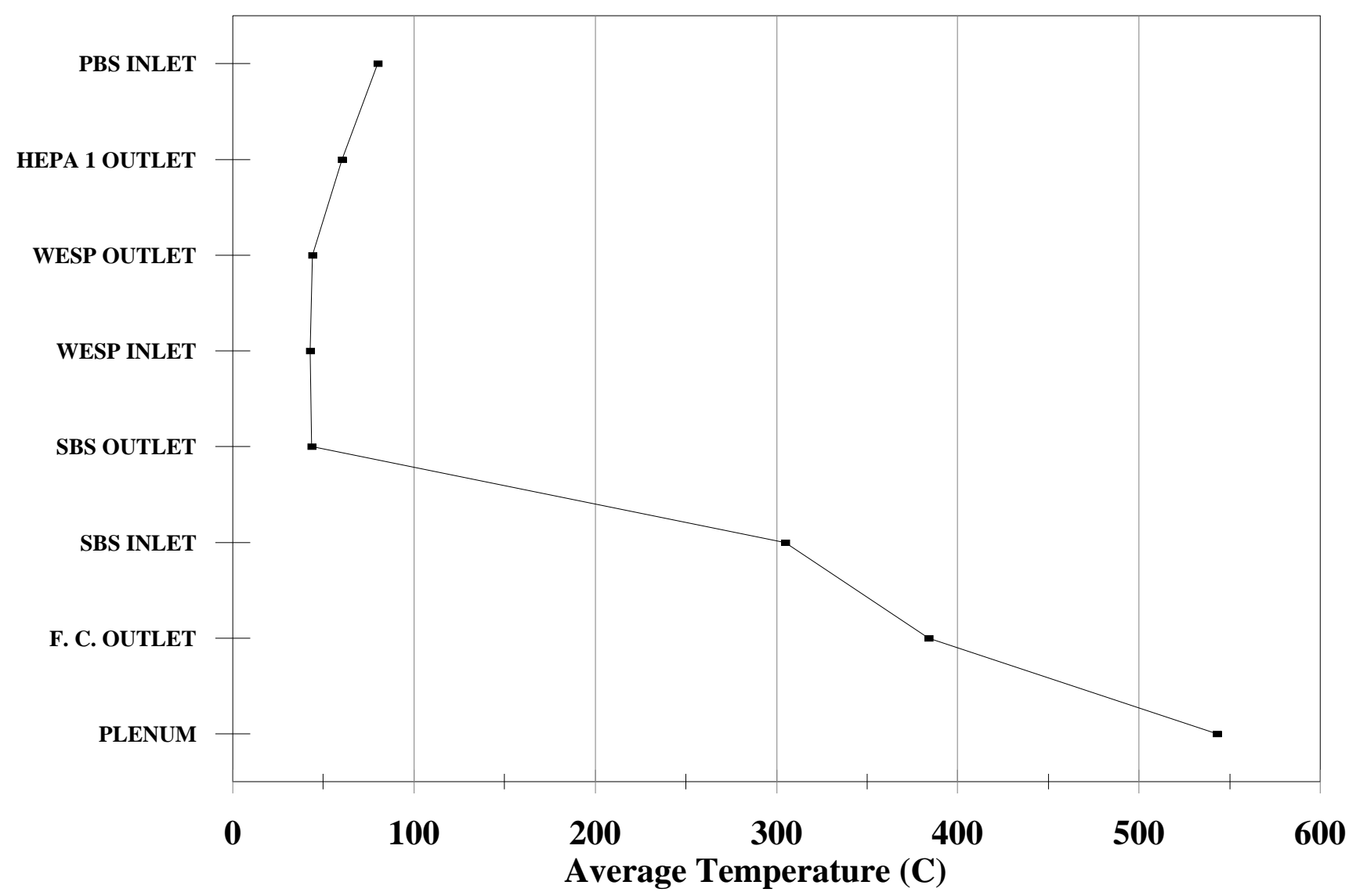

Figure 4.2. Average gas temperatures along the DM1200 off-gas train while processing with two double-outlet and one single-outlet bubblers, Test 2. 


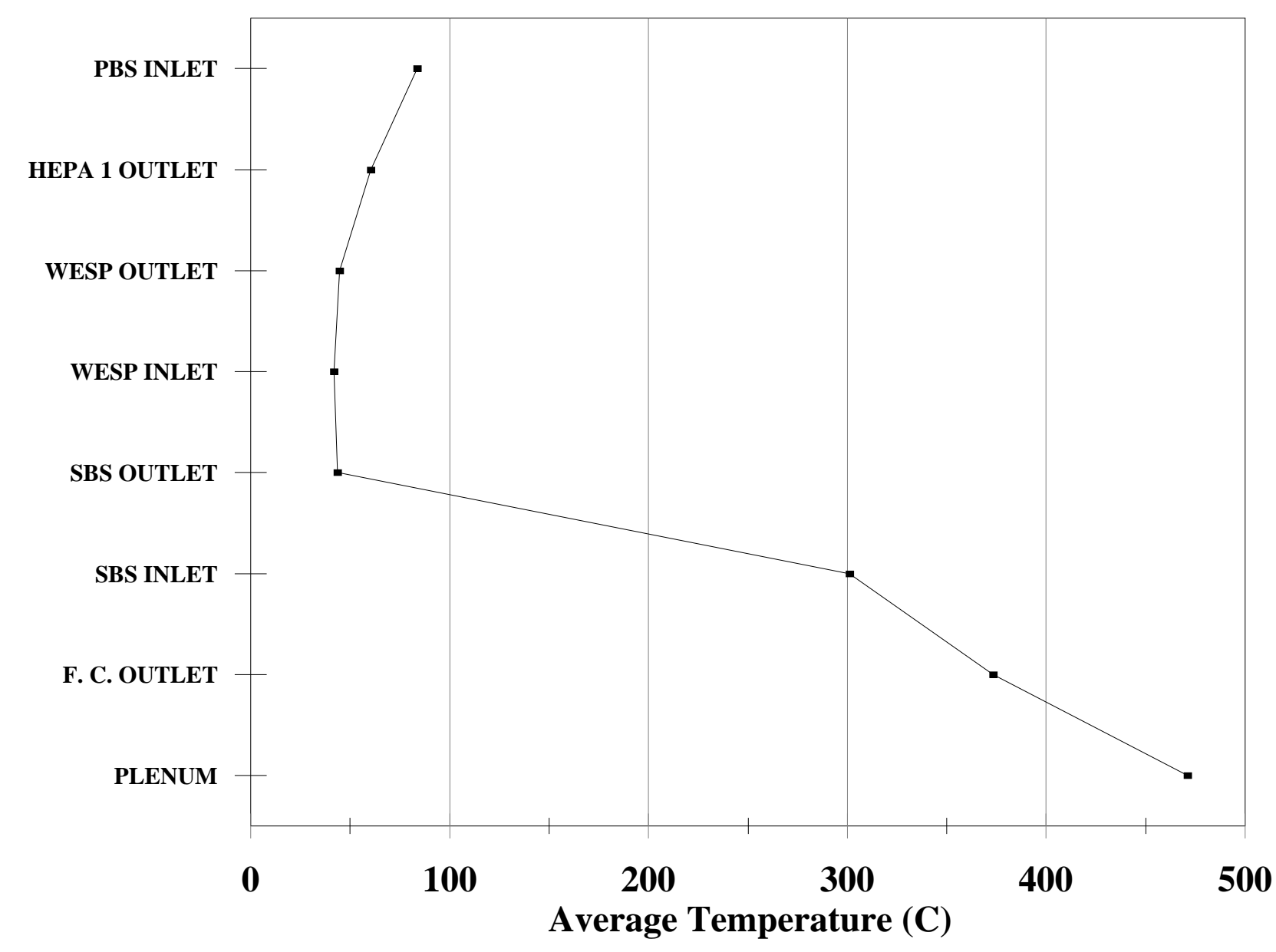

Figure 4.3. Average gas temperatures along the DM1200 off-gas train while processing with two double-outlet and two single-outlet bubblers, Test 3. 


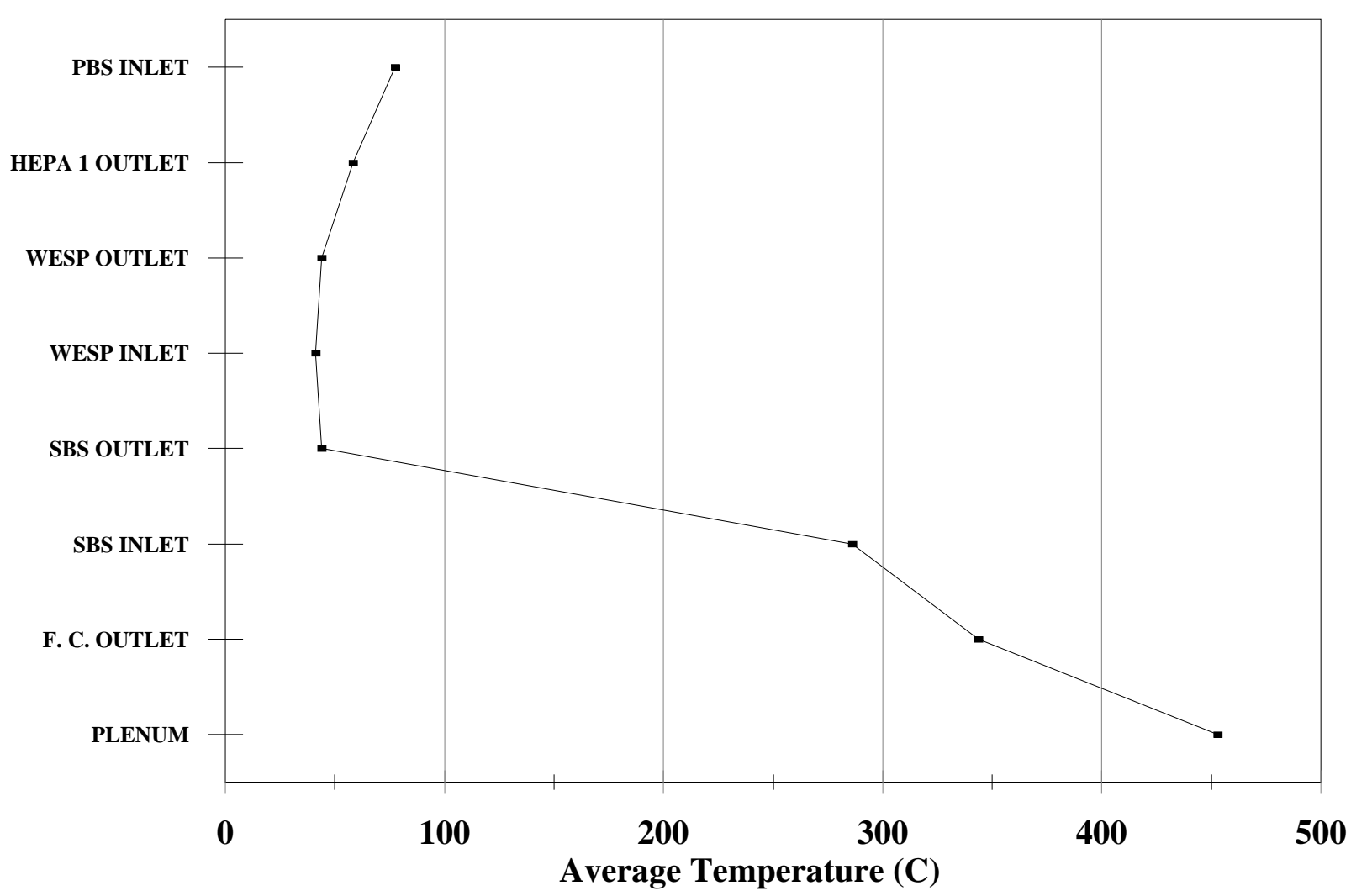

Figure 4.4. Average gas temperatures along the DM1200 off-gas train rates while processing with two double-outlet and two single-outlet bubblers, Test 4. 


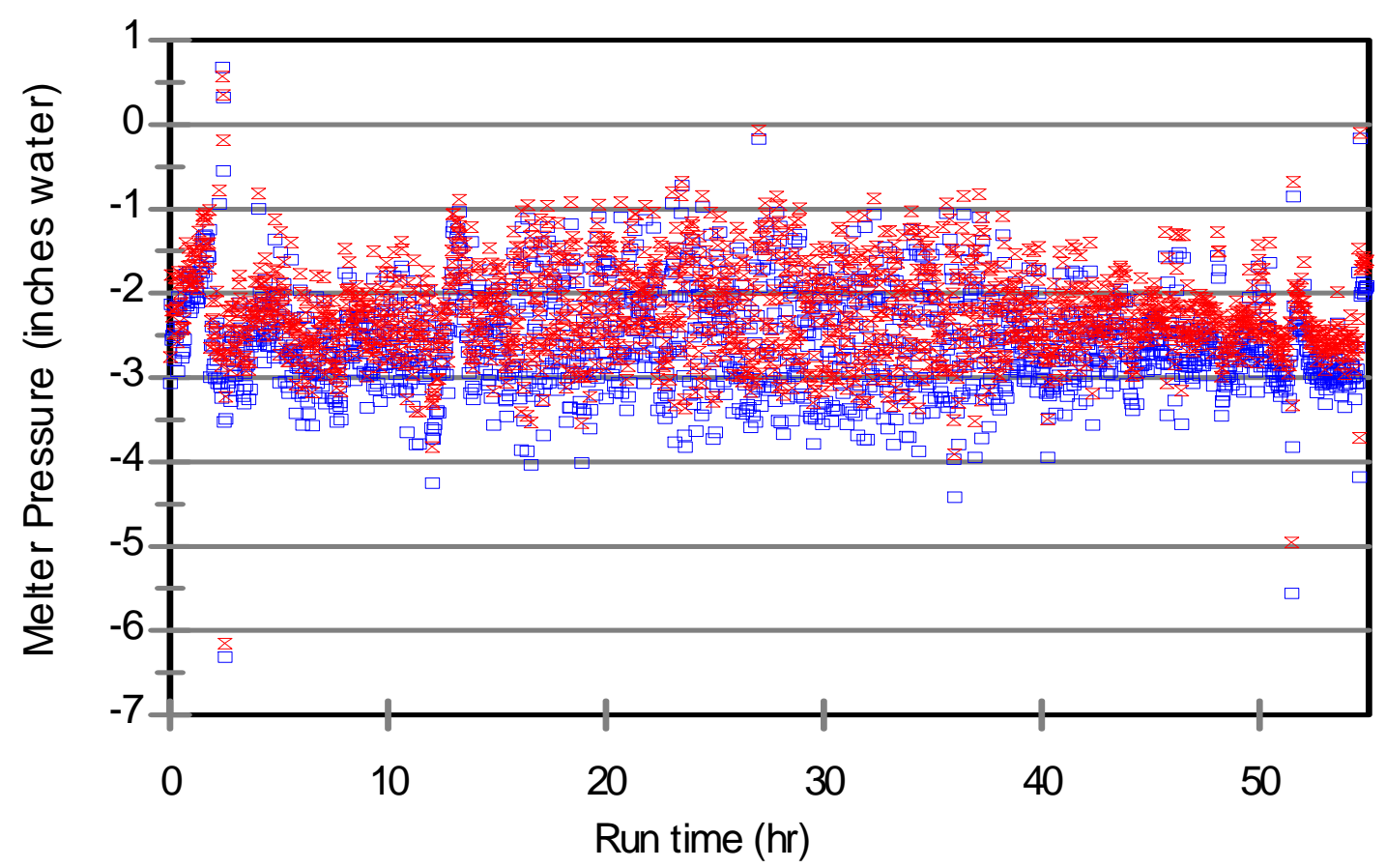

- @ instrument port

z@ level detector

Figure 4.5. Melter pressure while processing with two double-outlet bubblers, Test 1. 


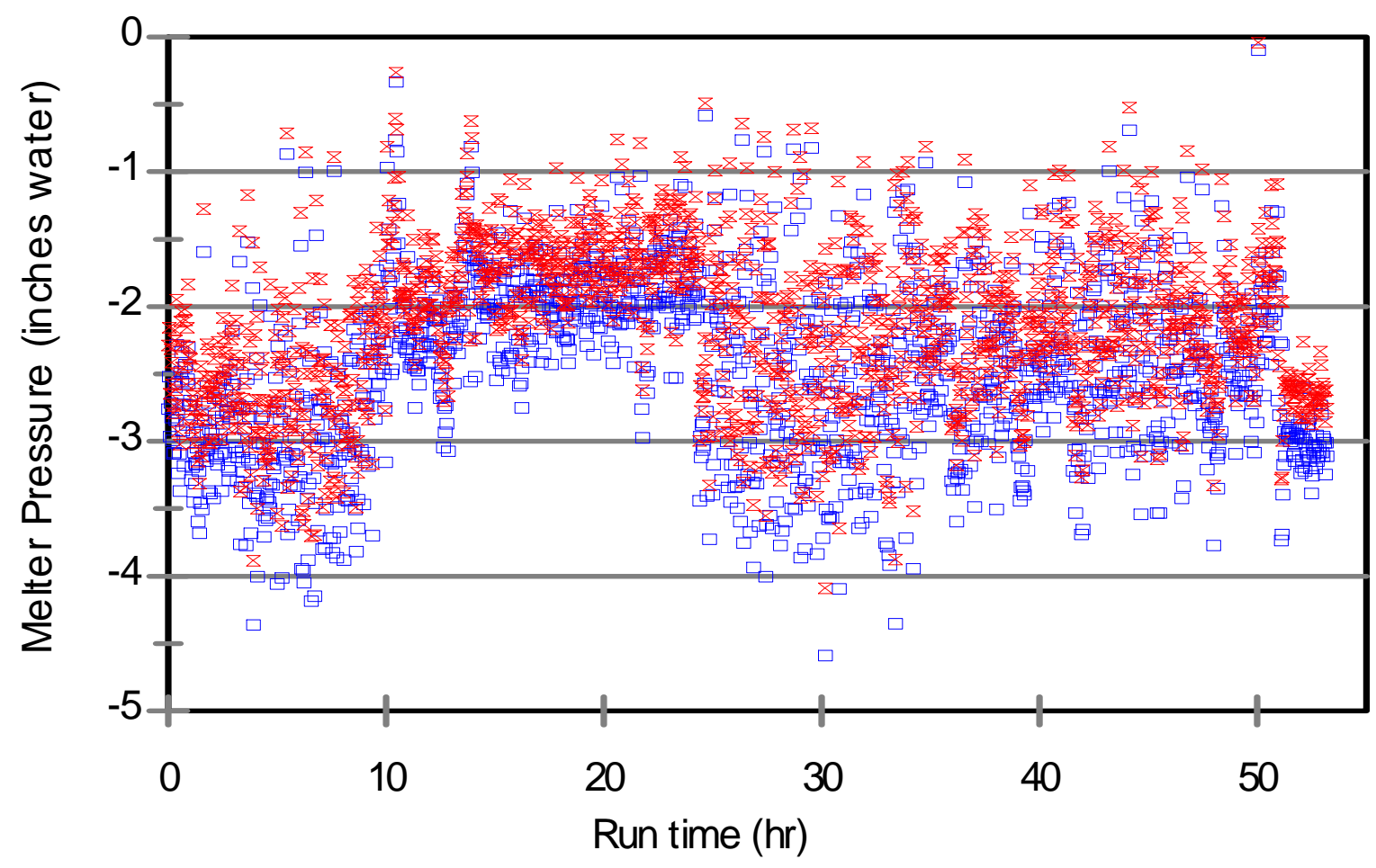

- @ instrument port

=@ level detector

Figure 4.6. Melter pressure while processing with two double-outlet and one single-outlet bubblers, Test 2. 


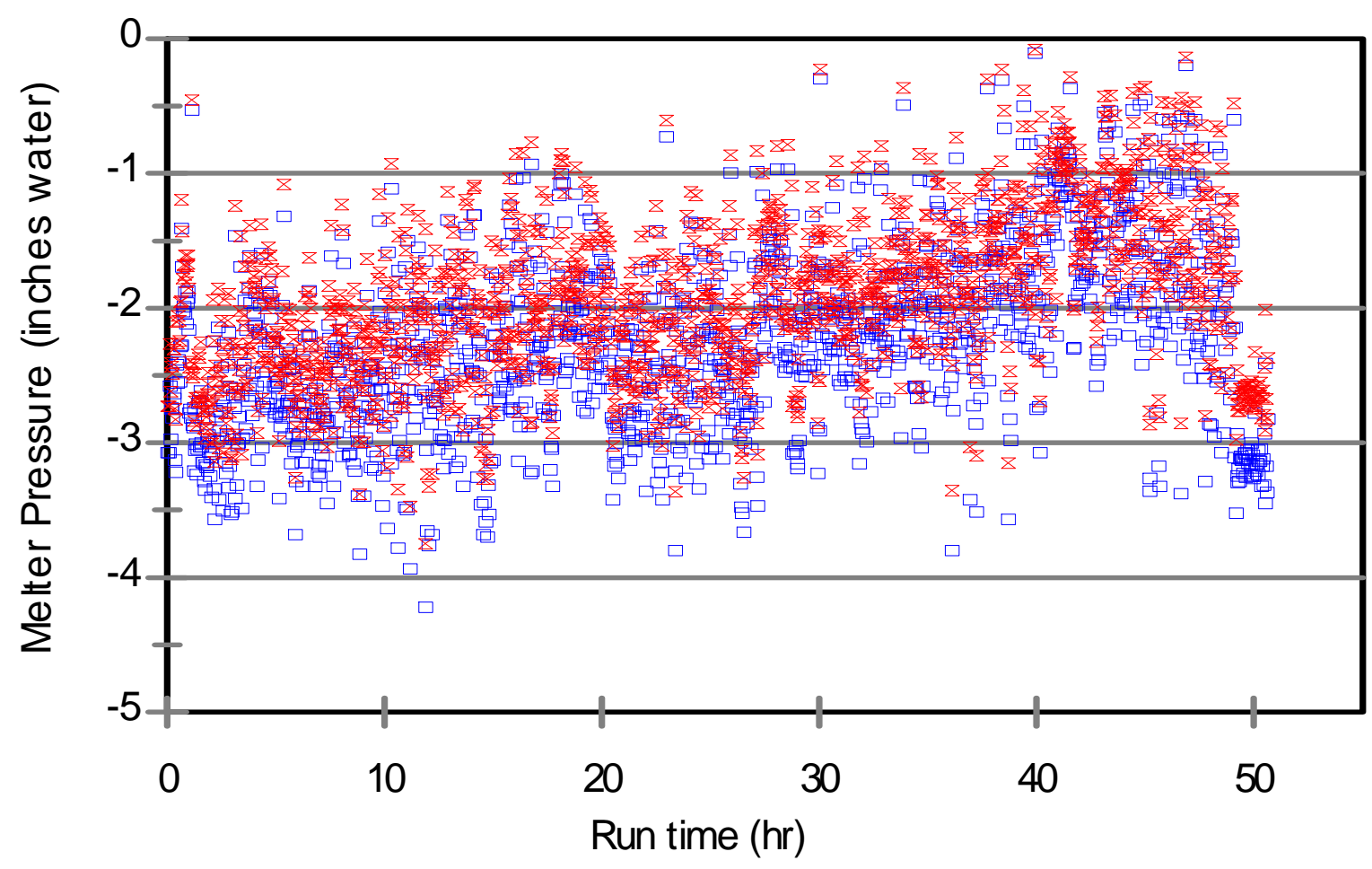

- @ instrument port

=@ level detector

Figure 4.7. Melter pressure while processing with two double-outlet and two single-outlet bubblers, Test 3. 


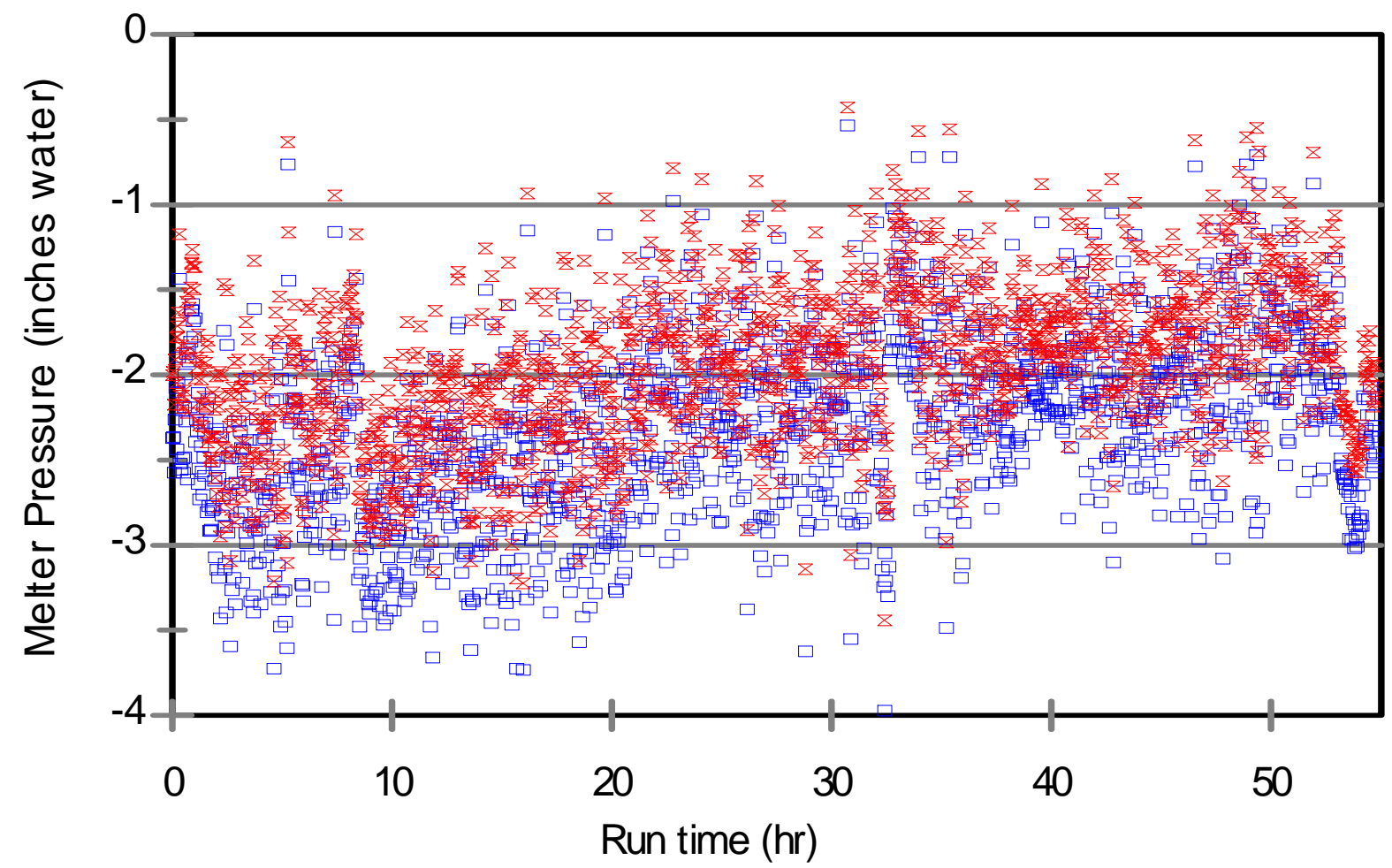

- @ instrument port

= @ level detector

Figure 4.8. Melter pressure while processing with two double-outlet and two single-outlet bubblers, Test 4. 


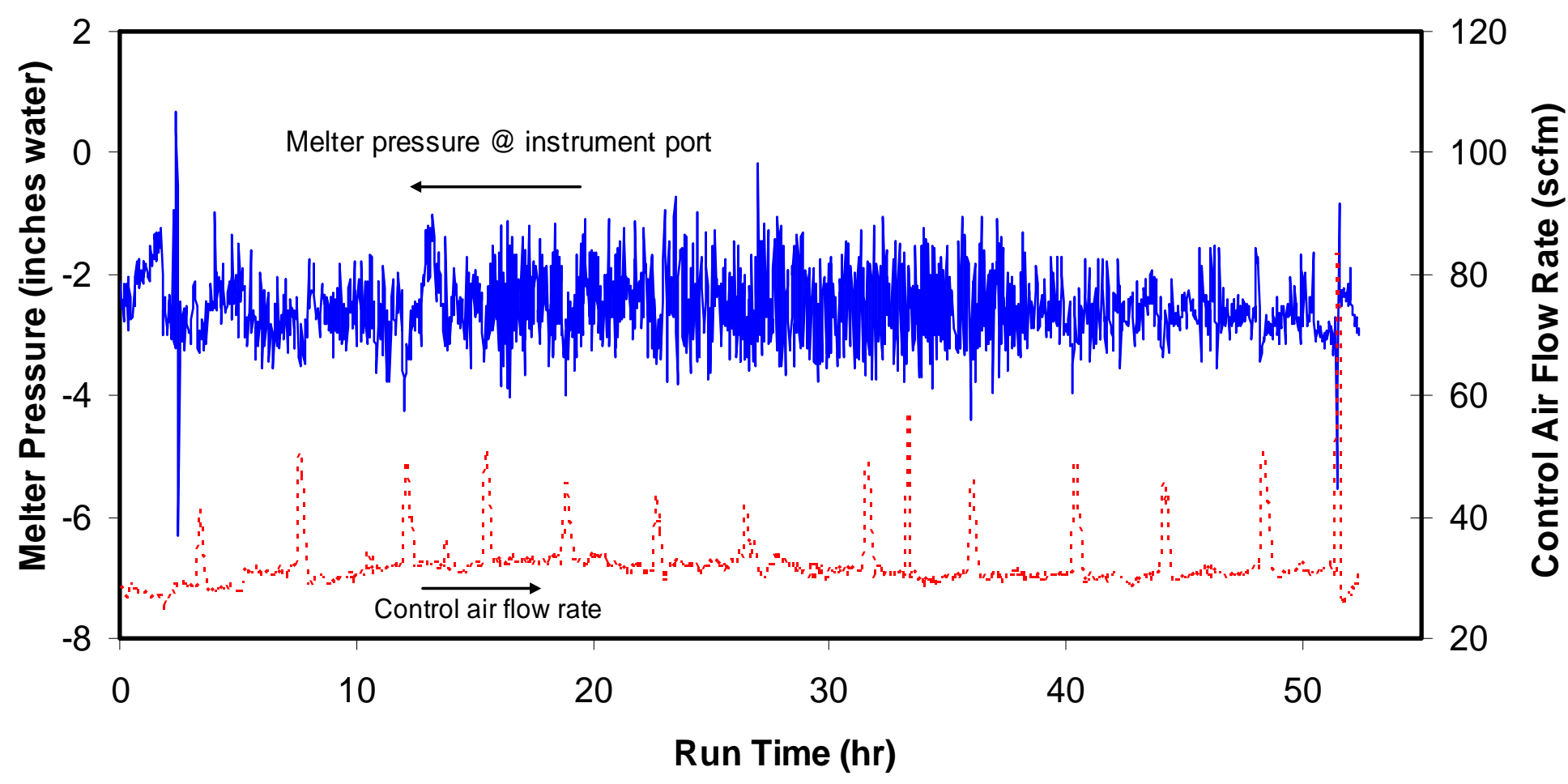

Figure 4.9. Melter pressure at instrument port and control air flow rate while processing with two double-outlet bubblers, Test 1. 


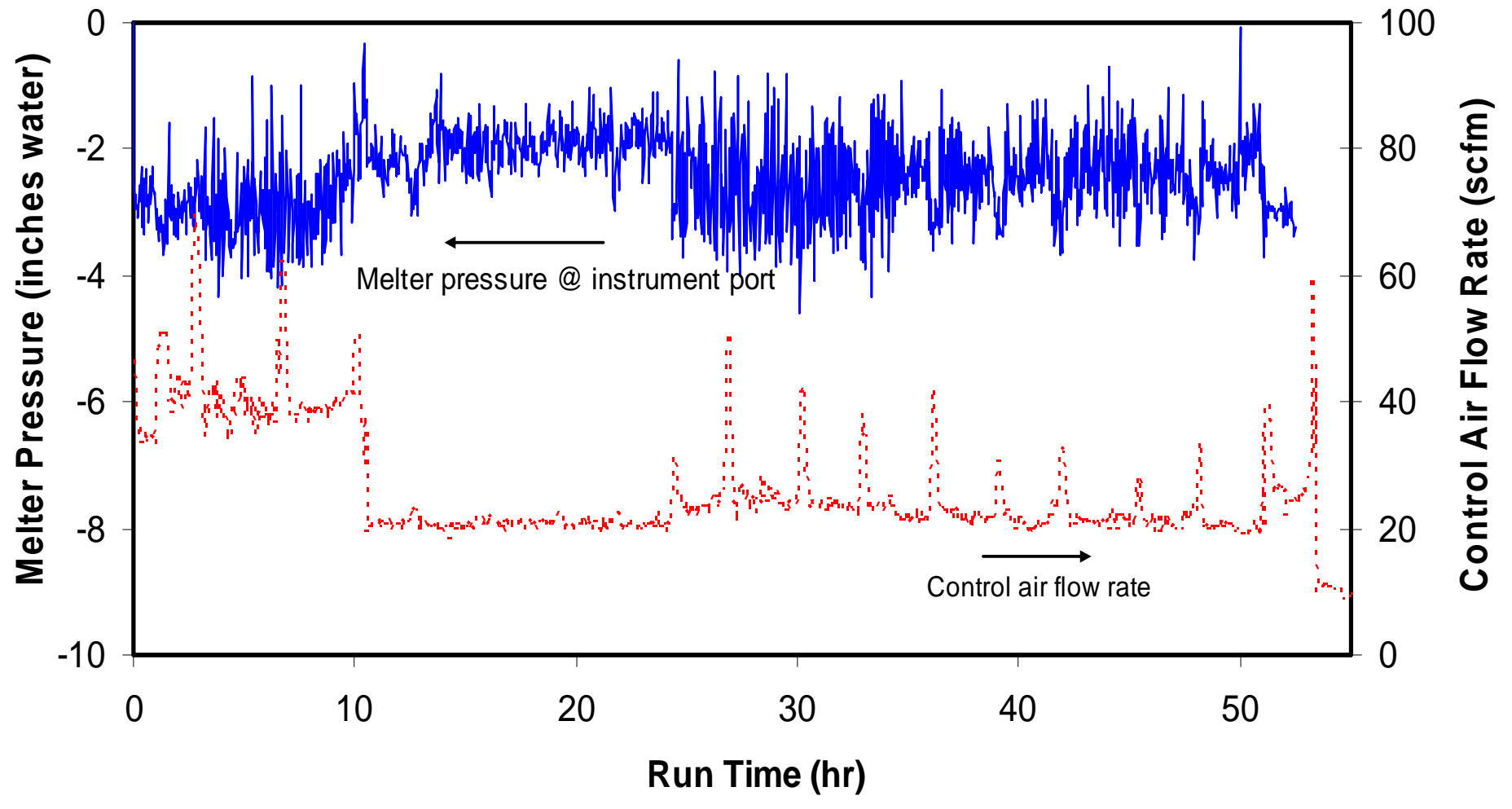

Figure 4.10. Melter pressure at instrument port and control air flow rate while processing with two double-outlet and one single-outlet bubblers, Test 2. 


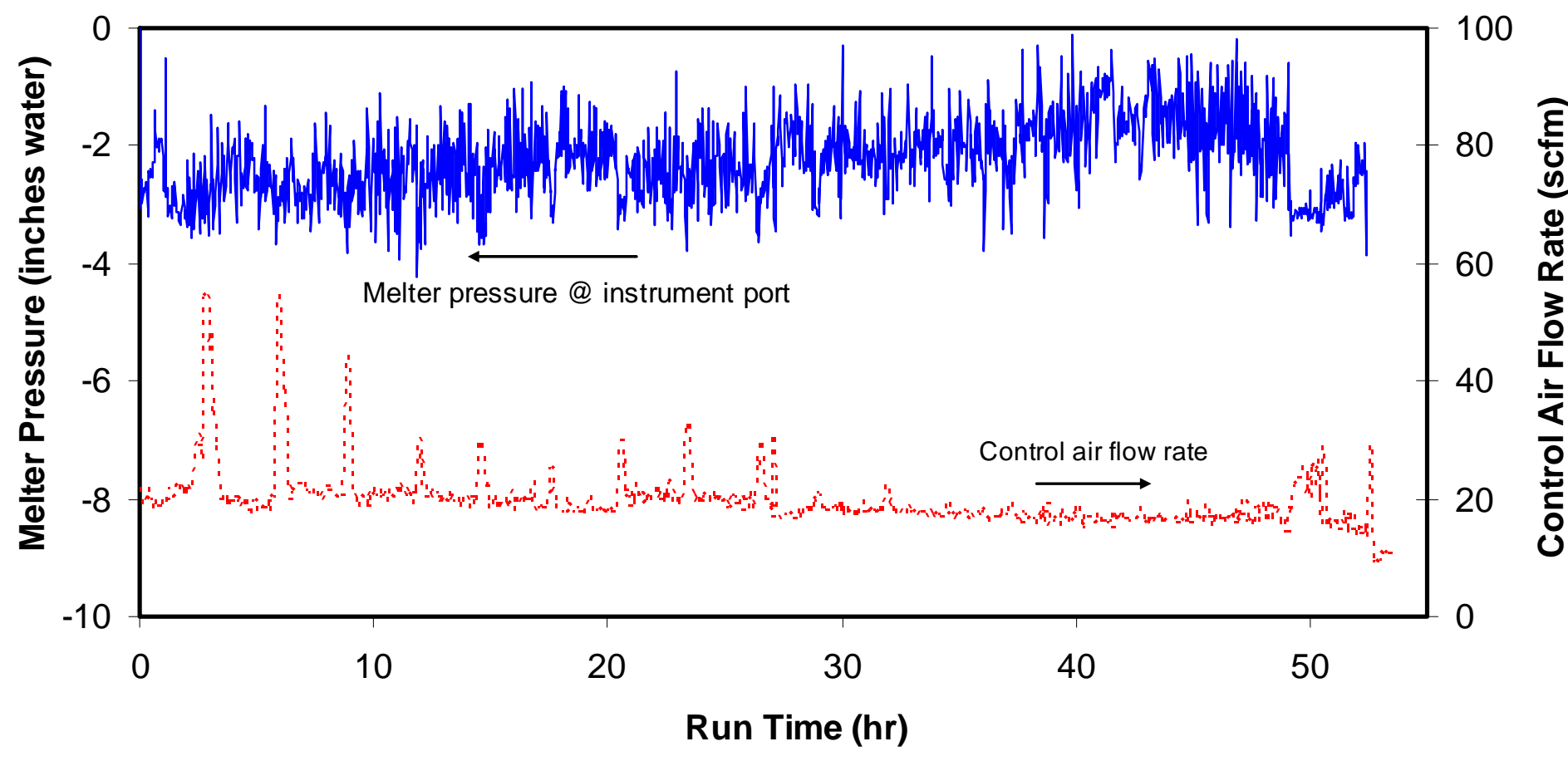

Figure 4.11. Melter pressure at instrument port and control air flow rate while processing with two double-outlet and two single-outlet bubblers, Test 3. 


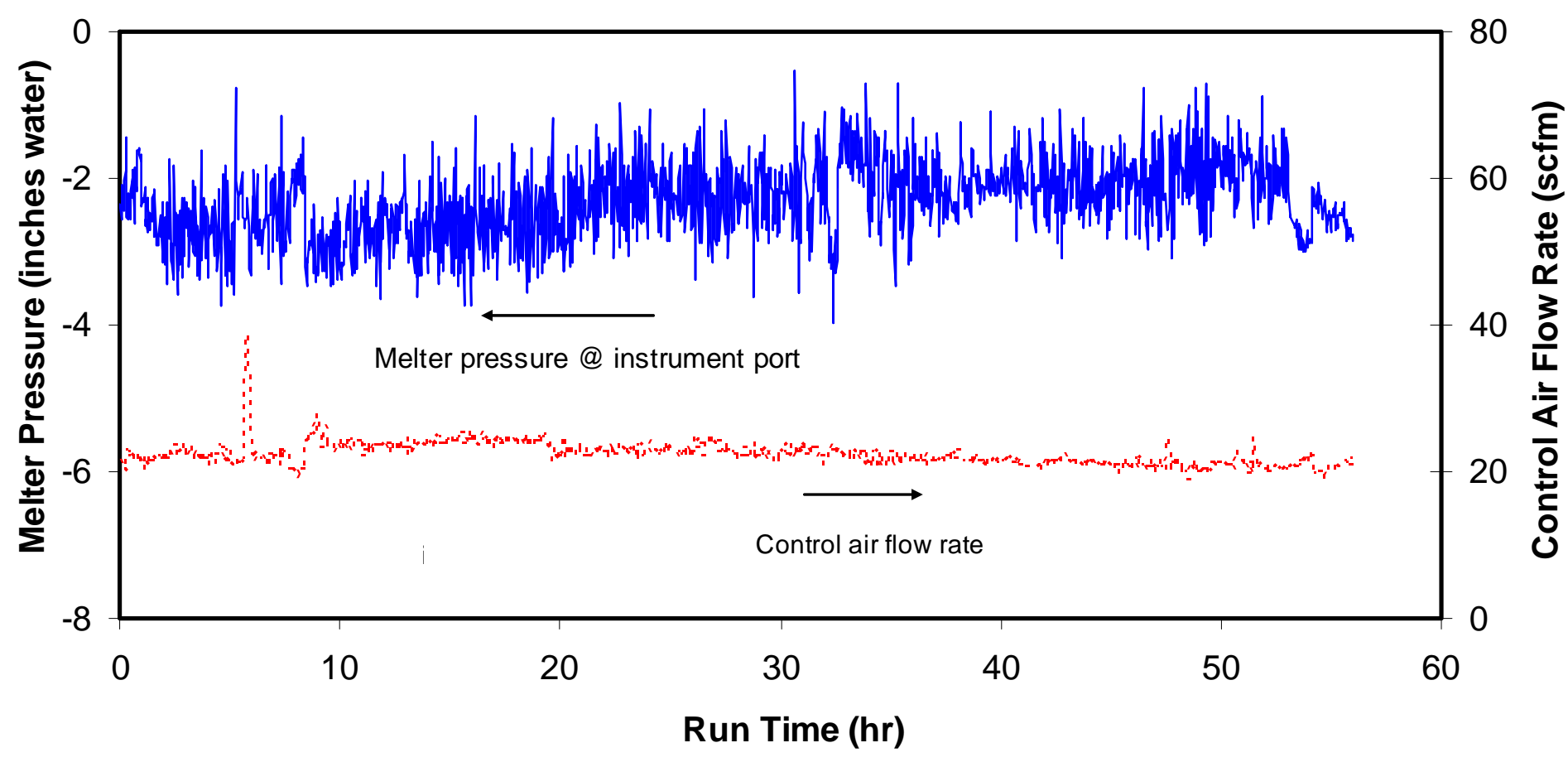

Figure 4.12. Melter pressure at instrument port and control air flow rate while processing with two double-outlet and two single-outlet bubblers, Test 4 . 


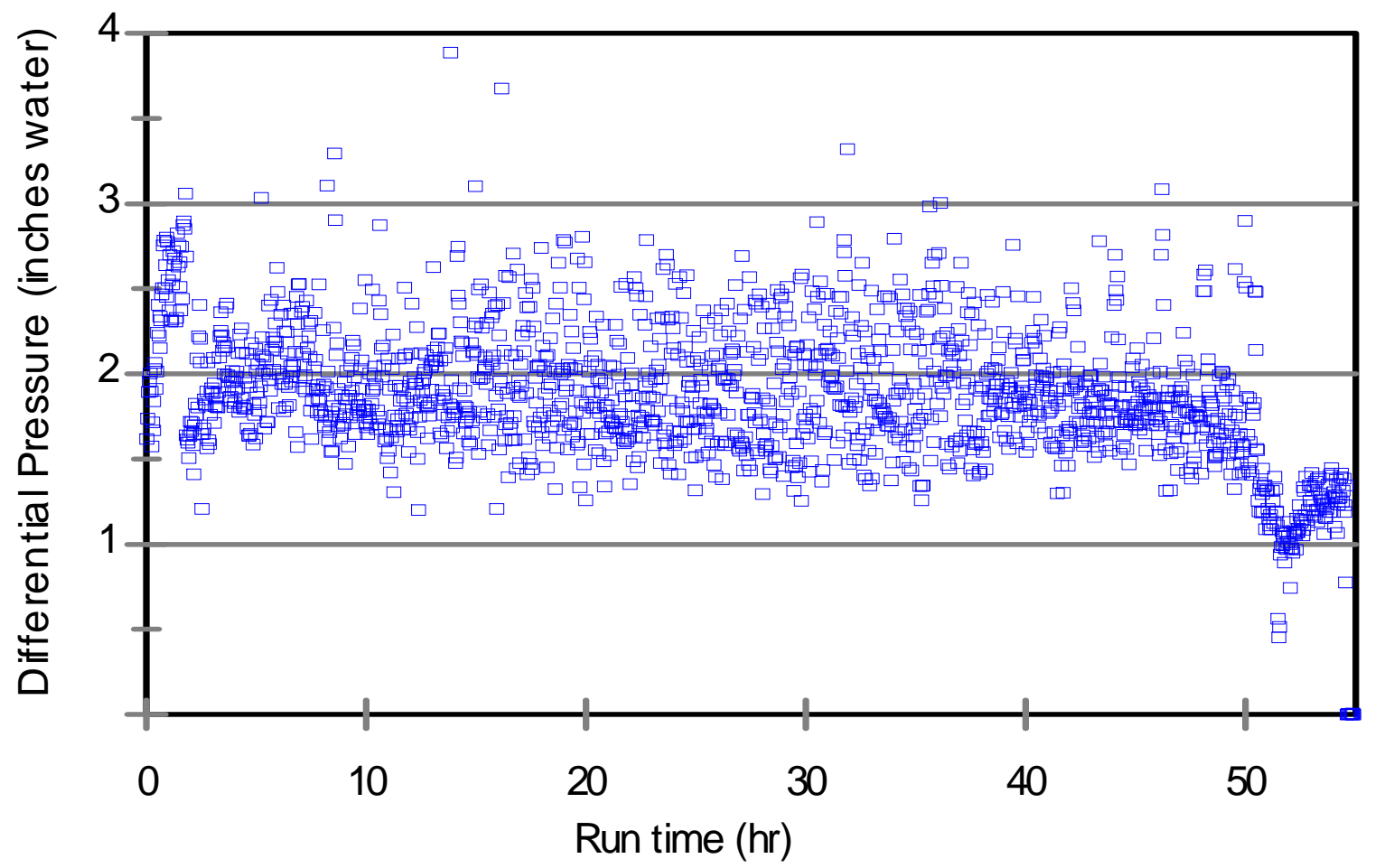

Figure 4.13. Differential pressure across the film cooler while processing with two double-outlet bubblers, Test 1. 


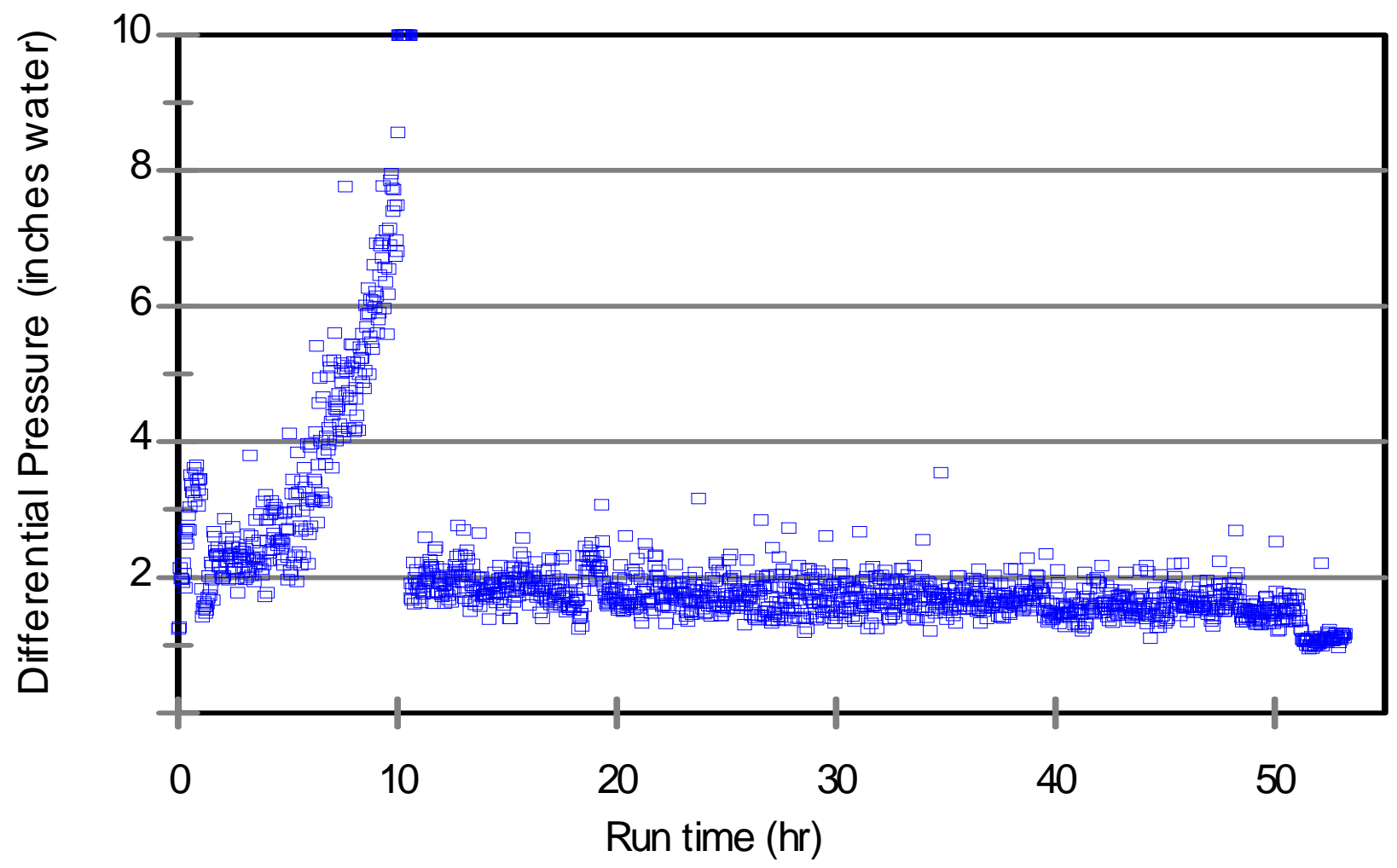

Figure 4.14. Differential pressure across the film cooler while processing with two double-outlet and one single-outlet bubblers. Test 2. 


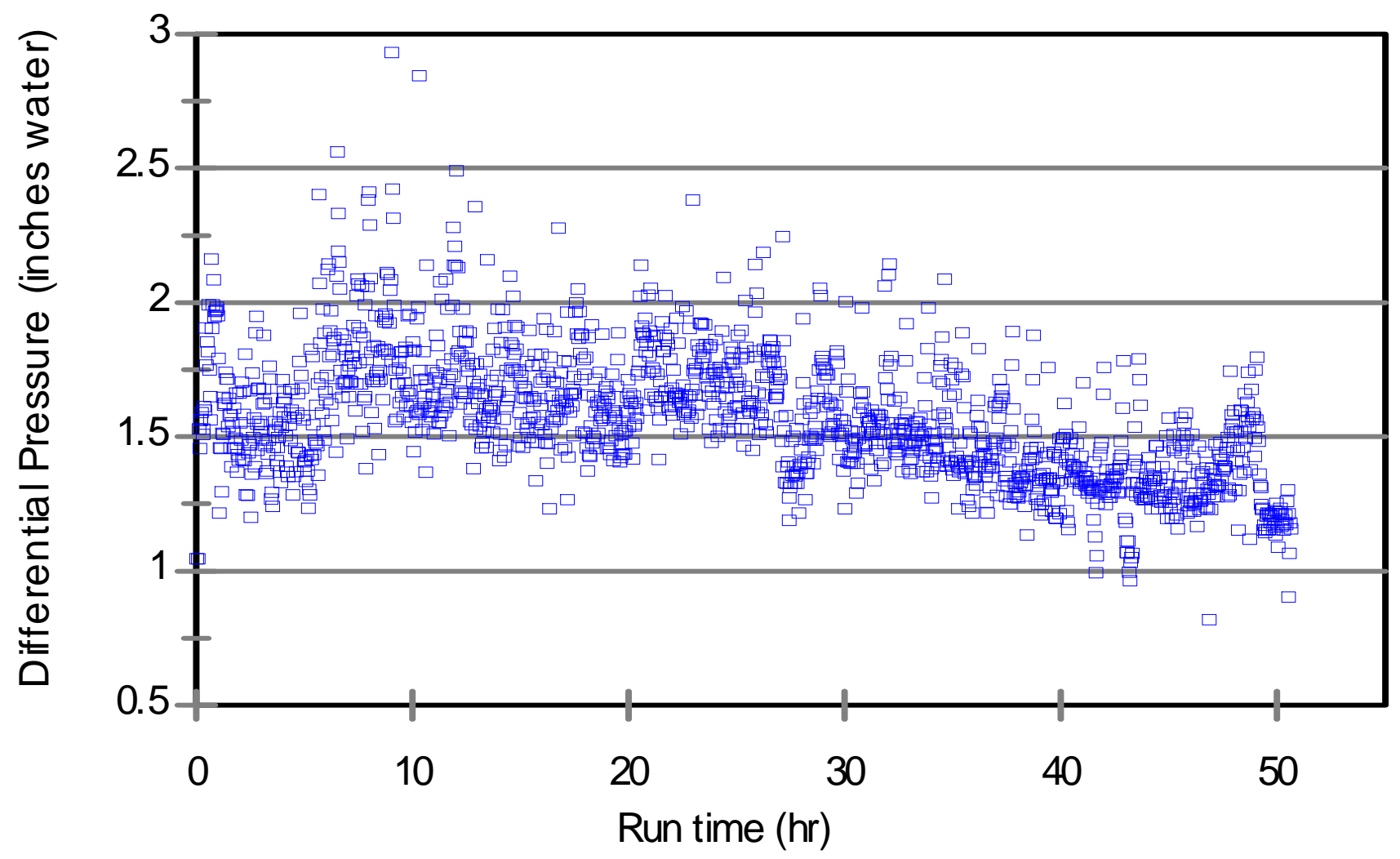

Figure 4.15. Differential pressure across the film cooler while processing with two double-outlet and two single-outlet bubblers. Test 3. 


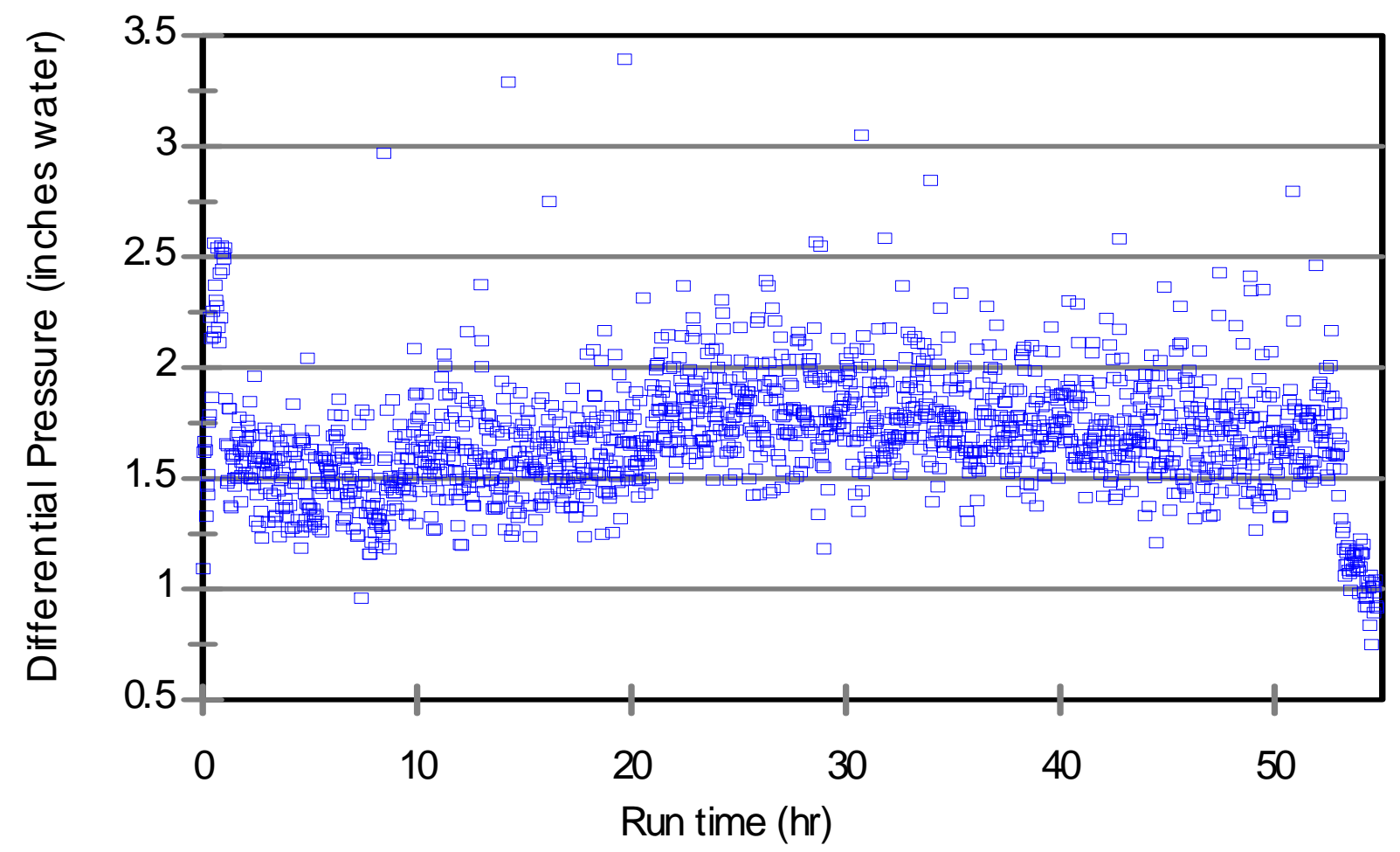

Figure 4.16. Differential pressure across the film cooler while processing with two double-outlet and two single-outlet bubblers. Test 4. 


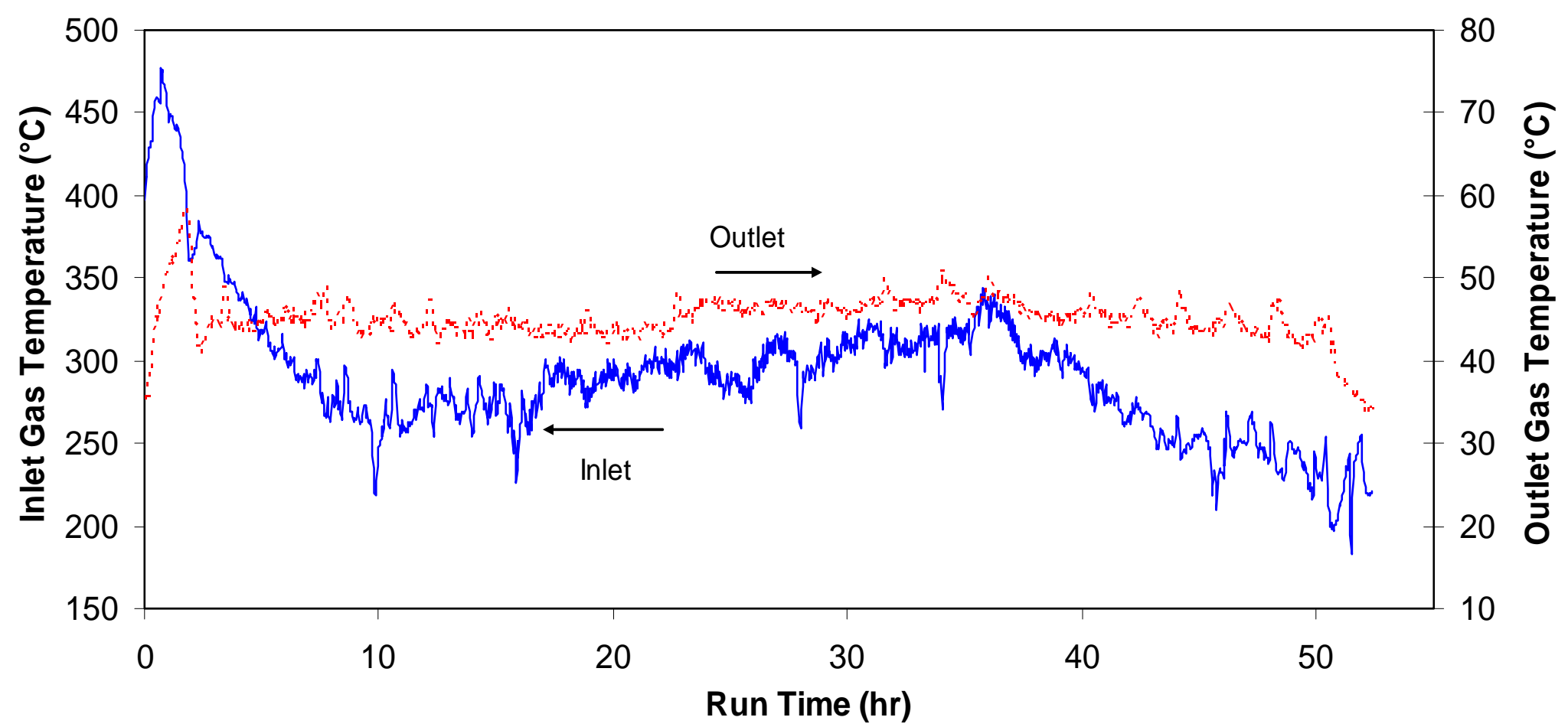

Figure 4.17. SBS inlet and outlet gas temperatures while processing with two double-outlet bubblers, Test 1. 


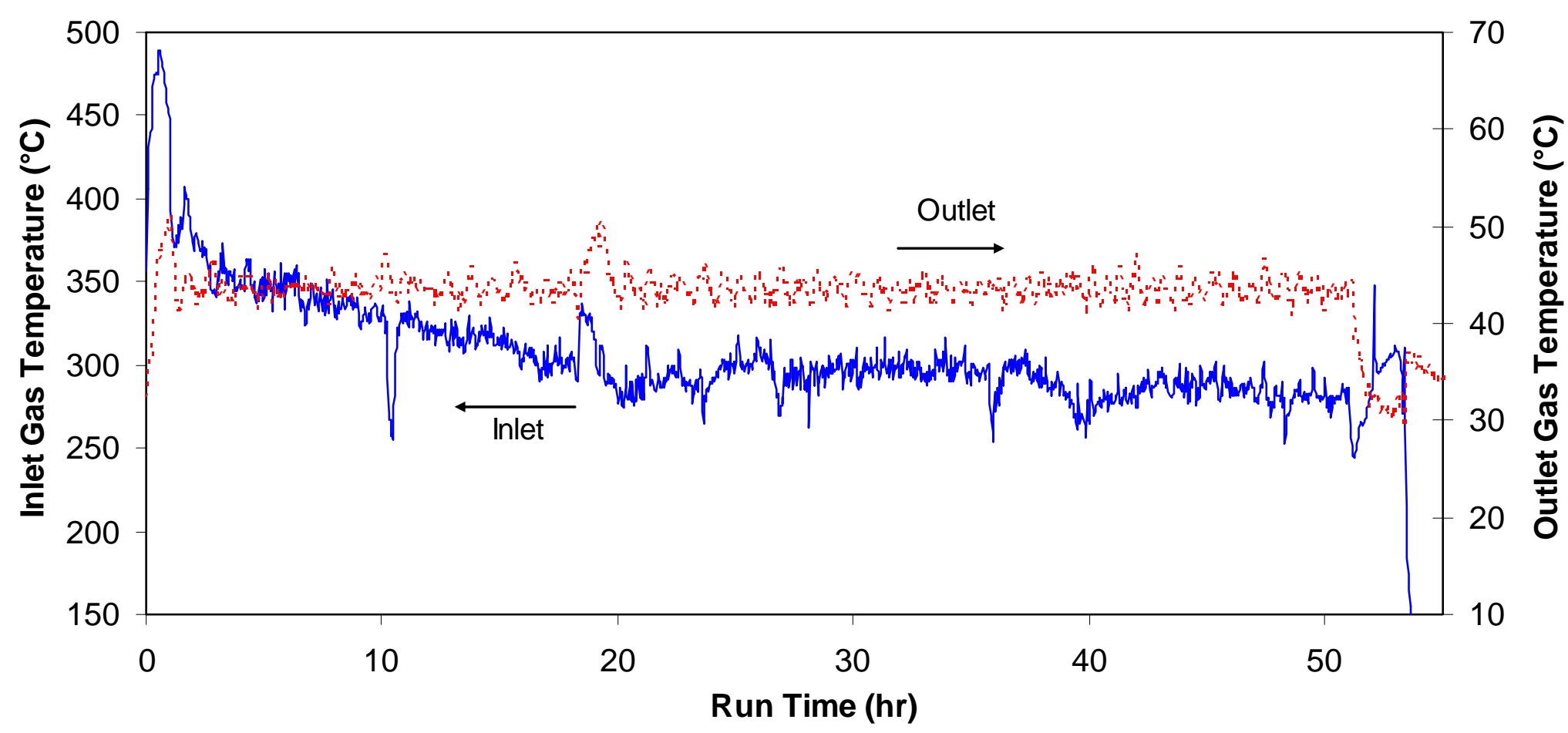

Figure 4.18. SBS inlet and outlet gas temperatures while processing with two double-outlet and one single-outlet bubblers, Test 2. 


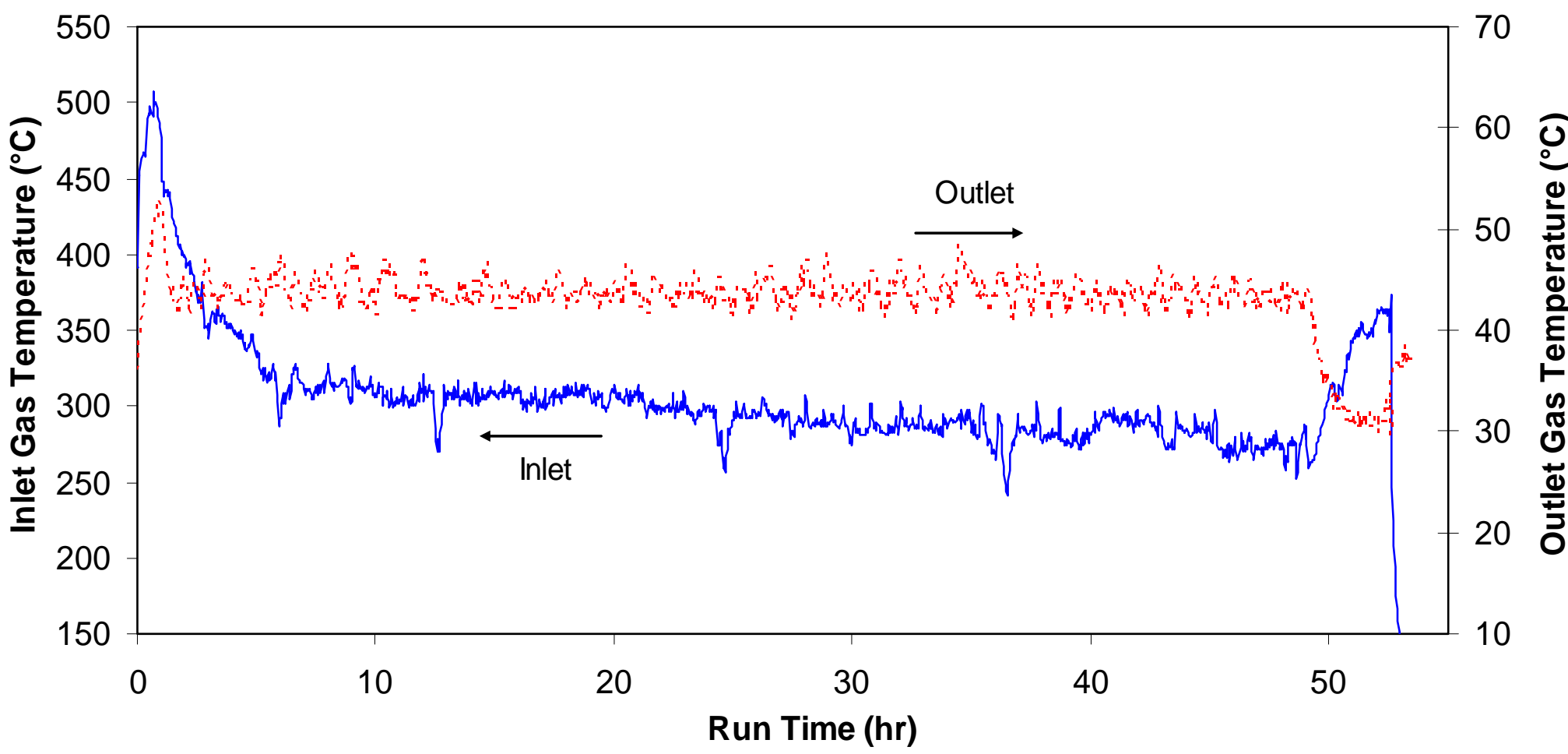

Figure 4.19. SBS inlet and outlet gas temperatures while processing with two double-outlet and two single-outlet bubblers, Test 3. 


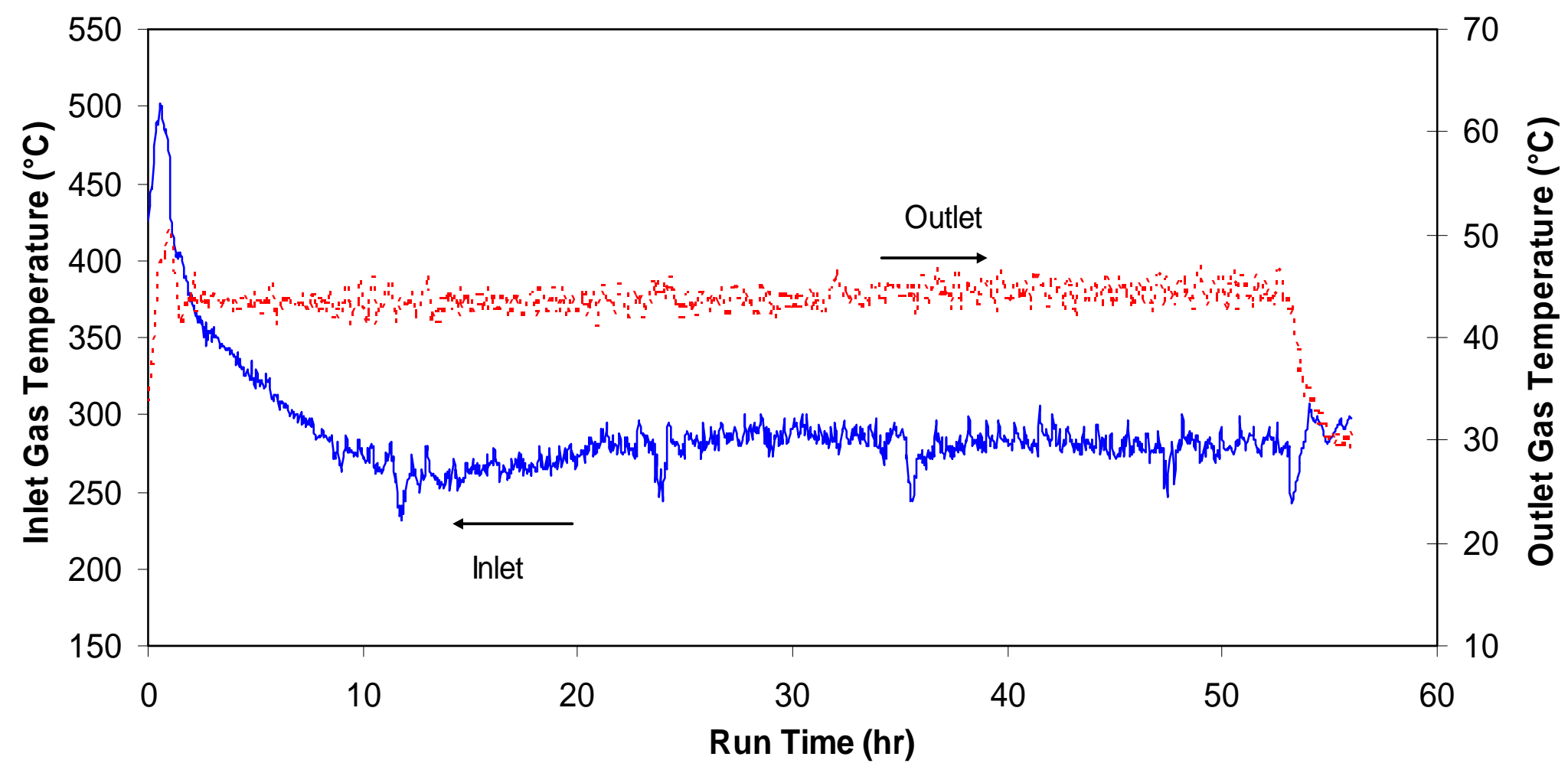

Figure 4.20. SBS inlet and outlet gas temperatures while processing with two double-outlet and two single-outlet bubblers, Test 4. 


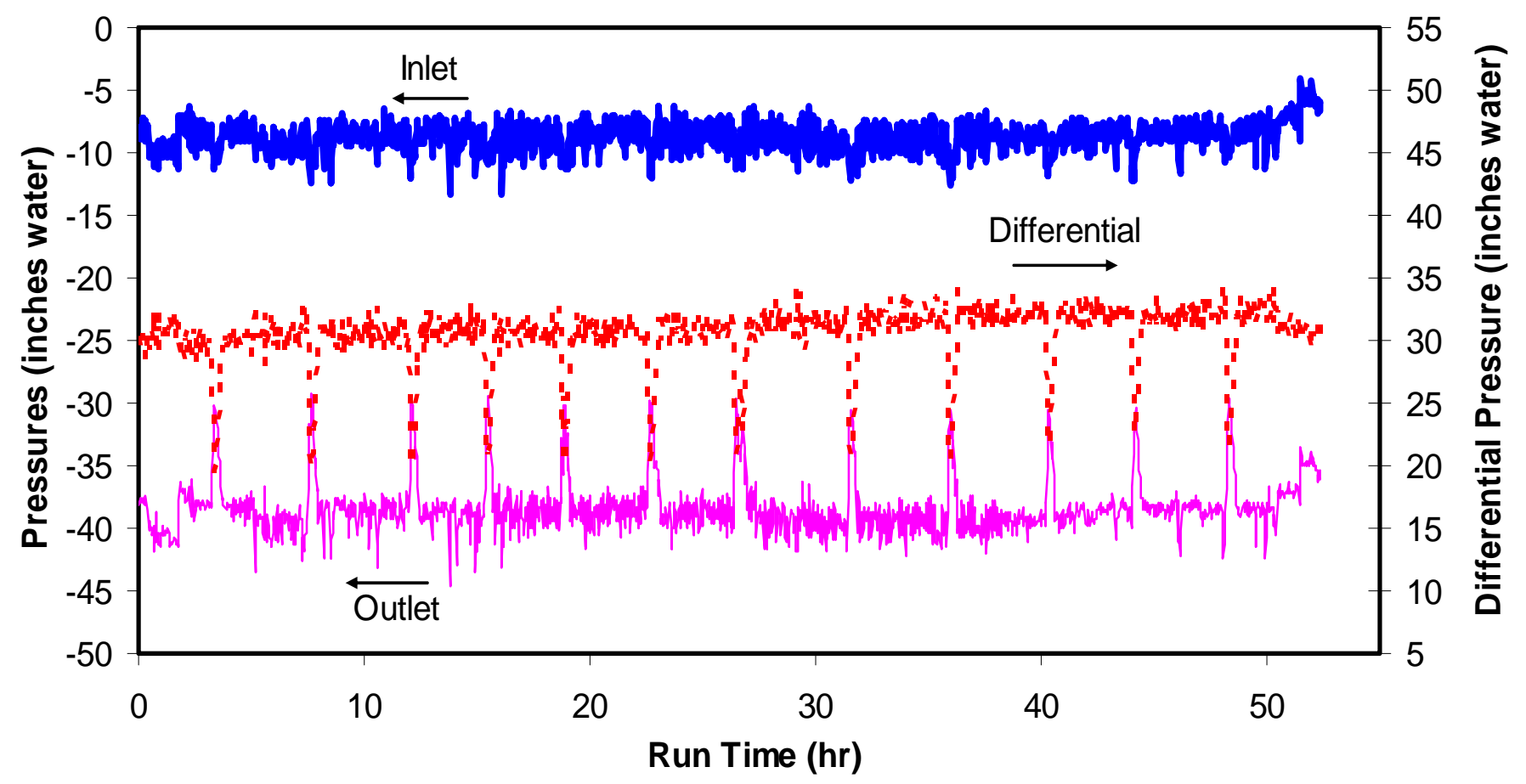

Figure 4.21. SBS inlet, outlet and differential pressures while processing with two double-outlet bubblers, Test 1. 


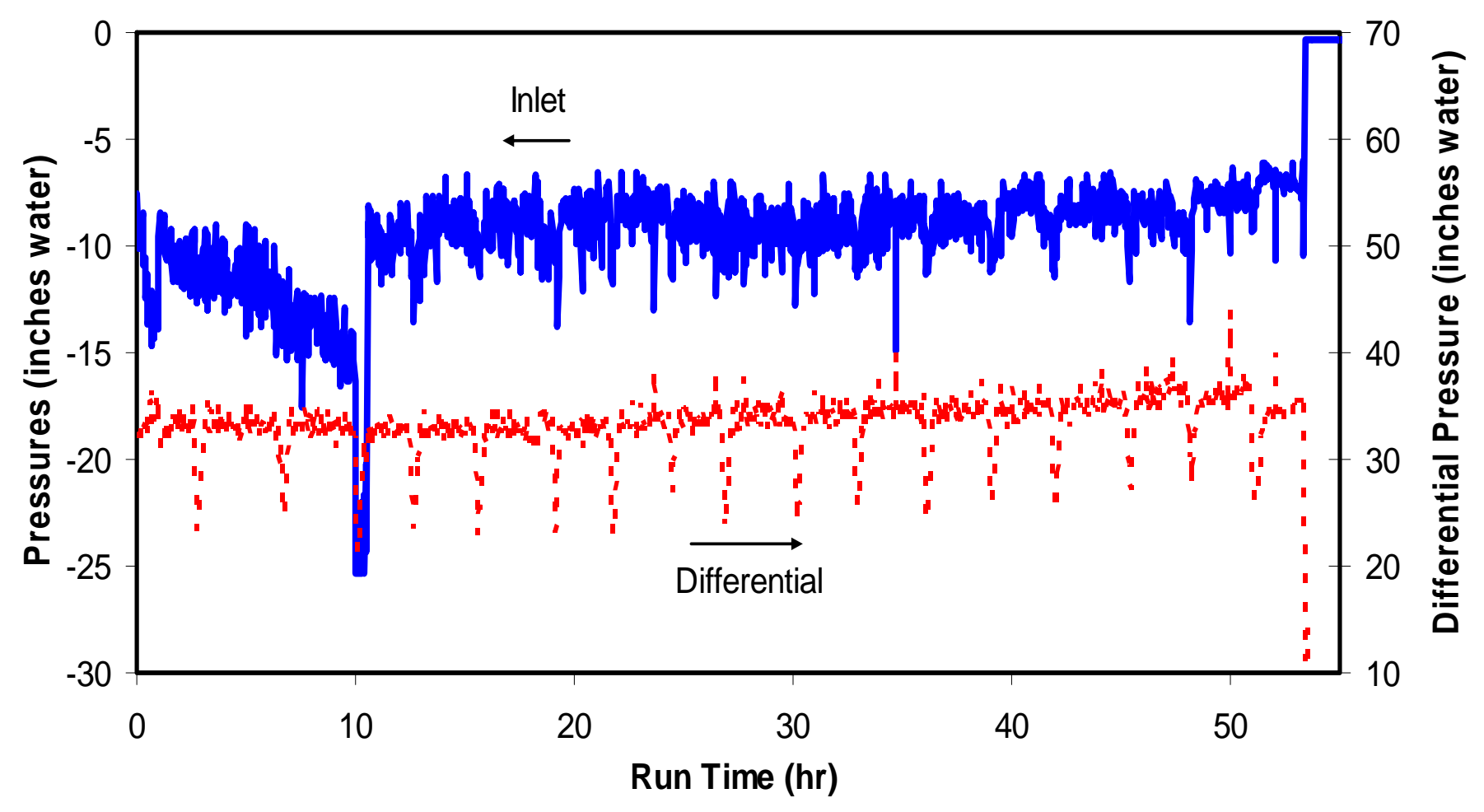

Figure 4.22. SBS inlet and differential pressures while processing with two double-outlet and one single-outlet bubblers, Test 2. 


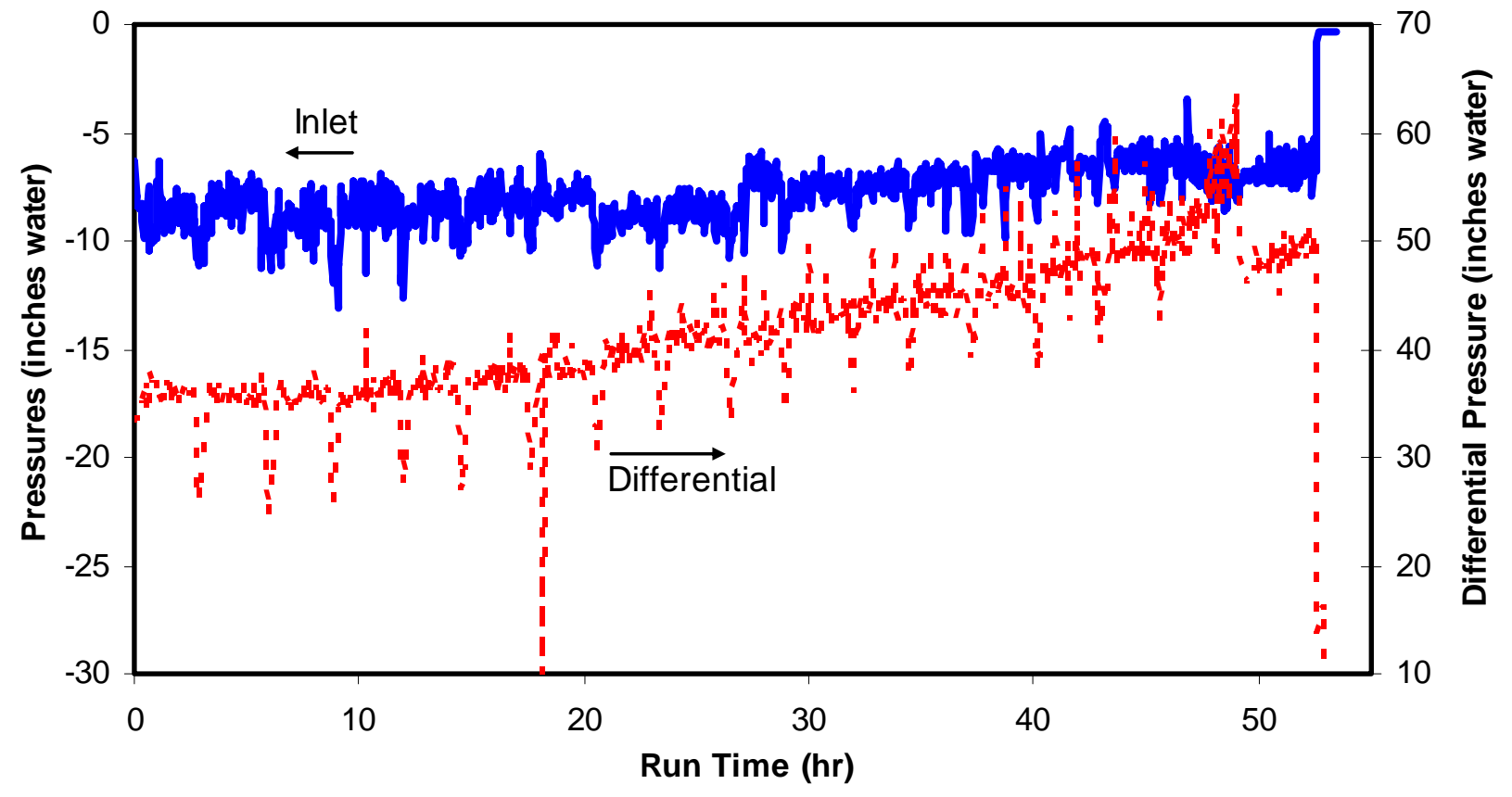

Figure 4.23. SBS inlet and differential pressures while processing with two double-outlet and two singleoutlet bubblers, Test 3. 


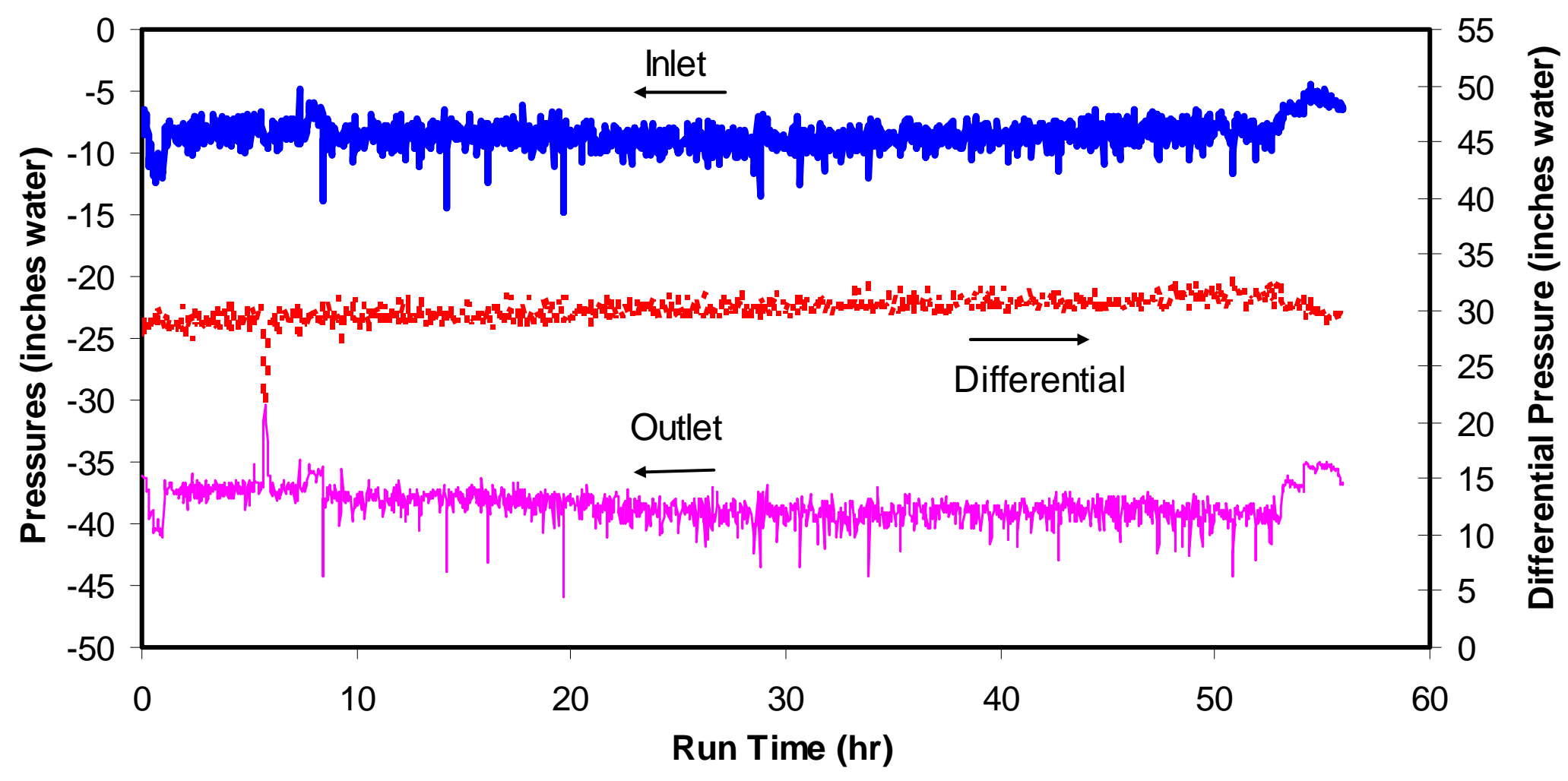

Figure 4.24. SBS inlet, outlet and differential pressures while processing with two double-outlet and two single-outlet bubblers, Test 4 . 


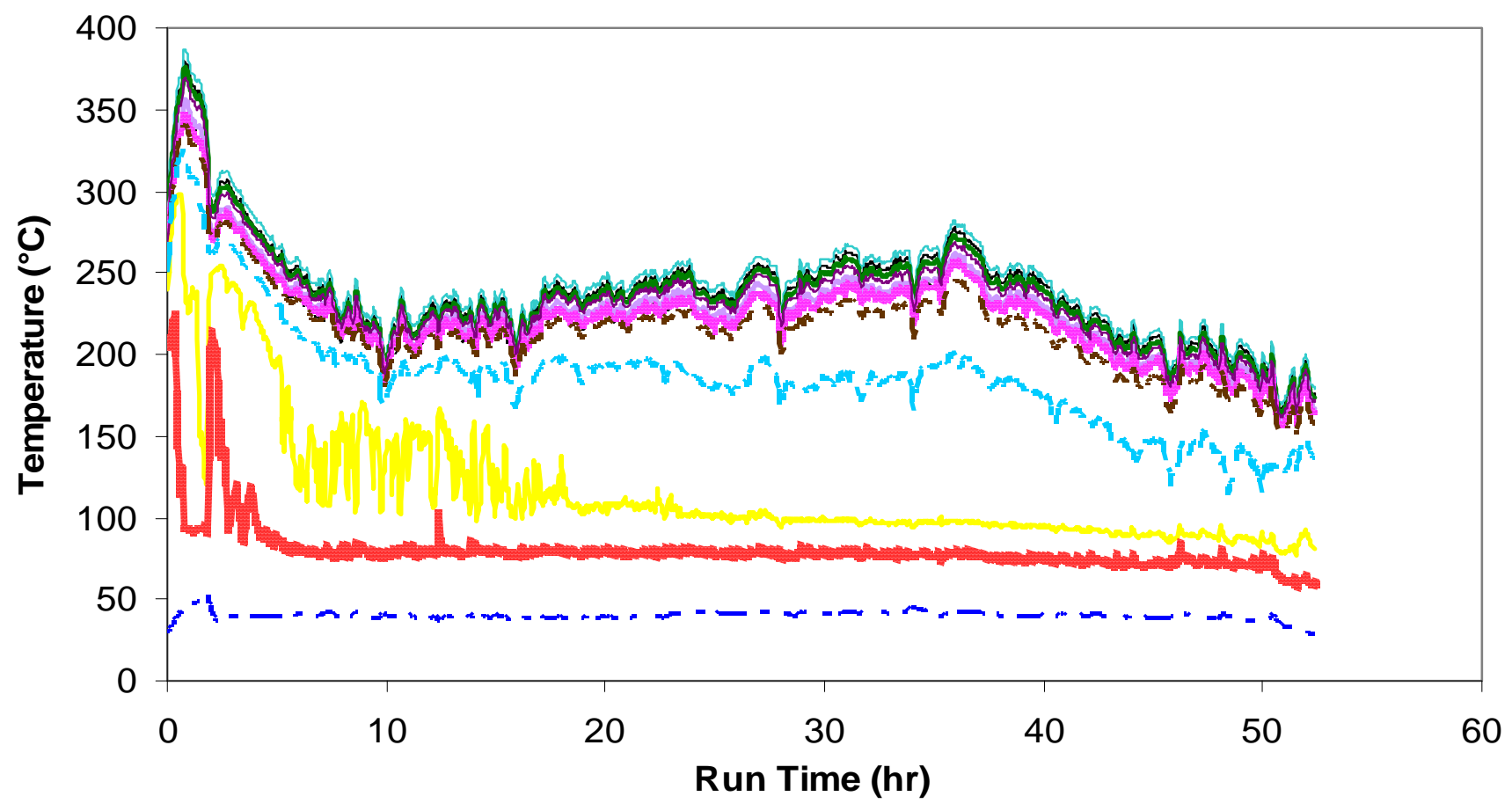

\begin{tabular}{|c|c|c|c|}
\hline ……@ 3 in. & - @ 8 in. & - @ 13 in. & - @ 18 in. \\
\hline$\ldots$ @ 53 in. & - - - SBS sump temp. & $— @ 23$ in. & $--@ 43$ in. \\
\hline
\end{tabular}

Figure 4.25. Off-gas temperatures in the SBS down-comer and sump water temperatures while processing with two double-outlet bubblers, Test 1. 


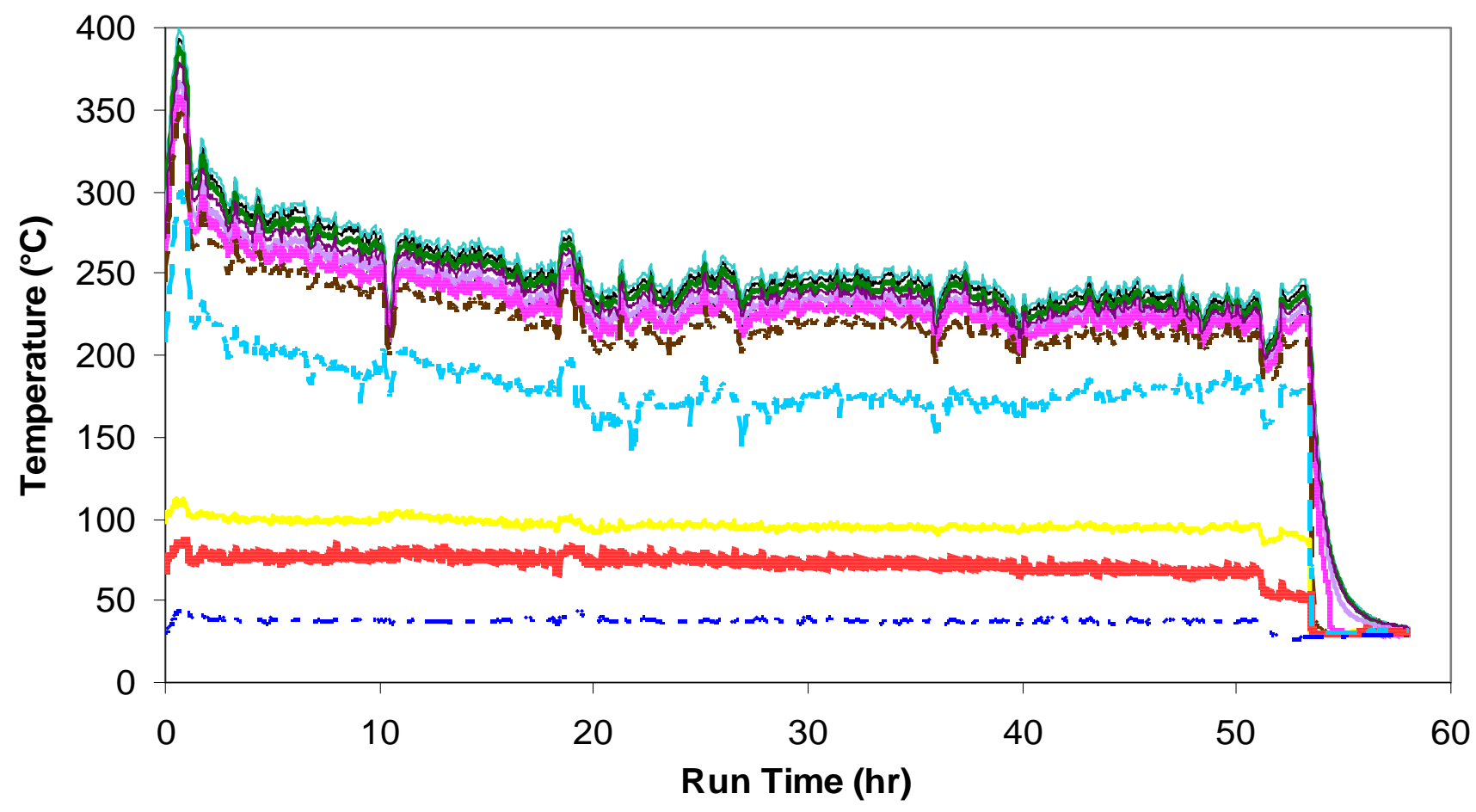

\begin{tabular}{|c|c|c|c|}
\hline $\begin{array}{l}\cdots \cdots \cdot 3 \text { in. } \\
-28 \text { in. }\end{array}$ & $\begin{array}{l}-@ 8 \text { in. } \\
-@ 33 \text { in. }\end{array}$ & $\begin{array}{r}-@ 13 \text { in. } \\
--@ 38 \text { in. }\end{array}$ & $\begin{array}{r}\longrightarrow 18 \text { in. } \\
@ 48 \text { in. }\end{array}$ \\
\hline - @ 53 in. & - - SBS sump temp. & $\longrightarrow @ 23$ in. & $--@ 43$ in. \\
\hline
\end{tabular}

Figure 4.26. Off-gas temperatures in the SBS down-comer and sump water temperatures while processing with two double-outlet and one single-outlet bubblers, Test 2. 


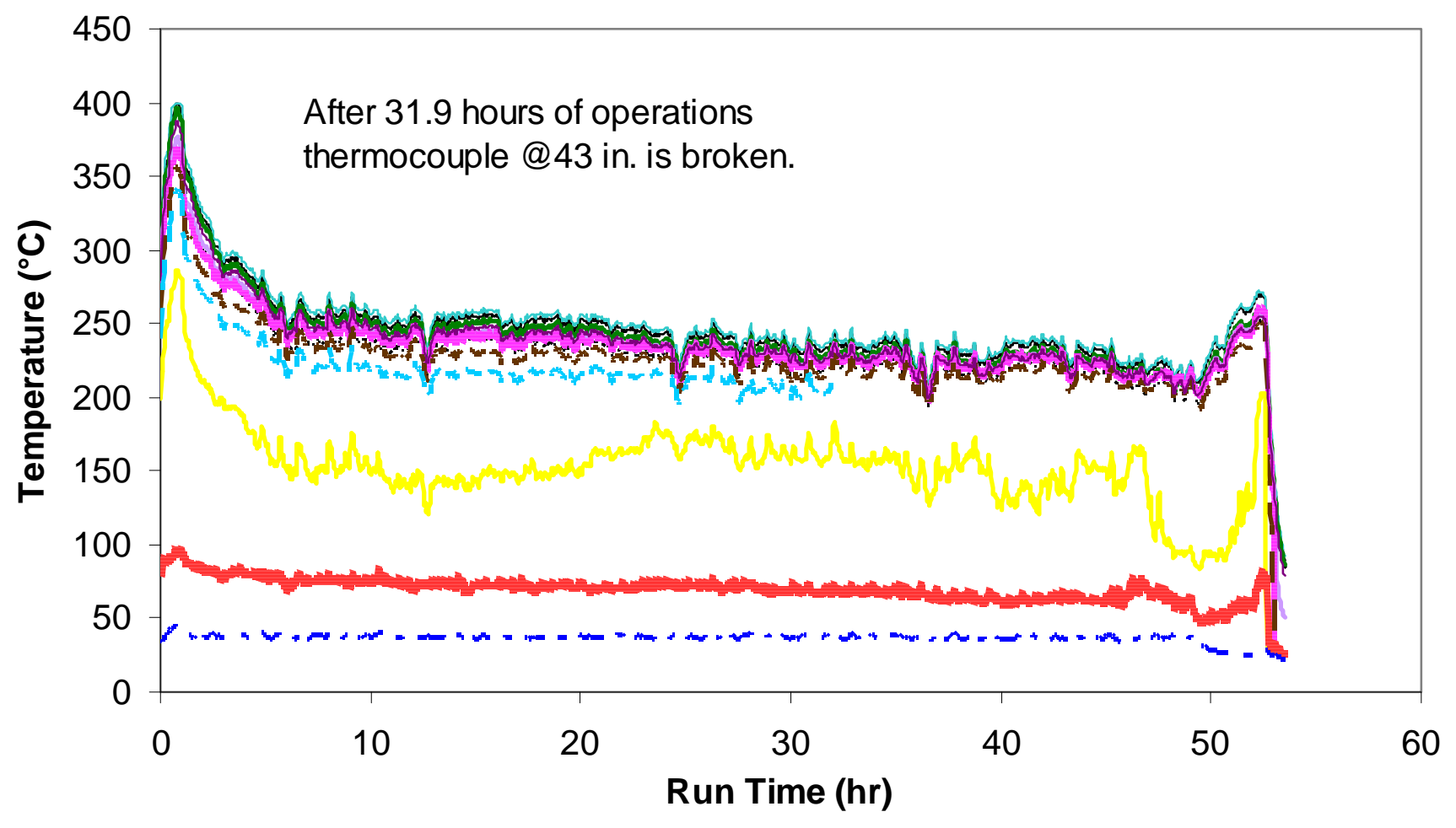

\begin{tabular}{|c|c|c|c|}
\hline .......@ 3 in. & — @ 8 in. & $— @ 13$ in. & $-@ 18$ in. \\
\hline$\longrightarrow @ 28$ in. & — @ 33 in. & $--@ 38$ in. & @ 48 in. \\
\hline =@ 53 in. & - - - SBS sump temp. & — @ 23 in. & $--@ 43$ in. \\
\hline
\end{tabular}

Figure 4.27. Off-gas temperatures in the SBS down-comer and sump water temperatures while processing with two double-outlet and two single-outlet bubblers, Test 3. 


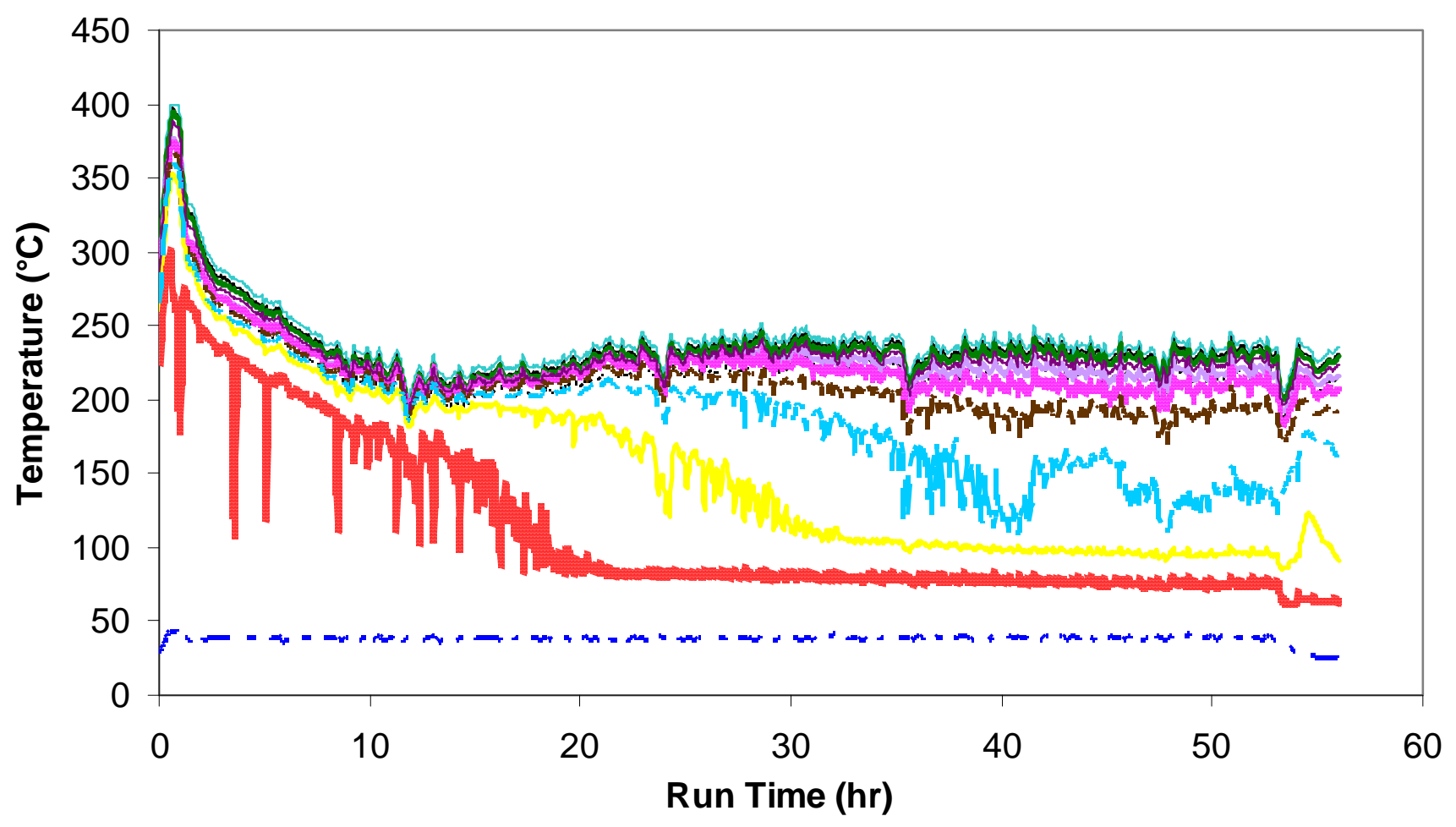

\begin{tabular}{|c|c|c|c|}
\hline .......@ 3 in. & — 08 in. & $— @ 13$ in. & — 18 in. \\
\hline$\longrightarrow @ 28$ in. & —@ 33 in. & $--@ 38$ in. & @ 48 in. \\
\hline —@ 53 in. & - - - SBS sump temp. & $-@ 23$ in. & $--@ 43$ in. \\
\hline
\end{tabular}

Figure 4.28. Off-gas temperatures in the SBS down-comer and sump water temperatures while processing with two double-outlet and two single-outlet bubblers, Test 4. 


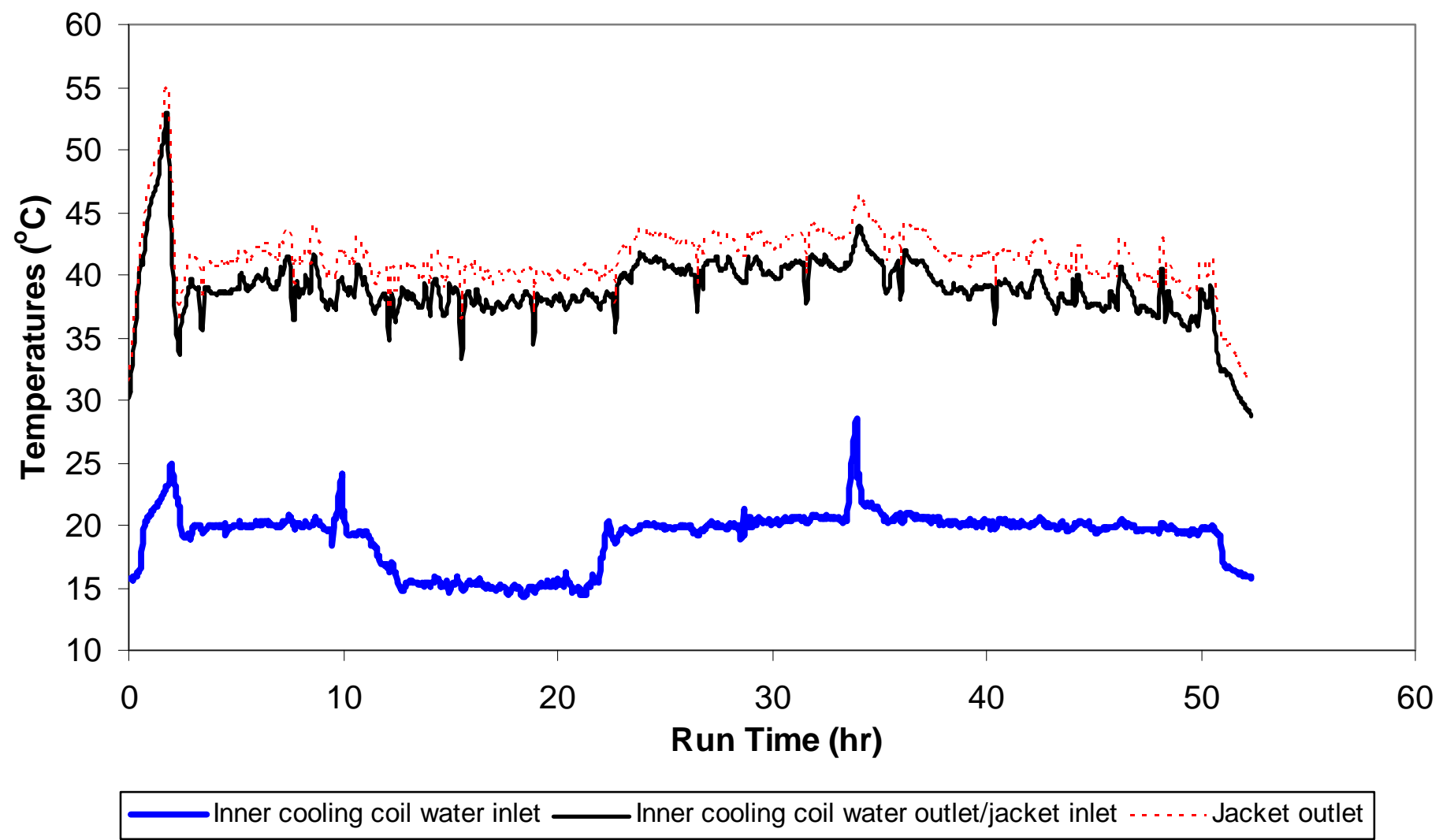

Figure 4.29. SBS cooling coil inlet, cooling coil outlet/jacket inlet and jacket outlet water temperatures while processing with two double-outlet bubblers, Test 1. 


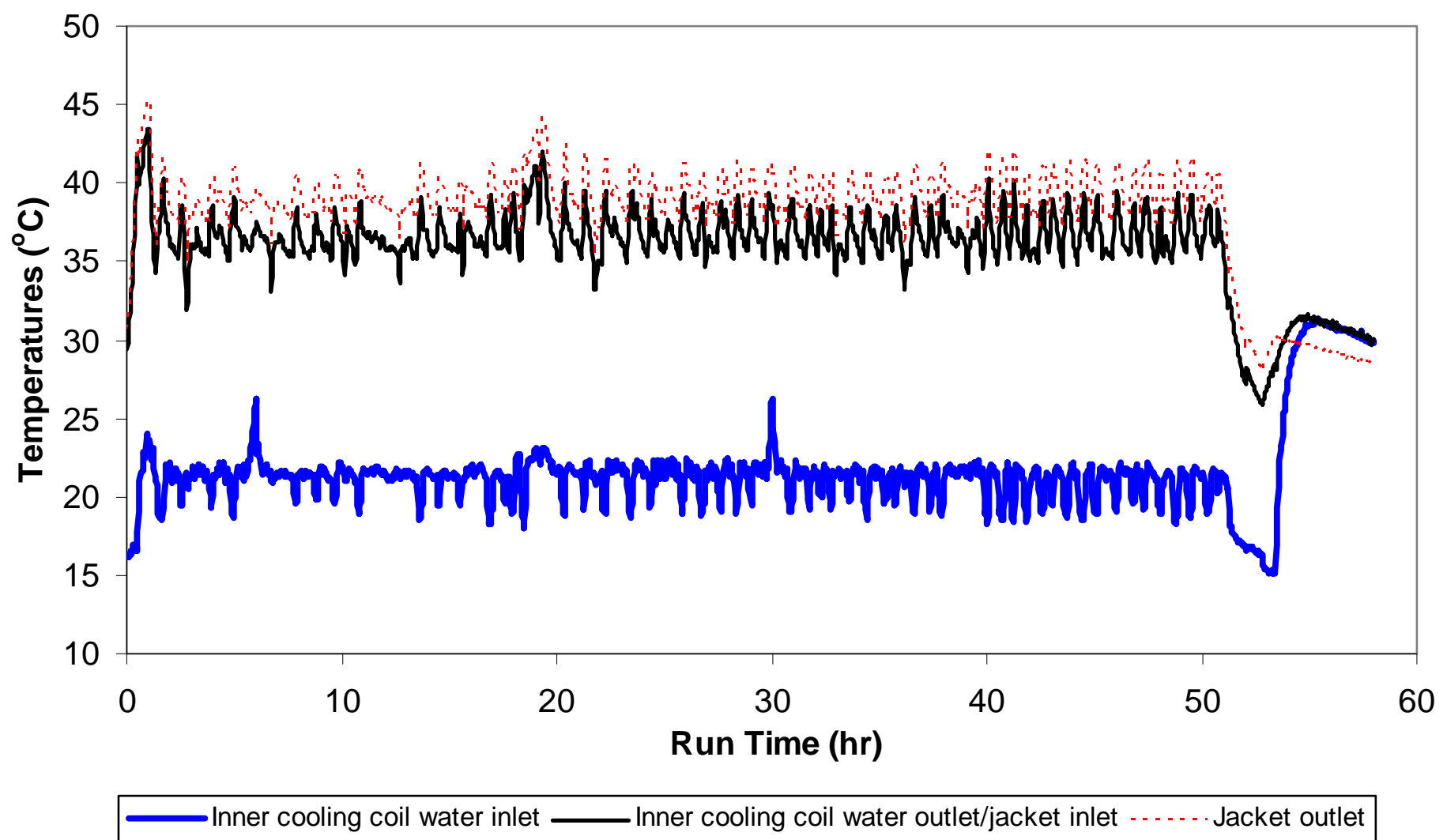

Figure 4.30. SBS cooling coil inlet, cooling coil outlet/jacket inlet and jacket outlet water temperatures while processing with two double-outlet and one single-outlet bubblers, Test 2. 


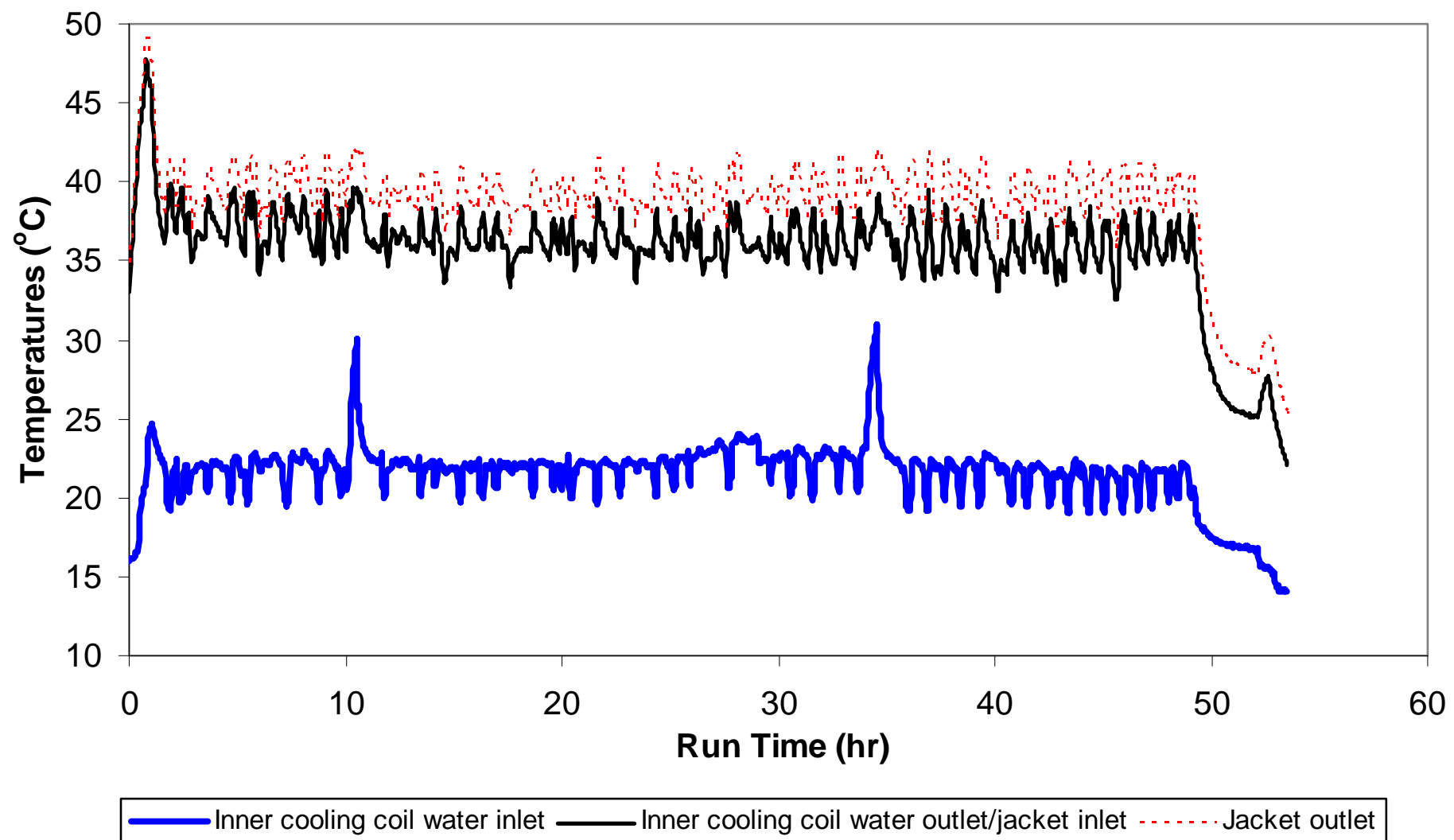

Figure 4.31. SBS cooling coil inlet, cooling coil outlet/jacket inlet and jacket outlet water temperatures while processing with two double-outlet and two single-outlet bubblers, Test 3. 


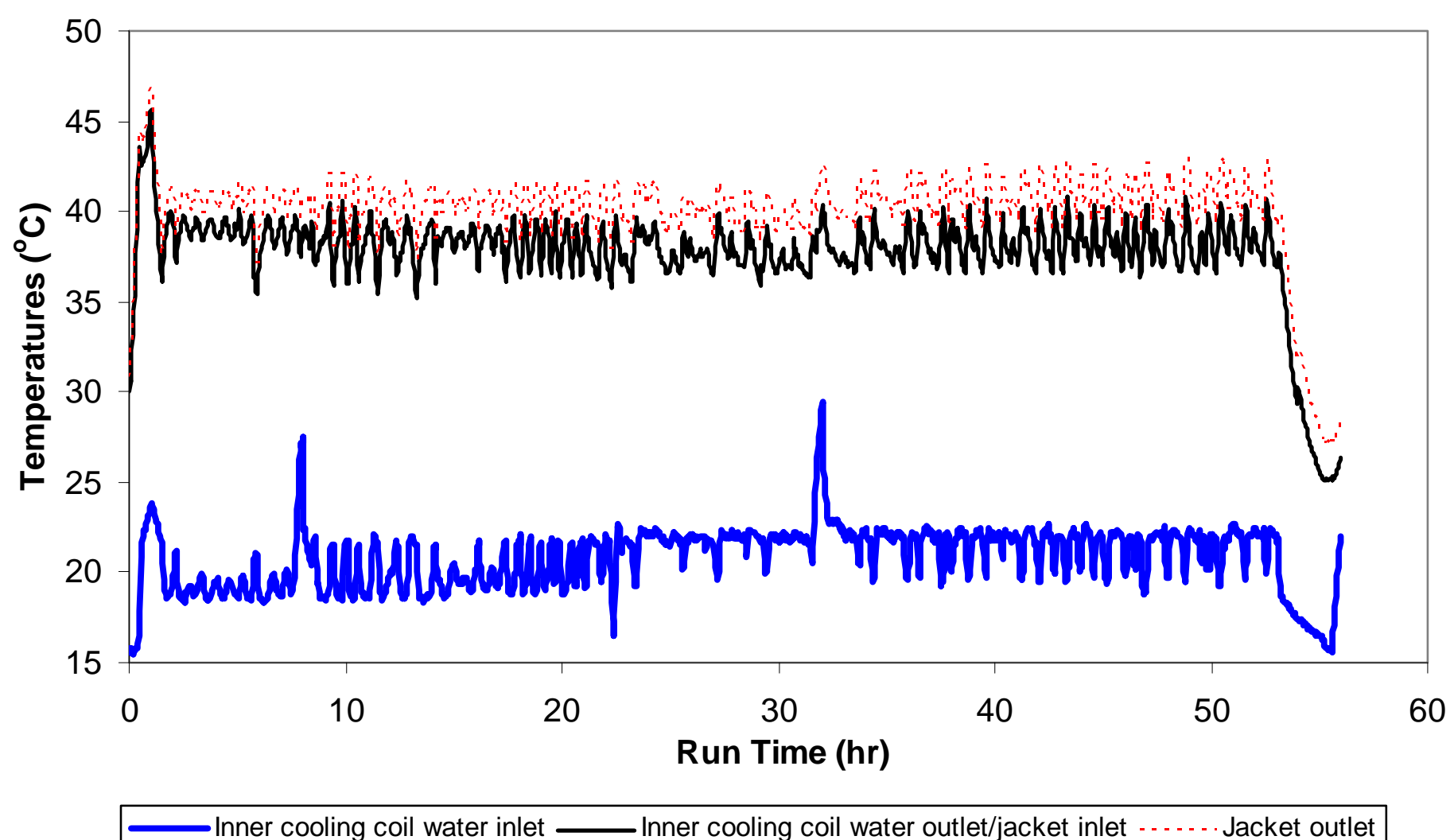

Figure 4.32. SBS cooling coil inlet, cooling coil outlet/jacket inlet and jacket outlet water temperatures while processing with two double-outlet and two single-outlet bubblers, Test 4. 


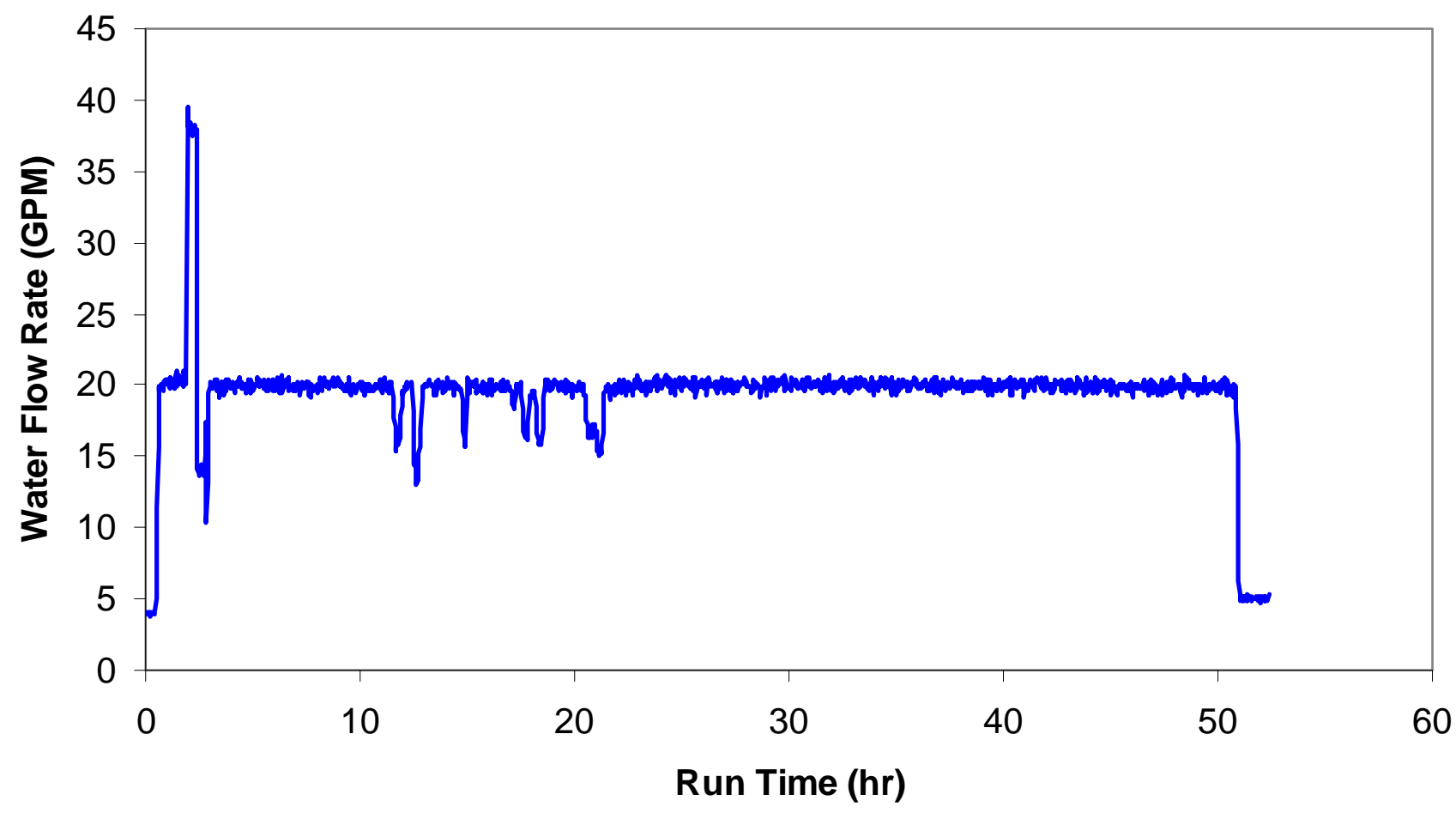

Figure 4.33. SBS cooling coil/jacket water flow rate while processing with two double-outlet bubblers, Test 1. 


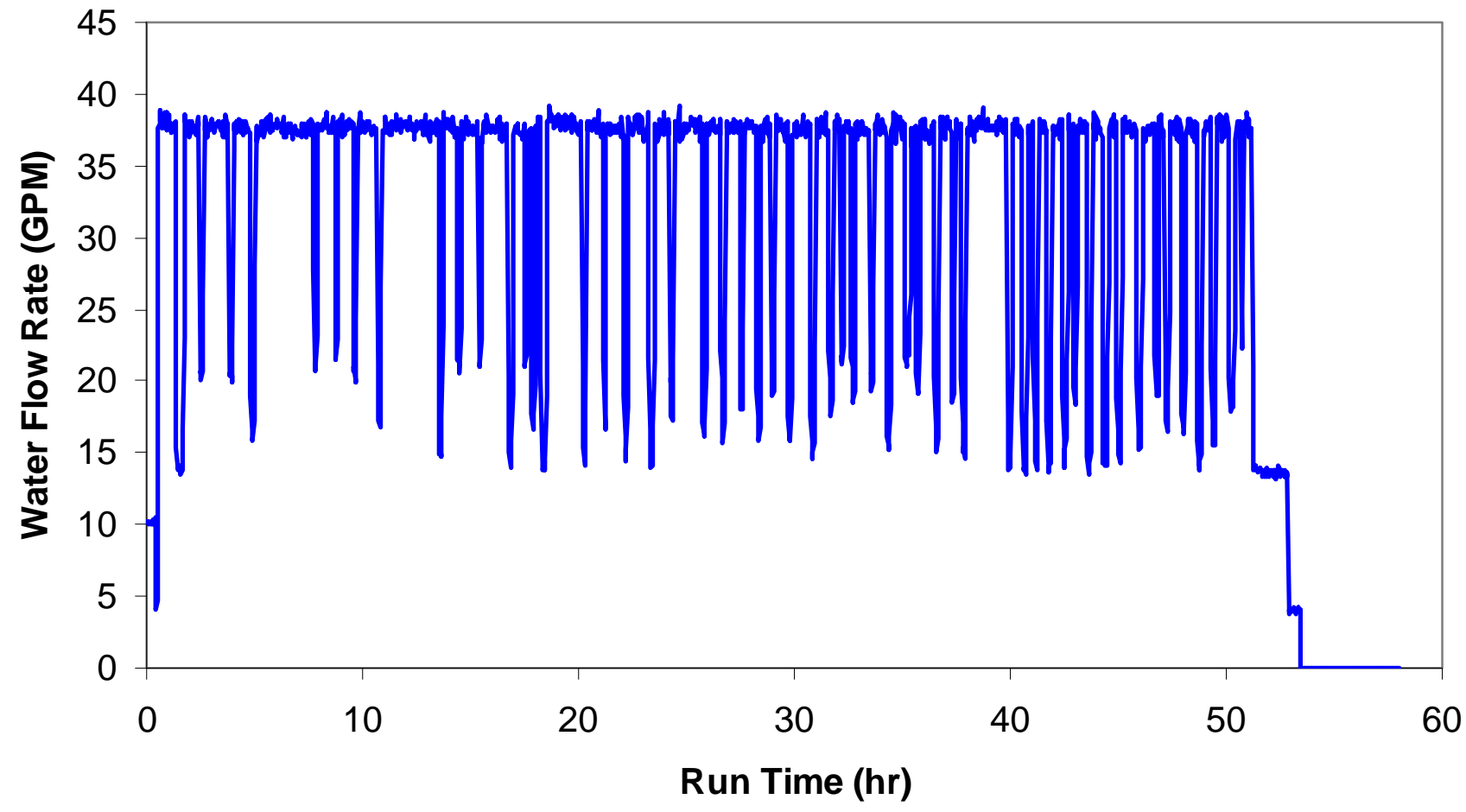

Figure 4.34. SBS cooling coil/jacket water flow rate during while processing with two double-outlet and one single-outlet bubblers, Test 2. 


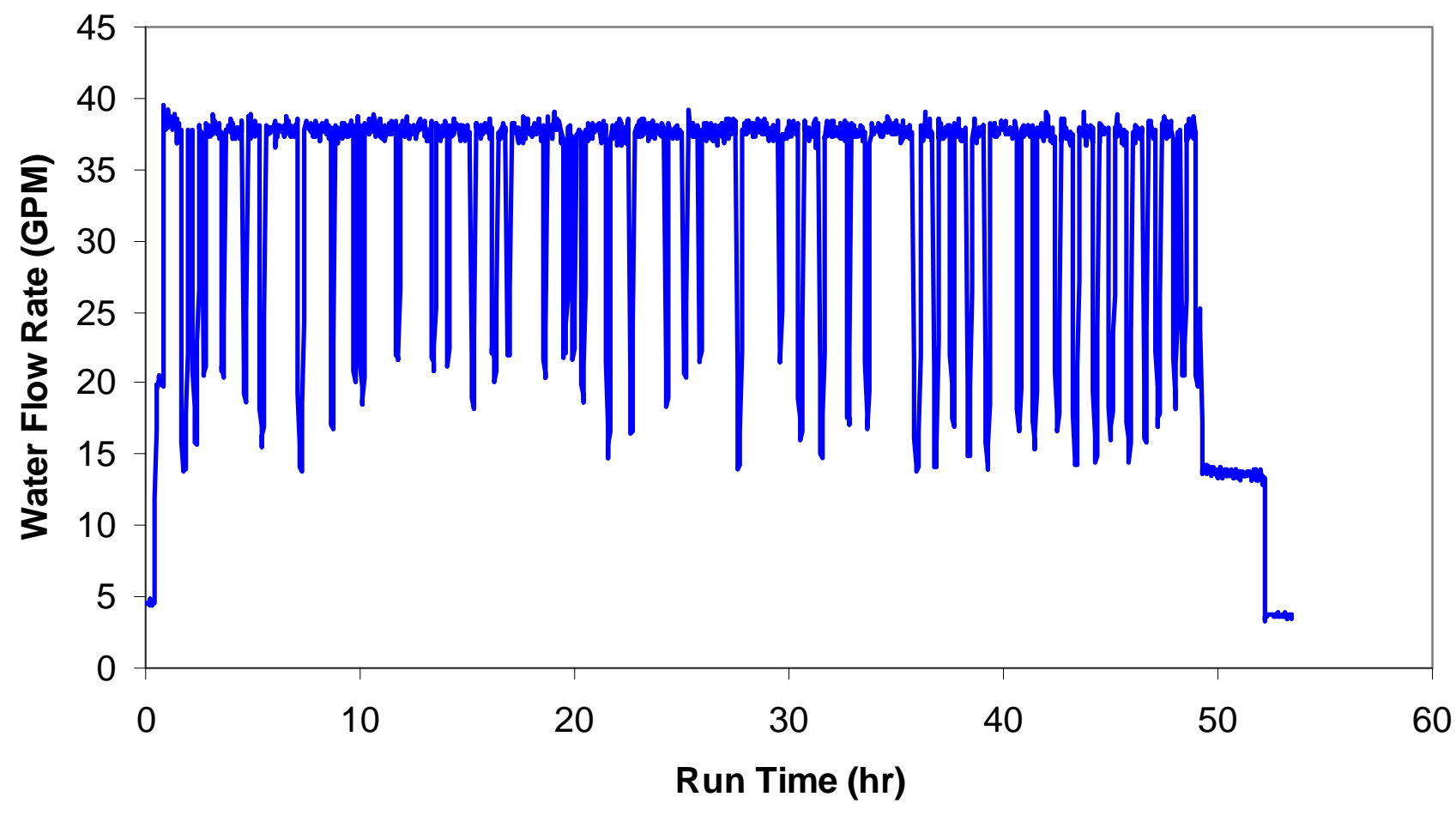

Figure 4.35. SBS cooling coil/jacket water flow rate while processing with two double-outlet and two single-outlet bubblers, Test 3. 


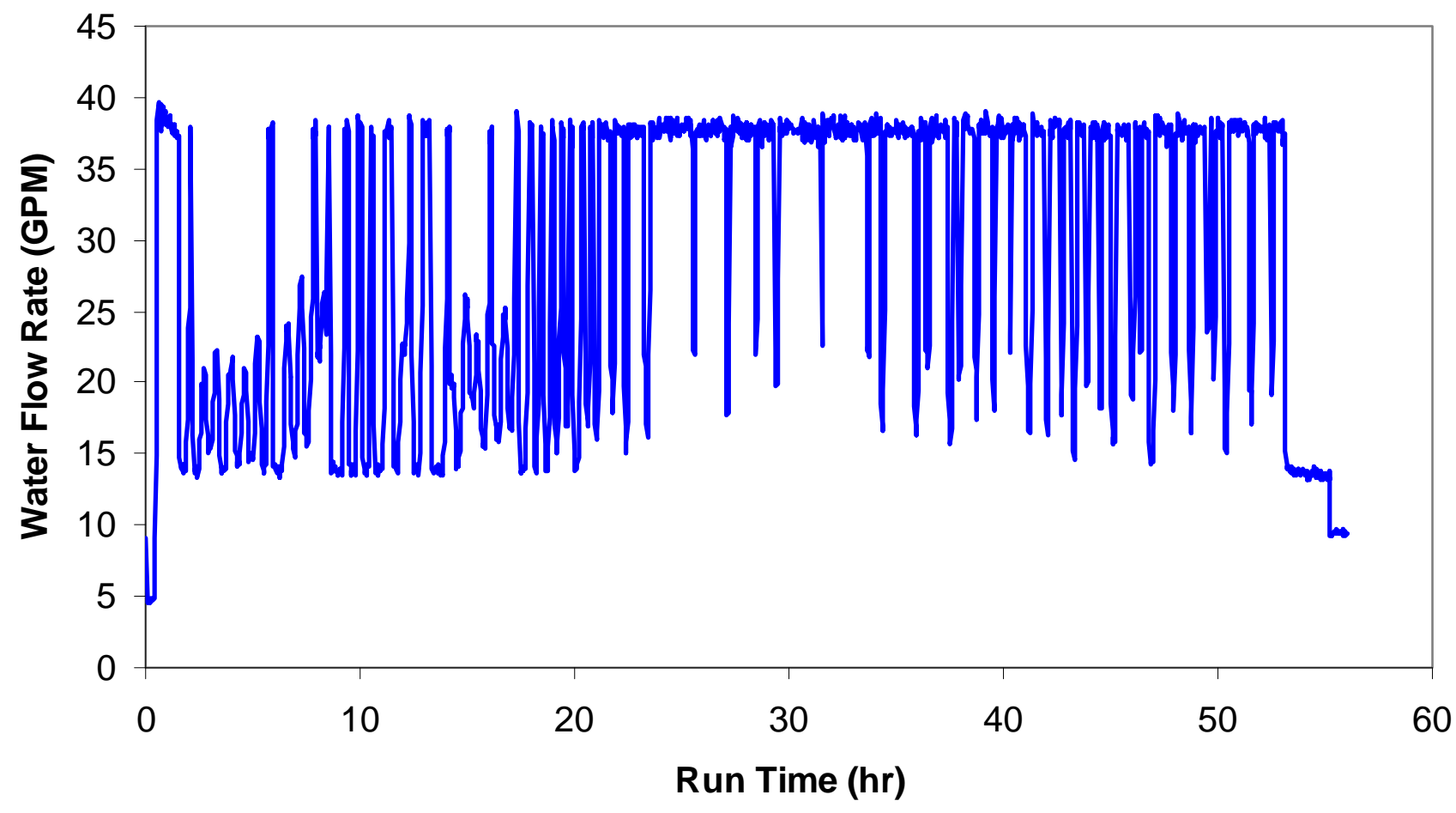

Figure 4.36. SBS cooling coil/jacket water flow rate while processing with two double-outlet and two single-outlet bubblers, Test 4. 


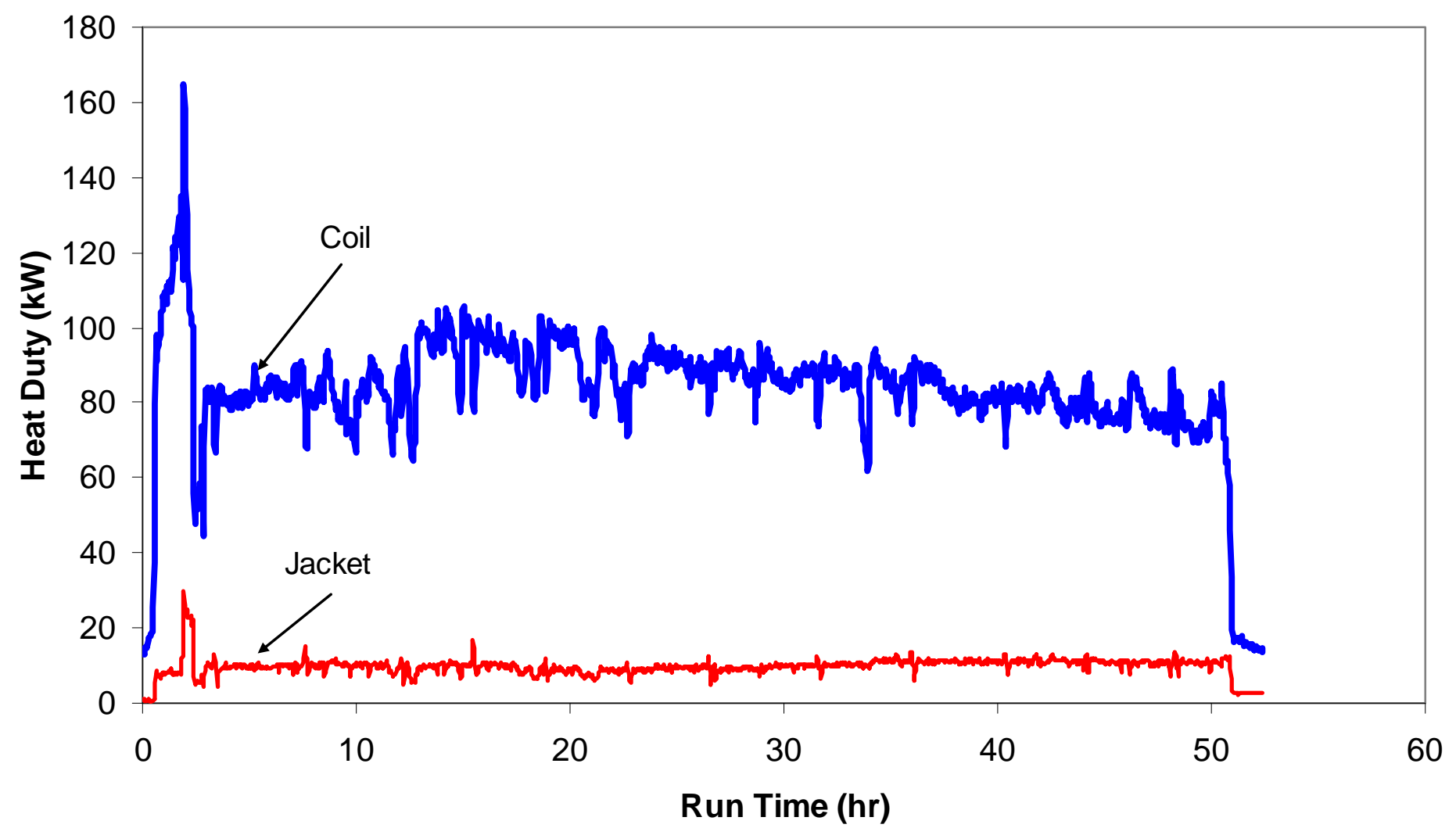

Figure 4.37. Calculated heat loads on the inner coil and jacket while processing with two double-outlet bubblers, Test 1. 


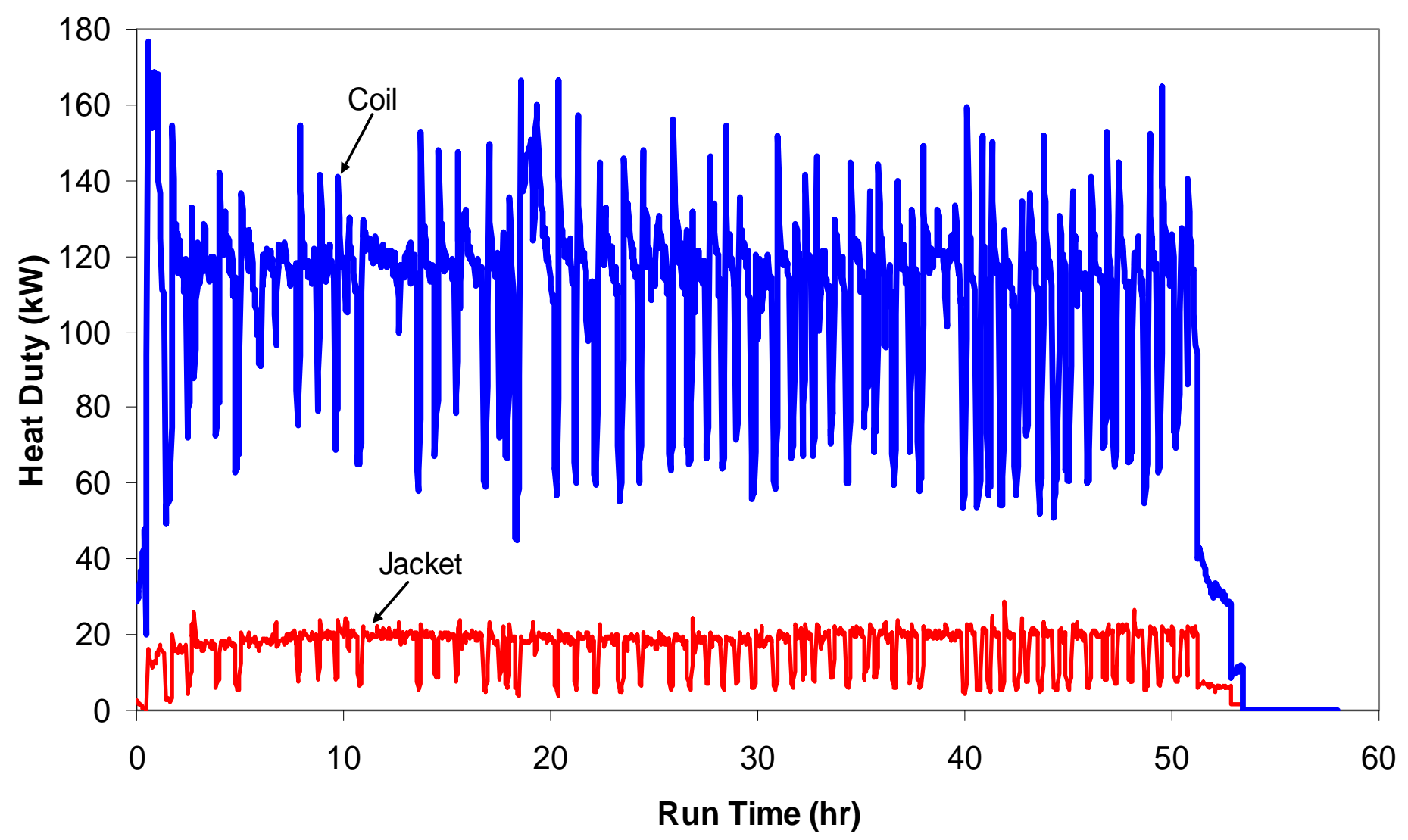

Figure 4.38. Calculated heat loads on the inner coil and jacket while processing with two double-outlet and one single-outlet bubblers, Test 2. 


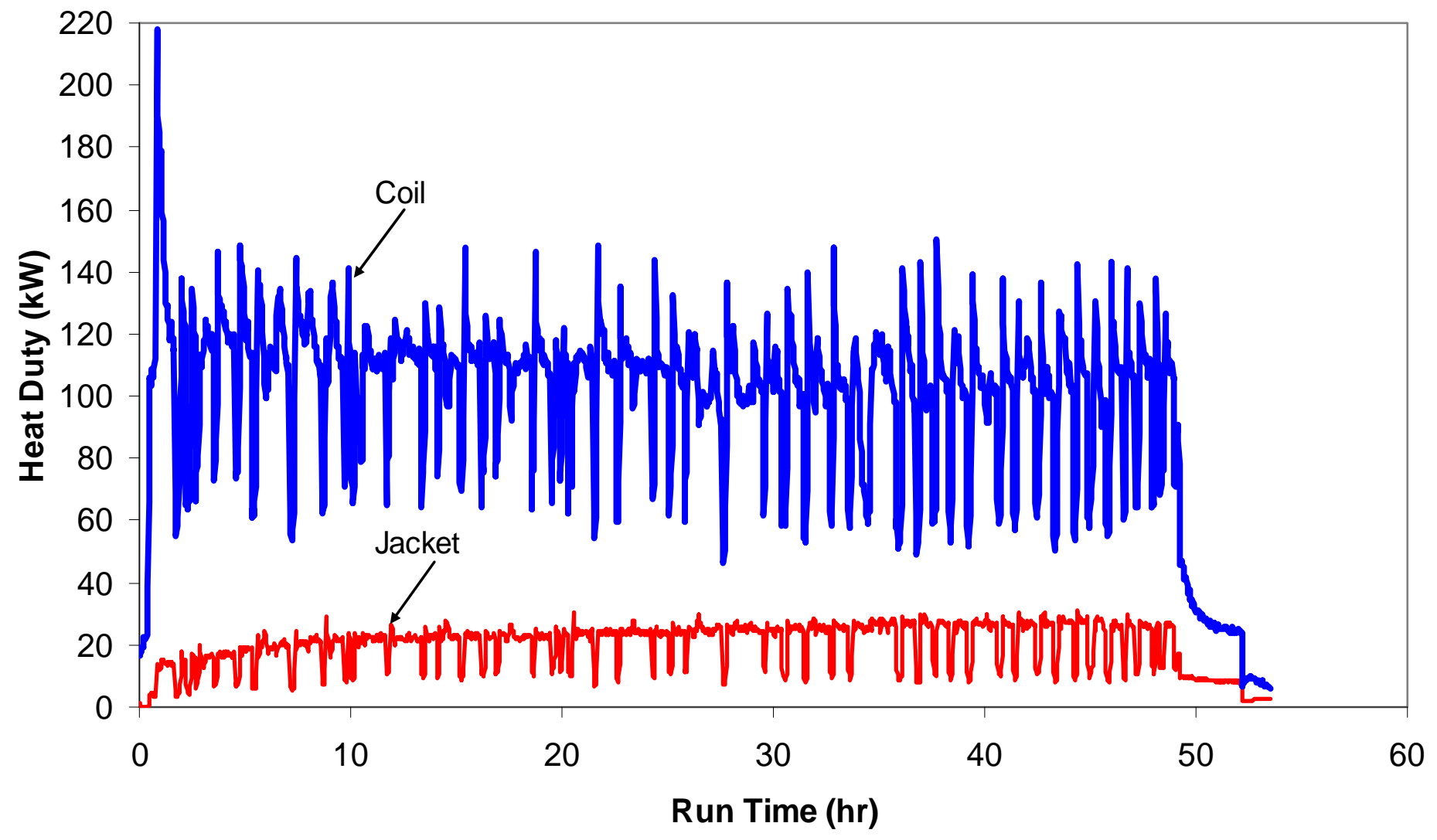

Figure 4.39. Calculated heat loads on the inner coil and while processing with two double-outlet and two single-outlet bubblers, Test 3. 


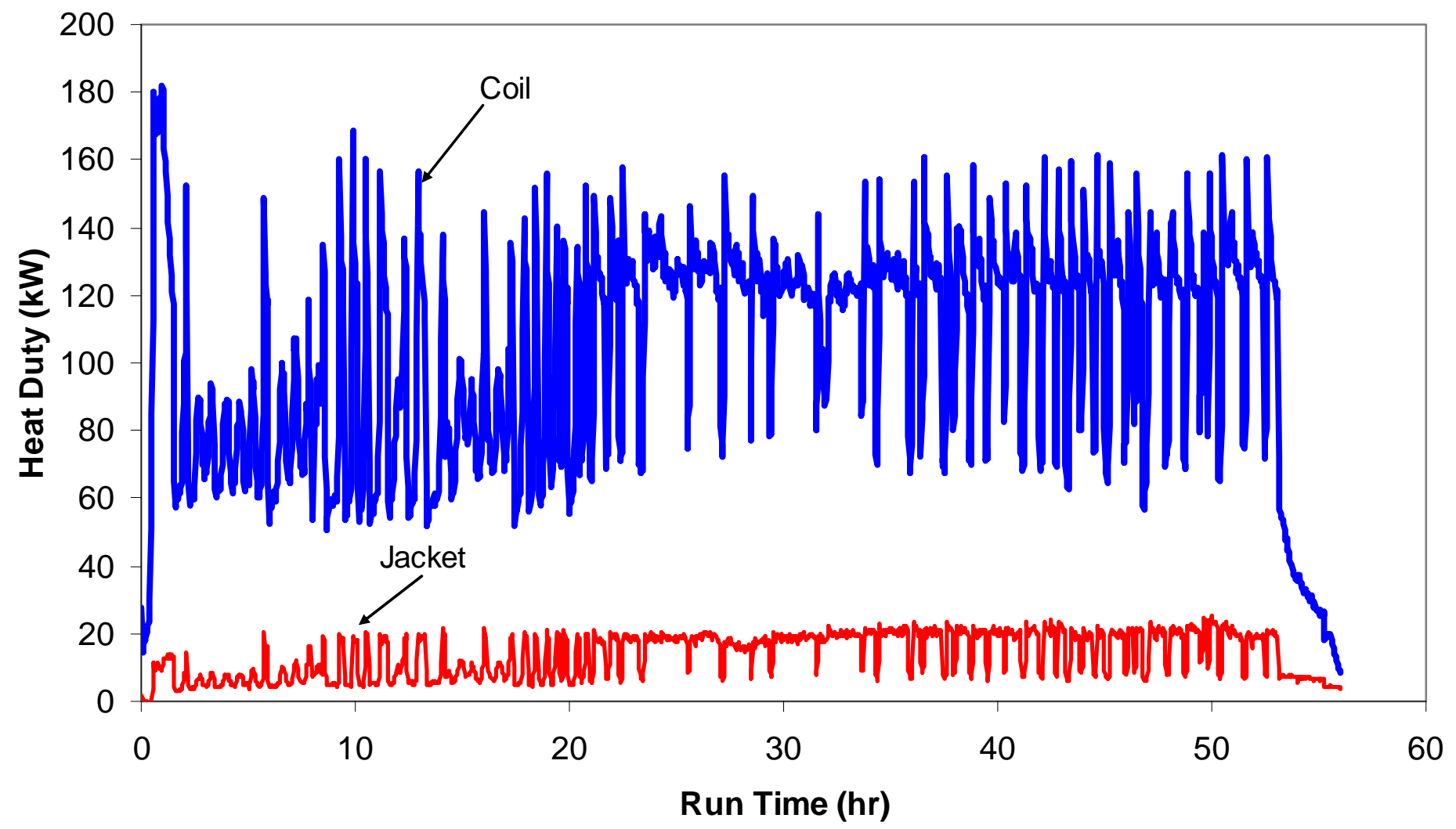

Figure 4.40. Calculated heat loads on the inner coil and jacket while processing with two double-outlet and two single-outlet bubblers, Test 4. 


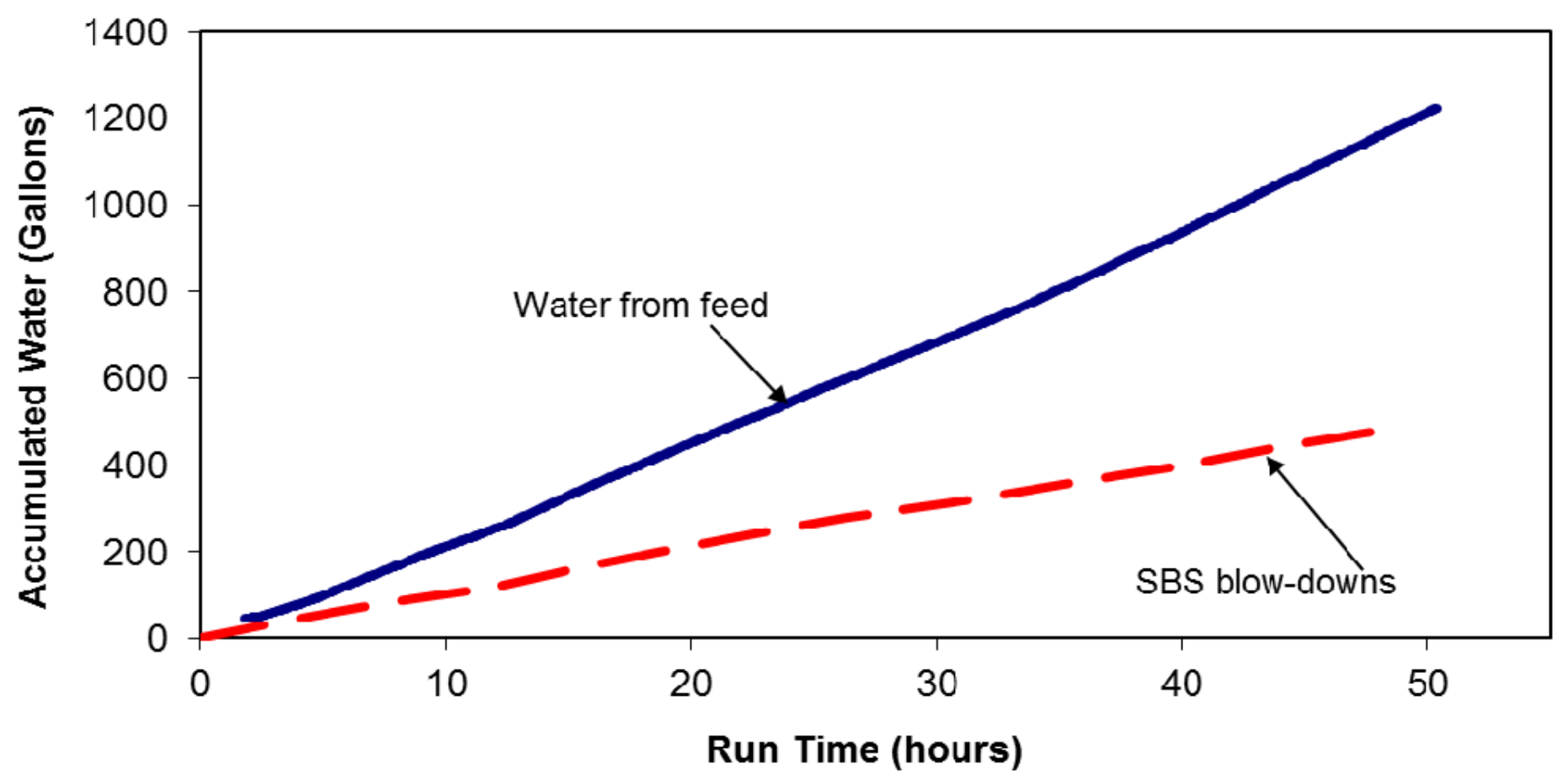

Figure 4.41. Accumulated SBS blow-down volume and accumulated feed water while processing with two double-outlet bubblers, Test 1. 


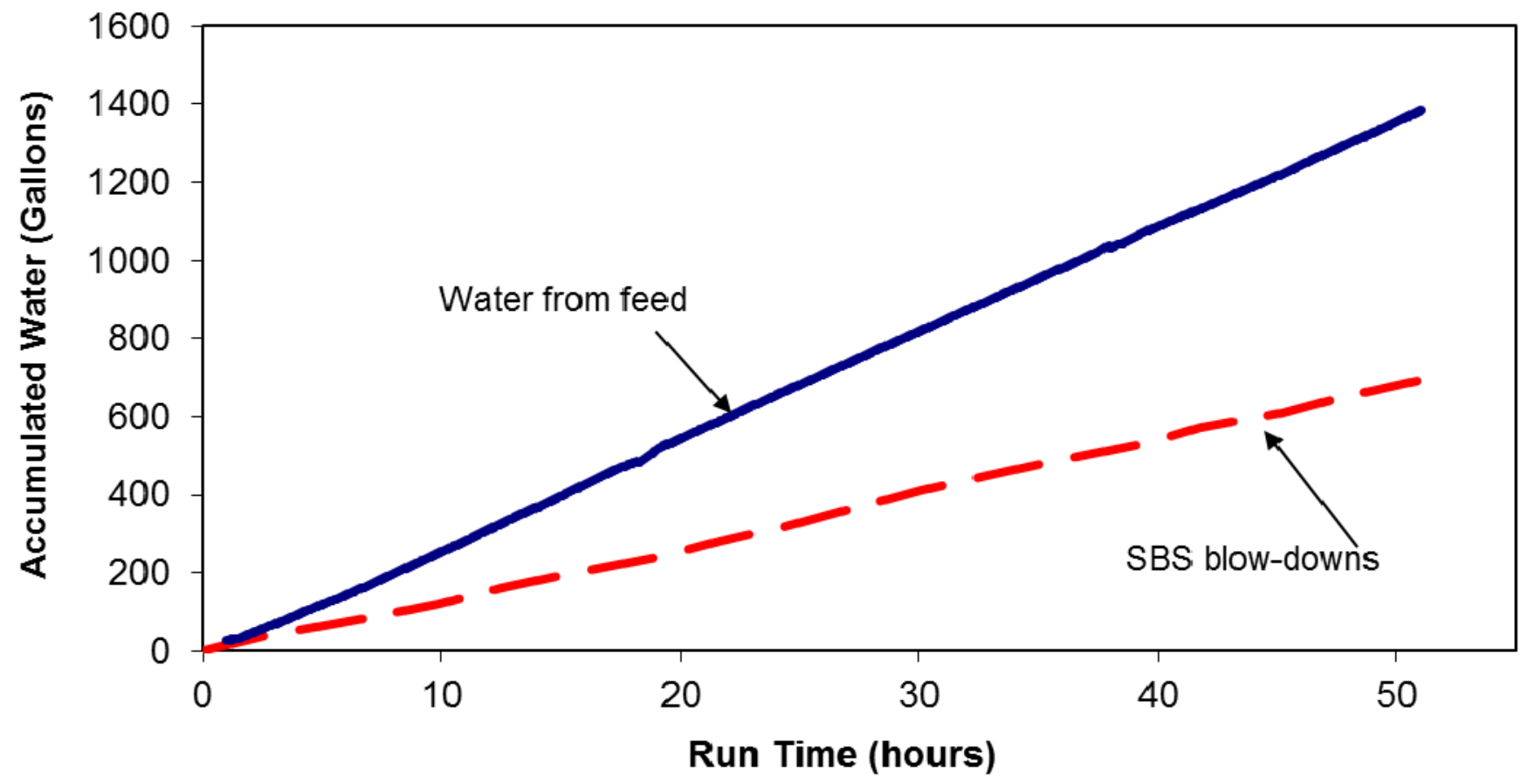

Figure 4.42. Accumulated SBS blow-down volume and accumulated feed water while processing with two double-outlet and one single-outlet bubblers, Test 2. 


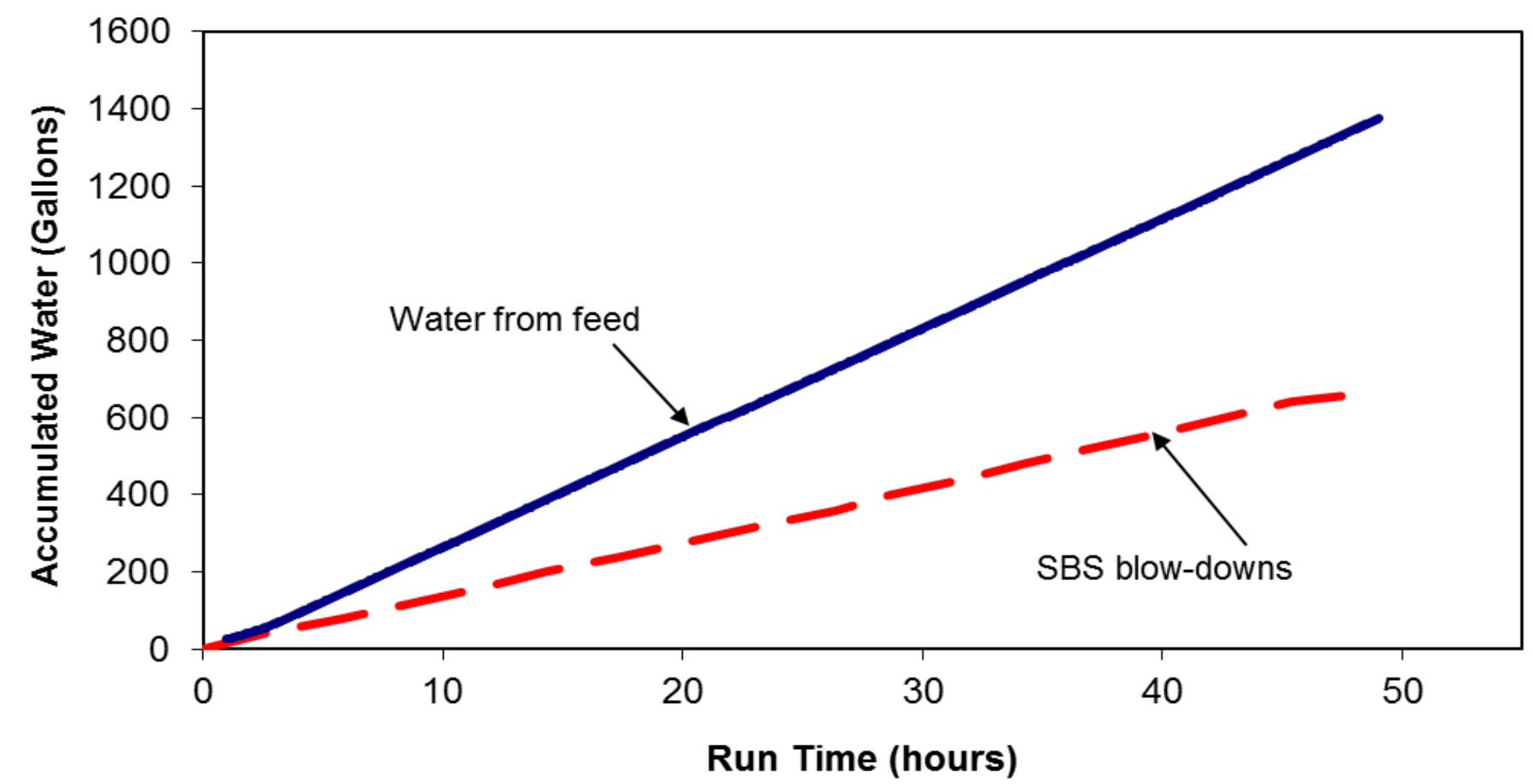

Figure 4.43. Accumulated SBS blow-down volume and accumulated feed water while processing with two double-outlet and two single-outlet bubblers, Test 3. 


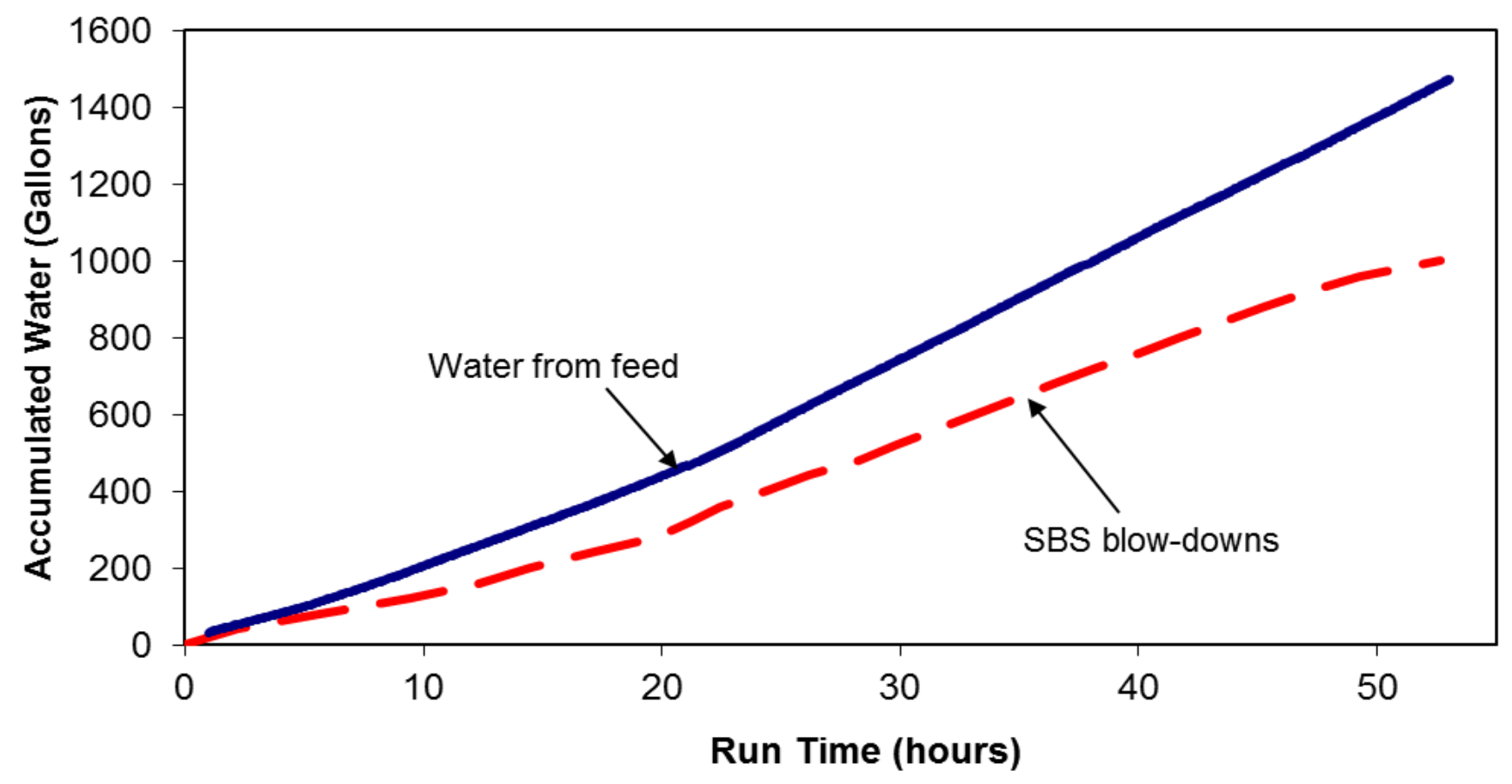

Figure 4.44. Accumulated SBS blow-down volume and accumulated feed water while processing with two double-outlet and two single-outlet bubblers, Test 4. 


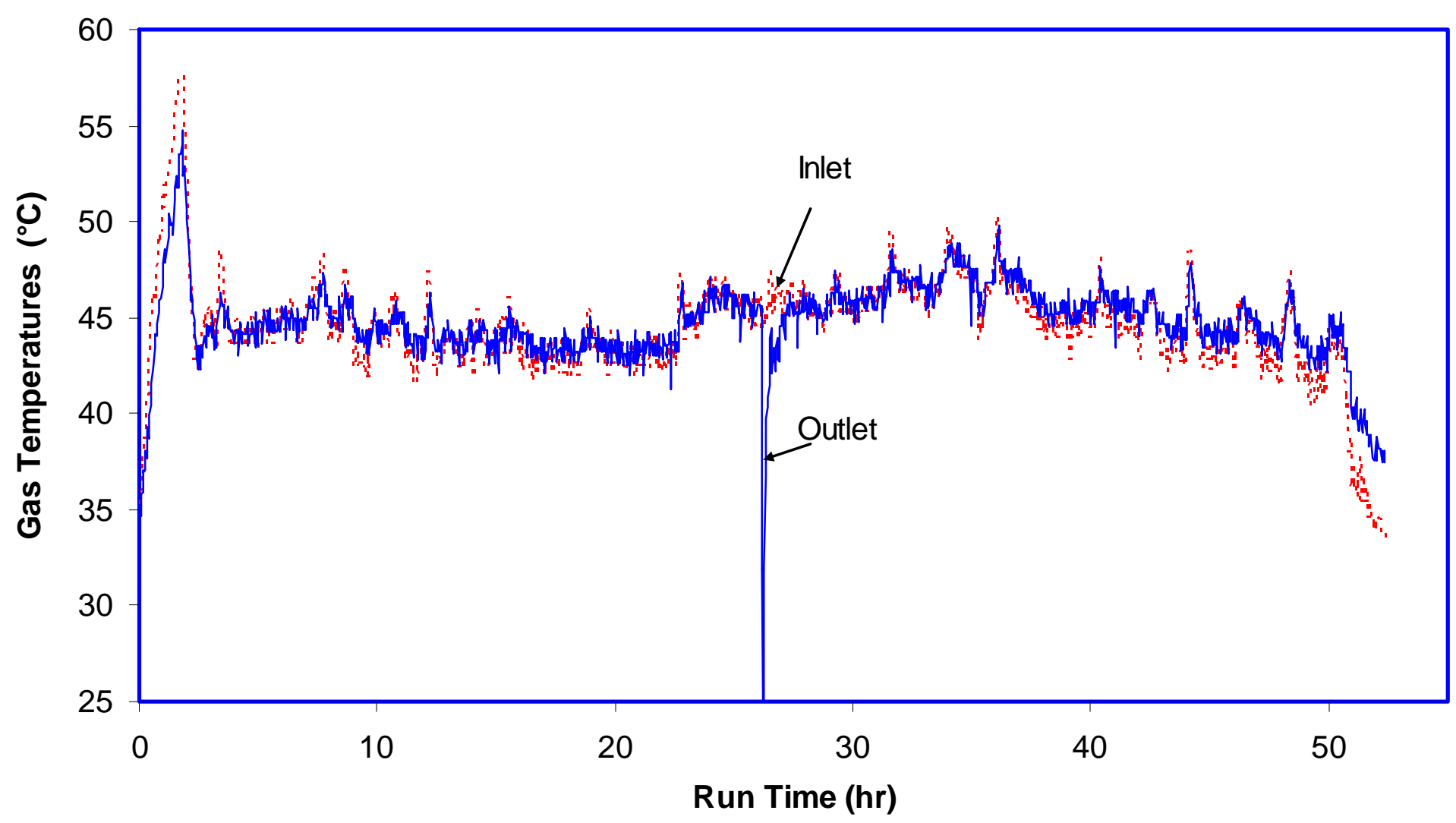

Figure 4.45. WESP inlet and outlet gas temperatures while processing with two double-outlet bubblers, Test 1.

(Note: Downward outlet temperature spikes are the result of WESP deluges.) 


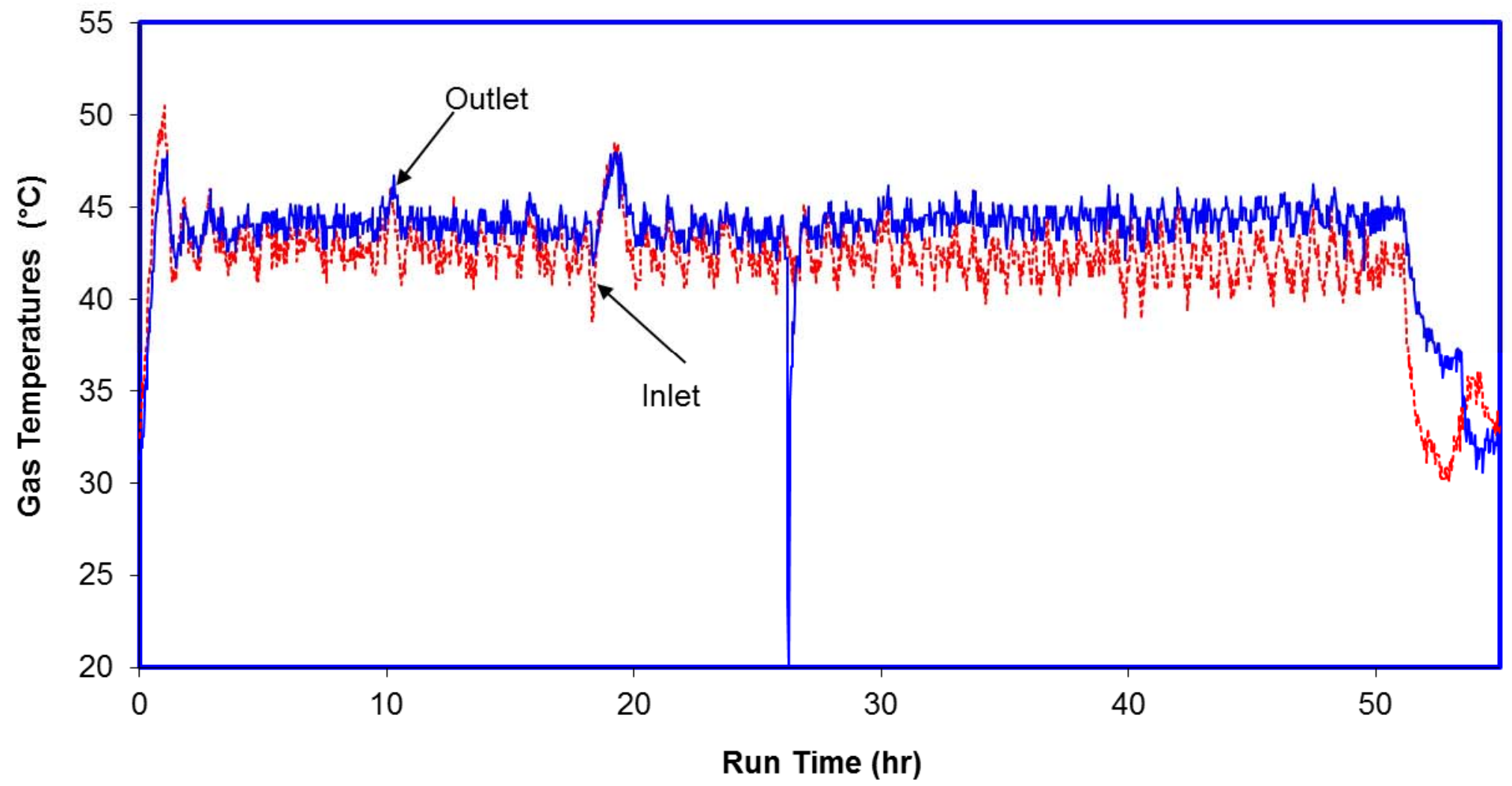

Figure 4.46. WESP inlet and outlet gas temperatures during while processing with two double-outlet and one single-outlet bubblers, Test 2.

(Note: Downward outlet temperature spikes are the result of WESP deluges.) 


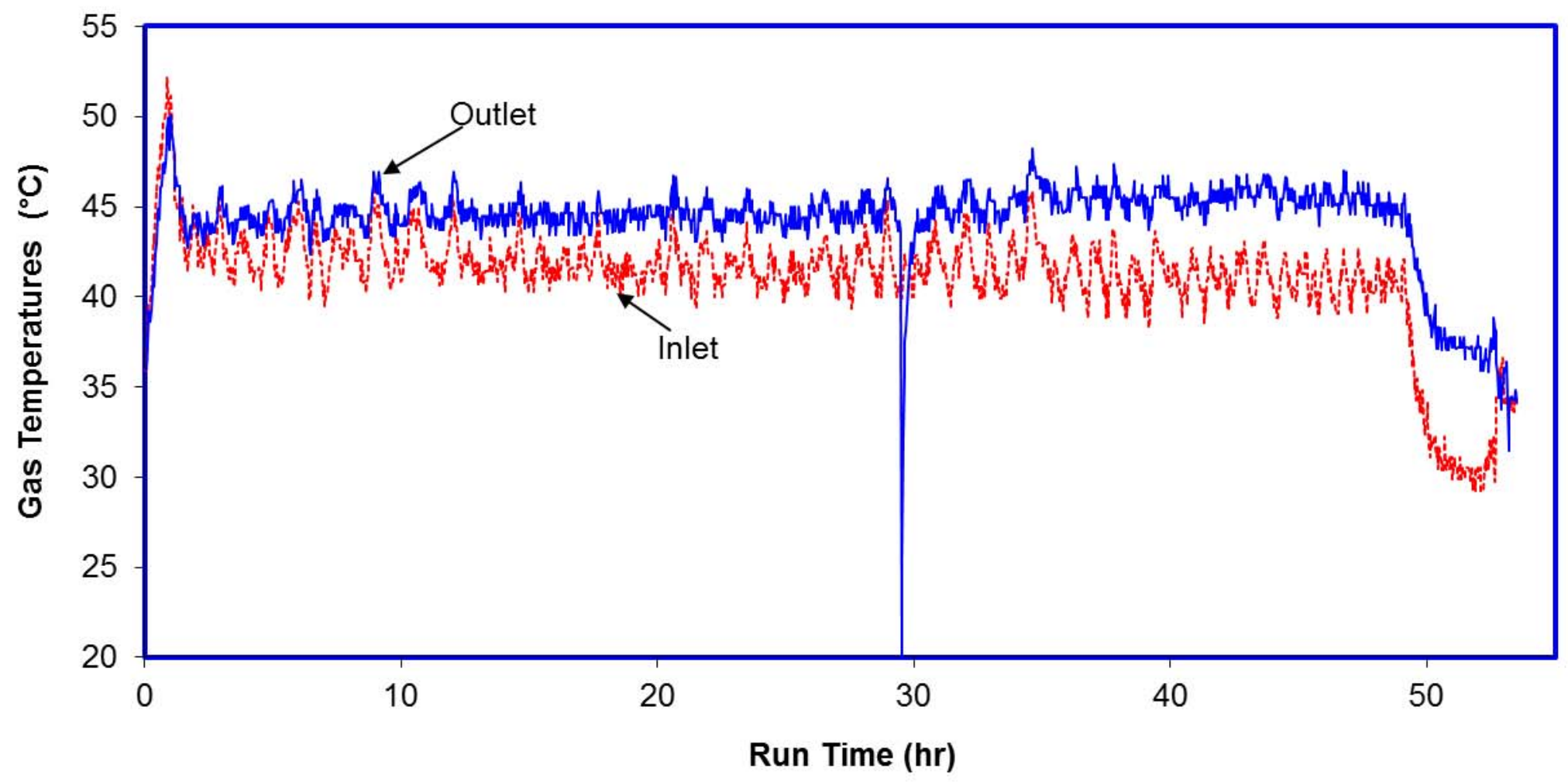

Figure 4.47. WESP inlet and outlet gas temperatures while processing with two double-outlet and two single-outlet bubblers, Test 3.

(Note: Downward outlet temperature spikes are the result of WESP deluges.) 


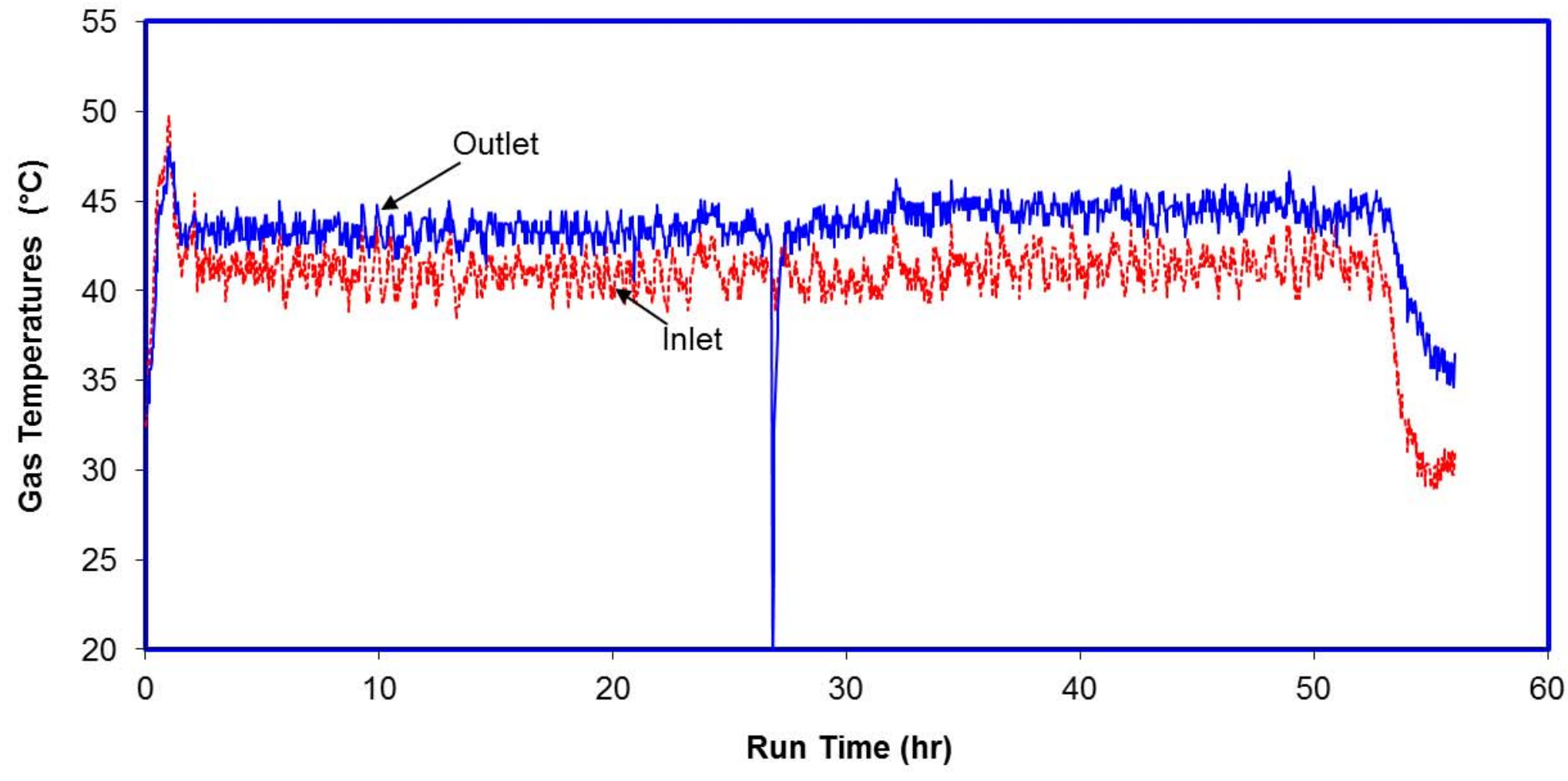

Figure 4.48. WESP inlet and outlet gas temperatures while processing with two double-outlet and two single-outlet bubblers, Test 4.

(Note: Downward outlet temperature spikes are the result of WESP deluges.) 


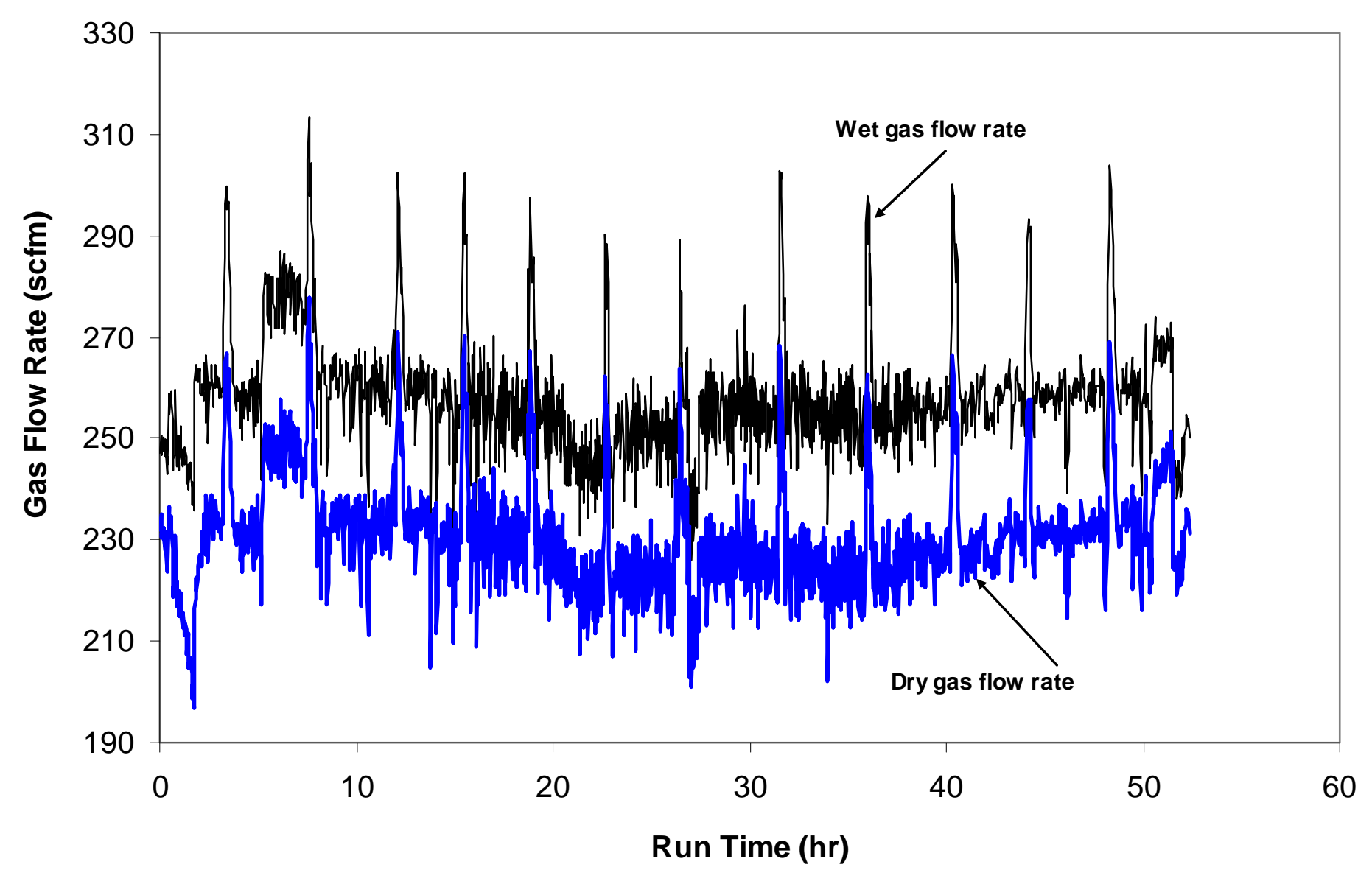

Figure 4.49. WESP outlet gas flow rate while processing with two double-outlet bubblers, Test 1. 


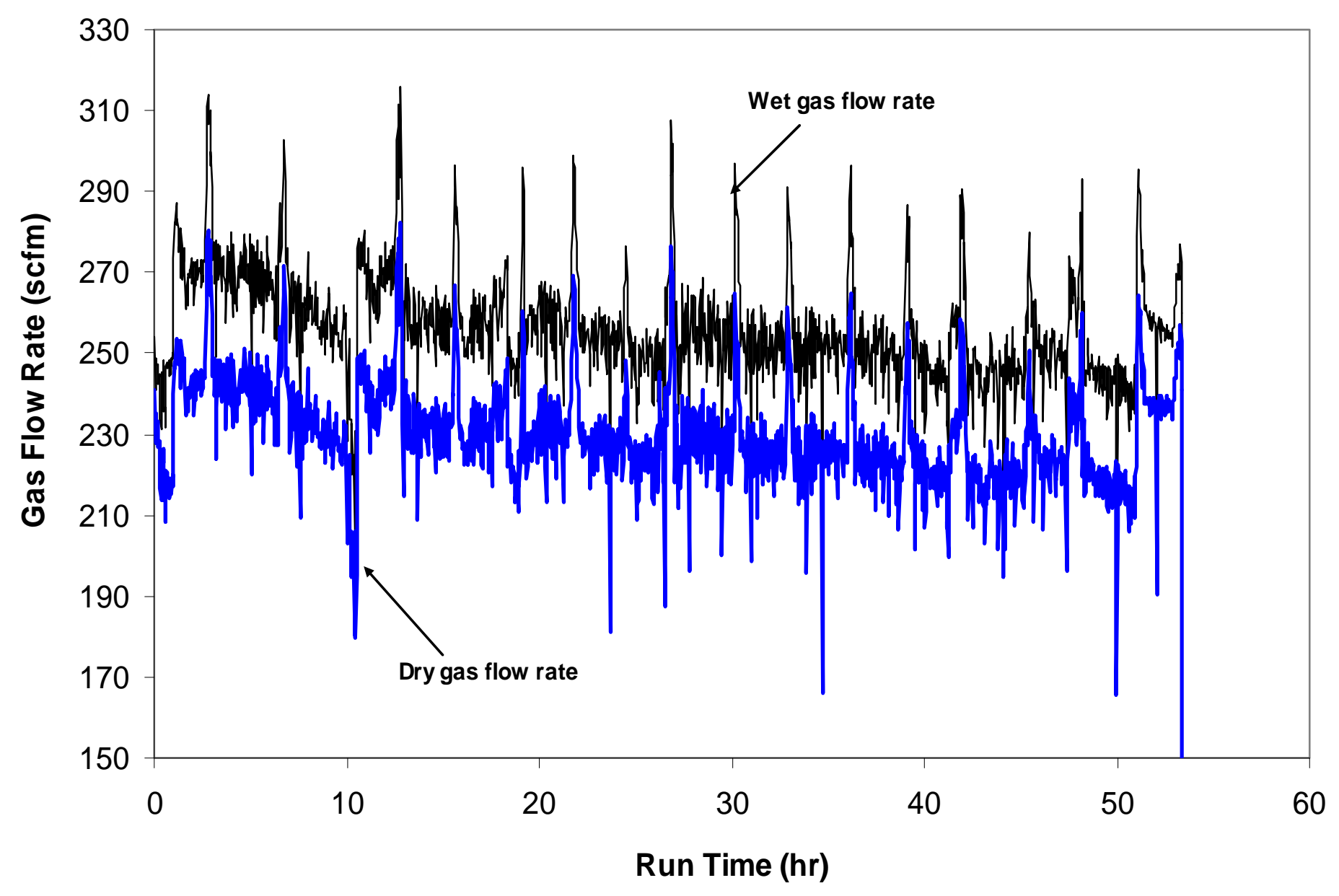

Figure 4.50. WESP outlet gas flow rate while processing with two double-outlet and one single-outlet bubblers, Test 2. 


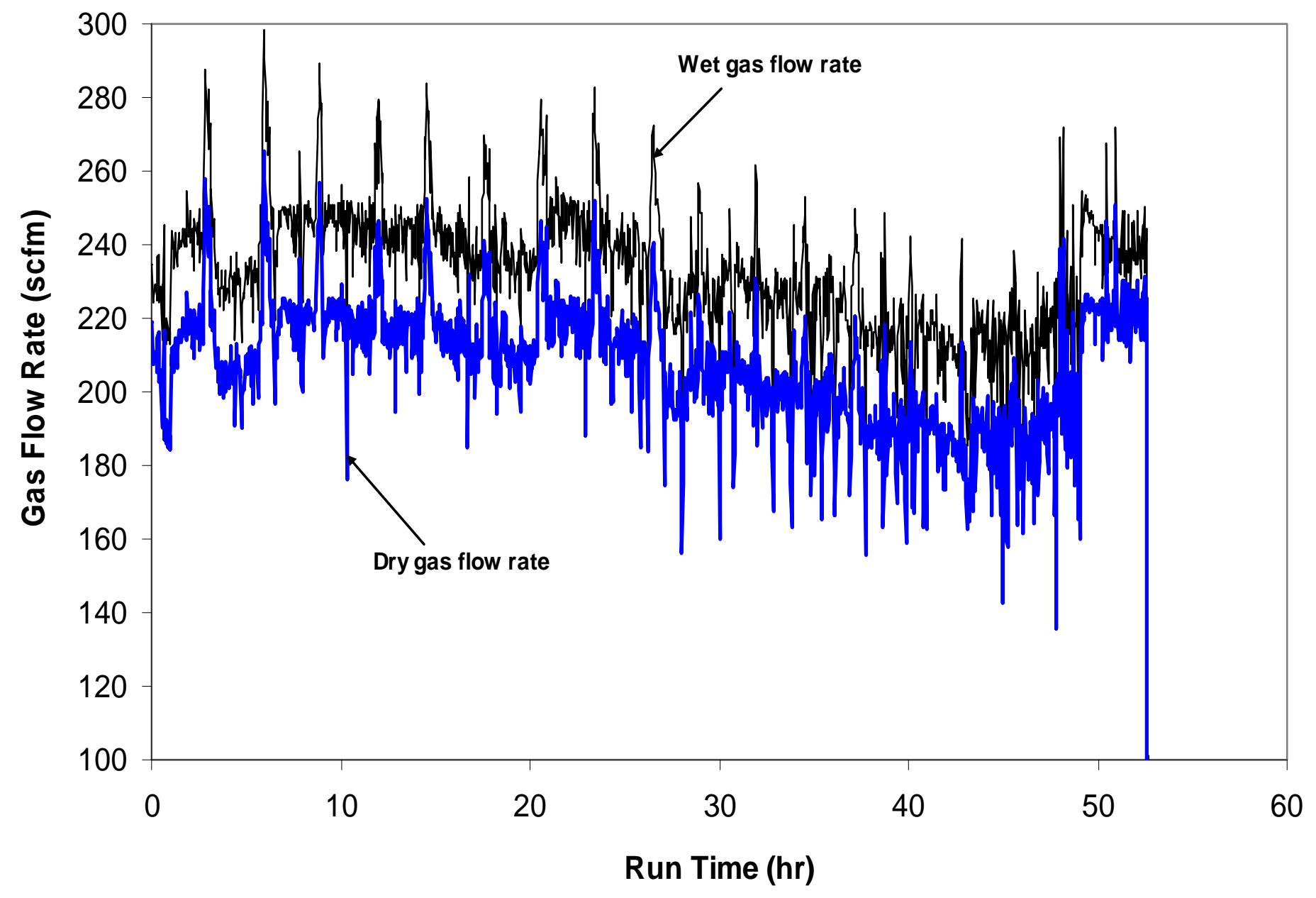

Figure 4.51. WESP outlet gas flow rate while processing with two double-outlet and two single-outlet bubblers, Test 3. 


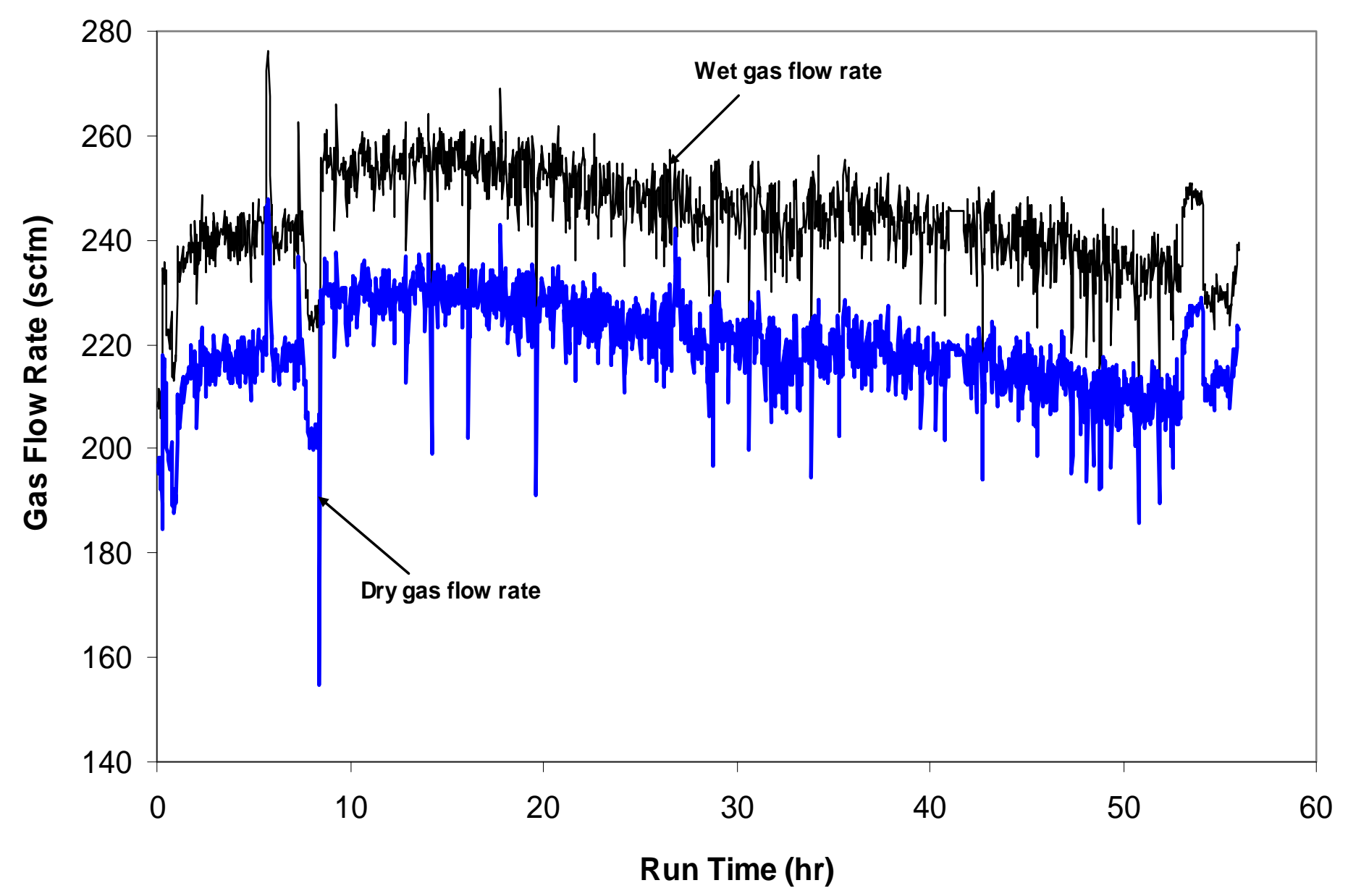

Figure 4.52. WESP outlet gas flow rate while processing with two double-outlet and two single-outlet bubblers, Test 4. 


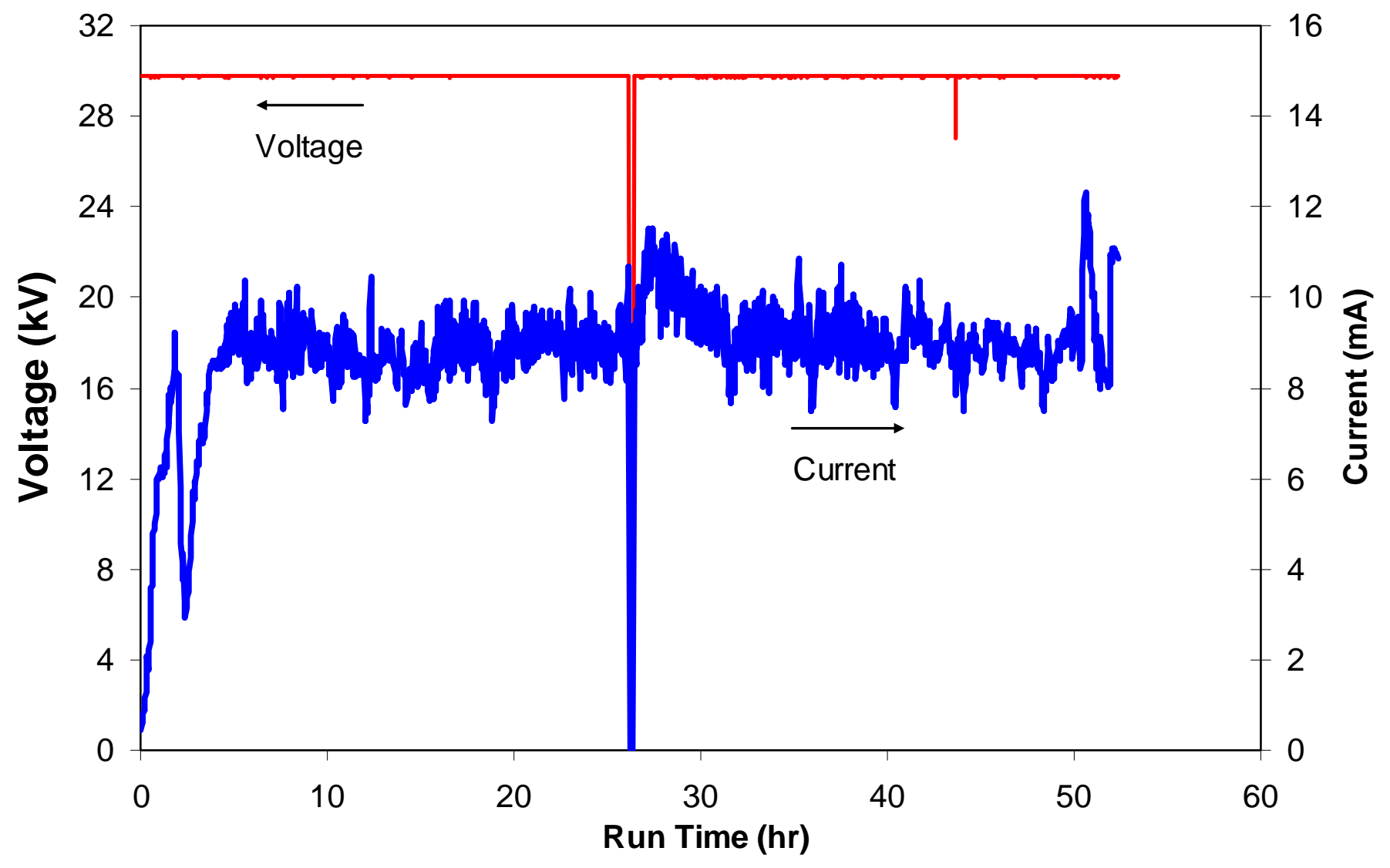

Figure 4.53. Voltage and current across the WESP while processing with two double-outlet bubblers, Test 1. 


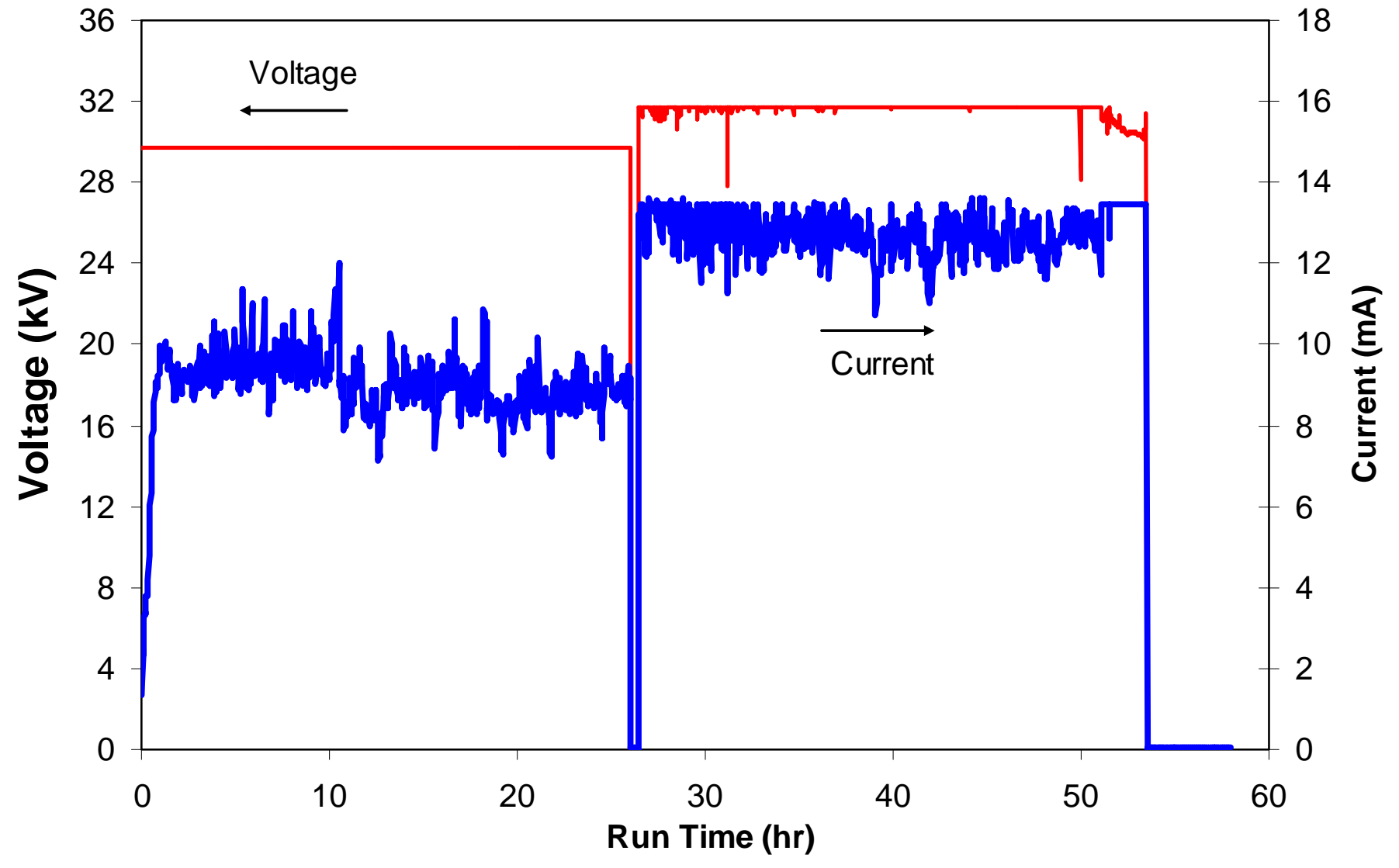

Figure 4.54. Voltage and current across the WESP while processing with two double-outlet and one single-outlet bubblers, Test 2. 


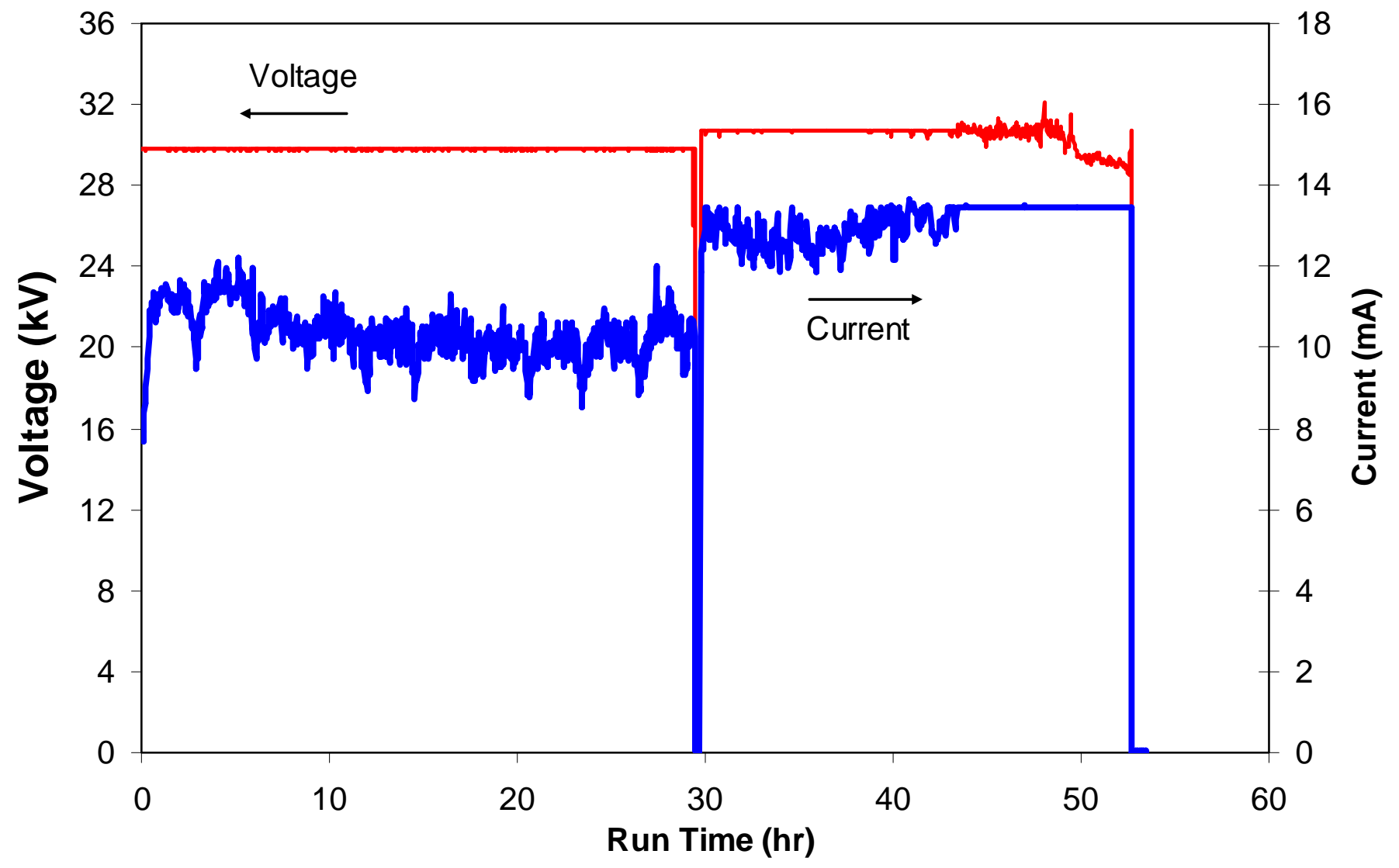

Figure 4.55. Voltage and current across the WESP while processing with two double-outlet and two single-outlet bubblers, Test 3. 


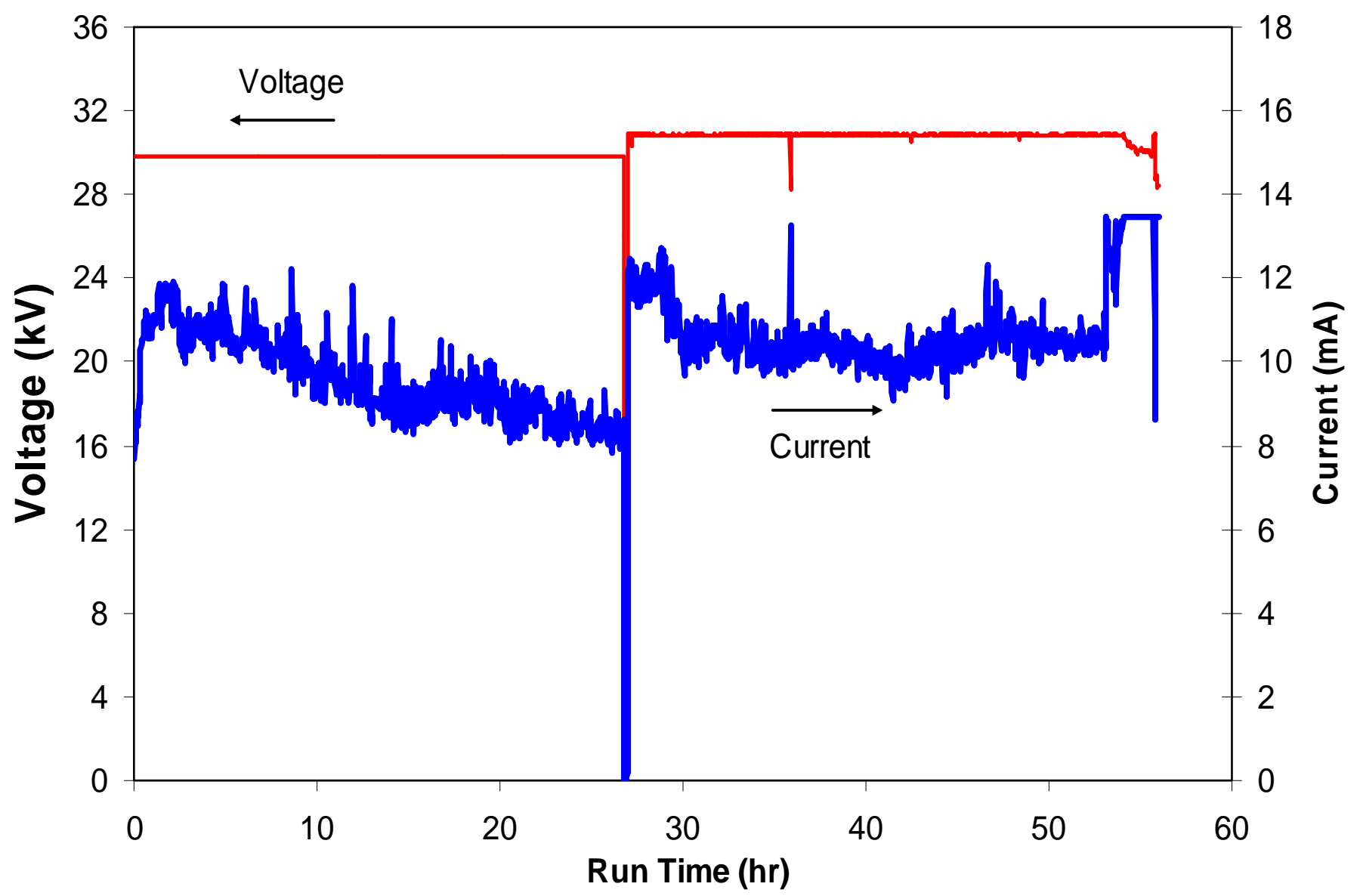

Figure 4.56. Voltage and current across the WESP while processing with two double-outlet and two single-outlet bubblers, Test 4. 


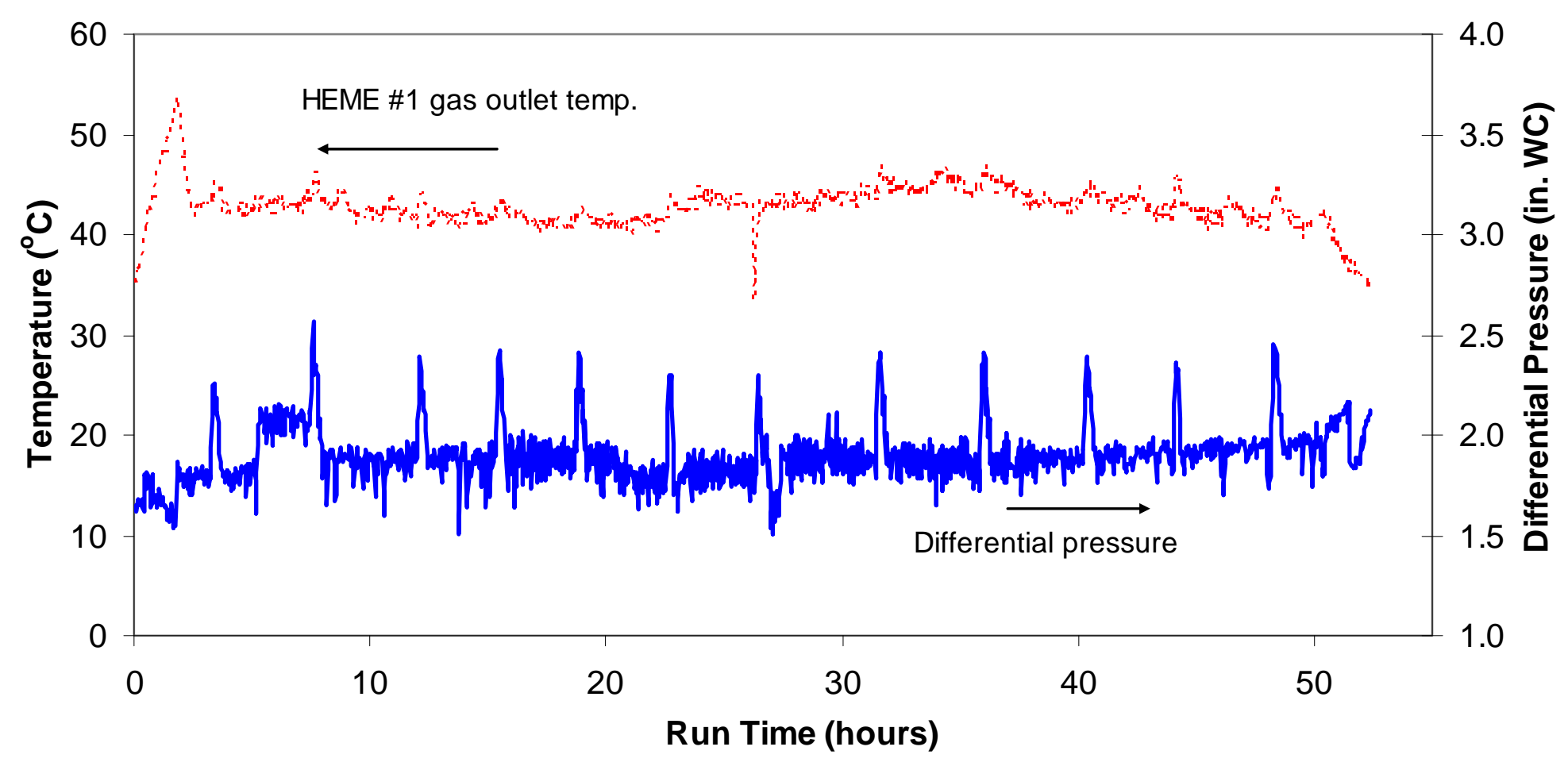

Figure 4.57. Outlet temperature and differential pressure for HEME \#1 while processing with two double-outlet bubblers, Test 1. 


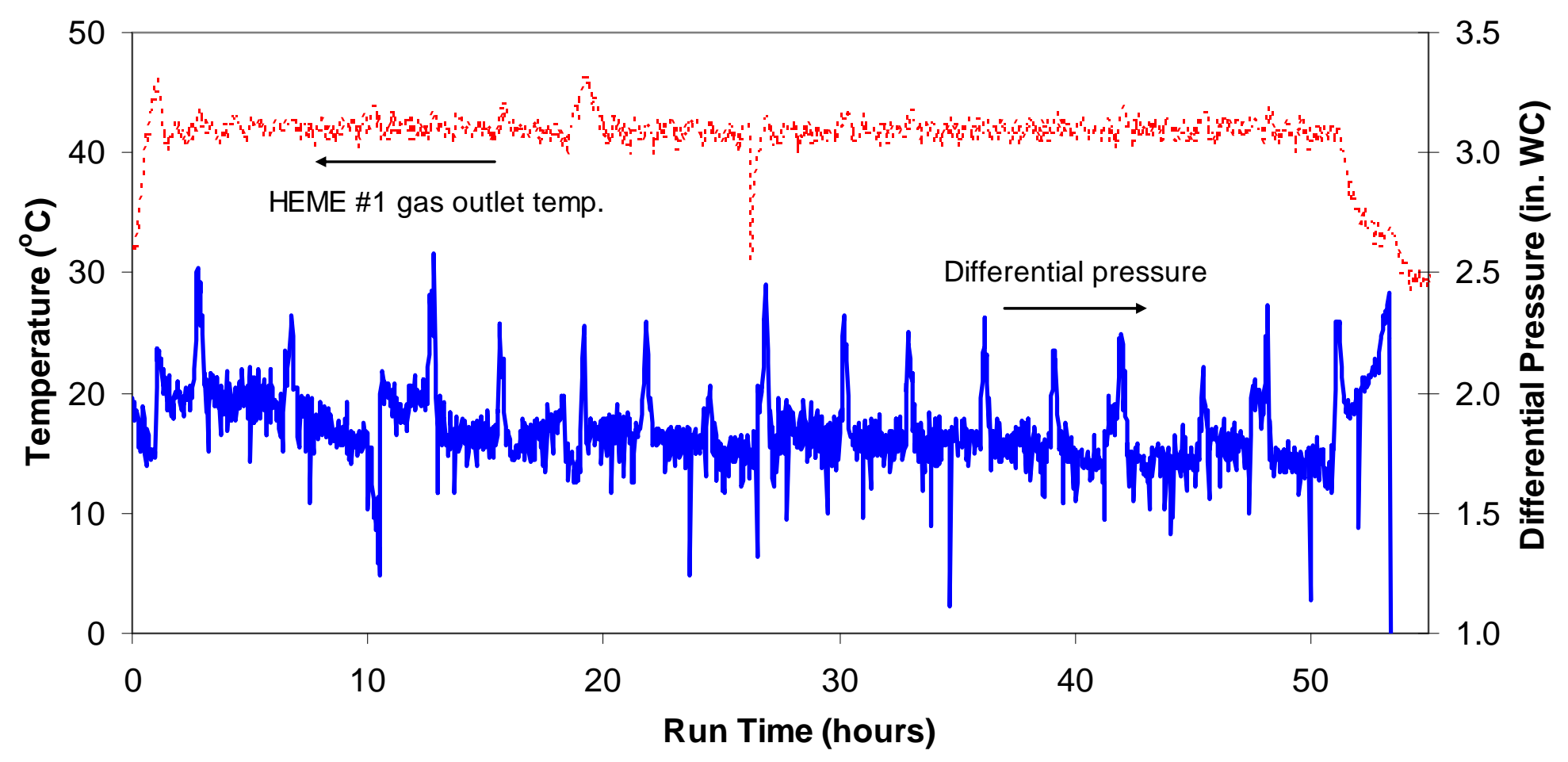

Figure 4.58. Outlet temperature and differential pressure for HEME \#1 while processing with two double-outlet and one single-outlet bubblers, Test 2. 


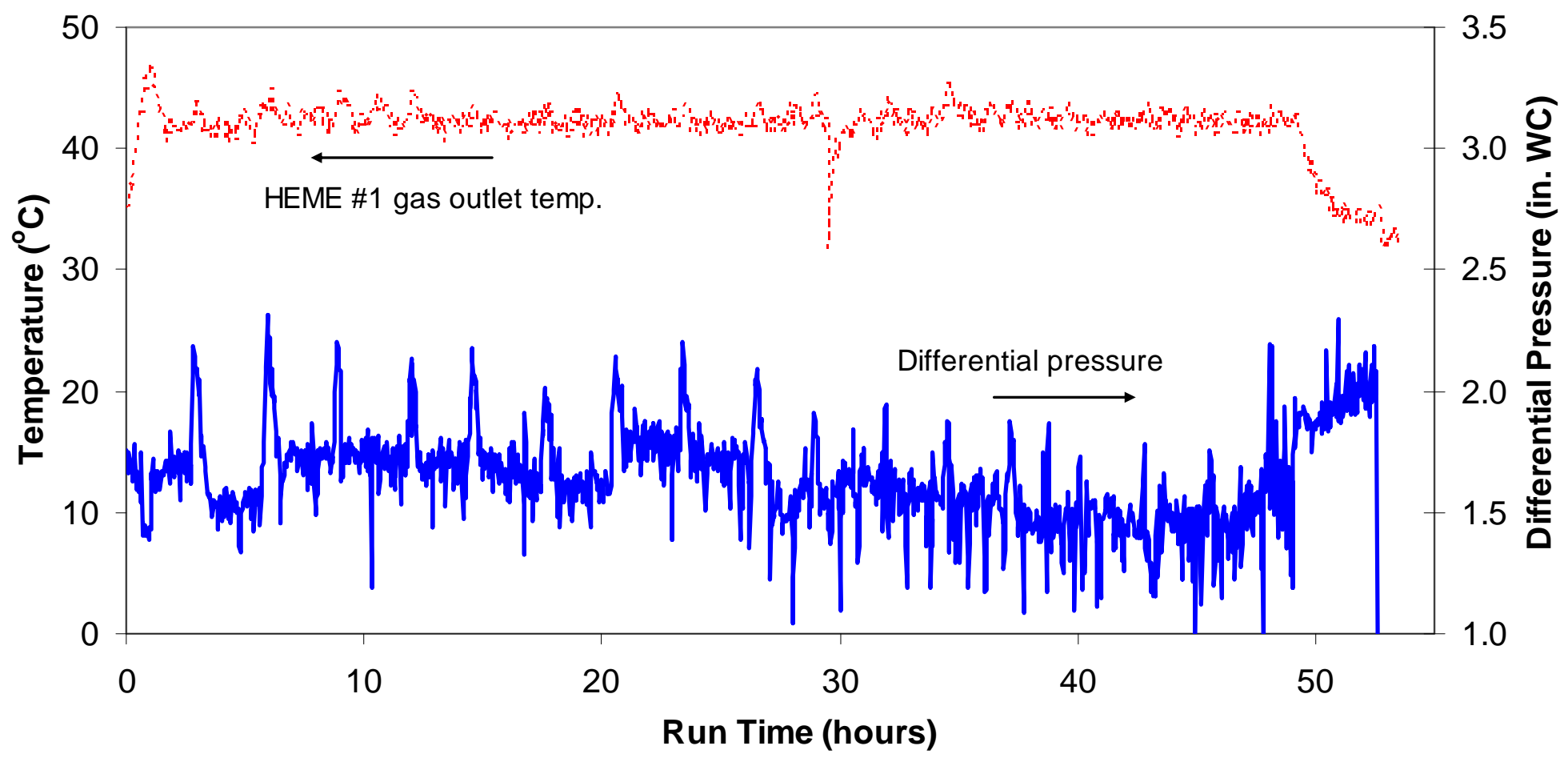

Figure 4.59. Outlet temperature and differential pressure for HEME \#1 while processing with two double-outlet and two single-outlet bubblers, Test 3. 


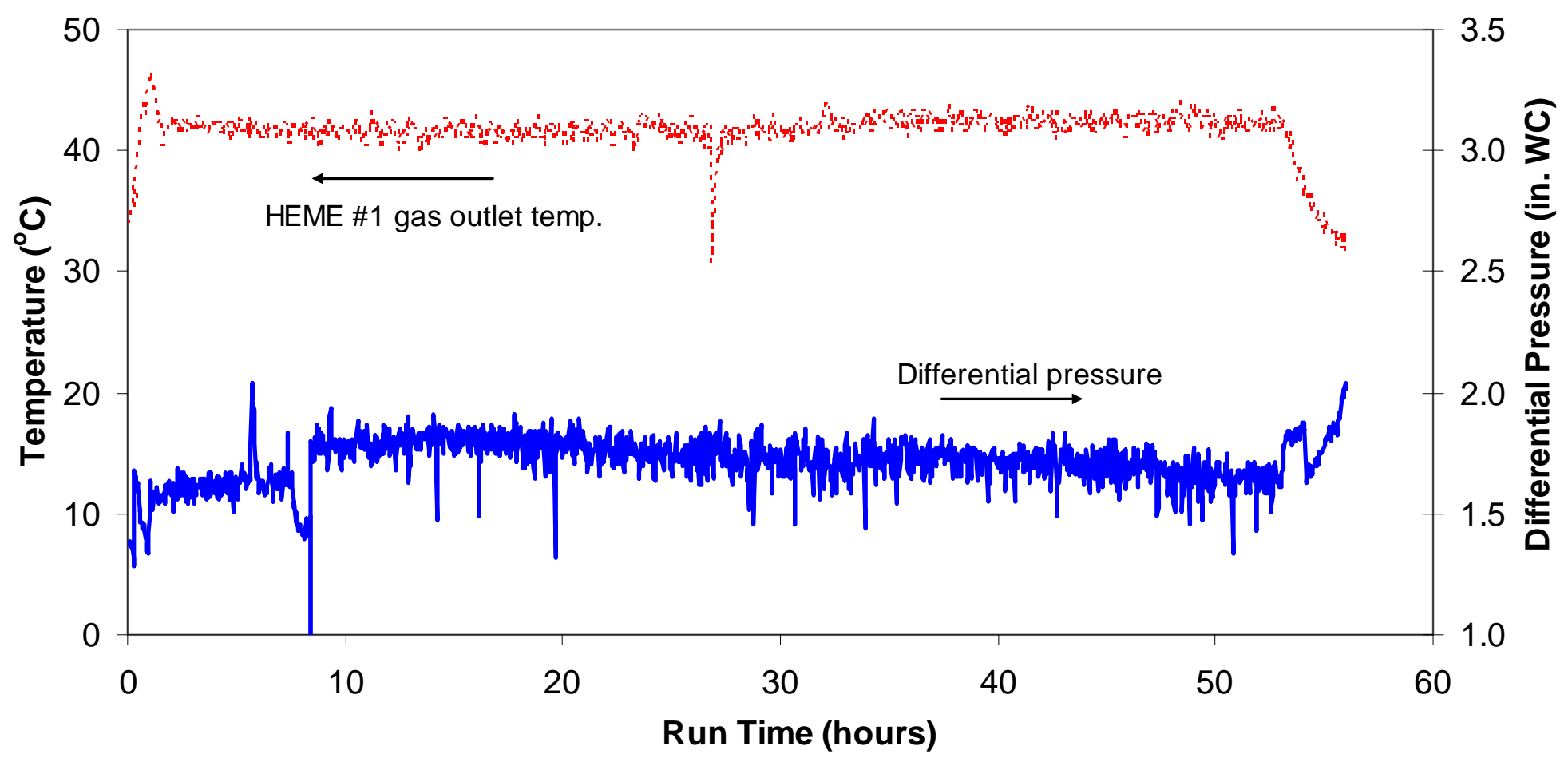

Figure 4.60. Outlet temperature and differential pressure for HEME \#1 while processing with two double-outlet and two single-outlet bubblers, Test 4. 


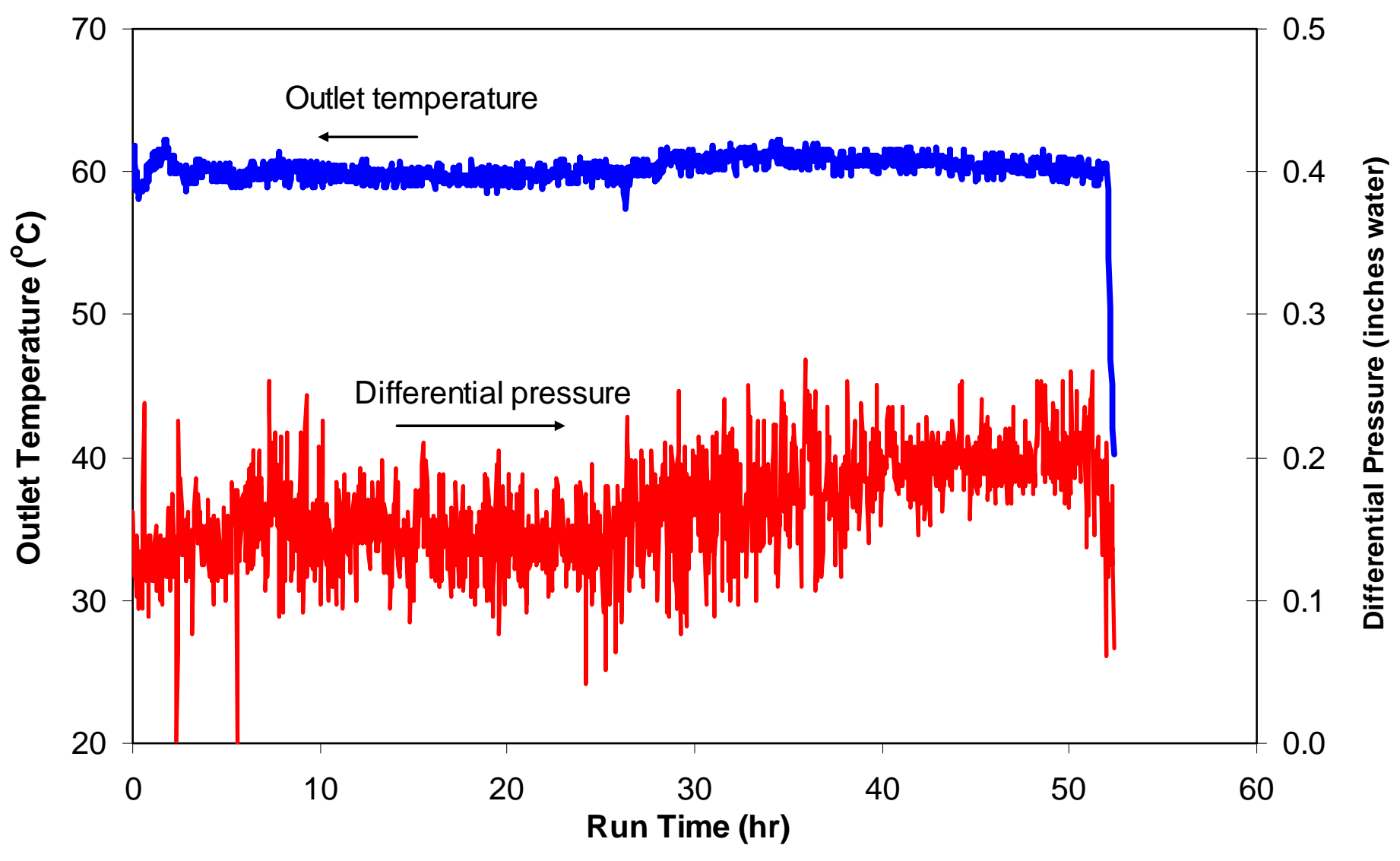

Figure 4.61. Outlet temperature and differential pressure for HEPA \#1 while processing with two double-outlet bubblers, Test 1. 


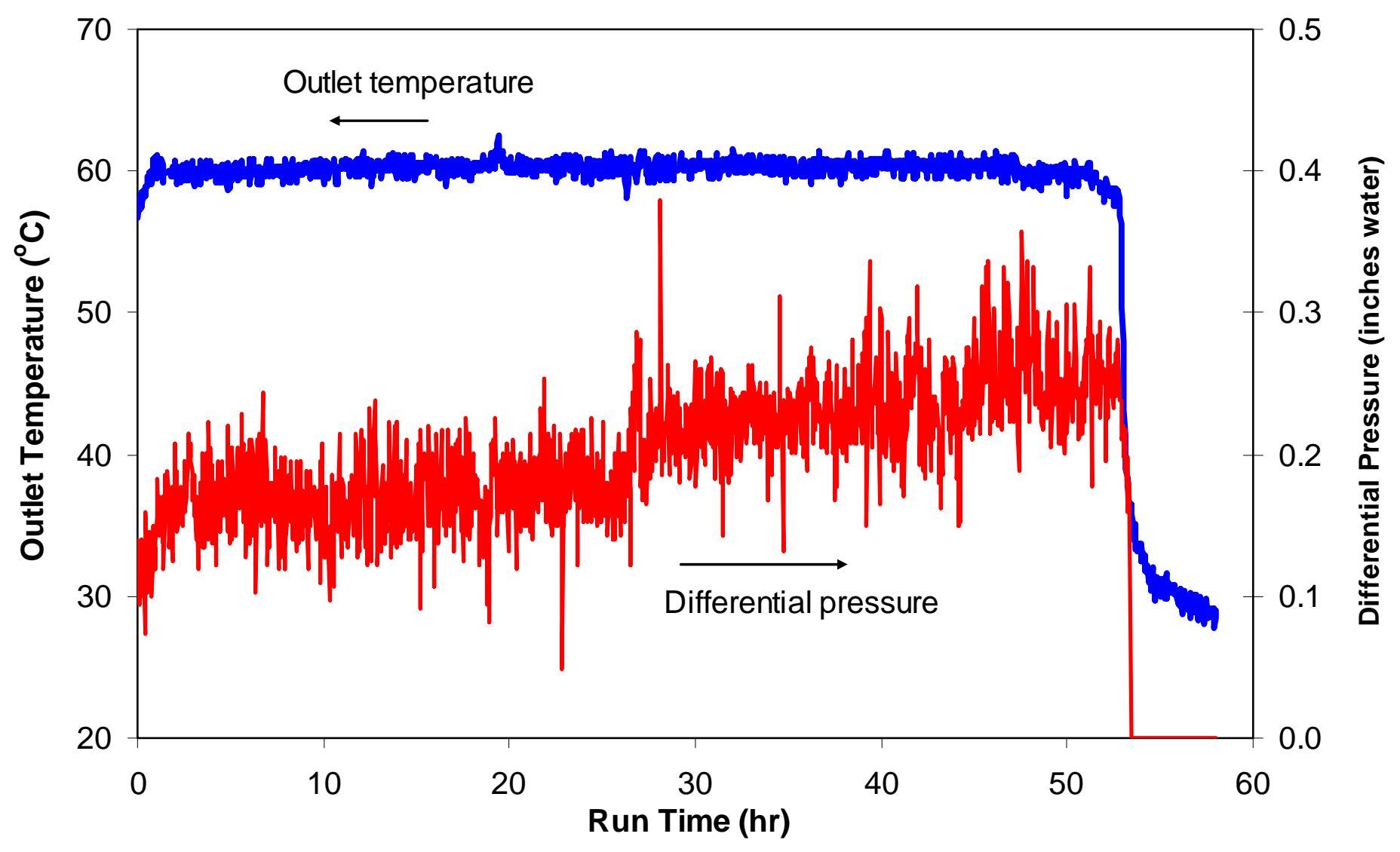

Figure 4.62. Outlet temperature and differential pressure for HEPA \#1 while processing with two double-outlet and one single-outlet bubblers, Test 2. 


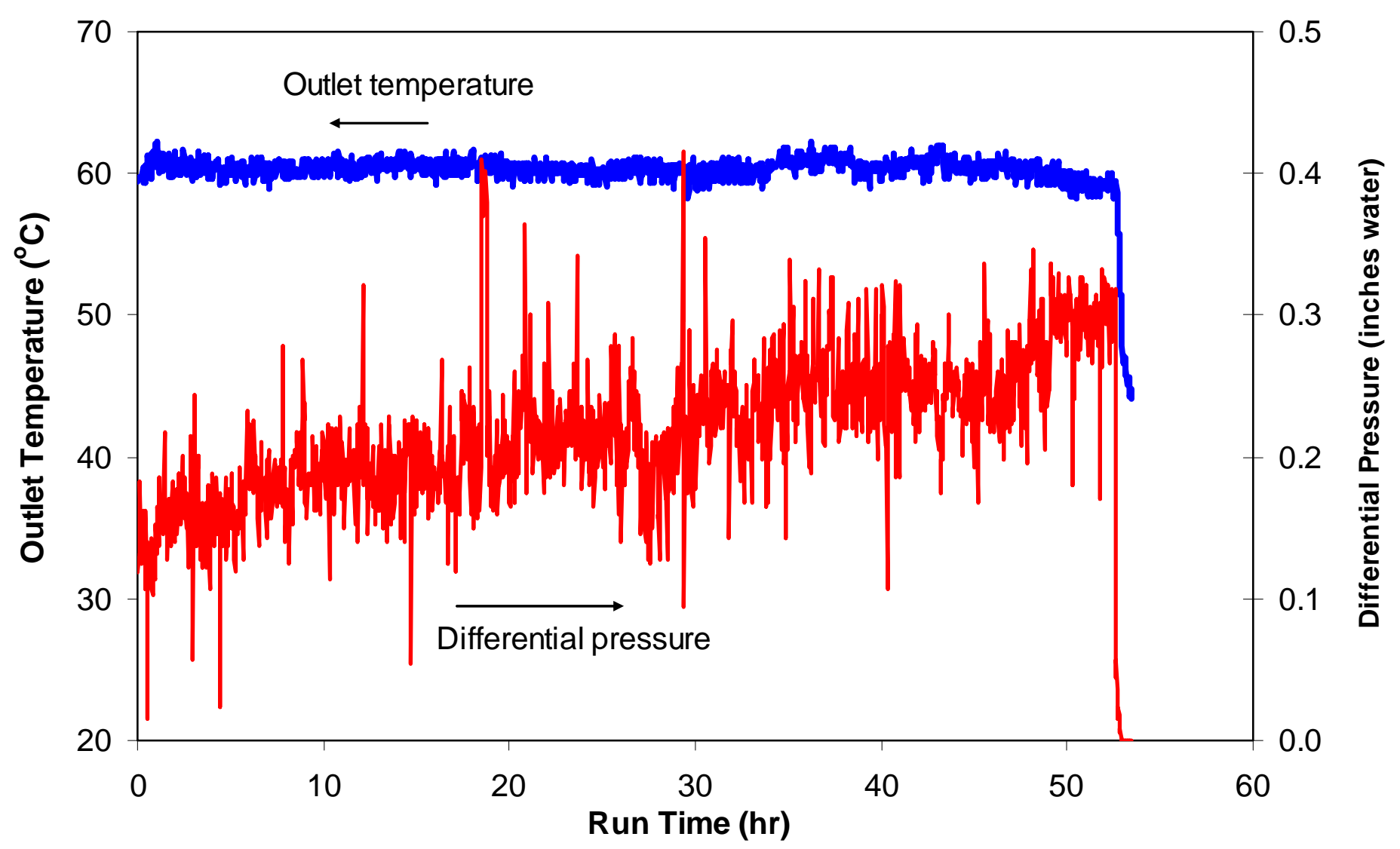

Figure 4.63. Outlet temperature and differential pressure for HEPA \#1 while processing with two double-outlet and two single-outlet bubblers, Test 3. 


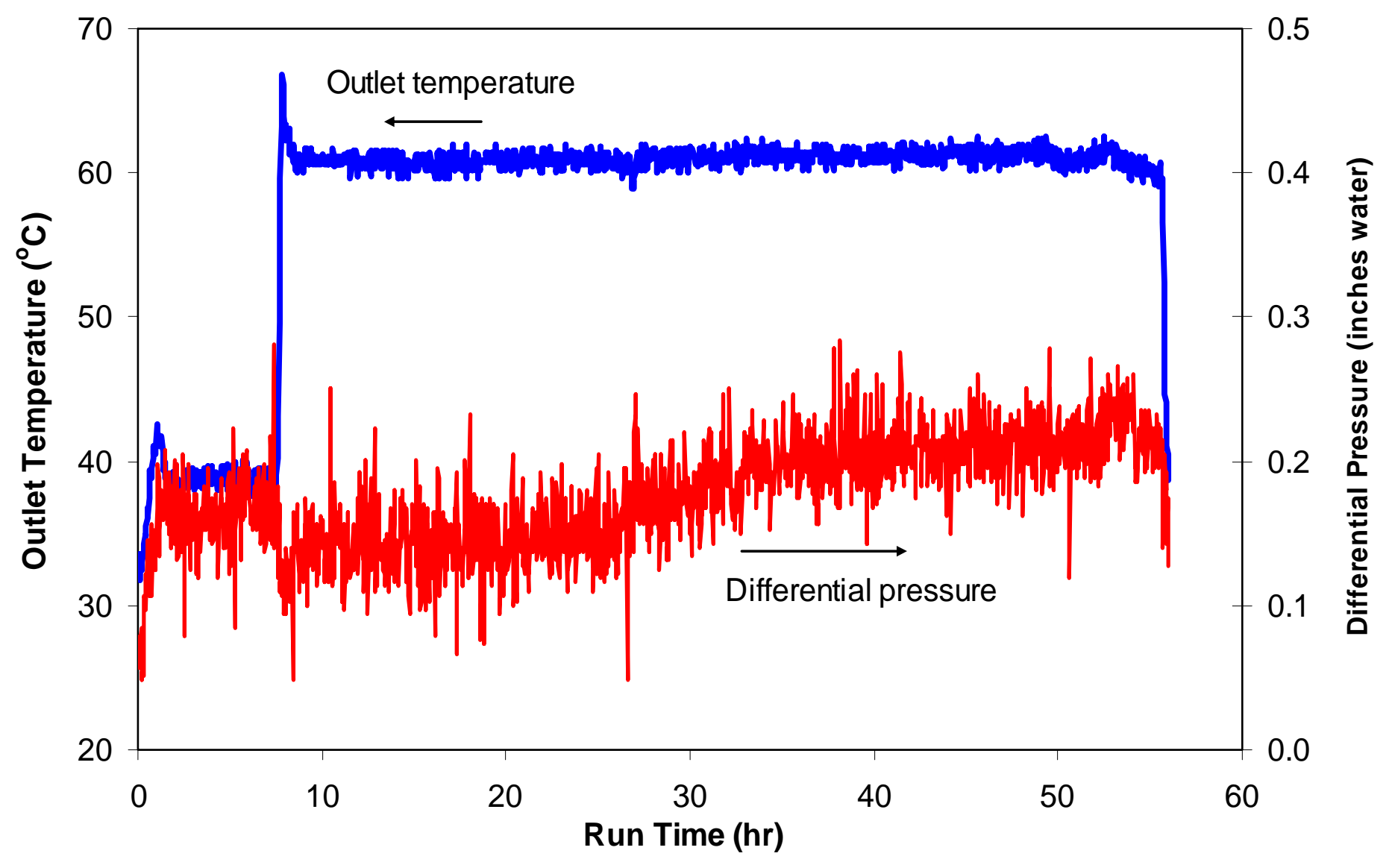

Figure 4.64. Outlet temperature and differential pressure for HEPA \#1 while processing with two double-outlet and two single-outlet bubblers, Test 4. 


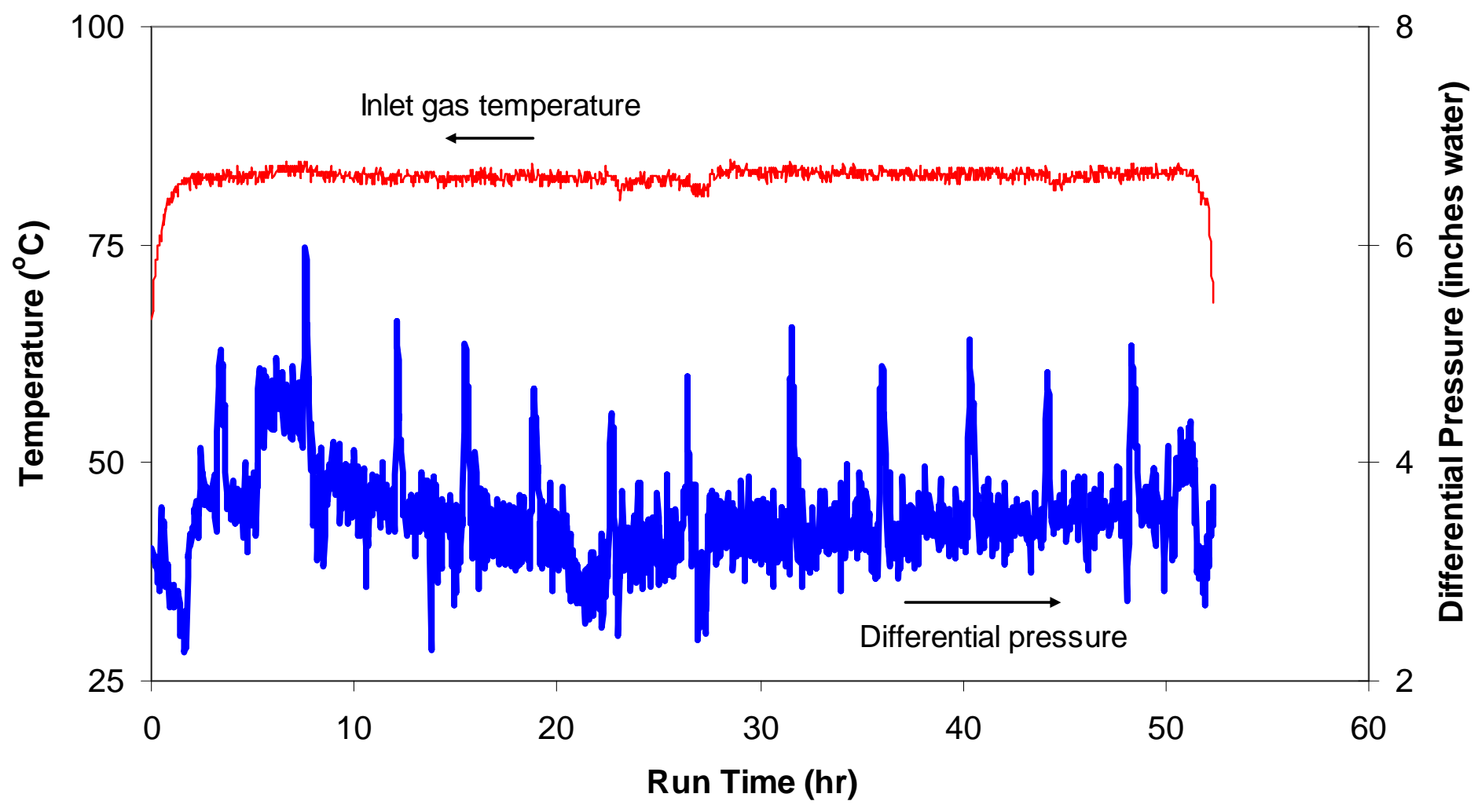

Figure 4.65. Inlet gas temperature and differential pressure for PBS while processing with two double-outlet bubblers, Test 1. 


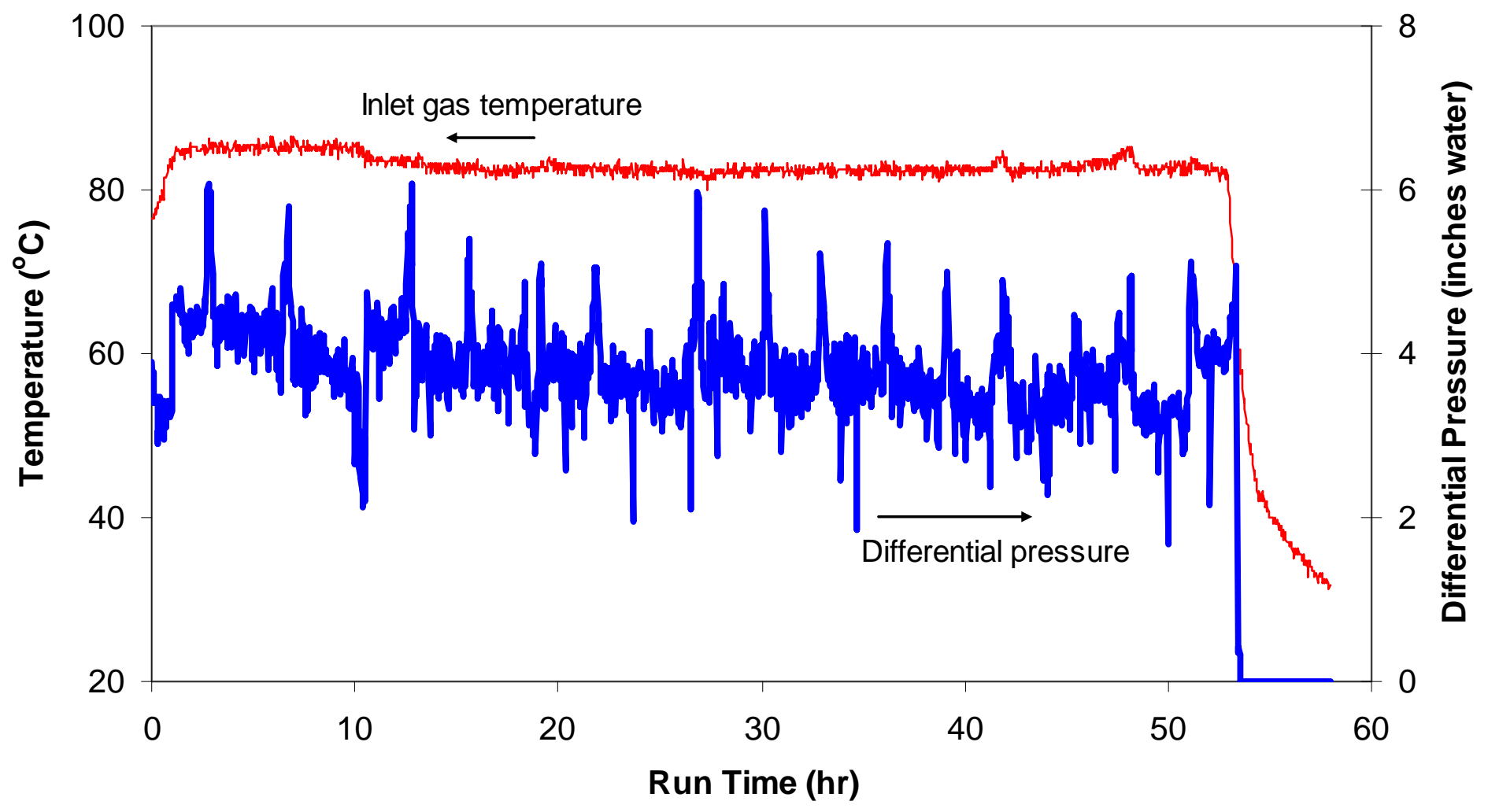

Figure 4.66. Inlet gas temperature and differential pressure for PBS for while processing with two double-outlet and one single-outlet bubblers, Test 2. 


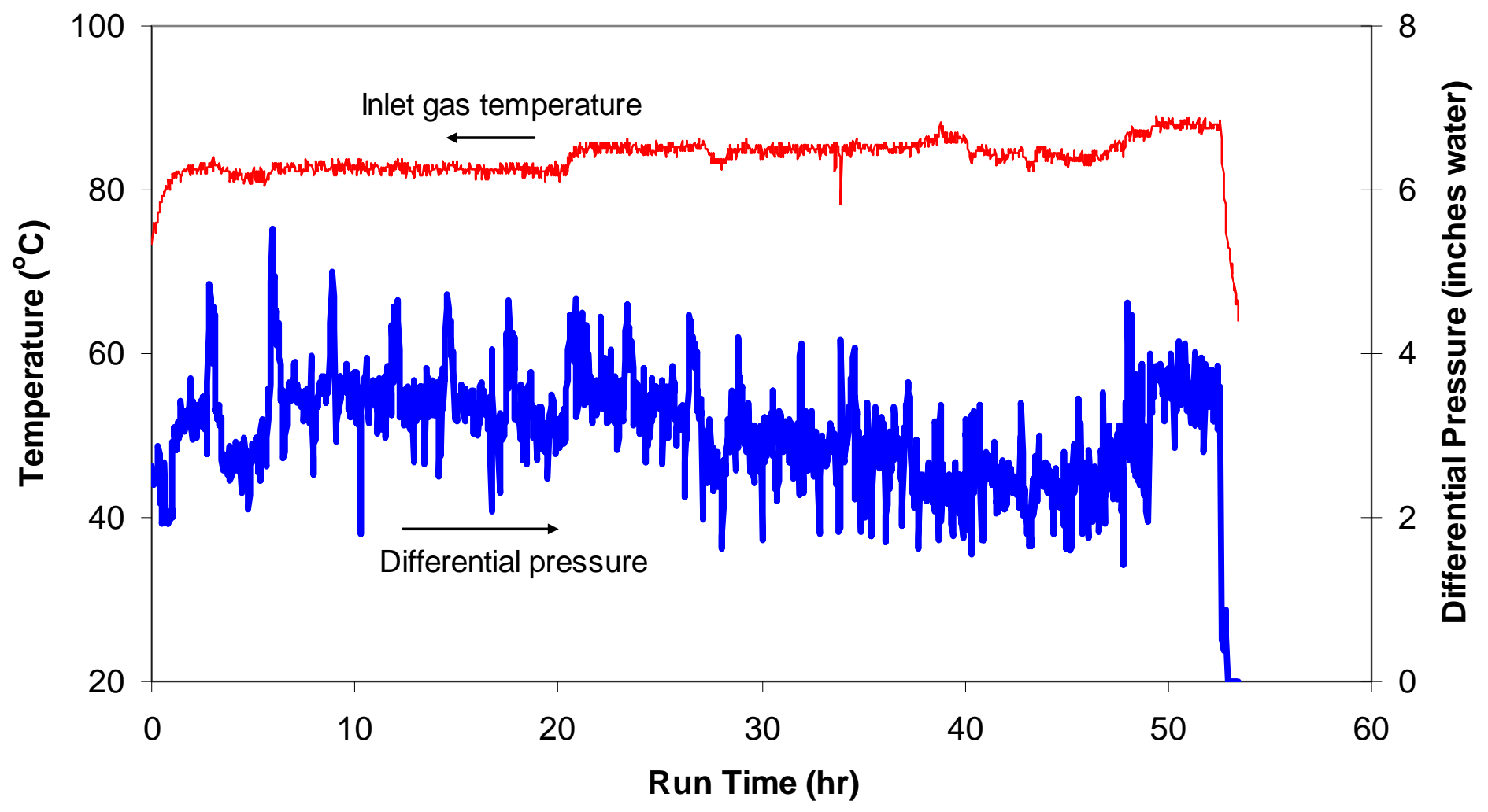

Figure 4.67. Inlet gas temperature and differential pressure for PBS while processing with two double-outlet and two single-outlet bubblers, Test 3. 


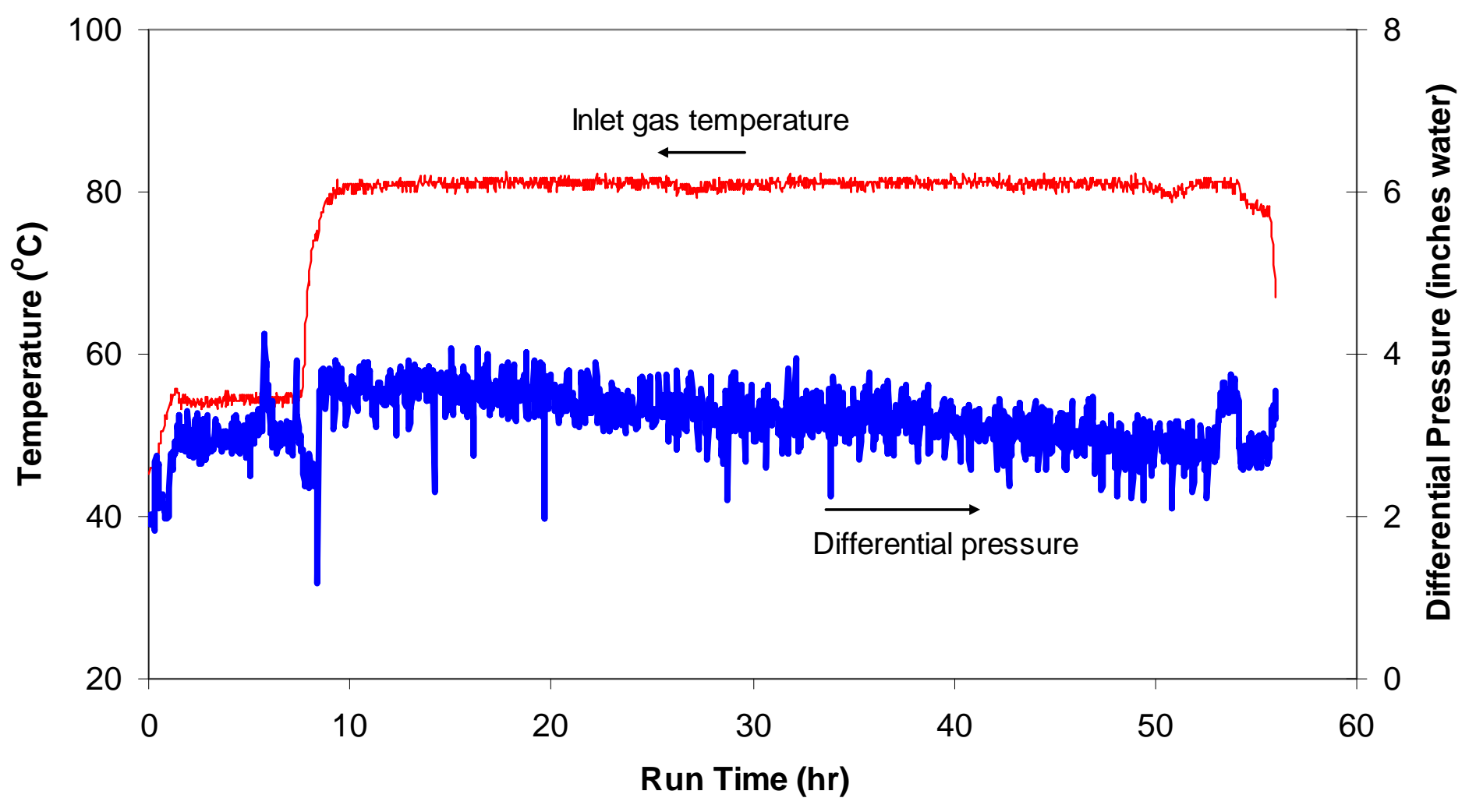

Figure 4.68. Inlet gas temperature and differential pressure for PBS while processing with two double-outlet and two single-outlet bubblers, Test 4. 


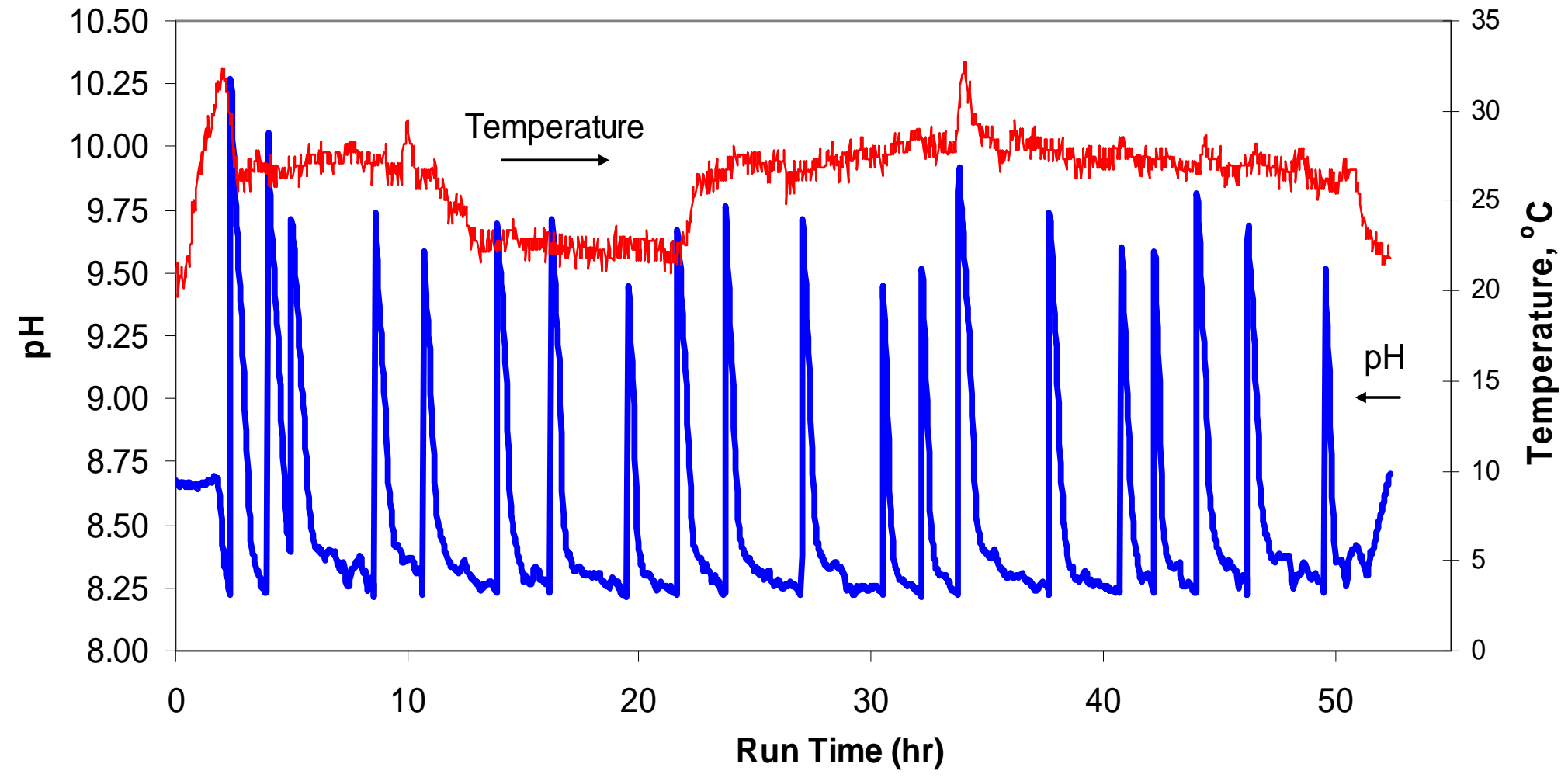

Figure 4.69. pH for PBS while processing with two double-outlet bubblers, Test 1. 


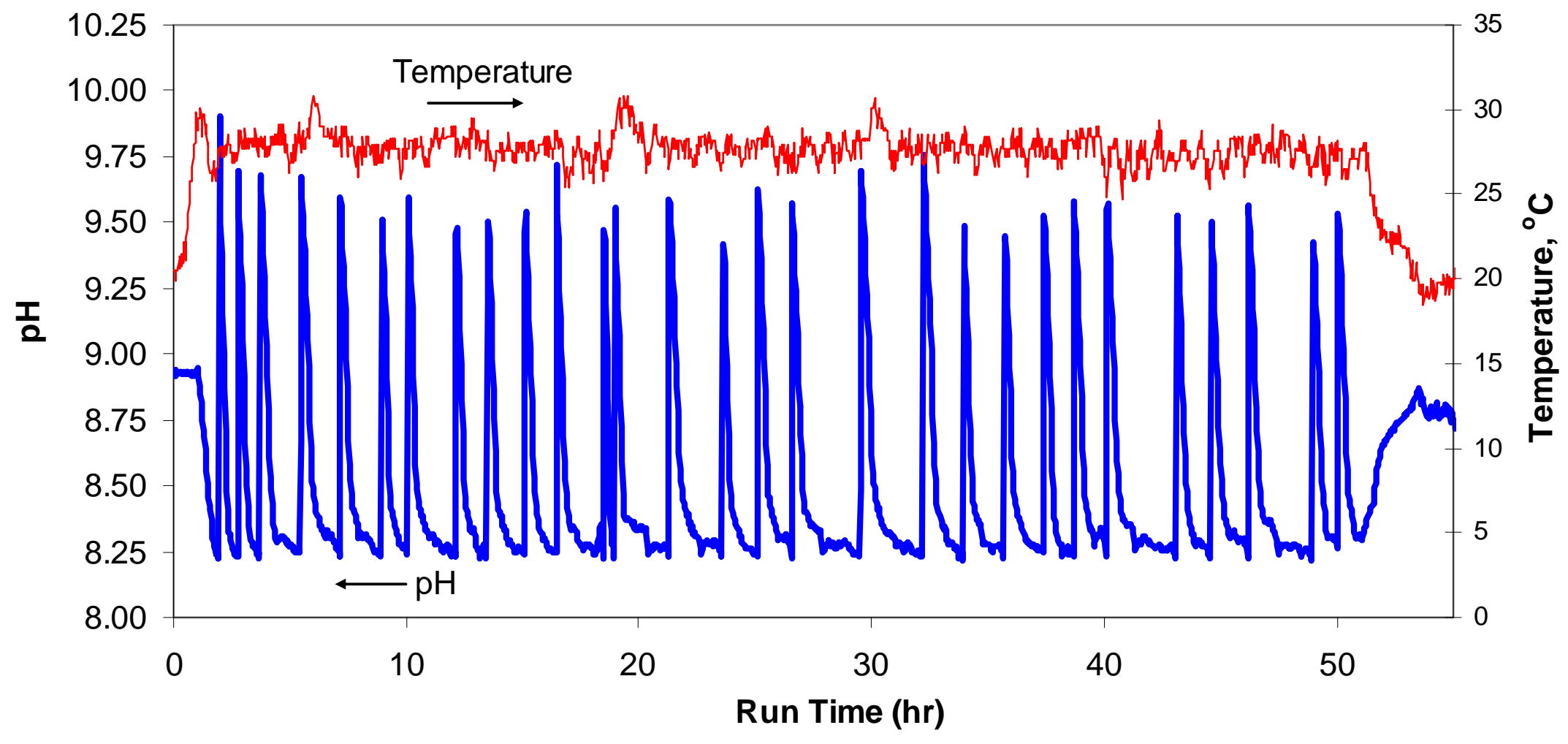

Figure 4.70. pH for PBS while processing with two double-outlet and one single-outlet bubblers, Test 2. 


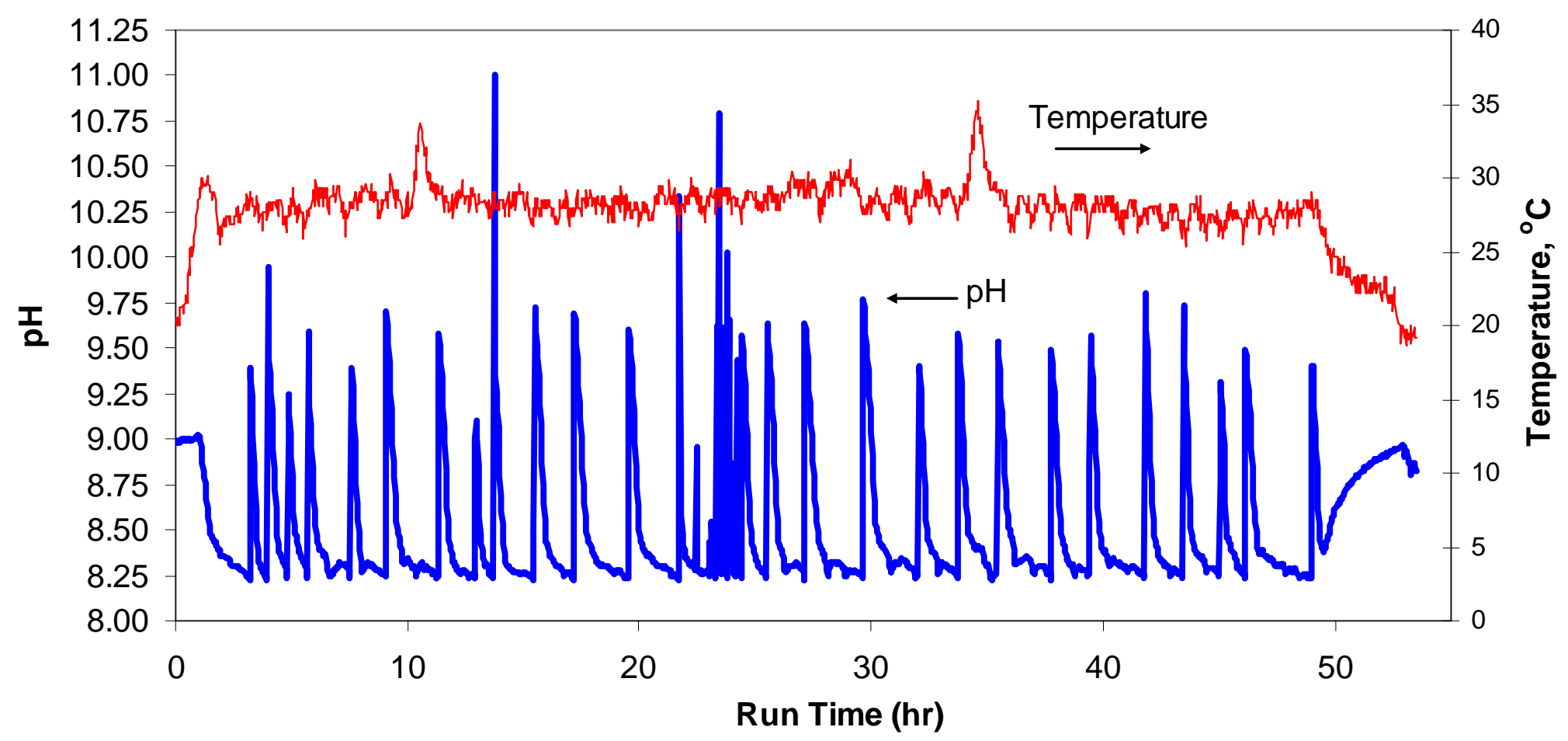

Figure 4.71. pH for PBS while processing with two double-outlet and two single-outlet bubblers, Test 3. 


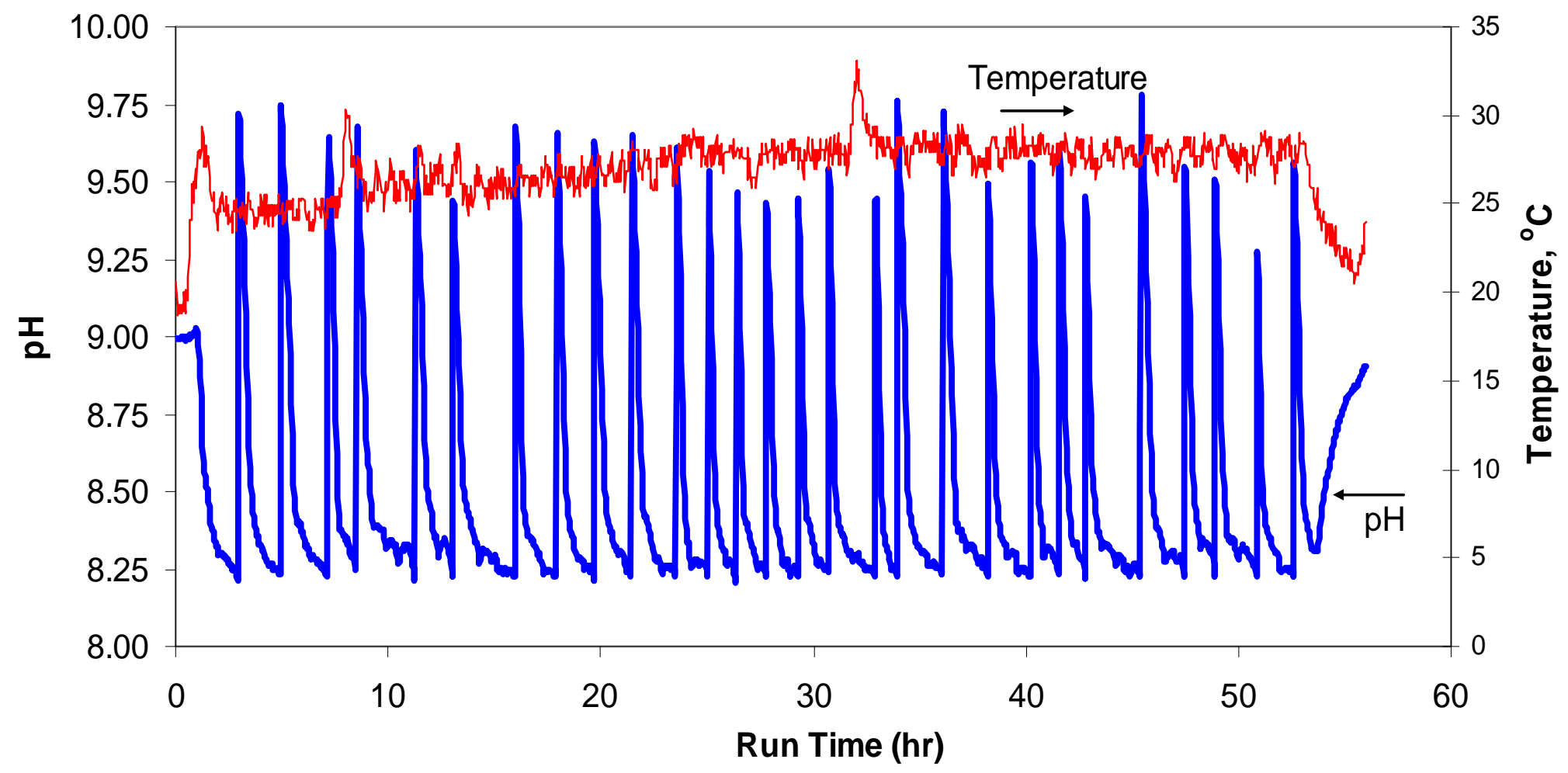

Figure 4.72. pH for PBS while processing with two double-outlet and two single-outlet bubblers, Test 4. 


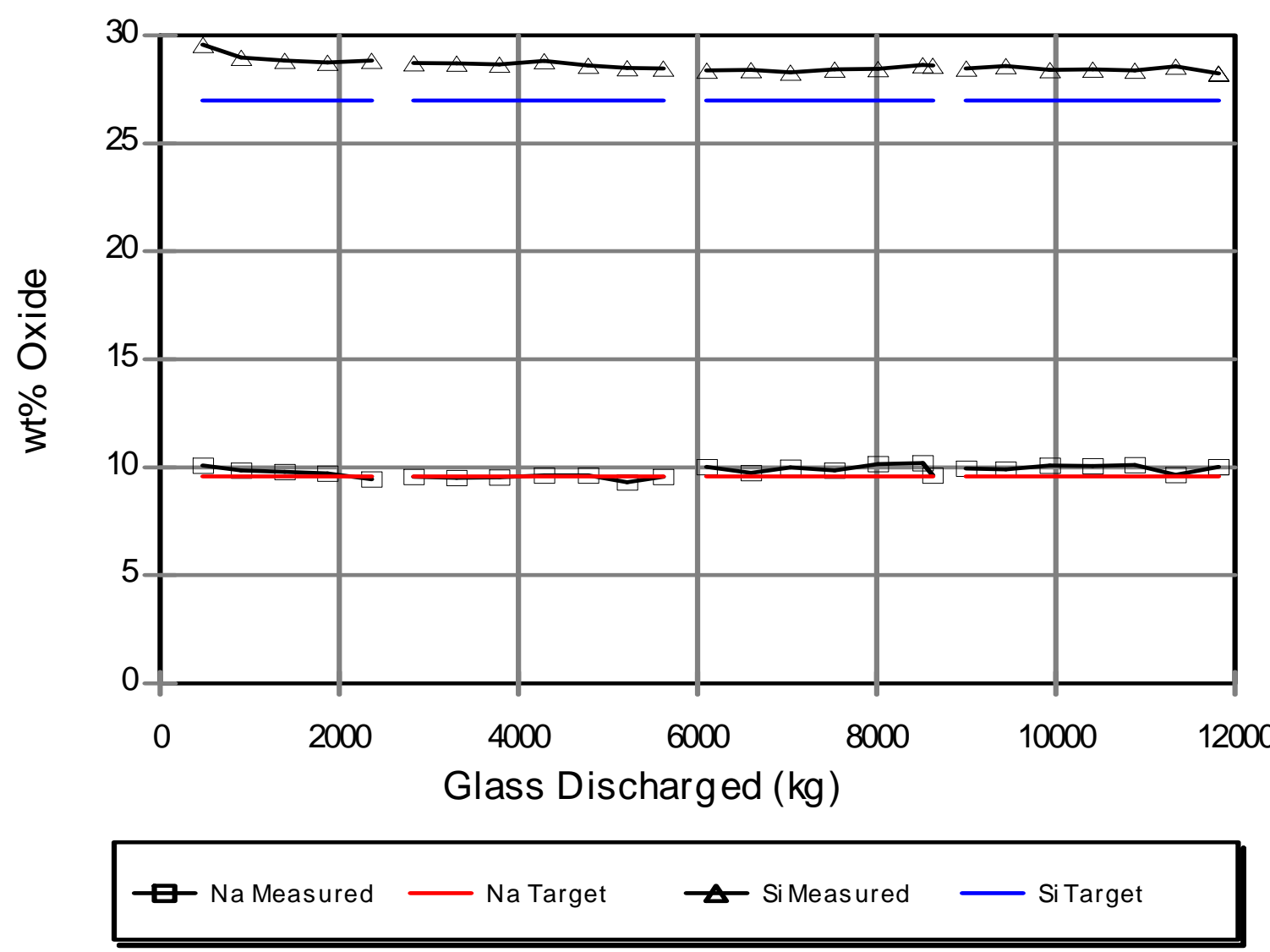

Figure 5.1.a. DM1200 product and target glass compositions determined by XRF. 

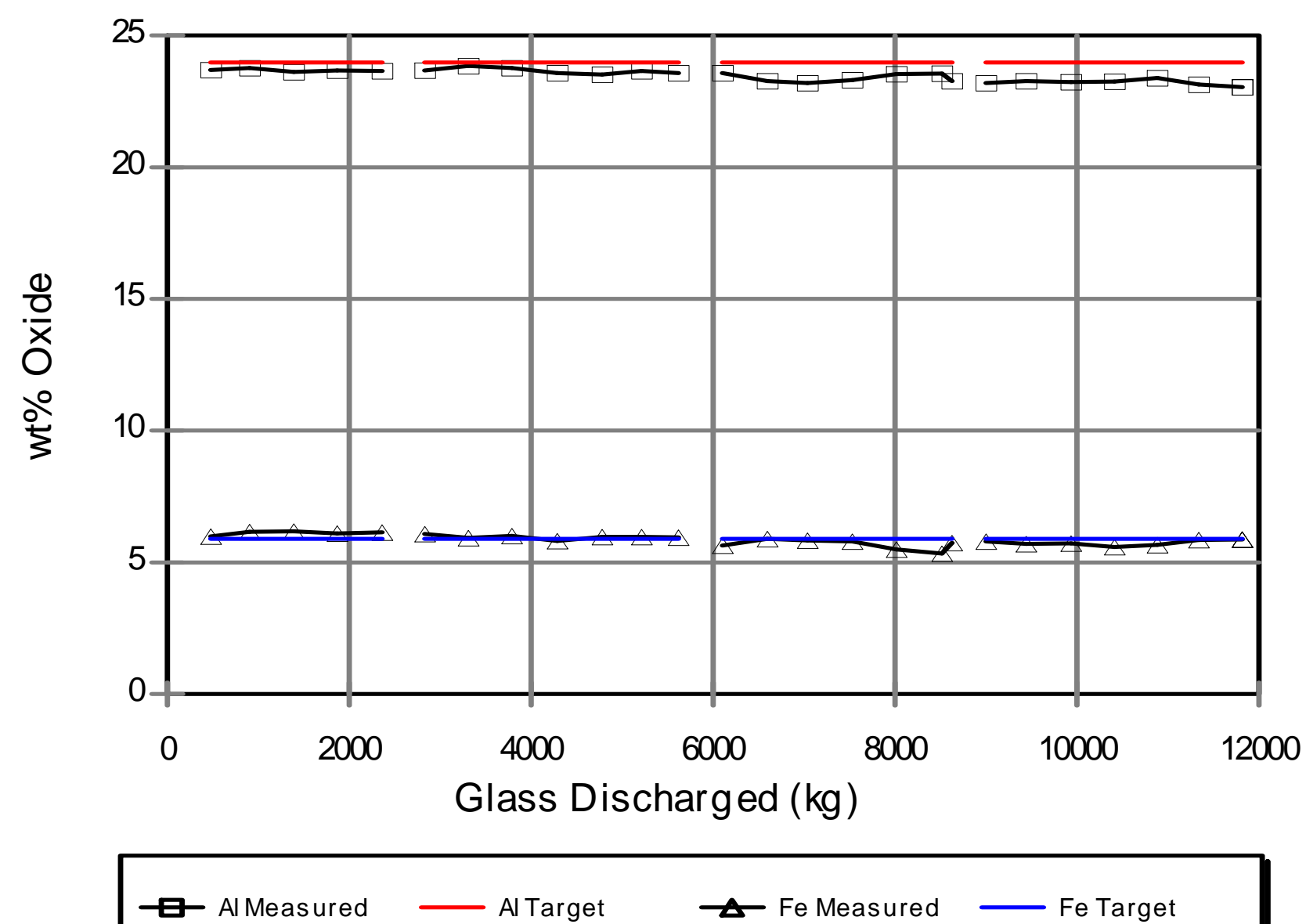

Figure 5.1.b. DM1200 product and target glass compositions determined by XRF. 


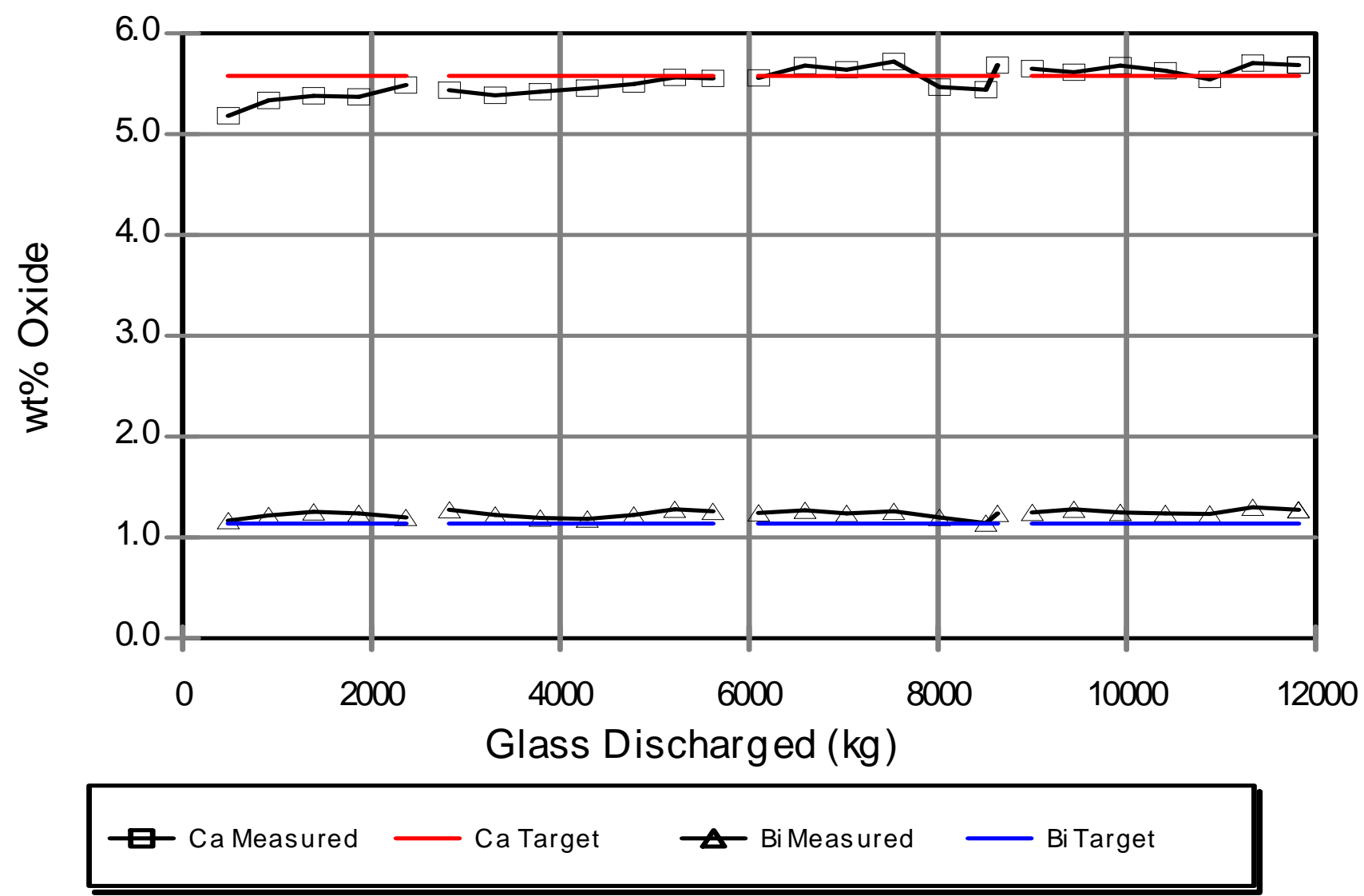

Figure 5.1.c. DM1200 product and target glass compositions determined by XRF. 

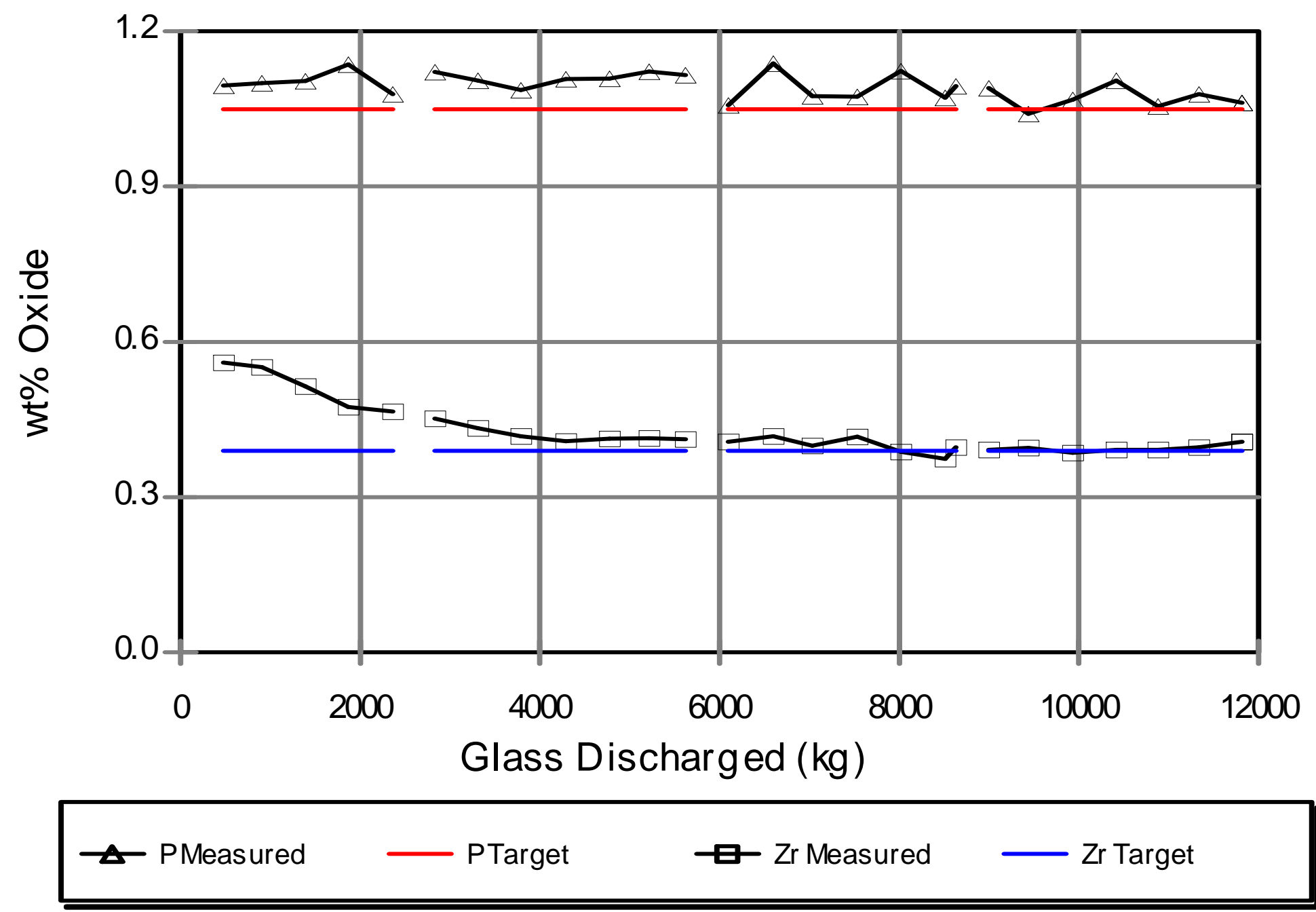

Figure 5.1.d. DM1200 product and target glass compositions determined by XRF. 

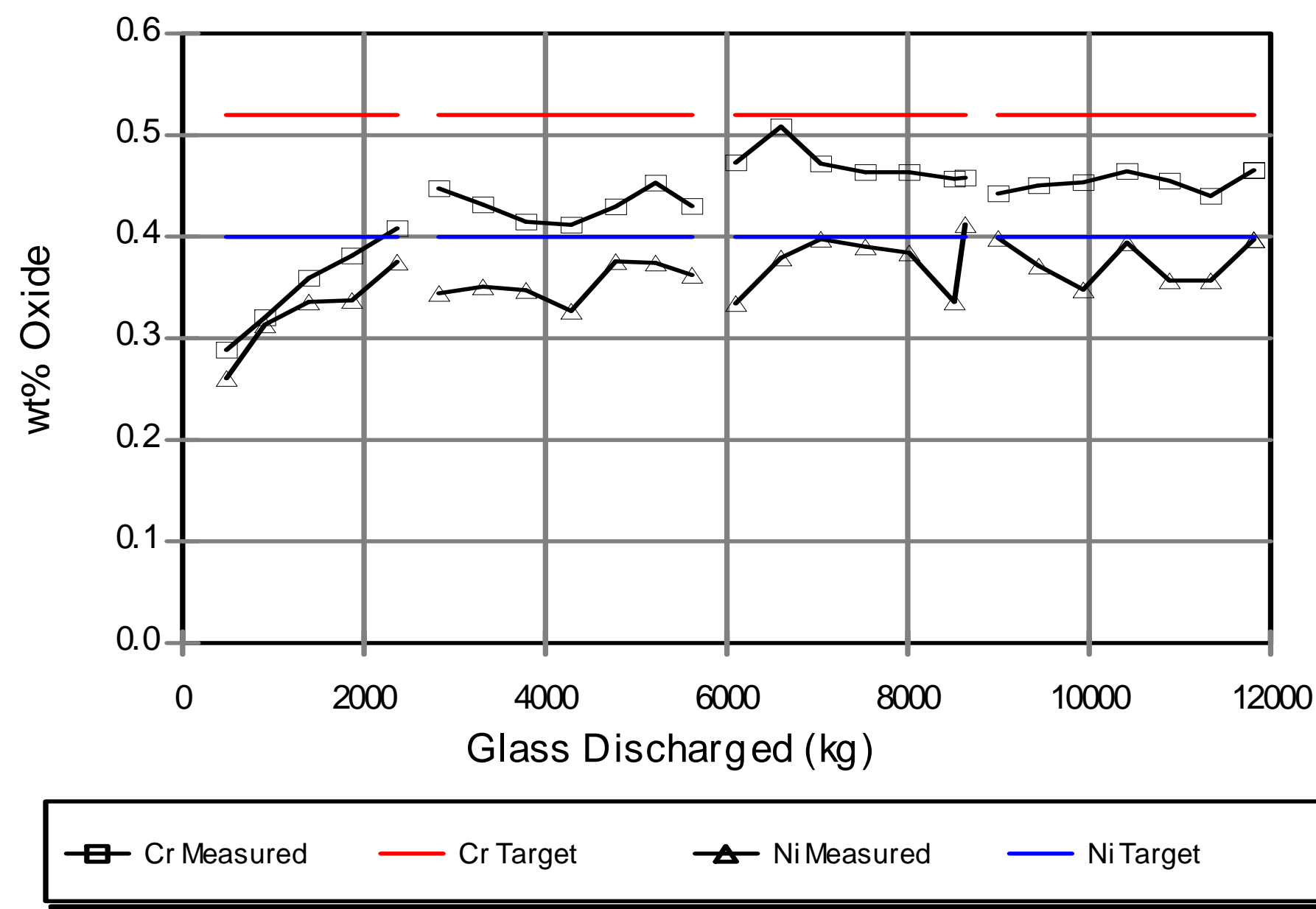

Figure 5.1.e. DM1200 product and target glass compositions determined by XRF. 


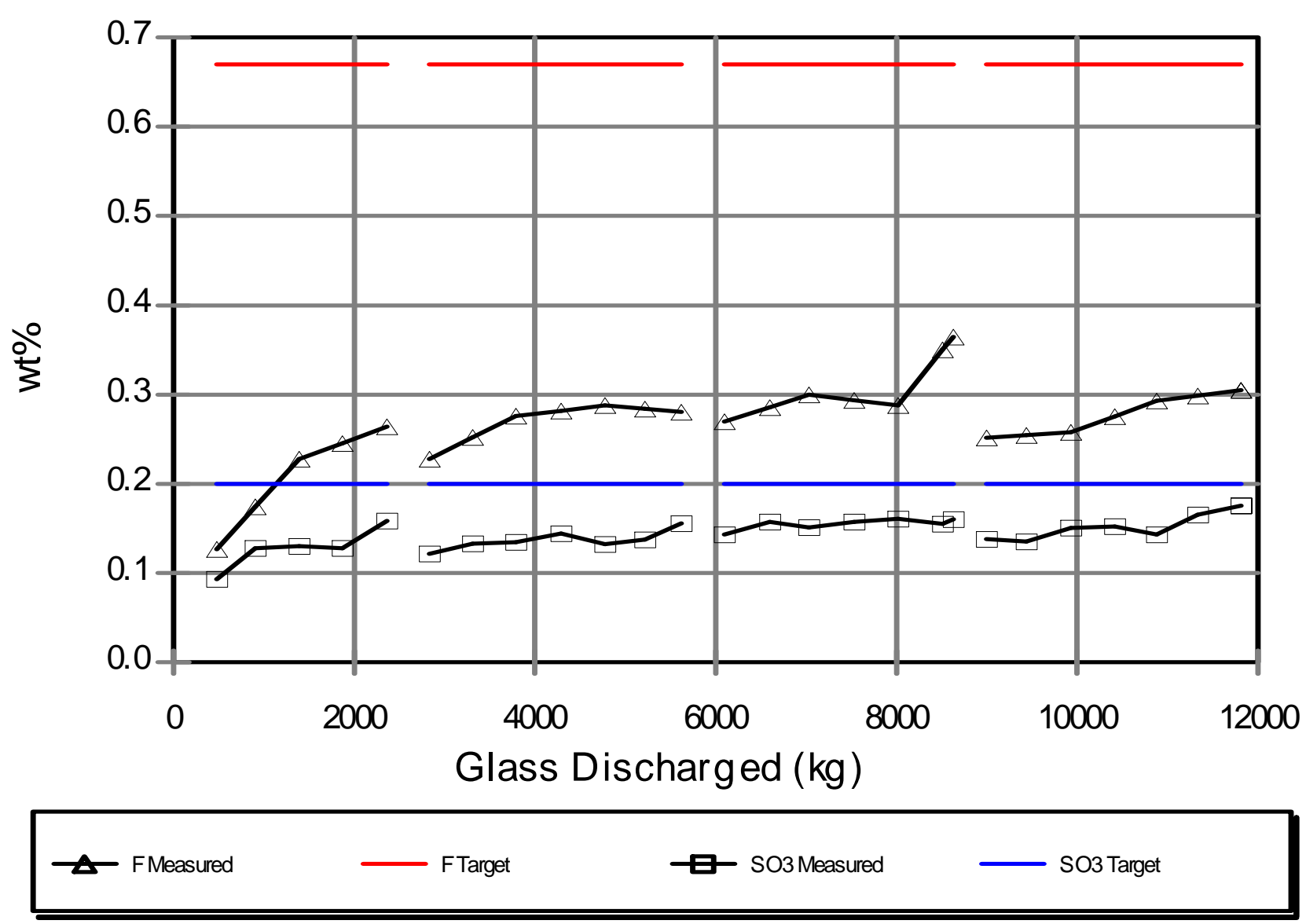

Figure 5.1.f. DM1200 product and target glass compositions determined by XRF. 


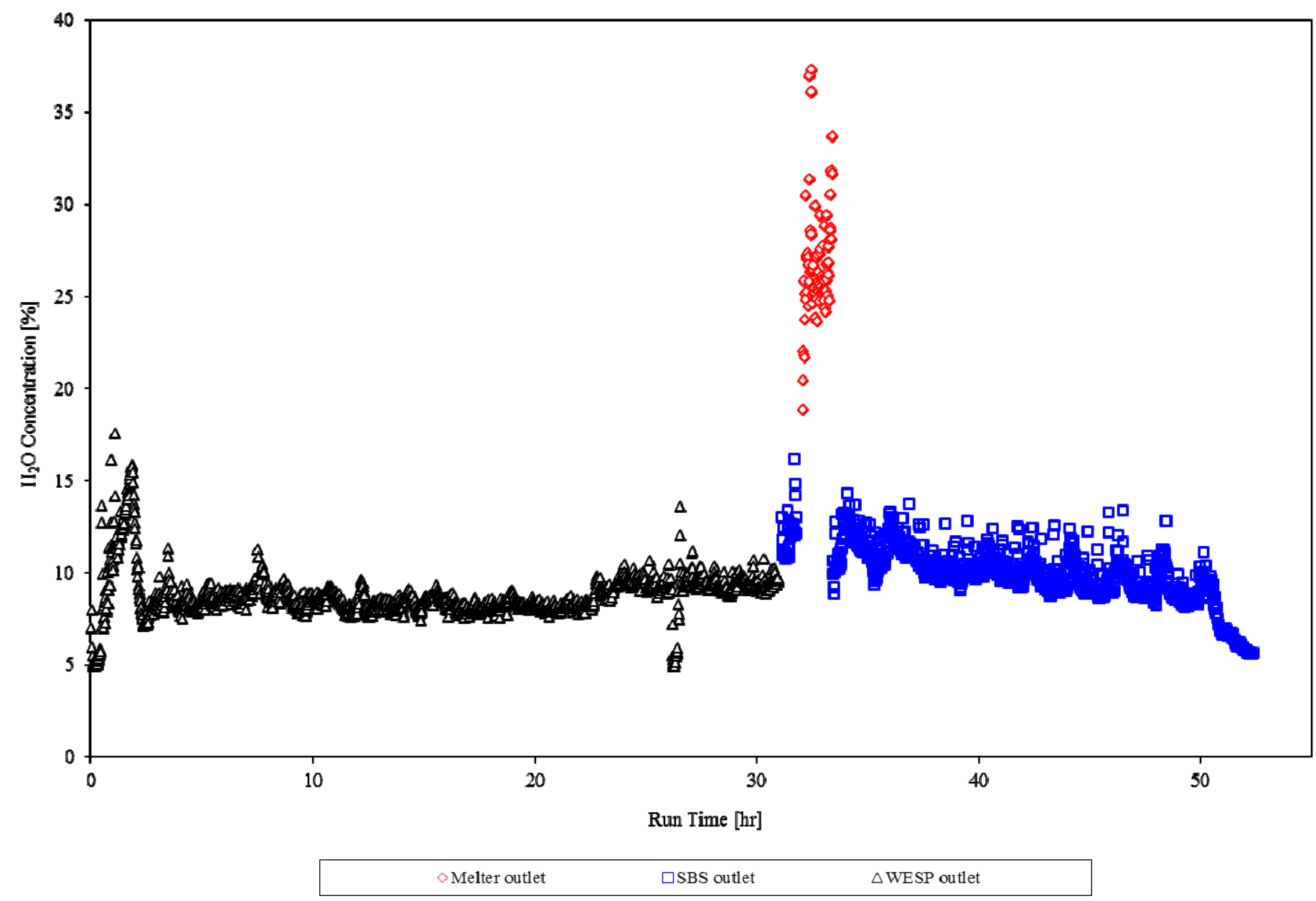

Figure 6.1.a. FTIR Monitored water emissions during Test 1. 


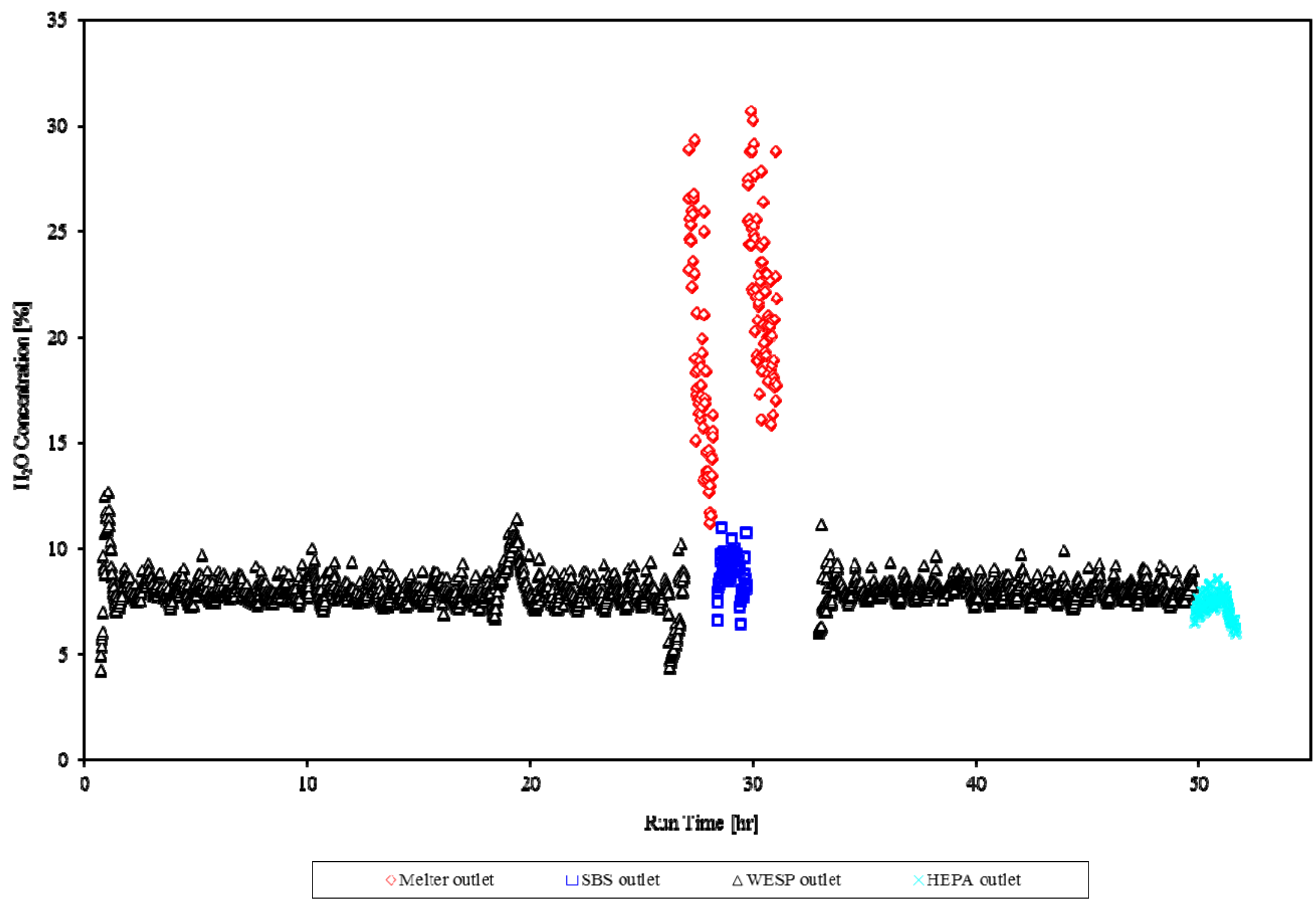

Figure 6.1.b. FTIR Monitored water emissions during Test 2. 


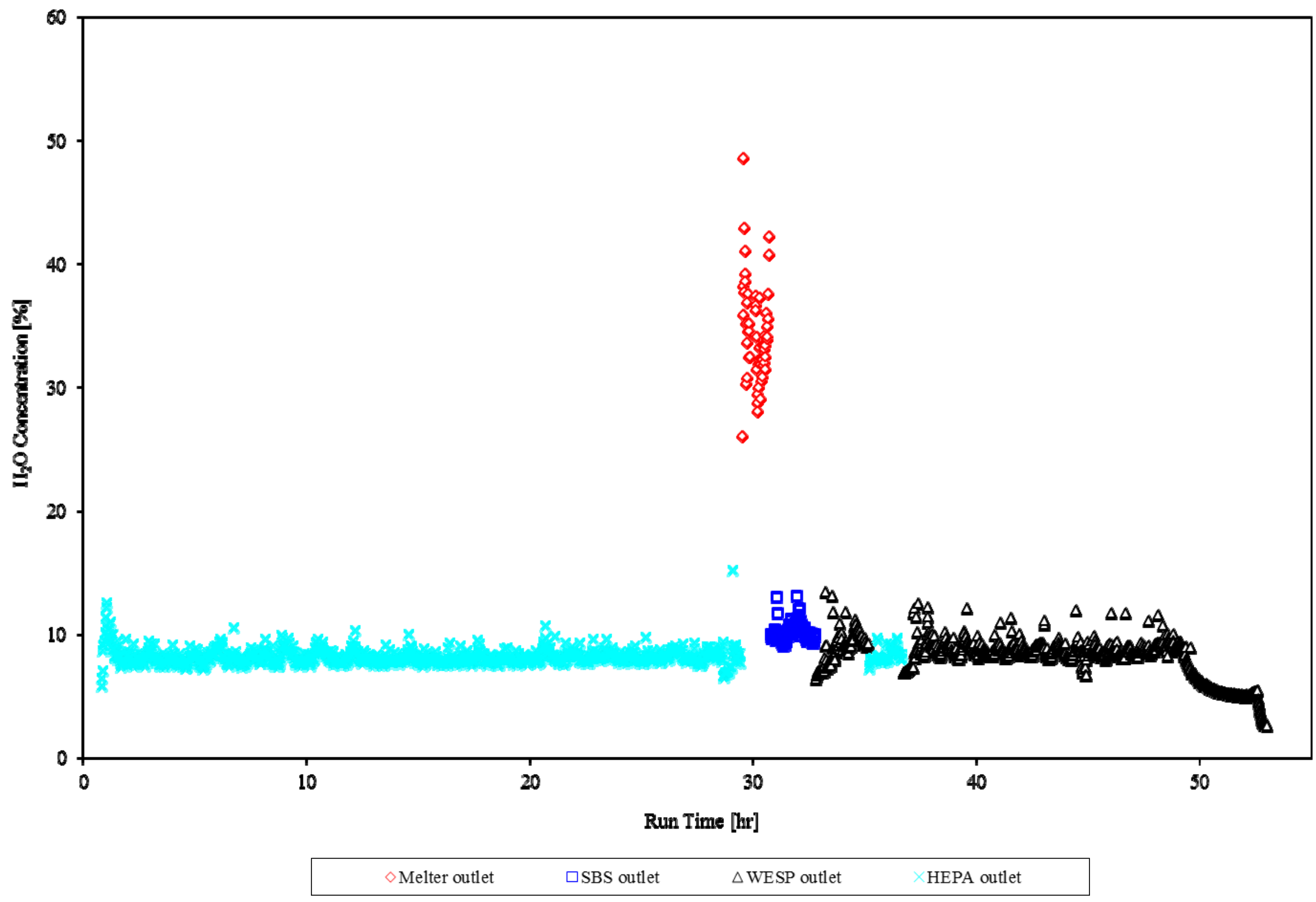

Figure 6.1.c. FTIR Monitored water emissions during Test 3. 


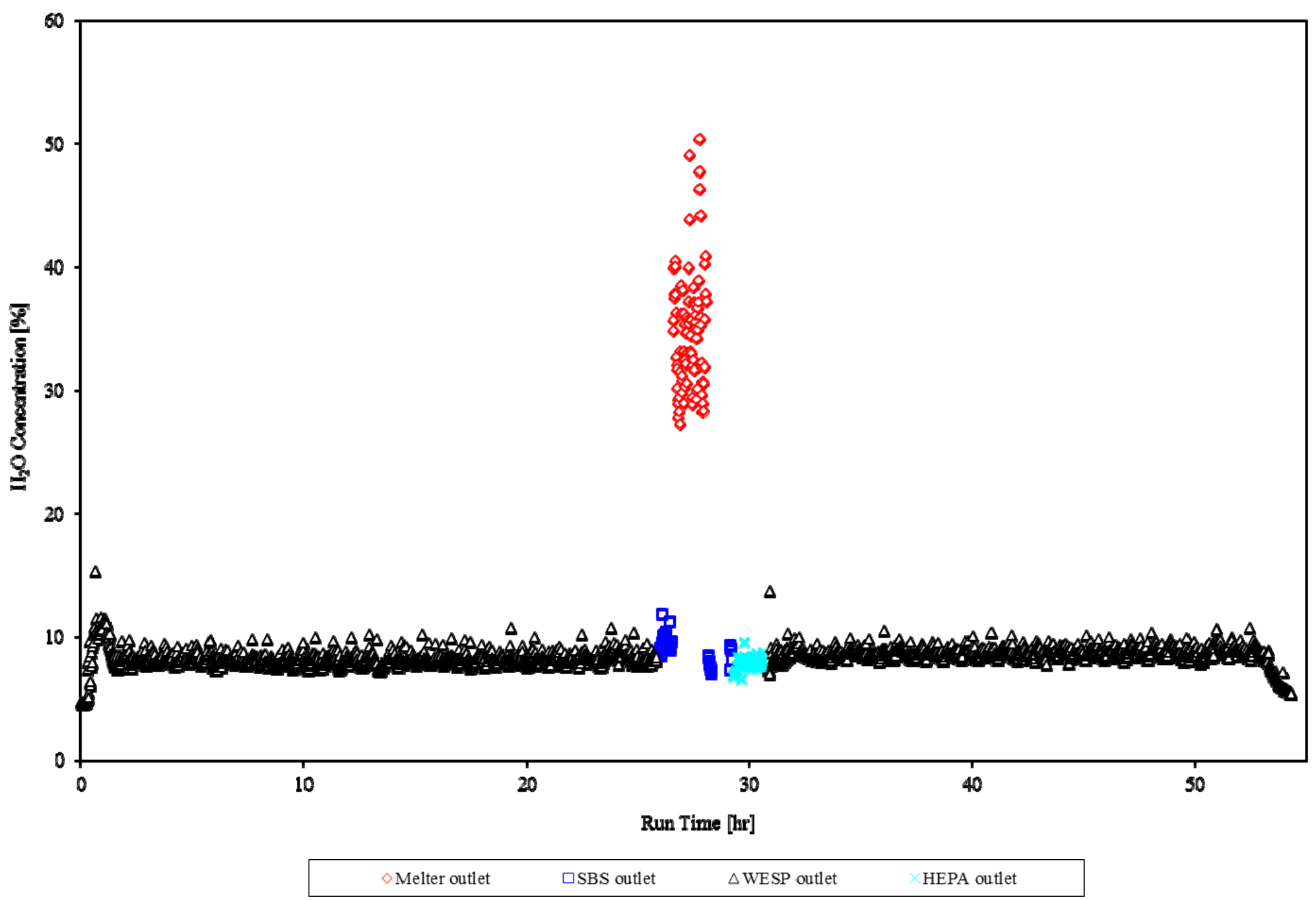

Figure 6.1.d FTIR Monitored water emissions during Test 4. 


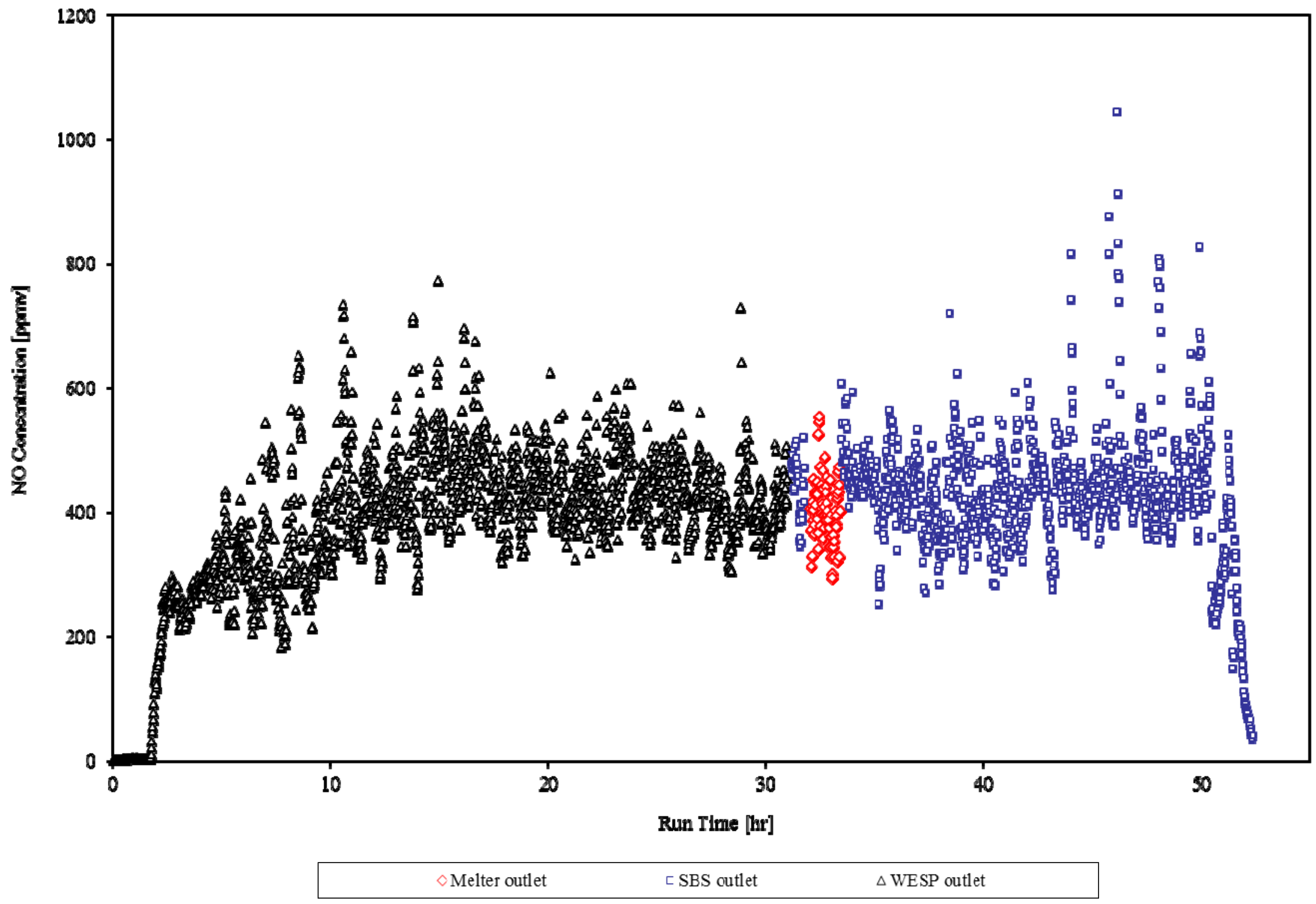

Figure 6.2.a. FTIR Monitored NO emissions during Test 1. 


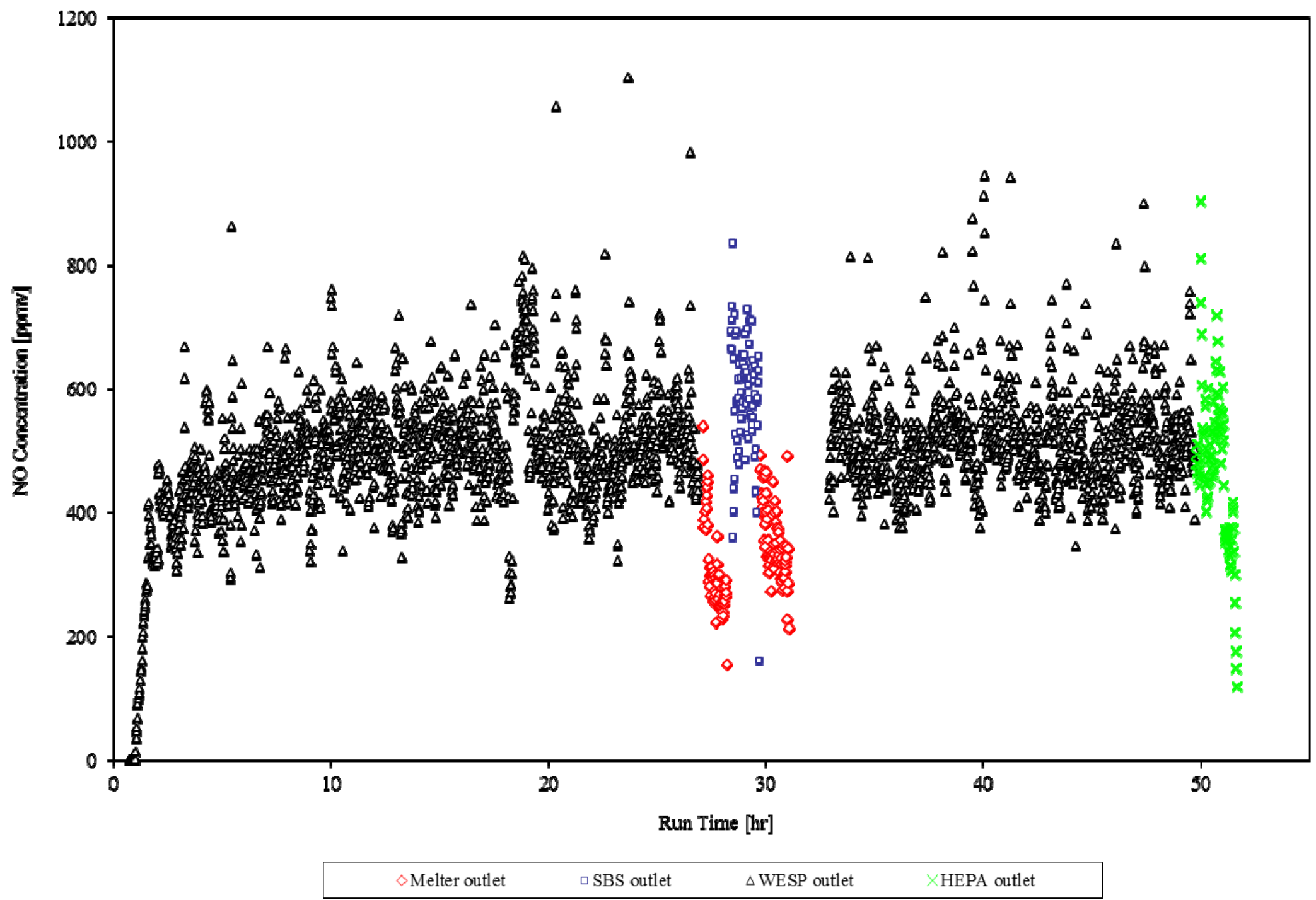

Figure 6.2.b. FTIR Monitored NO emissions during Test 2. 


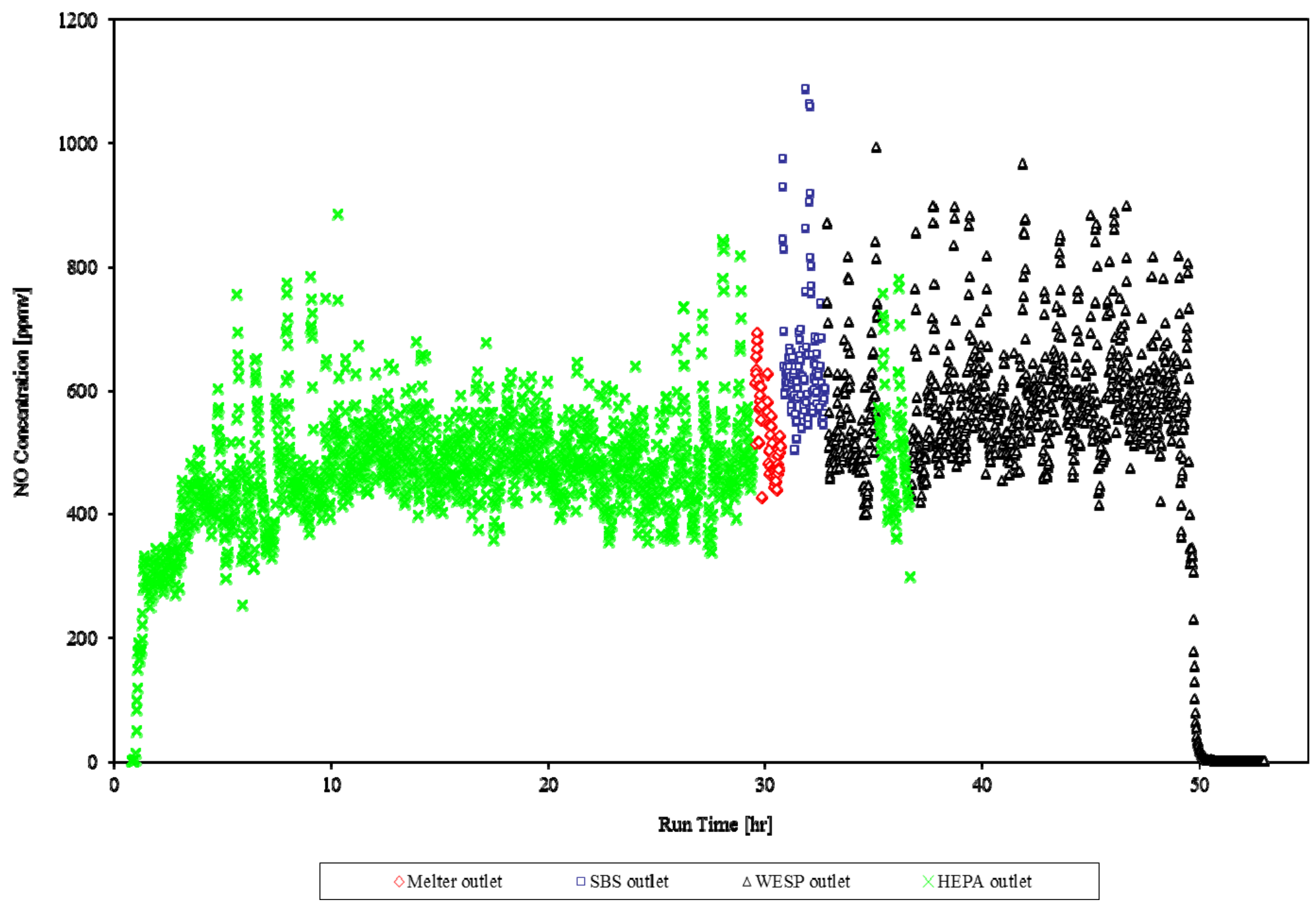

Figure 6.2.c. FTIR Monitored NO emissions during DM1200 Test 3. 


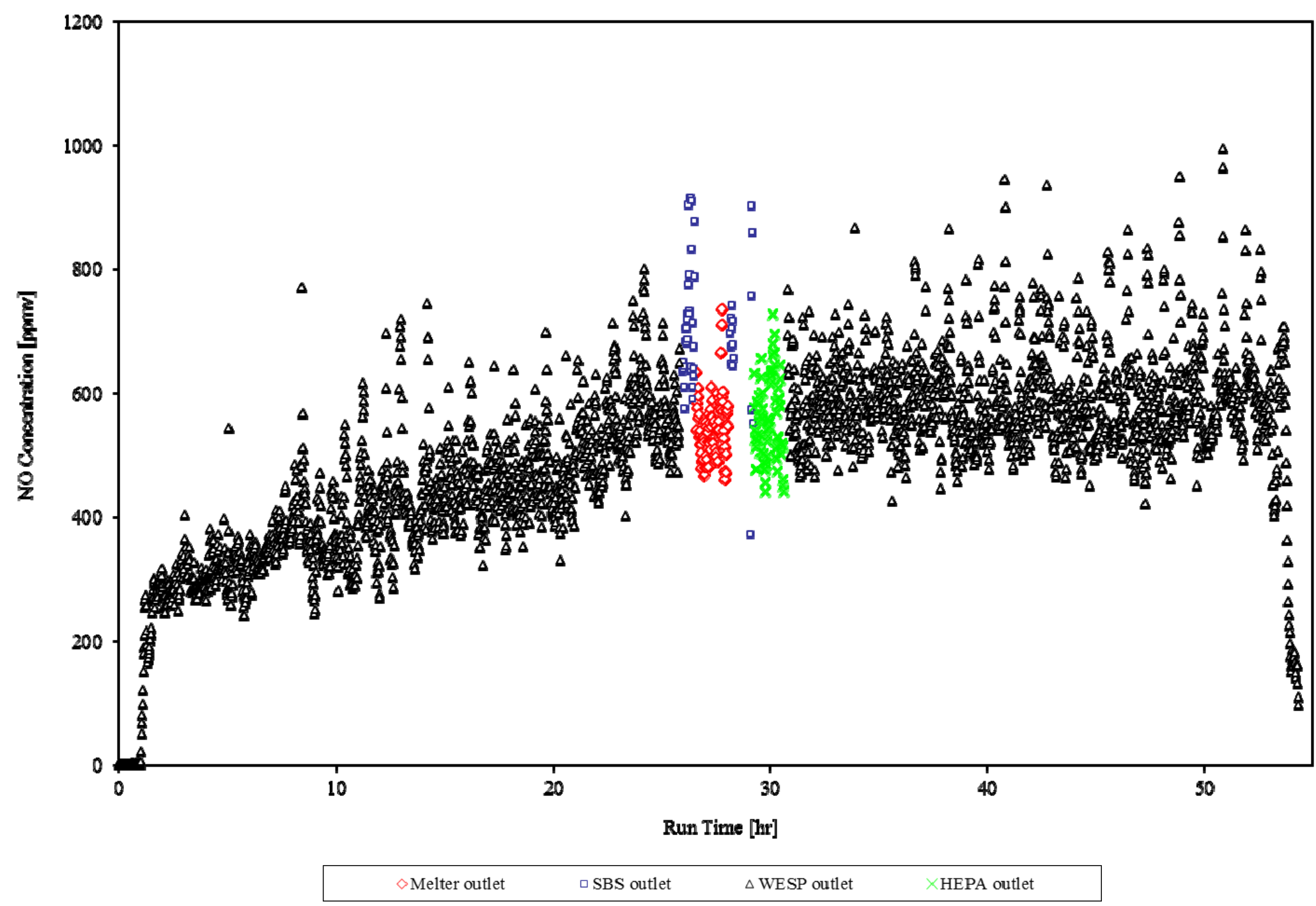

Figure 6.2.d. FTIR Monitored NO emissions during DM1200 Test 4. 